\title{
THE SYNTHESIS AND BIOLOGICAL EVALUATION OF GLYCOSPHINGOLIPIDS FOR IMPROVED CANCER IMMUNOTHERAPY
}

\author{
By \\ Janice Mei Hsia Cheng
}

\begin{abstract}
A thesis
submitted to the Victoria University of Wellington in fulfilment of the requirements for the degree of Doctor of Philosophy
\end{abstract}

Victoria University of Wellington

2014 


\section{Abstract}

The immune system plays a crucial role in providing the first line of defence against invading pathogens such as bacteria, viruses and parasites. It is activated when immune cells known as dendritic cells (DCs) detect specific molecules that are foreign to the host, and present them to $\mathrm{T}$ cells. This in turn causes the activation of $\mathrm{T}$ cells, which marks the start of an immune response leading to the clearance of the invader. The pathogen-derived molecules recognised by the immune cells are typically peptides and their role as activators of the immune system is well established. While T cells were originally thought to only recognise peptide antigens, it is now evident that $\mathrm{T}$ cells are also able to recognise nonpeptide antigens. Recognition of non-peptide antigens confers protection against pathogens that have cell surfaces that are highly functionalised with carbohydrate moieties, such as glycolipids, glycopeptides and polysaccharides.

Specifically, glycosphingolipids (GSLs) can activate invariant Natural Killer T (iNKT) cells via their $\mathrm{T}$ cell receptor (TCR) when presented by the CD1d molecule found on the surface of DCs. $\alpha$-Galactosylceramide ( $\alpha$-GalCer 1 , Figure 1), a synthetic analogue of a GSL extracted from the marine sponge Agelas mauritianus, was discovered to be a potent stimulator of iNKT cells when presented by CD1d.
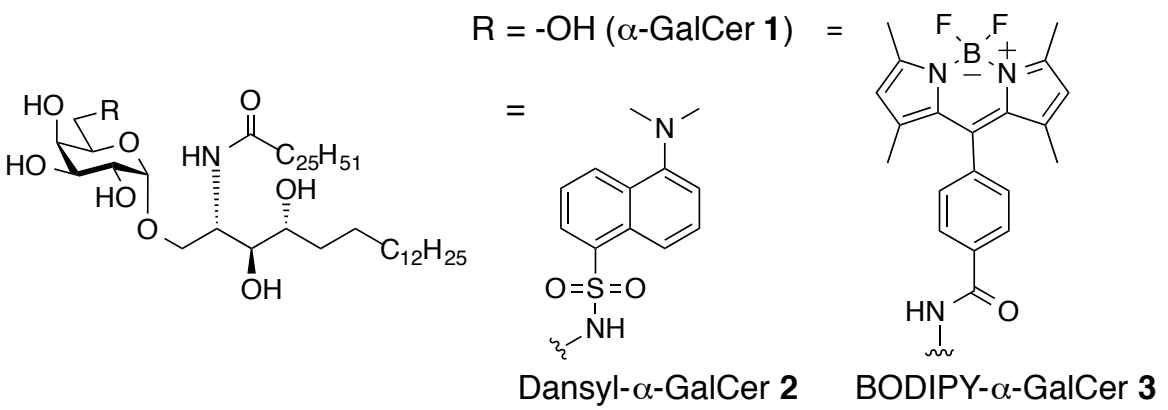

Figure 1. $\alpha$-GalCer and fluorescent probes.

$\alpha$-GalCer is currently being used in clinical trials as an adjuvant to boost the activation of immune cells during cancer immunotherapy. Although the molecular interaction of $\alpha$-GalCer with CD1d and iNKT cells is well established, it is not 
fully understood how the glycolipid interacts with different subsets of DCs. Greater understanding of the fate of the glycolipid during cancer immunotherapy will provide crucial information on how the current therapy can be improved.

In this thesis, the design and synthesis of two fluorescent $\alpha$-GalCer probes, dansyl- $\alpha$-GalCer (2, Figure 1) and BODIPY- $\alpha$-GalCer (3, Figure 1) is reported. Dansyl- $\alpha$-GalCer was able to activate DCs and iNKT cells in a similar fashion to the parent glycolipid $\alpha$-GalCer. Its activity was CD1d-dependent and DCs that have taken up $\alpha$-GalCer in vitro can be detected by flow cytometry. Unfortunately, the fluorescence of dansyl- $\alpha$-GalCer was too weak to be detected by fluorescent microscopy due to photobleaching of the dye. Accordingly, another $\alpha$-GalCer probe bearing a brighter fluorescent group, BODIPY, was synthesised. The $\alpha$-GalCer probes were made via two synthetic strategies and the benefits and shortcomings of each synthetic route are discussed.

Isoglobotrihexosylceramide (4, iGb3, Figure 2) is another GSL known to activate iNKT cells. Like $\alpha$-GalCer, it is presented by DCs in the context of a CD1d molecule. iGb3 contains a sphingosine lipid backbone $\beta$-linked to a trisaccharide head group, which is in contrast to the $\alpha$-linked phytosphingosine lipid found on $\alpha$-GalCer. Despite the structural difference, iGb3 can stimulate iNKT cells, though to a lesser extent than $\alpha$-GalCer. The intriguing activity of iGb3 provides a platform to further investigate the molecular interactions between CD1d, glycolipid and TCR of iNKT cell.

The crystal structure of iGb3 in complex with mouse CD1d and TCR of mouse iNKT cell show compelling evidence that the terminal galactose moiety is crucial for the observed activity and this is attributed to the hydrogen bond between the 6 "'-OH and Thr159 on the CD1d. To unambiguously determine the importance of the hydrogen bond conferred by 6 "' $-\mathrm{OH}, 6$ "' -deoxy-iGb3-sphingosine (5, Figure 2) was synthesised. 6" '-deoxy-iGb3-sphinganine 6 was also synthesised to study the role of the double bond on the sphingosine backbone. A novel synthetic route for the synthesis of iGb3 analogues was established. This allowed for the expedient synthesis of 6 "' -deoxy-iGb3 derivatives that will subsequently be 
crystallised with CD1d and TCR of iNKT cell, to provide further insight into the structural requirements of $\beta$-linked GSLs.
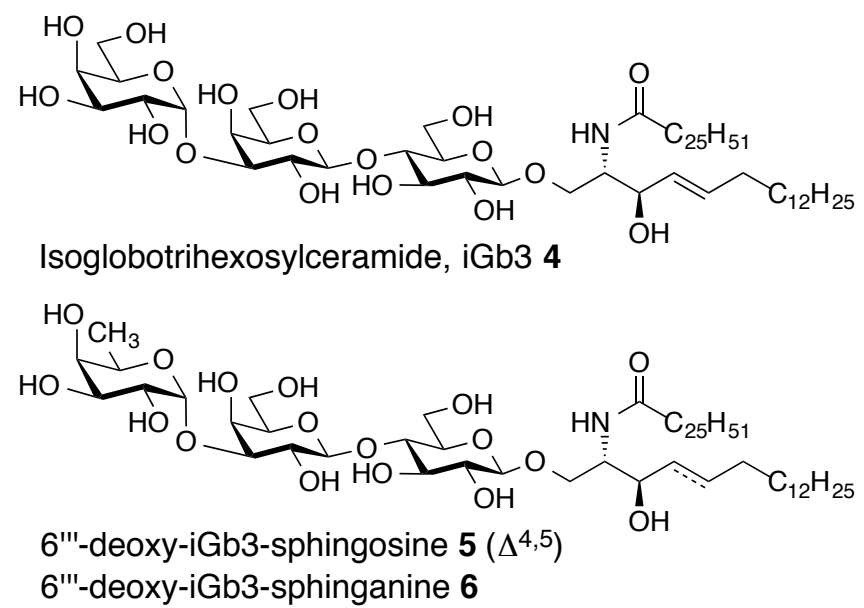

Figure 2. iGb3 and 6"'-deoxy-iGb3 derivatives.

Studies have also revealed that the length and saturation of the $N$-acyl chain of GSLs greatly influences their activity. It is speculated that varying the length of the acyl chain affects the processing and loading of the glycolipid onto CD1d and also TCR binding affinity. To this end, the syntheses of a series of acyl chain analogues of iGb3, including the shorter chain homologue C12:0 7 (Figure 3) and the unsaturated C20:2 derivative $\mathbf{8}$ are reported. A divergent synthetic route was employed, whereby a common intermediate from the synthesis of 6"'-deoxyiGb3 was used. This allowed for efficient syntheses of the acyl chain analogues that will facilitate a greater understanding of the structure-activity relationships.

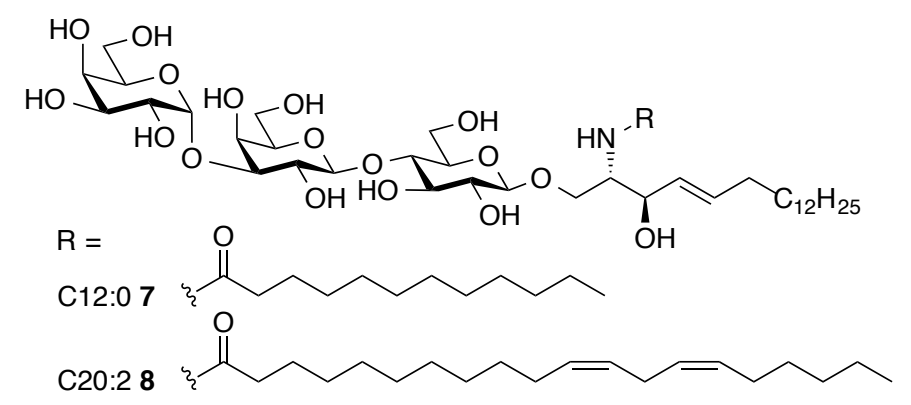

Figure 3. Acyl chain analogues of iGb3.

Taken together, the GSLs synthesised provide crucial insight into how they modulate the immune system and will guide future optimisation of cancer immunotherapy regimes. 


\section{Acknowledgements}

Many people have contributed to my $\mathrm{PhD}$ journey and it was an enriching experience because of the guidance, company, laughter and support of those around me. Firstly I would like to thank my supervisors Dr. Bridget Stocker and Dr. Mattie Timmer for their guidance and support. I have been challenged and stimulated by the both of you to strive for the best, to keep learning and not to give up. Thank you for your commitment to me as a researcher and your student. I would also like to thank Dr. Ian Herman for taking me under his wings, especially in the field of immunology. I would also like to express my gratitude to Prof. Graham LeGros who constantly pushes me to aim high and regularly reminds me of the big picture of scientific research.

Next, I would like to express my thanks to my group members, Alex, Amy, Hilary, Jessie, Kris, Kristal, Jamie, Rhia, Sarah, Stefan and Steph as well as past students of the immunoglycomics group, who have made the lab an enjoyable place to develop and grow together as scientists, with a good dose of humour. I would like to particularly thank Dr Emma Dangerfield who showed me the ropes in the lab, and also inspired me in my research journey. A big part of my research builds on work that she previously carried out in the group. I would also like to thank Steph for being generous with her BODIPY intermediates. In addition, I am grateful to all the support staff from SCPS and Malaghan Institute, without whom the research would not be as smooth-sailing. Specifically, thank you Ian Vorster for dedicating your time in helping me with the NMR and mass spectrometer. Also to Sally, Lisa, Grant, Alan, Nick, Manu and Darrell who ensures that we are well equipped to perform good research.

Beyond that, I would like to thank my family especially Mum, Dad, Vanessa, granddad and grandma, who are my constant pillars of support, and for believing in me. Also to my current and past flatmates, TK, Sharon, Ushma and Monique who are always encouraging, especially towards the end of my research journey- I appreciate your friendship. And lastly to the members of Truth Project thanks for walking alongside me in the last part of this journey. 


\section{Table of contents}

\section{CHAPTER 1: INTRODUCTION}

1.1 Tumour Immunology 1

1.2 Antigen presentation of tumour-associated peptides 2

1.3 Cancer immunotherapy 5

$\begin{array}{ll}\text { 1.4 DC-based cancer immunotherapy } & 7\end{array}$

1.5 DC-based vaccination at the molecular level 10

1.6 Fluorescent $\alpha$-GalCer probes to study cellular uptake and trafficking 16

$1.7 \beta$-linked glycolipids $\quad 19$

1.8 Crystal structures of $\beta$-linked glycolipids $\quad 27$

1.9 The significance of understanding $\beta$-linked self glycolipids $\quad 30$

1.10 Synthetic strategies for iGb3 and related analogues 31

1.11 Thesis outline 33

1.12 References 36

CHAPTER 2. THE SYNTHESIS AND BIOLOGICAL EVALUATION OF DANSYL $\alpha$-GALACTOSYLCERAMIDE AS A FLUORESCENT PROBE FOR THE MONITORING OF GLYCOLIPID UPTAKE BY CELLS

2.1 Introduction 43

2.2 Synthesis of dansyl- $\alpha$-GalCer $\quad 46$

2.3 The effects of dansyl- $\alpha-$ GalCer on the stimulation of iNKT cells in vitro and in vivo 53

2.4 Uptake of $\alpha$-GalCer by dendritic cells 56

2.5 Visualisation of dansyl $\alpha$-GalCer by fluorescence microscopy 57

2.6 Conclusion 58

2.7 Experimental $\quad 59$

$\begin{array}{ll}2.8 \text { References } & 78\end{array}$

CHAPTER 3. SYNTHESIS OF BODIPY- $\alpha$-GALACTOSYLCERAMIDE

3.1 Introduction $\quad 81$

3.2 Synthesis of BODIPY- $\alpha$-galactosylceramide 82

$\begin{array}{ll}3.3 \text { Conclusion } & 103\end{array}$

3.4 Experimental 103

3.5 References 117 
CHAPTER 4. SYNTHESIS OF TERMINAL SUGAR HOMOLOGUES OF iGB3

4.1 Introduction

4.2 Synthesis of 6 "' -deoxy homologues of iGb3

4.3 Conclusion

4.4 Experimental

4.5 References

CHAPTER 5. SYNTHESIS OF ACYL CHAIN HOMOLOGUES OF iGB3

5.1 Introduction

161

5.2 Synthesis of acyl chain homologues of iGb3

164

5.3 Conclusion

174

5.4 Experimental

175

5.5 References

CHAPTER 6. SUMMARY AND FUTURE PROSPECTS

APPENDIX

List of Publications

201

${ }^{1} \mathrm{H}$ and ${ }^{13} \mathrm{C}$ NMR spectra 


\section{List of Abbreviations}

$\alpha$-GalCer $\alpha$-galactosylceramide

Ac acetyl

$\mathrm{AcOH}$ acetic acid

APC antigen presenting cells

aq. Aqueous

$\beta$-GalCer $\beta$-galactosylceramide

$\beta$-GlcCer $\beta$-glucosylceramide

$\beta$-LacCer $\beta$-lactosylceramide

$\mathrm{Bn}$ benzyl

Boc tert-butyloxycarbonyl

br broad

$\mathrm{Bz}$ benzoyl

Calcd. calculated

CD cluster of differentiation

COSY correlated NMR spectrum

CTL cytotoxic T lymphocyte

CTLA-4 cytotoxic T-lymphocyte

Antigen 4

d doublet

dd doublet of doublets

DAB 1,4-dideoxy-1,4-imino-Darabinitol

DBU 1,8-diazabicyclo[5.4.0] undec-7-ene

DC dendritic cell

DCM dichloromethane

DiPEA diisopropylethylamine

DMAP 4-(dimethylamino)pyridine

DMDP 2,5-dihydroxymethyl-3,4dihydroxypyrrolidine

DMF dimethylformamide

DMSO dimethyl sulfoxide

DNA deoxyribonucleic acid

DPA arabinofuranosyl-1-mono phosphoryl-decaprenol

EDCI 1-ethyl-3-(3-

dimethylaminopropyl)

carbodiimide

eq. equivalents

ER endoplasmic reticulum

ESI electron spray ionization

Et ethyl

FITC fluorescein isothiocyanate

Gb3 globotrihexosylceramide

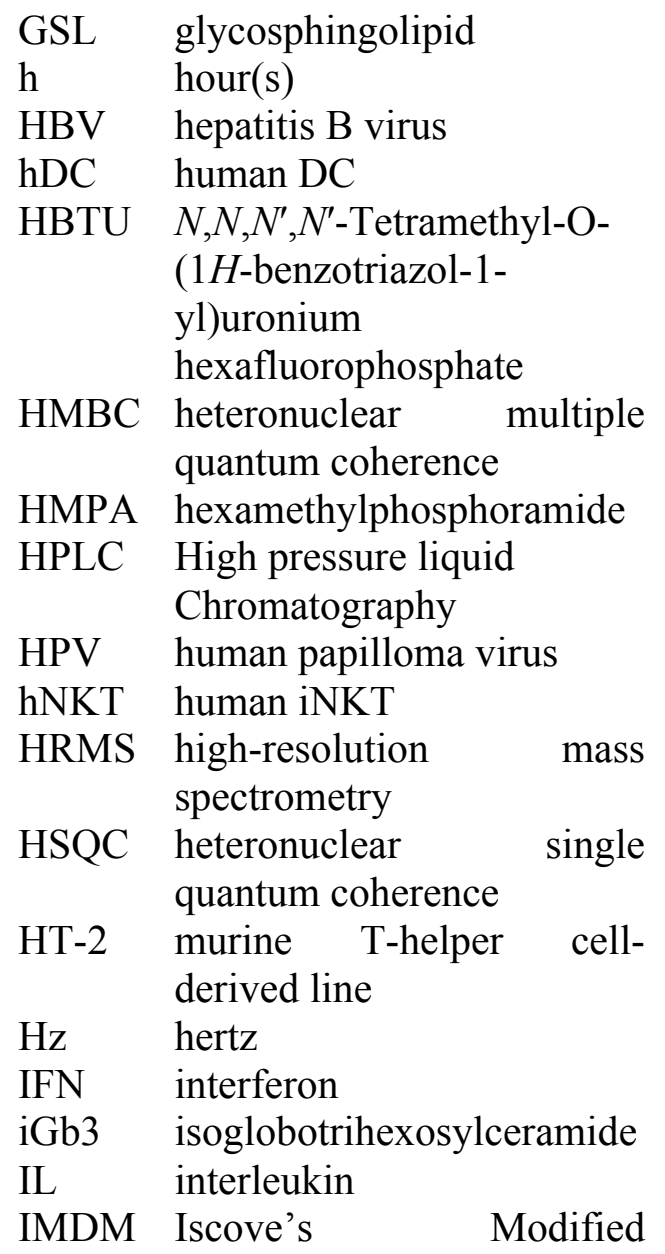

$\begin{array}{ll}\text { IMDM Iscove's M M } & \text { Dulbecco's Medium }\end{array}$

Imid. imidazole

iNKT invariant natural killer $\mathrm{T}$

$i \mathrm{Pr} \quad$ iso-propyl

IR infrared spectroscopy

i.v. intra venous

$J \quad$ coupling constant

KO knock out

LSD lysosomal storage disorder

$\mathrm{m} \quad$ multiplet

Me methyl

MHC major histocompatibility

complex

$\mathrm{MHz}$ mega hertz

MIC minimum inhibitory concentration

min minutes

mNKT mouse iNKT 


$\begin{array}{llll}\text { Mp } & \text { melting point } & \text { rt } & \text { room temperature } \\ \text { Ms } & \text { mesyl } & \mathrm{s} & \text { singlet } \\ m / z & \text { mass to charge ratio } & \text { sat. } & \text { saturated } \\ \text { NBD } & \text { 7-Nitrobenz-2-oxa-1,3- } & \text { SE } & \text { Standard Error } \\ & \text { diazol } & t & \text { triplet } \\ \text { NBS } & N \text {-bromosuccinimide } & \text { TAA } & \text { Tumour associated antigen } \\ \text { NIS } & N \text {-iodosuccinimide } & \text { TB } & \text { tuberculosis } \\ \text { NK } & \text { natural killer } & t \text {-Bu } & \text { tert-butyl } \\ \text { NMR } & \text { nuclear } & \text { TCR } & \text { T cell receptor } \\ & \text { resonance } & \text { TES } & \text { triethylsilane } \\ \text { Obsd. } & \text { observed } & \text { TFA } & \text { trifluoroacetic acid } \\ \text { PBMC } & \text { peripheral } & \text { THF } & \text { tetrahydrofuran } \\ & \text { mononuclear cells } & \text { TLC } & \text { thin layer chromatography } \\ \text { PyBOP } & \text { (Benzotriazol-1-yloxy) } & \text { TLR } & \text { toll-like receptor } \\ & \text { tripyrrolidinophosphonium } & \text { TMSOTf Trimethylsilyl } \\ & \text { hexafluorophosphate } & & \text { methanesulfonate } \\ \text { PBS } & \text { phosphate-buffered saline } & \text { Tf } & \text { trifluoromethanesulfonyl } \\ \text { PD-1 } & \text { programmed death 1 } & \text { TNF } & \text { tumour necrosis factor } \\ \text { PE } & \text { phycoerythrin } & \text { Tr } & \text { triphenylmethyl } \\ \text { Ph } & \text { phenyl } & & \\ \text { ppm } & \text { parts per million } & & \\ p \text { TsOH } & \text { para-toluenesulfonic acid } & & \\ \text { RBC } & \text { red blood cell } & & \\ \text { RPMI } & \text { Roswell Park Memorial } & & \\ & \text { Institute } & & \end{array}$





\section{Chapter 1. Introduction}

The term cancer refers to a family of diseases characterised by the uncontrolled growth and spread of abnormal cells. Cancer can be caused by inherited genetic factors or environmental (external) factors and it remains a leading cause of death worldwide, claiming 7.6 million lives in $2008 .{ }^{1}$ In New Zealand, approximately 21,000 people were diagnosed with cancer in $2010 .^{2}$ Treatment of cancer by surgical removal of the tumour can be traced back to ancient Egyptian times (1600 BC), as documented in the Edwin Smith Papyrus, ${ }^{3,4}$ while radiation therapy came into practice after the discovery of X-rays for medical diagnostics in $1896 .^{5-7}$ In the $20^{\text {th }}$ century, the need to treat World War I soldiers suffering from hematologic malignancies caused by nitrogen mustard gases prompted the development of chemical agents to treat cancer - a treatment referred to as chemotherapy. ${ }^{8}$ To this day, surgery, radiotherapy and chemotherapy continue to play a major role in the treatment of cancer, however, such practices often fail to induce complete tumour remission and are associated with unpleasant side effects.

\subsection{Tumour immunology}

The immune system plays a key role in homeostasis in the body. It responds in a regulated fashion to pathogens, but is trained not to react against self-tissues, a phenomenon known as self-tolerance. ${ }^{9}$ In the 1950 s, the scientific community generally accepted the theory that lymphocytes that are responsive to self-tissue were deleted in prenatal life during the development of the immune system. ${ }^{10}$ While self-tolerance is beneficial in the prevention of autoimmune diseases such as cystic fibrosis and rheumatoid arthritis, tumours often use this mechanism to evade immune recognition, which results in a weak natural protection against malignant tumours. In the 1960s, however, it was recognised that tumours, though being self-derived, contain tumour-associated antigens (TAAs), which the body's lymphocytes can detect - a concept called immunosurveillance. ${ }^{11}$ 
The importance of immunosurveillance in maintaining the health of an individual can be illustrated in many ways. For example, renal transplant patients receiving immunosuppressive treatment to prevent transplant rejection face a greater risk of developing skin cancer when exposed to UV rays from the sun. ${ }^{12}$ Also, the ability of the immune system to recognise specific TAAs is demonstrated by the ability of mice, which upon treatment of the first tumour challenge, were able to reject a second tumour challenge with the same tumour. ${ }^{13-16}$ Moreover, malignant tumours that are highly genetically unstable often generate a vast number of TAAs, which have not been seen by the host. ${ }^{17,18}$ This provides a range of candidate antigens for immune recognition. Nonetheless, due to the dynamic ability of cancerous cells to mutate and evade immunological checkpoints, the body's natural immune response is rarely robust enough to fight against aggressive tumours. ${ }^{19}$

\subsection{Antigen presentation of tumour-associated peptides}

Antigen presenting cells (APCs) patrol the body in the blood circulation and play a crucial role in sending signals to other immune cells upon encountering an antigen, thereby activating the immune system. There are two classes of APCs; professional and non-professional APCs, which are both capable of presenting peptide antigens. Professional APCs are very efficient at internalising antigens and displaying them on their cell surface. Dendritic cells (DCs) are an example of professional APCs and these specialised cells are distinct from non-professional APCs because they are capable of activating the immune cells that they present the antigen to. ${ }^{20}$

When an immature DC encounters an antigen, it matures and migrates to a nearby lymph node (Figure 1.1). At the lymph node, the DC cross presents the peptide antigen on a cell surface receptor, known as Major Histocompatibility Complex I (MHC I), to naïve T cells. ${ }^{21,22}$ These naïve $\mathrm{T}$ cells express $\mathrm{T}$ cell receptors (TCRs) on their cell surface and can recognise the peptide presented by MHC I. The interaction between TCR with MHC I and peptide antigen is highly specific and marks the initiation of an immune response, whereby naïve $\mathrm{T}$ cells become activated to produce the cytokine IL-2, causing them to proliferate and 
differentiate into mature, effector T cells. In addition to the formation of the TCRMHC I-peptide trimer, the interaction of co-stimulatory molecules on DCs and naïve $\mathrm{T}$ cells are also necessary for the maturation of $\mathrm{T}$ cells. ${ }^{23,24}$ The effector $\mathrm{T}$ cells then migrate from the lymph node to the site of infection and respond by eliminating the infection or by releasing other soluble mediators to recruit other immune cells. Different classes of T cells play distinct roles in the immune system and are often characterised by the expression of cell surface markers. CD8+ T cells, also known as cytotoxic T lymphocytes (CTLs), express the cluster of differentiation (CD) 8 protein on their surface and represent an important subset of $\mathrm{T}$ cells because they are capable of directly killing pathogens or tumour cells. The induction of CTLs is thus desired in cancer immunotherapy as CTLs are highly effective at killing tumour cells by releasing cytotoxic proteins such as perforin and granzymes. ${ }^{25}$ 


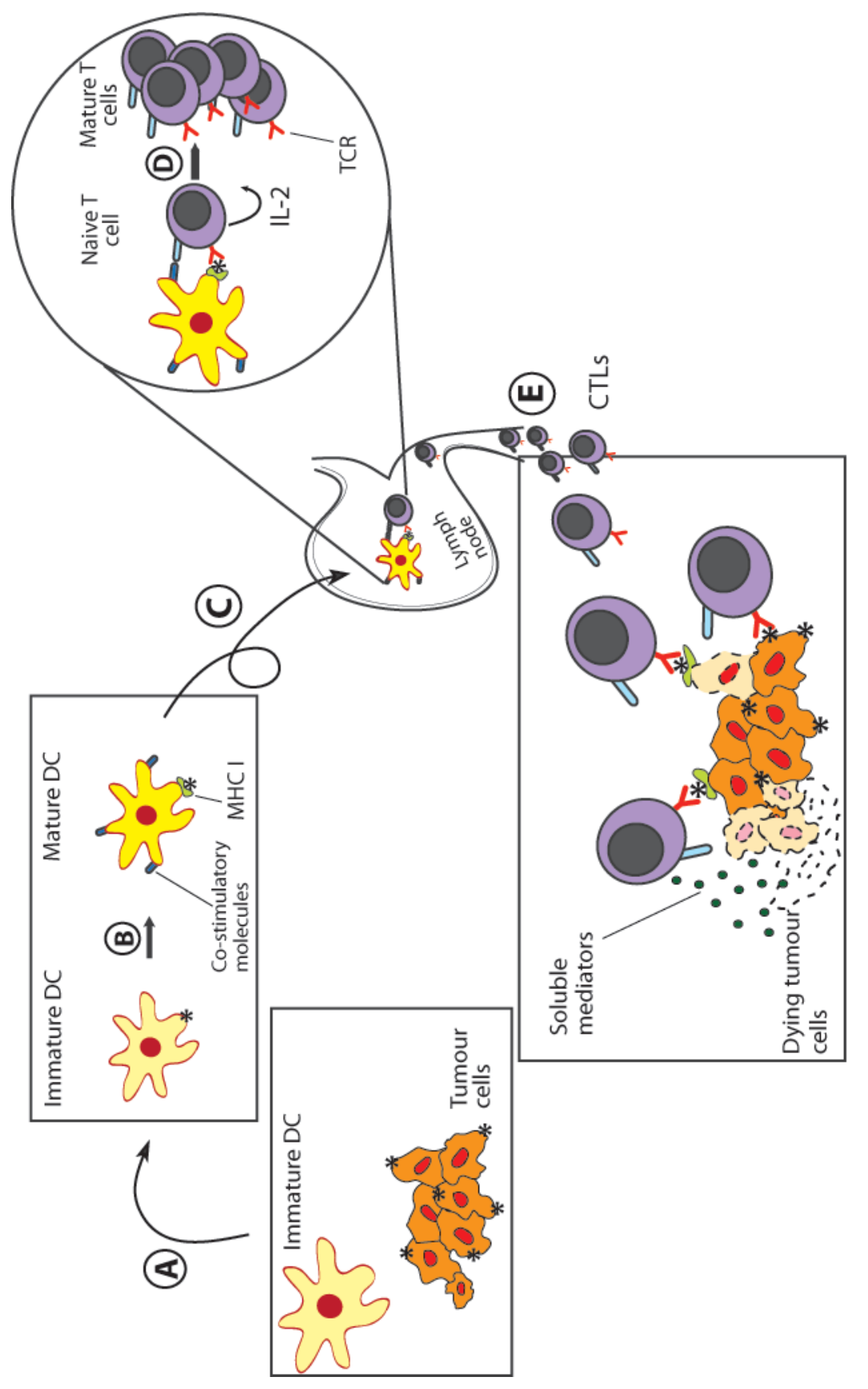

离家尊导

虽实草

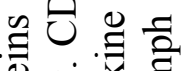

क्ष

运莕正

音

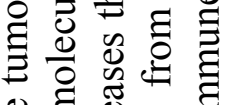

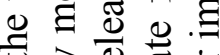

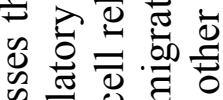

ठै

년

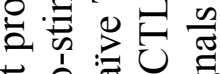

$=0$ 政

की कै 压

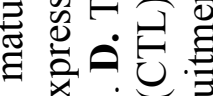

U

䟚

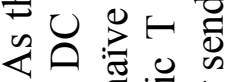

๑ 흥

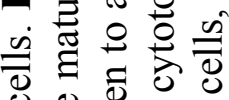

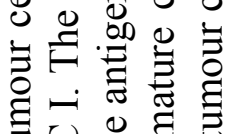

包导

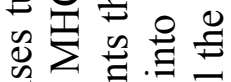

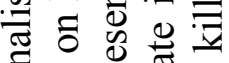

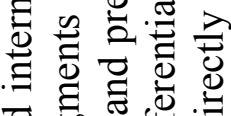

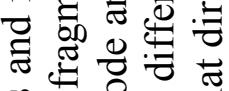

品

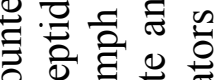

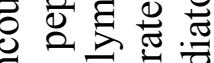

व

웡

년

है 5

毛

安过

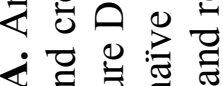

¿

$\exists \stackrel{0}{0}$

过品

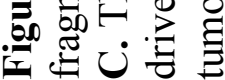




\subsection{Cancer immunotherapy}

Cancer immunotherapy is a promising new type of anti-cancer treatment that employs a patient's own immune system to fight cancers. The strategy for immunotherapy is to improve the immune response to tumours with the ultimate goal being the induction of a tumour-specific immune response. Although William B. Coley pioneered cancer immunotherapy more than 100 years ago by giving cancer patients bacterial infections to invoke their immune system, ${ }^{26}$ this method of treatment was subsequently abandoned because his results were not reproducible and other treatments for cancer became more mainstream. Nonetheless, in the mid-1980s a resurrection of cancer immunotherapy began. Since then, a greater understanding of the immune system aided by advances in technology has led to a boost in confidence, and the potential of cancer immunotherapy has become more widely accepted in the scientific and clinical community. The ability of the immune system to react in a very specific manner, hence with minimal side effects, makes it a promising treatment for malignant diseases. Moreover, the immune system is able to generate long-lasting tumour immunity when activated appropriately.

Cancer vaccines can be broadly classified into two types:

1. Prophylactic (preventive) vaccine: which prevents the onset of cancer in healthy people.

2. Therapeutic vaccine: which treats existing cancers by enhancing immune responses in patients.

In light of the successful immunisation against diseases such as polio and smallpox, many researchers are attempting to replicate similar immunisation strategies for malignant diseases. This led to the birth of prophylactic cancer vaccines effective against the hepatitis B virus (HBV) and the human papilloma virus (HPV), which are the causative agents for liver and cervical cancers, respectively. ${ }^{27-30}$ Though it would be ideal to develop prophylactic vaccines against all cancers, at present, this is unfeasible because of the vast number of rapidly mutating cancers. 
In addition to preventive vaccinations, various immunotherapy regimes have been developed to prime and boost a patient's immune system so that it can attack existing malignant tumours. Some therapeutic vaccination strategies currently under investigation include DNA vaccination, monoclonal antibody treatment, adoptive cellular therapy, peptide-based immunotherapy and of particular interest to us, dendritic cell (DC)-based vaccination. ${ }^{31-43}$ These are summarised in Table 1.1 .

Table 1.1. Therapeutic vaccinations currently under investigation.

\begin{tabular}{|c|c|}
\hline Vaccination & Description \\
\hline DNA va & $\begin{array}{l}\text { Naked DNA that encodes for a tumour antigen (protein) is } \\
\text { injected intramuscularly or intradermally into a cancer } \\
\text { patient. Cells in the body temporarily absorb the DNA } \\
\text { vaccine and produce the protein of interest. This leads to the } \\
\text { priming of the immune response against the target } \\
\text { protein. }{ }^{31-33}\end{array}$ \\
\hline $\begin{array}{l}\text { Monoclonal } \\
\text { antibody treatment }\end{array}$ & $\begin{array}{l}\text { A passive immunotherapy regime that involves the } \\
\text { administration of an antibody that specifically targets } \\
\text { molecules that are either tumour cell surface markers (e.g. } \\
\text { CTLA-4 or PD-1) or are directly responsible for tumour } \\
\text { growth (e.g. endothelial growth factor receptor). }{ }^{34-36}\end{array}$ \\
\hline $\begin{array}{l}\text { Adoptive cellular } \\
\text { therapy }\end{array}$ & $\begin{array}{l}\mathrm{T} \text { lymphocytes or tumour-infiltrating lymphocytes are } \\
\text { harvested from a patient, expanded in vitro with tumour } \\
\text { antigens and cytokines and readministered to the patient. } \\
\text { These effector T cells attack tumour cells in the patient. }{ }^{37,38}\end{array}$ \\
\hline $\begin{array}{l}\text { Peptide-based } \\
\text { immunotherapy }\end{array}$ & $\begin{array}{l}\text { Tumour-specific peptide fragments of defined sequence are } \\
\text { administered to the patient. The immune system mounts a } \\
\text { response against the peptide vaccine. }{ }^{39-41}\end{array}$ \\
\hline $\begin{array}{l}\text { Dendritic cell- } \\
\text { based vaccination }\end{array}$ & $\begin{array}{l}\text { Dendritic cells (DCs) are harvested from the patient, pulsed } \\
\text { (ie. incubated) with tumour antigens and sometimes } \\
\text { adjuvants and reinjected into the patient. This effectively } \\
\text { teaches the DCs to recognise the tumour (Section 1.4). }{ }^{42,43}\end{array}$ \\
\hline
\end{tabular}


In April 2010, the US Food and Drug Administration (FDA) approved the first anti-cancer therapy vaccine, which is a DC-based cancer immunotherapy (Provenge) against prostate cancer. ${ }^{44}$ Shortly after this, a monoclonal antibody was developed against a receptor found on the surface of $\mathrm{T}$ cells, known as the cytotoxic T-lymphocyte antigen 4 (CTLA-4) that is responsible for immunosuppression. ${ }^{35,45}$ Treatment of metastatic melanoma with the anti-CTLA-4 is highly promising and was approved by the US FDA in 2011, under the name ipilimumab. In addition, there are other immunotherapies in the pipeline, such as anti-programmed death (PD)-1, which block the molecules expressed on dying $\mathrm{T}$ cells known as PD-1 and it is currently being tested in clinical trials. ${ }^{36,46-48}$ Taken as a whole, the recent breakthroughs in cancer immunotherapy will pave the way for the release of many other immunotherapies into the market in the future.

\subsection{DC-based cancer immunotherapy}

In a typical DC-based immunotherapy programme, tumour cells are extracted from a patient and apoptosis (programmed cell death) is induced (Figure 1.2A). As previously discussed, tumour cells aberrantly express proteins which can serve as antigens for a $\mathrm{T}$ cell mediated anti-tumour immune response. ${ }^{20}$ At the same time, white blood cells containing precursors (peripheral blood mononuclear cells) to DCs are obtained from the same patient via a process called leukapheresis. The DC precursors are incubated in vitro with tumour cells that have undergone apoptosis and a cocktail of activation factors, which leads to DC maturation. The primed DCs are then reinjected into the patient to generate a systemic anti-tumour response. 


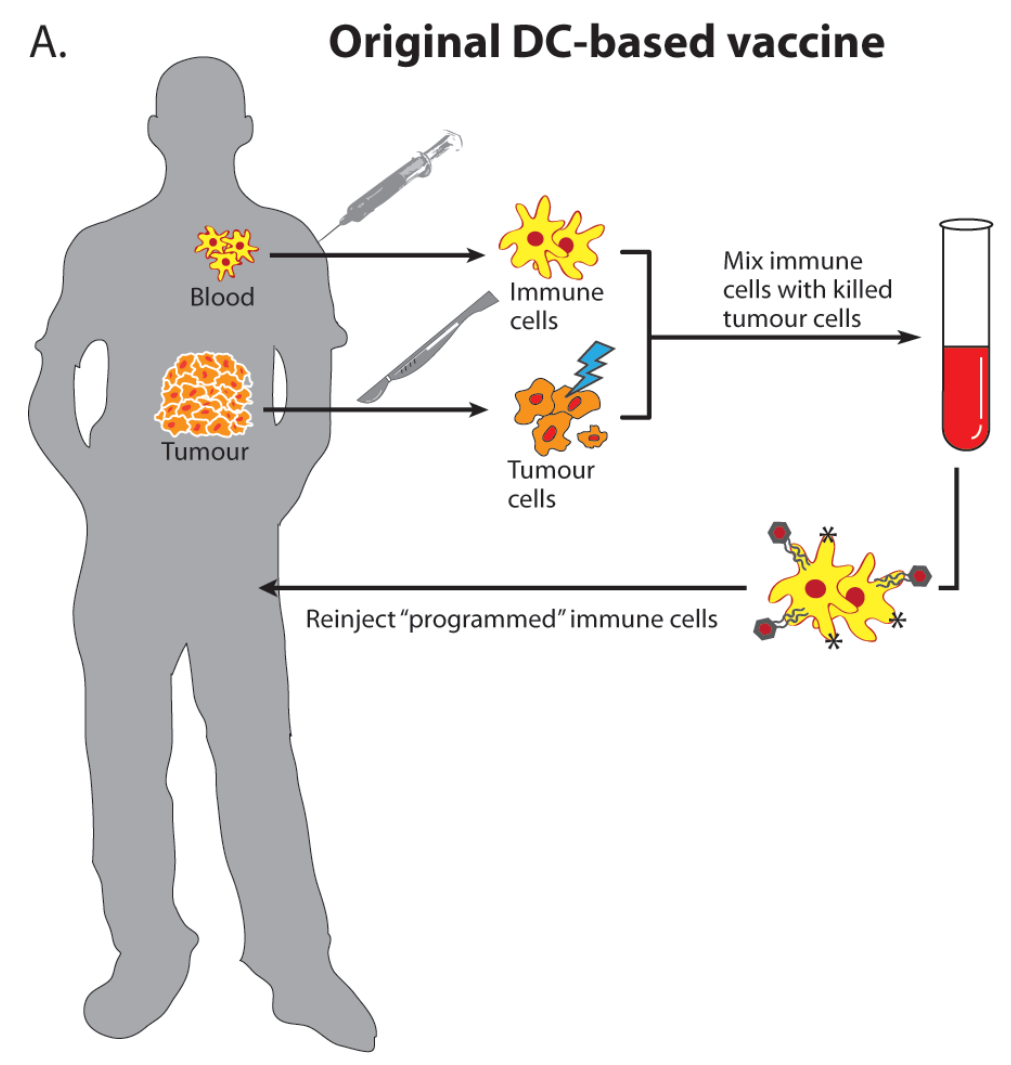

B. Improved DC-based vaccine

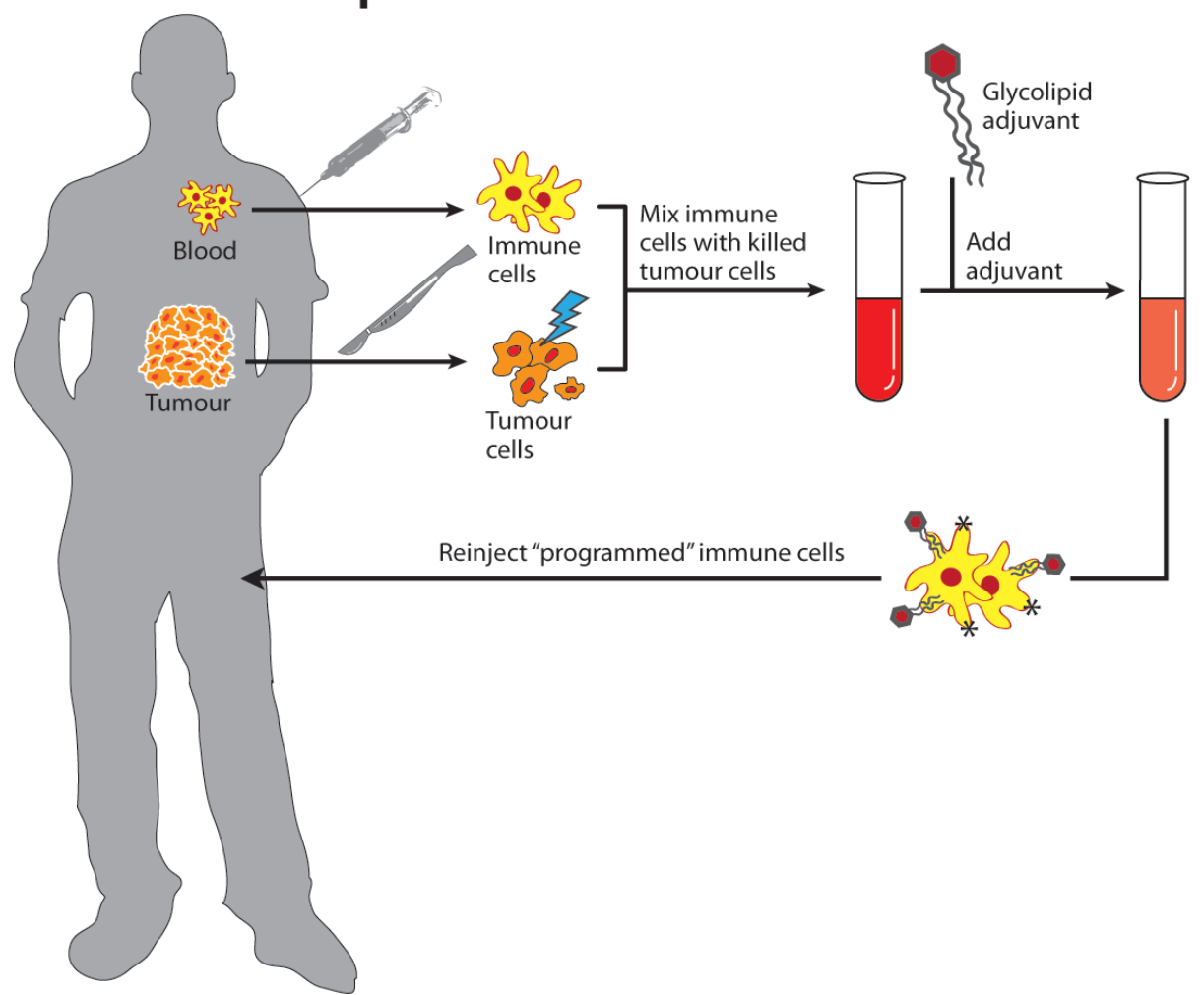

Figure 1.2. A. Original DC-based immunotherapy regime. B. Improved regime incorporating a glycolipid adjuvant. 
One may ask why the favourable anti-tumour immune response is not generated spontaneously in vivo considering that both DCs and tumour antigens are present in the body. It has been shown that most DC precursors found in or near malignant tissues are poor antigen presenters because they fail to be activated, possibly due to the immunosuppressive environment of the tumour. ${ }^{49,50}$ However, if the DC precursors are activated under in vitro culture conditions, they express vital co-stimulatory molecules (e.g. CD40, CD80 and CD86) and acquire the capacity to present antigens and activate naïve T cells.

Though tumour regression has been observed in a number of patients treated by this DC-based immunotherapy regime, the immune response generated can be further improved. ${ }^{51}$ It has been found that the addition of certain glycolipids, acting as adjuvants, boosts the immune response to elicit enhanced anti-tumour activity. ${ }^{51}$ Accordingly, an improved DC-based vaccination regime has been developed which involves the isolation of tumour cells and immune cells from the patient, apoptosis of the tumour cells by radiation, combination of the cells with a glycolipid adjuvant and reinjection of this mixture into the patient (Figure 1.2B). This leads to the stimulation of an enhanced tumour-specific response.

The original DC vaccination strategy, whereby DCs pulsed with peptide in vitro are reinjected into the patient, has been proven to generate a positive outcome in melanoma patients. ${ }^{52}$ The use of glycolipids in cancer immunotherapy has reached clinical trials but the glycolipid adjuvant has only ever been administered on its own, ${ }^{53}$ or administered after it was pre-loaded onto DCs. ${ }^{54-58}$ The improved protocol which incorporates the priming of DCs with both the peptide as well as the glycolipid adjuvant has yet to be tested in a clinical setting. 


\subsection{DC-based vaccination at the molecular level}

\subsubsection{Overview}

An explanation of the cancer immunotherapy protocol at the molecular level is given in Figure 1.3. Here the peptide antigen from the apoptosised tumour cell is presented on the MHC I molecule of the DC (as discussed in Section 1.2) and the presented antigen is then recognised by cytotoxic T lymphocytes (CTLs), which become activated to release soluble mediators that directly target the tumour, or trigger a cascade of immune activation leading to an anti-tumour response. ${ }^{59}$ This anti-tumour immune response can be modulated by the addition of a glycolipid adjuvant (for a comprehensive review see Wu et. al.). ${ }^{60}$ The glycolipid adjuvant is also presented on the surface of the DC, this time via another protein called CD1d. The CD1d-glycolipid complex in turn is detected by invariant natural killer $\mathrm{T}$ (iNKT) cells, a subset of $\mathrm{T}$ cells that play a crucial role in regulating immune responses. ${ }^{60-65}$ Upon stimulation, iNKT cells release cytokines that can promote either a favourable or unfavourable anti-tumour immune response, generally termed as $\mathrm{T}$ helper (Th) 1 or Th2 response, respectively. ${ }^{66}$ The 'Th1' response is characterised by the release of proinflammatory cytokines, such as interferon (IFN)- $\gamma$ and interleukin (IL)-12, which promote CTL and natural killer (NK) cell activation and therefore enhance the anti-tumour response. The 'Th2' response is immunomodulatory and produces cytokines such as IL-4, which are vital to prevent autoimmune diseases such as type-I diabetes and multiple sclerosis. However, activation of the Th2 response also leads to suppression of $\mathrm{T}$ cell mediated anti-tumour immunity. Hence, in developing adjuvants for anti-cancer immunotherapy, a glycolipid that is capable of selectively inducing a 'Th1' response is desired. 


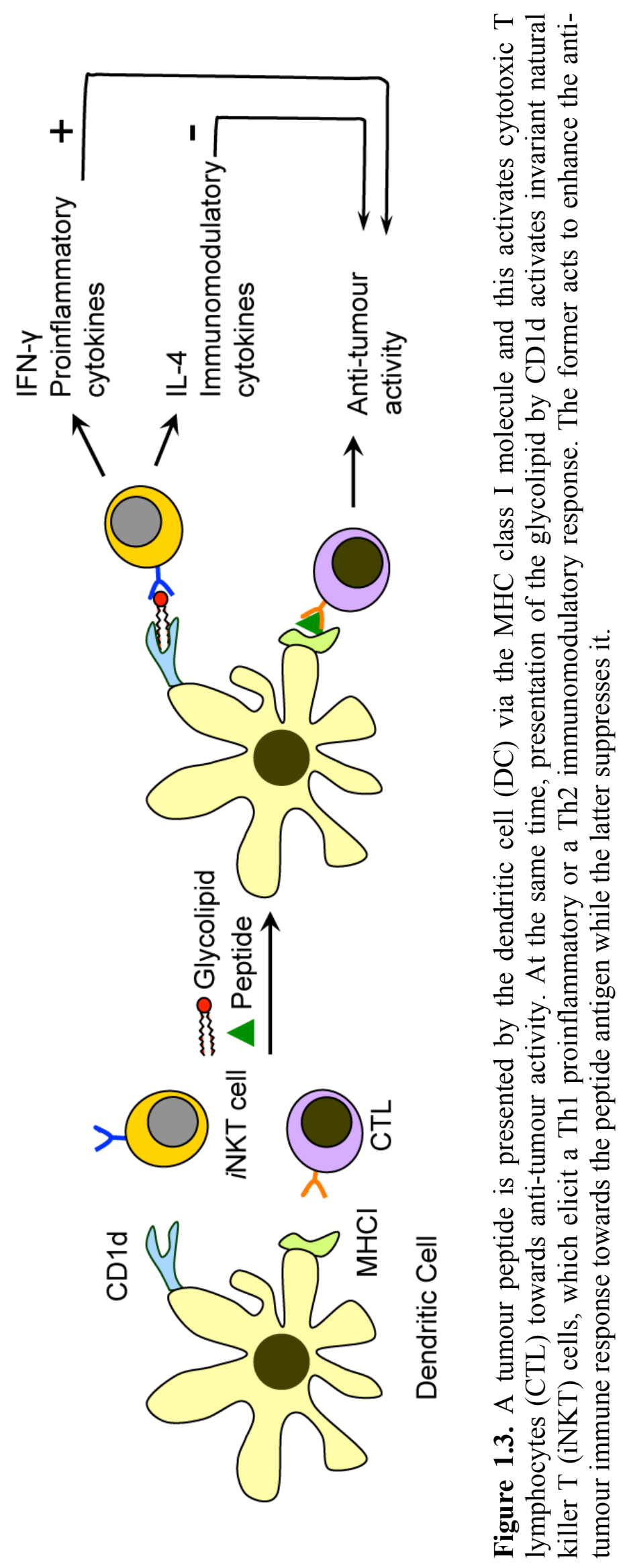




\subsection{2 $\alpha$-Galactosyl ceramide - A potent glycosphingolipid adjuvant}

In 1993, the pharmaceutical division of Kirin Breweries isolated a series of novel $\alpha$-galactosyl ceramides from the marine sponge Agelas mauritianus. ${ }^{67,68}$ Of these glycosphingolipids, agelasphin-9b (AGL-9b, 9, Figure 1.4), exhibited potent antitumour activity in in vivo models of several murine tumour cells and later served as the parent compound for subsequent analogue syntheses and structure-activity studies. ${ }^{69}$ The term "glycosphingolipid" refers to a class of glycolipids that contain the sphingoid base. AGL-9b is a glycosphingolipid that consists of a galactosyl moiety $\alpha$-linked to a ceramide portion that is made up of an $N$-acylated phytosphingosine backbone. During the course of this structure-activity work, KRN7000 (1) was found to have similar anti-tumour activity to 9 and, due to its easier synthesis, was deemed the more suitable candidate for clinical use. The abolishment of both the terminal methyl group on the phytosphingosine backbone and the hydroxyl group on the $\alpha$-position of the acyl chain did not affect the antitumour activity of the glycolipid. Since then, KRN7000, now widely known as $\alpha$ galactosyl ceramide ( $\alpha$-GalCer), has been reported to have potential in the treatment of several diseases including cancer, malaria, type-I diabetes, and multiple sclerosis. It has been found to exert its therapeutic activity via its ability to bind to CD1d and activate iNKT cells. ${ }^{70,71}$ This discovery was remarkable and provided the first evidence that glycolipids, like their protein counterparts, can be presented by APCs and recognised by T cells to invoke an immune response. The potential for $\alpha$-GalCer to treat various diseases lies in its potent ability to stimulate iNKT cells, which subsequently activates the Th1 and the Th2 response. Given the therapeutic potential of $\alpha$-GalCer, much effort has been spent in identifying other ligands that bind to CD1d and activate iNKT cells. ${ }^{60,69,72-80}$ 

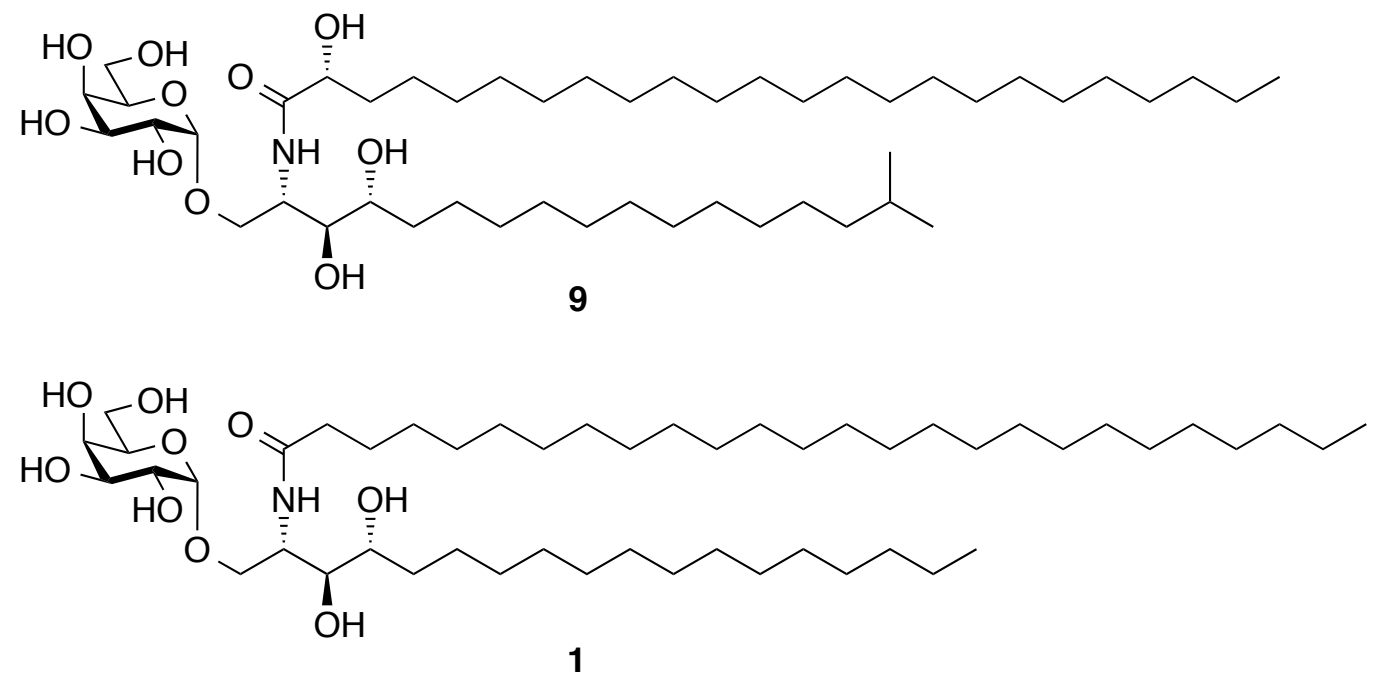

Figure 1.4. Agelasphin $9 b 9$ and $\alpha$-GalCer 1 .

\subsubsection{CD1d}

The CD1d protein is a member of a family of transmembrane glycoproteins consisting of a heavy chain with three extracellular domains ( $\alpha 1, \alpha 2$ and $\alpha 3)$ noncovalently linked to a $\beta_{2}$-microglobulin $\left(\beta_{2} \mathrm{~m}\right)$ chain. ${ }^{81}$ The non-polar amino acids in the $\alpha 1$ and $\alpha 2$ chains form two hydrophobic pockets, $\mathrm{A}^{\prime}$ and $\mathrm{F}^{\prime}$ that can accommodate the long lipid tails of a glycolipid, leaving the sugar head group exposed as depicted in Figure 1.5A. The protruding carbohydrate portion is then recognised by iNKT cells, via their TCR. The trimolecular interaction between the CD1d, $\alpha$-GalCer and TCR initiates a cascade of intracellular and extracellular signalling that results in the activation of iNKT cells. The precise molecular interaction of CD1d with glycolipid was elucidated when Rossjohn and coworkers crystallised a human iNKT cell TCR (blue and yellow) in complex with human CD1d (green) loaded with $\alpha$-GalCer (pink) (Figure 1.5B). ${ }^{82}$ In addition, crystal structures of mouse and human CD1d reveal that this protein is conserved across these two species (Figure 1.5C), whereby the human CD1d is shown in grey and the mouse CD1d in green. ${ }^{83}$ 
Given this similarity, it is generally accepted that the murine model is suitable when studying the potency of various analogues of $\alpha$-GalCer, however, considering the subtle, but non-negligible variation in DC subsets between mice and humans, ${ }^{84}$ care should be taken, and these glycosphingolipids should always be cross-tested against both species. The varying glycosphingolipid activities of triglycosyl ceramides such as isoglobotrihexosylceramide (iGb3) are attributed to the difference in CD1d structure between mice and humans. ${ }^{85}$ In contrast, differences in activities observed for glycosphingolipids that possess one sugar head group (i.e. $\alpha$-GalCer analogues) have been traced back to the variation in the TCR structure rather than the CD1d molecule. ${ }^{86-88}$

A.

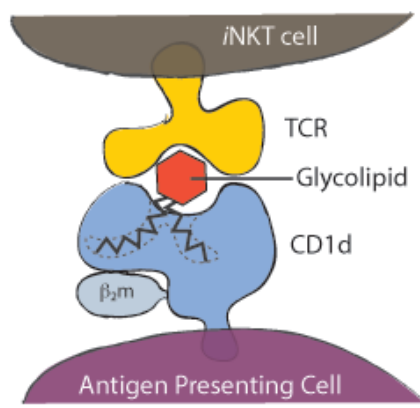

C.

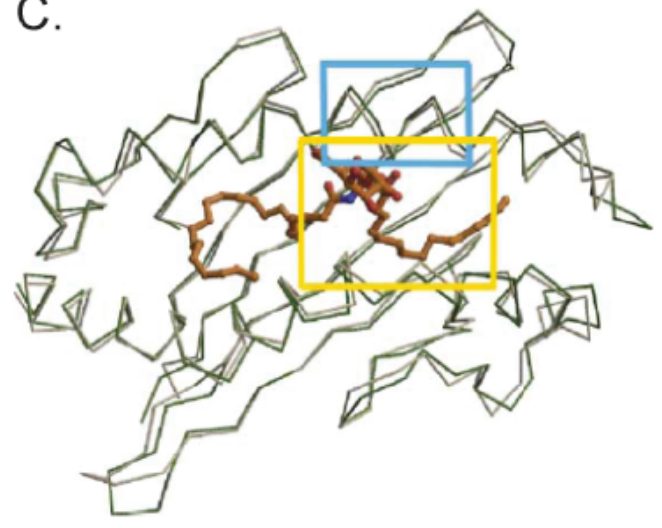

B.

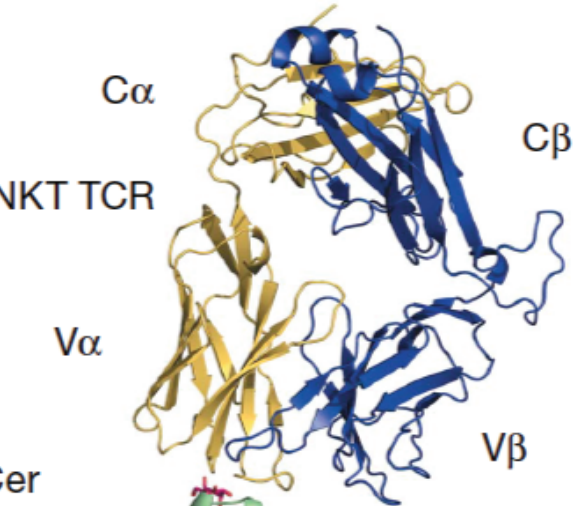

$\alpha$-GalCer

CD1d

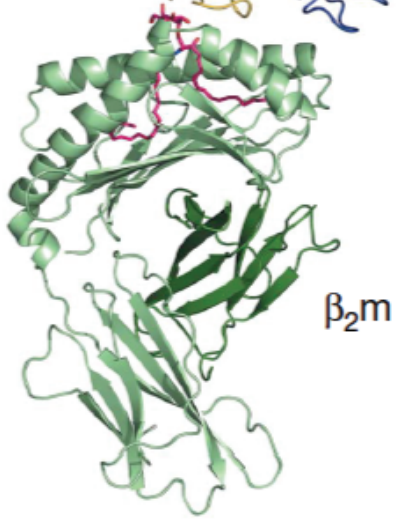

Figure 1.5. A. Representation of CD1d-glycolipid-TCR interaction during glycolipid presentation. B. Crystal structure of human NKT TCR (blue and yellow) interacting with CD1d (green)-glycolipid (pink) complex. ${ }^{82}$ (Adapted by permission from Macmillan Publishers Ltd: Nature Immunology, 2007, 448, 4449, (C2007) C. Structural comparison of human (grey) and mouse (green) CD1d with $\alpha$-GalCer bound (orange). ${ }^{83}$ (Adapted by permission from Macmillan Publishers Ltd: Nature Immunology, 2005, 6, 819-826, (C2005) 


\subsection{4 iNKT cells}

iNKT cells represent less than $1 \%$ of the total $\mathrm{T}$ cell population in humans and are a heterogeneous population of cells that express an invariant TCR and the natural killer (NK) 1.1 marker on the cell surface. ${ }^{61,63}$ They are commonly found in the thymus, spleen, liver and bone marrow. ${ }^{89}$ Unlike $\mathrm{CD} 4^{+}$and $\mathrm{CD}^{+} \mathrm{T}$ cells, which recognise peptide antigens, iNKT cells are restricted by the CD1d molecule and can only recognise glycolipids presented by CD1d. iNKT cells play an important role in bridging innate and adaptive immunity, ${ }^{89}$ for they not only have a key role in the activation of the immediate innate immune response, but also affect the subsequent, longer-lasting, adaptive immunity involving B and T cells. ${ }^{90-92}$ It is this unique characteristic that makes iNKT cells such a potent cell type with great potential in cancer immunotherapy. Of note, structure activity relationship studies and crystal structure analyses, have revealed that subtle differences exist between the TCR structures of humans and mice and can sometimes lead to speciesselective activity of glycolipids. ${ }^{86-88}$ 


\subsection{Fluorescent $\alpha$-GalCer probes to study cellular uptake and trafficking}

A comprehensive understanding of the effect of the glycosphingolipids on the immune system is crucial for the development of an effective DC-based cancer immunotherapy utilitising $\alpha$-GalCer or analogues thereof. The study of these glycosphingolipids in terms of their ability to be presented by CD1d molecules on DCs and to iNKT cells to produce specific cytokine profiles is important to provide information on how the structures of the glycosphingolipids can be altered to improve immune responses. In addition, it is important to understand the pharmacokinetics of glycosphingolipid adjuvants once they are administered as part of a cancer immunotherapy regime.

Within the human body, there are different subsets of DCs, residing in different compartments in vivo that play specific and sometimes overlapping roles in the immune system. ${ }^{93,94}$ Studies have shown that targeting of $\alpha$-GalCer adjuvants to these specific subsets of DCs (such as the CD8 $\alpha+$ DCs) can lead to enhanced antitumour responses. ${ }^{95,96}$ One of the methods to identify and quantify subsets of DCs that have taken up glycolipids and are responsible for the observed immune activation is by using a fluorescently labelled antibody that binds to the CD1dglycolipid complex. ${ }^{97-101}$ Unfortunately, these monoclonal antibodies are only useful in systems where $\alpha-G a l C e r$ is bound to CD1d and are less applicable when trying to study glycolipid trafficking. Therefore, an alternative approach is to tag the glycolipid itself with a fluorophore to directly visualise the glycolipid and its interaction with different immune cells. As such, a number of $\alpha$-GalCerderivatives containing a fluorophore ${ }^{102-104}$ or a biotinylated probe ${ }^{103,105,106}$ have been prepared. Though the ability of $\alpha$-GalCer to bind to CD1d and activate iNKT cells has been robustly studied, scant information is available on inter- and intracellular lipid trafficking and how this influences CD1d presentation and iNKT cell activation. ${ }^{107}$ Many groups have thus focussed on derivatising the lipid portion of $\alpha$-GalCer with a reporter probe in order to address such issues, as 
illustrated by syntheses of biotin-labelled $\alpha$-GalCer $\mathbf{1 0},{ }^{105}$ BODIPY-labelled $\alpha$-GalCer $\mathbf{1 1}^{102}$ and 7-nitro-benz-2-oxa-1,3-diazol-(NBD)-functionalised $\alpha$ GalCer $\mathbf{1 2}^{108}$ (Figure 1.6).

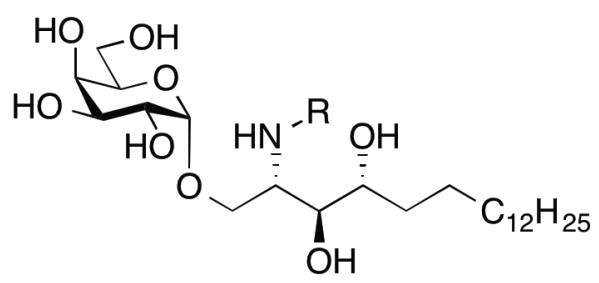

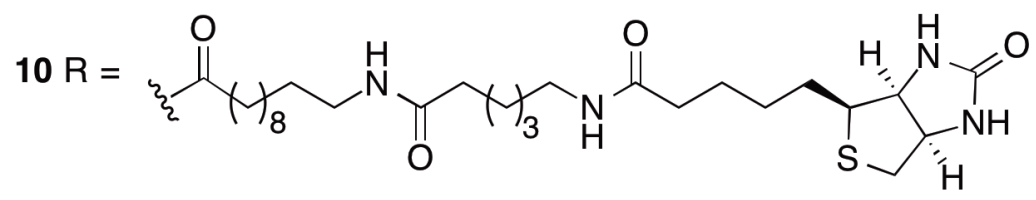<smiles></smiles>

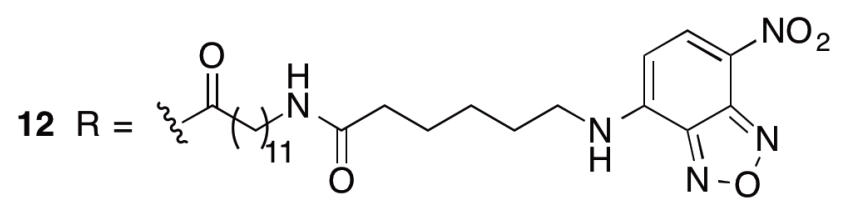

Figure 1.6. Lipid derivatised $\alpha$-GalCer.

However, as CD1d binds lipid chains within deep hydrophobic pockets, ${ }^{109}$ it has been proposed that the addition of a label on the lipid may interfere with $\alpha$ GalCer-CD1d association, ${ }^{110}$ and moreover, may influence intracellular trafficking. ${ }^{107,111-113}$ Conversely, modelling of the CD1d- $\alpha$-GalCer complex suggests that the hydroxyl groups at C-4' and C-6' on galactose are not involved in complex formation. ${ }^{114}$ This theory is also supported by the preparation of an $\alpha$ GalCer analogue containing an additional $\alpha$-linked galactose at the C6' position, which was shown to stimulate iNKT cells without the need for processing. ${ }^{115}$ Taken as a whole, it is generally accepted that the CD1d-glycolipid-iNKT cell TCR interaction tolerates the appendage of small molecules at $\mathrm{C6}^{\prime}$ and that this is an ideal position to attach a fluorescent reporter group. Indeed, this was the conclusion made by Zhou et al. who developed a strategy for the preparation of 
dansylated $\alpha$-GalCer derivative 2 (Figure 1.7). ${ }^{103}$ As such, biotin-labelled $\alpha$-GalCer $13^{103}$ and BODIPY-labelled $\alpha$-GalCer $14^{104}$ were also synthesised, with extensive confocal microscopy studies being performed on the latter to understand the intracellular trafficking on this glycolipid between endosomal compartments within a cell. These findings illustrate the versatility of using $\alpha$-GalCer derivatives with fluorophores attached on the $6^{\prime}$ position of the carbohydrate moiety. Therefore, attachment of small fluorescent groups at the $6^{\prime}$-position of $\alpha$ GalCer will provide useful probes to track and visualise these glycolipids in vitro and in vivo.

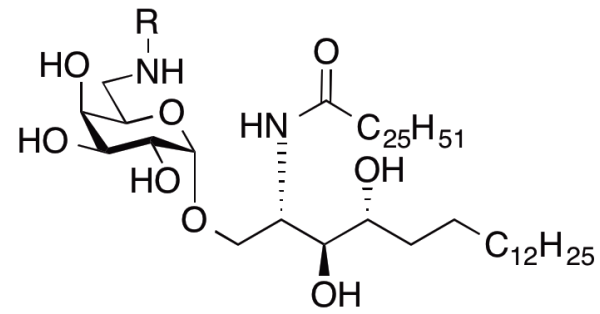<smiles>CN(C)c1cccc2c(S(C)(=O)=O)cccc12</smiles>

$13 \mathrm{R}=$<smiles>NC(=O)CCCC[C@H]1SC[C@@H]2NC(=O)N[C@@H]21</smiles><smiles>CC(=O)CCC1=[N+]2C(=Cc3c(C)cc(C)n3[B-]2(F)F)C=C1</smiles>

Figure 1.7. $6^{\prime}$-labelled $\alpha$-GalCer. 


\section{$1.7 \beta$-linked glycolipids}

In a general sense, the potent activity of exogenous glycolipids that stimulate the immune system requires an $\alpha$-linkage between the sugar moiety and the lipid portion. For example, bacterial glycolipids such as $\alpha$-glucosyldiacylglycerol from Streptococcus pneumoniae and $\alpha$-glycuronosylceramides from Sphingomonas spp. have $\alpha$-linkages and typically, $\beta$-analogues of immunoactive bacterial glycolipids have significantly lower stimulatory activity compared to their $\alpha$ linked couterparts. ${ }^{71,116-119}$ Indeed, the potent immunostimulatory activity attributed to $\alpha$-GalCer is significantly reduced when the glycosidic linkage is converted to give $\beta$-GalCer. In spite of this, $\beta$-linked glycolipids represent an important class of glycolipids because mammalian cells produce $\beta$-linked rather than $\alpha$-linked glycolipids.

iNKT cells represent a potent subset of $\mathrm{T}$ cells in both the innate and adaptive immune response and accordingly, much effort has focused on understanding iNKT cell development. The development of iNKT cells occurs in the thymus, where a T cell with a TCR that can recognise CD1d bound to a self glycolipid is positively selected. Mice lacking in the CD1d molecule (CD1d knockout mice) do not have iNKT cells, ${ }^{63,120}$ while mice with impaired intracellular CD1d trafficking have abnormal iNKT cell development. ${ }^{121}$ Because all endogenous glycolipids in mammalian cells are $\beta$-linked, this class of glycolipids has been studied extensively to understand how they shape the iNKT cell repertoir. That said, the exact identity of the self $\beta$-glycolipid remains elusive and it is unclear as to whether one or more glycolipids are responsible for the positive iNKT cell selection in the thymus. ${ }^{61,62}$

From a structural point of view, in a CD1d-glycolipid complex, the sugar headgroup of $\alpha$-linked monoglycosyl ceramides wraps closely to the $\alpha 2$-helix of CD1d in a parallel orientation, whereas the sugar headgroup of $\beta$-linked monoglycosyl ceramides protrude from CD1d at an angle perpendicular to the $\alpha 2$ - 
helix. For example, $\beta$-galactosylceramide ( $\beta$-GalCer), $\beta$-glucosylceramide ( $\beta$ GlcCer) and isoglobotrihexosylceramide (iGb3) are implicated in the development and activity of iNKT cells. ${ }^{122}$ The ability of the TCR to accommodate this difference is a source of fascination for many structural immunologists and much research is still underway to accurately determine the extent of their influence on iNKT cell biology.

\subsubsection{Isoglobotrihexosylceramide (iGb3)}

Isoglobotrihexosylceramide (iGb3, 4 Scheme 1.1) is a glycosphingolipid containing a trisaccharide headgroup attached to a sphingosine ceramide via a $\beta$ linkage. The prospect of iGb3 acting as the self antigen responsible for iNKT cell development was first reported by Bendelac and co-workers in 2004. Upon observing that mice lacking in $\beta$-hexosaminidase $\mathrm{B}$ have severely reduced number of iNKT cells, they synthesised and tested iGb3 to assess if it was indeed the endogenous ligand important for iNKT cell development. ${ }^{123} \beta$-Hexosaminidase B is the lysosomal enzyme responsible for cleaving terminal $\beta$-linked GalNAc residues from mammalian glycosphingolipids (GSLs) and one of the substrates, iGb4 (15) is converted to iGb3 (4) by this enzyme. While Bendelac and coworkers did not directly purify and characterise iGb3 from mammalian tissues, they were able to show that synthetic iGb3 stimulates the expansion of human iNKT cells from peripheral blood mononuclear cells (PBMCs), and the cytokine levels (IFN- $\gamma$ and IL-4) were comparable to that of $\alpha$-GalCer. ${ }^{123}$ They suggested that iGb3 was the major self glycolipid responsible for the selection of iNKT cells in the thymus of mammalian cells. 

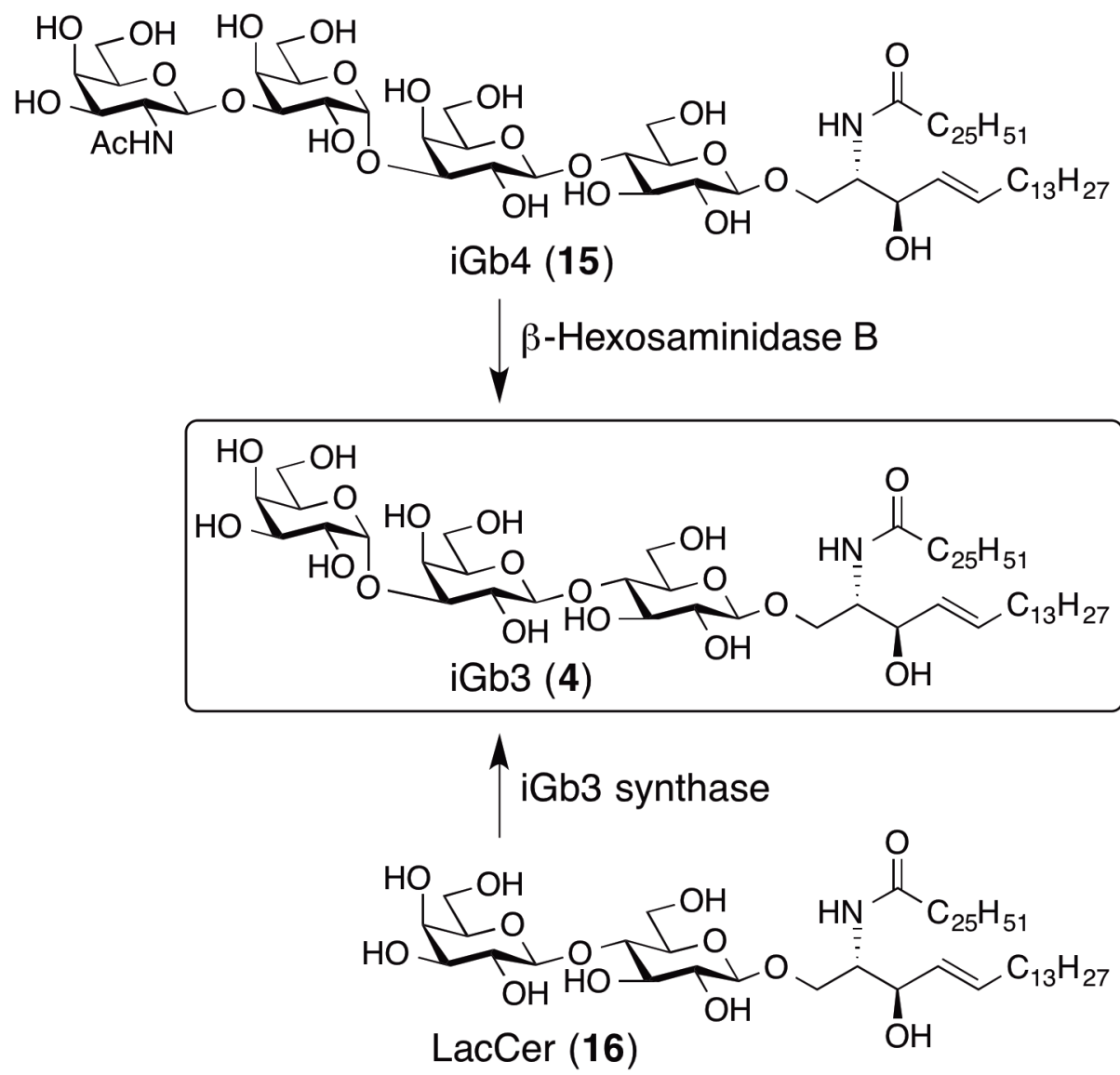

Scheme 1.1. Biosynthetic pathway for iGb3.

However, further studies by Speak et al. revealed that, iGb3 could not be detected in the thymus or in dendritic cells of humans and mice despite an extensive assessment using a highly sensitive HPLC assay. ${ }^{124}$ In addition, mice lacking in iGb3 synthase, a key enzyme that makes iGb3 (4) from LacCer (16) did not show reduced iNKT cell numbers or function (Scheme 1.1). ${ }^{125}$ Moreover, it was proposed that iGb3 is unlikely to be the self ligand responsible for the development of human iNKT cells because humans do not express functional iGb3 synthase ${ }^{126}$ and instead it was suggested that the loss of iNKT cells in $\beta$ hexasominidase B deficient mice was due to defective lysosomal function rather than the absence of iGb3. ${ }^{127}$ These studies, along with others, ${ }^{128}$ strongly argue against iGb3 being the major self glycolipid responsible for the expansion of iNKT cells. ${ }^{127}$ Nonetheless, the fact that both iGb3 and $\alpha$-GalCer, which are structurally very distinct, are able to activate iNKT cells is interesting and points towards the unique nature of the iNKT cell TCR to recognise diverse glycolipid ligands presented by CD1d. 
More recent findings by Brennan et al. revealed that $\beta$-glucosylceramide ( $\beta$ GlcCer, 17, Scheme 1.2) is an important self glycolipid whose expression is upregulated upon DC activation. ${ }^{129}$ During an infection, toll-like receptor (TLR)mediated activation of DCs leads to the upregulation of the enzyme glucosylceramide synthase that catalyses the synthesis of $\beta$-GlcCer (17) while the enzyme lactosylceramide synthase that converts $\beta$-GlcCer (17) to LacCer (16) is downregulated. The increased accumulation and presentation of $\beta$-GlcCer (17) by CD1d, in combination with the TLR-dependant production of cytokine IL-12, stimulates iNKT cells to produce IFN- $\gamma$. Brennan et al. also showed that the composition of the $N$-acyl lipid dictates the activity of $\beta$-GlcCer (17) and that the most abundant form found in mouse lymphoid organs is the C24:1 variant. This study, along with others thus illustrates that $\beta$-linked self glycolipids exert their effect not only during the development of iNKT cells, but that they are also responsible for iNKT cell activation during infection. ${ }^{101,130-134}$ The challenge remains to attribute the contribution of self $\beta$-linked glycolipids and foreign $\alpha$ linked glycolipids on the immunological profile during an infection.

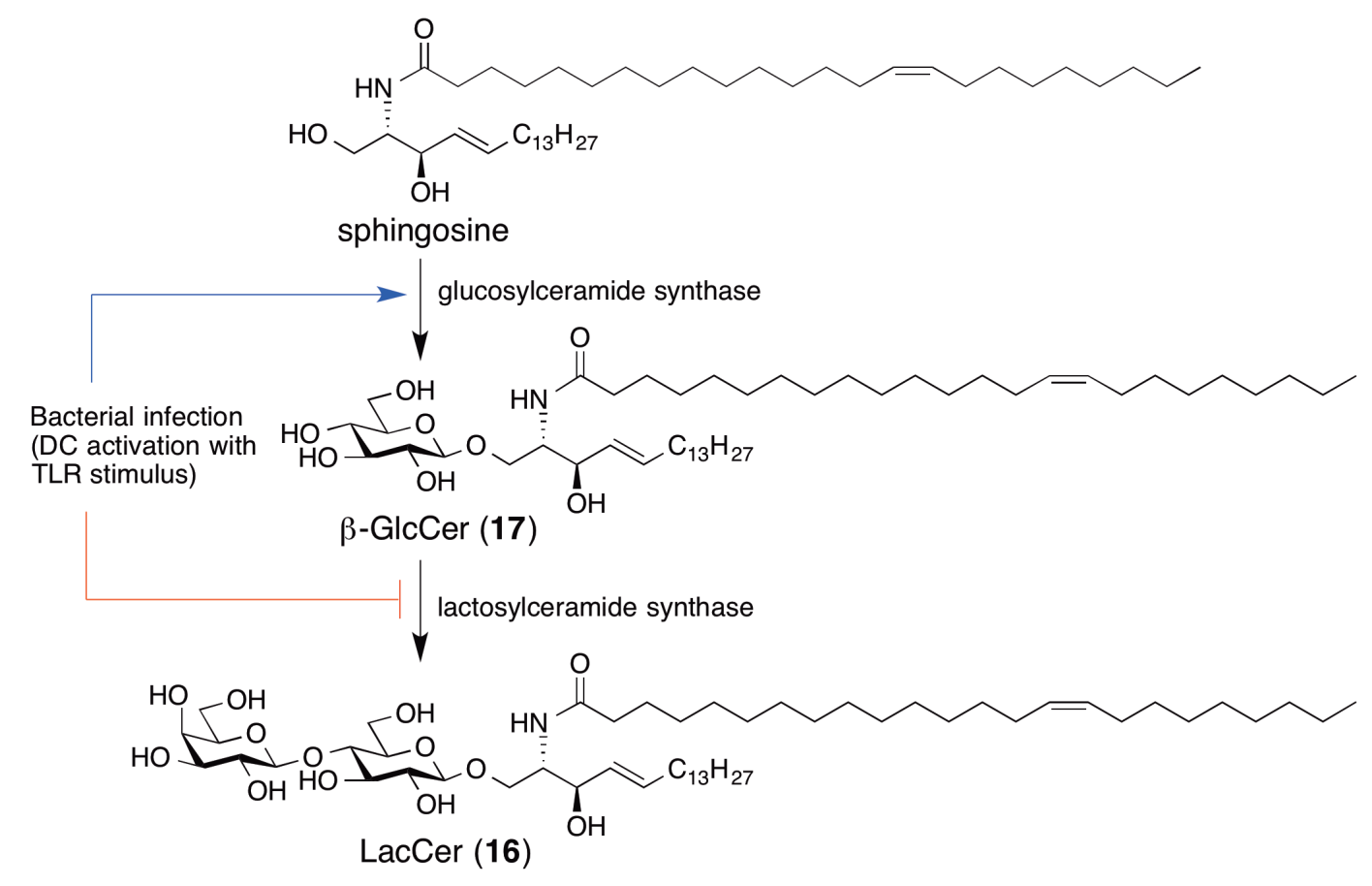

Scheme 1.2. Biosynthetic pathway for $\beta$-GlcCer (17). 


\subsubsection{Analogues of iGb3}

Several trisaccharide analogues of CD1d-binding iGb3 have been synthesised to probe the structural requirements of CD1d-binding $\beta$-linked glycosphingolipids. Modifications have been made to both the carbohydrate portion of the glycosphingolipid and to the lipid backbone. For example, Xia et al. synthesised two iGb3 analogues iGb3-phytosphingosine (18) and iGb3-sphinganine (19), each of which contain a modified ceramide backbone (Figure 1.8). ${ }^{135}$ In subsequent studies, they discovered that iGb3-phytosphingosine (18), which bears the same lipid backbone as $\alpha$-GalCer, was better at stimulating iNKT cells compared to the parent iGb3 (4) which bears a sphingosine backbone. Drawing from existing crystal structure studies of $\alpha$-GalCer with CD1d, the authors suggested that the 4-OH in phytosphingosine is able to provide an additional $\mathrm{H}$-bond with the $\mathrm{F}^{\prime}$ pocket of CD1d. However, it is important to note that this higher activity was only observed at low concentrations of the glycolipids $(10-100 \mathrm{ng} / \mathrm{mL})$. In addition, iGb3-sphinganine (19) also stimulated iNKT cells efficiently and it was rationalised that the absence of the $E$-alkene reduces the rigidity, of the lipid backbone, allowing for easier loading into the $\mathrm{F}^{\prime}$ pocket of CD1d. ${ }^{135}$

Savage and co-workers have also synthesised analogues of iGb3 and Gb3 with an $\alpha$-linkage between the proximal sugar and lipid portion, $\alpha \mathrm{GGb} 3(\mathbf{2 0})$ and $\alpha \mathrm{Gb} 3$ (21), and showed that akin to other monoglycosyl ceramides, the $\alpha$-versions were able to activate iNKT cells more effectively than their $\beta$-counterparts. ${ }^{136}$ 


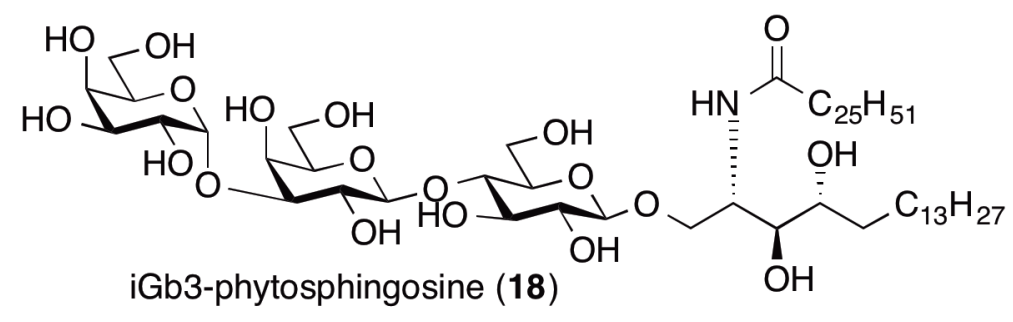

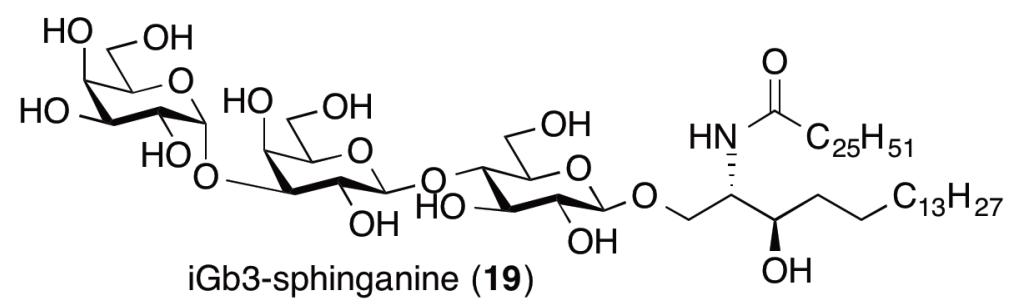

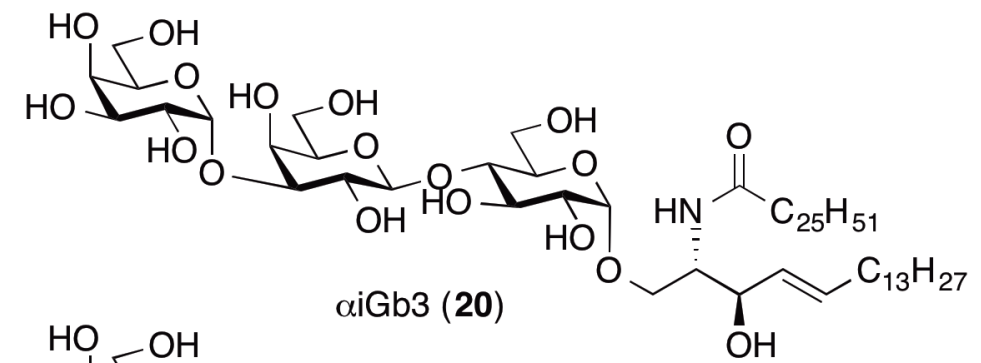

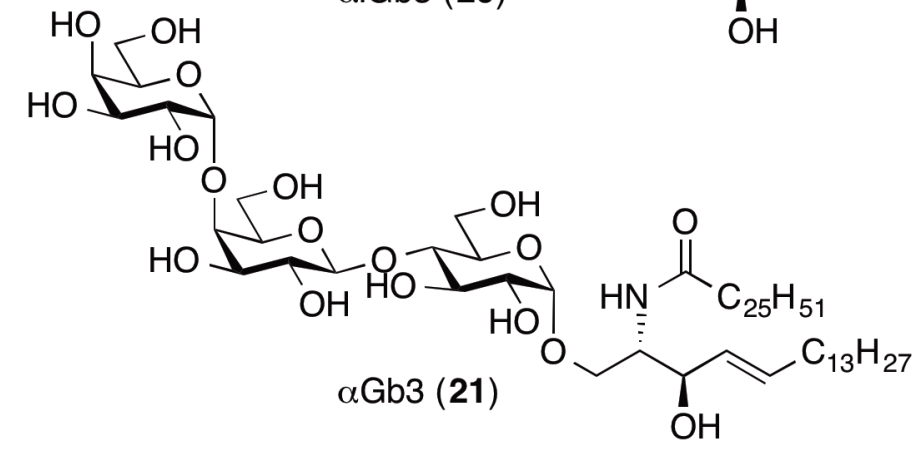

Figure 1.8. Analogues of iGb3. ${ }^{135,136}$

Despite the studies by Xia et. al. and the earlier work by Bendelac and coworkers, ${ }^{123,135}$ the ability of iGb3 to stimulate iNKT cells was still surprising as most known iNKT cell ligands were monoglycosyl ceramides. While it was already known that some diglycosyl ceramides, such as $\operatorname{Gal}(\alpha 1,2)-\alpha-\operatorname{GalCer}(\mathbf{2 2})$ (Figure 1.9) needed to be processed into the monoglycosyl version before iNKT cells can be stimulated. ${ }^{123}$ Here, the terminal galactose moiety of $\operatorname{Gal}(\alpha 1,2)-\alpha-$ GalCer (22) is hydrolysed by $\alpha$-galactosidase A in the lysosomal compartment of APCs, to give $\alpha$-GalCer, which is then loaded onto the CD1d and presented to an 
iNKT cell. While iGb3 can be processed into smaller fragments such as $\beta$-LacCer (23) and $\beta$-GlcCer (24) and stimulate iNKT cells, iGb3 was found to be able to activate iNKT cells in the absence of processing. ${ }^{117,123,137}$ Interestingly, a regioisomer of iGb3 that is found in mammalian cells, globotrihexosyl ceramide (Gb3, 25), varying only in the $\alpha 1,4$ linkage of the terminal galactose, was not able to stimulate iNKT cells. ${ }^{123,138}$ This indicated that the linkage of the terminal sugar is crucial for the observed activity of iGb3.

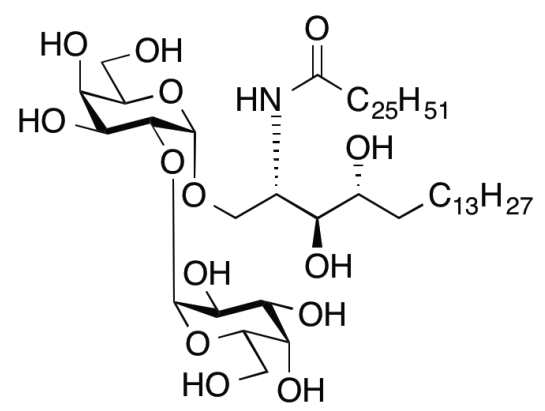

$\operatorname{Gal}(\alpha 1,2)-\alpha-$ GalCer (22)

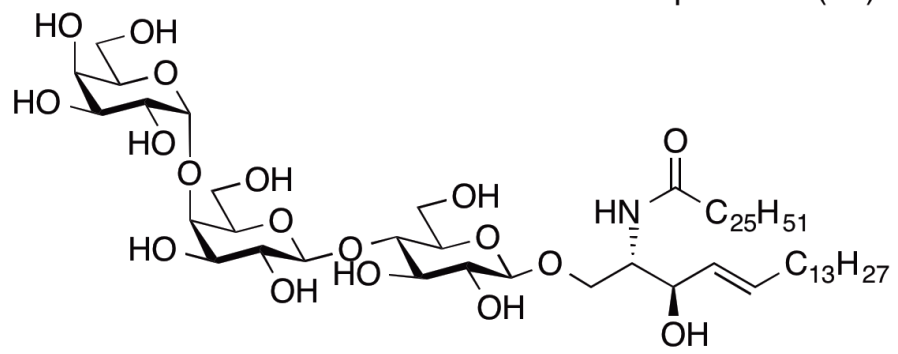

Gb3 (25)

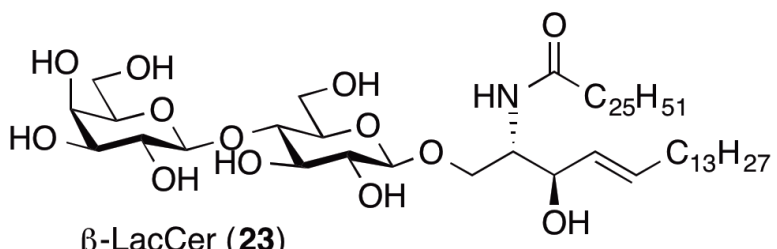

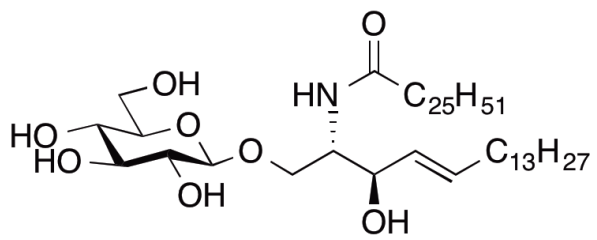

$\beta$-GlcCer (24)

Figure 1.9. Analogues of iGb3.

To probe the effect of the terminal galactose residue on the activity of iGb3, Chen et al. synthesised a series of dehydroxylated analogues of iGb3, whereby the hydroxyl groups on the terminal galactose were systematically removed to give 2'"'-deoxy- (26), 3"' -deoxy- (27), 4"''-deoxy- (28) and 6"'-deoxy-iGb3

(Figure 1.10). ${ }^{139}$ Of note, a phytosphingosine backbone was incorporated instead of the sphingosine lipid found on the original iGb3, with the intention of creating glycolipids with more potent iNKT cell stimulatory property. Testing of this series of iGb3 analogues revealed that the removal of hydroxyl groups at the 4"' and 6 "' -position did not significantly hamper the ability to stimulate iNKT cells, 
but the absence of the 2"'-OH or 3"' -OH reduced the agonist effect considerably. Thus, these studies further confirmed that the terminal sugar is critical for the activity of iGb3 and analogues thereof.

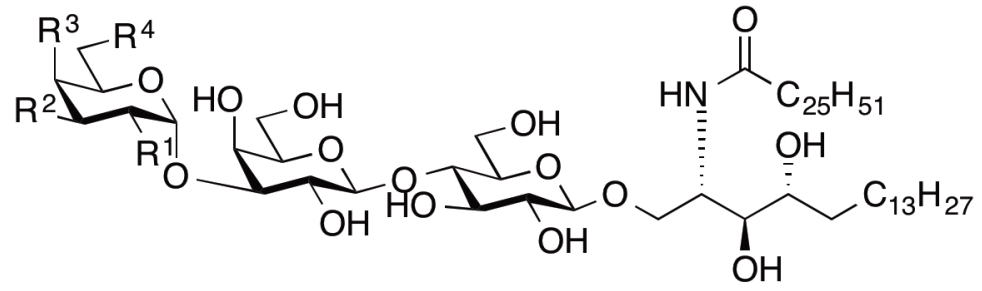

$2^{\prime \prime}$-deoxy-iGb3-phytosphingosine (26): $\mathrm{R}^{1}=\mathrm{H}, \mathrm{R}^{2}=\mathrm{OH}, \mathrm{R}^{3}=\mathrm{OH}, \mathrm{R}^{4}=\mathrm{OH}$ $3^{\prime \prime}$-deoxy-iGb3-phytosphingosine (27): $\mathrm{R}^{1}=\mathrm{OH}, \mathrm{R}^{2}=\mathrm{H}, \mathrm{R}^{3}=\mathrm{OH}, \mathrm{R}^{4}=\mathrm{Or}$ $4^{\prime \prime}$-deoxy-iGb3-phytosphingosine (28): $\mathrm{R}^{1}=\mathrm{OH}, \mathrm{R}^{2}=\mathrm{OH}, \mathrm{R}^{3}=\mathrm{H}, \mathrm{R}^{4}=\mathrm{Or}$ 6"'-deoxy-iGb3-phytosphingosine (29): $\mathrm{R}^{1}=\mathrm{OH}, \mathrm{R}^{2}=\mathrm{OH}, \mathrm{R}^{3}=\mathrm{OH}, \mathrm{R}^{4}=$ 卜

Figure 1.10. Deoxy analogues of iGb3 with phytosphingosine backbones. ${ }^{139}$ 


\subsection{Crystal structures of $\beta$-linked glycolipids}

Building from their seminal work in solving the crystal structure of $\alpha$-GalCer bound to CD1d, Rossjohn and co-workers solved the crystal structure of a mouse iNKT cell TCR in complex with mCD1d- $\beta$-GalCer and showed that the TCR docked onto the CD1d- $\beta-G a l C e r$ complex in a very similar manner to CD1d- $\alpha-$ GalCer (Figure 1.11). ${ }^{138}$ It is well established that when the lipid portion of $\alpha$ GalCer is embedded within the hydrophobic cavity of CD1d, the sugar headgroup that is exposed lies flat (parallel) against the $\alpha 2$-helix of $\mathrm{CD} 1 \mathrm{~d} .{ }^{82}$ One could anticipate that the sugar headgroup of $\beta$-GalCer protrudes out of the CD1d pocket so that it is perpendicular to the $\alpha 2$-helix, however, surprisingly, Rossjohn and coworkers observed that when the iNKT TCR engages with the CD1d- $\beta$-GalCer complex, the protruding sugar headgroup is "bulldozed" so that it lies flat against the $\alpha 2$-helix of CD1d, and hence occupies the space in a manner more similar to $\alpha$-GalCer. ${ }^{138}$ Of note, the hydrogen bond network between the $\beta$-linked galactose and the $\alpha 2$-helix of CD1d were analogous to the interactions found between CD1d and $\alpha$-GalCer, and moreover, the interactions between the iNKT TCR and CD1d were also conserved. Taken together, these crystal structures provide a molecular explanation for the ability of structurally distinct $\beta$-linked glycolipids to maintain their function as iNKT cell agonists. Nonetheless, the iNKT TCR has a lower affinity for $\beta$-GalCer compared to $\alpha$-GalCer, suggesting that an "energetic penalty" is imposed for "flattening" the $\beta$-linked glycolipid. ${ }^{138}$ 

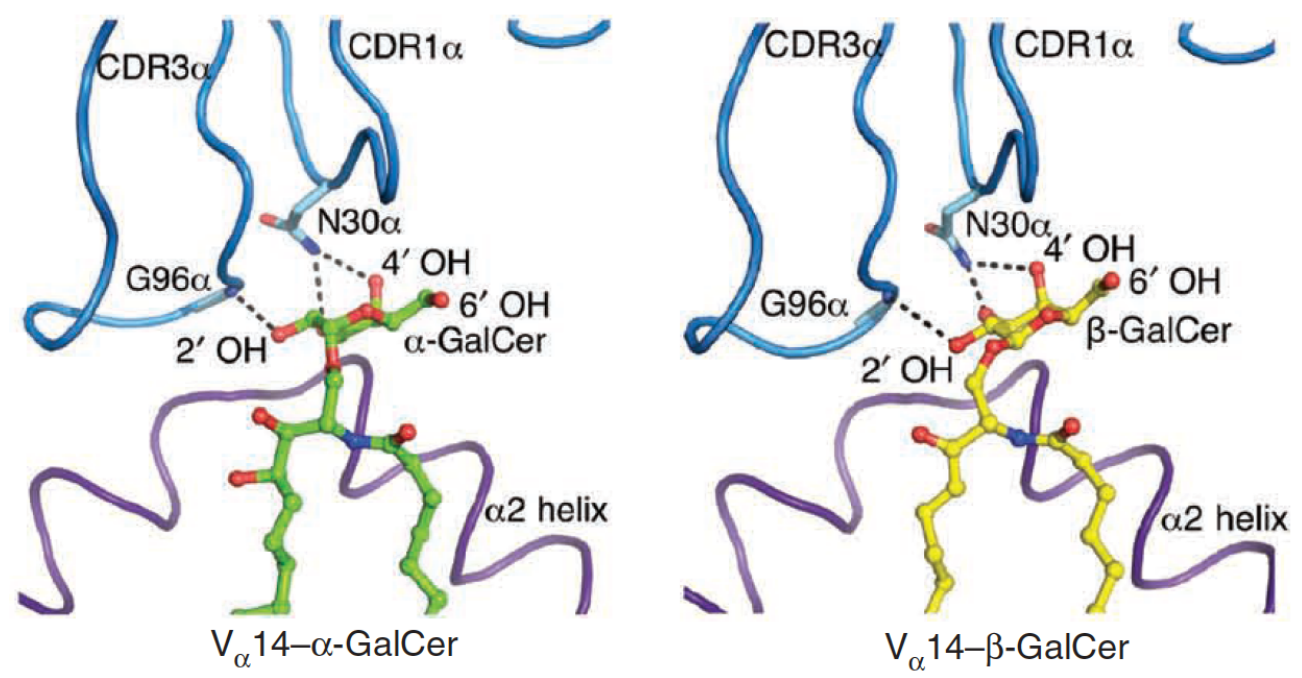

Figure 1.11. Crystal structure of mCD1d and TCR of iNKT cell with $\alpha$-GalCer (in green) and $\beta$-GalCer (in yellow). ${ }^{138}$ (Adapted by permission from Macmillan Publishers Ltd: Nature Immunology, 2011, 12, 827-833, (C2011)

The crystal structures of $\beta$-linked monoglycosyl ceramides formed the foundation for further studies of $\beta$-linked glycolipids with more than one sugar headgroup. In 2011, Yu et al. and Rossjohn and co-workers both reported the crystal structure of mouse iNKT cell TCR in complex with mCD1d-iGb3 and their results were in agreement. ${ }^{137,138}$ Both groups observed the flattening of the trisaccharide headgroup against the $\alpha 2$-helix of CD1d, similar to that observed for $\beta$-GalCer (Figure 1.12). The proximal glycosidic bond is "contorted" to resemble the $\alpha$-like conformation, with the $\beta$-Glc itself forming interactions with both CD1d and the iNKT cell TCR. The middle $\beta$-Gal also establishes many contacts with CD1d, and has some contact with the TCR. The terminal $\alpha$-Gal moiety, on the other hand, only interacts with the $\alpha 2$-helix of CD1d and not the TCR. Here, the terminal sugar is anchored by the hydrogen bond between the 6" $-\mathrm{OH}$ with Thr159 and the van der Waals interaction of 4 "'-OH and 6" $-\mathrm{OH}$ with Met162 of CD1d. In the absence of the terminal $\alpha$-Gal moiety, as in $\beta$-LacCer (23), the agonist activity of the glycolipid is abolished. In addition, a structural isomer Gb3 (25), does not stimulate iNKT cells because the terminal sugar $(\alpha 1,4-\mathrm{Gal})$ is oriented away from CD1d and does not form the interaction required to stabilise the trisaccharide headgroup. The loss of key interactions in the absence of the terminal $\alpha 1,3$-linked 
galactose results in the compromised avidity of $\beta$-LacCer (23) and Gb3 (25). Therefore, the engagement of the terminal sugar of iGb3 with CD1d is the prerequisite for the formation of a stable glycolipid conformation, giving rise to the iNKT cell agonist effect. ${ }^{138}$

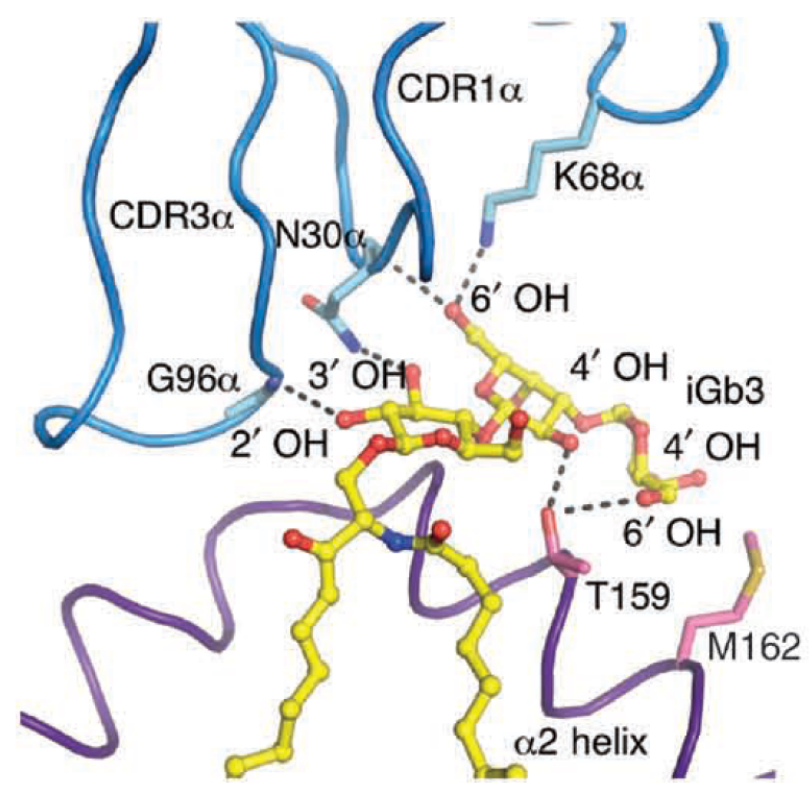

Figure 1.12. Crystal structure of mCD1d and TCR of iNKT cell with iGb3. ${ }^{138}$ (Adapted by permission from Macmillan Publishers Ltd: Nature Immunology, 2011, 12, 827-833, (C2011) 


\subsection{The significance of understanding $\beta$-linked self glycolipids}

The ability of iNKT cells to act as potent immune cells both for the innate and the adaptive immune response has driven much research to focus on developing glycolipid ligands that can invoke their activation. Current knowledge about CD1d presentation of glycolipids to iNKT cells and the associated downstream effects is largely attributed to the exogenous glycolipid $\alpha$-GalCer, however there is a growing appreciation of the ways in which $\beta$-linked self glycolipids modulate the immune response. Not only are $\beta$-linked self glycolipids responsible for the development of iNKT cells in the thymus, ${ }^{128,129}$ they also act as direct agonists during infection. ${ }^{101,131,133,134,140,141}$ Accordingly, the synergy of structure-activityrelationship studies and crystal structure analyses will provide a fuller picture of how $\beta$-linked glycolipids exert their effect on the immune system, with the view that this will then allow for the rational design of $\beta$-glycolipids for the treatment of diseases such as infections, autoimmune diseases and cancer. 


\subsection{Synthetic strategies for iGb3 and related analogues}

The first synthesis of trihexosyl ceramides dates back to 1987, when Koike et al. synthesised the iGb3 with a $\mathrm{C} 24 \mathrm{~N}$-acyl lipid along with Gb3 also bearing the C24 lipid (Scheme 1.3,n=22). ${ }^{142}$ The key step in this synthetic route was the coupling of a trisaccharide donor with a trichloroacetimidate leaving group 31 and the ceramide acceptor 32. The trisaccharide donor $\mathbf{3 1}$ was in turn obtained by coupling galactose donor $\mathbf{3 4}$ with a suitably protected lactose acceptor $\mathbf{3 5}$. It is important to note that the protecting group used at the 2-OH $\left(\mathrm{R}^{1} \mathrm{O}-\right)$ of lactose must be one that is capable of participating during the final glycosidation with the lipid acceptor 32 to facilitate the formation of the $\beta$-anomer. Schmidt and coworkers subsequently synthesised another iGb3 analogue with the $N$-palmitoyl (C16) lipid ( $n=14)$ using a very similar strategy, except that the trisaccharide donor was coupled in a stepwise fashion, first to the sphingosine acceptor, followed by functionalisation with the C16 acyl lipid. ${ }^{143}$ It was not until 2006, two years after iGb3 bearing a hexacosanoyl $(\mathrm{C} 26, n=24)$ lipid was first reported to be an important endogenous glycolipid for iNKT cell biology, that a flurry of syntheses of the prototypical iGb3-C26 analogue 4 were reported. ${ }^{144,145}$ Since then, the synthesis of various other derivatives such as the $\mathrm{C} 18$ homologue, ${ }^{146}$ thio-iGb3, ${ }^{147} 6^{\prime \prime \prime}$-deoxy analogues 26-29, ${ }^{139}$ phytosphingosine 18 and sphinganine 19 derivatives ${ }^{135}$ and $\alpha i G b 3$ analogue $\mathbf{2 0}^{139}$ have been published. Generally, the routes employed involve the coupling of a trisaccharide imidate donor with either a full ceramide lipid acceptor $\mathbf{3 2}$ or a 2-azido-sphingolipid acceptor 33. The alternative approach by first coupling the lactose donor to the ceramide backbone, followed by linkage of the terminal sugar has not been reported. While complete chemical synthesis of this glycolipid is the most common, some have also used enzymatic syntheses either to make the trisaccharide, ${ }^{145}$ or to furnish LacCer with the desired terminal galactose, in a highly stereospecific fashion. ${ }^{148}$ 


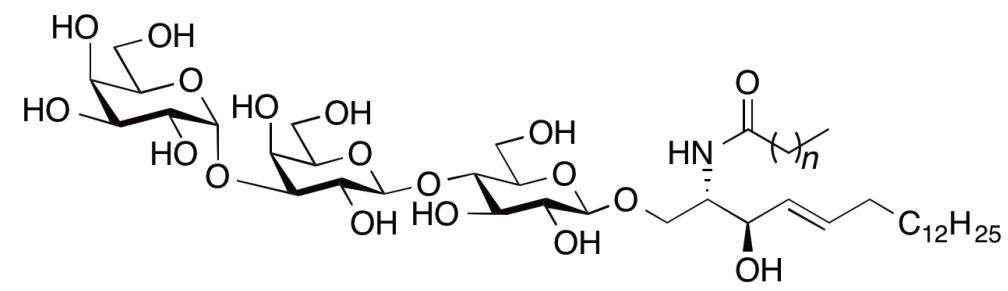

30

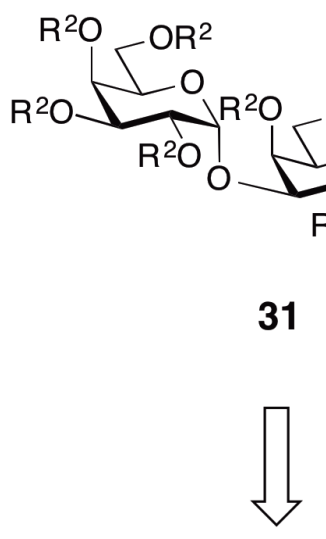

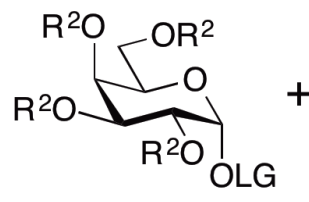

34

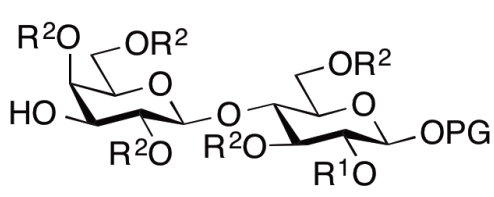

35<smiles>CCCCCCCCC(=O)NC(=O)OCCO</smiles>

or

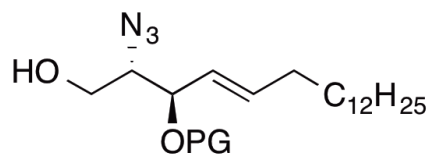

33

Scheme 1.3. Strategies for the synthesis iGb3 and analogues. 


\subsection{Thesis outline}

The research described in this thesis focuses on the synthesis of glycosphingolipids that can modulate the immune response, particularly in a cancer setting. To this end, the development of a variety of glycolipid probes which can be used as fluorescent tools to track the uptake and trafficking of $\alpha$-GalCer will be discussed, as will the synthesis of iGb3 derivatives that can be used in structure-activity studies to better understand the effects of these glycosphingolipids on iNKT cell activation. A brief outline of each thesis chapter is given below.

Chapter 2 describes the synthesis of the first $\alpha$-GalCer fluorescent probe, Dansyl- $\alpha$-GalCer 2 (Figure 1.13), which contains the dansyl fluorophore at the $6^{\prime}$ position of $\alpha$-GalCer. A new and highly efficient route for the synthesis of this probe is presented, and the ability of the probe to activate the immune response, as compared to the parent compound $\alpha-\mathrm{GalCer}$, is also discussed. Finally, the ability of the probe to act as a fluorescent tool to track the in vitro uptake of the glycolipids is also discussed.

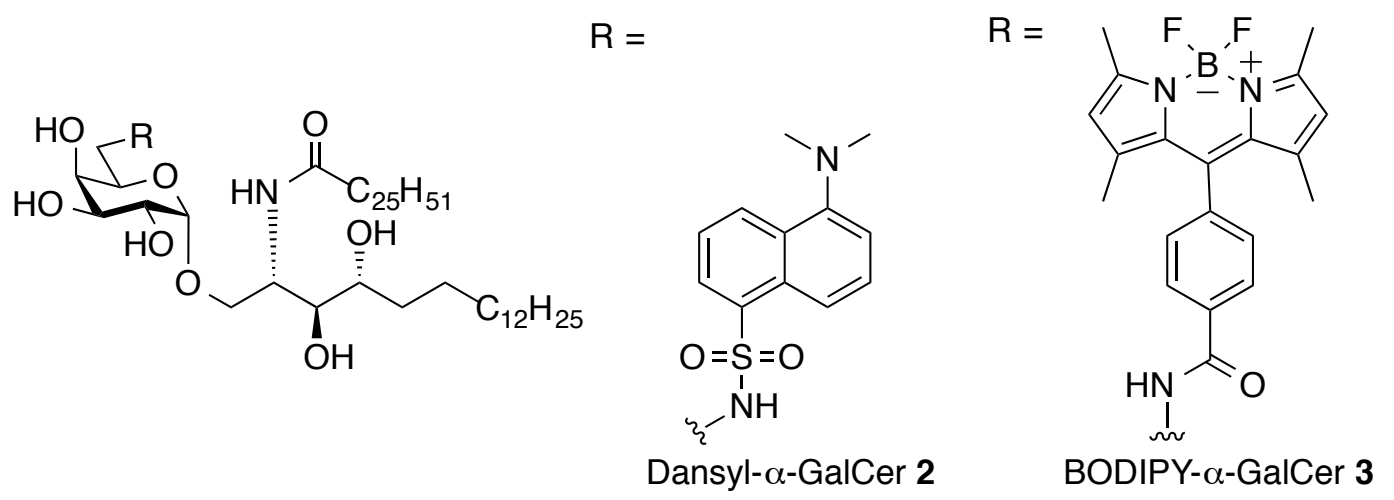

Figure 1.13. Fluorescent $\alpha$-GalCer probes

Chapter 3 then continues on with the development of fluorescent $\alpha$-GalCer probes and discusses the synthesis of BODIPY- $\alpha$-GalCer 3, which contains the BODIPY fluorophore at the 6'-position (Figure 1.13). A synthetic strategy, which 
is different to that used for the synthesis of dansyl- $\alpha$-GalCer, is presented and it is envisioned that the brighter BODIPY fluorophore will extend the range of biological experiments that can be undertaken using this probe. Finally, chapter 3 also discusses some of the limitations of the BODIPY fluorophore, the steps that need to be taken to ensure that the BODIPY- $\alpha$-GalCer 3 probe does not degrade, and initial studies into exploring the photo-reactivity of this fluorescent group.

Chapter 4 describes the synthesis of the first series of iGb3 derivatives, namely the 6 "'-deoxy analogues with either the sphingosine 5 or sphinganine $\mathbf{6}$ lipid backbone (Figure 1.14). This is the first time that such 6" '-deoxy homologues of iGb3 have been prepared and, in association with our collaborators, the subsequent biological evaluation and crystal structure studies using these substrates will allow for an assessment of the effect of the 6 "' $-\mathrm{OH}$ on the activity of iGb3, and also, how the lipid backbone effects glycolipid loading and iNKT cell activation. The route chosen for the synthesis of iGb3 sphingosine 5 or sphinganine $\mathbf{6}$ is highly efficient and moreover, allows for a convergent strategy that can be used to prepare not only sugar analogues, such as those discussed in this chapter, but also $N$-acyl chain derivatives. This is the first time that such a convergent route for the synthesis of iGb3 analogues will be described.
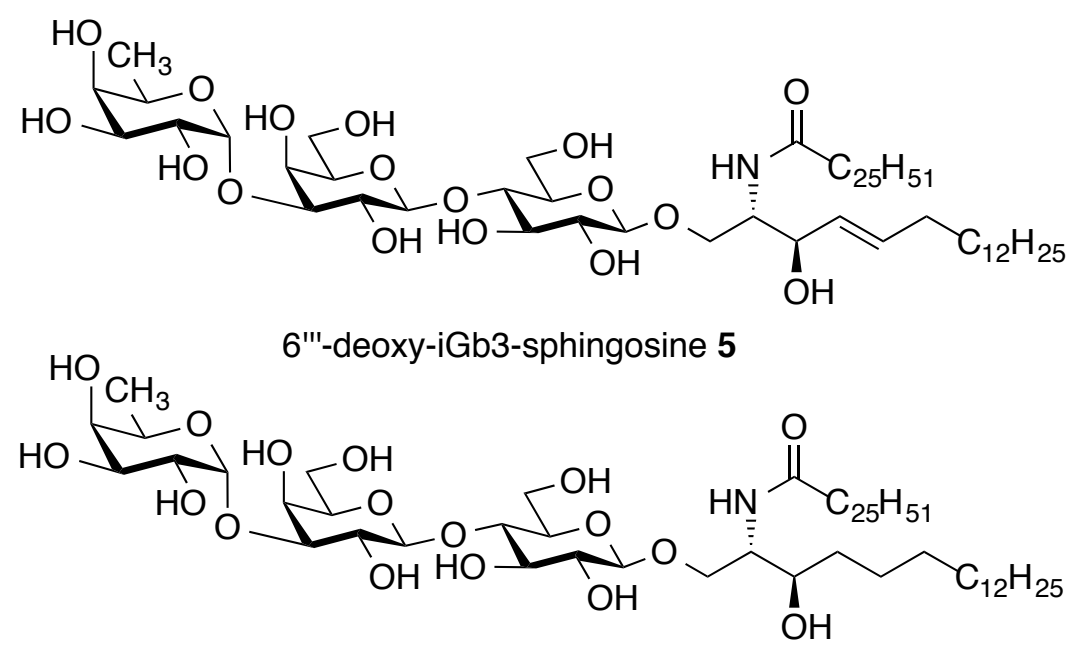

6"'-deoxy-iGb3-sphinganine 6

Figure 1.14. Target 6 "'-deoxy-iGb3 analogues. 
Chapter 5 describes the synthesis of two acyl chain homologues of iGb3. Key in the methodology is the use of a common intermediate presented in Chapter 4, which allows for the preparation of two $N$-acyl derivatives, namely the $\mathrm{C} 127$ and C20:2 8 homologues (Figure 1.15). The challenges and effectiveness of the synthetic route are presented, which can then be compared to the route used for the 6 "' -deoxy analogues. It is envisioned that the $\mathrm{N}$-acyl analogues, when compared to iGb3 itself, will allow for the effect of lipid length and saturation on iNKT cell activation to be determined.

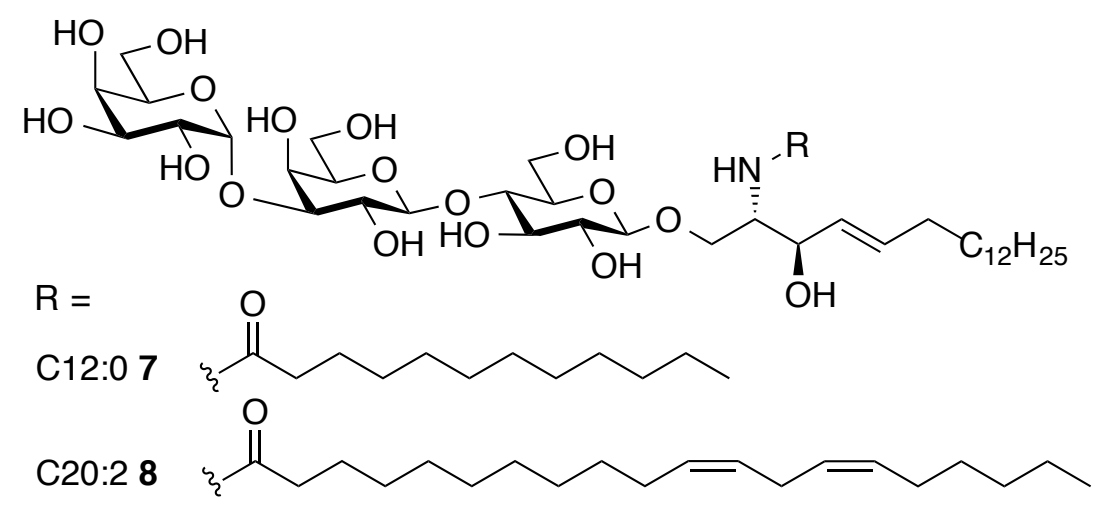

Figure 1.15. Target acyl chain homologues of iGb3.

Finally, Chapter 6 summarises the research performed in this thesis and highlights some future directions for each project area. 


\subsection{References}

1. WHO http://www.who.int/mediacentre/factsheets/fs297/en/index.html

2. Ministry of Health, Cancer: New Registrations and Deaths 2010 2013, Wellington: Ministry of Health.

3. Kardinal, C.; Yarbro, J., Semin. Oncol. 1979, 6, 396-408.

4. Wagener, D. J. T., The History of Oncology. Springer: Houten, The Netherlands, 2009.

5. Glasser, O., Wilhelm Conrad Röntgen and the early history of the Roentgen rays. Norman Publishing: California, 1993.

6. Swinton, A. A. C., Nature 1896, 53, 276-277.

7. Arthur, S., Nature 1896, 53, 274-276.

8. Goodman, L. S.; Wintrobe, M. M.; Dameshek, W.; Goodman, M. J.; Gilman, M. A.; McLennan, M. T., J. Am. Med. Assoc. 1946, 132, 126-132.

9. Van Parijs, L.; Abbas, A., Science 1998, 280, 243-248.

10. Billingham, R. E.; Brent, L.; Mendawar, P. B., Nature 1953, 172, 603-606.

11. Burnet, F. M., Lancet 1967, 1, 1171-1174.

12. Jensen, P.; Moller, B.; Hansen, S., J. Am. Acad. Dermatol. 2000, 42, 307.

13. Foley, E., Cancer Res. 1953, 13, 835-837.

14. Baldwin, R. W., Br. J. Cancer 1955, 9, 652-665.

15. Prehn, R. T.; Main, J. M., J. Natl. Cancer Inst. 1957, 18, 769-778.

16. Klein, G.; Sjogren, H. O.; Klein, E.; Hellstrom, K. E., Cancer Res. 1960, 1960, 1561-1572.

17. Boland, C. R.; Ricciardiello, L., Proc. Nat. Acad. Sci. 1999, 96, 1467514677.

18. Fenton, R. G.; Longo, D. L., J. Natl. Cancer Inst. 1995, 87, 241-243.

19. Visser, K. E., Cancer Immunol. Immun. 2008, 57, 1537-1539.

20. Janeway, C. A.; Travers, P.; Walport, M.; Shlomchik, M. J., In Immunobiology: the immune system in health and disease, Garland Science Publishing: New York, USA, 2005; pp 319-365.

21. Neefjes, J.; Jongsma, M. L. M.; Paul, P.; Bakke, O., Nat. Rev. Immunol. 2011, 11, 823-836.

22. Lin, M.-L.; Zhan, Y.; Villadangos, J. A.; Lew, A. M., Immunol. Cell Biol. 2008, $86,353-362$.

23. Reis e Sousa, C., Nat. Rev. Immunol. 2006, 6, 476-483.

24. Murugaiyan, G.; Martin, S.; Saha, B., Clin. Exp. Immunol. 2007, 149, 194202.

25. Trapani, J. A.; Smyth, M. J., Nat. Rev. Immunol. 2002, 2, 735-747.

26. Kaufman, H. L.; Wolchok, J. D., General principles of tumor immunotherapy: Basic and clinical applications of tumor immunology. Springer: Dordrecht, The Netherlands, 2007.

27. Davis, H. L., Adv. Drug Delivery. Rev. 1996, 21, 33-47.

28. Frazer, I. H., Nature 2004, 4, 46-54.

29. Michel, M. L.; Pontisso, P.; Sobczak, E.; Malpiece, Y.; Streeck, R. E.; Tiollais, P., Proc. Natl. Acad. Sci. U. S. A. 1984, 81, 7708-7712.

30. Moore, P. S.; Chang, Y., Nat Rev Cancer 2010, 10, 878-889. 
31. Rice, J.; Ottensmesier, C. H.; Stevenson, F. K., Nat. Rev. Cancer 2008, 8, 108-120.

32. Signori, E.; Iurescia, S.; Massi, E.; Fioretti, D.; Chiarella, P.; Robertis, M. D.; Rinaldi, M.; Tonon, G.; Fazio, V. M., Cancer Immuno. Immunoter. 2010, 59, 1583-1591.

33. Bins, A. D.; van den Berg, J. H.; Oosterhuis, K.; Haanen, J. B. A. G., Neth. J. Me. 2013, 71, 109-117.

34. Malero, I.; Hervas-Stubbs, S.; Glennie, M.; Pardoll, D. M.; Chen, L., Nat. Rev. Cancer 2007, 7, 95-106.

35. Peggs, K. S.; Quezada, S. A.; Korman, A. J.; Allison, J. P., Curr. Opin. Immunol. 2006, 18, 206-213.

36. Kamphorst, A. O.; Ahmed, R., Curr. Opin. Immunol. 2013, 25, 381-388.

37. Ho, W.; Blattman, J.; Dossett, M.; Yee, C.; Greenberg, P., Cancer Cell 2003, 3, 431-437.

38. Hinrichs, C. S.; Rosenberg, S. A., Immunol. Rev. 2014, 257, 56-71.

39. Perez, S. A.; Von Hofe, E.; Kallinteris, N. L.; Gritzapis, A. D.; Peoples, G. E.; Papamichail, M.; Baxevanis, C. N., Cancer 2010, 116, 2071-2080.

40. Pietersz, G. A.; Pouniotis, D. S.; Apostolopoulos, V., Curr. Med. Chem. 2006, 13, 1591-1607.

41. Noguchi, M.; Sasada, T.; Itoh, K., Cancer Immunol. Immunother. 2013, 62, 919-929.

42. Banchereau, J.; Palucka, A., Nat. Rev. Immunol. 2005, 5, 296-306.

43. Palucka, K.; Banchereau, J., Curr. Opin. Immunol. 2013, 25, 396-402.

44. Brower, V., 2010 2010, 102, 1108-1110.

45. Kavanagh, B.; O'Brien, S.; Lee, D.; Hou, Y.; Weinberg, V.; Rini, B.; Allison, J. P.; Small, E. J.; Fong, L., Blood 2008, 112, 1175-1183.

46. Fife, B. T.; Bluestone, J. A., Immunol. Rev. 2008, 224, 166-182.

47. Bachy, E.; Coiffier, B., Lancet Oncol 2014, 15, 7-8.

48. Westin, J. R.; Chu, F.; Zhang, M.; Fayad, L. E.; Kwak, L. W.; Fowler, N.; Romaguera, J.; Hagemeister, F.; Fanale, M.; Samaniego, F.; Feng, L.; Baladandayuthapani, V.; Wang, Z.; Ma, W.; Gao, Y.; Wallace, M.; Vence, L. M.; Radvanyi, L.; Muzzafar, T.; Rotem-Yehudar, R.; Davis, R. E.; Neelapu, S. S., Lancet Oncol. 2014, 15, 69-77.

49. Takamizawa, M.; Fagnoni, F.; Benike, C.; Kosek, J.; Hyakawa, H.; Engleman, E. G., J. Immunol. 1997, 158, 2134-2142.

50. Mesa, C.; Fernández, L. E., Immunol. Cell Biol. 2004, 82, 644-650.

51. Silk, J. D.; Hermans, I. F.; Gileadi, U.; Chong, T. W.; Shepherd, D.; Salio, M.; Mathew, B.; Schmidt, R. R.; Lunt, S. J.; Williams, K. J.; Stratford, I. J.; Harris, A. L.; Cerundolo, V., J. Clin. Invest. 2004, 114, 1800-1811.

52. Fay, J. W.; Palucka, A. K.; Paczesny, S.; Dhodapkar, M.; Johnston, D. A.; Burkeholder, S.; Ueno, H.; Banchereau, J., Cancer Immunol. Immun. 2006, $55,1209-1218$.

53. Giaccone, G.; Punt, C.; Ando, Y.; Ruijter, R.; Nishi, N.; Peters, M.; von Blomberg, B.; Scheper, R.; van der Vliet, H.; van den Eertwegh, A.; Roelvink, M.; Beijnen, J.; Zwierzina, H.; Pinedo, H., Clin. Cancer Res. 2002, 8, 3702-3709.

54. Chang, D. H.; Osman, K.; Connolly, J.; Kukreja, A.; Krasovsky, J.; Pack, M.; Hutchinson, A.; Geller, M.; Liu, N.; Annable, R.; Shay, J.; Kirchhoff, K.; Nishi, N.; Ando, Y.; Hayashi, K.; Hassoun, H.; Steinman, R. M.; Dhodapkar, M. V., J. Exp. Med. 2005, 201, 1503-1517. 
55. Ishikawa, A.; Motohashi, S.; Ishikawa, E.; Fuchida, H.; Higashino, K.; Otsuji, M.; Iizasa, T.; Nakayama, T.; Taniguchi, M.; Fujisawa, T., Clin. Cancer Res. 2005, 11, 1910-1917.

56. Nieda, M.; Okai, M.; Tazbirkova, A.; Lin, H.; Yamaura, A.; Ide, K.; Abraham, R.; Juji, T.; Macfarlane, D. J.; Nicol, A. J., Blood 2004, 103, 383 389.

57. Uchida, T.; Horiguchi, S.; Tanaka, Y.; Yamamoto, H.; Kunii, N.; Motohashi, S.; Taniguchi, M.; Nakayama, T.; Okamoto, Y., Cancer Immunol. Immun. 2008, 57, 337-345.

58. Motohashi, S.; Nagato, K.; Kunii, N.; Yamamoto, H.; Yamasaki, K.; Okita, K.; Hanaoka, H.; Shimizu, N.; Suzuki, M.; Yoshino, I.; Taniguchi, M.; Fujisawa, T.; Nakayama, T., J. Immunol. 2009, 182, 2492-2501.

59. Fujii, S.-I.; Shimizu, K.; Hemmi, H.; Steinman, R. M., Immunol. Rev. 2007, 220, 183-198.

60. Wu, D.; Fujio, M.; Wong, C.-H., Bioorg. Med. Chem. Lett. 2008, 16, 10731083.

61. Godfrey, D. I.; Stankovic, S.; Baxter, A. G., Nat. Immunol. 2010, 11, 197206.

62. Godfrey, D. I.; Pellicci, D. G.; Patel, O.; Kjer-Nielsen, L.; McCluskey, J.; Rossjohn, J., Semin. Immunol. 2010, 22, 61-67.

63. Godfrey, D. I.; MacDonald, H. R.; Kronenberg, M.; Smyth, M. J.; Van Kaer, L., Nat. Rev. Immunol. 2004, 4, 231-237.

64. Van Kaer, L., Nat. Rev. Immunol. 2005, 5, 31-42.

65. Zajonc, D. M.; Kronenberg, M., Curr. Opin. Struct. Biol. 2007, 17, 521-529.

66. Godfrey, D. I.; Pellicci, D. G.; Smyth, M. J., Science 2004, 306, 1687-1689.

67. Akimoto, K.; Natori, T.; Morita, M., Tetrahedron Lett. 1993, 34, 55935596.

68. Natori, T.; Koezuka, Y.; Higa, T., Tetrahedron Lett. 1993, 34, 5591-5592.

69. Morita, M.; Motoki, K.; Akimoto, K.; Natori, T.; Sakai, T.; Sawa, E.; Yamaji, K.; Koezuka, Y.; Kobayashi, E.; Fukushima, H., J. Med. Chem. 1995, 38, 2176-2187.

70. Bendelac, A., J. Exp. Med. 1995, 182, 2091-2096.

71. Kawano, T.; Cui, J.; Koezuka, Y.; Toura, I.; Kaneko, Y.; Motoki, K.; Ueno, H.; Nakagawa, R.; Sato, H.; Kondo, E.; Koseki, H.; Taniguchi, M., Science 1997, 278, 1626-1629.

72. Plettenburg, O.; Bodmer-Narkevitch, V.; Wong, C., J. Org. Chem. 2002, 67, 4559-4564.

73. Fan, G.; Pan, Y.; Lu, K.; Cheng, Y.; Lin, W.; Lin, S.; Lin, C.; Wong, C.; Fang, J.; Lin, C., Tetrahedron 2005, 61, 1855-1862.

74. Lee, A.; Farrand, K. J.; Dickgreber, N.; Hayman, C. M.; Jurs, S.; Hermans, I. F.; Painter, G. F., Carbohydr. Res. 2006, 341, 2785-2798.

75. Park, J.-J.; Lee, J. H.; Ghosh, S. C.; Bricard, G.; Venkataswamy, M. M.; Porcelli, S. A.; Chung, S.-K., Bioorg. Med. Chem. Lett. 2008, 18, 39063909.

76. Tashiro, T.; Hongo, N.; Nakagawa, R.; Seino, K.-i.; Watarai, H.; Ishii, Y.; Taniguchi, M.; Mori, K., Bioorg. Med. Chem. 2008, 16, 8896-8906.

77. Tashiro, T.; Nakagawa, R.; Inoue, S.; Shiozaki, M.; Watarai, H.; Taniguchi, M.; Mori, K., Tetrahedron Lett. 2008, 49, 6827-6830.

78. Tashiro, T.; Nakagawa, R.; Hirokawa, T.; Inoue, S.; Watarai, H.; Taniguchi, M.; Mori, K., Bioorg. Med. Chem. 2009, 17, 6360-6373. 
79. Banchet-Cadeddu, A.; Hénon, E.; Dauchez, M.; Renault, J.-H.; Monneaux, F.; Haudrechy, A., Org. Biomol. Chem. 2011, 9, 3080-3104.

80. Baek, D. J.; Seo, J.-H.; Lim, C.; Kim, J. H.; Chung, D. H.; Cho, W.-J.; Kang, C.-Y.; Kim, S., ACS Med. Chem. Lett. 2011, 2, 544-548.

81. Calabi, F.; Jarvis, J. M.; Martin, L.; Milstein, C., Eur. J. Immunol. 1989, 19, 285-292.

82. Borg, N. A.; Wun, K. S.; Kjer-Nielsen, L.; Wilce, M. C.; Pellicci, D. G.; Koh, R.; Besra, G. S.; Bharadwaj, M.; Godfrey, D. I.; McCluskey, J.; Rossjohn, J., Nature 2007, 448, 44-49.

83. Koch, M.; Stronge, V.; Shepherd, D.; Gadola, S.; Mathew, B.; Ritter, G.; Fersht, A.; Besra, G.; Schmidt, R.; Jones, E.; Cerundolo, V., Nat. Immunol. 2005, 6, 819-826.

84. Crozat, K.; Guiton, R.; Guilliams, M.; Henri, S.; Baranek, T.; SchwartzCornil, I.; Malissen, B.; Dalod, M., Immunol. Rev. 2010, 234, 177-198.

85. Sanderson, J. P.; Brennan, P. J.; Mansour, S.; Matulis, G.; Patel, O.; Lissin, N.; Godfrey, D. I.; Kawahara, K.; Zähringer, U.; Rossjohn, J.; Brenner, M. B.; Gadola, S. D., Eur. J. Immunol. 2013, 43, 815-825.

86. Dangerfield, E. M.; Cheng, J. M. H.; Knight, D. A.; Weinkove, R.; Dunbar, P. R.; Hermans, I. F.; Timmer, M. S. M.; Stocker, B. L., ChemBioChem 2012, 13, 1349-1356.

87. Li, X.; Shiratsuchi, T.; Chen, G.; Dellabona, P.; Casorati, G.; Franck, R. W.; Tsuji, M., J. Immunol. 2009, 183, 4415-4421.

88. Kjer-Nielsen, L.; Borg, N. A.; Pellicci, D. G.; Beddoe, T.; Kostenko, L.; Clements, C. S.; Williamson, N. A.; Smyth, M. J.; Besra, G. S.; Reid, H. H.; Bharadwaj, M.; Godfrey, D. I.; Rossjohn, J.; McCluskey, J., J. Exp. Med. 2006, 203, 661-673.

89. Cerundolo, V.; Silk, J. D.; Masri, S. H.; Salio, M., Nat. Rev. Immunol. 2009, 9, 28-38.

90. Hermans, I. F.; Silk, J. D.; Gileadi, U.; Salio, M.; Mathew, B.; Ritter, G.; Schmidt, R.; Harris, A. L.; Old, L.; Cerundolo, V., J. Immunol. 2003, 171, 5140-5147.

91. Gonzalez-Aseguinolaza, G.; Van Kaer, L.; Bergmann, C. C.; Wilson, J. M.; Schmieg, J.; Kronenberg, M.; Nakayama, T.; Taniguchi, M.; Koezuka, Y.; Tsuji, M., J. Exp. Med. 2002, 195, 617-624.

92. Fujii, S. I.; Shimizu, K.; Smith, C.; Bonifaz, L.; Steinman, R. M., J. Exp. Med. 2003, 198, 267-279.

93. Villadangos, J. A.; Schnorrer, P., Nat. Rev. Immunol. 2007, 7, 543-555.

94. Liu, K.; Nussenzweig, M. C., Eur. J. Immunol. 2010, 40, 2099-2102.

95. Palucka, K.; Ueno, H.; Zurawski, G.; Fay, J.; Banchereau, J., Curr. Opin. Immunol. 2010, 22, 258-263.

96. Palucka, K.; Banchereau, J.; Mellman, I., Immunity 2010, 33, 464-478.

97. Benlagha, K.; Weiss, A.; Beavis, A.; Teyton, L.; BendelaC, A., J. Exp. Med. 2000, 191, 1895-903.

98. Matsuda, J. L.; Naidenko, O. V.; Gapin, L.; Nakayama, T.; Taniguchi, M.; Wang, C. R.; Koezuka, Y.; Kronenberg, M., J. Exp. Med. 2000, 192, 74154.

99. Karadimitris, A.; Gadola, S.; Altamirano, M.; Brown, D.; Woolfson, A.; Klenerman, P.; Chen, J.; Koezuka, Y.; Roberts, I.; Price, D.; Dusheiko, G.; Milstein, C.; Fersht, A.; Luzzatto, L.; Cerundolo, V., Proc. Natl. Acad. Sci. U. S. A. 2001, 98, 3294-3298. 
100. Wu, D.; Zajonc, D.; Fujio, M.; Sullivan, B.; Kinjo, Y.; Kronenberg, M.; Wilson, I.; Wong, C., Proc. Natl. Acad. Sci. U. S. A. 2006, 103, 3972-3977.

101. Salio, M.; Speak, A. O.; Shepherd, D.; Polzella, P.; Illarionov, P. A.; Veerapen, N.; Besra, G. S.; Platt, F. M.; Cerundolo, V., Proc. Natl. Acad. Sci. U. S. A. 2007, 104, 20490-20495.

102. Vo-Hoang, Y.; Micouin, L.; Ronet, C.; Gachelin, G.; Bonin, M., ChemBioChem 2003, 4, 27-33.

103. Zhou, X.; Forestier, C.; Goff, R.; Li, C.; Teyton, L.; Bendelac, A.; Savage, P. B., Org. Lett. 2002, 4, 1267-1270.

104. Bai, L.; Sagiv, Y.; Liu, Y.; Freigang, S.; Yu, K. O. A.; Teyton, L.; Porcelli, S. A.; Savage, P. B.; Bendelac, A., Proc. Natl. Acad. Sci. U. S. A. 2009, 106, 10254-10259.

105. Sakai, T.; Naidenko, O. V.; Iijima, H.; Kronenberg, M.; Koezuka, Y., J. Med. Chem. 1999, 42, 1836-1841.

106. Xia, C.; Zhang, W.; Zhang, Y.; Woodward, R. L.; Wang, J.; Wang, P. G., Tetrahedron 2009, 65, 6390-6395.

107. Barral, D. C.; Brenner, M. B., Nat. Rev. Immunol. 2007, 7, 929-941.

108. Sakai, T.; Ehara, H.; Koezuka, Y., Org. Lett. 1999, 1, 359-361.

109. Zeng, Z.; Castaño, A. R.; Segelke, B. W.; Stura, E. A.; Peterson, P. A.; Wilson, I. A., Science 1997, 277, 339-345.

110. Naidenko, O. V.; Maher, J. K.; Ernst, W. A.; Sakai, T.; Modlin, R. L.; Kronenberg, M., J. Exp. Med. 1999, 190, 1069-1080.

111. Moody, D. B.; Briken, V.; Cheng, T. Y.; Roura-Mir, C.; Guy, M. R.; Geho, D. H.; Tykocinski, M. L.; Besra, G. S.; Porcelli, S. A., Nat. Immunol. 2002, 3, 435-442.

112. Mukherjee, S.; Soe, T.; Maxfield, F., J. Cell Biol. 1999, 144, 1271-1284.

113. Singh, R. D.; Liu, Y.; Wheatley, C. L.; Holicky, E. L.; Makino, A.; Marks, D. L.; Kobayashi, T.; Subramaniam, G.; Bittman, R.; Pagano, R. E., J. Biol. Chem. 2006, 281, 30660-30668.

114. Kamada, N.; Iijima, H.; Kimura, K.; Harada, M.; Shimizu, E.; Motohashi, S. I.; Kawano, T.; Shinkai, H.; Nakayama, T.; Sakai, T.; Brossay, L.; Kronenberg, M.; Taniguchi, M., Int. Immunol. 2001, 13, 853-861.

115. Prigozy, T.; Naidenko, O.; Qasba, P.; Elewaut, D.; Brossay, L.; Khurana, A.; Natori, T.; Koezuka, Y.; Kulkarni, A.; Kronenberg, M., Science 2001, 291, 664-667.

116. Brossay, L.; Naidenko, O.; Burdin, N.; Matsuda, J.; Sakai, T.; Kronenberg, M., J. Immunol. 1998, 161, 5124-8.

117. Ortaldo, J. R.; Young, H. A.; Winkler-Pickett, R. T.; Bere, E. W.; Murphy, W. J.; Wiltrout, R. H., J. Immunol. 2004, 172, 943-53.

118. Parekh, V. V.; Singh, A. K.; Wilson, M. T.; Olivares-Villagómez, D.; Bezbradica, J. S.; Inazawa, H.; Ehara, H.; Sakai, T.; Serizawa, I.; Wu, L.; Wang, C.-R.; Joyce, S.; Van Kaer, L., J. Immunol. 2004, 173, 3693-706.

119. Sidobre, S.; Hammond, K. J. L.; Bénazet-Sidobre, L.; Maltsev, S. D.; Richardson, S. K.; Ndonye, R. M.; Howell, A. R.; Sakai, T.; Besra, G. S.; Porcelli, S. A.; Kronenberg, M., Proc. Natl. Acad. Sci. U. S. A. 2004, 101, 12254-9.

120. Bendelac, A.; Savage, P. B.; Teyton, L., Annu. Rev. Immunol. 2007, 25, 297-336.

121. Chiu, Y.-H.; Park, S.-H.; Benlagha, K.; Forestier, C.; Jayawardena-Wolf, J.; Savage, P. B.; Teyton, L.; Bendelac, A., Nat. Immunol. 2002, 3, 55-60. 
122. Godfrey, D. I.; Rossjohn, J., J. Exp. Med. 2011, 208, 1121-1125.

123. Zhou, D.; Mattner, J.; Cantu, C.; Schrantz, N.; Yin, N.; Gao, Y.; Sagiv, Y.; Hudspeth, K.; Wu, Y.-P.; Yamashita, T.; Teneberg, S.; Wang, D.; Proia, R. L.; Levery, S. B.; Savage, P. B.; Teyton, L.; Bendelac, A., Science 2004, 306, 1786-1789.

124. Speak, A. O.; Salio, M.; Neville, D. C. A.; Fontaine, J.; Priestman, D. A.; Platt, N.; Heare, T.; Butters, T. D.; Dwek, R. A.; Trottein, F.; Exley, M. A.; Cerundolo, V.; Platt, F. M., Proc. Natl. Acad. Sci. U. S. A. 2007, 104, 59716.

125. Porubsky, S.; Speak, A. O.; Luckow, B.; Cerundolo, V.; Platt, F. M.; Gröne, H.-J., Proc. Natl. Acad. Sci. U. S. A. 2007, 104, 5977-5982.

126. Christiansen, D.; Milland, J.; Mouhtouris, E.; Vaughan, H.; Pellicci, D. G.; Mcconville, M. J.; Godfrey, D. I.; Sandrin, M. S., PLoS Biol. 2008, 6, e172.

127. Gadola, S. D.; Silk, J. D.; Jeans, A.; Illarionov, P. A.; Salio, M.; Besra, G. S.; Dwek, R.; Butters, T. D.; Platt, F. M.; Cerundolo, V., J. Exp. Med. 2006, 203, 2293-2303.

128. Porubsky, S.; Speak, A. O.; Salio, M.; Jennemann, R.; Bonrouhi, M.; Zafarulla, R.; Singh, Y.; Dyson, J.; Luckow, B.; Lehuen, A.; Malle, E.; Müthing, J.; Platt, F. M.; Cerundolo, V.; Gröne, H.-J., J. Immunol. 2012, 189, 3007-3017.

129. Brennan, P. J.; Tatituri, R. V. V.; Brigl, M.; Kim, E. Y.; Tuli, A.; Sanderson, J. P.; Gadola, S. D.; Hsu, F.-F.; Besra, G. S.; Brenner, M. B., Nat. Immunol. 2011, 12, 1202-1211.

130. Brigl, M.; Bry, L.; Kent, S. C.; Gumperz, J. E.; Brenner, M. B., Nat. Immunol. 2003, 4, 1230-1237.

131. Mattner, J.; Debord, K. L.; Ismail, N.; Goff, R. D.; Cantu, C.; Zhou, D.; Saint-Mezard, P.; Wang, V.; Gao, Y.; Yin, N.; Hoebe, K.; Schneewind, O.; Walker, D.; Beutler, B.; Teyton, L.; Savage, P. B.; Bendelac, A., Nature 2005, 434, 525-529.

132. Nagarajan, N. A.; Kronenberg, M., J. Immunol. 2007, 178, 2706-13.

133. Paget, C.; Mallevaey, T.; Speak, A. O.; Torres, D.; Fontaine, J.; Sheehan, K. C. F.; Capron, M.; Ryffel, B.; Faveeuw, C.; Leite de Moraes, M.; Platt, F.; Trottein, F., Immunity 2007, 27, 597-609.

134. Brigl, M.; Tatituri, R. V. V.; Watts, G. F. M.; Bhowruth, V.; Leadbetter, E. A.; Barton, N.; Cohen, N. R.; Hsu, F.-F.; Besra, G. S.; Brenner, M. B., J. Exp. Med. 2011, 208, 1163-1177.

135. Xia, C.; Schümann, J.; Emmanuel, R.; Zhang, Y.; Chen, W.; Zhang, W.; De Libero, G.; Wang, P. G., J. Med. Chem. 2007, 50, 3489-3496.

136. Yin, N.; Long, X.; Goff, R. D.; Zhou, D.; Cantu, C.; Mattner, J.; Mezard, P. S.; Teyton, L.; Bendelac, A.; Savage, P. B., ACS Chem. Biol. 2009, 4, 191197.

137. Yu, E. D.; Girardi, E.; Wang, J.; Zajonc, D. M., J. Immunol. 2011, 187, 2079-2083.

138. Pellicci, D. G.; Clarke, A. J.; Patel, O.; Mallevaey, T.; Beddoe, T.; Le Nours, J.; Uldrich, A. P.; McCluskey, J.; Besra, G. S.; Porcelli, S. A.; Gapin, L.; Godfrey, D. I.; Rossjohn, J., Nat. Immunol. 2011, 12, 827-833.

139. Chen, W.; Xia, C.; Wang, J.; Thapa, P.; Li, Y.; Talukdar, A.; Nadas, J.; Zhang, W.; Zhou, D.; Wang, P. G., J. Org. Chem. 2007, 72, 9914-9923. 
140. Stanic, A. K.; De Silva, A. D.; Park, J.-J.; Sriram, V.; Ichikawa, S.; Hirabyashi, Y.; Hayakawa, K.; Van Kaer, L.; Brutkiewicz, R. R.; Joyce, S., Proc. Natl. Acad. Sci. U. S. A. 2003, 100, 1849-1854.

141. Darmoise, A.; Teneberg, S.; Bouzonville, L.; Brady, R. O.; Beck, M.; Kaufmann, S. H. E.; Winau, F., Immunity 2010, 33, 216-228.

142. Koike, K.; Sugimoto, M.; Sato, S.; Ito, Y.; Nakahara, Y.; Ogawa, T., Carbohydr. Res. 1987, 163, 189-208.

143. Qiu, D.; Schmidt, R. R., Liebigs Ann. Chem. 1992, 1992, 217-224.

144. Xia, C.; Yao, Q.; Schumann, J.; Rossy, E.; Chen, W.; Zhu, L.; Zhang, W.; De Libero, G.; Wang, P., Bioorg. Med. Chem. Lett. 2006, 16, 2195-2199.

145. Yao, Q.; Song, J.; Xia, C.; Zhang, W.; Wang, P. G., Org. Lett. 2006, 8, 911914.

146. Kimura, A.; Imamura, A.; Ando, H.; Ishida, H.; Kiso, M., Synlett 2006, 2379-2382.

147. Xia, C.; Zhou, D.; Liu, C.; Lou, Y.; Yao, Q.; Zhang, W.; Wang, P. G., Org. Lett. 2006, 8, 5493-5496.

148. Adlercreutz, D.; Weadge, J. T.; Petersen, B. O.; Duus, J. Ø.; Dovichi, N. J.; Palcic, M. M., Carbohydr. Res. 2010, 345, 1384-1388. 


\section{Chapter 2. The synthesis and biological evaluation of dansyl $\alpha$-galactosylceramide as a fluorescent probe for the monitoring of glycolipid uptake by cells}

This chapter is an edited version of the following publication:

Cheng, J. M. H.; Chee, S. H.; Knight, D. A.; Acha-Orbea, H.; Hermans, I. F.; Timmer, M. S. M.; Stocker, B. L. 'An improved synthesis of dansylated $\alpha$-galactosylceramide and its use as a fluorescent probe for the monitoring of glycolipid uptake by cells.' Carbohydr. Res. 2011, 346, 914-926.

As the principal author, I carried out all the chemical transformations described in this paper and the biological testings were performed at the Malaghan Institute of Medical Research under the supervision of I. F. H. and D. A. K. and I played a major role in the writing this paper.

\subsection{Introduction}

The potent glycosphingolipid adjuvant, $\alpha$-galactosylceramide ( $\alpha$-GalCer 1, Figure 2.1 ) is a structural analogue of agelasphin-9b (AGL-9b, 9) that was isolated from the marine sponge Agelas mauritianus. ${ }^{1-3} \alpha-G a l C e r$ is able to activate a subset of $\mathrm{T}$ cells known as invariant natural killer $\mathrm{T}(i \mathrm{NKT})$ cells when it is presented in the context of CD1d [a member of the CD1 family of proteins found on the surface of antigen presenting cells (APCs)]. ${ }^{4-6} i \mathrm{NKT}$ cells represent a potent type of $\mathrm{T}$ cell, and the ability for glycosphingolipids to modulate their activation makes them useful targets for the treatment of several diseases including cancer, malaria, type I diabetes, and multiple sclerosis. 


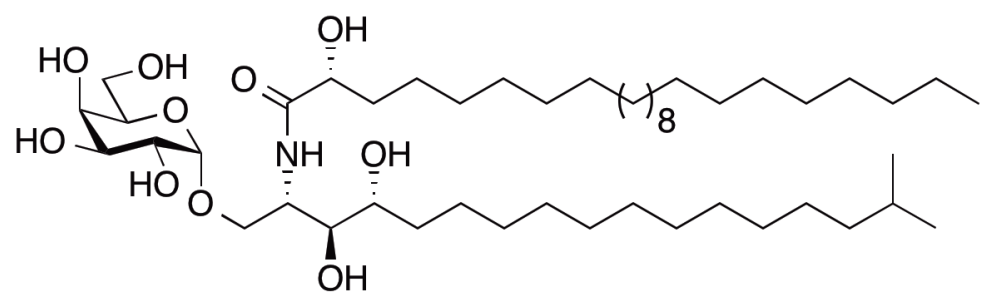

Agelasphin-9b (AGL-9b, 9)

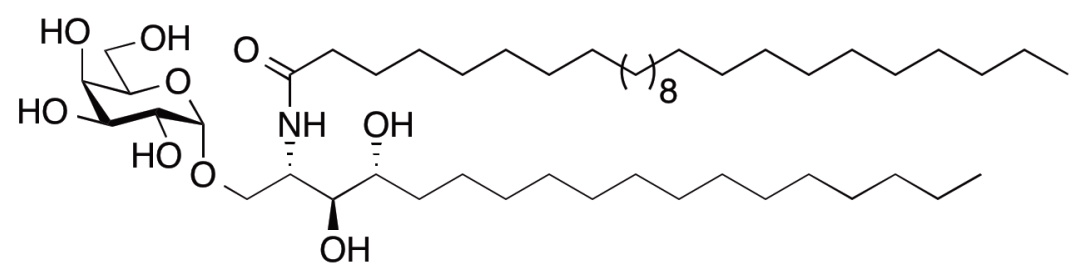

KRN7000 or $\alpha$-Galactosyl ceramide ( $\alpha$-GalCer, 1 )

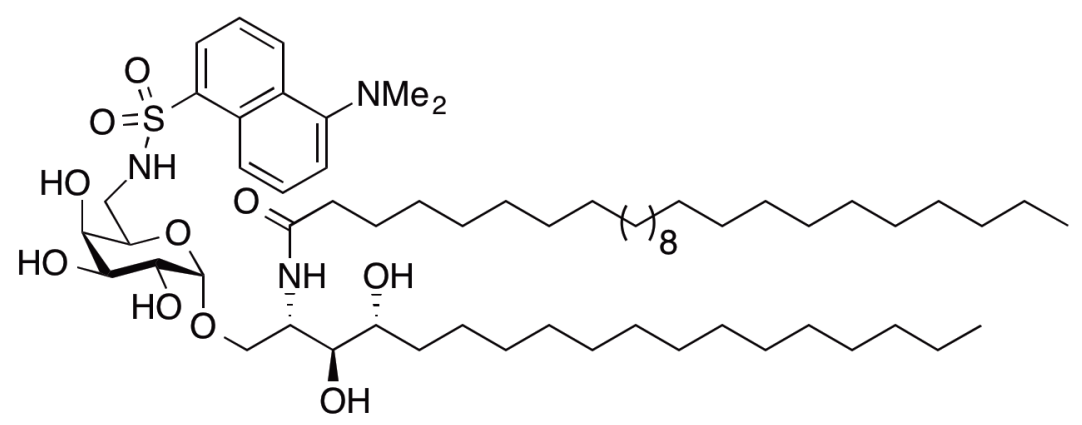

Dansyl $\alpha$-GalCer 2

Figure 2.1. $\alpha$-Galactosyl ceramide and derivatives.

Given the therapeutic potential of $\alpha$-GalCer, much effort has been spent in developing robust routes for its synthesis. ${ }^{3,7-10}$ In addition, a number of $\alpha$-GalCerderivatives containing a fluorophore ${ }^{11-13}$ or a biotinylated probe $\mathrm{e}^{11,14,15}$ have been prepared with the objective of using these substrates to better understand the mechanism of $i$ NKT cell activation by $\alpha$-GalCer. ${ }^{13,16,17}$ Though the ability of $\alpha$ GalCer to bind to CD1d and activate $i$ NKT cells has been robustly studied, only little information is known about lipid trafficking and how this influences presentation by CD1d and activation of $i$ NKT cells. ${ }^{18}$ Accordingly, many groups have focussed on modifying the lipid portion of $\alpha$-GalCer with a reporter probe in order to address such issues. ${ }^{12-14}$ However, as CD1d binds lipid chains within deep hydrophobic pockets, ${ }^{19}$ it has been proposed that the addition of a label on the 
lipid may interfere with $\alpha$-GalCer-CD1d association, ${ }^{17}$ and moreover, may influence intracellular trafficking. ${ }^{18,20-22}$ Conversely, modelling of the CD1d- $\alpha$ GalCer complex suggests that the hydroxyl groups at $\mathrm{C}^{\prime \prime}$ and $\mathrm{C}^{\prime \prime}$ on galactose are not involved in complex formation. ${ }^{23}$ This theory is also supported by the preparation of an $\alpha$-GalCer analogue containing an additional $\alpha$-linked galactose at the C6" position which was shown to stimulate $i$ NKT cells without the need for processing. ${ }^{24}$ Taken as a whole, it is generally accepted that the CD1dglycolipid-NKT cell receptor interaction tolerates the appendage of small molecules at $\mathrm{C}^{\prime \prime}$ and that this is an ideal position to attach a fluorescent reporter group. Indeed, this was the conclusion made by Zhou et al. who developed a strategy for the preparation of dansylated $\alpha$-GalCer derivative 2 . $^{11}$

Despite there being much interest in $\alpha$-GalCer, the need for a more efficient synthesis of an appropriately labelled fluorescent $\alpha$-GalCer derivative remains. An improved strategy for the synthesis of the dansylated $\alpha$-GalCer derivative 2 (Figure 2.1) is needed to facilitate studies of the mechanism by which $\alpha$-GalCer is transferred to resident dendritic cells (DCs) during cancer immunotherapy. ${ }^{25,26}$ The objective was to achieve a robust synthesis of $\mathbf{2}$ with high reaction yields throughout, and to assess the ability of $\mathbf{2}$ to both activate $i$ NKT cells and to serve as a fluorescent reporter group. The results of these studies are reported herein. 


\subsection{Synthesis of dansyl $\alpha$-GalCer}

\section{Retrosynthesis}

The retrosynthetic analysis for the preparation of dansylated $\alpha$-GalCer 2 is presented in Scheme 2.1. 2 was prepared from the mono-lipidated derivative 36, itself formed via the coupling of dansylated galactosyl iodide donor $\mathbf{3 7}$ to the phytosphingosine backbone $\mathbf{3 8}$. Donor $\mathbf{3 7}$ and acceptor $\mathbf{3 8}$ are in turn both readily prepared from D-galactose (39). The reasoning behind these key disconnects are two-fold. First, though it is possible to couple the complete ceramide lipid backbone to a suitably protected galactose donor, the ceramide is a particularly poor acceptor and yields for these glycosylations are typically modest (ca. 25$55 \%$ ). This is a general phenomenon largely independent of the type of galactose donor used. ${ }^{8,15,27-31}$ There are also advantages to be gained by incorporating the fluorescent dansyl group at an early stage in the synthesis for it has been well documented that molecules containing a chromophore are more easily monitored by TLC and purified by flash column chromatography. ${ }^{32}$ Accordingly, in contrast

to other syntheses of fluorescent $\alpha$-GalCer probes, ${ }^{11-13}$ a glycosylation reaction involving the use of a fluorescent galactose donor, such as $\mathbf{3 7}$ was employed. 

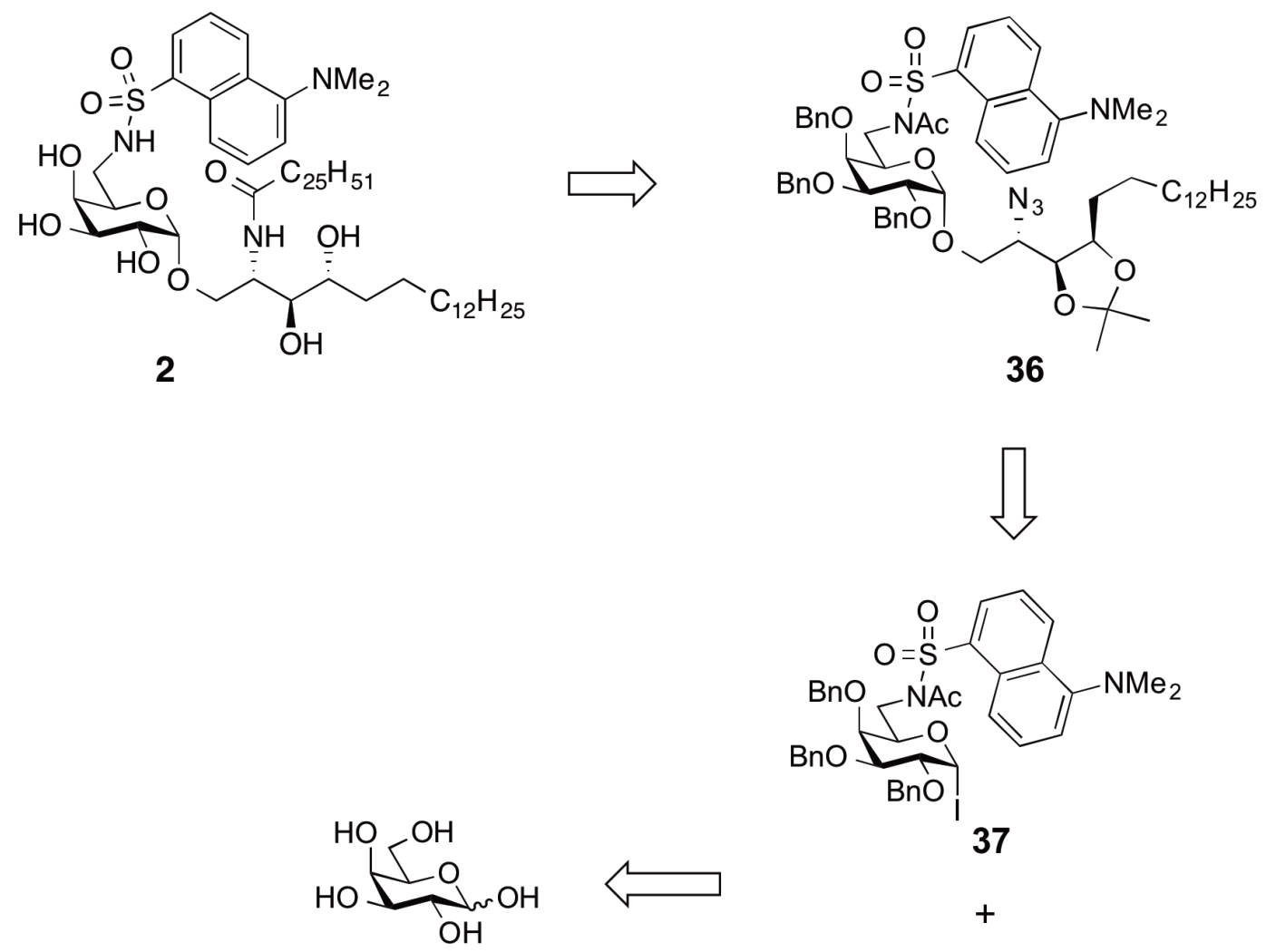

39

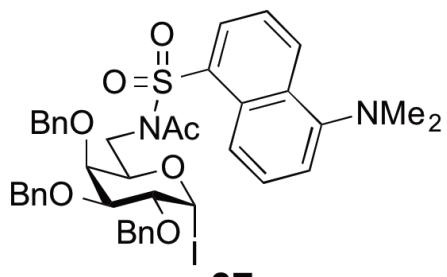

37
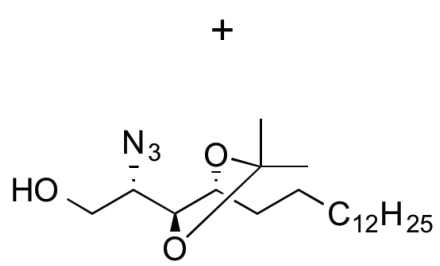

38

Scheme 2.1. Retrosynthetic analysis for the preparation of dansylated $\alpha$-GalCer 2 .

\section{Synthesis of phytosphingosine acceptor 6.}

With the synthetic strategy in place, the first goal was the preparation of the phytosphingosine backbone 38 (Scheme 2.2). Here, synthesis of the lipid portion commenced with the selective tritylation of D-galactose (39) at the primary position, ${ }^{33}$ followed by installation of an isopropylidene at the 3- and 4-positions to give the diol $\mathbf{4 0}$ in $85 \%$ (over two steps). ${ }^{34}$ Diol $\mathbf{4 0}$ was then treated with a solution of $\mathrm{NaIO}_{4}$ in $\mathrm{THF} / \mathrm{H}_{2} \mathrm{O}$, which resulted in cleavage of the diol and formation of the formate ester $\mathbf{4 1}$ in quantitative yield. As a temporary protecting group, the formate at the 4-position was a pivotal step in this strategy as it prevented cyclisation to the corresponding D-lyxofuranose derivative, which gave 
only modest yields (50-60\%) when subject to the subsequent Wittig reaction. Treatment of ester 41 with excess ylide (BuLi, 2.4 equiv., phosphonium bromide, 2.5 equiv.), however, gave the corresponding alkene $\mathbf{4 2}$ in good (78\%) yield and in an $E: Z$ ratio of approximately $1: 10$ based on ${ }^{1} \mathrm{H}$ NMR analysis.

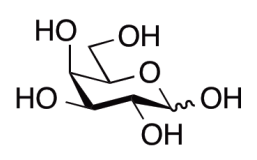

39

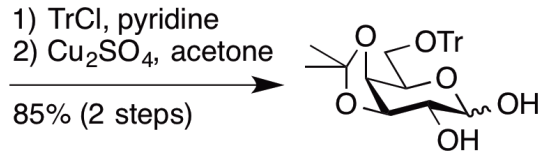

40

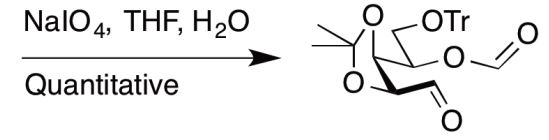

41 BuLi, THF $\mathrm{BrPh}_{3} \mathrm{PC}_{13} \mathrm{H}_{27}$ then $\mathbf{4 1}$ $78 \%$

$\checkmark$

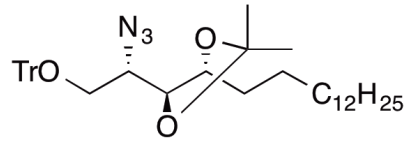

43

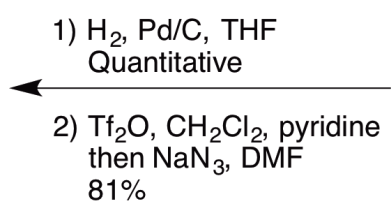

$81 \%$

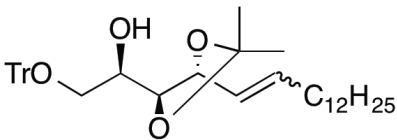

42

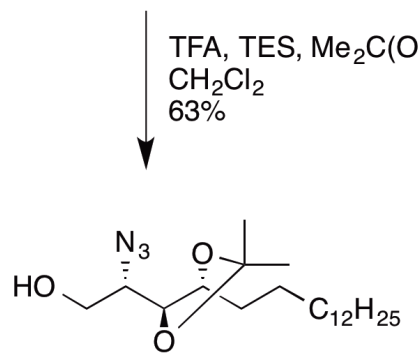

38

Scheme 2.2. Synthesis of phytosphingosine acceptor 38 .

Subsequent conversion of alkene $\mathbf{4 2}$ to the required phytosphingosine backbone then followed via quantitative hydrogenation, using $1.5 \mathrm{wt} \% \mathrm{Pd} / \mathrm{C}$, and treatment with triflic anhydride to give the intermediate triflate, which was converted to azide 43 in situ. During the installation of the azide functionality, it was found that the best yields were obtained when the reaction was preformed at $-15^{\circ} \mathrm{C}$ as higher temperatures resulted in the elimination of the triflate and the formation of the corresponding olefinic by-product. Selective removal of the trityl ether in the presence of the isopropylidene group was then attempted. This transformation has 
proven problematic in the past, ${ }^{7}$ and resulted in the authors adopting a 4-step deprotection/reprotection strategy to generate a modified 3-O, 4-O-benzylated phytosphingosine derivative. In view of the lability of the isopropylidene group, azide 43 was treated with TFA and TES, then added 2,2-dimethoxypropane to the reaction vessel to reinstall the isopropylidine, should it be cleaved under the acidic reaction conditions. Indeed, using this approach, phytosphingosine $\mathbf{3 8}$ was obtained in a respectable $63 \%$ yield. Numerous attempts were then made to further optimise the reaction including the addition of $\mathrm{MeOH}$ (to scavenge the liberated TES cation, which was sometimes found to be present on the 1-position of the lipid), however, this did not lead to any improvements in yield. The use of boron trichloride or formic acid to cleave the trityl group resulted in a lower yield of product. Despite this, in sum, the core phytosphingosine backbone $\mathbf{3 8}$ was prepared in 7-steps and in a good overall yield of 34\%. This represents one of the most efficient syntheses of the phytosphingosine backbone to date. ${ }^{35,36}$

\section{Synthesis of dansylated galactose donor and dansyl- $\alpha$-GalCer}

Having completed the synthesis of the required phytosphingosine lipid, attention was turned to the preparation of the dansylated galactose donor. The synthesis of the carbohydrate portion commenced with the formation of the common azide (45) ${ }^{11}$ using a modified sequence of reactions that first led to the formation of iodide 44 via the selective installation of isopropylidenes at the 1,2 and 3,4 positions of D-galactose (39), subsequent installation of the iodide at the primary position, and then simultaneous cleavage of the isopropylidenes and Fisher glycosylation using a $5 \%$ solution of $\mathrm{AcCl}$ in $\mathrm{MeOH}$ (Scheme 2.3). This route provided a convenient means by which to prepare gram quantities of iodide $\mathbf{4 5}$ in excellent overall yield $(72 \%, 3$ steps) and without the need for column chromatography. The iodide in $\mathbf{4 4}$ was then displaced to give the intermediate azide, which was subsequently benzylated to give the fully protected azide $\mathbf{4 5}$, again in excellent overall yield. 


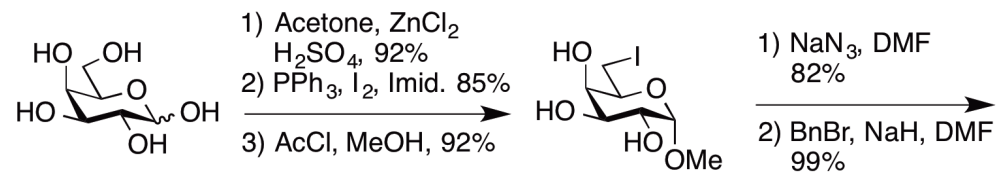

39

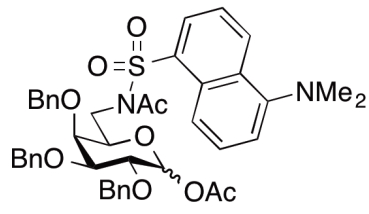

47

44

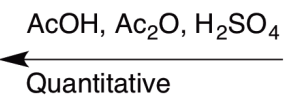

1) $\mathrm{TMSI}, \mathrm{CH}_{2} \mathrm{Cl}_{2}$

2) TBAI, DiPEA

38, benzene

$\checkmark$

$\mathrm{NaOMe}, \mathrm{MeOH}$ $\mathrm{CH}_{2} \mathrm{Cl}_{2}$

Quantitative

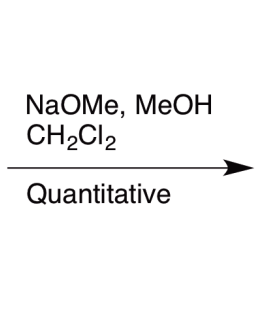

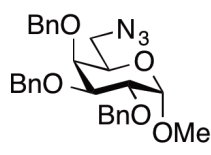

45

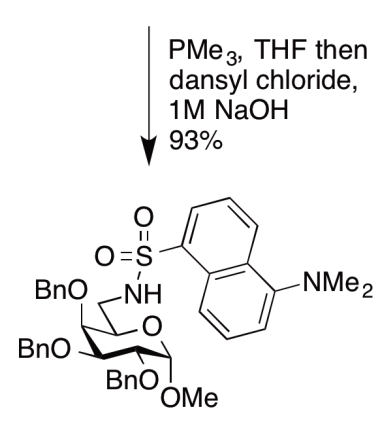

46

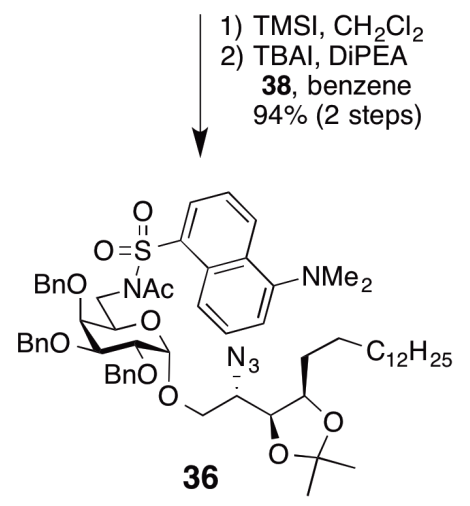

$\underset{74 \%}{\mathrm{AcOH}, \mathrm{H}_{2} \mathrm{O}}$

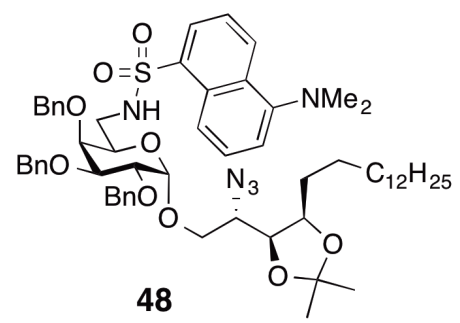

1) $\mathrm{PMe}_{3}, 1 \mathrm{M} \mathrm{NaOH}$

2) $\mathrm{HOC}(\mathrm{O}) \mathrm{C}_{25} \mathrm{H}_{51}, \mathrm{EDCl}$, DMAP, $\mathrm{CHCl}_{2}$ $68 \%$ (2 steps)

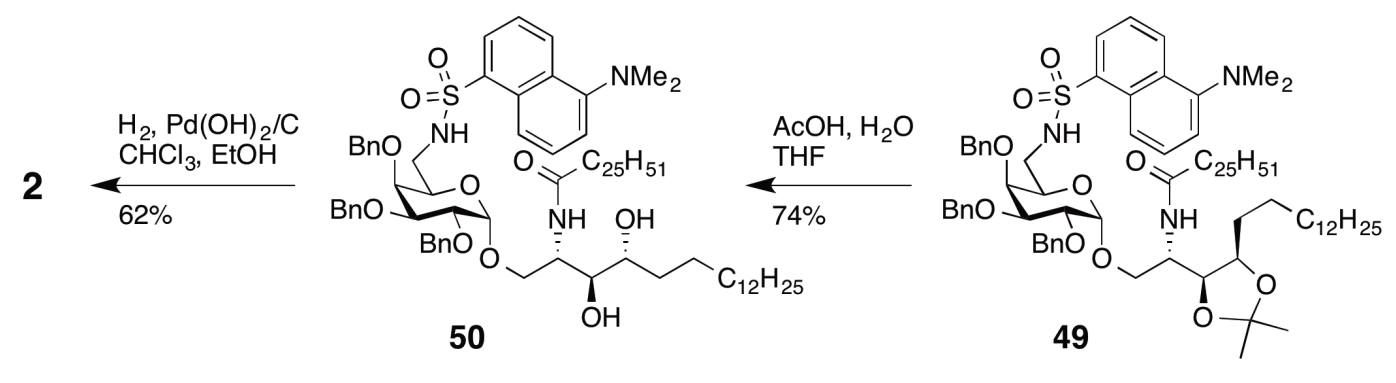

Scheme 2.3. Synthesis of dansyl- $\alpha$-GalCer 2 .

With the objective of installing the fluorescent group at an early stage in the synthesis, the dansyl group was then incorporated onto the galactose residue $\mathbf{4 5}$ in a two-step one-pot procedure involving the reduction of the azide to an amine 
using Staudinger conditions ${ }^{10}$ and subsequent treatment with dansyl chloride in the presence of $1 \mathrm{M} \mathrm{NaOH}$. Using this strategy, dansylated galactose 46 was prepared in excellent (93\%) yield. Dansylated galactose 46 is a bright-green oil, and as such, is readily identified during flash column chromatography whereby the yellow-green band can be followed by eye during the purification process. Furthermore, incorporation of the dansyl flurophore also aids in the monitoring of the reaction by TLC analysis with 46 glowing bright white under UV light $(\lambda=$ $254 \mathrm{~nm}$ ). Treatment of $\mathbf{4 6}$ with a solution of $\mathrm{AcOH}, \mathrm{Ac}_{2} \mathrm{O}$ and $\mathrm{H}_{2} \mathrm{SO}_{4}$ at $0{ }^{\circ} \mathrm{C}$ for 3 $\mathrm{h}$ then resulted in the quantitative formation of acetate 47 . Here, it should be noted that in addition to the conversion of the anomeric methoxy to the acetate, the amine of the dansyl group was also acetylated. Though not optimal, the overacetylation of dansyl groups has been previously reported ${ }^{37}$ and it was envisaged that the superfluous acetyl could be readily removed during a subsequent step of the synthesis. A dramatic change in colour was also observed upon monitoring the reaction by TLC analysis $(\lambda=254 \mathrm{~nm})$, with the methyl glycoside 46 fluorescing bright white, whilst the corresponding acetate 47 was mauve (see supporting information). As with all dansylated compounds prepared, acetate 47 was a fluorescent green colour, which allowed for its ready purification by flash column chromatography and illustrates the added value of the early incorporation of the dansyl-group into the synthetic plan.

Forging ahead, glycosyl donor $\mathbf{4 7}$ was converted to the corresponding glycosyl iodide via methodology based on seminal work by Gervay-Hague. ${ }^{38-41}$ Though a number of galactosyl donors have been prepared and coupled to the phytosphingosine backbone en route to the preparation of $\alpha$-GalCer, ${ }^{7,9,10,27-29,42-45}$ few of these glycosylation reactions have been reported to be both high-yielding and highly $\alpha$-selective. ${ }^{38,46-48}$ To this end, the glycosyl iodide route of GervayHague was employed and glycolipid 36 was prepared in 94\% yield and as only the $\alpha$-anomer, as determined by a ${ }^{1} \mathrm{H} J_{1,2}$ coupling constant of $3.2 \mathrm{~Hz}$ and a ${ }^{13} \mathrm{C}-{ }^{1} \mathrm{H}$ $\left({ }^{1} J_{\mathrm{CH}}\right)$ coupling of $172.2 \mathrm{~Hz}^{49}$

With glycolipid 36 in hand, the next step was the installation of the C26 acyl chain. First, the acetyl on the sulfonimide was removed using Zemplén 
conditions $^{50}$ to give the desired sulfonamide $\mathbf{4 8}$ in quantative yield. Both glycolipid 36 and sufonamide 48 had identical $\mathrm{R}_{f}$ values (0.40; PE/EA, 2/1, v/v), though were different colours when observed via TLC analysis. With glycolipid 36 being mauve in colour (UV light, $\lambda=254 \mathrm{~nm}$ ) and sulfonamide 48 bright white, this greatly aided in the monitoring of the reaction. The isopropylidene group was then removed and the diol product subjected to hydrogenation conditions using $\mathrm{Pd}(\mathrm{OH})_{2} / \mathrm{C}$ in the presence of $\mathrm{HCl}$ in an attempt to simultaneously remove the benzyl groups and reduce the azide to an amine. Numerous attempts were made to achieve this transformation, yet despite the addition of $\mathrm{HCl}$ to protonate the resultant amine and prevent poisoning of the palladium catalyst, only partial debenzylation was observed. In view of this, an alternative strategy was used whereby the azide in $\mathbf{4 8}$ was first reduced to the amine, using Staudinger methodology, then coupled the product to hexacosanoic acid via an EDCI/DMAP condensation protocol to give fully protected dansylated $\alpha$-GalCer 49 in good (68\%) overall yield. Removal of the isopropylidene group occurred smoothly to give $\mathbf{5 0}$ in 74\% yield. Finally, global deprotection, again using $\mathrm{Pd}(\mathrm{OH})_{2} / \mathrm{C}$ as the catalyst, gave dansyl $\alpha$-GalCer 2 in $62 \%$ yield, which glowed bright white under UV light $(\lambda=366 \mathrm{~nm})$. Though glycolipids such as 2 have been reported to be notoriously difficult to solubilise and hence assign, ${ }^{51,52}$ using a variety of 2D NMR spectroscopy techniques (COSY, HSQC, HMBC) with spectra recorded in pyridine- $d_{5}$, all ${ }^{13} \mathrm{C}$ and ${ }^{1} \mathrm{H}$ resonances were fully assigned. Furthermore, the dansyl group of $\mathbf{2}$ allowed for the ready detection of the compound by way of HPLC analysis (see supporting information). Thus, in summary, dansyl $\alpha$-GalCer 2 was prepared in 14 linear steps from the commercially available and inexpensive D-galactose and in $16 \%$ overall yield. This represents a highly efficient synthesis of this important biological probe and is comparable to the work of Zhou et al. whereby the probe was prepared in $<10 \%$ yield from 6-azido-6-deoxy-1,2:3,4-di- $O$-isopropylidene- $\alpha$-D-galactopyranose. ${ }^{11}$ 


\subsection{The effects of dansyl- $\alpha-$ GalCer on the stimulation of iNKT cells in vitro and in vivo}

Having developed an efficient synthesis of dansyl $\alpha$-GalCer 2, the next task was to investigate whether 2 could activate $i$ NKT cells in a manner similar to that of its parent compound, $\alpha$-GalCer (1)..$^{6,7,9,11,28,30,45}$ This was achieved by analysing levels of the cytokine IL-2 released by the ${\mathrm{V} \alpha 14^{+}} i \mathrm{NKT}$ cell hybridoma ${ }^{4}$ DN32.D3 following in vitro stimulation with the glycolipid compounds and by examining the expression of the co-stimulatory molecule, CD86, on splenic DC and B cells after injection of the compounds into mice. ${ }^{9}$ The latter assay is based on the observation that CD86 is upregulated as a consequence of $i \mathrm{NKT}$ cells interacting with, and subsequently driving, DC and B cell maturation. ${ }^{53,54}$

To assess the ability of $\mathbf{2}$ to stimulate DN32.D3 $i$ NKT cell hybridoma cells, titrated amounts of dansyl $\alpha$-GalCer 2 , or $\alpha$-GalCer 1 , were incubated for $24 \mathrm{~h}$ with the hybridoma cells in the presence of a constant number of murine DCs from the immortalised line, DC2114, ${ }^{55}$ and supernatants were then collected and analysed for IL-2. Proliferation of the IL-2-dependent cell line HT-2 was used as a read-out of IL-2 levels. As illustrated (Figure 2.2A), both $\alpha$-GalCer 1 and dansyl $\alpha$-GalCer 2 stimulated release of IL- 2 in a dose-dependent manner, with $\alpha$-GalCer 1 driving a higher level of this cytokine. This observation is consistent with literature precedent for weaker cytokine profiles generated by similar C6" substituted $\alpha$-GalCer derivatives, but is nonetheless indicative that dansyl $\alpha$ GalCer 2 is indeed capable of $i$ NKT cell activation. ${ }^{11,15}$ The ability of dansyl $\alpha$ GalCer 2 to stimulate $i$ NKT cells in vivo was assessed by injecting mice with 200 ng of dansyl $\alpha$-GalCer 2 or $\alpha$-GalCer 1 , and then determining the expression of the maturation marker CD86 on the surface of splenic B cells and DCs by flow cytometry $20 \mathrm{~h}$ later. As shown, administration of fluorescent analogue 2 stimulated the maturation of B cells and DCs (Figure 2.2B) with activities resembling that of the parent compound $\mathbf{1}$. To confirm that this in vivo activity was mediated by $i \mathrm{NKT}$ cells, the experiment was repeated in CD1d deficient 
mice. Here, no up-regulation of CD86 was observed on DCs (Figure 2.2C), confirming that dansyl $\alpha$-GalCer 2, like $\alpha$-GalCer 1, activates APCs by stimulating $i \mathrm{NKT}$ cells in a CD1d dependent manner. 


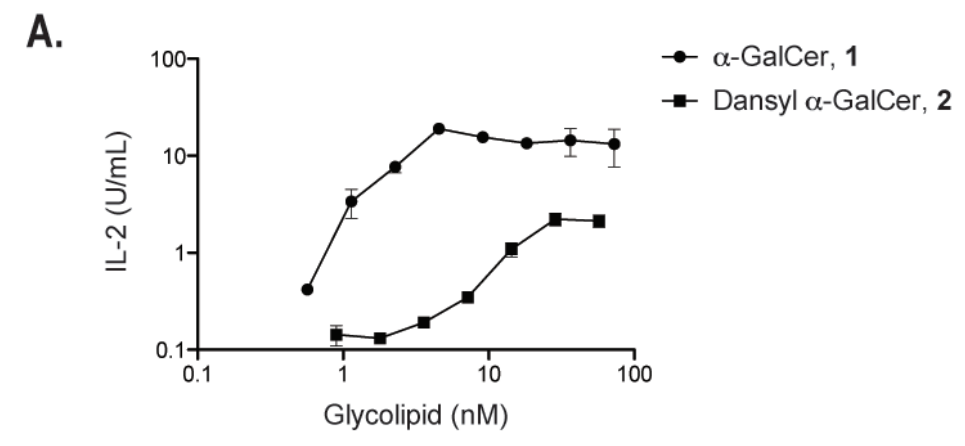

B.
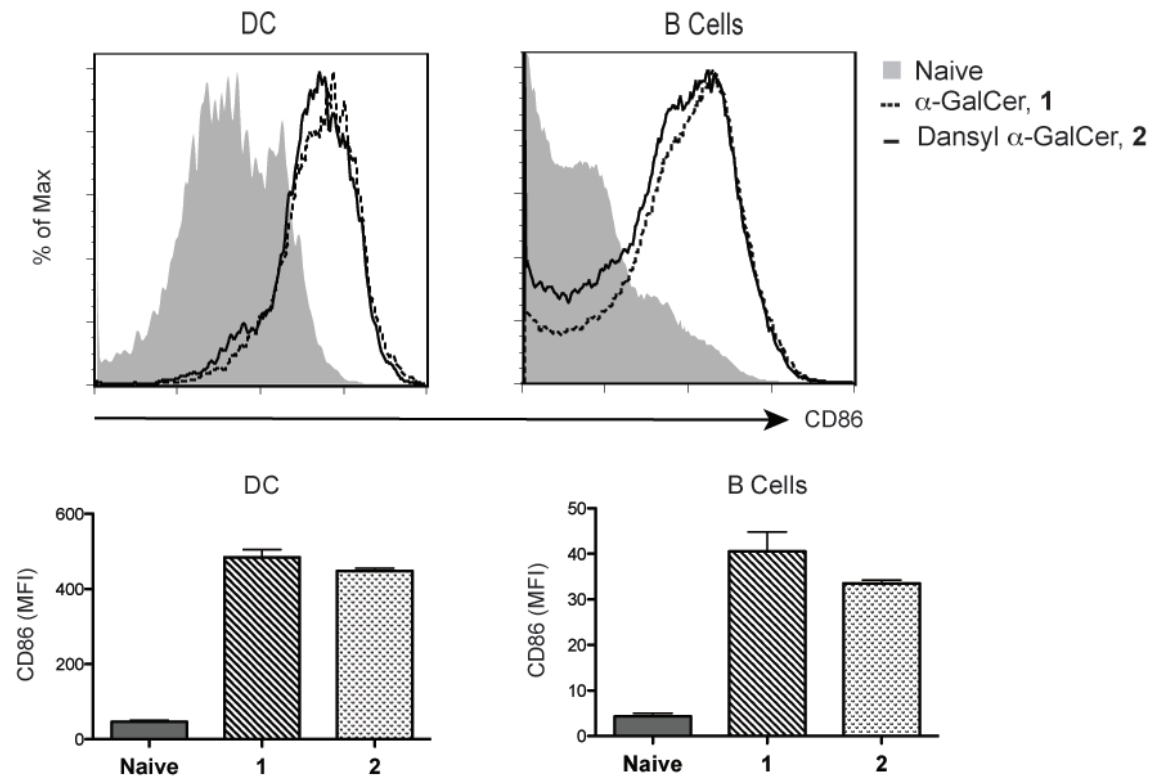

C.

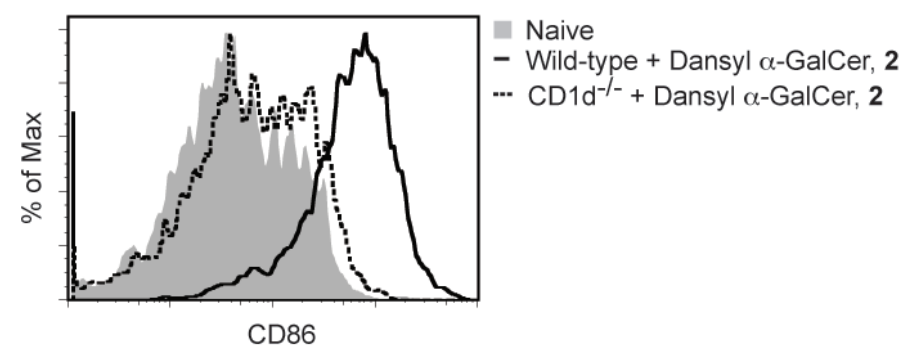

Figure 2.2. Dansyl $\alpha$-GalCer 2 stimulates $i \mathrm{NKT}$ cells in vitro and in vivo. (A). Production of IL-2 by $i$ NKT cells (DN32.D3) in the presence of titrated doses of $\alpha$-GalCer 1 or dansyl $\alpha$-GalCer 2; (B) Dansyl $\alpha$-GalCer 2 drives the maturation of splenic DCs and B cells in vivo. C57BL/6 mice were injected intravenously with $200 \mathrm{ng}$ of $\alpha$-GalCer $1(n=3)$ or dansyl $\alpha$-GalCer $2(n=3)$, and spleens were removed $20 \mathrm{~h}$ after injection for antibody labelling and flow cytometry. The expression of CD86 on CD11c ${ }^{+}$dendritic cells and B220 B cells were assessed; (C) $200 \mathrm{ng}$ of dansyl $\alpha$-GalCer 2 was injected intravenously into wild type $(\mathrm{C} 57 \mathrm{BL} / 6)$ and $\mathrm{CD} 1 \mathrm{~d}^{-/-}$mice, and spleens were removed $20 \mathrm{~h}$ after injection for antibody labelling and flow cytometry. The spleen of an untreated wild type $(\mathrm{C} 57 \mathrm{BL} / 6)$ mouse was used as control. The expression of CD86 on CD11 ${ }^{+}$ dendritic cells was assessed. Mean fluorescence intensities (MFI) \pm SD are presented. 


\subsection{Uptake of $\alpha$-GalCer by dendritic cells}

Having established that dansyl $\alpha$-GalCer 2 binds to CD1d and activates $i$ NKT cells in a manner similar to that of $\alpha$-GalCer $\mathbf{1}$, the ability of dansyl $\alpha$-GalCer $\mathbf{2}$ to function as a fluorescent reporter group was investigated. This has previously not been reported. The fluorescence quantum yield of dansyl $\alpha$-GalCer $2\left(\phi_{\mathrm{f}}\right)$ was found to be 0.22 as determined via a relative method against the standard, quinine sulfate $\left(\phi_{\mathrm{f}}=0.54\right.$ in $\left.1 \mathrm{M} \mathrm{NaOH}\right)$. The wavelengths of maximum absorbance and emission of 2 were determined by spectrofluorimetry to be $343 \mathrm{~nm}$ and $510 \mathrm{~nm}$, respectively, and these maxima were used in further analyses. The DC cell line, DC2114 was incubated with $4 \mu \mathrm{g}$ of dansyl $\alpha$-GalCer 2 for $24 \mathrm{~h}$ and uptake of the fluorescent glycolipid was analysed by flow cytometry (Figure 3). A distinct shift in mean fluorescence intensity was observed for cells treated with dansyl $\alpha$ GalCer 2, but not for those treated with $\alpha$-GalCer 1 or controls (untreated and vehicle), illustrating the ability of dansyl $\alpha$-GalCer 2 to be used as a fluorescent probe to detect sub-populations of DCs that present the glycolipid.

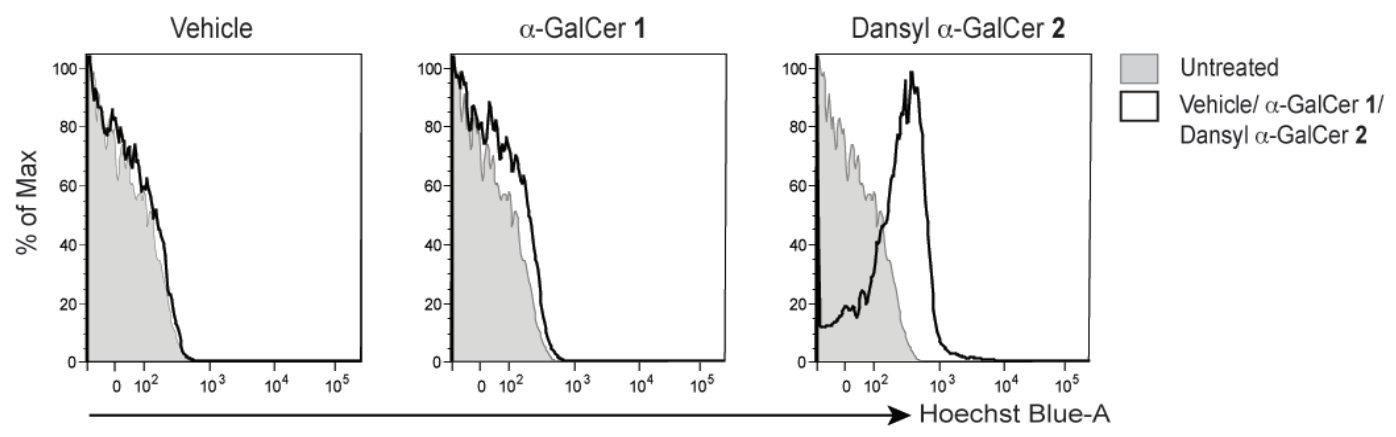

Figure 2.3. Cells that have taken up dansyl $\alpha$-GalCer 2 can be detected by flow cytometry. $1 \times 10^{6}$ cells (DC2114 cell line) were incubated for $24 \mathrm{~h}$ with $4 \mu \mathrm{g}$ of dansyl $\alpha$-GalCer 2 , $\alpha$-GalCer 1 or vehicle only. Cells were excited with the UV laser, and detected by the Hoechst Blue-A detector. 


\subsection{Visualisation of dansyl $\alpha$-GalCer by fluorescence microscopy}

Following the successful detection of dendritic cells that have taken up the fluorescent glycolipid by flow cytometry, the next objective was to visualise the location of glycolipid when incubated with dendritic cells. Initially, the DC2114 cell line was incubated at a concentration similar to that used for flow cytometry $\left(1 \times 10^{6}\right.$ cells incubated with $4 \mu \mathrm{g}$ of dansyl $\alpha$-GalCer 2$)$. Unfortunately, the fluorescence could not be detected, despite increasing the concentration of glycolipid by two-fold. In addition, two other cell types, bone marrow derived dendritic cells (BMDCs) and A20, a murine B lymphoma cell line used in in vivo vaccination, were also incubated with dansyl $\alpha$-GalCer 2 but again the fluorescence was no different when compared to the negative control containing cells only. When glycolipid concentrations that well exceed what would be relevant in in vitro and in vivo experiments were used, slight fluorescence was observed, but this rapidly disappeared due to quenching of the dye. Similarly, the probe was also quenched too rapidly when cells that have taken up the glycolipid was visualised under a confocal microscope. Efforts to amplify the fluorescence of dansyl $\alpha$-GalCer 2 by using the secondary antibody, anti-dansyl antibody was also met with little success. Unfortunately, while dansyl $\alpha$-GalCer $\mathbf{2}$ behaved in a very similar manner to the parent glycolipid $\alpha$-GalCer $\mathbf{1}$, it could not be visualised at the concentrations typically used in an in vivo setting. 


\subsection{Conclusion}

In summary, an efficient synthesis of the biologically important probe, dansyl $\alpha$ GalCer 2. En route to the synthesis of this glycolipid, a highly efficient synthesis of the phytosphingosine lipid acceptor was developed. In addition, it was found that the early incorporation of the fluorescent group greatly aids in the monitoring and purification of reaction products. The ability of dansyl $\alpha$-GalCer 2 to activate $i$ NKT cells and to be used as a fluorescent probe for the analysis of glycolipid uptake by DCs has also been illustrated. Unfortunately, the fluorophore was too weak and underwent photobleaching when subjected to the UV laser of the fluorescent microscope.

Given the importance of $\alpha$-GalCer $\mathbf{1}$, it is anticipated that similar derivatives with a fluorophore attached on the $6^{\prime \prime}$-position will find wide application as a molecular tool to better understand the trafficking of $\alpha$-GalCer and glycolipid presentation by CD1d. Extension of this work is presented in Chapter 3. 


\subsection{Experimental}

\subsubsection{General procedure}

Unless otherwise stated all reactions were performed under $\mathrm{N}_{2}$. Prior to use, THF (Pancreac) was distilled from $\mathrm{LiAlH}_{4}$, pyridine was dried over $4 \AA$ molecular sieves ( $4 \AA \mathrm{MS}$ ), acetone (Pure Science) was dried over $3 \AA$ MS, DCM (Pancreac) was distilled from $\mathrm{P}_{2} \mathrm{O}_{5}$, TMSI (Aldrich) was distilled from antimony powder $(\mathrm{M} \& \mathrm{~B})$ and stored over $\mathrm{Cu}(\mathrm{s})$ powder (Hopkin \& Williams), and $\mathrm{H}_{2} \mathrm{O}$ and benzene (Fisher Scientific) were distilled. Trityl chloride (Acros), $\mathrm{CuSO}_{4}$ (Fluka), $\mathrm{NaIO}_{4}(\mathrm{M} \& \mathrm{~B})$, anhydrous $\mathrm{Et}_{2} \mathrm{O}$ (Pancreac), n-BuLi (Aldrich), 1-bromotridecane (Aldrich), $\mathrm{PPh}_{3}$ (Merck), $\mathrm{Pd} / \mathrm{C}$ (Aldrich, 10 wt\%), $\mathrm{Tf}_{2} \mathrm{O}$ (Aldrich), $\mathrm{NaN}_{3}(\mathrm{BDH})$, anhydrous DMF (Acros), TFA (Aldrich), triethylsilane (Fluka), $\mathrm{Me}_{2} \mathrm{C}(\mathrm{OMe})_{2}$ (Aldrich), $\mathrm{ZnCl}_{2}$ (Aldrich), $\mathrm{H}_{2} \mathrm{SO}_{4}$ (Lab-Scan), $\mathrm{I}_{2}$ (BDH), imidazole (Aldrich), $\mathrm{AcCl}$ (B\&M), BnBr (Fluka), NaH (Avocado Research Chemicals, 60\% dispersion in mineral oil), $\mathrm{PMe}_{3}$ (Aldrich, $1 \mathrm{M}$ in THF), dansyl chloride (Acros), $\mathrm{AcOH}$ (Ajax Finechem), $\mathrm{Ac}_{2} \mathrm{O}$ (Peking Reagent), TBAI (Riedel-de Haen), DiPEA (Aldrich), $\mathrm{NaOMe}$ (Janssen Chimica), $\mathrm{C}_{25} \mathrm{H}_{51} \mathrm{COOH}$ (Acros), EDCI (Aldrich), DMAP (Merck), $\mathrm{Pd}(\mathrm{OH})_{2} / \mathrm{C}$ (Aldrich, $20 \mathrm{wt} \%$ ), EtOAc (Pancreac), hexanes (Fisher Scientific), petroleum ether (Pure Science), $\mathrm{MeOH}$ (Pure Science), $\mathrm{CHCl}_{3}$ (Pancreac), EtOH (absolute, Pure Science), $\mathrm{NaOH}$ (Pure Science), $\mathrm{NaHCO}_{3}$ (Pure Science), $\mathrm{NaCl}$ (Pancreac) were used as received. All solvents were removed by evaporation under reduced pressure. Reactions were monitored by TLC-analysis on Macherey-Nagel silica gel coated plastic sheets $(0.20 \mathrm{~mm}$, with fluorescent indicator $\mathrm{UV}_{254}$ ) with detection by UV-absorption (short wave UV - $254 \mathrm{~nm}$; long wave UV - $366 \mathrm{~nm}$ ), by dipping in $10 \% \mathrm{H}_{2} \mathrm{SO}_{4}$ in $\mathrm{EtOH}$ followed by charring at $\sim 150{ }^{\circ} \mathrm{C}$, by dipping in $\mathrm{I}_{2}$ in silica, or by dipping into a solution of ninhydrin in EtOH followed by charring at $\sim 150{ }^{\circ} \mathrm{C}$. Column chromatography was performed on Pure Science silica gel (40-63 micron). AccuBOND II ODS-C18 (Agilent) was used for reverse phase chromatography. High-resolution mass spectra were recorded on a Waters Q-TOF Premier ${ }^{\mathrm{TM}}$ Tandem Mass Spectrometer using postive electro-spray ionisation. Optical rotations were recorded using a PerkinElmer 241 polarimeter or Autopol II (Rudolph Research Analytical) at $589 \mathrm{~nm}$ 
(sodium D-line). Infrared spectra were recorded as thin films using a Bruker Tensor 27 FTIR spectrometer equipped with an Attenuated Total Reflectance (ATR) sampling accessory and are reported in wave numbers $\left(\mathrm{cm}^{-1}\right)$. Nuclear magnetic resonance spectra were recorded at $20^{\circ} \mathrm{C}$ in $\mathrm{D}_{2} \mathrm{O}$ or $\mathrm{CDCl}_{3}$ using either a Varian INOVA operating at $500 \mathrm{MHz}$ or Varian VNMRS operating at 600

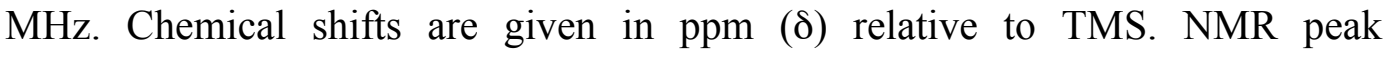
assignments were made using COSY, HSQC and HMBC 2D experiments. Melting points were obtained using the Gallenkamp Melting Point Apparatus. Spectrofluorimetry measurements were carried out on the Horiba Jobin-Yvon Fluorolog-3 spectrofluorometer using the three dimensional (3D) scanning mode. High-pressure liquid chromatography was performed on Waters 600 liquid chromatograph with a photodiode array detector (Waters 2996) and a C-18 column (Waters Xbridge $5 \mu \mathrm{m}$ ).

\section{3,4-O-Isopropylidene-6-O-triphenylmethyl-D-galactopyranoside (40).}

Trityl chloride (16.4 g, $58.8 \mathrm{mmol}$ ) was added to a solution of D-galactose (39) $(10 \mathrm{~g}, 55.5 \mathrm{mmol})$ in pyridine $(150 \mathrm{~mL})$. The reaction mixture was stirred at $50{ }^{\circ} \mathrm{C}$ for $48 \mathrm{~h}$ and concentrated in vacuo. The resulting residue was purified by chromatography through a short silica column and the tritylated galactose was eluted with acetone and crystallised from $\mathrm{EtOH}$ to afford the tritylated galactose (23.4 g, 55.4 mmol, quant.) [Rf: 0.17 (EtOAc)] as a white powder. To a solution of tritylated galactose $(15 \mathrm{~g}, 35.5 \mathrm{mmol})$ in anhydrous acetone $(750 \mathrm{~mL})$ was

added copper sulfate $(60 \mathrm{~g}, 380 \mathrm{mmol})$. After stirring the reaction mixture for $3 \mathrm{~d}$ at room temperature, carbon was then added and the solution filtered through celite. The filtrate was concentrated in vacuo and purified by flash column chromatography. Elution with 1:1 (v/v) hexanes/EtOAc afforded the title compound 40 (13.9 g, $30.1 \mathrm{mmol}, 85 \%, 2$ steps) as a clear oil. $\mathrm{R}_{f}: 0.65$ (EtOAc); $[\alpha]_{\mathrm{D}}^{20}=+18.0^{\circ}\left(c=1.0, \mathrm{CHCl}_{3}\right)$; IR (film) 3406, 3059, 3034, 2986, 2936, 1597, $1491,1449,1380,1219,1162,1132,1079,1032,909,872,814,776,732,705$ $\mathrm{cm}^{-1} ;{ }^{1} \mathrm{H}$ NMR $\left(500 \mathrm{MHz}, \mathrm{CDCl}_{3}\right) \delta 7.48-7.46\left(\mathrm{~m}, 6 \mathrm{H}, \mathrm{CPh}_{3}, \mathrm{CH}-o\right), 7.30\left(\mathrm{t}, J_{\mathrm{o}, \mathrm{m}}\right.$ $\left.=J_{\mathrm{m}, \mathrm{p}}=7.4 \mathrm{~Hz}, 6 \mathrm{H}, \mathrm{CPh}_{3}, \mathrm{CH}-m\right), 7.26-7.23\left(\mathrm{~m}, 3 \mathrm{H}, \mathrm{CPh}_{3}, \mathrm{CH}-p\right), 5.23\left(\mathrm{t}, J_{1,2}=\right.$ $\left.J_{1, \mathrm{OH}}=3.7 \mathrm{~Hz}, 1 \mathrm{H}, \mathrm{H}-1 \alpha\right), 4.34-4.29$ (m, 3H, H-3, H-4, H-5), 3.87-3.85 (m, 1H, $\mathrm{H}-2), 3.02\left(\mathrm{dd}, J_{6 \mathrm{a}, 6 \mathrm{~b}}=9.1 \mathrm{~Hz}, J_{6 \mathrm{a}, 5}=6.4 \mathrm{~Hz}, 1 \mathrm{H}, \mathrm{H}-6 \mathrm{a}\right), 3.33\left(\mathrm{dd}, J_{6 \mathrm{a}, 6 \mathrm{~b}}=9.1 \mathrm{~Hz}\right.$, 
$\left.J_{6 \mathrm{~b}, 5}=6.4 \mathrm{~Hz}, 1 \mathrm{H}, \mathrm{H}-6 \mathrm{a}\right), 3.02\left(\mathrm{~d}, J_{\mathrm{OH}, 1}=3.6 \mathrm{~Hz}, 1 \mathrm{H}, 1-\mathrm{OH}\right), 2.45-2.44(\mathrm{~m}, 1 \mathrm{H}$, 2-OH), 1.44 (s, 3H, $\left.\mathrm{CH}_{3} i \mathrm{Pr}\right), 1.36$ (s, 3H, $\left.\mathrm{CH}_{3} i \mathrm{Pr}\right) ;{ }^{13} \mathrm{C} \mathrm{NMR}\left(125 \mathrm{MHz}, \mathrm{CDCl}_{3}\right.$ ) $\delta 143.9\left(\mathrm{C}-i, \mathrm{CPh}_{3}\right), 128.8\left(\mathrm{C}-o, \mathrm{CPh}_{3}\right), 127.8\left(\mathrm{C}-m, \mathrm{CPh}_{3}\right), 127.0\left(\mathrm{C}-p, \mathrm{CPh}_{3}\right)$, 109.5 ( $\left.\mathrm{C}_{\mathrm{q}} i \mathrm{Pr}\right), 91.2$ (C-1), $86.8\left(\mathrm{C}_{\mathrm{q}} \mathrm{CPh}_{3}\right), 75.4$ (C-3), 72.9 (C-4), 69.1 (C-2), 67.9 (C-5), 62.8 (C-6), 27.4, $25.7\left(2 \times \mathrm{CH}_{3} i \mathrm{Pr}\right) ; \mathrm{HRMS}(\mathrm{ESI}) \mathrm{m} / \mathrm{z}$ calcd. for $\left[\mathrm{C}_{28} \mathrm{H}_{30} \mathrm{O}_{6}+\mathrm{Na}\right]^{+}:$485.1940, obsd.: 485.1949 .

4-O-Formyl-2,3- $O$-isopropylidene-5- $O$-triphenylmethyl-D-lyxose (41). A solution of $\mathrm{NaIO}_{4}(0.46 \mathrm{~g}, 2.14 \mathrm{mmol})$ in $\mathrm{H}_{2} \mathrm{O}(4 \mathrm{~mL})$ was added to $40(0.66 \mathrm{~g}$, $1.42 \mathrm{mmol}$ ) dissolved in $4 \mathrm{~mL}$ THF at $0{ }^{\circ} \mathrm{C}$. After stirring at room temperature overnight, the reaction mixture was diluted with EtOAc $(15 \mathrm{~mL})$ and washed with water $(20 \mathrm{~mL})$, saturated $\mathrm{NaHCO}_{3}$ solution $(20 \mathrm{~mL})$ and brine $(20 \mathrm{~mL})$, dried $\left(\mathrm{MgSO}_{4}\right)$, filtered and concentrated in vacuo to afford the title compound 41 (0.65 g, 1.42 mmol, quant.) as a clear oil, which was carried through without further purification. $\mathrm{R}_{f}: 0.48$ (PE/EA, 1/1, v/v); IR (film) 3450, 3060, 3025, 2989, 2938, 2360, 2341, 2252, 1732, 1597, 1491, 1449, 1383, 1218, 1160, 1076, 987, 907, 764, 729, 701, $648 \mathrm{~cm}^{-1} ;{ }^{1} \mathrm{H}$ NMR (500 MHz, $\left.\mathrm{CDCl}_{3}\right) \delta 9.60\left(\mathrm{~d}, J_{1,2}=2.4 \mathrm{~Hz}, 1 \mathrm{H}\right.$, $\mathrm{H}-1), 8.00\left(\mathrm{~s}, 1 \mathrm{H}, \mathrm{HCO}_{2}\right), 7.44-7.41\left(\mathrm{~m}, 6 \mathrm{H}, \mathrm{CPh}_{3}, \mathrm{CH}-o\right), 7.34-7.30(\mathrm{~m}, 6 \mathrm{H}$, $\left.\mathrm{CPh}_{3}, \mathrm{CH}-m\right), 7.27-7.24\left(\mathrm{~m}, 3 \mathrm{H}, \mathrm{CPh}_{3}, \mathrm{CH}-p\right), 5.18\left(\mathrm{q}, J_{3,4}=J_{4,5 \mathrm{a}}=J_{4,5 \mathrm{~b}}=4.9 \mathrm{~Hz}\right.$, $1 \mathrm{H}, \mathrm{H}-4), 4.74\left(\mathrm{dd}, J_{2,3}=7.7 \mathrm{~Hz}, J_{3,4}=4.2 \mathrm{~Hz}, 1 \mathrm{H}, \mathrm{H}-3\right), 4.31\left(\mathrm{dd}, J_{2,3}=7.7 \mathrm{~Hz}\right.$, $\left.J_{1,2}=2.4 \mathrm{~Hz}, 1 \mathrm{H}, \mathrm{H}-2\right), 3.40\left(\mathrm{dd}, J_{5 \mathrm{a}, 5 \mathrm{~b}}=9.8 \mathrm{~Hz}, J_{4,5 \mathrm{a}}=5.6 \mathrm{~Hz}, 1 \mathrm{H}, \mathrm{H}-5 \mathrm{a}\right), 3.37$ $\left(\mathrm{dd}, J_{5 \mathrm{a}, 5 \mathrm{~b}}=9.8 \mathrm{~Hz}, J_{4,5 \mathrm{~b}}=5.6 \mathrm{~Hz}, 1 \mathrm{H}, \mathrm{H}-5 \mathrm{~b}\right), 1.56$ (s, 3H, $\left.i \operatorname{Pr} \mathrm{CH}_{3}\right), 1.42$ (s, 3H, $\left.i \operatorname{Pr~} \mathrm{CH}_{3}\right) ;{ }^{13} \mathrm{C}$ NMR (125 MHz, $\mathrm{CDCl}_{3}$ ) $\delta 201.1$ (C-1), 159.7 (C-6), 128.6 (CH-o, $\left.\mathrm{CPh}_{3}\right), 127.9\left(\mathrm{CH}-m, \mathrm{CPh}_{3}\right), 127.2\left(\mathrm{CH}-p, \mathrm{CPh}_{3}\right), 111.1\left(\mathrm{C}_{\mathrm{q}} i \mathrm{Pr}\right), 87.2\left(\mathrm{C}_{\mathrm{q}} \mathrm{CPh}_{3}\right)$, 80.2 (C-2), 77.0 (C-3), 69.5 (C-4), 61.8 (C-5), 26.8, 25.1 (2 x $\mathrm{CH}_{3} i \mathrm{Pr}$ ); HRMS(ESI) $m / z$ calcd. for $\left[\mathrm{C}_{28} \mathrm{H}_{28} \mathrm{O}_{6}+\mathrm{Na}\right]^{+}: 483.1784$, obsd.: 483.1793 .

\section{3,4-O-Isopropylidene-1-O-triphenylmethyl-D-arabino-octadec-5-en-1,2,3,4-}

tetraol (42). A mixture of 1-bromotridecane $(7.0 \mathrm{~mL}, 27.4 \mathrm{mmol})$ and triphenylphosphine $(7.18 \mathrm{~g}, 27.4 \mathrm{mmol})$ was heated at $140{ }^{\circ} \mathrm{C}$ under $\mathrm{N}_{2}$ atmosphere for $5 \mathrm{~h}$. The reaction mixture was allowed to cool to room temperature and formed a solid gel. The gel was dissolved in boiling dry acetone $(36 \mathrm{~mL})$ and dry diethyl ether $(87 \mathrm{~mL})$ was added and the compound left to crystalise, which 
greatly improved in subsequent batches via the use of seed crystals. The crystals were filtered under $\mathrm{N}_{2}$ atmosphere to afford $\mathrm{BrPh}_{3} \mathrm{PC}_{13} \mathrm{H}_{27}(11.1 \mathrm{~g}, 21.1 \mathrm{~mol}$, $77 \%$ ) as white needles. $n$ - $\mathrm{BuLi}(6.6 \mathrm{~mL}, 13.2 \mathrm{mmol})$ was added to $\mathrm{BrPh}_{3} \mathrm{PC}_{13} \mathrm{H}_{27}$ $(7.0 \mathrm{~g}, 13.7 \mathrm{mmol})$ dissolved in distilled THF $(130 \mathrm{~mL})$ at $-78{ }^{\circ} \mathrm{C}$ and stirred for $15 \mathrm{~min}$. To the bright orange mixture, a solution of formate ester 41 (coevaporated with dry toluene $\mathrm{x} 3$ ) in THF $(55 \mathrm{~mL})$ was added. The reaction mixture was stirred at $-78{ }^{\circ} \mathrm{C}$ for $1 \mathrm{~h}$, then warmed to room temperature and stirred for a further $3.5 \mathrm{~h}$ before being quenched with $\mathrm{NH}_{4} \mathrm{Cl}$ solution $(150 \mathrm{~mL})$, concentrated, and redissolved in ethyl acetate $(100 \mathrm{~mL})$. The organic layer was washed with saturated $\mathrm{NaHCO}_{3}$ solution $(100 \mathrm{~mL})$, water $(100 \mathrm{~mL})$ and brine $(100 \mathrm{~mL})$, dried $\left(\mathrm{MgSO}_{4}\right)$, filtered and concentrated in vacuo. The resulting residue was purified by silica gel column chromatography and the desired product eluted with 8:1 (v/v) petroleum ether/EtOAc to afford alkene $\mathbf{4 2}$ as a clear oil $(2.57 \mathrm{~g}, 4.29 \mathrm{mmol}, 78 \%)$ in an approximate $E: Z, 1: 10 . \mathrm{R}_{f}: 0.33$ (PE/EA, 5/1, v/v); $[\alpha]_{\mathrm{D}}{ }^{20}=-22.2^{\circ}\left(c=1.0, \mathrm{CHCl}_{3}\right)$; IR (film) 3559, 3058, 3023, 2924, 2854, 1598, 1491, 1449, 1380, 1214, 1162, 1073, 898, 880, 763, 746, $705 \mathrm{~cm}^{-1} ;{ }^{1} \mathrm{H}$ NMR (500 MHz, $\left.\mathrm{CDCl}_{3}\right) \delta$ 7.48-7.44 (m, 6H, $\left.\mathrm{CPh}_{3}, \mathrm{CH}-o\right), 7.33-7.29\left(\mathrm{~m}, 6 \mathrm{H}, \mathrm{CPh}_{3}, \mathrm{CH}-m\right)$, 7.26-7.23 (m, 3H, CPh 3 , CH-p), $5.59\left(\mathrm{dd}, J_{5,6}=11.3 \mathrm{~Hz}, J_{6,7}=6.0 \mathrm{~Hz}, 1 \mathrm{H}, \mathrm{H}-6\right)$, $5.55\left(\mathrm{dd}, J_{5,6}=11.3 \mathrm{~Hz}, J_{4,5}=7.7 \mathrm{~Hz}, 1 \mathrm{H}, \mathrm{H}-5\right), 4.92\left(\mathrm{dd}, J_{4,5}=7.7 \mathrm{~Hz}, J_{3,4}=6.9\right.$ $\mathrm{Hz}, 1 \mathrm{H}, \mathrm{H}-4), 4.23$ (dd, $J_{3,4}=6.9 \mathrm{~Hz}, J_{2,3}=4.3 \mathrm{~Hz}, 1 \mathrm{H}, \mathrm{H}-3$ ), 3.77 (pent, $J_{1,2}=J_{2,3}$ $\left.=J_{2, \mathrm{OH}}=5.4 \mathrm{~Hz}, 1 \mathrm{H}, \mathrm{H}-2\right), 3.18\left(\mathrm{dd}, J_{1 \mathrm{a}, 1 \mathrm{~b}}=9.4 \mathrm{~Hz}, J_{1 \mathrm{a}, 2}=5.0 \mathrm{~Hz}, 1 \mathrm{H}, \mathrm{H}-1 \mathrm{a}\right), 3.13$ $\left(\mathrm{dd}, J_{1 \mathrm{a}, 1 \mathrm{~b}}=9.4 \mathrm{~Hz}, J_{1 \mathrm{~b}, 2}=6.4 \mathrm{~Hz}, 1 \mathrm{H}, \mathrm{H}-1 \mathrm{~b}\right), 2.03-1.98(\mathrm{~m}, 1 \mathrm{H}, \mathrm{H}-7 \mathrm{a}), 1.82-1.77$ (m, 1H, H-7a), 1.51 (s, 3H, CH $i \mathrm{Pr}$ ), 1.41 (s, 3H, $\mathrm{CH}_{3} i \mathrm{Pr}$ ), 1.33-1.23 (m, 20H, H8-H-17), 0.90 (t, $\left.J_{17,18}=7.0 \mathrm{~Hz}, 3 \mathrm{H}, \mathrm{H}-18\right) ;{ }^{13} \mathrm{C} \mathrm{NMR}\left(125 \mathrm{MHz}, \mathrm{CDCl}_{3}\right) \delta 143.9$ $\left(\mathrm{C}-i, \mathrm{CPh}_{3}\right), 135.4$ (C-6), $128.7\left(\mathrm{CH}-o, \mathrm{CPh}_{3}\right), 127.8\left(\mathrm{CH}-m, \mathrm{CPh}_{3}\right), 127.0(\mathrm{CH}-p$, $\mathrm{CPh}_{3}$ ), 125.0 (C-5), $108.4\left(\mathrm{C}_{\mathrm{q}} i \mathrm{Pr}\right), 86.7\left(\mathrm{C}_{\mathrm{q}} \mathrm{CPh}_{3}\right), 77.5$ (C-3), 73.0 (C-4), 69.2 (C-2), 65.0 (C-1), 31.9, 29.70, 29.67, 29.62, 29.52, 29.50, 29.4, 29.3, 27.6, 22.7 (C-7-C-17), 27.4, 25.1 (2 x CH 3 iPr), 14.2 (C-18); HRMS(ESI) $m / z$ calcd. for $\left[\mathrm{C}_{40} \mathrm{H}_{54} \mathrm{O}_{4}+\mathrm{Na}\right]^{+}:$621.3920, obsd.: 621.3926 . 


\section{2-Azido-3,4-O-isopropylidene-1- $O$-triphenylmethyl-D-arabino-octadecane-}

1,3,4-triol (43). $\mathrm{Pd} / \mathrm{C}(1.5 \mathrm{wt} \%)$ was added to a solution of 42 (2.52 g, 4.21 mmol) in distilled THF $(42 \mathrm{~mL})$ and stirred under $\mathrm{H}_{2}(\mathrm{~g})$ overnight at room temperature. The reaction mixture was then filtered through celite and concentrated to afford 3,4-O-Isopropylidene-1-O-triphenylmethyl-D-arabinooctadecane-1,2,3,4-tetraol as a clear oil (2.53 g, $4.21 \mathrm{mmol}$, quant.), which was used without further purification. $\mathrm{R}_{f}: 0.44(\mathrm{PE} / \mathrm{EA}, 5 / 1, \mathrm{v} / \mathrm{v}) ;[\alpha]_{\mathrm{D}}{ }^{20}=-4.0^{\circ}(c=$ 1.0, $\mathrm{CHCl}_{3}$ ); IR (film) 3560, 3060, 3023, 2923, 2853, 1598, 1491, 1449, 1379, 1216, 1073, 899, 872, 762, 746, $705 \mathrm{~cm}^{-1} ;{ }^{1} \mathrm{H}$ NMR (500 MHz, $\left.\mathrm{CDCl}_{3}\right) \delta 7.47(\mathrm{~d}$, $\left.J_{\mathrm{o}, \mathrm{m}}=7.7 \mathrm{~Hz}, 6 \mathrm{H}, \mathrm{CPh}_{3}, \mathrm{CH}-o\right), 7.31\left(\mathrm{t}, J_{\mathrm{o}, \mathrm{m}}=J_{\mathrm{m}, \mathrm{p}}=7.7 \mathrm{~Hz}, 6 \mathrm{H}, \mathrm{CPh}_{3}, \mathrm{CH}-m\right)$, 7.27-7.23 (m, 3H, CPh, $\mathrm{CH}-p), 4.15\left(\mathrm{dd}, J_{2,3}=6.0 \mathrm{~Hz}, J_{3,4}=3.8 \mathrm{~Hz}, 1 \mathrm{H}, \mathrm{H}-3\right)$, 4.11-4.07 (m, 1H, H-4), 3.74 (dt, $\left.J_{1,2}=9.5 \mathrm{~Hz}, J_{2,3}=6.0 \mathrm{~Hz}, 1 \mathrm{H}, \mathrm{H}-2\right), 3.24-3.19$ (m, 2H, H-1a, H-1b), 2.34 (d, $\left.J_{\mathrm{OH}, 2}=5.9 \mathrm{~Hz}, 1 \mathrm{H}, \mathrm{OH}\right), 1.71-1.64$ (m, 2H, H-5), 1.51-1.28 (m, 24H, H-6-H-17), 1.46 (s, 3H, $i \mathrm{Pr} \mathrm{CH}_{3}$ ), 1.37 (s, 3H, $i \operatorname{Pr} \mathrm{CH}_{3}$ ), 0.89 $\left(\mathrm{t}, J_{17,18}=6.9 \mathrm{~Hz}, 3 \mathrm{H}, \mathrm{H}-18\right) ;{ }^{13} \mathrm{C} \mathrm{NMR}\left(125 \mathrm{MHz}, \mathrm{CDCl}_{3}\right) \delta 143.9\left(\mathrm{C}-i, \mathrm{CPh}_{3}\right)$, $128.7\left(\mathrm{CH}-o, \mathrm{CPh}_{3}\right), 127.8\left(\mathrm{CH}-m, \mathrm{CPh}_{3}\right), 127.1\left(\mathrm{CH}-p, \mathrm{CPh}_{3}\right), 107.7\left(\mathrm{C}_{\mathrm{q}} i \mathrm{Pr}\right)$, $86.8\left(\mathrm{C}_{\mathrm{q}} \mathrm{CPh}_{3}\right), 77.5$ (C-4), 77.0 (C-3), 69.0 (C-2), 65.2 (C-1), 31.9, 29.72, 29.68, 29.63, 29.62, 29.57, 29.39, 26.8, 22.7 (C-5-C-17), 27.4, 25.2 (2 x CH 3 iPr), 14.2 (C-18); HRMS(ESI) $m / z$ calcd. for $\left[\mathrm{C}_{40} \mathrm{H}_{56} \mathrm{O}_{4}+\mathrm{Na}\right]^{+}:$623.4076, obsd.: 623.4080 . 3,4-O-Isopropylidene-1-O-triphenylmethyl-D-arabino-octadecane-1,2,3,4-tetraol $(1.59 \mathrm{~g}, 2.65 \mathrm{mmol})$ was then co-evaporated with dry toluene (x 3), dissolved in a mixture of dry DCM $(8 \mathrm{~mL})$ and distilled pyridine $(0.54 \mathrm{~mL})$ and cooled to -15 ${ }^{\circ} \mathrm{C}$. Triflic anhydride $(0.67 \mathrm{~mL}, 3.97 \mathrm{mmol})$ was added dropwise over 5 mins and stirred at $-15{ }^{\circ} \mathrm{C}$ for 45 mins to afford the triflate intermediate $\left[\mathrm{R}_{f}: 0.56(\mathrm{PE} / \mathrm{EA}\right.$, $5 / 1, \mathrm{v} / \mathrm{v})]$. Dry DMF (27 mL) was cooled to $-15{ }^{\circ} \mathrm{C}$ and added to the reaction mixture, followed by the addition of sodium azide $(0.69 \mathrm{~g}, 10.61 \mathrm{mmol})$. The reaction mixture was then warmed to room temperature, stirred overnight, and quenched by adding ice water $(120 \mathrm{~mL})$. The desired product was extracted with EtOAc $(2 \times 150 \mathrm{~mL})$ and the combined organic layers washed with saturated $\mathrm{NaHCO}_{3}$ solution $(200 \mathrm{~mL})$, water $(200 \mathrm{~mL})$ and brine $(200 \mathrm{~mL})$, dried $\left(\mathrm{MgSO}_{4}\right)$, filtered and concentrated in vacuo. The residue was purified by silica gel flash column chromatography and eluted with $1.5 \%$ EtOAc/hexanes to afford the title compound 43 (1.35 g, $2.16 \mathrm{mmol}, 81 \%$ ) as an amorphous white solid. $\mathrm{R}_{f}: 0.61$ 
$(\mathrm{PE} / \mathrm{EA}, 5 / 1, \mathrm{v} / \mathrm{v}) ;[\alpha]_{\mathrm{D}}{ }^{25}=+7.0^{\circ}\left(c=1.0, \mathrm{CHCl}_{3}\right), \mathrm{Lit}^{.58}[\alpha]_{\mathrm{D}}^{26}=+9.0^{\circ}(c=1.0$, $\mathrm{CHCl}_{3}$ ); IR (film) 3060, 2923, 2853, 2097, 1598, 1491, 1379, 1370, 1246, 1219, 1156, 1073, 1034, 900, 869, 762, 744, $703 \mathrm{~cm}^{-1}$; ${ }^{1} \mathrm{H}$ NMR $\left(500 \mathrm{MHz}, \mathrm{CDCl}_{3}\right) \delta$ $7.51\left(\mathrm{~d}, J_{\mathrm{o}, \mathrm{m}}=7.3 \mathrm{~Hz}, 6 \mathrm{H}, \mathrm{CPh}_{3}, \mathrm{CH}-o\right), 7.32\left(\mathrm{t}, J_{\mathrm{o}, \mathrm{m}}=J_{\mathrm{m}, \mathrm{p}}=7.6 \mathrm{~Hz}, 6 \mathrm{H}, \mathrm{CPh}_{3}\right.$, CH-m), 7.26 (d, $\left.J_{\mathrm{o}, \mathrm{m}}=7.1 \mathrm{~Hz}, 3 \mathrm{H}, \mathrm{CPh}_{3}, \mathrm{CH}-p\right), 4.13-4.09$ (m, 1H, H-4), 3.89 $\left(\mathrm{dd}, J_{2,3}=9.5 \mathrm{~Hz}, J_{3,4}=5.5 \mathrm{~Hz}, 1 \mathrm{H}, \mathrm{H}-3\right), 3.55\left(\mathrm{dd}, J_{1 \mathrm{a}, 1 \mathrm{~b}}=9.9 \mathrm{~Hz}, J_{1 \mathrm{a}, 2}=2.5 \mathrm{~Hz}\right.$, $1 \mathrm{H}, \mathrm{H}-1 \mathrm{a}), 3.50$ (dt, $\left.J_{2,3}=9.5 \mathrm{~Hz}, J_{1 \mathrm{a}, 2}=2.5 \mathrm{~Hz}, 1 \mathrm{H}, \mathrm{H}-2\right), 3.37$ (dd, $J_{1 \mathrm{a}, 1 \mathrm{~b}}=9.9$ $\left.\mathrm{Hz}, J_{1 \mathrm{~b}, 2}=7.8 \mathrm{~Hz}, 1 \mathrm{H}, \mathrm{H}-1 \mathrm{~b}\right), 1.59-1.28$ (m, 26H, H-5-H-17), 1.27 (s, 3H, $i \mathrm{Pr}$ $\left.\mathrm{CH}_{3}\right), 1.24$ (s, 3H, $i \operatorname{Pr~CH}$ ), 0.90 (t, $\left.J_{17,18}=6.9 \mathrm{~Hz}, 3 \mathrm{H}, \mathrm{H}-18\right) ;{ }^{13} \mathrm{C}$ NMR (125 $\left.\mathrm{MHz}, \mathrm{CDCl}_{3}\right) \delta 143.8\left(\mathrm{C}-i, \mathrm{CPh}_{3}\right), 128.7\left(\mathrm{CH}-o, \mathrm{CPh}_{3}\right), 127.9\left(\mathrm{CH}-m, \mathrm{CPh}_{3}\right)$, 127.1 (CH-p, $\left.\mathrm{CPh}_{3}\right), 108.1\left(\mathrm{C}_{\mathrm{q}} \mathrm{CPh}_{3}\right), 87.3\left(\mathrm{C}_{\mathrm{q}} i \operatorname{Pr}\right), 77.9$ (C-4), $75.9(\mathrm{C}-3), 64.6$ (C-1), 60.8 (C-2), 32.0, 29.73, 29.69, 29.66, 29.64, 29.57, 29.4, 29.3, 26.5, 22.7 (C-5-C-17), 28.0, 25.7 (2 x $\mathrm{CH}_{3} i \mathrm{Pr}$ ), 14.2 (C-18); HRMS(ESI) $\mathrm{m} / \mathrm{z}$ calcd. for $\left[\mathrm{C}_{40} \mathrm{H}_{55} \mathrm{~N}_{3} \mathrm{O}_{3}+\mathrm{Na}\right]^{+}$: 648.4141, obsd.: 648.4145.

2-Azido-3,4-O-isopropylidene-D-ribo-octadecane-1,3,4-triol (38). Triethylsilane (91.8 $\mu \mathrm{L}, 0.58 \mathrm{mmol})$ was added to a stirred solution of azide 43 (181 $\mathrm{mg}, 0.289$ mmol) (co-evaporated with dry toluene $\mathrm{x} 3$ ) in dry $\mathrm{DCM}(5 \mathrm{~mL})$ at $0{ }^{\circ} \mathrm{C}$. Trifluoroacetic acid (TFA) $(90 \mu \mathrm{L}, 1.17 \mathrm{mmol})$ was added very slowly, allowing the reaction mixture to return to a colourless solution before adding the next drop. After stirring for $2 \mathrm{~min}, 2$,2-dimethoxypropane ( $7 \mu \mathrm{L}, 0.057 \mathrm{mmol})$ was added and the reaction mixture stirred for a further $1 \mathrm{~min}$ before being quenched with saturated $\mathrm{NaHCO}_{3}$ solution $(50 \mathrm{~mL})$. The product was extracted with DCM $(2 \mathrm{x}$ $50 \mathrm{~mL})$ and the combined organic layers washed with water $(80 \mathrm{~mL})$ and brine $(80 \mathrm{~mL})$, dried $\left(\mathrm{MgSO}_{4}\right)$, filtered and concentrated in vacuo. The resulting residue was purified by silica gel flash column chromatography and eluted with 5:1 (v/v) petroleum ether/EtOAc to afford the title compound $38(69.3 \mathrm{mg}, 0.18 \mathrm{mmol}$, $63 \%)$ as an amorphous white solid. $\mathrm{R}_{f}: 0.21(\mathrm{PE} / \mathrm{EA}, 5 / 1, \mathrm{v} / \mathrm{v}) ;[\alpha]_{\mathrm{D}}{ }^{20}=+24.0^{\circ}(c$ $=1.0, \mathrm{CHCl}_{3}$ ); IR (film) 3426, 2923, 2853, 2098, 1465, 1370, 1247, 1220, 1170, 1063, 909, 869, $734 \mathrm{~cm}^{-1} ;{ }^{1} \mathrm{H}$ NMR (500 MHz, $\left.\mathrm{CDCl}_{3}\right) \delta 4.21-4.17\left(\mathrm{~m}, J_{3,4}=5.7\right.$ $\mathrm{Hz}, \mathrm{H}-4), 4.0\left(\mathrm{ddd}, J_{1 \mathrm{a}, 1 \mathrm{~b}}=11.8 \mathrm{~Hz}, J_{1 \mathrm{a}, \mathrm{OH}}=5.1 \mathrm{~Hz}, J_{1 \mathrm{a}, 2}=4.4 \mathrm{~Hz}, 1 \mathrm{H}, \mathrm{H}-1 \mathrm{a}\right), 3.97$ $\left(\mathrm{dd}, J_{2,3}=9.6 \mathrm{~Hz}, J_{3,4}=5.7 \mathrm{~Hz}, 1 \mathrm{H}, \mathrm{H}-3\right), 3.87\left(\mathrm{ddd}, J_{1 \mathrm{a}, 1 \mathrm{~b}}=11.8 \mathrm{~Hz}, J_{1 \mathrm{~b}, \mathrm{OH}}=6.6\right.$ $\left.\mathrm{Hz}, J_{1 \mathrm{~b}, 2}=5.6 \mathrm{~Hz}, 1 \mathrm{H}, \mathrm{H}-1 \mathrm{~b}\right), 3.48\left(\mathrm{ddd}, J_{2,3}=9.6 \mathrm{~Hz}, J_{1 \mathrm{~b}, 2}=5.6 \mathrm{~Hz}, J_{1 \mathrm{a}, 2}=4.4\right.$ 
$\mathrm{Hz}, 1 \mathrm{H}, \mathrm{H}-2), 2.20\left(\mathrm{dd}, J_{1 \mathrm{~b}, \mathrm{OH}}=6.6 \mathrm{~Hz}, J_{1 \mathrm{a}, \mathrm{OH}}=5.1 \mathrm{~Hz}, 1 \mathrm{H}, \mathrm{OH}\right), 1.63-1.55(\mathrm{~m}$, $3 \mathrm{H}, \mathrm{H}-5 \mathrm{a}, \mathrm{H}-5 \mathrm{~b}, \mathrm{H}-6 \mathrm{a}), 1.43$ (s, 3H, $i \operatorname{Pr} \mathrm{CH}_{3}$ ), 1.34 (s, 3H, $i \operatorname{Pr} \mathrm{CH}_{3}$ ), 1.41-1.26 (m, 23H, H-6b, H-7-H-17), 0.88 (t, $\left.J_{17,18}=7.0 \mathrm{~Hz}, 3 \mathrm{H}, \mathrm{H}-18\right) ;{ }^{13} \mathrm{C}$ NMR $(125$ $\left.\mathrm{MHz} \mathrm{CDCl}_{3}\right) \delta 108.4\left(\mathrm{C}_{\mathrm{q}} i \mathrm{Pr}\right), 77.7$ (C-4), 76.7 (C-3), 63.9 (C-1), $61.2(\mathrm{C}-2)$, $31.9,29.70,29.69,29.68,29.66,29.65,29.60,29.58,29.53,29.39,29.36,26.52$, 22.7 (C-5-C-17), 28.0, 25.5 (2 x CH CH$_{3}$ Pr), 14.1 (C-18); HRMS(ESI) $m / z$ calcd. for $\left[\mathrm{C}_{21} \mathrm{H}_{41} \mathrm{~N}_{3} \mathrm{O}_{3}+\mathrm{Na}\right]^{+}:$406.3046, obsd.: 406.3049 .

Methyl 6-deoxy-6-iodo- $\boldsymbol{\alpha}$-D-galactopyranoside (44). To a mixture of 1,2:3,4di-O-isopropylidene- $\alpha$-D-galactopyranose ${ }^{59,60}$ (2.6 g, $10 \mathrm{mmol}$ ), $\mathrm{PPh}_{3}$ (3.93 g, 15 $\mathrm{mmol})$ and imidazole $(1.36 \mathrm{~g}, 20 \mathrm{mmol})$ in dry THF $(100 \mathrm{~mL})$, was added iodine (3.81 g, $15 \mathrm{mmol})$ in small portions. After refluxing for $1 \mathrm{~h}$, the reaction mixture was cooled to room temperature, and quenched by the addition of $10 \%$ aq. $\mathrm{Na}_{2} \mathrm{~S}_{2} \mathrm{O}_{4}$. The product was extracted with EtOAc, and the combined organic layers were washed with brine, dried $\left(\mathrm{MgSO}_{4}\right)$, filtered and concentrated. Distillation of the residue gave 6-deoxy-6-iodo-1,2:3,4-di- $O$-isopropylidene- $\alpha$-Dgalactopyranose as a yellow oil $(3.14 \mathrm{~g}, 8.48 \mathrm{mmol}, 85 \%)$. AcCl $(1.8 \mathrm{~mL})$ was added dropwise to a mixture of 6-deoxy-6-iodo-1,2:3,4-di- $O$-isopropylidene- $\alpha$-Dgalactopyranose $(3.14 \mathrm{~g}, 8.48 \mathrm{mmol})$ in $\mathrm{MeOH}(60 \mathrm{~mL})$. After stirring for $2 \mathrm{~d}$, the mixture was concentrated in vacuo and the residue crystalised from $\mathrm{MeOH}$, to give the title compound $44(2.37 \mathrm{~g}, 7.80 \mathrm{mmol}, 92 \%)$ as white crystals. Mp 175.0-175.6 ${ }^{\circ} \mathrm{C}$, Lit: ${ }^{.7} 162{ }^{\circ} \mathrm{C} ; \mathrm{R}_{f}=0.43(\mathrm{MeOH} /$ EtOAc, $1 / 9, \mathrm{v} / \mathrm{v}) ;[\alpha]_{\mathrm{D}}{ }^{21}=$ $+129.0^{\circ}\left(c=1.0, \mathrm{H}_{2} \mathrm{O}\right),[\alpha]_{\mathrm{D}}{ }^{21}=+122.1^{\circ}(c=1.0, \mathrm{MeOH}), \mathrm{Lit}^{57}[\alpha]_{\mathrm{D}}{ }^{22}=+142.0^{\circ}$ $\left(c=1.0, \mathrm{H}_{2} \mathrm{O}\right.$ ); IR (film) 3354, 3227, 3007, 2953, 2935, 2906, 2836, 1454, 1417, 1360, 1347, 1297, 1257, 1200, 1145, 1132, 1102, 1078, 1026, 999, 935, 886, 854, 790, 721, 691, $657 \mathrm{~cm}^{-1} ;{ }^{1} \mathrm{H}$ NMR $\left(500 \mathrm{MHz}, \mathrm{D}_{2} \mathrm{O}\right) \delta 4.69\left(\mathrm{~d}, J_{1,2}=3.6 \mathrm{~Hz}, 1 \mathrm{H}\right.$, $\mathrm{H}-1), 3.97\left(\mathrm{dd}, J_{3,4}=3.3, J_{4,5}=1.2 \mathrm{~Hz}, 1 \mathrm{H}, \mathrm{H}-4\right), 3.90\left(\mathrm{ddd}, J_{4,5}=1.2 \mathrm{~Hz}, J_{5,6 \mathrm{a}}=\right.$ $\left.5.3, J_{5,6 \mathrm{~b}}=8.0 \mathrm{~Hz} 1 \mathrm{H}, \mathrm{H}-5\right), 3.75\left(\mathrm{dd}, J_{1,2}=3.6, J_{2,3}=10.2 \mathrm{~Hz}, 1 \mathrm{H}, \mathrm{H}-2\right), 3.69$ $\left(\mathrm{dd}, J_{2,3}=10.2, J_{3,4}=3.3 \mathrm{~Hz}, 1 \mathrm{H}, \mathrm{H}-3\right), 3.46(\mathrm{~s}, 3 \mathrm{H}, \mathrm{OMe}), 3.37\left(\mathrm{dd}, J_{5,6 \mathrm{a}}=5.3\right.$, $\left.J_{6 \mathrm{a}, 6 \mathrm{~b}}=10.2 \mathrm{~Hz}, 1 \mathrm{H}, \mathrm{H}-6 \mathrm{a}\right), 3.33\left(\mathrm{dd}, J_{5,6 \mathrm{~b}}=8.0, J_{6 \mathrm{a}, 6 \mathrm{~b}}=10.2 \mathrm{~Hz}, 1 \mathrm{H}, \mathrm{H}-6 \mathrm{~b}\right) ;{ }^{13} \mathrm{C}$ NMR (125 MHz, D 2 O) $\delta 100.2$ (C-1), 71.4 (C-5), 70.4 (C-4), 69.9 (C-2), 68.4 (C3), $54.6(\mathrm{OMe}), 2.3(\mathrm{C}-6)$. HRMS(ESI) $\mathrm{m} / z$ calcd. for $\left[\mathrm{C}_{7} \mathrm{H}_{13} \mathrm{IO}_{5}+\mathrm{Na}\right]^{+}$: 326.9700, obsd.: 326.9709 . 
Sodium azide ( $4.28 \mathrm{~g}, 0.066 \mathrm{mmol})$ was added to iodide $44(5.01 \mathrm{~g}, 0.016 \mathrm{mmol})$ dissolved in dry DMF (300 mL). After stirring overnight at room temperature, $\mathrm{MeOH}(150 \mathrm{~mL})$ was added and the reaction mixture was concentrated in vacuo. The residue was purified by silica gel flash column chromatography and the product eluted with $8 \% \mathrm{MeOH} / \mathrm{DCM}$. The residue was crystallised from methanol to afford methyl 6-azido-6-deoxy- $\alpha$-D-galactopyranoside (2.96 g, 14 mmol, 82\%) as white crystals. Mp 173.8-174.1 ${ }^{\circ} \mathrm{C}$, Lit: ${ }^{60} 172-173{ }^{\circ} \mathrm{C} ; \quad \mathrm{R}_{f}=0.43$ $(\mathrm{MeOH} /$ EtOAc, $1 / 9, \mathrm{v} / \mathrm{v}) ;[\alpha]_{\mathrm{D}}{ }^{23}=+144.0^{\circ}\left(c=1.0, \mathrm{H}_{2} \mathrm{O}\right), \mathrm{Lit}^{61}[\alpha]_{\mathrm{D}}{ }^{22}=+154.0^{\circ}$ $\left(c=0.8, \mathrm{H}_{2} \mathrm{O}\right)$; IR (film) 3371, 3238, 2934, 2091, 1642, 1459, 1349, 1297, 1245, 1137, 1107, 1080, 1029, 961, 791, 730, $698 \mathrm{~cm}^{-1} ;{ }^{1} \mathrm{H}$ NMR $\left(500 \mathrm{MHz}, \mathrm{CDCl}_{3}\right) \delta$ $4.73\left(\mathrm{~d}, J_{1,2}=3.7 \mathrm{~Hz}, 1 \mathrm{H}, \mathrm{H}-1\right), 3.90\left(\mathrm{ddd}, J_{5,6 \mathrm{a}}=8.8 \mathrm{~Hz}, J_{5,6 \mathrm{~b}}=4.0 \mathrm{~Hz}, J_{4,5}=1.1\right.$ $\mathrm{Hz}, 1 \mathrm{H}, \mathrm{H}-5), 3.79$ (dd, $\left.J_{3,4}=3.3 \mathrm{~Hz}, J_{4,5}=1.1 \mathrm{~Hz}, 1 \mathrm{H}, \mathrm{H}-4\right), 3.77$ (dd, $J_{2,3}=10.1$ $\left.\mathrm{Hz}, J_{1,2}=3.7 \mathrm{~Hz}, 1 \mathrm{H}, \mathrm{H}-2\right), 3.72\left(\mathrm{dd}, J_{2,3}=10.1 \mathrm{~Hz}, J_{3,4}=3.3 \mathrm{~Hz}, 1 \mathrm{H}, \mathrm{H}-3\right), 3.58$ $\left(\mathrm{dd}, J_{6 \mathrm{a}, 6 \mathrm{~b}}=12.8 \mathrm{~Hz}, J_{5,6 \mathrm{a}}=8.8 \mathrm{~Hz}, 1 \mathrm{H}, \mathrm{H}-6 \mathrm{a}\right), 3.44\left(\mathrm{~s}, 3 \mathrm{H}, \mathrm{OCH}_{3}\right), 3.27$ (dd, $J_{6 \mathrm{a}, 6 \mathrm{~b}}$ $\left.=12.8 \mathrm{~Hz}, J_{5,6 \mathrm{~b}}=4.0 \mathrm{~Hz}, 1 \mathrm{H}, \mathrm{H}-6 \mathrm{~b}\right) ;{ }^{13} \mathrm{C} \mathrm{NMR}\left(125 \mathrm{MHz}, \mathrm{CDCl}_{3}\right) \delta 100.2(\mathrm{C}-1)$, 70.0, 69.83, 69.77 (C-3, C-4, C-5), $68.6(\mathrm{C}-2), 54.3\left(\mathrm{OCH}_{3}\right), 51.3$ (C-6); HRMS(ESI) $m / z$ calcd. for $\left[\mathrm{C}_{7} \mathrm{H}_{13} \mathrm{~N}_{3} \mathrm{O}_{5}+\mathrm{Na}\right]^{+}:$242.0753, obsd.: 242.0749. Methyl 6-azido-6-deoxy- $\alpha$-D-galactopyranose $(1.53 \mathrm{~g}, 6.98 \mathrm{mmol})$ was then coevaporated from dry DMF (x 1), dissolved in dry DMF (150 mL) and cooled to 0 ${ }^{\circ} \mathrm{C}$. Benzyl bromide (3.3 mL, $27.9 \mathrm{mmol}$ ) was added followed by sodium hydride (60\% in oil suspension) (1.40 g, $34.9 \mathrm{mmol})$ and the reaction mixture allowed to warm to rt. After stirring for $2 \mathrm{~h}$, the reaction was quenched with $\mathrm{MeOH}(20 \mathrm{~mL})$ and concentrated in vacuo. The residue was taken up in EtOAc $(100 \mathrm{~mL})$ and the mixture was washed with water $(100 \mathrm{~mL})$, saturated $\mathrm{NaHCO}_{3}$ solution $(100 \mathrm{~mL})$, water $(100 \mathrm{~mL})$ and brine $(100 \mathrm{~mL})$, dried $\left(\mathrm{MgSO}_{4}\right)$, filtered and concentrated in vacuo. The residue was purified by silia gel flash column chromatography and eluted with 20:1 (v/v) petroleum ether/EtOAc to afford the title compound $\mathbf{4 5}$ $(3.37 \mathrm{~g}, 6.88 \mathrm{mmol}, 99 \%)$ as a clear oil. $\mathrm{R}_{f}: 0.72(\mathrm{PE} / \mathrm{EA}, 1 / 1, \mathrm{v} / \mathrm{v}) ;[\alpha]_{\mathrm{D}}{ }^{20}=+4.6^{\circ}$ $\left(c=1.0, \mathrm{CHCl}_{3}\right)$; IR (film) 2937, 2098, 1453, 1351, 1124, 1094, 1045, 789, 735 $\mathrm{cm}^{-1} ;{ }^{1} \mathrm{H}$ NMR $\left(500 \mathrm{MHz}, \mathrm{CDCl}_{3}\right) \delta 7.44-7.31\left(\mathrm{~m}, 15 \mathrm{H}, \mathrm{H}_{\text {arom }}\right), 5.03\left(\mathrm{~d}, J_{\mathrm{a}, \mathrm{b}}=\right.$ $11.4 \mathrm{~Hz}, 1 \mathrm{H}, \mathrm{CH}-\mathrm{a}, 4-O-\mathrm{Bn}), 4.93$ (d, $\left.J_{\mathrm{a}, \mathrm{b}}=11.7 \mathrm{~Hz}, 1 \mathrm{H}, \mathrm{CH}-\mathrm{a}, 3-O-\mathrm{Bn}\right), 4.87$ (d, 
$\left.J_{\mathrm{a}, \mathrm{b}}=12.1 \mathrm{~Hz}, 1 \mathrm{H}, \mathrm{CH}-\mathrm{a}, 2-O-\mathrm{Bn}\right), 4.78\left(\mathrm{~d}, J_{\mathrm{a}, \mathrm{b}}=11.7 \mathrm{~Hz}, 1 \mathrm{H}, \mathrm{CH}-\mathrm{b}, 3-O-\mathrm{Bn}\right)$, $4.72\left(\mathrm{~d}, J_{\mathrm{a}, \mathrm{b}}=12.1 \mathrm{~Hz}, 1 \mathrm{H}, \mathrm{CH}-\mathrm{b}, 2-O-\mathrm{Bn}\right), 4.72\left(\mathrm{~d}, J_{1,2}=3.7 \mathrm{~Hz}, 1 \mathrm{H}, \mathrm{H}-1\right), 5.03$ $\left(\mathrm{d}, J_{\mathrm{a}, \mathrm{b}}=11.4 \mathrm{~Hz}, 1 \mathrm{H}, \mathrm{CH}-\mathrm{b}, 4-O-\mathrm{Bn}\right), 4.07$ (dd, $J_{2,3}=10.1 \mathrm{~Hz}, J_{1,2}=3.7 \mathrm{~Hz}, 1 \mathrm{H}$, H-2), $3.96\left(\mathrm{dd}, J_{2,3}=10.1 \mathrm{~Hz}, J_{3,4}=2.6 \mathrm{~Hz}, 1 \mathrm{H}, \mathrm{H}-3\right), 3.83\left(\mathrm{dd}, J_{5,6 \mathrm{a}}=8.3 \mathrm{~Hz}\right.$, $\left.J_{5,6 \mathrm{~b}}=4.7 \mathrm{~Hz}, 1 \mathrm{H}, \mathrm{H}-5\right), 3.80\left(\mathrm{~d}, J_{3,4}=2.6 \mathrm{~Hz}, 1 \mathrm{H}, \mathrm{H}-4\right), 3.53\left(\mathrm{dd}, J_{6 \mathrm{a}, 6 \mathrm{~b}}=12.7\right.$ $\left.\mathrm{Hz}, J_{5,6 \mathrm{a}}=8.3 \mathrm{~Hz}, 1 \mathrm{H}, \mathrm{H}-6 \mathrm{a}\right), 3.43\left(\mathrm{~s}, 3 \mathrm{H}, \mathrm{OCH}_{3}\right), 2.94\left(\mathrm{dd}, J_{6 \mathrm{a}, 6 \mathrm{~b}}=12.7 \mathrm{~Hz}, J_{5,6 \mathrm{~b}}\right.$ $=4.7 \mathrm{~Hz}, 1 \mathrm{H}, \mathrm{H}-6 \mathrm{~b}) ;{ }^{13} \mathrm{C} \mathrm{NMR}\left(125 \mathrm{MHz}, \mathrm{CDCl}_{3}\right) \delta 138.7$ (C-i, 3-O-Bn), 138.4 (C-i, 2-O-Bn), 138.1 (C-i, 4-O-Bn), 128.52, 128.50, 128.4, 128.1, 128.0, 127.8, 127.7, 127.6 (8 x CH $\mathrm{CH}_{\text {arom}}$ ), 98.8 (C-1), 79.0 (C-3), 76.3 (C-2), 75.3 (C-4), 74.6 $\left(\mathrm{CH}_{2}, 4-O-\mathrm{Bn}\right), 73.70,73.65$ (2 x $\left.\mathrm{CH}_{2}, 2-O-\mathrm{Bn}, 3-O-\mathrm{Bn}\right), 69.8(\mathrm{C}-5), 55.5$ $\left(\mathrm{OCH}_{3}\right), 51.5$ (C-6); HRMS(ESI) $m / z$ calcd. for $\left[\mathrm{C}_{28} \mathrm{H}_{31} \mathrm{~N}_{3} \mathrm{O}_{5}+\mathrm{Na}\right]^{+}$: 512.2161, obsd.: 512.2159 .

Methyl 2,3,4-tri-O-benzyl-6-deoxy-6-[ $N$-(5-[dimethylamino]napth-1ylsulfonyl)amido]- $\alpha$-D-galactopyranoside (46). Trimethylphosphine (1M in THF) (10.2 mL, $10.2 \mathrm{mmol}$ ) was added to a solution of 45 (1.0 g, $2.04 \mathrm{mmol})$ (coevaporated with toluene $\mathrm{x} 3)$ in distilled THF $(10 \mathrm{~mL})$ at $0{ }^{\circ} \mathrm{C}$. After stirring for $15 \mathrm{~min}$ at $0{ }^{\circ} \mathrm{C}$ and $45 \mathrm{~min}$ at $\mathrm{rt}$, the reaction mixture was cooled to $0{ }^{\circ} \mathrm{C}$ and dansyl chloride $(1.38 \mathrm{~g}, 5.11 \mathrm{mmol})$ was added followed by the addition of $\mathrm{NaOH}$ $(1 \mathrm{M}, 8.17 \mathrm{~mL}, 8.17 \mathrm{mmol})$ over $10 \mathrm{~min}$. The reaction was then allowed to warm to rt and after $6 \mathrm{~h}$, further dansyl chloride $(0.83 \mathrm{~g}, 3.06 \mathrm{mmol})$ and $\mathrm{NaOH}(1 \mathrm{M}, 2.04$ $\mathrm{mL}, 2.04 \mathrm{mmol}$ ) was added. After stirring for a further $16 \mathrm{~h}$, the solution was diluted with EtOAc $(50 \mathrm{~mL})$, and the organic layer washed with water $(50 \mathrm{~mL})$ and brine $(50 \mathrm{~mL})$, dried $\left(\mathrm{MgSO}_{4}\right)$, filtered and concentrated in vacuo. The residue was purified by silica gel flash column chromatography and eluted with $1: 1(\mathrm{v} / \mathrm{v})$ petroleum ether/EtOAc to afford the title compound 46 (1.32 g, 1.89 mmol, 93\%) as a lime green foam. $\mathrm{R}_{f}$ : $0.61(\mathrm{PE} / \mathrm{EA}, 1 / 1, \mathrm{v} / \mathrm{v})$; Glows white on $\operatorname{TLC}(\lambda=254 \mathrm{~nm}) ;[\alpha]_{\mathrm{D}}{ }^{19}=+9.2^{\circ}\left(c=1.0, \mathrm{CHCl}_{3}\right)$; IR (film) 3293, 3063, 3030, 2988, 2349, 1588, 1574, 1497, 1326, 1200, 1143, 791, 735, $698 \mathrm{~cm}^{-1}$; ${ }^{1} \mathrm{H} \mathrm{NMR}$ $\left(500 \mathrm{MHz}, \mathrm{CDCl}_{3}\right) \delta 8.55\left(\mathrm{~d}, J_{\mathrm{CH}-2, \mathrm{CH}-3}=8.8 \mathrm{~Hz}, 1 \mathrm{H}, \mathrm{CH}-2 \mathrm{Dan}\right), 8.13\left(\mathrm{~d}, J_{\mathrm{CH}-3, \mathrm{CH}-}\right.$ $\left.{ }_{4}=7.4 \mathrm{~Hz}, 1 \mathrm{H}, \mathrm{CH}-4 \mathrm{Dan}\right), 8.08$ (d, $\left.J_{\mathrm{CH}-7, \mathrm{CH}-8}=8.7 \mathrm{~Hz}, 1 \mathrm{H}, \mathrm{CH}-8 \mathrm{Dan}\right), 7.54$ (dd, $\left.J_{\mathrm{CH}-7, \mathrm{CH}-8}=8.7 \mathrm{~Hz}, J_{\mathrm{CH}-6, \mathrm{CH}-7}=7.7 \mathrm{~Hz}, 1 \mathrm{H}, \mathrm{CH}-7 \mathrm{Dan}\right), 7.50\left(\mathrm{~d}, J_{\mathrm{CH}-2, \mathrm{CH}-3}=8.8\right.$ $\mathrm{Hz}, 1 \mathrm{H}, \mathrm{CH}-8 \mathrm{Dan}), 7.38-7.26$ (m, 15H, $\left.\mathrm{H}_{\text {arom}}\right), 7.20-7.17$ (m, 3H, $\mathrm{H}_{\text {arom }}$, CH-6 
Dan), $4.84\left(\mathrm{~d}, J_{\mathrm{a}, \mathrm{b}}=11.7 \mathrm{~Hz}, 1 \mathrm{H}, \mathrm{CH}-\mathrm{a}, 4-O-\mathrm{Bn}\right), 4.77\left(\mathrm{~d}, J_{\mathrm{a}, \mathrm{b}}=12.0 \mathrm{~Hz}, 1 \mathrm{H}, \mathrm{CH}-\right.$ a, 2-O-Bn), $4.76\left(\mathrm{~d}, J_{\mathrm{a}, \mathrm{b}}=11.5 \mathrm{~Hz}, 1 \mathrm{H}, \mathrm{CH}-\mathrm{a}, 3-O-\mathrm{Bn}\right), 4.61\left(\mathrm{~d}, J_{\mathrm{a}, \mathrm{b}}=12.0 \mathrm{~Hz}\right.$, $2 \mathrm{H}, \mathrm{CH}-\mathrm{b}, 2-O-\mathrm{Bn}, \mathrm{CH}-\mathrm{b}, 3-O-\mathrm{Bn}), 4.47$ (d, $\left.J_{1,2}=3.8 \mathrm{~Hz}, 1 \mathrm{H}, \mathrm{H}-1\right), 4.29(\mathrm{dd}$, $\left.J_{\mathrm{NH}, 6 \mathrm{a}}=8.4 \mathrm{~Hz}, J_{\mathrm{NH}, 6 \mathrm{~b}}=4.6 \mathrm{~Hz}, 1 \mathrm{H}, \mathrm{NH} \operatorname{Dan}\right), 3.86\left(\mathrm{dd}, J_{1,2}=3.8 \mathrm{~Hz}, J_{2,3}=9.4\right.$ $\mathrm{Hz}, 1 \mathrm{H}, \mathrm{H}-2), 3.65$ (dd, $\left.J_{2,3}=9.4 \mathrm{~Hz}, J_{3,4}=2.7 \mathrm{~Hz}, 1 \mathrm{H}, \mathrm{H}-3\right), 3.64$ (bs, 1H, H-4), $3.48\left(\mathrm{t}, J_{5,6}=6.6 \mathrm{~Hz}, 1 \mathrm{H}, \mathrm{H}-5\right), 3.23\left(\mathrm{~s}, 3 \mathrm{H}, \mathrm{OCH}_{3}\right), 2.99-2.77(\mathrm{~m}, 2 \mathrm{H}, \mathrm{H}-6 \mathrm{a}, \mathrm{H}-$ 6b), $2.88\left(\mathrm{~s}, 6 \mathrm{H}, \mathrm{NMe}_{2}\right) ;{ }^{13} \mathrm{C} \mathrm{NMR}\left(125 \mathrm{MHz}, \mathrm{CDCl}_{3}\right) \delta 138.5,138.3$ (2 x C-i, 2$O-\mathrm{Bn}, 3-O-\mathrm{Bn}$ ), 137.9 (C-i, 4-O-Bn), 134.9 (C-4a Dan), 130.4 (C-2 Dan), 129.5, 128.9, 128.6, 128.5, 128.4, 128.3, 128.1, 127.8, 127.7, 127.6 (10 x $\left.\mathrm{CH}_{\text {arom }}\right), 123.4$ (C-3 Dan), 118.9 (C-8 Dan), 115.4 (C-6 Dan), 98.7 (C-1), 79.0 (C-3), 76.1 (C-2), $74.1\left(\mathrm{CH}_{2}, 4-\mathrm{O}-\mathrm{Bn}\right), 73.7$ (C-4), 73.63, 73.59 (2 x $\left.\mathrm{CH}_{2}, 2-O-\mathrm{Bn}, 3-O-\mathrm{Bn}\right), 68.5$ (C-5), $55.4\left(\mathrm{OCH}_{3}\right), 45.5\left(\mathrm{NMe}_{2}\right), 43.3$ (C-6); HRMS(ESI) $m / z$ calcd. for $\left[\mathrm{C}_{40} \mathrm{H}_{44} \mathrm{~N}_{2} \mathrm{O}_{7} \mathrm{~S}+\mathrm{Na}\right]^{+}:$719.2756, obsd.: 719.2760.

\section{1-O-Acetyl-2,3,4-tri-O-benzyl-6-deoxy-6-[ $N$-(5-[dimethylamino]napth-1-}

ylsulfonyl)acetimido]- $\alpha$-D-galactopyranoside (47). Concentrated sulfuric acid $(63.1 \mu \mathrm{L}, 1.18 \mathrm{mmol})$ was added dropwise to a solution of $46(550 \mathrm{mg}, 0.79$ mmol) in acetic anhydride $(2.4 \mathrm{~mL}, 25.3 \mathrm{mmol})$ and acetic acid $(0.32 \mathrm{~mL}, 5.53$ mmol) at $0{ }^{\circ} \mathrm{C}$. After stirring at $0^{\circ} \mathrm{C}$ for $1 \mathrm{~h}$ and at $\mathrm{rt}$ for $2 \mathrm{~h}$, the solution was diluted with DCM $(50 \mathrm{~mL})$ and washed with water $(50 \mathrm{~mL})$, saturated $\mathrm{NaHCO}_{3}$ solution $(50 \mathrm{~mL})$ and brine $(100 \mathrm{~mL})$, dried $\left(\mathrm{MgSO}_{4}\right)$, filtered and concentrated in vacuo. The residue was purified by silica gel flash column chromatography and eluted with 3:1 (v/v) petroleum ether/EtOAc to afford the title compound 47 (605 $\mathrm{mg}, 0.79 \mathrm{mmol})$ as a lime green foam. $\mathrm{R}_{f}: 0.66(\mathrm{PE} / \mathrm{EA}, 1 / 1, \mathrm{v} / \mathrm{v})$; Glows mauve on $\operatorname{TLC}(\lambda=254 \mathrm{~nm}) ;[\alpha]_{\mathrm{D}}^{23}=+65.8^{\circ}\left(c=1.0, \mathrm{CHCl}_{3}\right)$; IR (film) 3064, 3030, 2940, 2872, 1750, 1705, 1454, 1342, 1229, 1135, 1102, 1051, 1009, 788, 739, 699 $\mathrm{cm}^{-1} ;{ }^{1} \mathrm{H}$ NMR $\left(500 \mathrm{MHz}, \mathrm{CDCl}_{3}\right) \delta 8.64$ (bs, $\left.1 \mathrm{H}, \mathrm{CH}-2 \mathrm{Dan}\right), 8.30$ (d, $J_{\mathrm{CH}-3, \mathrm{CH}-4}=$ 7.4 Hz, 1H, CH-4 Dan), 7.85 (bs, 1H, CH-8 Dan), 7.58-7.53 (m, 2H, CH-3, CH-7 Dan), 7.44-7.29 (m, 15H, $\mathrm{H}_{\text {arom }}$ ), 7.23 (bs, 1H, CH-6 Dan), 6.35 (d, J $\mathrm{J}_{1,2}=3.7 \mathrm{~Hz}$, $1 \mathrm{H}, \mathrm{H}-1), 5.11\left(\mathrm{~d}, J_{\mathrm{a}, \mathrm{b}}=11.6 \mathrm{~Hz}, 1 \mathrm{H}, \mathrm{CH}-\mathrm{a}, 4-O-\mathrm{Bn}\right), 4.91$ (d, $J_{\mathrm{a}, \mathrm{b}}=11.6 \mathrm{~Hz}, 1 \mathrm{H}$, $\mathrm{CH}-\mathrm{a}, 3-O-\mathrm{Bn}), 4.82$ (d, $\left.J_{\mathrm{a}, \mathrm{b}}=11.6 \mathrm{~Hz}, 1 \mathrm{H}, \mathrm{CH}-\mathrm{b}, 3-\mathrm{O}-\mathrm{Bn}\right), 4.72$ (s, 2H, CH-a, CH-b, 2-O-Bn), 4.69 (d, $\left.J_{\mathrm{a}, \mathrm{b}}=11.6 \mathrm{~Hz}, 1 \mathrm{H}, \mathrm{CH}-\mathrm{b}, 4-O-\mathrm{Bn}\right), 4.29$ (dd, $J_{5,6 \mathrm{a}}=8.6$ $\left.\mathrm{Hz}, J_{5,6 \mathrm{~b}}=2.5 \mathrm{~Hz}, 1 \mathrm{H}, \mathrm{H}-5\right), 4.18\left(\mathrm{dd}, J_{2,3}=10.0 \mathrm{~Hz}, J_{1,2}=3.7 \mathrm{~Hz}, 1 \mathrm{H}, \mathrm{H}-2\right), 4.10$ 
$\left(\mathrm{dd}, J_{6 \mathrm{a}, 6 \mathrm{~b}}=15.2 \mathrm{~Hz}, J_{5,6}=8.6 \mathrm{~Hz}, 1 \mathrm{H}, \mathrm{H}-6 \mathrm{a}\right), 4.07$ (bd, $\left.J_{3,4}=2.6 \mathrm{~Hz}, 1 \mathrm{H}, \mathrm{H}-4\right)$, $3.96\left(\mathrm{dd}, J_{2,3}=10.0 \mathrm{~Hz}, J_{3,4}=2.6 \mathrm{~Hz}, 1 \mathrm{H}, \mathrm{H}-3\right), 3.85\left(\mathrm{dd}, J_{6 \mathrm{a}, 6 \mathrm{~b}}=15.2 \mathrm{~Hz}, J_{5,6 \mathrm{~b}}=\right.$ $2.5 \mathrm{~Hz}, \mathrm{H} 1, \mathrm{H}-6 \mathrm{~b}$ ), 2.93 (s, 6H, NMe $), 2.18$ (s, 3H, NAc), 2.15 (s, 3H, OAc); ${ }^{13} \mathrm{C}$ NMR (125 MHz, $\left.\mathrm{CDCl}_{3}\right) \delta 170.9$ (C=O NAc), 169.7 (C=O OAc), 138.4 (C-i, 3$O-\mathrm{Bn}), 138.3$ (C-i, 4-O-Bn), 137.9 (C-i, 2-O-Bn), 134.7 (C-4a Dan), 131.2 (C-2 Dan), 129.2 (C-1/C-8a Dan), 128.53, 128.50, 128.45, 128.42, 128.40, 128.38, $128.01,127.94,127.89,127.86,127.82,127.74,127.72,127.61,127.5(15 \mathrm{x}$ $\mathrm{CH}_{\text {arom}}$ ), 123.5 (C-3 Dan), 115.5 (C-6 Dan), 90.2 (C-1), 78.9 (C-3), 75.2 (C-2), 74.9 (C-4), $74.6\left(\mathrm{CH}_{2}, 4-\mathrm{O}-\mathrm{Bn}\right), 73.5\left(\mathrm{CH}_{2}, 3-\mathrm{O}-\mathrm{Bn}\right), 73.4\left(\mathrm{CH}_{2}, 2-O-\mathrm{Bn}\right), 72.1$ (C-5), 47.7 (C-6), 45.5 ( $\mathrm{NMe}_{2}$ ), 24.7 (NAc), 21.2 (OAc), HRMS(ESI) m/z calcd. for $\left[\mathrm{C}_{43} \mathrm{H}_{46} \mathrm{~N}_{2} \mathrm{O}_{9} \mathrm{~S}+\mathrm{Na}\right]^{+}$: 789.2822, obsd.: 789.2819 .

\section{2-Azido-3,4-O-isopropylidene-1- $O$-(2,3,4-tri- $O$-benzyl-6-deoxy-6-[ $N$-(5-}

\section{[dimethylamino]napth-1-ylsulfonyl)acetimido]- $\alpha$-D-galactopyranosyl)-D-ribo-} octadecane-1,3,4-triol (36). Iodotrimethylsilane $(65.3 \mu \mathrm{L}, 0.46 \mathrm{mmol})$ was added to a solution of $47(237 \mathrm{mg}, 0.31 \mathrm{mmol}$ ) (co-evaporated with toluene $\mathrm{x} 3$ ) in dry $\operatorname{DCM}(2 \mathrm{~mL})$ at $0{ }^{\circ} \mathrm{C}$. After stirring for $1 \mathrm{~h}$ at $0{ }^{\circ} \mathrm{C}$, the reaction mixture was concentrated in vacuo, azeotroped with toluene (x 3) and redissolved in dry benzene $(3 \mathrm{~mL})$. In a separate flask, molecular sieves $(4 \AA, 50 \mathrm{mg})$, TBAI (342 $\mathrm{mg}, 0.93 \mathrm{mmol})$ and DiPEA $(53.8 \mu \mathrm{L}, 0.31 \mathrm{mmol})$ were added to a solution of the lipid acceptor 38 (39.1 mg, $0.10 \mathrm{mmol}$ ) (co-evaporated from toluene x 3) in dry benzene $(1 \mathrm{~mL})$ at room temperature. After stirring the reaction mixture at $70{ }^{\circ} \mathrm{C}$ for $15 \mathrm{~min}$, the glycosyl iodide was cannulated into the flask containing the lipid acceptor and stirred at $70{ }^{\circ} \mathrm{C}$ for $20 \mathrm{~h}$. The reaction mixture was then diluted with EtOAc $(10 \mathrm{~mL})$, cooled to $0{ }^{\circ} \mathrm{C}$ and filtered. The filtrate was concentrated and the resulting residue purified by silica gel flash column chromatography. Elution with 7:1 (v/v) hexanes/EtOAc afforded the desired glycolipid 36 (103.9 mg, 0.095 mmol, 94\%) as a lime green oil. $\mathrm{R}_{f}: 0.40$ (PE/EA, 2/1, v/v); Glows mauve on TLC $(\lambda=254 \mathrm{~nm}) ;[\alpha]_{\mathrm{D}}^{24}=+40.9^{\circ}\left(c=1.0, \mathrm{CHCl}_{3}\right) ; \mathrm{IR}$ (film) 3064, 3031, 2923, 2853, 2789, 2099, 1704, 1572, 1455, 1347, 1231, 1147, 1096, 1045, 787, 735, $697 \mathrm{~cm}^{-1}$; ${ }^{1} \mathrm{H}$ NMR $\left(500 \mathrm{MHz}, \mathrm{CDCl}_{3}\right) \delta 8.58\left(\mathrm{~d}, J_{\mathrm{CH}-2, \mathrm{CH}-3}=8.6 \mathrm{~Hz}, 1 \mathrm{H}, \mathrm{CH}-2 \mathrm{Dan}\right), 8.29$ $\left(\mathrm{d}, J_{\mathrm{CH}-3, \mathrm{CH}-4}=7.3 \mathrm{~Hz}, 1 \mathrm{H}, \mathrm{CH}-4 \mathrm{Dan}\right), 7.75\left(\mathrm{~d}, J_{\mathrm{CH}-7, \mathrm{CH}-8}=8.6 \mathrm{~Hz}, 1 \mathrm{H}, \mathrm{CH}-8\right.$ Dan), 7.53 (t, $J_{\mathrm{CH}-2, \mathrm{CH}-3}=J_{\mathrm{CH}-7, \mathrm{CH}-8}=8.6 \mathrm{~Hz}, 2 \mathrm{H}, \mathrm{CH}-3, \mathrm{CH}-7$ Dan), 7.43-7.2.8 
(m, 15H, $\mathrm{H}_{\text {arom }}$ ), 7.19 (d, $\left.J_{\mathrm{CH}-6, \mathrm{CH}-7}=7.5 \mathrm{~Hz}, 1 \mathrm{H}, \mathrm{CH}-6 \mathrm{Dan}\right), 5.11\left(\mathrm{~d}, J_{\mathrm{a}, \mathrm{b}}=11.7\right.$ $\left.\mathrm{Hz}, 1 \mathrm{H}, \mathrm{CH}-\mathrm{a}, 4^{\prime}-O-\mathrm{Bn}\right), 5.00\left(\mathrm{~d}, J_{1,2}=3.2 \mathrm{~Hz}, 1 \mathrm{H}, \mathrm{H}-1\right), 4.91\left(\mathrm{~d}, J_{\mathrm{a}, \mathrm{b}}=11.7 \mathrm{~Hz}\right.$, $\left.1 \mathrm{H}, \mathrm{CH}-\mathrm{a}, 2^{\prime}-O-\mathrm{Bn}\right), 4.80$ (d, $\left.J_{\mathrm{a}, \mathrm{b}}=12.0 \mathrm{~Hz}, 1 \mathrm{H}, \mathrm{CH}-\mathrm{a}, 33^{\prime}-O-\mathrm{Bn}\right), 4.79$ (d, $J_{\mathrm{a}, \mathrm{b}}=$ $\left.11.7 \mathrm{~Hz}, 1 \mathrm{H}, \mathrm{CH}-\mathrm{b}, 2^{\prime}-O-\mathrm{Bn}\right), 4.74$ (d, $\left.J_{\mathrm{a}, \mathrm{b}}=12.0 \mathrm{~Hz}, 1 \mathrm{H}, \mathrm{CH}-\mathrm{b}, 3^{\prime}-O-\mathrm{Bn}\right), 4.68$ $\left(\mathrm{d}, J_{\mathrm{a}, \mathrm{b}}=11.7 \mathrm{~Hz}, 1 \mathrm{H}, \mathrm{CH}-\mathrm{b}, 4^{\prime}-O-\mathrm{Bn}\right), 4.25-4.18$ (m, 3H, H-1a, H-5', H-3), 4.154.05 (m, 4H, H-6'a, H-4, H-3', H-2'), 4.00 (bs, 1H, H-4'), 3.85 (dd, $J_{6 \mathrm{a}^{\prime}, 6 \mathrm{~b}^{\prime}}=14.8$ $\left.\mathrm{Hz}, J_{5,6 \mathrm{~b}^{\prime}}=2.9 \mathrm{~Hz}, 1 \mathrm{H}, \mathrm{H}-6^{\prime} \mathrm{b}\right), 3.74\left(\mathrm{dd}, J_{1 \mathrm{a}, 1 \mathrm{~b}}=10.8 \mathrm{~Hz}, J_{1 \mathrm{~b}, 2}=5.2 \mathrm{~Hz}, 1 \mathrm{H}, \mathrm{H}-\right.$ 1b), $3.41\left(\mathrm{ddd}, J_{2,3}=9.5 \mathrm{~Hz}, J_{1 \mathrm{a}, 2}=5.2 \mathrm{~Hz}, J_{1 \mathrm{~b}, 2}=2.5 \mathrm{~Hz}, 1 \mathrm{H}, \mathrm{H}-2\right), 2.89$ (s, 6H, $\mathrm{NMe}_{2}$ ), 2.18 (s, 3H, NAc), 1.67-1.54 (m, 4H, H-5, H-6), 1.43 (s, 3H, CH $i_{3} \mathrm{Pr}$ ), 1.30 (s, 3H, $\left.\mathrm{CH}_{3} i \mathrm{Pr}\right), 1.41-1.27$ (m, 22H, H-7-H-17), 0.89 (t, $J_{17,18}=7.0 \mathrm{~Hz}, 3 \mathrm{H}$, $\mathrm{H}-18) ;{ }^{13} \mathrm{C}$ NMR (125 MHz, $\left.\mathrm{CDCl}_{3}\right) \delta 170.8$ (C=O NAc), 152.2 (C-5 Dan), 138.9 (C-i, 3'-O-Bn), 138.8 (C-i, 2'-O-Bn), 138.6 (C-i, 4'-O-Bn), 134.5 (C-4a Dan), 131.5 (C-2 Dan), 131.4 (C-4 Dan), 129.9, (C-1 Dan), 129.6 (C-8a Dan), 128.7 (C7 Dan), 128.43, 128.40, 128.37, 128.2, 127.7, 127.6, 127.53, 127.52, 127.43 (9 x $\mathrm{CH}_{\text {arom}}$ ), 123.1 (C-3 Dan), 117.8 (C-8 Dan), 115.2 (C-6 Dan), 108.1 ( $\left.\mathrm{C}_{\mathrm{q}} i \operatorname{Pr}\right), 98.5$ (C-1'), 78.8 (C-2'), 77.8 (C-4), 76.5 (C-3'), 75.5 (C-4'), 75.0 (C-3), $74.5\left(\mathrm{CH}_{2}, 4^{\prime}\right.$ $O-\mathrm{Bn}), 73.7\left(\mathrm{CH}_{2}, 2^{\prime}-\mathrm{O}-\mathrm{Bn}\right), 72.7\left(\mathrm{CH}_{2}, 3^{\prime}-O-\mathrm{Bn}\right), 70.0$ (C-5'), $69.2(\mathrm{C}-1), 59.3$ (C-2), 47.8 (C-6'), $45.4\left(\mathrm{NMe}_{2}\right), 31.9,29.71,29.68,29.67,29.66,29.64,29.61$, 29.4, 26.6, 22.7 (C-5-C-17), 28.3, 25.8 (2 x CH 3 iPr), 24.8 (NAc), 14.1 (C-18); HRMS(ESI) $m / z$ calcd. for $\left[\mathrm{C}_{62} \mathrm{H}_{83} \mathrm{~N}_{5} \mathrm{O}_{10} \mathrm{~S}+\mathrm{Na}\right]^{+}: 1112.5758$, obsd.: 1112.5767 .

\section{2-Azido-3,4-O-isopropylidene-1- $O$-(2,3,4-tri- $O$-benzyl-6-deoxy-6-[ $N$-(5-}

\section{[dimethylamino]napth-1-ylsulfonyl)amido]- $\alpha$-D-galactopyranosyl)-D-ribo-}

octadecane-1,3,4-triol (48). Glycolipid 36 (42.5 mg, $0.039 \mathrm{mmol}$ ) was dissolved in a mixture of methanol/DCM $(3: 1, \mathrm{v} / \mathrm{v})$ and to this was added $\mathrm{NaOMe}$ until the solution reached $\mathrm{pH}$ 9.0. After stirring at room temperature for $3 \mathrm{~d}$ in the dark, while maintaining $\mathrm{pH} 9.0$ via the addition of $\mathrm{NaOMe}$. The solution was then diluted with EtOAc $(15 \mathrm{~mL})$, and washed with saturated $\mathrm{NH}_{4} \mathrm{Cl}$ solution $(20 \mathrm{~mL})$, water $(20 \mathrm{~mL})$ and brine $(20 \mathrm{~mL})$. The organic layer was dried $\left(\mathrm{MgSO}_{4}\right)$, filtered and concentrated in vacuo to afford the title compound 48 (41 mg, quant.) as a lime green oil, which was used without further purification. $\mathrm{R}_{f}: 0.40$ (PE/EA, 2/1, $\mathrm{v} / \mathrm{v})$; Glows white on $\operatorname{TLC}(\lambda=254 \mathrm{~nm}) ;[\alpha]_{\mathrm{D}}^{21}=+10.5^{\circ}\left(c=1.0, \mathrm{CHCl}_{3}\right)$; IR (film) 3309, 3031, 2923, 2853, 2360, 2099, 1574, 1454, 1328, 1220, 1145, 1094, 
1047, 791, 734, $697 \mathrm{~cm}^{-1}$; ${ }^{1} \mathrm{H}$ NMR $\left(500 \mathrm{MHz}, \mathrm{CDCl}_{3}\right) \delta 8.55\left(\mathrm{~d}, J_{\mathrm{CH}-2, \mathrm{CH}-3 \text { Dan }}=\right.$ $8.4 \mathrm{~Hz}, 1 \mathrm{H}, \mathrm{CH}-2 \mathrm{Dan}), 8.17$ (d, $\left.J_{\mathrm{CH}-3, \mathrm{CH}-4 \text { Dan }}=7.3 \mathrm{~Hz}, 1 \mathrm{H}, \mathrm{CH}-4 \mathrm{Dan}\right), 8.09$ (d, $\left.J_{\mathrm{CH}-7, \mathrm{CH}-8 \text { Dan }}=8.7 \mathrm{~Hz}, 1 \mathrm{H}, \mathrm{CH}-8 \mathrm{Dan}\right), 7.56\left(\mathrm{dd}, J_{\mathrm{CH}-7, \mathrm{CH}-8 \mathrm{Dan}}=8.7 \mathrm{~Hz}, J_{\mathrm{CH}-6, \mathrm{CH}-7}\right.$ Dan $=7.6 \mathrm{~Hz}, 1 \mathrm{H}, \mathrm{CH}-7 \mathrm{Dan}), 7.53\left(\mathrm{dd}, J_{\mathrm{CH}-2, \mathrm{CH}-3 \mathrm{Dan}}=8.4 \mathrm{~Hz}, J_{\mathrm{CH}-3, \mathrm{CH}-4 \mathrm{Dan}}=7.3\right.$ Hz, 1H, CH-3 Dan),7.38-7.26 (m, 13H, $\mathrm{H}_{\text {arom }}$ ), 7.20-7.18 (m, 3H, Harom, CH-6 Dan), $4.86\left(\mathrm{~d}, J_{\mathrm{a}, \mathrm{b}}=11.8 \mathrm{~Hz}, 1 \mathrm{H}, \mathrm{CH}-\mathrm{a}, 4^{\prime}-O-\mathrm{Bn}\right), 4.79$ (d, $J_{\mathrm{a}, \mathrm{b}}=12.0 \mathrm{~Hz}, 1 \mathrm{H}, \mathrm{CH}-$ a, 3'-O-Bn), 4.77 (d, $\left.J_{1,2}=3.6 \mathrm{~Hz}, 1 \mathrm{H}, \mathrm{H}^{\prime} 1^{\prime}\right), 4.75$ (d, $J_{\mathrm{a}, \mathrm{b}}=11.9 \mathrm{~Hz}, 1 \mathrm{H}, \mathrm{CH}-\mathrm{a}, 2-$ $O-\mathrm{Bn}), 4.66\left(\mathrm{~d}, J_{\mathrm{a}, \mathrm{b}}=11.9 \mathrm{~Hz}, 1 \mathrm{H}, \mathrm{CH}-\mathrm{b}, 2-O-\mathrm{Bn}\right), 4.63\left(\mathrm{~d}, J_{\mathrm{a}, \mathrm{b}}=12.0 \mathrm{~Hz}, 1 \mathrm{H}\right.$, CH-b, 3-O-Bn), 4.53 (d, $\left.J_{\mathrm{a}, \mathrm{b}}=11.8 \mathrm{~Hz}, 1 \mathrm{H}, \mathrm{CH}-\mathrm{b}, 4-O-\mathrm{Bn}\right), 4.34$ (dd, $J_{6^{\prime} \mathrm{b}, \mathrm{NH}}=8.3$ $\left.\mathrm{Hz}, J_{6^{\prime} \mathrm{a}, \mathrm{NH}}=4.5 \mathrm{~Hz}, 1 \mathrm{H}, \mathrm{NH}\right), 4.13$ (p, $\left.J_{4,5}=4.2 \mathrm{~Hz}, 1 \mathrm{H}, \mathrm{H}-4\right), 4.04$ (dd, $J_{2,3}=9.8$ $\left.\mathrm{Hz}, J_{3,4}=5.4 \mathrm{~Hz}, 1 \mathrm{H}, \mathrm{H}-3\right), 3.95\left(\mathrm{dd}, J_{1 \mathrm{a}, 1 \mathrm{~b}}=10.6 \mathrm{~Hz}, J_{1 \mathrm{a}, 2}=2.5 \mathrm{~Hz}, 1 \mathrm{H}, \mathrm{H}-1 \mathrm{a}\right)$, $3.91\left(\mathrm{dd}, J_{2^{\prime}, 3^{\prime}}=10.1 \mathrm{~Hz}, J_{1,2}=3.6 \mathrm{~Hz}, 1 \mathrm{H}, \mathrm{H}-2^{\prime}\right), 3.79\left(\mathrm{dd}, J_{2^{\prime}, 3^{\prime}}=10.1 \mathrm{~Hz}, J_{3^{\prime}, 4^{\prime}}=\right.$ $\left.2.7 \mathrm{~Hz}, 1 \mathrm{H}, \mathrm{H}-3^{\prime}\right), 3.70$ (bs, 1H, H-4'), 3.69-3.68 (m, 1H, H-5'), 3.61 (dd, $J_{1 \mathrm{a}, 1 \mathrm{~b}}=$ $\left.10.6 \mathrm{~Hz}, J_{1 \mathrm{~b}, 2}=6.4 \mathrm{~Hz}, 1 \mathrm{H}, \mathrm{H}-1 \mathrm{~b}\right), 3.38\left(\mathrm{ddd}, J_{2,3}=9.8 \mathrm{~Hz}, J_{1 \mathrm{~b}, 2}=6.4 \mathrm{~Hz}, J_{1 \mathrm{a}, 2}=\right.$ $2.5 \mathrm{~Hz}, 1 \mathrm{H}, \mathrm{H}-2), 2.92-2.81\left(\mathrm{dd}, J_{5,6^{\prime} \mathrm{a}}=7.1 \mathrm{~Hz}, J_{6^{\prime} \mathrm{a}, \mathrm{NH}}=4.5 \mathrm{~Hz}, 1 \mathrm{H}, \mathrm{H}-6^{\prime} \mathrm{a}\right), 2.83$ $\left(\mathrm{ddd}, J_{6^{\prime} \mathrm{a}, 6^{\prime} \mathrm{b}}=13.6 \mathrm{~Hz}, J_{6^{\prime} \mathrm{b}, \mathrm{NH}}=8.3 \mathrm{~Hz}, J_{5,6^{\prime} \mathrm{b}}=6.1 \mathrm{~Hz}, 1 \mathrm{H}, \mathrm{H}-6^{\prime} \mathrm{b}\right), 2.89(\mathrm{~s}, 6 \mathrm{H}$, $\mathrm{NMe}_{2}$ ), 1.64-1.27 (m, 26H, H-5-H-17), 1.45 (s, 3H, CH $\left.{ }_{3} \mathrm{Pr}\right), 1.31$ (s, 3H, $\mathrm{CH}_{3}$ $i \operatorname{Pr}), \quad 0.89\left(\mathrm{t}, J_{17,18}=7.0 \mathrm{~Hz}, 3 \mathrm{H}, \mathrm{H}-18\right) ;{ }^{13} \mathrm{C} \mathrm{NMR}\left(125 \mathrm{MHz}, \mathrm{CDCl}_{3}\right) \delta 152.1$ (C-5 Dan), 138.7, 138.6 (2 x C-i, 2'-O-Bn, 3'-O-Bn), 138.0 (C-i, 4'-O-Bn), 134.7 (C-4a Dan), 130.5 (C-2 Dan), 129.9 (C-1/C-8a Dan), 129.6 (C-4 Dan), 129.5 (C1/C-8a Dan), 128.9, 128.6, 128.4 (3 x CHarom) 128.30 (C-7 Dan), 128.25, 128.22, 127.64, 127.63, 127.54, 127.48 (6 x CHarom), 123.1 (C-3 Dan), 118.8 (C-8 Dan), 115.2 (C-6 Dan), $108.3\left(\mathrm{C}_{\mathrm{q}} i \mathrm{Pr}\right), 98.5\left(\mathrm{C}-1^{\prime}\right), 78.6\left(\mathrm{C}-3^{\prime}\right), 77.8$ (C-4), $76.3\left(\mathrm{C}-2^{\prime}\right)$, 75.2 (C-3), $74.1\left(\mathrm{CH}_{2}, 4^{\prime}-\mathrm{O}-\mathrm{Bn}\right), 73.8\left(\mathrm{C}-4^{\prime}\right), 73.7\left(\mathrm{CH}_{2}, 3^{\prime}-\mathrm{O}-\mathrm{Bn}\right), 72.9\left(\mathrm{CH}_{2}, 2^{\prime}-\right.$ O-Bn), 69.5 (C-1), 69.2 (C-5'), 59.5 (C-2), $45.4\left(\mathrm{NMe}_{2}\right), 43.3$ (C-6'), 31.9, 29.72, 29.69, 29.68, 29.64, 29.61, 29.4, 29.3, 26.6, 22.7 (C-5-C-17), 28.3, 25.7 (2 x CH $i$ Pr), 14.1 (C-18); HRMS(ESI) $m / z$ calcd. for $\left[\mathrm{C}_{60} \mathrm{H}_{81} \mathrm{~N}_{5} \mathrm{O}_{9} \mathrm{~S}+\mathrm{Na}\right]^{+}:$1070.5653, obsd.: 1070.5657 . 


\section{1-O-(2,3,4-tri-O-benzyl-6-deoxy-6-[ $N$-(5-[dimethylamino]napth-1-}

ylsulfonyl)amido]- $\alpha$-D-galactopyranosyl)-2-hexacosanoylamido-3,4-O-

isopropylidene-D-ribo-octadecane-1,3,4-triol (49). Trimethylphosphine $(1 \mathrm{M}$ in THF) (0.17 mL, $0.17 \mathrm{mmol})$ was added to a solution of $48(34.7 \mathrm{mg}, 0.033 \mathrm{mmol})$ (co-evaporated with toluene $\mathrm{x} 3$ ) in distilled THF $(0.2 \mathrm{~mL})$ at $0{ }^{\circ} \mathrm{C}$. After stirring the reaction mixture for $15 \mathrm{~min}$ at $0{ }^{\circ} \mathrm{C}$ and $45 \mathrm{~min}$ at $\mathrm{rt}$, the solution was cooled to $0{ }^{\circ} \mathrm{C}$ and $1 \mathrm{M} \mathrm{NaOH}(\mathrm{aq})$ solution $(0.33 \mathrm{~mL}, 0.33 \mathrm{mmol})$ added drop wise. The reaction mixture was then stirred at $\mathrm{rt}$ for $21 \mathrm{~h}$, then diluted with EtOAc $(20 \mathrm{~mL})$, washed with water $(2 \times 20 \mathrm{~mL})$ and brine $(20 \mathrm{~mL})$, dried $\left(\mathrm{MgSO}_{4}\right)$ and filtered. Concentration in vacuo afforded the amine [ $\mathrm{R}_{f}: 0.11$ (Toluene:EtOAc, 3/2, v/v)] as a green oil, which was used without further purification. A solution of hexacosanoic acid (33.5 mg, $0.084 \mathrm{mmol})$, EDCI (16.2 $\mathrm{mg}, 0.084 \mathrm{mmol})$, and DMAP $(0.4 \mathrm{mg}, 0.0034 \mathrm{mmol})$ in dry DCM $(1 \mathrm{~mL})$ was added to the amine dissolved in dry DCM $(1 \mathrm{~mL})$. After stirring at room temperature for $43 \mathrm{~h}$, the reaction mixture was diluted with EtOAc $(20 \mathrm{~mL})$ and washed with saturated $\mathrm{NaHCO}_{3}$ solution $(20 \mathrm{~mL})$, water $(20 \mathrm{~mL})$ and brine $(20 \mathrm{~mL})$, dried $\left(\mathrm{MgSO}_{4}\right)$ and filtered. The filtrate was concentrated in vacuo and the residue was purified by silica gel column chromatography. Elution with 5:1 (v/v) hexanes/EtOAc afforded the desired product 49 (31.9 $\mathrm{mg}, 0.023 \mathrm{mmol}, 68 \%)$ as a lime green oil. $\mathrm{R}_{f}: 0.79$ (Toluene/EtOAc, 3/2, v/v); Glows white on TLC $(\lambda=254 \mathrm{~nm}) ;[\alpha]_{\mathrm{D}}^{22}=+4.3^{\circ}(c$ $=1.0, \mathrm{CHCl}_{3}$ ); IR (film) 2923, 2853, 2361, 1650, 1541, 1455, 1330, 1218, 1145, 1092, 1052, 791, $697 \mathrm{~cm}^{-1} ;{ }^{1} \mathrm{H}$ NMR $\left(500 \mathrm{MHz}, \mathrm{CDCl}_{3}\right) \delta 8.55\left(\mathrm{~d}, J_{\mathrm{CH}-2, \mathrm{CH}-3}=7.4\right.$ $\mathrm{Hz}, 1 \mathrm{H}, \mathrm{CH}-2 \mathrm{Dan}), 8.19$ (bs, 1H, CH-4 Dan), 8.16 (d, $J_{\mathrm{CH}-7, \mathrm{CH}-8}=7.3 \mathrm{~Hz}, 1 \mathrm{H}$, CH-8 Dan), $7.56\left(\mathrm{t}, J_{\mathrm{CH}-6, \mathrm{CH}-7}=J_{\mathrm{CH}-7, \mathrm{CH}-8}=8.1 \mathrm{~Hz}, 1 \mathrm{H}, \mathrm{CH}-7 \mathrm{Dan}\right), 7.52\left(\mathrm{t}, J_{\mathrm{CH}-}\right.$ 2,CH-3 $\left.=J_{\mathrm{CH}-3, \mathrm{CH}-4}=8.0 \mathrm{~Hz}, 1 \mathrm{H}, \mathrm{CH}-3 \mathrm{Dan}\right), 7.41-7.23\left(\mathrm{~m}, 15 \mathrm{H}, \mathrm{H}_{\text {arom }}\right), 7.20(\mathrm{~d}$, $\left.J_{\mathrm{CH}-6, \mathrm{CH}-7}=7.1 \mathrm{~Hz}, 1 \mathrm{H}, \mathrm{CH}-6 \mathrm{Dan}\right), 5.89\left(\mathrm{~d}, J_{\mathrm{NH}, 2}=9.5 \mathrm{~Hz}, 1 \mathrm{H}, \mathrm{NH} \mathrm{C}_{26}\right), 4.92(\mathrm{bs}$, 1H, NH Dan), 4.88 (d, $\left.J_{\mathrm{a}, \mathrm{b}}=11.5 \mathrm{~Hz}, 1 \mathrm{H}, \mathrm{CH}-\mathrm{a}, 4^{\prime \prime}-O-\mathrm{Bn}\right), 4.89\left(\mathrm{~d}, J_{1,2}=3.6 \mathrm{~Hz}\right.$, $\left.1 \mathrm{H}, \mathrm{H}-1^{\prime \prime}\right), 4.79\left(\mathrm{~d}, J_{\mathrm{a}, \mathrm{b}}=11.4 \mathrm{~Hz}, 1 \mathrm{H}, \mathrm{CH}-\mathrm{a}, 2^{\prime \prime}-O-\mathrm{Bn}\right), 4.76\left(\mathrm{~d}, J_{\mathrm{a}, \mathrm{b}}=11.7 \mathrm{~Hz}\right.$, $\left.1 \mathrm{H}, \mathrm{CH}-\mathrm{a}, 3^{\prime \prime}-O-\mathrm{Bn}\right), 4.71\left(\mathrm{~d}, J_{\mathrm{a}, \mathrm{b}}=11.7 \mathrm{~Hz}, 1 \mathrm{H}, \mathrm{CH}-\mathrm{b}, 3^{\prime \prime}-O-\mathrm{Bn}\right), 4.64\left(\mathrm{~d}, J_{\mathrm{a}, \mathrm{b}}=\right.$ $\left.11.4 \mathrm{~Hz}, 1 \mathrm{H}, \mathrm{CH}-\mathrm{b}, 2^{\prime \prime}-O-\mathrm{Bn}\right), 4.53$ (d, $\left.J_{\mathrm{a}, \mathrm{b}}=11.5 \mathrm{~Hz}, 1 \mathrm{H}, \mathrm{CH}-\mathrm{b}, 4^{\prime \prime}-O-\mathrm{Bn}\right), 4.18-$ $4.14(\mathrm{~m}, 1 \mathrm{H}, \mathrm{H}-2), 4.07$ (t, $\left.J_{2,3}=J_{3,4}=6.9 \mathrm{~Hz}, 1 \mathrm{H}, \mathrm{H}-3\right), 4.04-4.01(\mathrm{~m}, 1 \mathrm{H}, \mathrm{H}-4)$, $3.96\left(\mathrm{dd}, J_{2^{\prime \prime}, 3^{\prime \prime}}=10.0 \mathrm{~Hz}, J_{1^{\prime \prime}, 2^{\prime \prime}}=3.6 \mathrm{~Hz}, 1 \mathrm{H}, \mathrm{H}-2^{\prime \prime}\right), 3.85\left(\mathrm{dd}, J_{1 \mathrm{a}, 1 \mathrm{~b}}=11.6 \mathrm{~Hz}\right.$, $\left.J_{1 \mathrm{a}, 2}=4.6 \mathrm{~Hz}, 1 \mathrm{H}, \mathrm{H}-1 \mathrm{a}\right), 3.82$ (bs, $\left.1 \mathrm{H}, \mathrm{H}-4^{\prime \prime}\right), 3.77$ (dd, $J_{2^{\prime \prime}, 3^{\prime \prime}}=10.0 \mathrm{~Hz}, J_{3^{\prime \prime}, 4^{\prime \prime}}=$ 
$\left.2.7 \mathrm{~Hz}, 1 \mathrm{H}, \mathrm{H}-3^{\prime \prime}\right), 3.71\left(\mathrm{t}, J_{5^{\prime \prime}, 6^{\prime \prime} \mathrm{a}}=6.6 \mathrm{~Hz}, 1 \mathrm{H}, \mathrm{H}-5^{\prime \prime}\right), 3.59\left(\mathrm{dd}, J_{1 \mathrm{a}, 1 \mathrm{~b}}=11.6 \mathrm{~Hz}\right.$, $J_{1 \mathrm{~b}, 2}=2.6 \mathrm{~Hz}, 1 \mathrm{H}, \mathrm{H}-1 \mathrm{~b}$ ), 2.90 (s, 6H, NMe $), 2.87-2.85$ (m, 2H, H-6"'a, H-6"b), $2.14\left(\mathrm{dt}, J_{2^{\prime} \mathrm{a}, 2^{\prime} \mathrm{b}}=14.7 \mathrm{~Hz}, J_{2^{\prime} \mathrm{a}, 3^{\prime}}=7.5 \mathrm{~Hz}, 1 \mathrm{H}, \mathrm{H}-2^{\prime} \mathrm{a}\right), 2.06\left(\mathrm{dt}, J_{2^{\prime} \mathrm{a}, 2^{\prime} \mathrm{b}}=14.7 \mathrm{~Hz}\right.$, $\left.J_{2^{\prime} \mathrm{a}, 3^{\prime}}=7.5 \mathrm{~Hz}, 1 \mathrm{H}, \mathrm{H}-2^{\prime} \mathrm{b}\right), 1.71$ (bs, 2H, $\left.\mathrm{CH}_{2}, \mathrm{H}-5\right), 1.59$ (m, 2H, H-3'), 1.47-1.43 (m, 68H, CH 2 , H-6 - H-17, H-4'-H-25'), 1.43 (s, 3H, $\left.\mathrm{CH}_{3} i \mathrm{Pr}\right), 1.32$ (s, 3H, $\mathrm{CH}_{3}$ $i \operatorname{Pr}$ ), $0.89\left(\mathrm{t}, \mathrm{J}_{17,18}=J_{25^{\prime}, 26^{\prime}}=7.0 \mathrm{~Hz}, 6 \mathrm{H}, \mathrm{H}-18, \mathrm{H}-26^{\prime}\right) ;{ }^{13} \mathrm{C} \mathrm{NMR}(125 \mathrm{MHz}$, $\left.\mathrm{CDCl}_{3}\right) \delta 172.6(\mathrm{C}=\mathrm{O}), 151.8$ (C-5 Dan), 138.4 (C-i, 3"'-O-Bn), 138.2 (C-i, 2"'-OBn), 138.1 (C-i, 4"-O-Bn), 134.7 (C-4a/C-8a Dan), 130.3 (C-2 Dan), 129.8 (C4a/C-8a Dan), 129.6 (C-8 Dan), 128.8, 128.72, 128.66, 128.60, 128.56, 128.5, $128.4,128.15,128.1,128.0,127.94,127.88,127.7,127.6,127.5\left(15 \mathrm{x} \mathrm{CH}_{\text {arom }}\right)$, 128.21 (C-7 Dan), 123.3 (C-3 Dan), 119.1 (C-1 Dan), 115.2 (C-6 Dan), 107.8 (C $i \operatorname{Pr}), 99.3\left(\mathrm{C}-1^{\prime \prime}\right), 79.1\left(\mathrm{C}-3^{\prime \prime}\right), 77.7(\mathrm{C}-4), 76.6(\mathrm{C}-3), 76.5\left(\mathrm{C}-2^{\prime \prime}\right), 74.2\left(\mathrm{CH}_{2}, 4^{\prime \prime}-\right.$ $O-\mathrm{Bn}), 73.7\left(\mathrm{CH}_{2}, 2^{\prime \prime}-O-\mathrm{Bn}\right), 73.4\left(\mathrm{C}-4^{\prime \prime}\right), 73.0\left(\mathrm{CH}_{2}, 3^{\prime \prime}-O-\mathrm{Bn}\right), 69.4(\mathrm{C}-1), 69.1$ (C-5"), 49.1 (C-2), $45.5\left(\mathrm{NMe}_{2}\right), 43.3\left(\mathrm{C}-6^{\prime \prime}\right), 36.9$ (C-2'), 31.95, 31.94, 31.6, 29.8, 29.73, 29.71, 29.70, 29.67, 29.65, 29.50, 29.47, 29.42, 29.39, 29.37, 28.83, 26.8, 22.7 (C-5-C-17, C-3'-C-25'), 27.8, 25.73 (2 x CH $3 i \mathrm{Pr}), 25.72\left(\mathrm{CH}_{2}, \mathrm{C}-3^{\prime \prime}\right)$, $14.1\left(2 \times \mathrm{CH}_{3}, \mathrm{C}-18, \mathrm{C}-26{ }^{\prime}\right)$; HRMS(ESI) $m / z$ calcd. for $\left[\mathrm{C}_{86} \mathrm{H}_{133} \mathrm{~N}_{3} \mathrm{O}_{10} \mathrm{~S}+\mathrm{Na}\right]^{+}$: 1422.9609, obsd.: 1422.9615.

\section{1-O-(2,3,4-tri- $O$-benzyl-6-deoxy-6-[ $N$-(5-[dimethylamino]napth-1-}

ylsulfonyl)amido]- $\alpha$-D-galactopyranosyl)-2-hexacosanoylamido-D-ribo-

octadecane-1,3,4-triol (50). Glycolipid 49 (31.9 mg, $0.023 \mathrm{mmol}$ ) was dissolved in a mixture of $8: 4: 1(\mathrm{v} / \mathrm{v} / \mathrm{v}) \mathrm{AcOH} / \mathrm{H}_{2} \mathrm{O} /$ toluene and stirred at $50{ }^{\circ} \mathrm{C}$ for $1 \mathrm{~d}$ after which analysis by TLC revealed presence of starting material. The reaction mixture was concentrated in vacuo (to remove the acetone by product), then redissolved in the same mixture of solvents and stirred for a further day. This procedure was repeated until all starting material was converted to product as indicated by TLC analysis. The reaction mixture was then concentrated in vacuo and the residue was purified by silica gel flash column chromatography. Elution with 1:2 (v/v) hexanes/EtOAc afforded the diol 50 (23.0 mg, $0.017 \mathrm{mmol}, 74 \%)$ as a lime green oil. $\mathrm{R}_{f}: 0.22(\mathrm{PE} / \mathrm{EA}, 3 / 2, \mathrm{v} / \mathrm{v})$; Glows white on TLC $(\lambda=254 \mathrm{~nm})$; $[\alpha]_{\mathrm{D}}^{21}=+13.7^{\circ}\left(c=1.0, \mathrm{CHCl}_{3}\right)$; IR (film) 3357, 3032, 2922, 2852, 2360, 1643, 1536, 1498, 1312, 1143, 1092, 1050, 909, 789, 733, $697 \mathrm{~cm}^{-1} ;{ }^{1} \mathrm{H}$ NMR (500 
$\left.\mathrm{MHz}, \mathrm{CDCl}_{3}\right) \delta 8.54\left(\mathrm{~d}, J_{\mathrm{CH}-2, \mathrm{CH}-3}=8.5 \mathrm{~Hz}, 1 \mathrm{H}, \mathrm{CH}-2 \mathrm{Dan}\right), 8.10\left(\mathrm{~d}, J_{\mathrm{CH}-3, \mathrm{CH}-4}=\right.$ $7.4 \mathrm{~Hz}, 1 \mathrm{H}, \mathrm{CH}-4 \mathrm{Dan}), 8.07$ (d, $\left.J_{\mathrm{CH}-7, \mathrm{CH}-8}=8.6 \mathrm{~Hz}, 1 \mathrm{H}, \mathrm{CH}-8 \mathrm{Dan}\right), 7.56$ (dd, $J_{\mathrm{CH}-}$ $\left.7, \mathrm{CH}-8=8.6 \mathrm{~Hz}, J_{\mathrm{CH}-6, \mathrm{CH}-7}=7.8 \mathrm{~Hz}, 1 \mathrm{H}, \mathrm{CH}-7 \mathrm{Dan}\right), 7.51\left(\mathrm{dd}, J_{\mathrm{CH}-2, \mathrm{CH}-3}=8.5 \mathrm{~Hz}\right.$, $\left.J_{\mathrm{CH}-3, \mathrm{CH}-4}=7.4 \mathrm{~Hz}, 1 \mathrm{H}, \mathrm{CH}-3 \mathrm{Dan}\right), 7.41-7.27$ (m, 13H, $\left.\mathrm{H}_{\text {arom }}\right), 7.22-7.18(\mathrm{~m}, 3 \mathrm{H}$, $\mathrm{H}_{\text {arom }}$ CH-6 Dan), 6.31 (d, $\left.J_{\mathrm{NH}, 2}=8.3 \mathrm{~Hz}, 1 \mathrm{H}, \mathrm{NH} \mathrm{C} 26\right), 4.88\left(\mathrm{~d}, J_{\mathrm{a}, \mathrm{b}}=11.7 \mathrm{~Hz}\right.$, $\left.1 \mathrm{H}, \mathrm{CH}-\mathrm{a}, 4^{\prime \prime}-O-\mathrm{Bn}\right), 4.83\left(\mathrm{~d}, J_{\mathrm{a}, \mathrm{b}}=11.7 \mathrm{~Hz}, 1 \mathrm{H}, \mathrm{CH}-\mathrm{a}, 2^{\prime \prime}-O-\mathrm{Bn}\right), 4.81\left(\mathrm{~d}, J_{\mathrm{a}, \mathrm{b}}=\right.$ $\left.11.2 \mathrm{~Hz}, 1 \mathrm{H}, \mathrm{CH}-\mathrm{a}, 3^{\prime \prime}-O-\mathrm{Bn}\right), 4.75$ (d, $\left.J_{\mathrm{a}, \mathrm{b}}=11.2 \mathrm{~Hz}, 1 \mathrm{H}, \mathrm{CH}-\mathrm{b}, 3^{\prime}-O-\mathrm{Bn}\right), 4.74$ $\left(\mathrm{d}, J_{1^{\prime \prime}, 2^{\prime \prime}}=3.7 \mathrm{~Hz}, 1 \mathrm{H}, \mathrm{H}-1^{\prime \prime}\right), 4.66\left(\mathrm{~d}, J_{\mathrm{a}, \mathrm{b}}=11.7 \mathrm{~Hz}, 1 \mathrm{H}, \mathrm{CH}-\mathrm{b}, 2^{\prime \prime}-O-\mathrm{Bn}\right), 4.53$ (d, $\left.J_{\mathrm{a}, \mathrm{b}}=11.7 \mathrm{~Hz}, 1 \mathrm{H}, \mathrm{CH}-\mathrm{b}, 4^{\prime \prime}-O-\mathrm{Bn}\right), 4.51-4.49$ (m, 1H, NH Dan), 4.30 (dd, $J_{2,3}$ $\left.=8.1 \mathrm{~Hz}, J_{1 \mathrm{a} / \mathrm{b}, 2}=3.7 \mathrm{~Hz}, 1 \mathrm{H}, \mathrm{H}-2\right), 3.95\left(\mathrm{dd}, J_{2^{\prime \prime}, 3^{\prime \prime}}=10.0 \mathrm{~Hz}, J_{1^{\prime \prime}, 2^{\prime \prime}}=3.7 \mathrm{~Hz}, 1 \mathrm{H}\right.$, H-2"), 3.87-3.84 (m, 3H, H-1a, H-4" , H-5"), 3.80 (dd, $J_{2^{\prime \prime}, 3^{\prime \prime}}=10.0 \mathrm{~Hz}, J_{3^{\prime \prime}, 4^{\prime \prime}}=$ $\left.2.4 \mathrm{~Hz}, 1 \mathrm{H}, \mathrm{H}-3^{\prime \prime}\right), 3.76\left(\mathrm{dd}, J_{1 \mathrm{a}, 1 \mathrm{~b}}=10.5 \mathrm{~Hz}, J_{1 \mathrm{~b}, 2}=4.1 \mathrm{~Hz}, 1 \mathrm{H}, \mathrm{H}-1 \mathrm{~b}\right), 3.65-3.64$ (m, 1H, 3-OH), 3.54-3.51 (m, 2H, H-3, H-4), 2.89 (s, 6H, $\left.\mathrm{NMe}_{2}\right), 2.83$ (ddd, $\left.J_{6 " \mathrm{a}, 6^{\prime \prime} \mathrm{b}}=14.1 \mathrm{~Hz}, J_{5,6^{\prime \prime} \mathrm{a}}=7.1 \mathrm{~Hz}, J_{6^{\prime \prime} \mathrm{a}, \mathrm{NH}}=4.4 \mathrm{~Hz}, 1 \mathrm{H}, \mathrm{H}-6^{\prime \prime} \mathrm{a}\right), 2.77$ (ddd, $J_{66^{\prime \prime} \mathrm{a}, 6^{\prime \prime} \mathrm{b}}$ $\left.=14.1 \mathrm{~Hz}, J_{6^{\prime} \mathrm{b}, \mathrm{NH}}=8.1 \mathrm{~Hz}, J_{5,6^{\prime} \mathrm{b}}=6.1 \mathrm{~Hz}, 1 \mathrm{H}, \mathrm{H}-6^{\prime \prime} \mathrm{b}\right), 2.56-2.55(\mathrm{~m}, 1 \mathrm{H}, 4-$ $\mathrm{OH}), 2.25\left(\mathrm{dt}, J_{2^{\prime} \mathrm{a}, 2^{\prime} \mathrm{b}}=14.9 \mathrm{~Hz}, J_{2^{\prime} \mathrm{a}, 3^{\prime}}=7.5 \mathrm{~Hz}, 1 \mathrm{H}, \mathrm{H}-2^{\prime} \mathrm{a}\right), 2.19\left(\mathrm{dt}, J_{2^{\prime} \mathrm{a}, 2^{\prime} \mathrm{b}}=14.9\right.$ $\left.\mathrm{Hz}, J_{2^{\prime} \mathrm{b}, 3^{\prime}}=7.5 \mathrm{~Hz}, 1 \mathrm{H}, \mathrm{H}-2^{\prime} \mathrm{b}\right), 1.67-1.15$ (m, 74H, CH $\left.2, \mathrm{H}-5-\mathrm{H}-17, \mathrm{H}-3^{\prime}-\mathrm{H}-25^{\prime}\right)$, 0.89 (t, J J $\left.17,18=J_{25^{\prime}, 26^{\prime}}=7.0 \mathrm{~Hz}, 6 \mathrm{H}, \mathrm{H}-18, \mathrm{H}-26^{\prime}\right) ;{ }^{13} \mathrm{C} \mathrm{NMR}\left(125 \mathrm{MHz}, \mathrm{CDCl}_{3}\right) \delta$ 173.5 (C=O), 152.0 (C-5 Dan), 138.2 (C-i, 3"'-O-Bn), 137.9 (C-i, 4"'-O-Bn), 137.8 (C-i, 2"-O-Bn), 134.1 (C-4a Dan), 130.6 (C-2 Dan), 129.9 (C-1 Dan), 129.6 (C-4 Dan), 129.5 (C-8a Dan), 128.9, 128.6, 128.54, 128.52, 128.2, 128.05, 128.00, 127.8, 127.5 (9 x CHarom), 128.4 (C-7 Dan), 123.1 (C-3 Dan), 118.8 (C-8 Dan), 115.2 (C-6 Dan), 98.8 (C-1"), $79.4\left(\mathrm{C}-3^{\prime \prime}\right), 76.3(\mathrm{C}-3), 75.9\left(\mathrm{C}-2^{\prime \prime}\right), 74.2\left(\mathrm{CH}_{2}\right.$, $\left.4^{\prime \prime}-O-\mathrm{Bn}\right), 74.0\left(\mathrm{CH}_{2}, 2^{\prime \prime}-O-\mathrm{Bn}\right), 73.3\left(\mathrm{CH}_{2}, 3^{\prime \prime}-O-\mathrm{Bn}\right), 73.2(\mathrm{C}-4), 73.1\left(\mathrm{C}-4^{\prime \prime}\right)$, $69.6\left(\mathrm{C}-5^{\prime \prime}\right), 69.2$ (C-1), 49.9 (C-2), $45.4\left(\mathrm{NMe}_{2}\right), 43.2$ (C-6"), 36.8 (C-2'), 33.5 (C-5), 31.9, 29.73, 29.71, 29.68, 29.67, 29.6, 29.44, 29.38, 25.9, 25.7, 22.7 (C-6C-17, C-3'-C-25'), 14.1 (2 x CH $\left.3, \mathrm{C}-18, \mathrm{C}-26^{\prime}\right)$; HRMS(ESI) $m / z$ calcd. for $\left[\mathrm{C}_{83} \mathrm{H}_{129} \mathrm{~N}_{3} \mathrm{O}_{10} \mathrm{~S}+\mathrm{Na}\right]^{+}$: 1382.9296, obsd.: 1382.9296 . 


\section{1-O-[6-deoxy-6-[N-(5-[dimethylamino]napth-1-ylsulfonyl)amido]- $\alpha$-D-}

\section{galactopyranosyl]-2-hexacosanoylamido-D-ribo-octadecane-1,3,4-triol}

$\mathrm{Pd}(\mathrm{OH})_{2} / \mathrm{C}(2.5 \mathrm{~mol} \%)$ was added to a solution of diol 50 (9.9 $\left.\mathrm{mg}, 0.0073 \mathrm{mmol}\right)$ in a mixture of $\mathrm{CHCl}_{3} / \mathrm{EtOH}(1 \mathrm{~mL}, 3 / 2, \mathrm{v} / \mathrm{v})$ and the reaction stirred under $\mathrm{H}_{2}(\mathrm{~g})$ for $17 \mathrm{~h}$ at $\mathrm{rt}$. The reaction mixture was then filtered through Celite, the Celite washed thoroughly with $\mathrm{CHCl}_{3} / \mathrm{EtOH}(3 / 2, \mathrm{v} / \mathrm{v})$, and the filtrate concentrated in vacuo. The residue was purified by silica gel flash column chromatography $(10 \%$ $\mathrm{MeOH} / \mathrm{DCM}$ ), followed by reverse phase chromatography (ODS-C18 resin, product eluted with $\mathrm{MeOH}$ ), and finally silica gel column chromatography by elution with 5\% MeOH/DCM. This afforded the target compound 2 (4.9 mg, 4.49 mmol, 62\%) as a yellow oil. $\mathrm{R}_{f}$ : $0.22(\mathrm{DCM} / \mathrm{MeOH}, 92 / 8, \mathrm{v} / \mathrm{v})$; Glows white on $\operatorname{TLC}(\lambda=366 \mathrm{~nm}) ;[\alpha]_{\mathrm{D}}^{22}=+40.0^{\circ}(c=0.1$, pyridine); IR (film) 3320, 2918, 2851, 2359, 1653, 1634, 1560, 1457, 1310, 1144, 1030, 791, $684 \mathrm{~cm}^{-1}$; UV $\lambda_{\max } \mathrm{Abs}=343 \mathrm{~nm}, \lambda_{\max } \mathrm{Em}=510 \mathrm{~nm} ;{ }^{1} \mathrm{H}$ NMR $\left(600 \mathrm{MHz}\right.$, Pyridine- $\left.d_{5}\right) \delta 9.04(\mathrm{~d}$, $\left.J_{\mathrm{CH}-2, \mathrm{CH}-3}=8.7 \mathrm{~Hz}, 1 \mathrm{H}, \mathrm{CH}-2 \mathrm{Dan}\right), 8.61-8.57$ (m, 2H, NH C26, $\mathrm{CH}-4$ Dan), 8.54 $\left(\mathrm{d}, J_{\mathrm{CH}-7, \mathrm{CH}-8}=8.6 \mathrm{~Hz}, 1 \mathrm{H}, \mathrm{CH}-8 \mathrm{Dan}\right), 7.54\left(\mathrm{dd}, J_{\mathrm{CH}-7, \mathrm{CH}-8}=8.6 \mathrm{~Hz}, J_{\mathrm{CH}-6, \mathrm{CH}-7}=\right.$ $7.4 \mathrm{~Hz}, 1 \mathrm{H}, \mathrm{CH}-7 \mathrm{Dan}), 7.51\left(\mathrm{dd}, J_{\mathrm{CH}-2, \mathrm{CH}-3}=8.7 \mathrm{~Hz}, J_{\mathrm{CH}-3, \mathrm{CH}-4}=7.6 \mathrm{~Hz}, 1 \mathrm{H}, \mathrm{CH}-\right.$ $3 \mathrm{Dan}), 7.12$ (d, $\left.J_{\mathrm{CH}-6, \mathrm{CH}-7}=7.4 \mathrm{~Hz}, 1 \mathrm{H}, \mathrm{CH}-6 \mathrm{Dan}\right), 5.45$ (d, $J_{1^{\prime \prime}, 2^{\prime \prime}}=3.9 \mathrm{~Hz}, 1 \mathrm{H}$, H-1"), $5.23(\mathrm{~m}, 1 \mathrm{H}, \mathrm{H}-2), 4.54\left(\mathrm{dd}, J_{1 \mathrm{a}, 1 \mathrm{~b}}=10.9 \mathrm{~Hz}, J_{1 \mathrm{a}, 2}=5.6 \mathrm{~Hz}, 1 \mathrm{H}, \mathrm{H}-1 \mathrm{a}\right)$, $4.52\left(\mathrm{dd}, J_{2^{\prime \prime}, 3^{\prime \prime}}=9.9 \mathrm{~Hz}, J_{1^{\prime \prime}, 2^{\prime \prime}}=3.9 \mathrm{~Hz}, 1 \mathrm{H}, \mathrm{H}-2^{\prime \prime}\right), 4.51\left(\mathrm{t}, J_{5,6 \mathrm{a}}=J_{5,6 \mathrm{~b}}=6.6 \mathrm{~Hz}\right.$, $\left.1 \mathrm{H}, \mathrm{H}-5^{\prime \prime}\right), 4.35-4.30\left(\mathrm{~m}, 3 \mathrm{H}, \mathrm{H}-3, \mathrm{H}-4, \mathrm{H}-4^{\prime \prime}\right), 4.26\left(\mathrm{dd}, J_{2^{\prime \prime}, 3^{\prime \prime}}=9.9 \mathrm{~Hz}, J_{3^{\prime \prime}, 4^{\prime \prime}}=\right.$ $\left.3.3 \mathrm{~Hz}, 1 \mathrm{H}, \mathrm{H}-3^{\prime \prime}\right), 4.19\left(\mathrm{dd}, J_{1 \mathrm{a}, 1 \mathrm{~b}}=10.9 \mathrm{~Hz}, J_{1 \mathrm{~b}, 2}=4.8 \mathrm{~Hz}, 1 \mathrm{H}, \mathrm{H}-1 \mathrm{~b}\right), 3.85$ (bs, $\left.2 \mathrm{H}, \mathrm{H}-6^{\prime \prime} \mathrm{a}, \mathrm{H}-6^{\prime \prime} \mathrm{b}\right), 2.50\left(\mathrm{dt}, J_{2^{\prime} \mathrm{a}, 2^{\prime} \mathrm{b}}=14.8 \mathrm{~Hz}, J_{2^{\prime} \mathrm{a}, 3^{\prime}}=7.8 \mathrm{~Hz}, 1 \mathrm{H}, \mathrm{H}-2^{\prime} \mathrm{a}\right), 2.46(\mathrm{dt}$, $\left.J_{2^{\prime} \mathrm{a}, 2^{\prime} \mathrm{b}}=14.8 \mathrm{~Hz}, J_{2^{\prime} \mathrm{b}, 3^{\prime}}=7.8 \mathrm{~Hz}, 1 \mathrm{H}, \mathrm{H}-2^{\prime} \mathrm{b}\right), 2.34-1.24\left(\mathrm{~m}, 72 \mathrm{H}, \mathrm{CH}_{2}, \mathrm{H}-5-\mathrm{H}-17\right.$, H-3'-H-25'), $0.86\left(\mathrm{t}, \mathrm{J}_{17,18}=J_{25^{\prime}, 26^{\prime}}=7.0 \mathrm{~Hz}, 6 \mathrm{H}, \mathrm{H}-18, \mathrm{H}-26^{\prime}\right) ;{ }^{13} \mathrm{C}$ NMR $(150$ MHz, Pyridine- $\left.d_{5}\right) \delta 173.7$ (C=O), 152.4 (C-5 Dan), 137.8 (C-4a Dan), 130.8 (C-1 Dan), 130.6 (C-8a Dan), 130.3 (C-8 Dan), 129.3 (C-4 Dan), 128.5 (C-3 Dan), 123.5 (C-7 Dan), 120.7 (C-2 Dan), 115.9 (C-6 Dan), 101.4 (C-1"), 76.8 (C-3), 72.8 (C-4), 71.42 (C-5'), $71.37\left(\mathrm{C}-3^{\prime \prime}\right), 71.2\left(\mathrm{C}-4^{\prime \prime}\right), 70.2$ (C-2'), 68.5 (C-1), 51.5 (C-2), $45.52\left(\mathrm{NMe}_{2}\right), 45.16\left(\mathrm{C}-6^{\prime \prime}\right), 37.1$ (C-2'), 34.6 (C-5), 32.42, 32.41, 30.8, $30.7,30.5,30.4,30.34,30.33,30.32$, 30.31, 30.29, 303.27, 30.26, 30.23, 30.21, $30.19,30.14,30.10,29.92,29.90,26.8,26.7,23.24,23.23$ (C-6-C-17, C-3'-C- 
25'), $14.6 \quad\left(2 \times \mathrm{CH}_{3}, \quad \mathrm{C}-18, \quad \mathrm{C}-26^{\prime}\right) ; \quad \mathrm{HRMS}(\mathrm{ESI}) \mathrm{m} / z$ calcd. for $\left[\mathrm{C}_{62} \mathrm{H}_{111} \mathrm{~N}_{3} \mathrm{O}_{10} \mathrm{~S}+\mathrm{Na}\right]^{+}:$1112.7888, obsd.: 1112.7878 .

\subsubsection{Materials and methods for cell proliferation (IL-2) assay, in vivo DC maturation assay and in vitro treatment of dendritic cells}

Mice. Breeding pairs of the inbred strains C57BL/6 were obtained from The Jackson Laboratories and from the Animal Resource Centre. All mice were maintained in the Biomedical Research Unit of the Malaghan Institute of Medical Research. CD1d ${ }^{-/-}$mice, which are devoid of CD1d-restricted $i$ NKT cells, were also used. $^{62}$ Experiments were approved by the NZ national animal ethics committee and performed according to established national guidelines.

Solubilisation of glycolipid. $\alpha$-GalCer (Industrial Research Ltd, New Zealand) and synthesised glycolipids were tested to be endotoxin-free at the sensitivity of $0.125 \mathrm{EU} / \mathrm{mL}$ with an endotoxin kit (Pyrotell, Limulus Amebocyte Lysate). Each glycolipid (1 mg) was dissolved in $200 \mu \mathrm{L}$ of $\mathrm{CHCl}_{3} / \mathrm{MeOH} / \mathrm{H}_{2} \mathrm{O}$ (10:10:3), heated at $37^{\circ} \mathrm{C}$ for $15 \mathrm{~min}$ followed by sonication for $10 \mathrm{~min}$. The solution was then diluted to $200 \mu \mathrm{g} / \mathrm{mL}$ in $0.5 \%$ Tween/phosphate-buffered saline (PBS) and left to sit at $-4{ }^{\circ} \mathrm{C}$ overnight. The glycolipid was then heated at $80{ }^{\circ} \mathrm{C}$ for $5 \mathrm{~min}$ followed by sonication for $5 \mathrm{~min}$ then cooled at $0{ }^{\circ} \mathrm{C}$ for $5 \mathrm{mins}$ (x 2) and left to sit at $-4{ }^{\circ} \mathrm{C}$ overnight.

Cell proliferation (IL-2) assay. Dendritic cells (DC2114; $;^{53} 2.5 \times 10^{4}$ cells) and murine NKT hybridoma cells (DN32.D3; ${ }^{4} 1 \times 10^{5}$ cells) were incubated with various concentration of glycolipid ( $\alpha$-GalCer 1: $583 \mathrm{nM}-0.569 \mathrm{nM}$; Dansyl $\alpha$ GalCer 2: $458 \mathrm{nM}-0.447 \mathrm{nM}$ in two fold dilution) in $200 \mu \mathrm{L}$ of complete medium consisting of Iscove's Modified Dulbecco's Medium (IMDM) supplemented with $2 \mathrm{mM}$ glutamine (Invitrogen), $1 \%$ penicillin-streptomycin (Invitrogen), $5 \times 10^{-5} \mathrm{M}$ 2-mercaptoethanol (Invitrogen) and 5\% foetal bovine serum (Sigma-Aldrich) at $37{ }^{\circ} \mathrm{C}$ for 24 h. $100 \mu \mathrm{L}$ of supernatant was added to $5 \times 10^{3}$ HT-2 cells in a flatbottom 96-well plate, incubated at $37^{\circ} \mathrm{C}$ for $24 \mathrm{~h}$ and incorporation of 
$\left[{ }^{3} \mathrm{H}\right]$ thymidine over the final $6 \mathrm{~h}$ was measured with a beta-counter (Wallac). A standard curve was constructed by incubating HT-2 cells with known concentrations of IL-2 (200-0 U/mL in five fold dilution). Each sample was analysed in triplicate.

Antigen presenting cell maturation assay. Glycolipid diluted to $2 \mu \mathrm{g} / \mathrm{mL}$ and $200 \mathrm{ng}$ (in $100 \mu \mathrm{L}$ ) was administered i.v. to groups of C57BL/6 mice $(n=3)$ and spleens were removed $20 \mathrm{~h}$ later. Spleens were teased through a cell strainer, red blood cells (RBC) were lysed with RBC lysis buffer followed by antibody labelling for CD11c (clone HL3; BD Pharmingen), B220 (clone RA3-6B2 conjugated to A488; BD Pharmingen), CD86 (clone GL1 conjugated to PE; eBioscience) and propidium iodide (BD Pharmingen) and analysis by flow cytometry (BD FACSort). The same protocol was used for assessing expression of CD86 on $\mathrm{CD} 11 \mathrm{c}^{+}$dendritic cells by $\mathrm{CD} 1 \mathrm{~d}^{-/-}$mice.

Uptake of glycolipid by dendritic cells in vitro. $1 \times 10^{6}$ DC2114 cells were incubated with $4 \mu \mathrm{g}$ of glycolipid in $1 \mathrm{~mL}$ cIMDM in a flat-bottom 6-well plate at $37^{\circ} \mathrm{C}$ for $24 \mathrm{~h}$. Cells were resuspended in FACS buffer, stained with viability dye (PI; BD Pharmingen) and analysed by flow cytometry (BD LSRII SORP; UV laser, filter 525/50, Hoechst Blue detector) 


\subsection{References}

1. Natori, T.; Koezuka, Y.; Higa, T. Tetrahedron Lett. 1993, 34, 5591-5592.

2. Akimoto, K.; Natori, T.; Morita, M. Tetrahedron Lett. 1993, 34, 5593-5596.

3. Morita, M.; Motoki, K.; Akimoto, K.; Natori, T. et al. J. Med. Chem. 1995, $38,2176-2187$.

4. Bendelac, A.; Lantz, O.; Quimby, M. E.; Yewdell, J. W.; Bennink,J. R.; Randy R. Brutkiewicz, Science, 1995, 268, 863-865.

5. Kawano, T.; Cui, J.; Koezuka, Y.; Toura, I.; Kaneko, Y.; Motoki, K.; Ueno, H.; Nakagawa,R.; Sato, H.; Kondo, E.; Koseki, H.; Taniguchi, M. Science, 1997, 278, 1626-1629.

6. For some recent reviews see: a) Godfrey, D. I.; Stankovic, S.; Baxter, A. G. Nat. Rev. Immunol. 2010, 11, 197-206, b) Wu, D.; Fujio, M.; Wong, C.-H. Bioorg. Med. Chem. 2008, 16, 1073-1083, c) Speak, A. O.; Cerundolo, V.; Platt, F. M. Immunol. Cell. Biol. 2008, 86, 588-597. d) Godfrey, D. I.; Berzins, S. P. Nat. Rev. Immunol. 2007, 7, 505-518, e) Van Kaer, L. Nat. Rev. Immunol. 2005, 5, 31-42, f) Zajonc, D. M.; Kronenberg, M. Curr. Opin. Struct. Biol. 2007, 17, 521-529, g) Godfrey, D. I.; Kronenbreg, M. J. Clin. Invest. 2004, 114, 1379-1388.

7. Fan, G.; Pan, Y. S.; Lu, K. C.; Cheng, Y. P.; Lin, W.-C.; Lin, S.; Lin, C.-H.; Wong, C.-H.; Fang, J.-M.; Lin, C.-C. Tetrahedron, 2005, 61, 1855-1862.

8. Park, J. J.; Lee, J. H.; Ghosh, S. C.; Bricard, G.; Vebjataswamy, M. M.; Porcelli, S. A.; Chung, S.-K. Bioorg. Med. Chem. Lett. 2008, 18, 3906-3909.

9. Lee, A.; Farrand, K. J.; Dickgreber, N.; Hayman, C. M.; Jürs, S.; Hermans, I. F.; Painter, G. F. Carbohydr. Res. 2006, 341, 2785-2798.

10. Plettenburg, O.; Bodmer-Narkevitch, V.; Wong, C.-H. J. Org. Chem. 2002, 67, 4559-4564.

11. Zhou, X.-T.; Forestier, C.; Goff, R. D.; Li, C.; Teyton, L.; Bendelac, A.; Savage, P. V. Org. Lett. 2002, 4, 1267-1270.

12. Sakai, T.; Ehara, H.; Koezuka, Y. Org. Lett. 1999, 1, 359-361.

13. Vo-Hoang, Y.; Micouin, L.; Ronet, C.; Gachelin, G.; Bonin, M. Chem. Bio. Chem. 2003, 4, 27-33.

14. Sakai, T.; Naidenko, O. V.; Iijima, H.; Kronenberg, M.; Koezuka, Y. J. Med. Chem. 1999, 42, 1836-1841.

15. Xia, C.; Zhang, W.; Zhang, Y.; Woodward, R. L.; Wang, J.; Wang, P. G. Tetrahedron, 2009, 65, 6390-6395.

16. Sagiv, Y.; Hudspeth, K.; Mattner, J.; Schrantz, N.; Stern, R. K.; Zhou, D.; Savage, P. B.; Teyton, L.; Bendelac, A. J. Immunol. 2006, 177, 26-30.

17. Naidenko, O. V.; Maher, J. K.; Ernst, W. A.; Sakai, T.; Modlin, R. L.; Kronenberg, M. J. Exp. Med. 1999, 190, 1069-1080.

18. Barral, D. C.; Brenner, M. B. Nature Rev. Immunol. 2007, 7, 929-941.

19. Zheng, Z.-H.; Castaño, A. R.; Segelke, B. W.; Stura, E. A.; Peterson, P. A.; Wilson, I. A. Science 1997, 277, 339-345.

20. Mukherjee, S.; Soe, T. T; Maxfield, F. R. J. Cell. Biol. 1999, 144, 12711284.

21. Moody, D. B.; Briken, V.; Cheng, T.-Y.; Roura-Mir, C.; Guy, M. R.; Geho, D. H.; Tykocinski, M. L.; Besra, G. S.; Porcelli, S. A. Nat. Immunol. 2002, 3, 435-442. 
22. Singh, R. D.; Liu, Y.; Wheatley, C. L.; Holicky, E. L.; Makino, A.; Marks, D. L.; Kobayashi, T.; Subramaniam, G.; Bittman, R.; Pagano, R. E. J. Biol. Chem. 2006, 281, 30660-30668.

23. Kamada, N.; Iijima, H.; Kimura, K.; Harada, M.; Shimizu, E.; Motohashi, S.; Kawano, T.; Shinkai, H.; Nakayama, T.; Sakai, T.; Brossay, L.; Kronenberg, M.; Taniguchi, M. Internat. Immunol. 2001, 13, 853-861.

24. Prigozy, T. I.; Naidenko, O.; Qasba, P.; Elewaut, D.; Brossay, L.; Khurana, A.; Natori, T.; Koezuka, Y.; Kulkarni, A.; Kronenberg, M. Science 2001, 291, 664-667.

25. Farrand, K. J.; Dickgreber, N.; Stoitzner, P.; Ronchese, F.; Petersen, T. R.; Hermans, I. F. J. Immunol. 2009, 183, 7732-7742.

26. Shin-ichiro Fujii, S.-H.; Goto, A.; Shimizu, K. Blood, 2009, 113, 4262-4272.

27. Velmourougane, G.; Raju, R.; Bricard, G.; Im, J. S.; Besra, G. S.; Porcelli, S. A.; Howell, A. R. Bioorg. Med. Chem. Lett. 2009, 19, 3386-3388.

28. Raju, R.; Castillo, B. F.; Richardson, S. K.; Thakur, M.; Severins, R.; Kronenbrg, M.; Howell, A. R. Bioorg. Med. Chem. Lett. 2009, 19, 41224125.

29. Takikawa, H.; Muto, S.-E.; Mori, K. Tetrahedron, 1998, 54, 3141-3150.

30. Ndonye, R. M.; Izmirian, D. P.; Dunn, M. F.; Yu, K. O. A.; Porcelli, S. A.; Khurana, A.; Kronenberg, M.; Richardson, S. K.; Howell, A. R. J. Org. Chem. 2005, 70, 10260-10270.

31. For a recent review highlighting glycosylation reactions using sphingosines and ceramides see: Morales-Serna, J. A.; Boutureira, O.; Diaz, Y.; Matheu, M. I.; Castillon, S. Carbohydr. Res. 2007, 342, 1595-1612.

32. For examples see: a) Timmer, M. S. M.; Stocker, B. L.; Northcote, P. T.; Burkett, B. A. Tetrahedron Lett. 2009, 50, 7199-7204, b) Caton-Williams, J.; Huang, Z. Angew. Chem., Int. Ed. 2008, 47, 1723-1725, c) Aumüller, I.; Lindhorst, T. K. Eur. J. Org. Chem. 2006, 1103-1108, d) Sekine, M.; Aoyagi, M.; Ushioda, M.; Ohkubo, A.; Seio, K. J. Org. Chem. 2005, 70, 8400-8408.

33. Bhattacharjee, A. K.; Zissis, E.; Glaudemans, C. P. J. Carbohydr. Res. 1981, 89, 249-254.

34. Morgenlie, S. Carbohydr. Res. 1975, 41, 77-83.

35. The preparation of a 3-O, 4-O benzyl protected phytosphingosine lipid by Wong and co-workers (ref. 10) is comparably efficient having been prepared from 2-deoxy galactose in 9 steps and $36 \%$ overall yield.

36. For a highly efficient synthesis of the fully deprotected phytosphingosine lipid (6-steps, 29\% overall yield), see: Lin, C.-C.; Fan, G.-T.; Fang, J.-M. A. Tetrahedron Lett. 2003, 44, 5281-5283.

37. Yeager, A. R.; Finney, N. S. J. Org. Chem. 2005, 70, 1269-1275.

38. Du, W.; Gervay-Hague, J. Org. Lett. 2005, 7, 2063-2065.

39. Gervay, J.; Hadd, M. J. J. Org. Chem. 1997, 62, 6961-6967.

40. Gervay, J.; Nguyen, T. N.; Hadd, M. J. Carbohydr. Res. 1997, 300, 119-125.

41. Hadd, M. J.; Gervay, J. Carbohydr. Res. 1999, 320, 61-69.

42. Natori, T.; Morita, M.; Akimoto, K.; Koezuka, Y. Tetrahedron, 1994, 50, 2771-2784.

43. Graziani, A.; Passacantilli, P.; Piancatelli, G.; Tani, S. Tetrahedron Asymm. 2000, 11, 3921-3937.

44. Murata, K.; Toba, T.; Nakanishi, K.; Takahashi, B.; Yamamura, T.; Miyake, S.; Annoura, H. J. Org. Chem. 2005, 70, 2398-2401. 
45. Goff, R. D.; Gao, Y.; Mattner, J.; Zhou, D.; Yin, N.; Cantu, C.; Teyton, L.; Bendelac, A.; Savage, P. B. J. Am. Chem. Soc. 2004, 126, 13602-13603.

46. Franchini, L.; Matto, P.; Ronchetti, F.; Panza, L.; Barbieri, L.; Costantine, V.; Mangoni, A.; Cavallari, M.; Mori, L.; De Libero, G. Bioorg. Med. Chem. 2007, 15, 55529-5536.

47. Wenjum D.; Kulkarni, S. S.; Gervay-Hague, J. Chem. Commun. 2007, 23362338.

48. Schombs, M.; Park, F. E.; Du, W.; Kulkarni, S. S.; Gervay-Hague, J. J. Org. Chem. 2010, 75, 4891-4898.

49. Gorin, P. A. J.; Mazuzek, M. Can. J. Chem. 1975, 53, 1212-1223.

50. Zemplén, G.; Pascu, E. Ber. Dtsch. Chem. Ges. 1929, 1613-1614.

51. Liu, Y.; Goff, R. D.; Zhou, D.; Mattner, J.; Sullivan, B. A.; Khurana, A.; Cantu III, C.; Ravkov, E. V.; Ibegbu, C. C.; Altman, J. D.; Teyton, L.; Bendelac, A.; Savage, P. B. J. Immunol. Methods 2006, 312, 34-39.

52. Taki, T.; Kuroyanagi, M.; Yoshioka, H.; Handa, S. J. Biochem. 1992, 111, 614-619.

53. Fujii, S.-I.; Shimizu, K.; Smith, C.; Bonifaz, L.; Steinman, R. M. J. Exp. Med. 2003, 198, 267-279.

54. Hermans, I. F.; Silk, J. D.; Gileadi, U.; Salio, M.; Mathew, B.; Ritter, G.; Schmidt, R.; Harris, A. L.; Old, L.; Cerundolo, V. J. Immunol. 2003, 171, 5140-5147.

55. Steiner, Q. G.; Otten, L. A.; Hicks, M. J.; Kaya, G.; Grosjean, F.; Saeuberli, E.; Lavanchy, C.; Beermann, F.; McClain, K. L.; Acha-Orbea, H. Blood. 2008, 111, 2073-2082.

56. Lin, C.-C.; Fan, G.-T.; Fang, J.-M. Tett. Lett. 2003, 44, 5281-5283.

57. Banks, M. R.; Blake, A. J.; Cadogan, J. I. G.; Dawson, I. M.; Gaur, S. Gosney, I.; Gould, R. O.; Grant, K. J.; Hodgson, P. K. G. J. Chem. Soc., Chem. Commun. 1993, 1146-1148.

58. Ballou, C. E.; Fischer, H. O. L. J. Am. Chem. Soc. 1954, 76, 3188-3193.

59. Lehmann, J.; Weckerle, W. Carbohydr. Res. 1972, 22, 23-35.

60. Hanessian, S. J. Org. Chem. 1969, 34, 675-681.

61. Zaliz, C. L. R.; Varela, O. J. Carb. Chem. 2001, 20, 689-701.

62. Chen, Y.-H.; Chiu, N. M.; Mandal, M.; Wang, N.; Wang, C.-R. Immunity 1997, 6, 459-467. 


\section{Chapter 3. Synthesis of BODIPY- $\alpha-$ galactosylceramide}

\subsection{Introduction}

Fluorescently labelled $\alpha$-galactosylceramide ( $\alpha$-GalCer) probes (eg. Dansyl- $\alpha$ GalCer 2 and BODIPY- $\alpha$-GalCer 3, Figure 3.1) are valuable tools that can be used to further understand the mechanism of iNKT cell activation by $\alpha$-GalCer (1). While crystal structure analysis and the results from structure activity relationships provide a clear understanding of the specific molecular interactions between $\alpha$-GalCer, CD1d and TCR, which gives rise to the activation of iNKT cells, little is known about the trafficking of $\alpha$-GalCer.
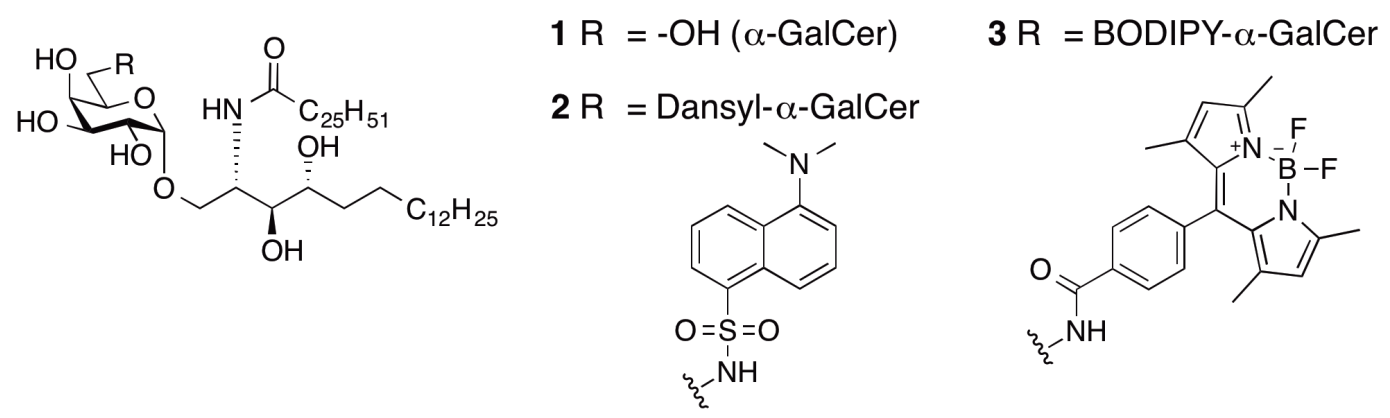

Figure 3.1. $\alpha$-GalCer 1, dansyl- $\alpha$-GalCer 2 and BODIPY- $\alpha-G a l C e r ~ 3$.

Tagging $\alpha$-GalCer with a fluorophore will allow for further understanding about the behaviour of the glycolipid during cancer immunotherapy. The value of fluorescently labeled $\alpha$-galactosylceramide has been illustrated in Chapter 2, whereby the synthesis and biological evaluation of dansyl- $\alpha$-GalCer 2 were described. While the uptake of dansyl- $\alpha$-GalCer $\mathbf{2}$ by dendritic cells (DCs) can be monitored by flow cytometry, the dansyl fluorophore is not bright enough to be detected by fluorescent and confocal microscopy. This is because the fluorescent glycolipid underwent rapid photobleaching when it was subjected under the UV laser used to excite the fluorophore. Moreover, it was found that when DCs were 
isolated from mice injected with dansyl- $\alpha$-GalCer $\mathbf{2}$, and analysed, no glycolipid could be detected by flow cytometry. Therefore, to meet the need for a brighter fluorophore, boron dipyrromethene (BODIPY)- $\alpha$-GalCer 3 was developed. BODIPY is a boron dipyrromethene dye that has a wavelength of maximum absorbance at around $500 \mathrm{~nm}$ and emits light at $510 \mathrm{~nm}$. It was chosen as a fluorophore due to its higher fluorescence quantum yield $(\Phi=0.40-0.60)$ compared to the dansyl group $(\Phi=0.20){ }^{1}$

\subsection{Synthesis of BODIPY- $\alpha$-galactosylceramide}

\section{Retrosynthesis}

To synthesise BODIPY- $\alpha-G a l C e r ~ 3$, a strategy that differed to that used for the synthesis of dansyl- $\alpha$-GalCer 2 was undertaken. Instead of the early incorporation of the fluorescent group onto the galactose sugar head group, the route was designed such that the BODIPY group was introduced at the end of the synthesis, by coupling BODIPY-COOH 52 with the fully deprotected glycolipid, 6'-amino$\alpha$-GalCer 51 (Scheme 3.1). It was envisaged that the development of the late stage intermediate, 6 '-amino- $\alpha$-GalCer 51 will be useful not only for making BODIPY$\alpha$-GalCer, but also for attaching other functionalities to the $6^{\prime}$-position of $\alpha$ GalCer, such as other fluorescent groups, biotin tags, or peptides. ${ }^{2-7}$ The BODIPY-COOH 52 could in turn be synthesised in six steps from terephthalic acid 53 and dimethylpyrrole 54 using a strategy developed in the Stocker-Timmer group, ${ }^{1,8}$ while the deprotected glycolipid $\mathbf{5 1}$ could be obtained from $6^{\prime}$-azido- $\alpha$ GalCer 55 via removal of the ceramide protecting groups with simultaneous hydrogenolysis of the benzyl ethers and reduction of the azide to an amine. The fully protected glycolipid $\mathbf{5 5}$ could be made by coupling galactosyl imidate $\mathbf{5 6}$, itself obtained from D-galactose $\mathbf{3 9},{ }^{9,10}$ with a suitably protected lipid acceptor $\mathbf{5 7}$, prepared in a few steps from phytosphingosine 58. In contrast to the synthetic route employed for the synthesis of dansyl- $\alpha$-GalCer $\mathbf{2}$, the glycosylation strategy employed for the preparation of BODIPY- $\alpha$-GalCer 3 would involve the coupling of galactose donor 56 with the complete ceramide acceptor $\mathbf{5 7}$. While the yields of glycosylations with $\mathrm{N}$-acylated ceramide acceptors are typically lower (ca. 25$55 \%$ ) compared to coupling with the ceramide backbone, this strategy is necessary 
to avoid the presence of two amine groups, which would be difficult to selectively acylate after coupling. ${ }^{7}$

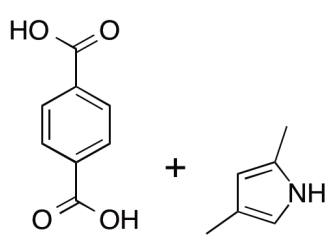

53

54

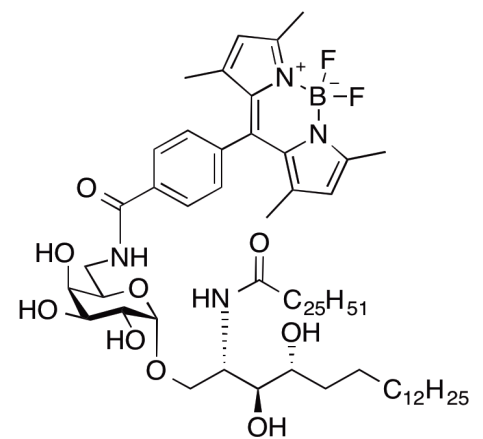

3

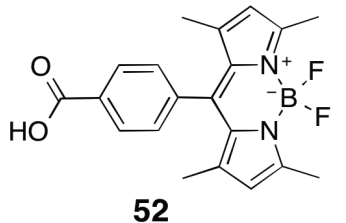

$+$

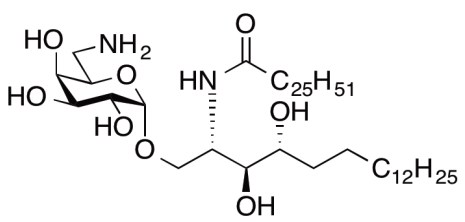

51

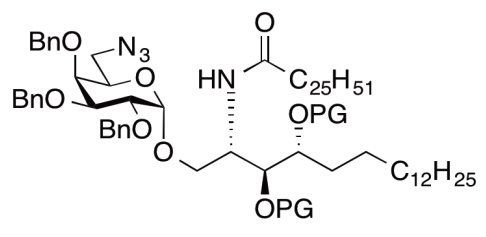

55

Scheme 3.1. Retrosynthetic scheme for BODIPY- $\alpha-G a l C e r 3$. 


\section{Synthesis of 6-azido-galactosyl imidate donor 56.}

The synthesis of BODIPY- $\alpha$-GalCer 3 commenced with the preparation of galactosyl imidate donor 56 (Scheme 3.2). Here, methyl 6-azido galactoside 45, a convenient intermediate previously described in Chapter 2 (Scheme 2.3), was converted to lactol 59 via acid-mediated hydrolysis of the anomeric methyl group. The trichloroacetimidate moiety was installed via the treatment of lactol $\mathbf{5 9}$ with trichloroacetonitrile in the presence of DBU to provide imidate donor 56, as the $\alpha$-anomer only, in $89 \%$ yield. Taken together, galactosyl imidate donor 56 was made from D-galactose (39) in 33\% overall yield over seven steps, which is more efficient compared to the route reported by Li et al., which produced the imidate donor 56 in $11 \%$ overall yield over seven steps, starting from a later stage intermediate, $1-S$ - $\beta$-D-galactosyl toluene. ${ }^{10}$

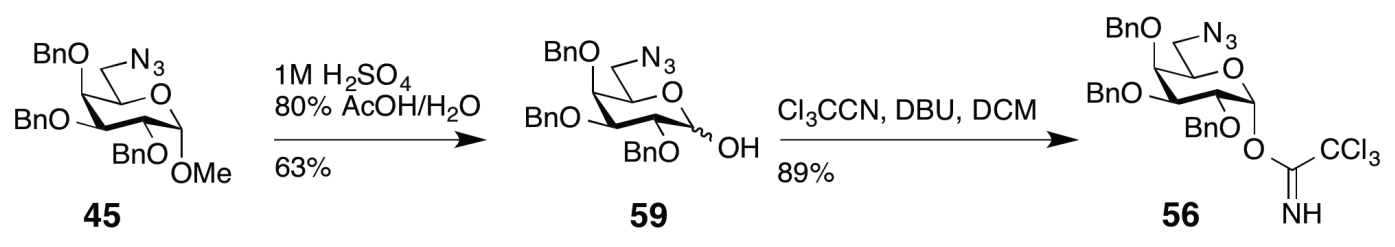

Scheme 3.2. Synthesis of 6-azido-galactosyl imidate donor 56.

\section{Synthesis of BODIPY-COOH 52}

A robust and efficient route for the synthesis of BODIPY-COOH $\mathbf{5 2}$ was developed by Ms. Stephanie Chee within the group (Scheme 3.3). ${ }^{11}$ Briefly, the synthesis began with the methylation and partial hydrolysis of terephthalic acid $\mathbf{5 3}$ to give monoester 60 in $85 \%$ yield over two steps. Next, the acid chloride $\mathbf{6 1}$ was generated in the presence of thionyl chloride, followed immediately by condensation with dimethylpyrrole 54 to form dipyrrole $\mathbf{6 2}$ in 77\% yield over two steps. The boron functionality was then introduced by reacting dipyrrole $\mathbf{6 2}$ with $\mathrm{BF}_{3} . \mathrm{OEt}_{2}$ to give BODIPY ester $\mathbf{6 3}$ in an excellent $98 \%$ yield. Finally, hydrolysis of the methyl ester under basic conditions afforded BODIPY-COOH 52 in quantitative yield. For this thesis, methyl ester $\mathbf{6 3}$ was kindly provided by Ms. 
Stephanie Chee, which upon hydrolysis afforded the desired BODIPY-COOH 52 in identical yield to that previously reported. ${ }^{11}$<smiles>O=C(O)c1ccc(C(=O)O)cc1</smiles>

53

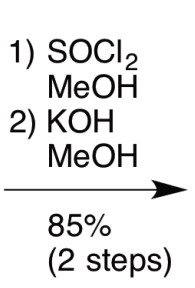

(2 steps)

60<smiles>COC(=O)c1ccc(C(=O)O)cc1</smiles>

6<smiles>COC(=O)c1ccc(C(=O)Cl)cc1</smiles>

61

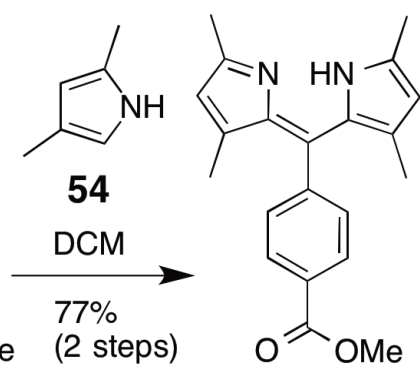

62

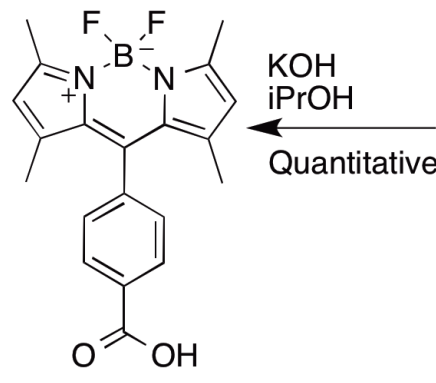

52<smiles>COC(=O)c1ccc(C2=C3C(C)=CC(C)=[N+]3[B-](F)(F)n3c(C)cc(C)c32)cc1</smiles>

63

Scheme 3.3. Synthesis of BODIPY-COOH 52. ${ }^{11}$

Synthesis of 3,4-isopropylidene protected ceramide acceptor 65 and coupling to galactosyl donor 56

With the sugar building block and BODIPY-COOH in hand, attention then turned to the synthesis of the ceramide acceptor. Again, an existing intermediate from the established route for the synthesis of dansyl- $\alpha$-GalCer 2 , namely the 2-azido-3,4isopropylidene protected phytosphingosine 43 (Chapter 2), was used (Scheme 3.4). ${ }^{9}$ The azide functionality in $\mathbf{4 3}$ was reduced using trimethylphosphine and $1 \mathrm{M}$ $\mathrm{NaOH}$ and the resultant amine coupled to hexacosanoic acid using an EDCI/DMAP-mediated protocol to give the fully protected lipid 64. Next, the 
selective removal of the trityl group was attempted under acidic conditions, but despite the controlled and careful addition of AcOH or TFA, hydrolysis of the isopropylidene protecting group could not be prevented. Therefore, a Birch reaction was used as an alternative method to selectively cleave the trityl group, which yielded the 3,4-isopropylidene protected acceptor $\mathbf{6 5}$ in 70\% yield. Here, it should be noted that the synthesis of acceptor 65 starting from 2-azido phytosphingosine, which has previously been described by Schmidt and coworkers, ${ }^{12,13}$ is a shorter and more direct route than the one used herein, however intermediate $\mathbf{4 3}$ was chosen as it was readily available from previous work with dansyl- $\alpha$-GalCer $2{ }^{9}$

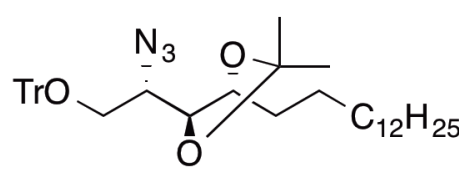

43
1) $\mathrm{PMe}_{3}, \mathrm{THF}$ $1 \mathrm{M} \mathrm{NaOH}$

2)

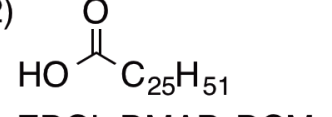

EDCI, DMAP, DCM

$75 \%$ (2 steps)

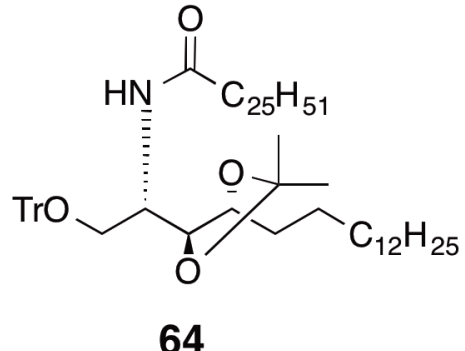

64

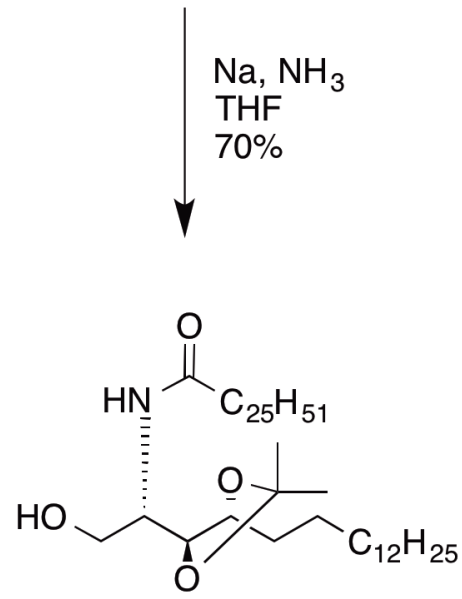

65

Scheme 3.4. Synthesis of 3,4-isopropylidene protected ceramide acceptor 65.

With acceptor 65 in hand, attempts were then made to perform the glycosylation reaction using imidate donor $\mathbf{5 6}$ and TMSOTf as the promoter. Glycosylation reactions with imidate donors using TMSOTf as the activator are typically performed at temperatures of $0{ }^{\circ} \mathrm{C}$ or lower, ${ }^{14}$ however, when the coupling was attempted with a 2:1 mixture of DCM/THF as the solvent (Table 3.1, Entry 1), the 
reaction mixture containing both donor and acceptor appeared cloudy, indicating poor solubility of the lipid acceptor. As a result, no desired glycolipid was observed and instead the hydrolysed donor $\mathbf{5 9}$ was obtained upon workup of the reaction, along with the recovery of uncoupled acceptor $\mathbf{6 5}$.

Table 3.1. Glycosylation reaction between imidate donor 56 and 3,4isopropylidene acceptor $\mathbf{6 5}$.

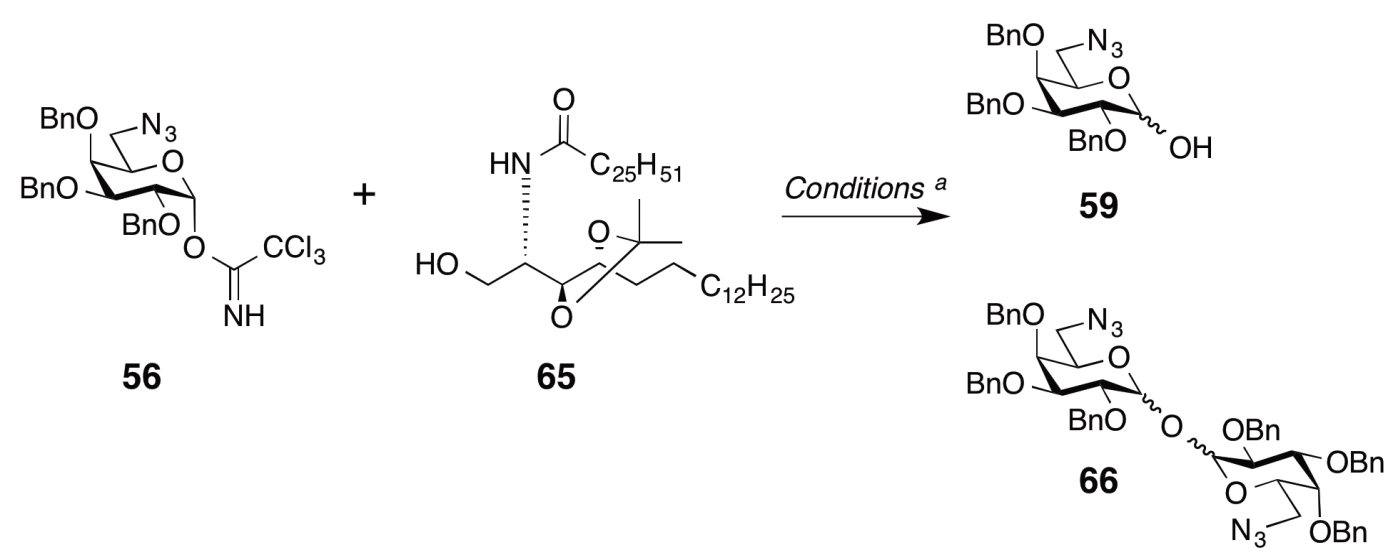

\begin{tabular}{|c|c|c|c|c|}
\hline Entry & Activator & Solvent $^{b}$ & Temperature & Product $^{c}$ \\
\hline 1 & TMSOTf & DCM/THF (2/1) & $0{ }^{\circ} \mathrm{C}$ to $\mathrm{rt}$ & 59 \\
\hline 2 & TMSOTf & $\begin{array}{l}\text { Toluene/DCM/DMF } \\
(2 / 1 / 0.1)\end{array}$ & $\mathrm{rt}$ & 59 \\
\hline 3 & TMSOTf & Toluene/DCM (2/1) & $30{ }^{\circ} \mathrm{C}$ & $59+66$ \\
\hline 4 & TMSOTf & $\mathrm{Et}_{2} \mathrm{O} / \mathrm{THF}(5 / 1)$ & $\mathrm{rt}$ & $59+66$ \\
\hline 5 & $\mathrm{BF}_{3} . \mathrm{OEt}_{2}$ & $\mathrm{Et}_{2} \mathrm{O} / \mathrm{THF}(5 / 1)$ & $-20^{\circ} \mathrm{C}$ & $59+66$ \\
\hline
\end{tabular}

${ }^{a}$ Donor and acceptor were co-evaporated with dry toluene (x3) and dissolved in the solvent and stirred with $4 \AA$ molecular sieves for 30 mins. Then the reaction was set to the desired temperature and the promoter was added slowly.

${ }^{b}$ Solvent and ratio (v/v) in parentheses.

${ }^{c}$ Uncoupled lipid acceptor $\mathbf{6 5}$ was recovered from all unsuccessful coupling reactions.

During co-evaporation of the donor and acceptor prior to the glycosylation reactions, it was observed that toluene, which was used as the solvent for azeotropic removal of water, was able to dissolve the lipid acceptor well. Therefore, the glycosylation was attempted using a mixture of toluene/DCM/DMF $(2 / 1 / 0.1, v / v / v)$ as the solvent (Entry 2). While the reaction mixture was completely clear at room temperature, unfortunately the glycosylation was 
unsuccessful, presumably because the highly reactive donor was being activated at a temperature that was too high. Again only the hydrolysed donor $\mathbf{5 9}$ was obtained. Similarly, glycosylation with toluene and DCM as a solvent at $30{ }^{\circ} \mathrm{C}$ was to no avail and gave 1,1'-digalactoside 66 (Entry 3). While all effort was made to ensure that dry conditions were used for the sensitive coupling reactions, unfortunately the severe unreactivity of the lipid acceptor $\mathbf{6 5}$ meant that trace amounts of water in the reaction mixture led to the formation of lactol $\mathbf{5 9}$, which then reacted with another molecule of imidate $\mathbf{5 6}$ to form 1,1'-disaccharide $\mathbf{6 6}$. A diethyl ether/THF solvent system, which is commonly used in coupling reactions between lipid acceptors and glycoside donors, ${ }^{15}$ but again the lipid acceptor was only soluble at room temperature and as a result, gave 1,1'-digalactoside 66 (Entry 4). In accordance with Wong and co-workers' procedure, ${ }^{15}$ a milder promoter $\mathrm{BF}_{3} . \mathrm{OEt}_{2}$ was used with the same solvent system, though disappointingly, this also only gave a mixture of lactol 59 and 1,1'-digalactoside 66 (Entry 5). From this series of glycosylation attempts, it was concluded that the solubility of the ceramide acceptor $\mathbf{6 5}$, and hence its poor reactivity, was a major issue and that even trace amounts of water would thus preferentially react with the imidate donor $\mathbf{5 6}$ to form the lactol. Here, it should also be noted that although Schmidt and co-workers have reported the synthesis of lipid acceptor $\mathbf{6 5}$, it has not been used directly as a glycosyl acceptor and instead was converted to a 1phosphite derivative for the synthesis of glycophosphatidylinositols (GPIs). ${ }^{12,13}$

\section{Synthesis of benzoyl protected ceramide acceptor 57 and coupling to galactosyl donor 56}

Due to the poor reactivity of isopropylidene protected lipid acceptor $\mathbf{6 5}$, alternative protecting groups on the phytosphingosine backbone were evaluated. Xia et al. reported on the successful coupling of the lipid acceptor when the 3-and 4-hydroxyls were protected as either benzyl ethers or benzoate esters (yield 57$59 \%) .{ }^{16}$ It was therefore proposed that a change of protecting groups would aid with the solubility of the lipid, and thus the glycosylation reaction. Furthermore, Xia et. al. observed that when the 3-and 4- hydroxyls of the phytosphingosine backbone were protected with benzyl groups, upon glycosylation with a 
galactosyl imidate donor, the $\beta$-coupled glycolipid was obtained. This was a consequence of the high reactivity of the acceptor due to the arming effect of the electron-donating benzyl groups. Alternatively, when the secondary alcohols were protected as benzoate esters, only the $\alpha$-isomer was obtained as the major product. From the above results, it was therefore proposed that the glycosylation reaction would be more successful if a benzoate-protected lipid was used.

To this end, the synthesis of the target $\mathrm{N}$-acylated phytosphingosine lipid began with a similar approach to that previously reported, whereby commercially available phytosphingosine $\mathbf{5 8}$ was first $N$-acylated at the 2-position with hexacosanoic (C26) acid (Scheme 3.5). ${ }^{16,17}$ Various strategies such as direct coupling of the amine $\mathbf{5 8}$ and the $\mathrm{C} 26$ acid with $\mathrm{EDCI}^{18-21}$ as the coupling agent or coupling to the $\mathrm{C} 26$ acid chloride ${ }^{20}$ or the $\mathrm{C} 26-N$-hydroxysuccinimidyl ester ${ }^{16,22}$ have been employed to obtain the trihydroxy ceramide 67.

Initial attempts to carry out this reaction according to Gervay-Hague and coworkers' protocol utilizing EDCI as a coupling agent in the presence of DMAP afforded trihydroxy ceramide 67 in $70 \%$ yield (Scheme 3.5 ). ${ }^{18}$ During the reaction, it was observed that the $\mathrm{C} 26$ acid was poorly soluble in pyridine solvent used. The poor solubility of the lipid leading to its lower reactivity, as well as the ability of EDCI to facilitate the formation of both esters and amides were suspected to be the reason for this average yield. Therefore, to improve the yield of the reaction, an alternative coupling agent selective only for the acylation of amines but not alcohols, PyBOP, in the presence of DiPEA and DMF was used. Due to the difficulty in monitoring the disappearance of the starting materials, phytosphingosine $\mathbf{6 7}$ and $\mathrm{C} 26$ acid by TLC, two reactions were set up and allowed to stir for 1 day or 3 days, which upon purification by crystallisation afforded the ceramide 67 in $37 \%$ and $73 \%$, respectively. The purity of the lipid was confirmed by ${ }^{1} \mathrm{H}$ NMR. It was concluded that the reaction proceeded slowly, and so when it was allowed to react for 7 days, the triol $\mathbf{6 7}$ was obtained in quantitative yield. This is the first report of PyBOP being used as the coupling agent for phytosphingosine-based ceramides and has by far the best yield compared to other coupling protocols available. ${ }^{16,19-24}$ 
Next, the primary hydroxyl was regioselectively protected as a trityl ether to give diol 68 in 79\% yield. Subsequent benzoylation of the secondary hydroxyls, followed by the selective removal of the trityl group then afforded the ceramide acceptor 57 in $67 \%$ over two steps. ${ }^{16,23}$ This strategy thus allowed for the synthesis of the complete ceramide acceptor in an excellent overall yield of $53 \%$ over four steps. The results obtained are comparable to Xia and co-workers' route, which began with succinimide-activated acyl lipid $(77 \%$ and $65 \%$ overall yield over five steps for the C26 and C8 lipids, respectively) ${ }^{16,17}$ and Morita et al.'s route, which began with 2-azido-tri-O-benzyl-phytosphingosine (42\% overall yield over 5 steps). ${ }^{23}$ 


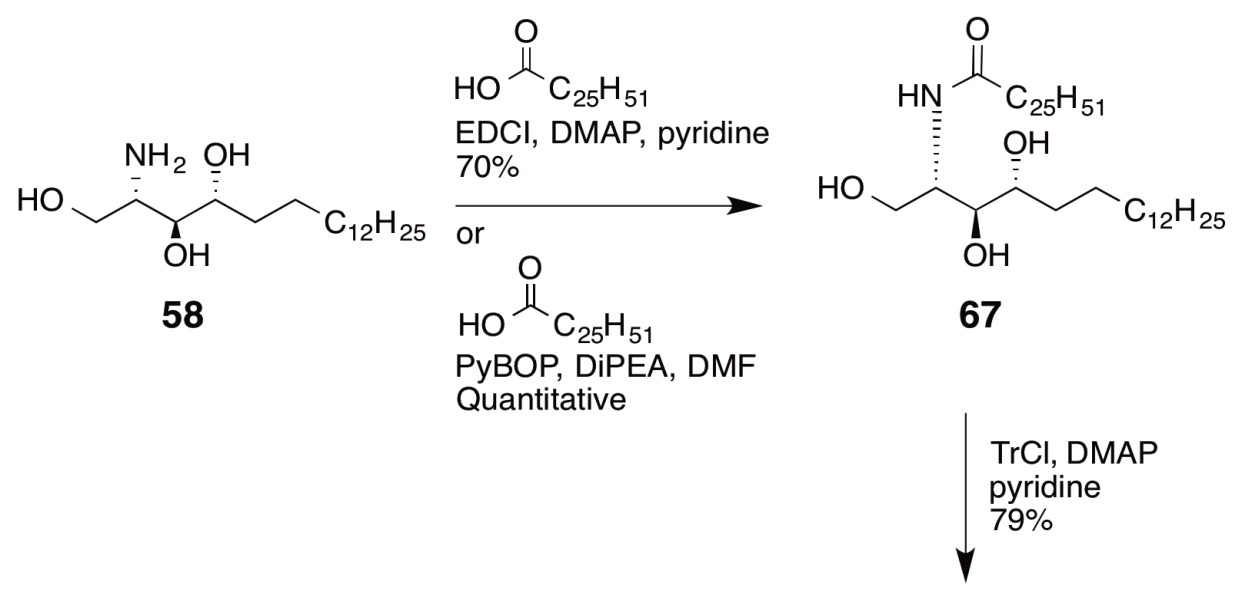

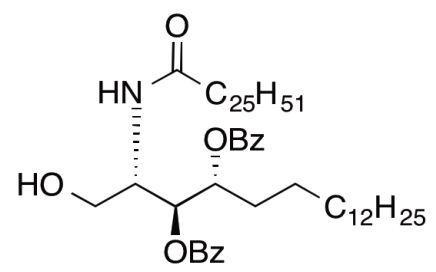

57
1) $\mathrm{BzCl}, \mathrm{DMAP}$ pyridine

2) $\mathrm{pTsOH} . \mathrm{H}_{2} \mathrm{O}$ $\mathrm{MeOH} / \mathrm{DCM}$

$67 \%$ (2 steps)<smiles>BC(=O)N[C@@H](CO[CH])[C@H](O)C(O)CCCC</smiles>

68

56, TMSOTf, $4 \AA$ MS $\mathrm{Et}_{2} \mathrm{O} / \mathrm{THF}(5: 1)$ $(\alpha: \beta ; 3: 1 ; 68 \%: 23 \%)$

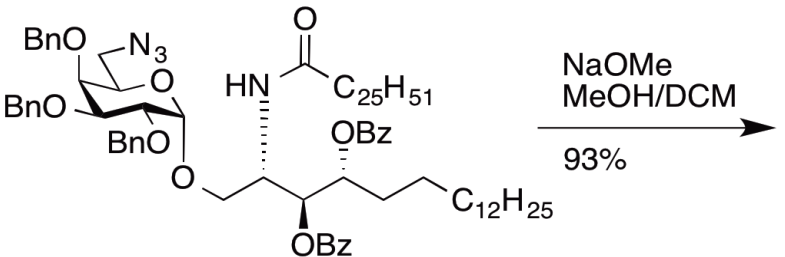

$55 \alpha$

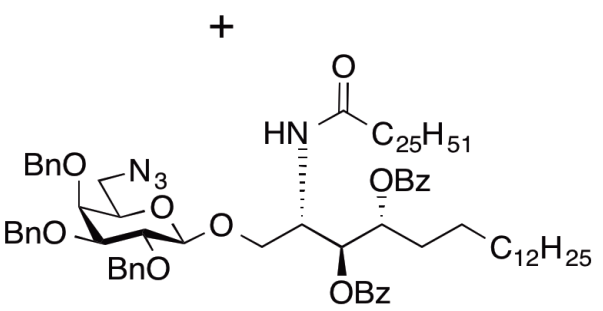

$55 \beta$

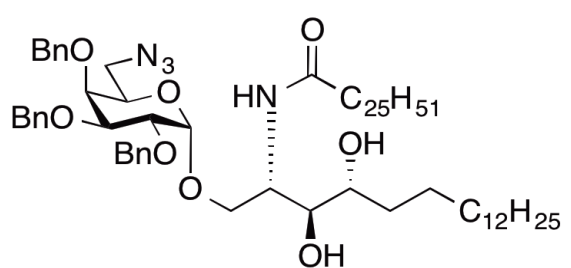

69

Scheme 3.5. Synthesis of $\alpha$-GalCer diol 69.

With the benzoate protected lipid acceptor $\mathbf{5 7}$ and the galactose donor $\mathbf{5 6}$ in hand, the glycosylation was then attempted using TMSOTf as the activator. It was found that $0{ }^{\circ} \mathrm{C}$ was the lowest temperature that could be used for optimal activation of 
the trichloroacetimidate donor 56, while keeping the highly lipophilic lipid acceptor 57 dissolved. Indeed, this afforded the protected 6'-azido glycolipid $\mathbf{5 5}$ in excellent yield $(91 \%)$ and satisfactory $\alpha$-selectivity $(\alpha: \beta=3: 1)$, as confirmed by a coupling constant of $3.6 \mathrm{~Hz}$ for the anomeric signal in the ${ }^{1} \mathrm{H}$ NMR and a one bond coupling $\left({ }^{1} J_{\mathrm{CH}}\right)$ of $168.6 \mathrm{~Hz}$ for the $\alpha$-anomer. ${ }^{25}$ In contrast, the $\beta$-anomer had an anomeric coupling constant of $7.8 \mathrm{~Hz}$ and a one bond $\mathrm{CH}$-coupling of 159.4 Hz. Due to the poor solubility of the lipid, glycosylation reactions using the complete ceramide acceptor typically result in a modest yield (ca. 40-50\%) for the $\alpha$-anomer, ${ }^{16,19}$ thus the results observed here were encouraging, particularly as the $\alpha$ and $\beta$-anomers could be isolated in $68 \%$ and $23 \%$ yields, respectively. The removal of the protecting groups on $\alpha$-linked glycolipid $\mathbf{5 5}$ was initially attempted with a Birch reduction, which would remove the benzyl groups as well as the benzoyl groups upon quenching of the reaction with methanol to form sodium methoxide in situ. However, difficulty in isolating the product upon neutralisation with Dowex $+\mathrm{H}^{+}$meant that stepwise deprotection was needed and the 3- and 4-Obenzoyl groups were first removed under basic conditions to afford the diol glycolipid 69 in an excellent yield of 93\%.

Next, the simultaneous hydrogenolysis of the three benzyl ethers and the reduction of the azide to the amine was carried out using Pearlman's catalyst with the addition of $5 \%$ formic acid to prevent catalyst poisoning by the amine that is formed during the reaction (Scheme 3.6). While the reaction appeared to proceed to completion by TLC analysis, the isolation and purification of amine $\mathbf{5 1}$ proved difficult. Despite using copious amounts of $\mathrm{CHCl}_{3} / \mathrm{EtOH} / \mathrm{MeOH}$ and pyridine to wash the celite plug that was used to filter the catalyst upon completion of the reaction, only a small amount of material was recovered. The difficulty in isolating the glycolipid from the reaction mixture was attributed to the poor solubility of the fully deprotected 6'-amino glycolipid $\mathbf{5 1}$ and its affinity for the palladium hydroxide on carbon catalyst. Initial attempts to directly couple the crude mixture of amine $\mathbf{5 1}$ to BODIPY-COOH 52 was unsuccessful, as confirmed by the absence of new product when the reaction mixture was analysed by TLC. Indeed, both starting materials were re-isolated. 

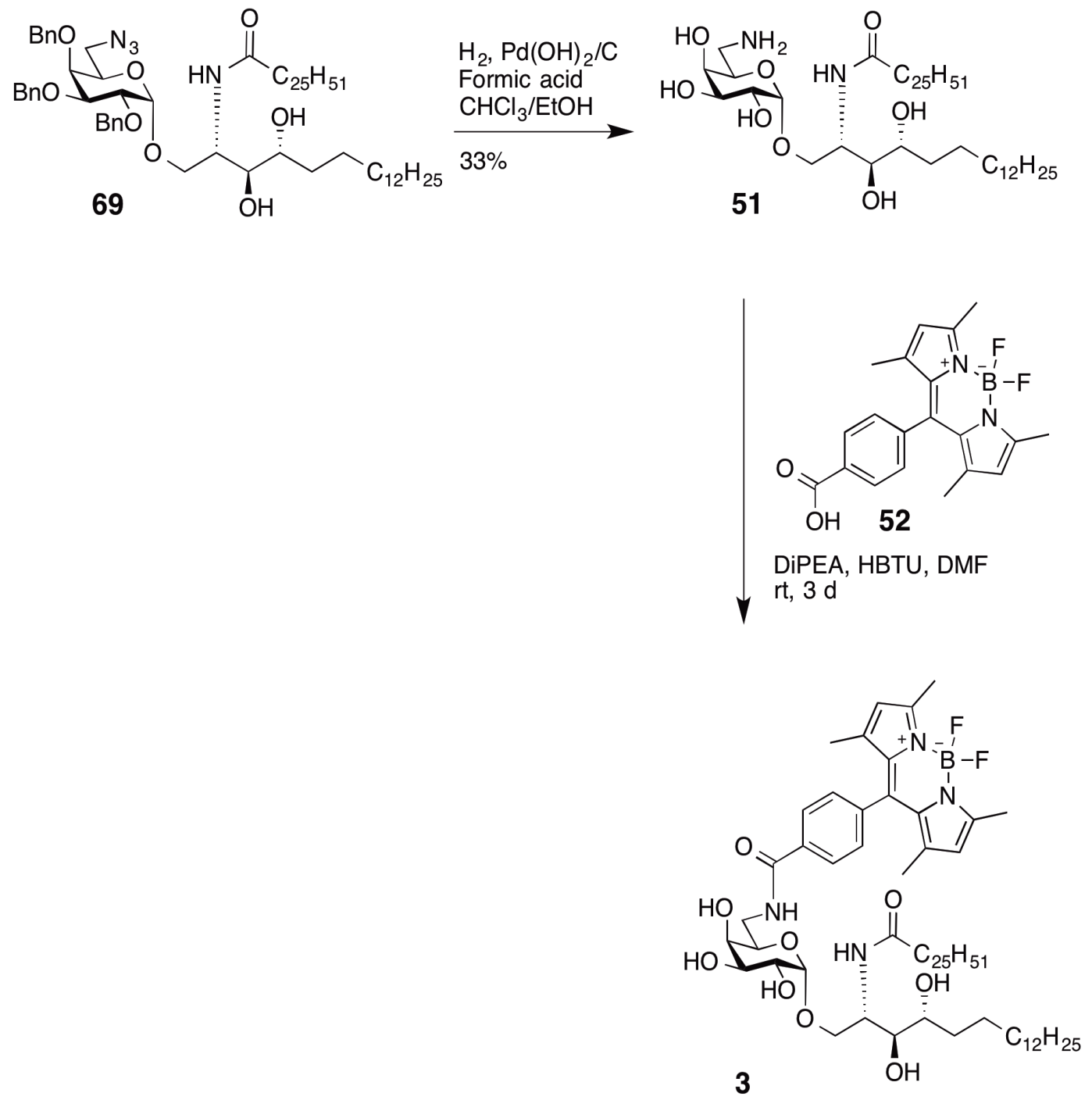

Scheme 3.6. Synthesis of BODIPY- $\alpha$-GalCer 3 using BODIPY-COOH 52.

To understand why the coupling reaction was unsuccessful, some of the crude mixture of glycolipid $\mathbf{5 1}$ that was used in the coupling reaction was analysed by mass spectrometry. The presence of the desired product $6^{\prime}$-amino- $\alpha$-GalCer 51 was detected (m/z calcd. for $\left[\mathrm{C}_{50} \mathrm{H}_{100} \mathrm{~N}_{2} \mathrm{O}_{8}+\mathrm{H}\right]^{+}:$857.7552, obsd.: 857.7533) along with an unexpected $\mathrm{m} / \mathrm{z}$ of 869.7521 . Upon careful analysis, this was attributed to the formation of $\underline{c i s}$-decalin $\mathbf{E}$ (Scheme 3.7) due to the presence of trace formaldehyde present in the methanol that was used to wash, filter and transfer the glycolipid (m/z calcd. for $\left[\mathrm{C}_{51} \mathrm{H}_{100} \mathrm{~N}_{2} \mathrm{O}_{8}+\mathrm{H}\right]^{+}:$869.7552, obsd.: 869.7521). The mechanism for the formation of cis-decalin $\mathbf{E}$ is depicted in Scheme 3.7, whereby the amine $\mathbf{5 1}$ attacks a molecule of formaldehyde to form alcohol $\mathbf{B}$, which upon 
elimination of water gives the iminium intermediate D. Subsequent attack of the 4-OH on the iminium carbon followed by the loss of $\mathrm{H}^{+}$generates the cis-decalin by-product $\mathbf{E}$.

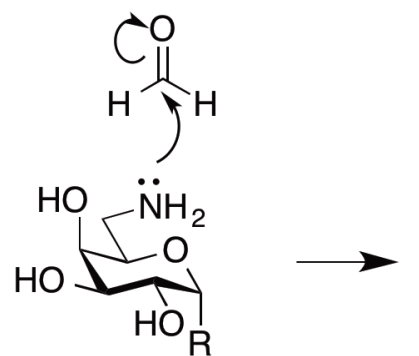

51

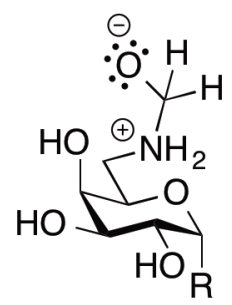

A

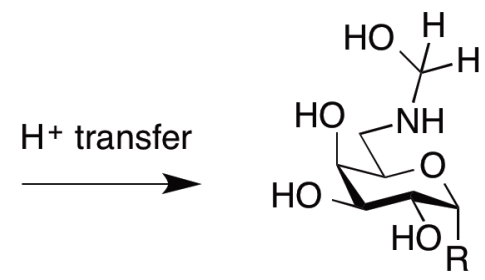

B

$\left[\mathrm{C}_{50} \mathrm{H}_{100} \mathrm{~N}_{2} \mathrm{O}_{8}+\mathrm{H}\right]^{+}$

$\mathrm{m} / \mathrm{z}$ calcd.: 857.7552

$\mathrm{m} / \mathrm{z}$ obsd.: 857.7533<smiles>[R]C1OC2CNC(CO2)C(O)C1O</smiles>

E

$\left[\mathrm{C}_{51} \mathrm{H}_{100} \mathrm{~N}_{2} \mathrm{O}_{8}+\mathrm{H}\right]^{+}$

$\mathrm{m} / \mathrm{z}$ calcd.: 869.7552

$\mathrm{m} / \mathrm{z}$ obsd.: 869.7521

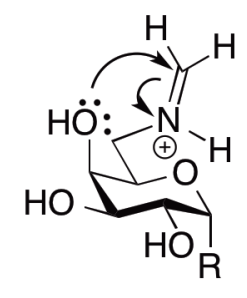

D

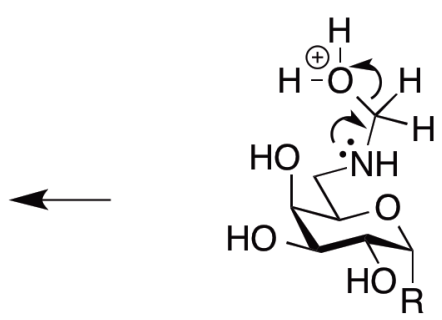

C

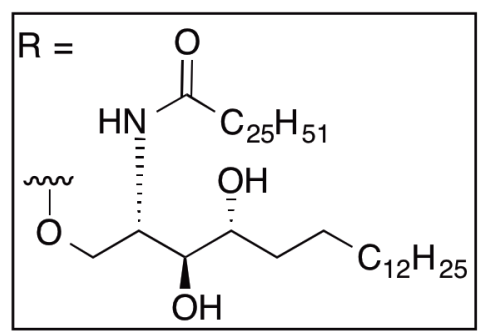

Scheme 3.7. Mechanism for the formation of cis-decalin $\mathbf{E}$ by-product.

In an attempt to regenerate the free amine (ie. to hydrolyse the cis-decalin $\mathbf{E}$ ), the crude mixture was co-evaporated with $1 \mathrm{M} \mathrm{HCl}$ (aq). Though somewhat successful, unfortunately, the reaction did not go to completion. Careful purification of the mixture of amine $\mathbf{5 1}$ and cis-decalin $\mathbf{E}$ by silica gel column chromatography using a solvent system of DCM, $\mathrm{MeOH}, \mathrm{EtOH}$ and $\mathrm{NH}_{3}$ was also unsuccessful and instead caused the formation of ammonium chloride salt when 
the ammonia in the eluting solvent reacted with trace $\mathrm{HCl}$ from the hydrolysis step. Efforts to separate the salt from the glycolipid by reverse phase column chromatography were futile as the glycolipid was insoluble in water and had to be loaded onto the column with a few drops of pyridine, which caused the product to be eluted together with the salts. Removal of the salts by dialysis was also attempted, but again proved unviable because the glycolipid was only soluble in a mixture of $\mathrm{DCM} / \mathrm{MeOH} /$ water $(2 / 1 / 5, \mathrm{v} / \mathrm{v} / \mathrm{v})$, which was not compatible with the cellulose dialysis tube. Fortunately, most of the ammonium chloride was successfully removed by crystallisation from a mixture of warm $30 \% \mathrm{MeOH}$ in DCM and filtered. Due to the challenges in obtaining pure 6 -amino- $\alpha$-GalCer (ie. without salts), it was decided that the semi-pure mixture would be used for the subsequent coupling to BODIPY-COOH 52 and the yield for conversion of diol 69 to fully deprotected amine $\mathbf{5 1}$ was estimated at 33\%. The identity of amine $\mathbf{5 1}$ was confirmed by HRMS and the ${ }^{1} \mathrm{H}$ NMR data matched that reported by Zhou et $a l{ }^{2}$ The synthetic approach used here is similar to Zhou et al.'s strategy whereby a galactosyl donor is coupled to the complete ceramide acceptor leading to the generation of the fully deprotected $6^{\prime}$-amino- $\alpha$-GalCer 51 and subsequent functionalisation with a fluorophore. However, the protecting group strategy used in the synthesis reported herein allowed for the simultaneous removal of benzyl protecting groups and azide reduction leading to the generation of amine $\mathbf{5 1}$ in comparable overall yield of $21 \%$ over 3 steps from the ceramide lipid acceptor. In contrast, Zhou et al. reported an overall yield of 19\% over 4 steps from the ceramide acceptor. ${ }^{2}$

Next, amine 51 was coupled to BODIPY-COOH 52 using HBTU as the coupling agent (Scheme 3.6). The reaction appeared promising based on TLC analysis, however, to obtain BODIPY- $\alpha$-GalCer 3 of sufficient purity and free from the coupling reagents and by-products, repeated purification by silica gel flash column chromatography was required. Unfortunately, all attempts to purify BODIPY- $\alpha$-GalCer 3 proved futile. Indeed, the more time taken to purify the desired product, the more decomposition was observed. 
As a fluorescent dye, BODIPY has many advantages including its high quantum yield and relatively small size. ${ }^{1}$ It is also fairly inert, although some decomposition of the dye has been observed under rather harsh photo-oxidation conditions. For example, some early analyses of the dye in the 1970's revealed that complete fragmention can occur when a slow stream of oxygen is allowed to bubble through a solution of 3,5,3',5'-tetramethyl-4,4'-diethyldipyrrylmethene exposed to photolysis under a halogen lamp (500W). ${ }^{26,27}$ Similar photo-oxidation was proposed to occur on boron-containing dipyrrylmethenes $\mathbf{F}$ (Scheme 3.8) ${ }^{28}$ Here, addition of a singlet oxygen species across the double bond at C-7a and C-8 of the BODIPY core gave dioxetanes $\mathbf{G}$, which subsequently broke down to the ring opened intermediates $\mathbf{H}$, which upon hydrolysis of the boron-nitrogen bonds produce dimethylketopyrroles $\mathbf{I}$ and ketones $\mathbf{K}$. Tautomerisation of dimethylketopyrroles $\mathbf{I}$ then leads to the formation of lactam $\mathbf{J}$, while further oxidation of ketone $\mathbf{K}$ produces 3-methylmaleimide $\mathbf{M}$ via dealkylation of the endoperoxide intermediate $\mathbf{L} .^{28,29}$ 


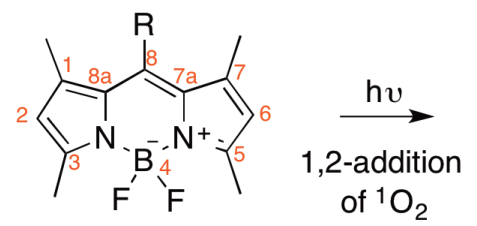

$\mathbf{F}$

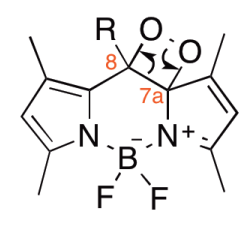

G<smiles></smiles>

H<smiles>C=C1C=C(C)C(=O)NC1=O</smiles>

$\mathbf{J}$<smiles>[R]C(=O)c1[nH]c([X])cc1C</smiles>

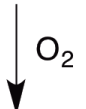

$\mathrm{R}$

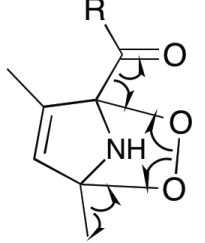

$\mathbf{L}$<smiles>CC1=CC(=O)NC1=O</smiles>

M

Scheme 3.8. Proposed mechanism of photo-oxidation of BODIPY core following photolysis with halogen lamp and $\mathrm{O}_{2}$ stream. ${ }^{28}$

The degradation of the BODIPY dye under more ambient conditions, however, has not been previously reported, yet TLC, HRMS and ${ }^{1} \mathrm{H}$ NMR analysis of the product mixture following the repeated purification of BODIPY- $\alpha$-GalCer 3 revealed the presence of several species. Given the small quantities of material available, it was difficult to conclusively prove the structures of the degradation products, however, by TLC, all products had very similar $\mathrm{R}_{f}$ values. Notably, analysis by HRMS revealed two by-products containing either one or two chlorine atoms, as evidenced by characteristic isotopic distributions arising from ${ }^{35} \mathrm{Cl}$ and ${ }^{37} \mathrm{Cl}$ atoms. Further analysis revealed the $[\mathrm{M}+\mathrm{H}]^{+}$peak of 1241.8541 , which 
correlated to the calculated value of 1241.8565 for $\mathrm{C}_{70} \mathrm{H}_{117} \mathrm{BClF}_{2} \mathrm{~N}_{4} \mathrm{O}_{9}{ }^{+}$. Thus, it has been tentatively proposed that BODIPY- $\alpha$-GalCer 3 undergoes photooxidation with subsequent addition of $\mathrm{Cl}^{-}$, which is formed from the decomposition of the NMR solvent $\mathrm{CDCl}_{3}$, to yield the cholorinated adduct $\mathbf{Q}$ (Scheme 3.9). In this proposed mechanism, a singlet oxygen species is added across C-3 and C-8a of the BODIPY core to give endoperoxide $\mathbf{N}$, which subsequently breaks down to give adduct $\mathbf{O}$ that is set up for attack by $\mathrm{Cl}^{-}$at the 2position. Loss of $\mathrm{H}_{2} \mathrm{O}_{2}$ then reinstates the aromaticity of the BODIPY core to produce the chlorinated BODIPY- $\alpha$-GalCer $\mathbf{Q}$. While there is a possibility of chlorination at the 3- and 5- methyl carbons of the BODIPY core, this is unlikely to be the by-product in this case because halogenation at the 3-and 5-positions typically occurs in the presence of a cationic halogen, such as $N$ bromosuccinimide, to form a 3-brominated BODIPY core. ${ }^{30}$ 


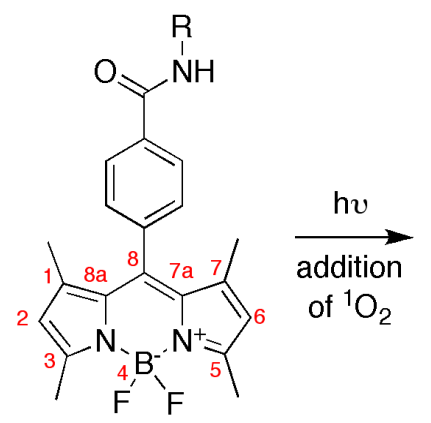

3

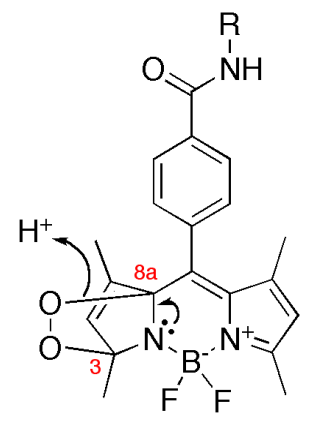

$\mathbf{N}$

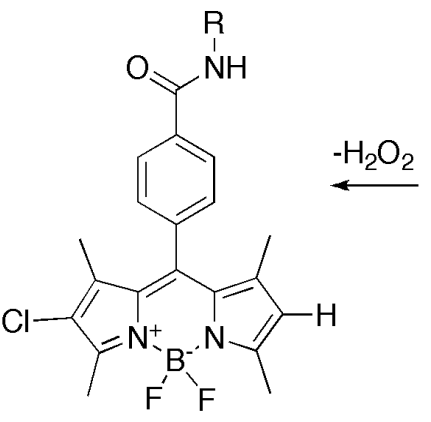

Q

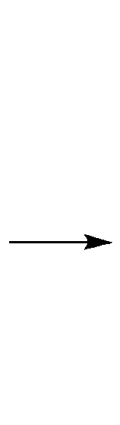

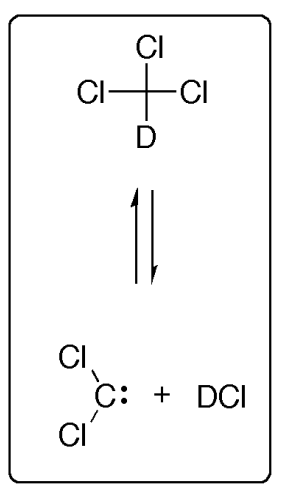

(1)

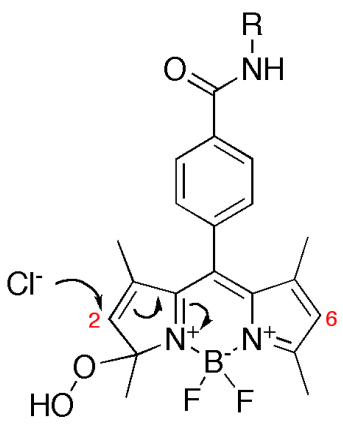

O

$\mathrm{HO}$

$$
\downarrow " \mathrm{Cl}^{-"}
$$<smiles>[R]NC(=O)c1ccc(-c2c3c(C)cc(C)[n+]-3p(F)n2C(C)(O)C(O)(O)Cl)cc1</smiles>

$\mathbf{P}$

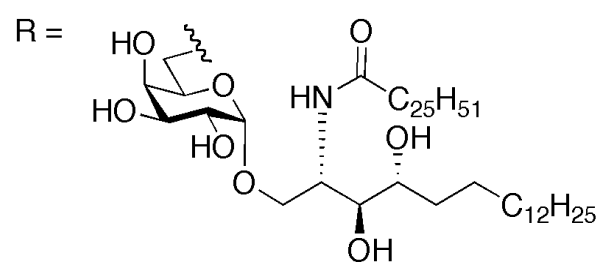

Scheme 3.9. Proposed mechanism for the formation of chlorinated BODIPY- $\alpha-$ GalCer $\mathbf{Q}$.

To support the assumption that the BODIPY group can be oxidized under relatively mild conditions, a small sample of the dye itself, BODIPY-COOH 52, was exposed to UV light (12 Watt lamp) for 10 minutes. Analysis by HRMS once again revealed an oxidised product $\left(\mathrm{m} / \mathrm{z}\right.$ calcd. for $\left[\mathrm{C}_{20} \mathrm{H}_{18} \mathrm{BF}_{2} \mathrm{~N}_{2} \mathrm{O}_{3}\right]+: 383.1373$, obsd.: 383.1385). A mechanism, similar to that proposed for the chlorination adduct $\mathbf{Q}$, is proposed to explain the formation of this product, whereby attack of $\mathrm{H}_{2} \mathrm{O}$ in intermediate $\mathbf{O}$ leads to an alcohol with net addition of atomic oxygen to the BODIPY core. 
In addition to the oxidised chlorinated adduct, which was determined by HRMS, ${ }^{1} \mathrm{H}$ NMR of the reaction mixture of BODIPY- $\alpha$-GalCer 3 after repeated purification also revealed that the proton signal belonging to H-2 and H-6 of the pyrrole ring was significantly reduced compared to the other signals. Upon analysis by mass spectrometry, two masses corresponding to the substitution of one and two hydrogen(s) with deuterium(s) were detected (Scheme 3.10). This increase in mass came about due to the insolubility of the BODIPY- $\alpha$-GalCer 3 in $\mathrm{CDCl}_{3}$ alone, which necessitated the need for a mixture of $10 \%$ deuterated methanol $\left(\mathrm{CD}_{3} \mathrm{OD}\right)$ in $\mathrm{CDCl}_{3}$ for $\mathrm{NMR}$ analyses. Electrophilic aromatic subsitution is most likely to occur at the 2- and 6- positions of the BODIPY core, giving rise to deuteration at one $(\rightarrow \mathbf{S})$ or both $(\rightarrow \mathbf{T})$ of these sites. The protic nature of $\mathrm{CD}_{3} \mathrm{OD}$ means that a $\mathrm{D}^{+}$is a readily available electrophile. Accordingly, it was thus determined that non-protic deuterated solvents, such as pyridine- $\mathrm{d}_{5}$, should be used as the NMR solvent for future analyses.

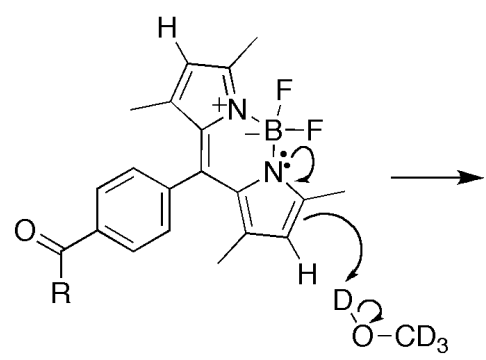

3

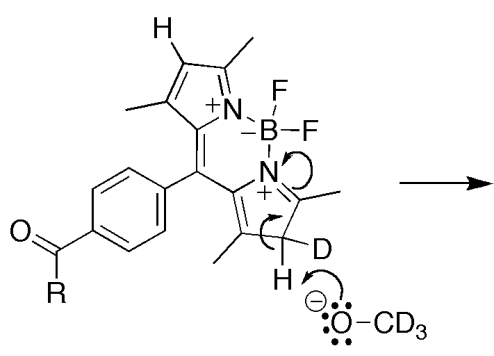

$\mathbf{R}$<smiles>[2H]C(=O)c1ccc(C2=C(C)C(C)=C(C)C2=[N+]2C(C)=C(C)C(C)=C2F)cc1</smiles>

$\mathbf{S}$

$\mathrm{C}_{70} \mathrm{H}_{177} \mathrm{DBF}_{2} \mathrm{~N}_{4} \mathrm{O}_{9}{ }^{+}$ $\mathrm{m} / \mathrm{z}$ calcd.: 1208.9017 $\mathrm{m} / \mathrm{z}$ obsd.: 1208.8995

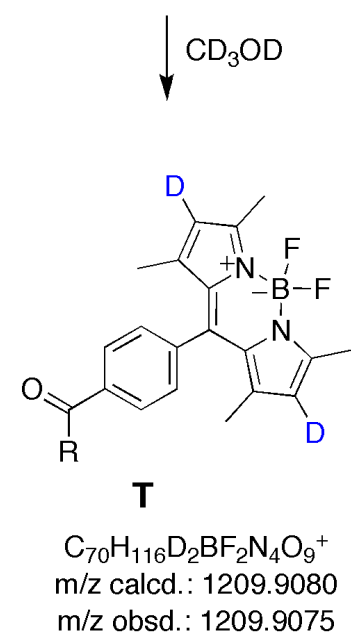

Scheme 3.10. Deuteration of BODIPY- $\alpha-G a l C e r 3$. 
To circumvent the aforementioned issues and to develop a BODIPY dye that would allow for easier purficiation (and thus limit exposure to light and oxygen), BODIPY-COOH 52 was derivatised as the $N$-hydroxysuccimidyl ester $\mathbf{7 0}$ (Scheme 3.11). In this way, only a base, such as DiPEA, would need to be added to the reaction vessel, while the cleaved $N$-hydroxysuccinimide could be more readily separated from the desired BODIPY- $\alpha-$ GalCer 3 . Indeed, the coupling of the amine 51 with BODIPY-NHS 70 proceeded smoothly and the product could be readily purified by silica gel flash column chromatography (gradient from 1\% to $3 \% \mathrm{MeOH}$ in DCM) to afford BODIPY- $\alpha$-GalCer 3 as a red solid in $44 \%$ yield. This compromised yield is largely attributed to the loss of material when purifying the fully deprotected amine $\mathbf{5 1}$, as the $44 \%$ yield obtained is comparable to amide couplings performed on the $6^{\prime}$ position of the $\alpha$-GalCer which typically range from 33-53\% yield. ${ }^{2,3}$ Unfortunately, limited quantities of fully protected $6^{\prime}$ azido- $\alpha$-GalCer 55 prevented further optimisation of the last two steps of this route. Nonetheless, enough quantity of the final target BODIPY- $\alpha-$ GalCer 3 was obtained for the purpose of characterisation. The identity of the BODIPY- $\alpha-$ GalCer 3 was confirmed by ${ }^{1} \mathrm{H}$ and ${ }^{13} \mathrm{C}$ NMR and mass spectrometry analysis $\left(\mathrm{m} / \mathrm{z}\right.$ calcd. for $\left[\mathrm{C}_{70} \mathrm{H}_{117} \mathrm{BF}_{2} \mathrm{~N}_{4} \mathrm{O}_{9}+\mathrm{H}\right]^{+}:$1207.8954, obsd.: 1207.8958), including the characteristic three-bond correlation (HMBC) between H-6' and carbonyl carbon of BODIPY amide, and there was no evidence of oxidized, chlorinated or deuterated by-products by NMR or HRMS. BODIPY- $\alpha$-GalCer $\mathbf{3}$ has subsequently been sent to collaborators Dr. Hans van der Vliet and co-workers for use as a fluorescent probe to study DC processing of $\alpha$-GalCer, and also to determine the loading of glycolipids on tumour cells. 


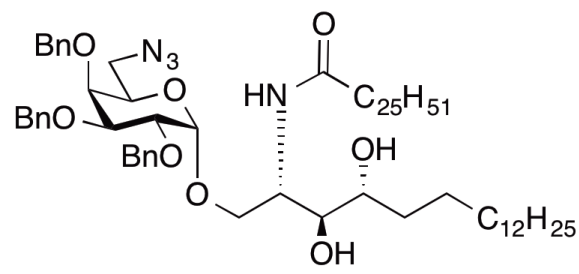

69

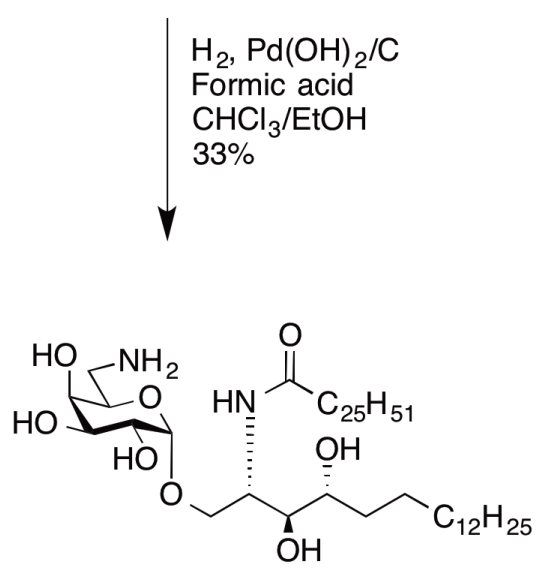

51

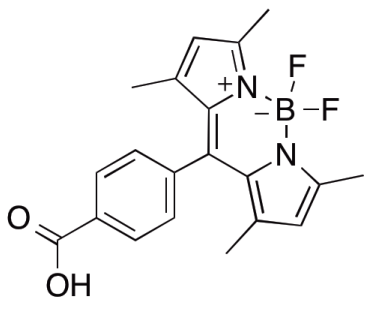

52

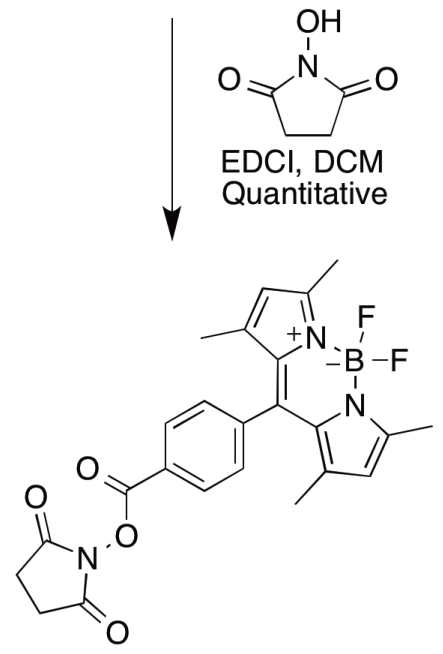

70

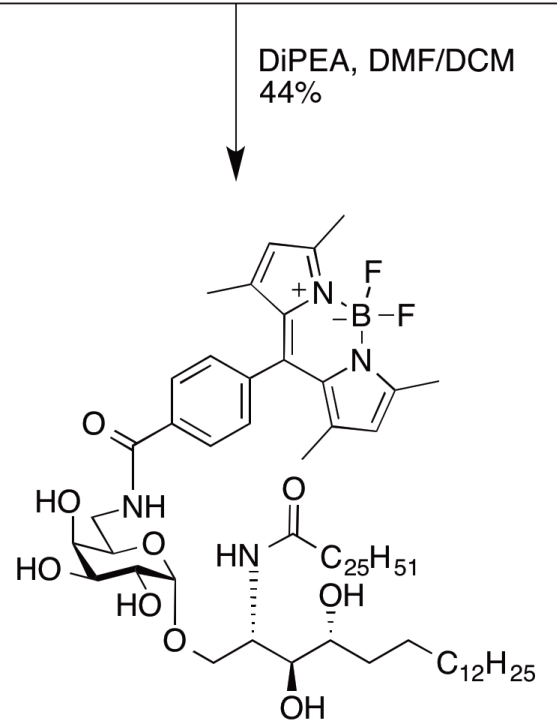

3

Scheme 3.11. Synthesis of BODIPY- $\alpha$-GalCer 3 using BODIPY-NHS 70. 


\subsection{Conclusion}

In conclusion BODIPY- $\alpha$-GalCer 3 was successfully synthesised from the fully deprotected amine 51 and BODIPY-NHS 70. It was discovered that the solubility of the ceramide lipid acceptor $\mathbf{5 7}$ was crucial for its reactivity in the glycosylation with imidate donor 56. Though the glycosylation yield obtained when using the $\mathrm{N}$-acylated ceramide acceptor is generally lower compared to the yields when the phytosphingosine acceptor, remarkably a good $68 \%$ yield of the desired $\alpha$-anomer was observed. Moreover, the synthetic strategy employed here is more convergent and provides the fully deprotected glycolipid $\mathbf{5 1}$ bearing a reactive amine on the 6 '-position that can be readily derivatised. The main challenge, however, is the purification of the amine glycolipid 51, of which can be optimised with the availability of more of the fully protected glycolipid 55. In the course of purification of the final product, BODIPY- $\alpha$-GalCer 3, limitations due to the sensitivity of the dye to photo-oxidation were also discovered. Indeed, this is an area that is currently undergoing further investigation in the group to provide more conclusive evidence for the oxidation products observed. BODIPY- $\alpha-$ GalCer 3 has been sent to collaborators who will use the probe to study the role of $\alpha$-GalCer in cancer immunotherapy in more depth.

\subsection{Experimental}

\section{General procedure}

Unless otherwise stated all reactions were performed under $\mathrm{N}_{2}$. Prior to use, THF (Pancreac) was distilled from sodium and benzophenone, pyridine was distilled and dried over $4 \AA \AA$ molecular sieves (4Å MS), DCM (Pancreac) was distilled from $\mathrm{P}_{2} \mathrm{O}_{5}$, and $\mathrm{H}_{2} \mathrm{O}$ and benzene (Fisher Scientific) were distilled. Trityl chloride (Acros), anhydrous $\mathrm{Et}_{2} \mathrm{O}$ (Pancreac), $\mathrm{PPh}_{3}$ (Merck), $\mathrm{Pd}(\mathrm{OH})_{2} / \mathrm{C}$ (Aldrich, 20 $\mathrm{wt} \%$ ), anhydrous DMF (Acros), TFA (Aldrich), trichloroacetonitrile (Aldrich), triethylsilane (Fluka), TMSOTf (Aldrich), $\mathrm{H}_{2} \mathrm{SO}_{4}$ (Lab-Scan), Formic acid (Aldrich), $\mathrm{AcCl}(\mathrm{B} \& \mathrm{M}), \mathrm{BnBr}$ (Fluka), $\mathrm{PMe}_{3}$ (Aldrich, 1M in THF), AcOH (Ajax Finechem), $\mathrm{Ac}_{2} \mathrm{O}$ (Peking Reagent), TMSOTf (Aldrich), DiPEA (Aldrich), 
$\mathrm{NaOMe}$ (Janssen Chimica), phytosphingosine (TCI), $\mathrm{C}_{25} \mathrm{H}_{51} \mathrm{COOH}$ (Acros), $\mathrm{BzCl}$ (Aldrich, distilled an stored under argon), HBTU (Acros), PyBOP (Aldrich), EDCI (Aldrich), DMAP (Merck), sodium (Aldrich), $\mathrm{Pd}(\mathrm{OH})_{2} / \mathrm{C}$ (Aldrich, 20 $w t \%)$, EtOAc (Pancreac), hexanes (Fisher Scientific), petroleum ether (Pure Science), $\mathrm{MeOH}$ (Pure Science), $\mathrm{CHCl}_{3}$ (Pancreac), EtOH (absolute, Pure Science), $\mathrm{NaOH}$ (Pure Science), $\mathrm{NaHCO}_{3}$ (Pure Science), $\mathrm{NaCl}$ (Pancreac) were used as received. All solvents were removed by evaporation under reduced pressure. Reactions were monitored by TLC-analysis on Macherey-Nagel silica gel coated plastic sheets $\left(0.20 \mathrm{~mm}\right.$, with fluorescent indicator $\left.\mathrm{UV}_{254}\right)$ with detection by UV-absorption (short wave UV - $254 \mathrm{~nm}$; long wave UV - $366 \mathrm{~nm}$ ), by dipping in $10 \% \mathrm{H}_{2} \mathrm{SO}_{4}$ in $\mathrm{EtOH}$ followed by charring at $\sim 150{ }^{\circ} \mathrm{C}$, by dipping in $\mathrm{I}_{2}$ in silica, or by dipping into a solution of ninhydrin in EtOH followed by charring at $\sim 150{ }^{\circ} \mathrm{C}$. Column chromatography was performed on Pure Science silica gel (40-63 micron). AccuBOND II ODS-C18 (Agilent) was used for reverse phase chromatography. Sephadex ${ }^{\circledR}$ LH-20 (Aldrich) was used for size exclusion chromatography. Infrared spectra were recorded as thin films using a Bruker Tensor 27 FTIR spectrometer equipped with an Attenuated Total Reflectance (ATR) sampling accessory and are reported in wave numbers $\left(\mathrm{cm}^{-1}\right)$. Nuclear magnetic resonance spectra were recorded at $20{ }^{\circ} \mathrm{C}$ in $\mathrm{CD}_{3} \mathrm{OD}, \mathrm{CDCl}_{3}$, or pyridine-d5 using either a Varian INOVA operating at $500 \mathrm{MHz}$ or Varian

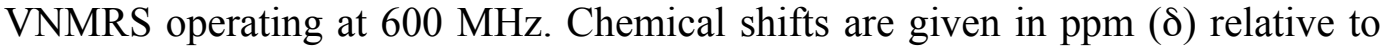
TMS. NMR peak assignments were made using COSY, HSQC and HMBC 2D experiments.

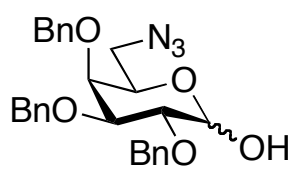

\section{6-Azido-2,3,4-tri-O-benzyl-6-deoxy- $\alpha$-D-galactose (59)}

Methyl 6-azido-2,3,4-tri-O-benzyl-6-deoxy- $\alpha$-D-galactoside $\mathbf{4 5}^{2,9}$ (360 $\mathrm{mg}, 0.74 \mathrm{mmol}$ ) was dissolved in aqueous acetic acid $(11 \mathrm{~mL}, 80 \% \mathrm{v} / \mathrm{v})$ in water followed by the addition of $1 \mathrm{M} \mathrm{H}_{2} \mathrm{SO}_{4}(2.72 \mathrm{~mL}$, $2.72 \mathrm{mmol}$ ). The reaction was stirred at $100{ }^{\circ} \mathrm{C}$ for $4.5 \mathrm{~h}$, then $120^{\circ} \mathrm{C}$ for a further $1.5 \mathrm{~h}$. The reaction mixture was poured into ice water and extracted with EtOAc $(2 \times 50 \mathrm{~mL})$ and the combined organic layers were washed with water $(100 \mathrm{~mL})$, saturated $\mathrm{NaHCO}_{3}$ solution $(2 \times 100 \mathrm{~mL})$ and brine $(100 \mathrm{~mL})$, dried $\left(\mathrm{MgSO}_{4}\right)$, filtered and concentrated in vacuo. The residue was purified by silica gel flash 
column chromatography and eluted with $5: 1(\mathrm{v} / \mathrm{v})$ petroleum ether/EtOAc to afford lactol 59 (219 mg, $0.46 \mathrm{mmol}, 63 \%)$ as a clear oil. $\mathrm{R}_{f}: 0.43$ (PE/EA, 2:1, $\mathrm{v} / \mathrm{v}) ;[\alpha]_{\mathrm{D}}{ }^{25}=+9.0^{\circ}\left(\mathrm{c}=1.0, \mathrm{CHCl}_{3}\right)$; IR (film) 3410, 3064, 3031, 2871, 2101, 1497, 1454, 1362, 1281, 1214, 1062, 1027, 912, 736, $697 \mathrm{~cm}^{-1} ;{ }^{1} \mathrm{H}$ NMR (500 $\left.\mathrm{MHz}, \mathrm{CDCl}_{3}\right) \delta 7.45-7.31\left(\mathrm{~m}, 15 \mathrm{H}, \mathrm{H}_{\text {arom }}\right), 5.31$ (t, $\left.J_{1,2}=J_{1, \mathrm{OH}}=2.6 \mathrm{~Hz}, 1 \mathrm{H}, \mathrm{H}-1\right)$, $5.03\left(\mathrm{~d}, J_{\mathrm{a}, \mathrm{b}}=11.2 \mathrm{~Hz}, 1 \mathrm{H}, \mathrm{CH}-\mathrm{a}, 4-O-\mathrm{Bn}\right), 4.88\left(\mathrm{~d}, J_{\mathrm{a}, \mathrm{b}}=12.0 \mathrm{~Hz}, 1 \mathrm{H}, \mathrm{CH}-\mathrm{a}, 2-\right.$ $O-\mathrm{Bn}), 4.86\left(\mathrm{~d}, J_{\mathrm{a}, \mathrm{b}}=11.8 \mathrm{~Hz}, 1 \mathrm{H}, \mathrm{CH}-\mathrm{a}, 3-O-\mathrm{Bn}\right), 4.81\left(\mathrm{~d}, J_{\mathrm{a}, \mathrm{b}}=12.0 \mathrm{~Hz}, 1 \mathrm{H}\right.$, CH-b, 2-O-Bn), 4.74 (d, $\left.J_{\mathrm{a}, \mathrm{b}}=11.8 \mathrm{~Hz}, 1 \mathrm{H}, \mathrm{CH}-\mathrm{b}, 3-O-\mathrm{Bn}\right), 4.64\left(\mathrm{~d}, J_{\mathrm{a}, \mathrm{b}}=11.2\right.$ $\mathrm{Hz}, 1 \mathrm{H}, \mathrm{CH}-\mathrm{b}, 4-O-\mathrm{Bn}), 4.08-4.06$ (m, 2H, H-2, H-5), 3.97 (dd, $J_{2,3}=9.9 \mathrm{~Hz}, J_{3,4}$ $=2.6 \mathrm{~Hz}, 1 \mathrm{H}, \mathrm{H}-3), 3.86(\mathrm{bs}, 1 \mathrm{H}, \mathrm{H}-4), 3.53\left(\mathrm{dd}, J_{6 \mathrm{a}, 6 \mathrm{~b}}=12.5 \mathrm{~Hz}, J_{5,6 \mathrm{a}}=7.6 \mathrm{~Hz}\right.$, $1 \mathrm{H}, \mathrm{H}-6 \mathrm{a}), 3.33\left(\mathrm{~d}, J_{1, \mathrm{OH}}=2.6 \mathrm{~Hz}, 1 \mathrm{H}, \mathrm{OH}\right), 3.13\left(\mathrm{dd}, J_{6 \mathrm{a}, 6 \mathrm{~b}}=12.5 \mathrm{~Hz}, J_{5,6 \mathrm{~b}}=5.9\right.$ $\mathrm{Hz}, 1 \mathrm{H}, \mathrm{H}-6 \mathrm{~b}) ;{ }^{13} \mathrm{C} \mathrm{NMR}\left(125 \mathrm{MHz}, \mathrm{CDCl}_{3}\right) \delta 138.5$ (C-i, 2-O-Bn), 138.2 (C-i, 4$O-\mathrm{Bn}), 138.1$ (C-i, 3-O-Bn), 128.6, 128.53, 128.47, 128.2, 128.1, 128.0, 127.8, 127.6 (9 x C $\mathrm{arom}_{\text {ar }}, 91.9$ (C-1), 78.6 (C-3), 76.5 (C-2), 74.74 (C-4), $74.67\left(\mathrm{CH}_{2}, 4-\right.$ $O-\mathrm{Bn}), 73.6\left(\mathrm{CH}_{2}, 2-O-\mathrm{Bn}\right), 73.3\left(\mathrm{CH}_{2}, 3-\mathrm{O}-\mathrm{Bn}\right), 69.7(\mathrm{C}-5), 51.2(\mathrm{C}-6)$; HRMS(ESI) m/z calcd. for $\left[\mathrm{C}_{27} \mathrm{H}_{29} \mathrm{~N}_{3} \mathrm{O}_{5}+\mathrm{Na}\right]^{+}:$498.1999, obsd.: 498.2005.

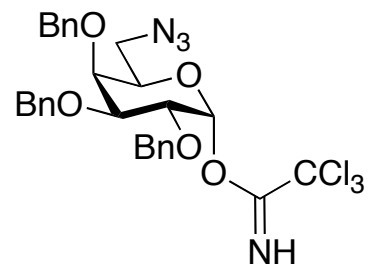

$O$-(6-Azido-2,3,4-tri-O-benzyl-6-deoxy- $\alpha$-D-galactosyl) trichloroacetimidate (56)

Trichloroacetonitrile $(448 \mu \mathrm{L}, 4.47 \mathrm{mmol})$ and DBU (100 $\mu \mathrm{L}, 0.67 \mathrm{mmol})$ were added to lactol $59(213 \mathrm{mg}, 0.45$ mmol) (co-evaporated with toluene x 3) in dry $\mathrm{CH}_{2} \mathrm{Cl}_{2}(5 \mathrm{~mL})$. After stirring for $1.5 \mathrm{~h}$ at $\mathrm{rt}$, the reaction mixture was concentrated in vacuo and purified by silica gel flash column chromatography (silica gel was pre-washed with $1 \%$ triethylamine in 20:1 v/v petroleum ether/EtOAc). Elution with 20:1 (v/v) petroleum ether/EtOAc afforded imidate 56 (246 $\mathrm{mg}, 0.40 \mathrm{mmol}, 89 \%)$ as a clear oil. $\mathrm{R}_{f}$ : $0.62(\mathrm{PE} / \mathrm{EA}, 2: 1, \mathrm{v} / \mathrm{v}) ;[\alpha]_{\mathrm{D}}{ }^{24}=+34.0^{\circ}\left(\mathrm{c}=1.0, \mathrm{CHCl}_{3}\right)$; IR (film) 3338, 3064, 3031, 2916, 2101, 1732, 1672, 1497, 1454, 1351, 1287, 1107, 1071, 1027, 838, 794, 736, $697 \mathrm{~cm}^{-1} ;{ }^{1} \mathrm{H}$ NMR $\left(500 \mathrm{MHz}, \mathrm{CDCl}_{3}\right) \delta 8.61(\mathrm{~s}, 1 \mathrm{H}, \mathrm{NH}), 7.41-$ $7.29\left(\mathrm{~m}, 15 \mathrm{H}, \mathrm{H}_{\text {arom }}\right), 6.56\left(\mathrm{~d}, J_{1,2}=3.3 \mathrm{~Hz}, 1 \mathrm{H}, \mathrm{H}-1\right), 5.06\left(\mathrm{~d}, J_{\mathrm{a}, \mathrm{b}}=11.3 \mathrm{~Hz}, 1 \mathrm{H}\right.$, CH-a, 4-O-Bn), 4.91 (d, $\left.J_{\mathrm{a}, \mathrm{b}}=11.8 \mathrm{~Hz}, 1 \mathrm{H}, \mathrm{CH}-\mathrm{a}, 3-O-\mathrm{Bn}\right), 4.81\left(\mathrm{~d}, J_{\mathrm{a}, \mathrm{b}}=11.8 \mathrm{~Hz}\right.$, $1 \mathrm{H}, \mathrm{CH}-\mathrm{b}, 3-\mathrm{O}-\mathrm{Bn}$ ), 4.78 (bs, 2H, CH-a, CH-b, 2-O-Bn), 4.65 (d, $J_{\mathrm{a}, \mathrm{b}}=11.3 \mathrm{~Hz}$, $1 \mathrm{H}, \mathrm{CH}-\mathrm{b}, 4-\mathrm{O}-\mathrm{Bn}), 4.27$ (dd, $\left.J_{2,3}=10.0 \mathrm{~Hz}, J_{1,2}=3.3 \mathrm{~Hz}, 1 \mathrm{H}, \mathrm{H}-2\right), 4.06-4.05$ 
(m, 2H, H-3, H-5), 3.92 (bs, 1H, H-4), 3.53 (dd, $J_{6 \mathrm{a}, 6 \mathrm{~b}}=12.6 \mathrm{~Hz}, J_{5,6 \mathrm{a}}=7.4 \mathrm{~Hz}$, $1 \mathrm{H}, \mathrm{H}-6 \mathrm{a}), 3.14$ (dd, $\left.J_{6 \mathrm{a}, 6 \mathrm{~b}}=12.6 \mathrm{~Hz}, J_{5,6 \mathrm{~b}}=5.8 \mathrm{~Hz}, 1 \mathrm{H}, \mathrm{H}-6 \mathrm{~b}\right) ;{ }^{13} \mathrm{C}$ NMR $(125$ $\left.\mathrm{MHz}, \mathrm{CDCl}_{3}\right) \delta 161.1(\underline{\mathrm{C}}=\mathrm{NH}), 138.4$ (C-i, 3-O-Bn), 138.3 (C-i, 2-O-Bn), 138.1 (C-i, 4-O-Bn), 128.54, 128.52, 128.51 (3 x C-m, 2-O-Bn, 3-O-Bn, 4- $O-\mathrm{Bn}$ ), 128.4 (C-o, 4-O-Bn), 127.8 (C-m, 3-O-Bn), 127.6 (C-m, 2-O-Bn), 128.1, 127.9, 127.7 (3 x C-p, 2-O-Bn, 3-O-Bn, 4-O-Bn), 94.9 (C-1), 78.0 (C-3), 75.8 (C-2), $74.9\left(\mathrm{CH}_{2}\right.$, 4-O-Bn), $74.7(\mathrm{C}-4), 73.6\left(\mathrm{CH}_{2}, 3-O-\mathrm{Bn}\right), 73.1\left(\mathrm{CH}_{2}, 2-O-\mathrm{Bn}\right), 72.4(\mathrm{C}-5), 50.9$ (C-6); HRMS(ESI) $\mathrm{m} / \mathrm{z}$ calcd. for $\left[\mathrm{C}_{29} \mathrm{H}_{29} \mathrm{~N}_{4} \mathrm{O}_{5} \mathrm{Cl}_{3}+\mathrm{Na}\right]^{+}$: 641.1096, obsd.: 641.1113.

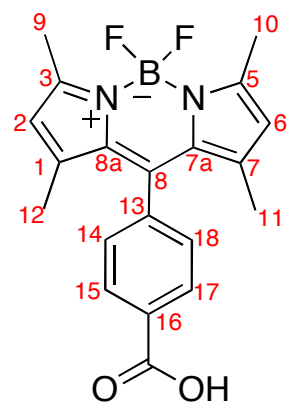

4-(4,4-Difluoro-1,3,5,7-tetramethyl-3a,4a-diaza-4-bora-sindacen-8-yl)benzoic acid (52)

To a solution of the methyl ester $\mathbf{6 3}^{11}(0.148 \mathrm{~g}, 0.387 \mathrm{mmol})$ in isopropanol $(15 \mathrm{~mL})$ under an argon atmosphere, an aqueous solution of $0.1 \mathrm{M} \mathrm{KOH}(15 \mathrm{~mL})$ was added. The reaction mixture was stirred at room temperature until complete consumption of $\mathbf{6 3}$ by TLC analysis was observed. The reaction mixture was then acidified with $\mathrm{H}^{+}$dowex until $\mathrm{pH}=2-3$, filtered and evaporated to dryness in vacuo. The residue was purified by silica gel gradient column chromatography $\left(\mathrm{CH}_{2} \mathrm{Cl}_{2} / \mathrm{MeOH}, 99: 1\right.$ to $\left.96: 4, \mathrm{v} / \mathrm{v}\right)$ to afford the BODIPY-COOH $52(0.142 \mathrm{~g}$, $0.387 \mathrm{mmol}$, quantitative). The obtained red-brown solid is stored in the dark. Mp 182.5-182.8 ${ }^{\circ} \mathrm{C} ; \mathrm{R}_{f}=0.74(\mathrm{MeOH} /$ EtOAc, 1/4, v/v); IR (film) 2924, 1723, 1544, 1510, 1309, 1194, 1157, 983, $739 \mathrm{~cm}^{-1} ;{ }^{1} \mathrm{H}$ NMR (500 MHz, $\left.\mathrm{CDCl}_{3}\right) \delta 8.25(\mathrm{~d}$, $\left.J_{14,15}=J_{17,18}=8.5 \mathrm{~Hz}, 8.2 \mathrm{~Hz}, 2 \mathrm{H}, \mathrm{H}-15, \mathrm{H}-17\right), 7.44\left(\mathrm{~d}, J_{14,15}=J_{17,18}=8.2,2 \mathrm{H}\right.$, H-14, H-18), 6.01 (s, 2H, H-2, H-6), 2.56 (s, 6H, H-9, H-10), 1.37 (s, 6H, H-11, $\mathrm{H}-12) ;{ }^{13} \mathrm{C}$ NMR (125 MHz, $\mathrm{CDCl}_{3}$ ) $\delta 130.9$ (C-15, C-17), 128.5 (C-14, C-18), 121.5 (C-2), 14.6 (C-9, C-10, C-11, C-12); HRMS(ESI) $\mathrm{m} / z$ calcd. for $\left[\mathrm{C}_{20} \mathrm{H}_{19} \mathrm{BF}_{2} \mathrm{~N}_{2} \mathrm{O}_{2} \mathrm{Na}\right]^{+}$: 391.1405, obsd.: 391.1413 . 


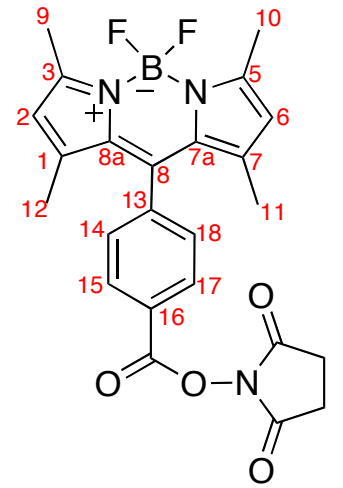

\section{2,5-Dioxopyrrolidin-1-yl-4-(4,4-difluoro-1,3,5,7-} tetramethyl-3a,4a-diaza-4-bora-s-indacen-8-yl)benzoate (70)

To a solution of BODIPY-COOH $52(60.4 \mathrm{mg}, 0.164$ mmol) in $\mathrm{CH}_{2} \mathrm{Cl}_{2}(6.5 \mathrm{ml})$ was added NHS (37.8 mg, 0.328 mmol) and EDCI (62.9 mg, $0.328 \mathrm{mmol})$ and the solution stirred in the dark overnight. After completion of the reaction by TLC analysis, the mixture is diluted with distilled water, extracted with $\mathrm{CH}_{2} \mathrm{Cl}_{2}(2 \times 25 \mathrm{~mL})$ and washed with brine $(1 \times 25 \mathrm{~mL})$. The combined organic phase was dried over anhydrous $\mathrm{MgSO}_{4}$, filtered and concentrated in vacuo. Purification of the crude residue by silica gel gradient column chromatography (petroleum ether to $\mathrm{CH}_{2} \mathrm{Cl}_{2}$ ) afforded BODIPY-NHS 70 (76.0 $\mathrm{mg}, 0.163 \mathrm{mmol}$, quantitative) as a red solid. $\mathrm{R}_{f}=0.83(\mathrm{MeOH} / \mathrm{EtOAc}, 1 / 4, \mathrm{v} / \mathrm{v})$; ${ }^{1} \mathrm{H}$ NMR $\left(500 \mathrm{MHz}, \mathrm{CDCl}_{3}\right) \delta 8.29\left(\mathrm{~d}, J_{14,15}=J_{17,18}=8.5 \mathrm{~Hz}, 2 \mathrm{H}, \mathrm{H}-15, \mathrm{H}-17\right)$, $7.51\left(\mathrm{~d}, J_{14,15}=J_{17,18}=8.5,2 \mathrm{H}, \mathrm{H}-14, \mathrm{H}-18\right), 6.02(\mathrm{~s}, 2 \mathrm{H}, \mathrm{H}-2, \mathrm{H}-6), 3.50(\mathrm{~s}, 4 \mathrm{H}$, $\mathrm{CH}_{2}$ NHS x 2), 2.57 (s, 6H, H-9, H-10), 1.39 (s, 6H, H-11, H-12); ${ }^{13} \mathrm{C}$ NMR (125 $\left.\mathrm{MHz}, \mathrm{CDCl}_{3}\right) \delta 169.2$ (C=O NHS), 160.3 (C=O BOD), 156.2 (C-3, C-5), 142.1 (C-1, C-7), 142.0 (C-13), 139.3 (C-16), 131.3 (C-15, C-17), 131.1 (C-7a, C-8a), 129.0 (C-14, C-18), 125.8 (C-8), 121.7 (C-2, C-6), 53.4 ( $\underline{\mathrm{CH}}_{2}$ NHS x 2), 14.8 (C9, C-10, C-11, C-12); HRMS(ESI) m/z calcd. for $\left[\mathrm{C}_{24} \mathrm{H}_{23} \mathrm{BF}_{2} \mathrm{~N}_{3} \mathrm{O}_{4}\right]^{+}: 466.1744$, obsd.: 466.1740 .

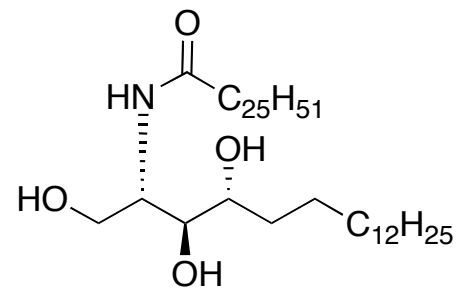

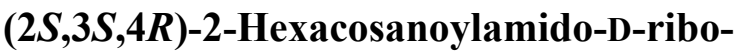
octadecane-1,3,4-triol (67)

Phytosphingosine 58 (200 $\mathrm{mg}, 0.63 \mathrm{mmol})$ and hexacosanoic acid $(275 \mathrm{mg}, 0.69 \mathrm{mmol})$ were coevaporated together with toluene (x 3) and dry DMF (6.5 mL) was added followed by PyBOP (656 mg, $1.26 \mathrm{mmol})$ and DiPEA (0.28 $\mathrm{mL}, 1.58 \mathrm{mmol}$ ). The white suspension was stirred at $\mathrm{rt}$ for $7 \mathrm{~d}$, at which time the solvent was removed under reduced pressure and the desired product crystallised from EtOAc/MeOH (30 mL, 1:1, v/v). The suspension was spun down in a centrifuge, the filtrate removed and the white solid washed twice with 
EtOAc/MeOH, before it was dried in vacuo to afford triol $\mathbf{6 7}$ as a white solid (440 $\mathrm{mg}, 0.63 \mathrm{mmol}$, quantitative). $\mathrm{R}_{f}: 0.60\left(15 \% \mathrm{MeOH} / \mathrm{CHCl}_{3}\right.$, v/v, charred with cerium ammonium molybdate dip); $[\alpha]_{\mathrm{D}}^{25}=-2.9^{\circ}(\mathrm{c}=1.0$, pyridine); IR (film) 3313, 2956, 2917, 2850, 1642, 1543, 1471, 1086, 1036, 981, 717, $573 \mathrm{~cm}^{-1} ;{ }^{1} \mathrm{H}$ $\operatorname{NMR}\left(600 \mathrm{MHz}, 10 \% \mathrm{CD}_{3} \mathrm{OD}\right.$ in $\left.\mathrm{CDCl}_{3}\right) 6.90\left(\mathrm{~d}, J_{\mathrm{NH}, 2}=8.2 \mathrm{~Hz}, 1 \mathrm{H}, \mathrm{NH}\right), 3.99$ $\left(\mathrm{dd}, J_{\mathrm{NH}, 2}=8.2 \mathrm{~Hz}, J_{1 \mathrm{a}, 2}=J_{1 \mathrm{~b}, 2}=4.5 \mathrm{~Hz}, 1 \mathrm{H}, \mathrm{H}-2\right), 3.71\left(\mathrm{dd}, J_{1 \mathrm{a}, 1 \mathrm{~b}}=11.4 \mathrm{~Hz}, J_{1 \mathrm{a}, 2}\right.$ $=4.5 \mathrm{~Hz}, 1 \mathrm{H}, \mathrm{H}-1 \mathrm{a}), 3.61\left(\mathrm{dd}, J_{1 \mathrm{a}, 1 \mathrm{~b}}=11.4 \mathrm{~Hz}, J_{1 \mathrm{~b}, 2}=4.5 \mathrm{~Hz}, 1 \mathrm{H}, \mathrm{H}-1 \mathrm{~b}\right), 3.49$ 3.42 (m, 2H, H-3, H-4), 2.13 (t, $\left.J_{\alpha, \beta}=7.6 \mathrm{~Hz}, 2 \mathrm{H}, \mathrm{CH}_{2}-\alpha\right), 1.56-1.51(\mathrm{~m}, 2 \mathrm{H}$, $\left.\mathrm{CH}_{2}-\beta\right), 1.36-1.30$ (m, 2H, H-5), 1.61-1.56 (m, 1H, H-6a), 1.48-1.44 (m, 1H, H6b), $1.23-1.81(\mathrm{~m}, 66 \mathrm{H}, \mathrm{H}-7-\mathrm{H}-17, \mathrm{H}-\gamma-\mathrm{H}-(\omega-1)), 0.80\left(\mathrm{t}, J_{17,18}=J_{\omega-1, \omega}=7.0\right.$ $\mathrm{Hz}, 6 \mathrm{H}, \mathrm{H}-18, \mathrm{H}-\omega) ;{ }^{13} \mathrm{C}$ NMR $\left(150 \mathrm{MHz}, 10 \% \mathrm{CD}_{3} \mathrm{OD}\right.$ in $\left.\mathrm{CDCl}_{3}\right) \delta 174.6$ $(\mathrm{HN} \underline{\mathrm{C}}=\mathrm{O}), 75.6$ (C-3), 72.4 (C-4), 61.1 (C-1), 51.9 (C-2), 36.5 (C- $\alpha$ ), 33.0 (C-6), $31.84,29.64,29.62$, 29.60, 29.57, 29.56, 29.45, 29.30, 29.26, 29.23, 22.58 (C-7C-17, C- $\gamma-\mathrm{C}-(\omega-1)), 25.77,25.72$ (C-5, C- $\beta$ ), 13.9 (C-18, C- $\omega)$; HRMS(ESI) m/z calcd. for $\left[\mathrm{C}_{44} \mathrm{H}_{89} \mathrm{NO}_{4}+\mathrm{H}\right]^{+}:$696.6864, obsd.: 696.6837 .

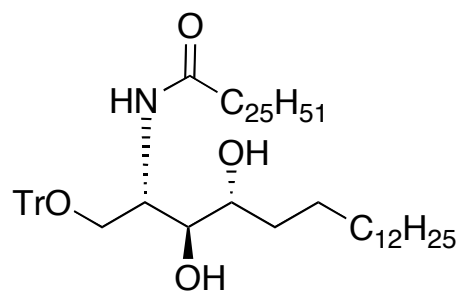

(2S,3S,4R)-2-Hexacosanoylamido-1triphenylmethoxy-octadecane-3,4-diol (68)

Trityl chloride (213 mg, $0.76 \mathrm{mmol}$ ) and DMAP (1.9 $\mathrm{mg}, 0.015 \mathrm{mmol})$ were added to triol $67(106 \mathrm{mg}$, $0.15 \mathrm{mmol}$ ) (co-evaporated with toluene $\mathrm{x} 3$ ) dissolved in dry pyridine $(1.2 \mathrm{~mL})$. The reaction was stirred for $20 \mathrm{~h}$ at $\mathrm{rt}$, at which point further trityl chloride $(128 \mathrm{mg}, 0.46 \mathrm{mmol})$ and pyridine $(0.7 \mathrm{~mL})$ were added. Stirring was continued for $4 \mathrm{~h}$, then at $40{ }^{\circ} \mathrm{C}$ for $5 \mathrm{~h}$ to ensure complete conversion as monitored by TLC. The reaction mixture was then diluted with warm $\mathrm{CHCl}_{3}(50 \mathrm{~mL})$, washed with brine $(30 \mathrm{~mL})$, dried $\left(\mathrm{MgSO}_{4}\right)$, filtered and concentrated in vacuo. The residue was purified by silica gel flash column chromatography and eluted with $0.3 \% \mathrm{MeOH} / \mathrm{CHCl}_{3}$ to afford diol $68(113 \mathrm{mg}$, $0.12 \mathrm{mmol}, 79 \%)$ as a clear oil. $\mathrm{R}_{f}: 0.53(\mathrm{PE} / \mathrm{EA}, 2: 1, \mathrm{v} / \mathrm{v}) ;[\alpha]_{\mathrm{D}}{ }^{26}=+17.0^{\circ}(\mathrm{c}=$ 1.0, $\mathrm{CHCl}_{3}$ ); IR (film) 3305, 2918, 2850, 1643, 1536, 1491, 1468, 1449, 1217, 1071, 900, 760, 704, $633 \mathrm{~cm}^{-1} ;{ }^{1} \mathrm{H}$ NMR $\left(500 \mathrm{MHz}, \mathrm{CDCl}_{3}\right) \delta 7.42\left(\mathrm{~d}, J_{\mathrm{CH}-o, \mathrm{CH}-m}=\right.$ $\left.7.3 \mathrm{~Hz}, 6 \mathrm{H}, \mathrm{CPh}_{3}, \mathrm{CH}-o\right), 7.32\left(\mathrm{t}, J_{\mathrm{CH}-o, \mathrm{CH}-m}=J_{\mathrm{CH}-m, \mathrm{CH}-p}=7.6 \mathrm{~Hz}, 6 \mathrm{H}, \mathrm{CPh}_{3}, \mathrm{CH}-\right.$ $m), 7.25\left(\mathrm{t}, J_{\mathrm{CH}-m, \mathrm{CH}-p}=7.2 \mathrm{~Hz}, 3 \mathrm{H}, \mathrm{CPh}_{3}, \mathrm{CH}-p\right), 6.11\left(\mathrm{~d}, J_{\mathrm{NH}, 2}=8.3 \mathrm{~Hz}, 1 \mathrm{H}\right.$, 
$\mathrm{NH}), 4.36\left(\mathrm{dd}, J_{\mathrm{NH}, 2}=8.3 \mathrm{~Hz}, J_{1 \mathrm{a}, 2}=J_{1 \mathrm{~b}, 2}=4.1 \mathrm{~Hz}, 1 \mathrm{H}, \mathrm{H}-2\right), 3.60-3.59(\mathrm{~m}, 1 \mathrm{H}$, H-3), 3.51 (dd, $\left.J_{1 \mathrm{a}, 1 \mathrm{~b}}=10.0 \mathrm{~Hz}, J_{1 \mathrm{a}, 2}=4.1 \mathrm{~Hz}, 1 \mathrm{H}, \mathrm{H}-1 \mathrm{a}\right), 3.40-3.36$ (m, 1H, H-4), $3.35\left(\mathrm{dd}, J_{1 \mathrm{a}, 1 \mathrm{~b}}=10.0 \mathrm{~Hz}, J_{1 \mathrm{~b}, 2}=4.1 \mathrm{~Hz}, 1 \mathrm{H}, \mathrm{H}-1 \mathrm{~b}\right), 3.23\left(\mathrm{~d}, J_{3,3-\mathrm{OH}}=8.6 \mathrm{~Hz}, 1 \mathrm{H}\right.$, 3-OH), $2.46\left(\mathrm{~d}, J_{4,4-\mathrm{OH}}=4.1 \mathrm{~Hz}, 1 \mathrm{H}, 4-\mathrm{OH}\right), 2.15\left(\mathrm{t}, J_{\alpha, \beta}=7.7 \mathrm{~Hz}, 2 \mathrm{H}, \mathrm{CH}_{2}-\alpha\right)$, 1.90-1.64 (m, 1H, H-5a), 1.64-1.58 (m, 2H, $\left.\mathrm{CH}_{2}-\beta\right), 1.48-1.38$ (m, 3H, H-6, H5b), 1.32-1.15 (m, 66H, H-7-H-17, H- $\gamma-\mathrm{H}-(\omega-1)), 0.89$ (t, $J_{17,18}=J_{\omega-1, \omega}=6.8$ $\mathrm{Hz}, 6 \mathrm{H}, \mathrm{H}-18, \mathrm{H}-\omega) ;{ }^{13} \mathrm{C} \mathrm{NMR}\left(125 \mathrm{MHz}, \mathrm{CDCl}_{3}\right) \delta 173.3(\mathrm{HNC}=\mathrm{O}), 143.3$ (C-i, $\left.\mathrm{CPh}_{3}\right), 128.6\left(\mathrm{C}-o, \mathrm{CPh}_{3}\right), 128.2\left(\mathrm{C}-m, \mathrm{CPh}_{3}\right), 127.5\left(\mathrm{C}-p, \mathrm{CPh}_{3}\right), 87.8\left(\mathrm{C}_{\mathrm{q}} \mathrm{CPh}_{3}\right)$, 75.8 (C-3), 73.3 (C-4), 63.0 (C-1), 50.5 (C-2), 37.0 (C- $\alpha$ ), 33.4 (C-5), 32.1, 29.84, 20.83, 29.82, 29.80, 29.79, 29.76, 29.6, 29.54, 29.50, 29.49, 29.48, 22.8 (C-7-C17, C- $\gamma-\mathrm{C}-(\omega-1)), 26.0$ (C-6), 25.9 (C- $\beta$ ), 14.3 (C-18, C- $\omega)$; HRMS(ESI) m/z calcd. for $\left[\mathrm{C}_{63} \mathrm{H}_{103} \mathrm{NO}_{4}+\mathrm{Na}\right]^{+}: 960.7779$, obsd.: 960.7781 .

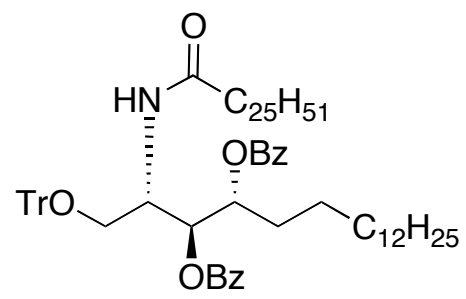

\section{$(2 S, 3 S, 4 R)-3,4-D i b e n z o y l o x y-2-$}

\section{hexacosanoylamido-1-triphenylmethyoxy- octadecane}

Benzoyl chloride $(94 \mu \mathrm{L}, 0.81 \mathrm{mmol})$ and DMAP (1.6 mg, $0.013 \mathrm{mmol})$ were added to diol 68 (126 $\mathrm{mg}, 0.13 \mathrm{mmol}$ ) (co-evaporated with toluene $\mathrm{x} 3$ ) dissolved in dry pyridine (1.3 $\mathrm{mL}$ ) and stirred at $\mathrm{rt}$ for $15 \mathrm{~h}$. The pyridine was removed in vacuo, the residue redissolved in EtOAc $(70 \mathrm{~mL})$ and the organic layer then washed with water $(70$ $\mathrm{mL})$, saturated $\mathrm{NaHCO}_{3}$ solution $(70 \mathrm{~mL})$, brine $(70 \mathrm{~mL})$, dried $\left(\mathrm{MgSO}_{4}\right)$, filtered and concentrated in vacuo. This afforded the title compound, which was used without further purification. $\mathrm{R}_{f}: 0.49(\mathrm{PE} / \mathrm{EA}, 5: 1, \mathrm{v} / \mathrm{v}) ;[\alpha]_{\mathrm{D}}{ }^{26}=+12.0^{\circ}(\mathrm{c}=1.0$, $\mathrm{CHCl}_{3}$ ); IR (film) 2923, 2853, 1719, 1671, 1540, 1450, 1315, 1280, 1109, 1069, $1027,757,707,632 \mathrm{~cm}^{-1} ;{ }^{1} \mathrm{H}$ NMR $\left(500 \mathrm{MHz}, \mathrm{CDCl}_{3}\right) \delta 7.96\left(\mathrm{~d}, J_{\mathrm{CH}-o, \mathrm{CH}-m}=7.5\right.$ $\mathrm{Hz}, 2 \mathrm{H}, \mathrm{CH}-o, 4-O-\mathrm{Bz}), 7.89$ (d, $\left.J_{\mathrm{CH}-o, \mathrm{CH}-m}=7.5 \mathrm{~Hz}, 2 \mathrm{H}, \mathrm{CH}-o, 3-O-\mathrm{Bz}\right), 7.59$ (t, $\left.J_{\mathrm{CH}-m, \mathrm{CH}-p}=7.3 \mathrm{~Hz}, 1 \mathrm{H}, \mathrm{CH}-p, 3-O-\mathrm{Bz}\right), 7.55\left(\mathrm{t}, J_{\mathrm{CH}-m, \mathrm{CH}-p}=7.6 \mathrm{~Hz}, 1 \mathrm{H}, \mathrm{CH}-p, 4-\right.$ $O-\mathrm{Bz}), 7.42\left(\mathrm{t}, J_{\mathrm{CH}-o, \mathrm{CH}-m}=J_{\mathrm{CH}-m, \mathrm{CH}-p}=8.3 \mathrm{~Hz}, 2 \mathrm{H}, \mathrm{CH}-m, 3-O-\mathrm{Bz}\right), 7.41\left(\mathrm{t}, J_{\mathrm{CH}-}\right.$ $\left.o, \mathrm{CH}-m=J_{\mathrm{CH}-m, \mathrm{CH}-p}=8.3 \mathrm{~Hz}, 2 \mathrm{H}, \mathrm{CH}-m, 4-O-\mathrm{Bz}\right), 7.31-7.30\left(\mathrm{~m}, 6 \mathrm{H}, \mathrm{CPh}_{3}, \mathrm{CH}-o\right)$, 7.15-7.20 (m, 9H, $\left.\mathrm{CPh}_{3}, \mathrm{CH}-m, \mathrm{CH}-p\right), 6.03$ (d, $\left.J_{\mathrm{NH}, 2}=9.3 \mathrm{~Hz}, 1 \mathrm{H}, \mathrm{NH}\right), 5.81$ $\left(\mathrm{dd}, J_{2,3}=9.1 \mathrm{~Hz}, J_{3,4}=2.6 \mathrm{~Hz}, 1 \mathrm{H}, \mathrm{H}-3\right), 5.35\left(\mathrm{dt}, J_{4,5}=10.1 \mathrm{~Hz}, J_{3,4}=2.6 \mathrm{~Hz}\right.$, $1 \mathrm{H}, \mathrm{H}-4), 4.59\left(\mathrm{tt}, J_{2, \mathrm{NH}}=J_{2,3}=9.3 \mathrm{~Hz}, J_{1 \mathrm{a}, 2}=J_{1 \mathrm{~b}, 2}=3.2 \mathrm{~Hz}, 1 \mathrm{H}, \mathrm{H}-2\right), 3.34(\mathrm{dd}$, 
$\left.J_{1 \mathrm{a}, 1 \mathrm{~b}}=9.9 \mathrm{~Hz}, J_{1 \mathrm{a}, 2}=3.2 \mathrm{~Hz}, 1 \mathrm{H}, \mathrm{H}-1 \mathrm{a}\right), 3.29\left(\mathrm{dd}, J_{1 \mathrm{a}, 1 \mathrm{~b}}=9.9 \mathrm{~Hz}, J_{1 \mathrm{~b}, 2}=3.2 \mathrm{~Hz}\right.$, $1 \mathrm{H}, \mathrm{H}-1 \mathrm{~b}), 2.18\left(\mathrm{dt}, J_{\mathrm{a}, \mathrm{b}}=14.5 \mathrm{~Hz}, J_{\alpha, \beta}=7.7 \mathrm{~Hz}, 2 \mathrm{H}, \mathrm{CH}_{2}-\alpha\right), 1.93-1.81(\mathrm{~m}, 2 \mathrm{H}$, H-5), $1.68-1.58$ (m, 2H, $\left.\mathrm{CH}_{2}-\beta\right), 1.40-1.14$ (m, 68H, H-6-H-17, H- $\gamma-\mathrm{H}-$ $(\omega-1)), 0.89\left(\mathrm{t}, J_{17,18}=J_{\omega-1, \omega}=7.0 \mathrm{~Hz}, 6 \mathrm{H}, \mathrm{H}-18, \mathrm{H}-\omega\right) ;{ }^{13} \mathrm{C}$ NMR $(125 \mathrm{MHz}$, $\left.\mathrm{CDCl}_{3}\right) \delta 172.8(\mathrm{HNC}=\mathrm{O}), 166.4(\mathrm{C}=\mathrm{O}, 4-O-\mathrm{Bz}), 165.0(\mathrm{C}=\mathrm{O}, 3-O-\mathrm{Bz}), 143.3$ (C-i, $\left.\mathrm{CPh}_{3}\right), 133.1$ (C-p, 3-O-Bz), 132.9 (C-p, 4-O-Bz), 130.2 (C-i, 4-O-Bz), 129.82 (C-i, 3-O-Bz), 129.78 (C-o, 3-O-Bz), 129.75 (C-o, 4-O-Bz), 128.5 (C-o, $\mathrm{CPh}_{3}$ ), 128.40 (C-m, 3-O-Bz), 128.35 (C-m, 4-O-Bz), 127.8 (C-m, $\left.\mathrm{CPh}_{3}\right), 127.0$ $\left(\mathrm{C}-p, \mathrm{CPh}_{3}\right), 86.8\left(\mathrm{C}_{\mathrm{q}} \mathrm{CPh}_{3}\right), 74.2(\mathrm{C}-4), 72.7$ (C-3), $61.6(\mathrm{C}-1), 48.5(\mathrm{C}-2), 36.9$ (C- $\alpha), 31.9,29.74,29.73,29.72,29.69,29.68,29.67,29.66,29.63,29.58,29.55$, 29.53, 29.45, 29.39, 29.38, 25.72 (C-6-C-17, C- $\gamma-C-(\omega-1)), 28.4$ (C-5), 25.70 (C$\beta), 14.1(\mathrm{C}-18, \mathrm{C}-\omega)$; HRMS(ESI) m/z calcd. for $\left[\mathrm{C}_{77} \mathrm{H}_{111} \mathrm{NO}_{6}+\mathrm{Na}\right]^{+}:$1168.8304, obsd.: 1168.8302 .

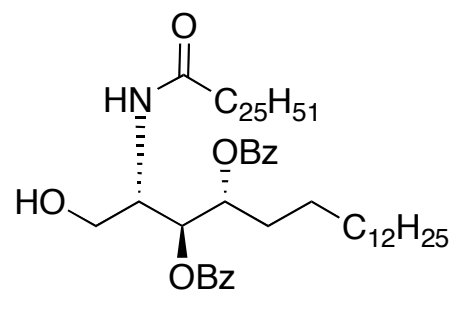

\section{$(2 S, 3 S, 4 R)-3,4-D i b e n z o y l o x y-2-$}

hexacosanoylamido-octadecan-1-ol (57)

(2S,3S,4R)-3,4-Dibenzoyloxy-2-hexacosanoylamido1-triphenylmethyoxy-octadecane was dissolved in 1:1 (v/v) $\mathrm{MeOH} / \mathrm{CH}_{2} \mathrm{Cl}_{2}$ and $p$ TsOH. $\mathrm{H}_{2} \mathrm{O}(7.7 \mathrm{mg}$, addition of further $p$ TsOH. $\mathrm{H}_{2} \mathrm{O}(7.7 \mathrm{mg}, 0.040 \mathrm{mmol})$, and stirring was contiunued for $6 \mathrm{~h}$. The reaction mixture was diluted with EtOAc $(70 \mathrm{~mL})$, washed with saturated $\mathrm{NaHCO}_{3}$ solution $(2 \times 70 \mathrm{~mL})$, brine $(70 \mathrm{~mL})$, dried $\left(\mathrm{MgSO}_{4}\right)$, filtered and concentrated in vacuo. The crude mixture was purified by silica gel flash column chromatography and eluted with $\mathrm{MeOH} / \mathrm{CH}_{2} \mathrm{Cl}_{2}(3 \%$, v/v) to afford the lipid acceptor $\mathbf{5 7}$ as an amorphous white solid (81 $\mathrm{mg}, 0.090 \mathrm{mmol}$, $67 \%$ over two steps). $\mathrm{R}_{f}: 0.25(\mathrm{PE} / \mathrm{EA}, 2: 1, \mathrm{v} / \mathrm{v}) ;[\alpha]_{\mathrm{D}}{ }^{27}=+54.0^{\circ}\left(\mathrm{c}=1.0, \mathrm{CHCl}_{3}\right)$; IR (film) 2918, 2850, 1721, 1649, 1541, 1468, 1315, 1280, 1177, 1111, 1070, 1027, 757, $710 \mathrm{~cm}^{-1} ;{ }^{1} \mathrm{H} \mathrm{NMR}\left(500 \mathrm{MHz}, \mathrm{CDCl}_{3}\right) \delta 8.05\left(\mathrm{~d}, J_{\mathrm{CH}-o, \mathrm{CH}-m}=7.7 \mathrm{~Hz}\right.$, $2 \mathrm{H}, \mathrm{CH}-o, 3-O-\mathrm{Bz}), 7.94\left(\mathrm{~d}, J_{\mathrm{CH}-o, \mathrm{CH}-m}=7.5 \mathrm{~Hz}, 2 \mathrm{H}, \mathrm{CH}-o, 4-O-\mathrm{Bz}\right), 7.63$ (t, $J_{\mathrm{CH}-}$ $m, \mathrm{CH}-p=7.7 \mathrm{~Hz}, 1 \mathrm{H}, \mathrm{CH}-p, 3-O-\mathrm{Bz}), 7.52\left(\mathrm{t}, J_{\mathrm{CH}-m, \mathrm{CH}-p}=7.5 \mathrm{~Hz}, 1 \mathrm{H}, \mathrm{CH}-p, 4-O-\right.$ $\mathrm{Bz}), 7.49\left(\mathrm{t}, J_{\mathrm{CH}-m, \mathrm{CH}-p}=J_{\mathrm{CH}-o, \mathrm{CH}-m}=7.7 \mathrm{~Hz}, 2 \mathrm{H}, \mathrm{CH}-m, 3-O-\mathrm{Bz}\right), 7.37\left(\mathrm{t}, J_{\mathrm{CH}-m, \mathrm{CH}-}\right.$ $\left.p=J_{\mathrm{CH}-o, \mathrm{CH}-m}=7.5 \mathrm{~Hz}, 2 \mathrm{H}, \mathrm{CH}-m, 4-O-\mathrm{Bz}\right), 6.48\left(\mathrm{~d}, J_{\mathrm{NH}, 2}=9.3 \mathrm{~Hz}, 1 \mathrm{H}, \mathrm{NH}\right)$, 
$5.44\left(\mathrm{dd}, J_{2,3}=9.6 \mathrm{~Hz}, J_{3,4}=2.3 \mathrm{~Hz}, 1 \mathrm{H}, \mathrm{H}-3\right), 5.37\left(\mathrm{dt}, J_{4,5}=9.1 \mathrm{~Hz}, J_{3,4}=2.9\right.$ $\mathrm{Hz}, 1 \mathrm{H}, \mathrm{H}-4), 4.40$ (t, $\left.J_{2,3}=9.6 \mathrm{~Hz}, 1 \mathrm{H}, \mathrm{H}-2\right), 3.68-3.59$ (m, 2H, H-1a, H-1b), $2.91\left(\mathrm{dd}, J_{\mathrm{OH}, 1 \mathrm{a}}=8.5 \mathrm{~Hz}, J_{\mathrm{OH}, 1 \mathrm{~b}}=5.1 \mathrm{~Hz}, 1 \mathrm{H}, \mathrm{OH}\right), 2.28\left(\mathrm{t}, J_{\alpha, \beta}=7.6 \mathrm{~Hz}, 2 \mathrm{H}\right.$, $\mathrm{CH}_{2}-\alpha$ ), 2.03-2.00 (m, 2H, H-5), 1.72-1.66 (m, 2H, $\left.\mathrm{CH}_{2}-\beta\right), 1.45-1.13$ (m, 68H, H-6-H-17, H- $\gamma-\mathrm{H}-(\omega-1)), 0.88\left(\mathrm{t}, J_{17,18}=J_{\omega-1, \omega}=7.0 \mathrm{~Hz}, 6 \mathrm{H}, \mathrm{H}-18, \mathrm{H}-\omega\right)$;

${ }^{13} \mathrm{C}$ NMR (125 MHz, $\left.\mathrm{CDCl}_{3}\right) \delta 173.3(\mathrm{HNC}=\mathrm{O}), 167.1(\mathrm{C}=\mathrm{O}, 3-\mathrm{O}-\mathrm{Bz}), 166.3$ $(\mathrm{C}=\mathrm{O}, 4-O-\mathrm{Bz}), 133.8$ (C-p, 3-O-Bz), 133.1 (C-p, 4-O-Bz), 130.0 (C-o, 3-O-Bz), 129.9 (C-i, 4-O-Bz), 129.6 (C-o, 4-O-Bz), 129.1 (C-i, 3-O-Bz), 128.7 (C-m, 3-O$\mathrm{Bz}), 128.4$ (C-m, 4-O-Bz), 74.0 (C-4), 73.6 (C-3), 61.6 (C-1), 49.9 (C-2), 36.9 (C$\alpha)$, 31.9, 29.73, 29.71, 29.68, 29.66, 29.65, 29.60, 29.58, 29.55, 29.40, 29.37, 29.34, 25.85, 22.7 (C-6-C-17, C- $\gamma-\mathrm{C}-(\omega-1)), 28.4$ (C-5), 25.75 (C- $\beta$ ), 14.1 (C-18, C- $\omega)$; HRMS(ESI) m/z calcd. for $\left[\mathrm{C}_{58} \mathrm{H}_{97} \mathrm{NO}_{6}+\mathrm{Na}\right]^{+}$: 926.7208 , obsd.: 926.7220.

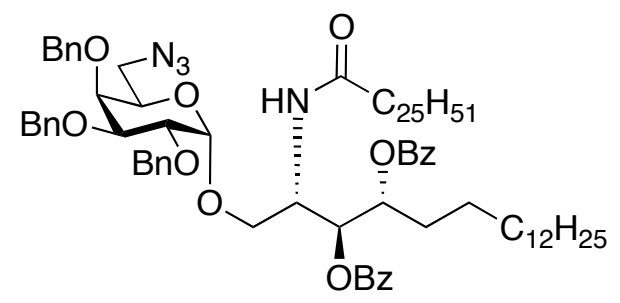

(2S,3S,4R)-3,4-Dibenzoyloxy-1-(2,3,4-tri$O$-benzyl-6-deoxy-6-azido- $\alpha$-Dgalactopyranosyloxy)-2hexacosanoylamido-octadecane (55)

Imidate donor $56(88 \mathrm{mg}, 0.14 \mathrm{mmol})$ and lipid acceptor 57 (64 mg, $0.071 \mathrm{mmol}$ ) were co-evaporated with toluene (x 3), dissolved in dry $\mathrm{Et}_{2} \mathrm{O} / \mathrm{THF}(2.7 \mathrm{~mL}, 5: 1, \mathrm{v} / \mathrm{v})$ and stirred with activated $4 \AA$ molecular sieves for $1 \mathrm{~h}$. The reaction mixture was cooled to $0{ }^{\circ} \mathrm{C}$, freshly distilled TMSOTf (stock of $0.055 \mathrm{M}$ in $\mathrm{Et}_{2} \mathrm{O}, 76.8 \mu \mathrm{L}, 0.0042 \mathrm{mmol}$ ) was added dropwise and the resulting solution stirred at $0{ }^{\circ} \mathrm{C}$ for $1.5 \mathrm{~h}$. The reaction was quenched with triethylamine $(50 \mu \mathrm{L}, 0.36 \mathrm{mmol})$, diluted with EtOAc $(20 \mathrm{~mL})$, filtered through a celite pad and concentrated in vacuo. The residue was first purified by size exclusion column chromatography ( $\mathrm{LH} 20, \mathrm{CH}_{2} \mathrm{Cl}_{2} / \mathrm{MeOH}, 1: 1$, $\mathrm{v} / \mathrm{v})$ to isolate a mixture of $\alpha$ - and $\beta$-coupled glycolipid, which was further purified by silica gel gradient column chromatography (petroleum ether/EtOAc, $15: 1$ to $5: 1, \mathrm{v} / \mathrm{v})$ to give the $\alpha$-coupled glycolipid $\mathbf{5 5} \boldsymbol{\alpha}$ as a colourless oil (65.7 $\mathrm{mg}, 0.048 \mathrm{mmol}, 68 \%$ ) and the $\beta$-coupled glycolipid $55 \beta$ (22.6 mg, $0.017 \mathrm{mmol}$, $23 \%)$ also as a colourless oil. $\alpha$ : $\mathrm{R}_{f}: 0.58(8 \% \mathrm{EA} /$ Toluene $) ;[\alpha]_{\mathrm{D}}{ }^{27}=-36.0^{\circ}(\mathrm{c}=$ 1.0, $\mathrm{CHCl}_{3}$ ); IR (film) 3063, 3030, 2922, 2852, 2362, 2338, 2102, 1722, 1673, 
1530, 1453, 1278, 1095, 1068, 1027, 756, 711, $699 \mathrm{~cm}^{-1}$; ${ }^{1} \mathrm{H}$ NMR (500 MHz, $\left.\mathrm{CDCl}_{3}\right) \delta 8.03\left(\mathrm{~d}, J_{\mathrm{CH}-o, \mathrm{CH}-m}=7.1 \mathrm{~Hz}, 2 \mathrm{H}, \mathrm{CH}-o, 3-O-\mathrm{Bz}\right), 7.96\left(\mathrm{~d}, J_{\mathrm{CH}-o, \mathrm{CH}-m}=7.0\right.$ $\mathrm{Hz}, 2 \mathrm{H}, \mathrm{CH}-o, 4-O-\mathrm{Bz}), 7.60$ (t, $\left.J_{\mathrm{CH}-m, \mathrm{CH}-p}=7.5 \mathrm{~Hz}, 1 \mathrm{H}, \mathrm{CH}-p, 3-O-\mathrm{Bz}\right), 7.54$ (t, $\left.J_{\mathrm{CH}-m, \mathrm{CH}-p}=7.5 \mathrm{~Hz}, 1 \mathrm{H}, \mathrm{CH}-p, 4-O-\mathrm{Bz}\right), 7.48-7.19\left(\mathrm{~m}, 19 \mathrm{H}, \mathrm{H}_{\text {arom }}\right), 6.66\left(\mathrm{~d}, J_{\mathrm{NH}, 2}\right.$ $=9.5 \mathrm{~Hz}, 1 \mathrm{H}, \mathrm{NH}), 5.64\left(\mathrm{dd}, J_{2,3}=9.3 \mathrm{~Hz}, J_{2,3}=2.7 \mathrm{~Hz}, 1 \mathrm{H}, \mathrm{H}-3\right), 5.39-5.36(\mathrm{~m}$, $1 \mathrm{H}, \mathrm{H}-4), 4.96\left(\mathrm{~d}, J_{\mathrm{a}, \mathrm{b}}=11.5 \mathrm{~Hz}, 1 \mathrm{H}, \mathrm{CH}-\mathrm{a}, 4^{\prime}-O-\mathrm{Bn}\right), 4.78\left(\mathrm{~d}, J_{\mathrm{a}, \mathrm{b}}=11.7 \mathrm{~Hz}, 1 \mathrm{H}\right.$, $\left.\mathrm{CH}-\mathrm{a}, 3^{\prime}-O-\mathrm{Bn}\right), 4.75\left(\mathrm{~d}, J_{1^{\prime}, 2^{\prime}}=3.6 \mathrm{~Hz}, 1 \mathrm{H}, \mathrm{H}-1^{\prime}\right), 4.78\left(\mathrm{~d}, J_{\mathrm{a}, \mathrm{b}}=11.7 \mathrm{~Hz}, 1 \mathrm{H}\right.$, $\left.\mathrm{CH}-\mathrm{a}, 3^{\prime}-O-\mathrm{Bn}\right), 4.68$ (d, $\left.J_{\mathrm{a}, \mathrm{b}}=11.7 \mathrm{~Hz}, 1 \mathrm{H}, \mathrm{CH}-\mathrm{b}, 3^{\prime}-O-\mathrm{Bn}\right), 4.64$ (s, 2H, CH-a, CH-b, 2'-O-Bn), 4.61 (t, $\left.J_{1,2}=3.2 \mathrm{~Hz}, 1 \mathrm{H}, \mathrm{H}-2\right), 4.57$ (d, $J_{\mathrm{a}, \mathrm{b}}=11.5 \mathrm{~Hz}, 1 \mathrm{H}, \mathrm{CH}-$ b, $\left.4^{\prime}-O-\mathrm{Bn}\right), 3.98\left(\mathrm{dd}, J_{2^{\prime}, 3^{\prime}}=10.1 \mathrm{~Hz}, J_{1^{\prime}, 2^{\prime}}=3.6 \mathrm{~Hz}, 1 \mathrm{H}, \mathrm{H}-2^{\prime}\right), 3.93\left(\mathrm{dd}, J_{5^{\prime}, 6^{\prime} \mathrm{a}}=\right.$ $\left.8.3 \mathrm{~Hz}, J_{5^{\prime}, 6^{\prime} \mathrm{b}}=5.2 \mathrm{~Hz}, 1 \mathrm{H}, \mathrm{H}-5^{\prime}\right), 3.90\left(\mathrm{dd}, J_{1 \mathrm{a}, 1 \mathrm{~b}}=11.6 \mathrm{~Hz}, J_{1 \mathrm{a}, 2}=3.2 \mathrm{~Hz}, 1 \mathrm{H}, \mathrm{H}-\right.$ 1a), $3.83\left(\mathrm{dd}, J_{2^{\prime}, 3^{\prime}}=10.1 \mathrm{~Hz}, J_{3^{\prime}, 4^{\prime}}=2.7 \mathrm{~Hz}, 1 \mathrm{H}, \mathrm{H}-3^{\prime}\right), 3.78$ (bs, $1 \mathrm{H}, \mathrm{H}-4^{\prime}$ ), 3.67 $\left(\mathrm{dd}, J_{1 \mathrm{a}, 1 \mathrm{~b}}=11.6 \mathrm{~Hz}, J_{1 \mathrm{~b}, 2}=3.2 \mathrm{~Hz}, 1 \mathrm{H}, \mathrm{H}-1 \mathrm{~b}\right), 3.55\left(\mathrm{dd}, J_{6^{\prime} \mathrm{a}, 6^{\prime} \mathrm{b}}=12.4 \mathrm{~Hz}, J_{5^{\prime}, 6^{\prime} \mathrm{a}}=\right.$ $\left.8.3 \mathrm{~Hz}, 1 \mathrm{H}, \mathrm{H}-6^{\prime} \mathrm{a}\right), 3.04$ (dd, $\left.J_{6^{\prime} \mathrm{a}, 6^{\prime} \mathrm{b}}=12.4 \mathrm{~Hz}, J_{5^{\prime}, 6^{\prime} \mathrm{b}}=5.2 \mathrm{~Hz}, 1 \mathrm{H}, \mathrm{H}-6^{\prime} \mathrm{a}\right), 2.20$ (t, $\left.J_{\alpha, \beta}=7.6 \mathrm{~Hz}, 2 \mathrm{H}, \mathrm{CH}_{2}-\alpha\right), 1.95-1.90$ (m, 2H, H-5), 1.67-1.64 (m, 2H, H $\beta$ ), 1.39$1.21(\mathrm{~m}, 68 \mathrm{H}, \mathrm{H}-6-\mathrm{H}-17, \mathrm{H}-\gamma-\mathrm{H}-(\omega-1)), 0.89\left(\mathrm{t}, J_{17,18}=J_{\omega-1, \omega}=6.9 \mathrm{~Hz}, 6 \mathrm{H}, \mathrm{H}-\right.$ 18, $\mathrm{H}-\omega) ;{ }^{13} \mathrm{C}$ NMR $\left(125 \mathrm{MHz}, \mathrm{CDCl}_{3}\right) \delta 173.1$ (HNC $\left.=\mathrm{O}\right), 166.2(\mathrm{C}=\mathrm{O}, 4-O-\mathrm{Bz})$, $165.3(\mathrm{C}=\mathrm{O}, 3-O-\mathrm{Bz}), 138.6$ (C- $\left.i, 3^{\prime}-O-\mathrm{Bn}\right), 138.2$ (C-i, 2' $\left.-O-\mathrm{Bn}\right), 138.0\left(\mathrm{C}-i, 4^{\prime}-\right.$ $O-\mathrm{Bn}), 133.3$ (C-p, 3-O-Bz), 132.9 (C-p, 4-O-Bz), 130.1, 129.8 (C-i, 3-O-Bz, 4$O-\mathrm{Bz}), 129.8$ (C-o, 3-O-Bz), 129.8 (C-o, 4-O-Bz), 128.6, 128.5, 128.42, 128.41, 128.34, 128.32, 128.1, 128.0, 127.7, 127.62, 127.56 (21 x $\left.\mathrm{CH}_{\text {arom}}\right), 99.7$ (C-1', $\left.{ }^{1} J_{\mathrm{CH}}=168.5 \mathrm{~Hz}\right), 78.5\left(\mathrm{C}-3^{\prime}\right), 76.4\left(\mathrm{C}-2^{\prime}\right), 74.8\left(\mathrm{C}-4^{\prime}\right), 74.6\left(\mathrm{CH}_{2}, 4^{\prime}-O-\mathrm{Bn}\right), 73.8$ (C-4), $73.5\left(\mathrm{CH}_{2}, 3^{\prime}-O-\mathrm{Bn}\right), 73.3\left(\mathrm{CH}_{2}, 2^{\prime}-O-\mathrm{Bn}\right), 72.4$ (C-3), 70.2 (C-5'), 69.7 (C-1), 51.3 (C-6'), 48.6 (C-2), 36.8 (C- $\alpha$ ), 28.3 (C-5), 25.8 (C- $\beta$ ), 31.9, 29.8, 29.72, 29.70, 29.67, 29.64, 29.61, 29.60, 29.56, 29.50, 29.4, 25.7, 22.7 (C-6-C17, C- $\gamma-\mathrm{C}-(\omega-1)), \quad 14.1 \quad(\mathrm{C}-18, \quad \mathrm{C}-\omega) ; \mathrm{HRMS}(\mathrm{ESI}) \mathrm{m} / \mathrm{z}$ calcd. for $\left[\mathrm{C}_{85} \mathrm{H}_{124} \mathrm{~N}_{4} \mathrm{O}_{10}+\mathrm{H}\right]^{+}:$1361.9390, obsd.: 1361.9399 . 


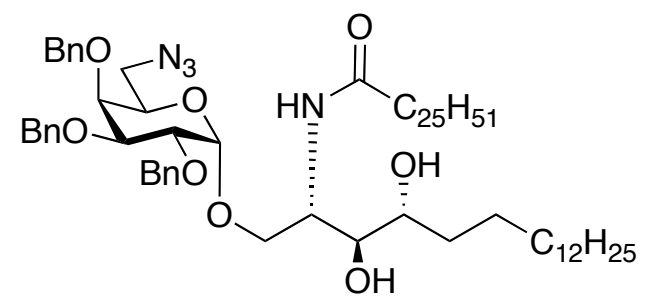

$(2 S, 3 S, 4 R)$-2-Hexacosanoylamido-

(2,3,4-tri-O-benzyl-6-deoxy-6-azido- $\alpha$ -

D-galactopyranosyloxy)-octadecane-

3,4-diol (69)

Glycolipid 55 $\boldsymbol{\alpha}$ (66 mg, $0.048 \mathrm{mmol}$ ) was

dissolved in $\mathrm{MeOH} / \mathrm{CH}_{2} \mathrm{Cl}_{2}(3 \mathrm{~mL}, 2: 1(\mathrm{v} / \mathrm{v})$ and sodium methoxide was added until the reaction mixture reached $\mathrm{pH} 9.0$. After stirring for $12 \mathrm{~h}$ at $\mathrm{rt}$, the reaction was quenched with Dowex $-\mathrm{H}^{+}$, filtered and concentrated under reduced pressure. The residue was purified by silica gel gradient column chromatography (petroleum ether/EtOAc, 10:1 to 3:1, v/v) to afford glycolipid diol 69 as an amorphous white solid (52 mg, $0.045 \mathrm{mmol}, 93 \%) . \mathrm{R}_{f}: 0.28$ (PE/EA, 2:1, v/v); $[\alpha]_{\mathrm{D}}^{24}=+6.0^{\circ}\left(\mathrm{c}=1.0, \mathrm{CHCl}_{3}\right)$; IR (film) 3332, 3065, 3032, 2918, 2850, 2100, 1717, 1636, 1623, 1542, 1469, 1455, 1350, 1284, 1216, 1078, 1041, 909, 757, 696 $\mathrm{cm}^{-1} ;{ }^{1} \mathrm{H}$ NMR $\left(500 \mathrm{MHz}, \mathrm{CDCl}_{3}\right) \delta$ 7.40-7.29 (m, 15H, $\left.\mathrm{H}_{\text {arom }}\right), 6.29\left(\mathrm{~d}, J_{\mathrm{NH}, 2}=\right.$ $8.3 \mathrm{~Hz}, 1 \mathrm{H}, \mathrm{NH}), 4.98$ (d, $\left.J_{\mathrm{a}, \mathrm{b}}=11.3 \mathrm{~Hz}, 1 \mathrm{H}, \mathrm{CH}-\mathrm{a}, 4^{\prime}-O-\mathrm{Bn}\right), 4.884$ (d, $J_{\mathrm{a}, \mathrm{b}}=11.6$ $\left.\mathrm{Hz}, 1 \mathrm{H}, \mathrm{CH}-\mathrm{a}, 2^{\prime}-O-\mathrm{Bn}\right), 4.885$ (d, $\left.J_{1,2}=3.7 \mathrm{~Hz}, 1 \mathrm{H}, \mathrm{H}-1^{\prime}\right), 4.82$ (d, $J_{\mathrm{a}, \mathrm{b}}=11.7 \mathrm{~Hz}$, $\left.1 \mathrm{H}, \mathrm{CH}-\mathrm{a}, 3^{\prime}-O-\mathrm{Bn}\right), 4.78\left(\mathrm{~d}, J_{\mathrm{a}, \mathrm{b}}=11.7 \mathrm{~Hz}, 1 \mathrm{H}, \mathrm{CH}-\mathrm{b}, 3^{\prime}-O-\mathrm{Bn}\right), 4.70\left(\mathrm{~d}, J_{\mathrm{a}, \mathrm{b}}=\right.$ $\left.11.7 \mathrm{~Hz}, 1 \mathrm{H}, \mathrm{CH}-\mathrm{b}, 2^{\prime}-O-\mathrm{Bn}\right), 4.59$ (d, $\left.J_{\mathrm{a}, \mathrm{b}}=11.3 \mathrm{~Hz}, 1 \mathrm{H}, \mathrm{CH}-\mathrm{b}, 4^{\prime}-O-\mathrm{Bn}\right), 4.27$ $\left(\mathrm{dd}, J_{\mathrm{NH}, 2}=8.3 \mathrm{~Hz}, J_{1 \mathrm{a}, 2}=3.4 \mathrm{~Hz}, 1 \mathrm{H}, \mathrm{H}-2\right), 4.05\left(\mathrm{dd}, J_{2^{\prime}, 3^{\prime}}=10.0 \mathrm{~Hz}, J_{1^{\prime}, 2^{\prime}}=3.7\right.$ $\left.\mathrm{Hz}, 1 \mathrm{H}, \mathrm{H}-2^{\prime}\right), 3.93\left(\mathrm{dd}, J_{1 \mathrm{a}, 1 \mathrm{~b}}=10.4 \mathrm{~Hz}, J_{1 \mathrm{a}, 2}=3.4 \mathrm{~Hz}, 1 \mathrm{H}, \mathrm{H}-1 \mathrm{a}\right), 3.86$ (dd, $J_{2^{\prime}, 3^{\prime}}$ $\left.=10.0 \mathrm{~Hz}, J_{3^{\prime}, 4^{\prime}}=2.4 \mathrm{~Hz}, 1 \mathrm{H}, \mathrm{H}-3^{\prime}\right), 3.84-3.82\left(\mathrm{~m}, 2 \mathrm{H}, \mathrm{H}-1 \mathrm{~b}, \mathrm{H}-4^{\prime}\right), 3.73$ (t, $J_{5^{\prime}, 6^{\prime}}$ $\left.=6.6 \mathrm{~Hz}, 1 \mathrm{H}, \mathrm{H}-5^{\prime}\right), 3.52-3.46$ (m, 3H, H-4, H-6'a, H-3), 3.03 (dd, $J_{6^{\prime} \mathrm{a}, 6^{\prime} \mathrm{b}}=12.6$ $\left.\mathrm{Hz}, J_{5^{\prime}, 6^{\prime} \mathrm{b}}=5.5 \mathrm{~Hz}, 1 \mathrm{H}, \mathrm{H}-6^{\prime} \mathrm{b}\right), 2.16\left(\mathrm{t}, J_{\alpha, \beta}=7.6 \mathrm{~Hz}, 2 \mathrm{H}, \mathrm{CH}_{2}-\alpha\right), 1.60-1.50(\mathrm{~m}$, $\left.3 \mathrm{H}, \mathrm{CH}_{2}-\beta \mathrm{a}, \mathrm{H}-5\right), 1.48-1.21$ (m, 69H, H-6-H-17, H- $\left.\beta \mathrm{b}-\mathrm{H}-(\omega-1)\right), 0.88$ (t, $J_{17,18}$ $\left.=J_{\omega-1, \omega}=7.0 \mathrm{~Hz}, 6 \mathrm{H}, \mathrm{H}-18, \mathrm{H}-\omega\right) ;{ }^{13} \mathrm{C} \mathrm{NMR}\left(125 \mathrm{MHz}, \mathrm{CDCl}_{3}\right) \delta 173.9$ $(\mathrm{HNC}=\mathrm{O}), 138.2$ (C-i, 3' $-O-\mathrm{Bn}), 138.0$ (C-i, 4'-O-Bn), 137.8 (C-i, 2'-O-Bn), $128.69,128.67,128.6,128.5,128.3,128.2,128.1,128.0,127.7$ (15 x $\left.\mathrm{CH}_{\text {arom }}\right)$, $99.0\left(\mathrm{C}-1^{\prime}\right), 79.3$ (C-3'), 76.3 (C-3), $75.9\left(\mathrm{C}-2^{\prime}\right), 74.8\left(\mathrm{CH}_{2}, 4^{\prime}-O-\mathrm{Bn}\right), 74.5(\mathrm{C}-$ $\left.4^{\prime}\right), 74.4\left(\mathrm{CH}_{2}, 2^{\prime}-\mathrm{O}-\mathrm{Bn}\right), 73.5(\mathrm{C}-4), 73.2\left(\mathrm{CH}_{2}, 3^{\prime}-O-\mathrm{Bn}\right), 70.4$ (C-5'), 69.9 (C1), 51.2 (C-6'), 49.2 (C-2), 37.0 (C- $\alpha$ ), 33.5, 32.1, 29.9, 29.85, 29.81, 29.7, 29.6, 29.51, 29.46, 22.8 (C-6-C-17, C- $\gamma-\mathrm{C}-(\omega-1)), 26.0,25.9$ (C-5, C- $\beta$ ), 14.3 (C-18, 
C- $\omega)$; HRMS(ESI) $\mathrm{m} / \mathrm{z}$ calcd. for $\left[\mathrm{C}_{71} \mathrm{H}_{117} \mathrm{~N}_{4} \mathrm{O}_{8}+\mathrm{H}\right]^{+}$: 1153.8866, obsd.: 1153.8871 .

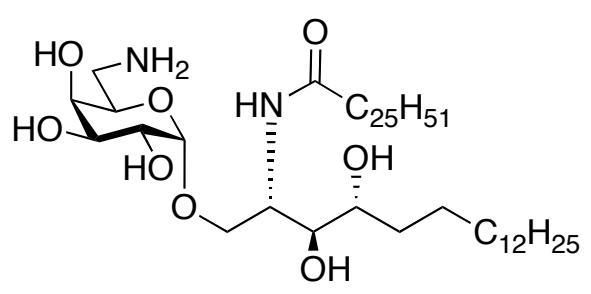

$(2 S, 3 S, 4 R)-1-(6-A m i n o-6-d e o x y-\alpha-D-$

galactopyranosyloxy)-2-hexacosanoylamido-octadecane-3,4-diol (51)

To a solution of glycolipid diol $69(25.1 \mathrm{mg}, 0.022 \mathrm{mmol})$ dissolved in $\mathrm{CHCl}_{3} / \mathrm{EtOH}(2.5 \mathrm{ml}, 3: 2, \mathrm{v} / \mathrm{v})$ was added formic acid $(0.13 \mathrm{~mL}, 0.0034 \mathrm{mmol})$ and $\mathrm{Pd}(\mathrm{OH})_{2} / \mathrm{C}\left(20 \mathrm{wt} \%\right.$ loading, $50 \mathrm{wt} \% \mathrm{H}_{2} \mathrm{O}, 36 \mathrm{mg}, 0.033 \mathrm{mmol}$ ) and the reaction stirred under $\mathrm{H}_{2}(\mathrm{~g})$ for $15 \mathrm{~h}$ at $\mathrm{rt}$. The reaction mixture was filtered through a celite pad and washed successively with $\mathrm{CHCl}_{3} / \mathrm{EtOH} / \mathrm{MeOH}(20 \mathrm{~mL}$, $3: 1: 1, \mathrm{v} / \mathrm{v} / \mathrm{v})$ and pyridine $(10 \mathrm{~mL})$ and concentrated in vacuo. The residue was semi-purified by silica gel column chromatography $\left(\mathrm{CH}_{2} \mathrm{Cl}_{2} \rightarrow \mathrm{CH}_{2} \mathrm{Cl}_{2} / \mathrm{MeOH} / \mathrm{EtOH} / \mathrm{NH}_{3} \quad(35 \%\right.$ aq $) \rightarrow 55: 2: 2: 1 \rightarrow 35: 2: 2: 1 \rightarrow 15: 2: 2: 1$, $\mathrm{v} / \mathrm{v} / \mathrm{v} / \mathrm{v})$ to afford amine 51 as an amorphous white solid (6.2 $\mathrm{mg}, 0.0072 \mathrm{mmol}$, 33\%). $\mathrm{R}_{f}: 0.50$ (DCM/MeOH/EtOH/ $\left.\mathrm{NH}_{3}-\mathrm{H}_{2} \mathrm{O}, 5: 2: 2: 1, \mathrm{v} / \mathrm{v} / \mathrm{v} / \mathrm{v}\right) ;{ }^{1} \mathrm{H}$ NMR (500 $\mathrm{MHz}, 10 \% \mathrm{CD}_{3} \mathrm{OD}$ in $\left.\mathrm{CDCl}_{3}\right) \delta 4.84\left(\mathrm{~d}, J_{1^{\prime}, 2^{\prime}}=3.9 \mathrm{~Hz}, 1 \mathrm{H}, \mathrm{H}-1^{\prime}\right), 4.12\left(\mathrm{dd}, J_{\mathrm{NH}, 2}\right.$ $\left.=9.1 \mathrm{~Hz}, J_{1 \mathrm{a}, 2}=J_{1 \mathrm{~b}, 2}=4.9 \mathrm{~Hz}, 1 \mathrm{H}, \mathrm{H}-2\right), 3.82-3.79\left(\mathrm{~m}, 2 \mathrm{H}, \mathrm{H}-1 \mathrm{a}, \mathrm{H}-4{ }^{\prime}\right), 4.74-$ $4.73\left(\mathrm{~m}, 1 \mathrm{H}, \mathrm{H}-5^{\prime}\right), 3.71\left(\mathrm{dd}, J_{2^{\prime}, 3^{\prime}}=10.1 \mathrm{~Hz}, J_{1^{\prime}, 2^{\prime}}=3.9 \mathrm{~Hz}, 1 \mathrm{H}, \mathrm{H}-2^{\prime}\right), 3.63$ (dd, $\left.J_{2^{\prime}, 3^{\prime}}=10.1 \mathrm{~Hz}, J_{3^{\prime}, 4^{\prime}}=3.4 \mathrm{~Hz}, 1 \mathrm{H}, \mathrm{H}-3^{\prime}\right), 3.53\left(\mathrm{dd}, J_{1 \mathrm{a}, 1 \mathrm{~b}}=10.6 \mathrm{~Hz}, J_{1 \mathrm{~b}, 2}=4.9 \mathrm{~Hz}\right.$, $1 \mathrm{H}, \mathrm{H}-1 \mathrm{~b}), 3.47-3.42(\mathrm{~m}, 2 \mathrm{H}, \mathrm{H}-3, \mathrm{H}-4), 3.01\left(\mathrm{dd}, J_{6^{\prime} \mathrm{a}, 6^{\prime} \mathrm{b}}=13.0 \mathrm{~Hz}, J_{5,6^{\prime} \mathrm{a}}=7.6\right.$ $\left.\mathrm{Hz}, 1 \mathrm{H}, \mathrm{H}-6^{\prime} \mathrm{a}\right), 2.85\left(\mathrm{dd}, J_{6^{\prime} \mathrm{a}, 6^{\prime} \mathrm{b}}=13.0 \mathrm{~Hz}, J_{5,6^{\prime} \mathrm{b}}=3.6 \mathrm{~Hz}, 1 \mathrm{H}, \mathrm{H}-6^{\prime} \mathrm{b}\right), 2.12(\mathrm{t}$, $\left.J_{\alpha, \beta}=7.6 \mathrm{~Hz}, 2 \mathrm{H}, \mathrm{CH}_{2}-\alpha\right), 1.63-1.47\left(\mathrm{~m}, 4 \mathrm{H}, \mathrm{CH}_{2}-\beta, \mathrm{H}-5\right), 1.30-1.06$ (m, 68H, H-6-H-17, H- $\gamma-\mathrm{H}-(\omega-1)), 0.80\left(\mathrm{t}, J_{17,18}=J_{\omega-1, \omega}=6.9 \mathrm{~Hz}, 6 \mathrm{H}, \mathrm{H}-18, \mathrm{H}-\omega\right)$; HRMS(ESI) m/z calcd. for $\left[\mathrm{C}_{50} \mathrm{H}_{100} \mathrm{~N}_{2} \mathrm{O}_{8}+\mathrm{H}\right]^{+}$: 857.7552, obsd.: 857.7533. 


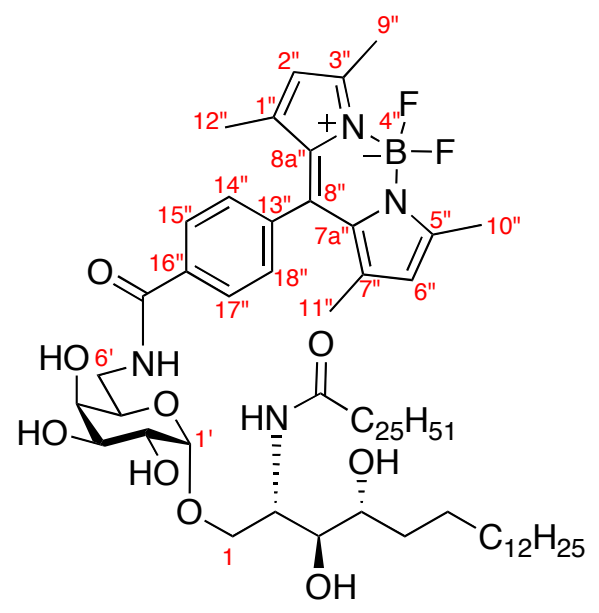

(2S,3S,4R)-1-(6-Deoxy-6-[4-(4,4-difluoro-

1,3,5,7-tetramethyl-3a,4a-diaza-4-bora-s-

indacen-8-yl)benzylamido])- $\alpha$-D-

galactopyranosyloxy)-2-

hexacosanoylamido-octadecane-3,4-diol (3) Amine 51 (4.1 mg, $0.0048 \mathrm{mmol})$ was coevaporated with DMF (x 3), dissolved in dry DMF $(0.6 \mathrm{~mL})$ and the solution was warmed to $45{ }^{\circ} \mathrm{C}$ followed by the addition of DiPEA

(3.3 $\mu \mathrm{L}, 0.019 \mathrm{mmol})$. BODIPY-NHS 70 (9.2 $\mathrm{mg}, 0.020 \mathrm{mmol})$ was coevaporated with DMF (x 3) and added as a solution in DMF $(0.6 \mathrm{~mL})$ and stirred for $1 \mathrm{~h}$ at $45{ }^{\circ} \mathrm{C}$, at which point additional BODIPY-NHS 70 (4.6 mg, 0.010 mmol $)$ in dry $\mathrm{CH}_{2} \mathrm{Cl}_{2}(0.6 \mathrm{~mL})$ and DiPEA $(3.3 \mu \mathrm{L}, 0.019 \mathrm{mmol})$ was added and the reaction mixture stirred for further $14 \mathrm{~h}$ at $45{ }^{\circ} \mathrm{C}$. The reaction mixture was then concentrated in vacuo and purified immediately by silica gel gradient column chromatography $\left(\mathrm{CH}_{2} \mathrm{Cl}_{2}\right.$ to $3 \% \mathrm{MeOH}$ in $\left.\mathrm{CH}_{2} \mathrm{Cl}_{2}\right)$ to afford BODIPY- $\alpha$-GalCer 3 as a red solid (2.5 mg, $0.0021 \mathrm{mmol}, 44 \%)$. $\mathrm{R}_{f}: 0.38(10 \% \mathrm{MeOH} / \mathrm{DCM}, \mathrm{v} / \mathrm{v})$; IR (film) 3346, 2851, 1642, 1631, 1608, 1547, 1513, 1468, 1450, 1409, 1308, 1192, 1157, 1071, 1044, 983, 759, 700, $635 \mathrm{~cm}^{-1} ;{ }^{1} \mathrm{H}$ NMR (600 MHz, pyridine-d $\left.\mathrm{d}_{5}\right) \delta$ $9.74\left(\mathrm{t}, J_{6^{\prime} \mathrm{a}, \mathrm{NH}}=J_{6^{\prime} \mathrm{b}, \mathrm{NH}}=5.6 \mathrm{~Hz}, 1 \mathrm{H}, \mathrm{NH}-\mathrm{BOD}\right), 8.67\left(\mathrm{~d}, J_{2, \mathrm{NH}}=8.5 \mathrm{~Hz}, 1 \mathrm{H}, \mathrm{NH}-\right.$ lipid), 8.46 (d, $\left.J_{14^{\prime \prime}, 15^{\prime \prime}}=J_{17^{\prime \prime}, 18^{\prime \prime}}=8.2 \mathrm{~Hz}, 2 \mathrm{H}, \mathrm{H}-15^{\prime \prime}, \mathrm{H}-17^{\prime \prime}\right), 7.34$ (d, $J_{14^{\prime \prime}, 15^{\prime \prime}}=$ $\left.J_{17^{\prime \prime}, 18^{\prime \prime}}=8.2 \mathrm{~Hz}, 2 \mathrm{H}, \mathrm{H}-14^{\prime \prime}, \mathrm{H}-18^{\prime \prime}\right), 5.99$ (s, 2H, H-2", $\left.\mathrm{H}-6^{\prime \prime}\right), 5.57$ (d, $J_{1^{\prime}, 2^{\prime}}=$ $\left.3.9 \mathrm{~Hz}, 1 \mathrm{H}, \mathrm{H}-1^{\prime}\right), 5.29-5.27(\mathrm{~m}, 1 \mathrm{H}, \mathrm{H}-2), 4.70\left(\mathrm{t}, J_{5^{\prime}, 6^{\prime} \mathrm{a}}=J_{5^{\prime}, 6^{\prime} \mathrm{b}}=6.3 \mathrm{~Hz}, 1 \mathrm{H}, \mathrm{H}-\right.$ $\left.5^{\prime}\right), 4.68-4.65\left(2 \mathrm{H}, \mathrm{H}-1 \mathrm{a}, \mathrm{H}-2^{\prime}\right), 4.49$ (dd, $J_{5^{\prime}, 6^{\prime} \mathrm{a}}=6.3 \mathrm{~Hz}, J_{6^{\prime} \mathrm{a}, 6^{\prime} \mathrm{b}}=13.4 \mathrm{~Hz}, 1 \mathrm{H}, \mathrm{H}-$ $\left.6^{\prime} \mathrm{a}\right), 4.46\left(\mathrm{~d}, J_{3^{\prime}, 4^{\prime}}=1.3 \mathrm{~Hz}, 1 \mathrm{H}, \mathrm{H}-4^{\prime}\right), 4.40-4.37$ (m, 2H, H-1b, H-3'), 4.35-4.23 (m, 2H, H-3, H-4), 4.22-4.19 (m, 1H, H-6'b), 2.71 (s, 6H, H-9' , H-10' ), 2.48 (t, $\left.J_{\alpha, \beta}=5.6 \mathrm{~Hz}, 2 \mathrm{H}, \mathrm{CH}_{2}-\alpha\right), 2.30-2.27\left(\mathrm{~m}, 1 \mathrm{H}, \mathrm{CH}_{2}\right), 1.97-1.91\left(\mathrm{~m}, 3 \mathrm{H}, \mathrm{H}-5, \mathrm{CH}_{2}\right)$, 1.85-1.80 (m, 3H, $\left.\mathrm{CH}_{2}-\beta, \mathrm{CH}_{2}\right), 1.71-1.69$ (m, 2H, $\left.\mathrm{CH}_{2}\right), 1.36$ (s, 6H, H-11' ", H$\left.12^{\prime \prime}\right), 1.45-1.17$ (m, 63H, H-6-H-17, H- $\left.\gamma-\mathrm{H}-(\omega-1)\right), 0.88$ (t, 6H, H-18, H- $\left.\omega\right)$; ${ }^{13} \mathrm{C}$ NMR (150 MHz, pyridine-d $\left.{ }_{5}\right) \delta 173.7$ (HNC=O lipid), 168.1 (HNC=O BOD),

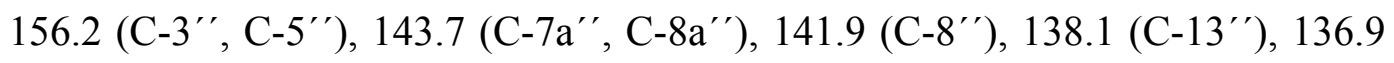

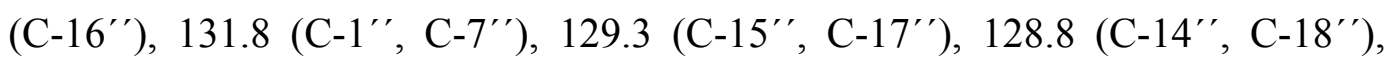


122.2 (C-2"', C-6"'), 77.1 (C-3/C-4), 72.9 (C-3/C-4), 71.64 (C-3'), 71.57 (C-4'), 70.8 (C-5'), 70.4 (C-2'), 69.1 (C-1), 51.8 (C-2), 72.3 (C-6'), 37.2 (C- $\alpha$ ), 34.7, $32.5,30.8,30.5,30.41,30.40,30.39,30.37,30.36,30.34,30.33,30.29,30.27$, 30.26, 30.18, 30.14, 29.98, 29.97, 23.29 (C-6-C-17, C- $\gamma-\mathrm{C}-(\omega-1)), 26.89$ (C-5), 26.79 (C- $\beta$ ) $, 15.1,14.9$ (C-9", C-10"', C-11"', C-12"'), 14.6 (C-18, C$\omega) ; \operatorname{HRMS}(E S I) \quad \mathrm{m} / \mathrm{z}$ calcd. for $\left[\mathrm{C}_{70} \mathrm{H}_{117} \mathrm{BF}_{2} \mathrm{~N}_{4} \mathrm{O}_{9}+\mathrm{H}\right]^{+}:$1207.8954, obsd.: 1207.8958 . 


\subsection{References}

1. $\quad$ Loudet, A.; Burgess, K., Chem. Rev. 2007, 107, 4891-932.

2. Zhou, X.; Forestier, C.; Goff, R. D.; Li, C.; Teyton, L.; Bendelac, A.; Savage, P. B., Org. Lett. 2002, 4, 1267-1270.

3. Trappeniers, M.; Van Beneden, K.; Decruy, T.; Hillaert, U.; Linclau, B.; Elewaut, D.; Van Calenbergh, S., J. Am. Chem. Soc. 2008, 130, 1646816469.

4. Bai, L.; Sagiv, Y.; Liu, Y.; Freigang, S.; Yu, K. O. A.; Teyton, L.; Porcelli, S. A.; Savage, P. B.; Bendelac, A., Proc. Natl. Acad. Sci. U. S. A. 2009, 106, 10254-10259.

5. Aspeslagh, S.; Li, Y.; Yu, E. D.; Pauwels, N.; Trappeniers, M.; Girardi, E.; Decruy, T.; Van Beneden, K.; Venken, K.; Drennan, M.; Leybaert, L.; Wang, J.; Franck, R. W.; Van Calenbergh, S.; Zajonc, D. M.; Elewaut, D., EMBO J. 2011, 30, 2294-305.

6. Pauwels, N.; Aspeslagh, S.; Vanhoenacker, G.; Sandra, K.; Yu, E. D.; Zajonc, D. M.; Elewaut, D.; Linclau, B.; Van Calenbergh, S., Org. Biomol. Chem. 2011, 9, 8413-8421.

7. Huang, Y.-C.; Chiang, L.-W.; Chang, K.-S.; Su, W.-C.; Lin, Y.-H.; Jeng, K.-C.; Lin, K.-I.; Liao, K.-Y.; Huang, H.-L.; Yu, C.-S., Molecules 2012, 17, 3058-3081.

8. Brellier, M.; Duportail, G.; Baati, R., Tetrahedron Lett. 2010, 51, 12691272.

9. Cheng, J. M. H.; Chee, S. H.; Knight, D. A.; Acha-Orbea, H.; Hermans, I. F.; Timmer, M. S. M.; Stocker, B. L., Carbohydr. Res. 2011, 346, 914-26.

10. Li, C.; Sun, Y.; Zhang, J.; Zhao, Z.; Yu, G.; Guan, H., Carbohydr. Res. 2013, 376, 15-23.

11. Chee, S. BODIPY Fluorescent probes to study glycolipid uptake and trafficking in cancer immunotherapy. Honours Project, Victoria University of Wellington 2012.

12. Mayer, T.; Kratzer, B.; Schmidt, R., Angew. Chem. Int. Ed. 1994, 33, $2177-$ 2181.

13. Kratzer, B.; Mayer, T.; Schmidt, R., Eur J Org Chem 1998, 291-298.

14. Zhu, X.; Schmidt, R. R., Angew. Chem. Int. Ed. 2009, 48, 1900-34.

15. Plettenburg, O.; Bodmer-Narkevitch, V.; Wong, C., J. Org. Chem. 2002, 67, 4559-4564.

16. Xia, C.; Yao, Q.; Schumann, J.; Rossy, E.; Chen, W.; Zhu, L.; Zhang, W.; De Libero, G.; Wang, P., Bioorg. Med. Chem. Lett. 2006, 16, 2195-2199.

17. Xia, C.; Zhang, W.; Zhang, Y.; Woodward, R. L.; Wang, J.; Wang, P. G., Tetrahedron 2009, 65, 6390-6395.

18. Du, W.; Kulkarni, S. S.; Gervay-Hague, J., Chem. Commun. 2007, 2336-8.

19. Khaja, S. D.; Kumar, V.; Ahmad, M.; Xue, J.; Matta, K. L., Tetrahedron Lett. 2010, 51, 4411-4414.

20. Ramjit, H. G.; Newton, R.; Guare, J. P., Rapid Commun. Mass Spectrom. 2005, 19, 1257-1262.

21. Yen, Y.-F.; Sawant, R.; Luo, S.-Y., Synthesis 2013, 45, 511-517.

22. Kim, S.; Song, S.; Lee, T.; Jung, S.; Kim, D., Synthesis 2004, 847-850. 
23. Morita, M.; Motoki, K.; Akimoto, K.; Natori, T.; Sakai, T.; Sawa, E.; Yamaji, K.; Koezuka, Y.; Kobayashi, E.; Fukushima, H., J. Med. Chem. 1995, 38, 2176-2187.

24. Xarnod, C.; Huang, W.; Ren, R.-G.; Liu, R.-C.; Wei, B.-G., Tetrahedron 2012, 68, 6688-6695.

25. Bubb, W. A., Concepts Magn. Reson. 2003, 19A, 1-19.

26. Lightner, D. A.; Crandall, D. C., Tetrahedron Lett. 1973, 14, 1799-1802.

27. Norris, R. D.; Lightner, D. A., J. Heterocycl. Chem. 1979, 16, 263-272.

28. Jones, G.; Klueva, O.; Kumar, S.; Pacheco, D. P., Solid State Lasers X, SPIE 2001, 4267, 24-35.

29. Quistad, G. B.; Lightner, D. A., Tetrahedron Lett. 1971, 12, 4417-4420.

30. Ulrich, G.; Ziessel, R.; Haefele, A., J. Org. Chem. 2012, 77, 4298-311. 


\section{Chapter 4. Synthesis of terminal sugar homologues of iGb3}

\subsection{Introduction}

The glycolipid isoglobotrihexosylceramide (iGb3 4, Figure 4.1) is a triglycosylated ceramide that can activate iNKT cells when presented by CD1d. Structurally, it differs greatly from the prototype potent foreign antigen $\alpha$ galactosylceramide in that it contains a trisaccharide head group, possesses a $\beta$ linkage between the proximal sugar and the lipid portion, and contains a sphingosine backbone bearing an E-alkene at C4-C5. Despite this structural difference, crystal structure studies have revealed that the T cell receptor (TCR) of iNKT cells is able to interact with the iGb3 presented on CD1d, ${ }^{1,2}$ hence confirming that the activity observed in iNKT cell stimulation assays was indeed exerted by the intact iGb3, rather than truncated versions which may occur after lysosomal processing.

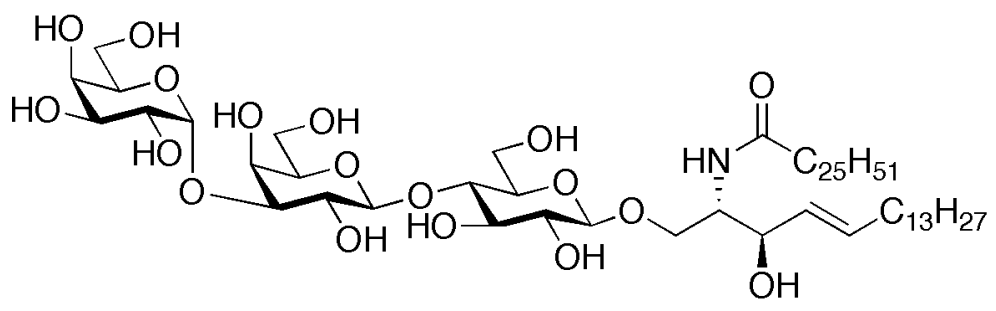

Figure 4.1. Isoglobotrihexosylceramide (iGb3, 4).

The ability of the iNKT cell to accommodate this structural diversity is fascinating and it provides a platform to further understand the structural requirements for iNKT cell agonists. In 2011, both Rossjohn and co-workers and $\mathrm{Yu}$ et al. simultaneously reported the crystal structure of the ternary complex, iNKT TCRiGb3-CD1d and suggested that the main reason for $\beta$-linked glycolipids activating iNKT cells in a manner similar to $\alpha$-linked glycolipids is because the TCR of the 
iNKT cell is able to "bulldoze" the protruding headgroup so that it sits flat against the $\alpha 2$-helix of CD1d. ${ }^{2,3}$ The internal glycosidic bond is thus forced to resemble the $\alpha$-conformation. Indeed, the crystal structure studies reveal that the terminal galactose sugar forms crucial interactions with CD1d. Specifically, the 6"'hydroxyl of iGb3 forms a hydrogen bond with Thr159 of CD1d, and both the 4"' and 6"'-hydroxyls partake in van der Waals interactions with Thr159 and Met162 (Figure 4.2).

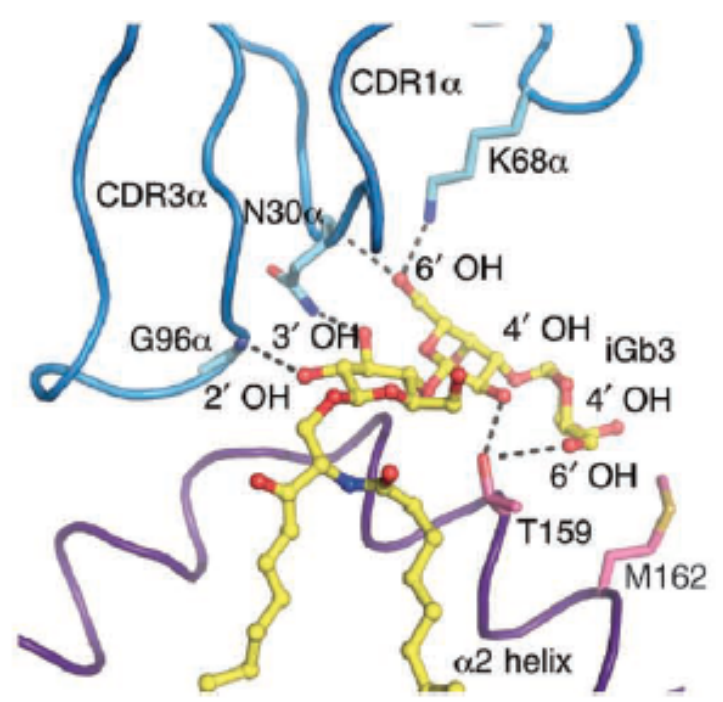

Figure 4.2. Crystal structure of iNKT cell TCR (blue) in engagement with iGb3 (yellow) and murine CD1d (purple). ${ }^{2}$ (Adapted by permission from Macmillan Publishers Ltd: Nature Immunology, 2011, 12, 827-833, C2011)

To unambiguously ascertain the importance of the 6 "' -hydroxyl in iGb3 binding and the activation of iNKT cells, the synthesis of 6 "'-deoxy-iGb3-sphingosine 5 (Figure 4.3) was envisioned so that its activity as an iNKT cell ligand can be established. 6""'-deoxy-iGb3-sphingosine 5 will also allow for crystal structure studies to be performed by the groups of Jamie Rossjohn and Dale Godfrey with whom we are collaborating. A similar derivative, the 6"'-deoxy-iGb3phytosphingosine, ${ }^{4}$ which contains the lipid found in $\alpha$-GalCer, has been synthesised by Chen et al. but no crystal structure data was presented. Herein, however, it is envisioned that our target $6^{\prime \prime \prime}$-deoxy homologue 5, bearing the same sphingosine backbone as the parent iGb3, will be a more useful tool to 
systematically probe the role of the terminal sugar without changing the lipid portion.
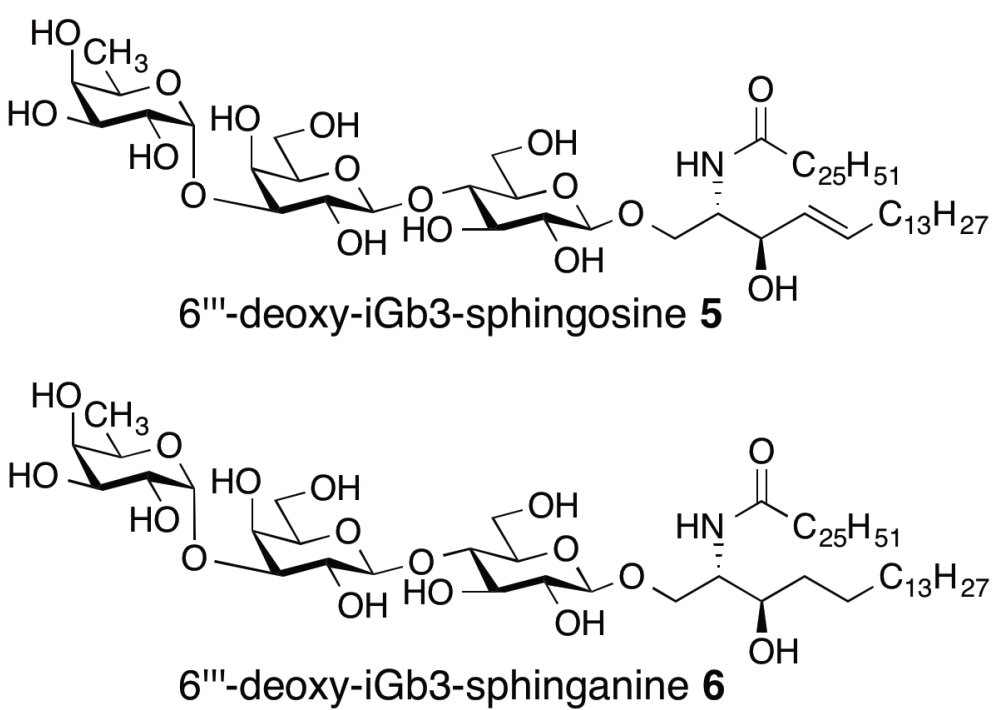

Figure 4.3. Target iGb3 analogues, 5 and 6.

In addition to the $6{ }^{\prime \prime \prime}$-deoxy homologue 5, the 6"'-deoxy-iGb3-sphinganine 6 (Figure 4.3) is also a useful tool to determine if removal of the rigid $E$-alkene on the lipid backbone alters activity of the glycolipid. Hereby, it should also be noted that the iGb3-sphinganine analogue (with galactose as the terminal sugar) has been synthesised and found to activate iNKT cells more efficiently than the original iGb3-sphingosine. ${ }^{5}$ Various studies have also shown that changes to the lipid backbone of a glycolipid can alter the presentation of the sugar head group. ${ }^{6-8}$ Thus, the biological assessment of $6^{\prime \prime \prime}$-deoxy-iGb3-sphinganine 6 will help determine if the loss of rigidity in the lipid backbone will compensate for the loss of H-bond through the 6"' -hydroxyl of the terminal sugar. 


\subsection{Synthesis of 6"'-deoxy homologues of iGb3}

\section{Retrosynthesis}

To synthesise 6"'-deoxy-iGb3-sphingosine 5 and 6"'-deoxy-iGb3-sphinganine 6 a convergent strategy was employed whereby the fully protected 6 "' -deoxy-iGb3sphingosine derivative $\mathbf{7 1}$ could be selectively deprotected to give either target (Scheme 4.1). Here, global deprotection via Birch reduction will afford sphingosine 5 with the double bond still intact, while catalytic hydrogenation followed by hydrolysis will afford sphinganine $\mathbf{6}$. The fully protected glycolipid 71 could in turn be obtained via the glycosylation of lactosylceramide (LacCer) acceptor $\mathbf{7 3}$ with the D-fucosyl donor $\mathbf{7 2}$, itself obtained in several steps from Dgalactose 39. LacCer acceptor $\mathbf{7 3}$ could be obtained from lactosyl 2-azidosphingosine 74 via coupling with hexacosanoic acid followed by hydrolysis of the isopropylidene protecting group and selective acetylation of the 4"-OH. Lactosyl 2-azido-sphingosine $\mathbf{7 4}$ could be formed by the coupling of lactosyl donor $\mathbf{7 5}$ and sphingosine acceptor 76. Finally, the lactosyl donor 75 could be prepared from Dlactose (77) and the sphingosine acceptor $\mathbf{7 6}$ from D-arabinose (78) according to published procedures. ${ }^{9,10}$ 

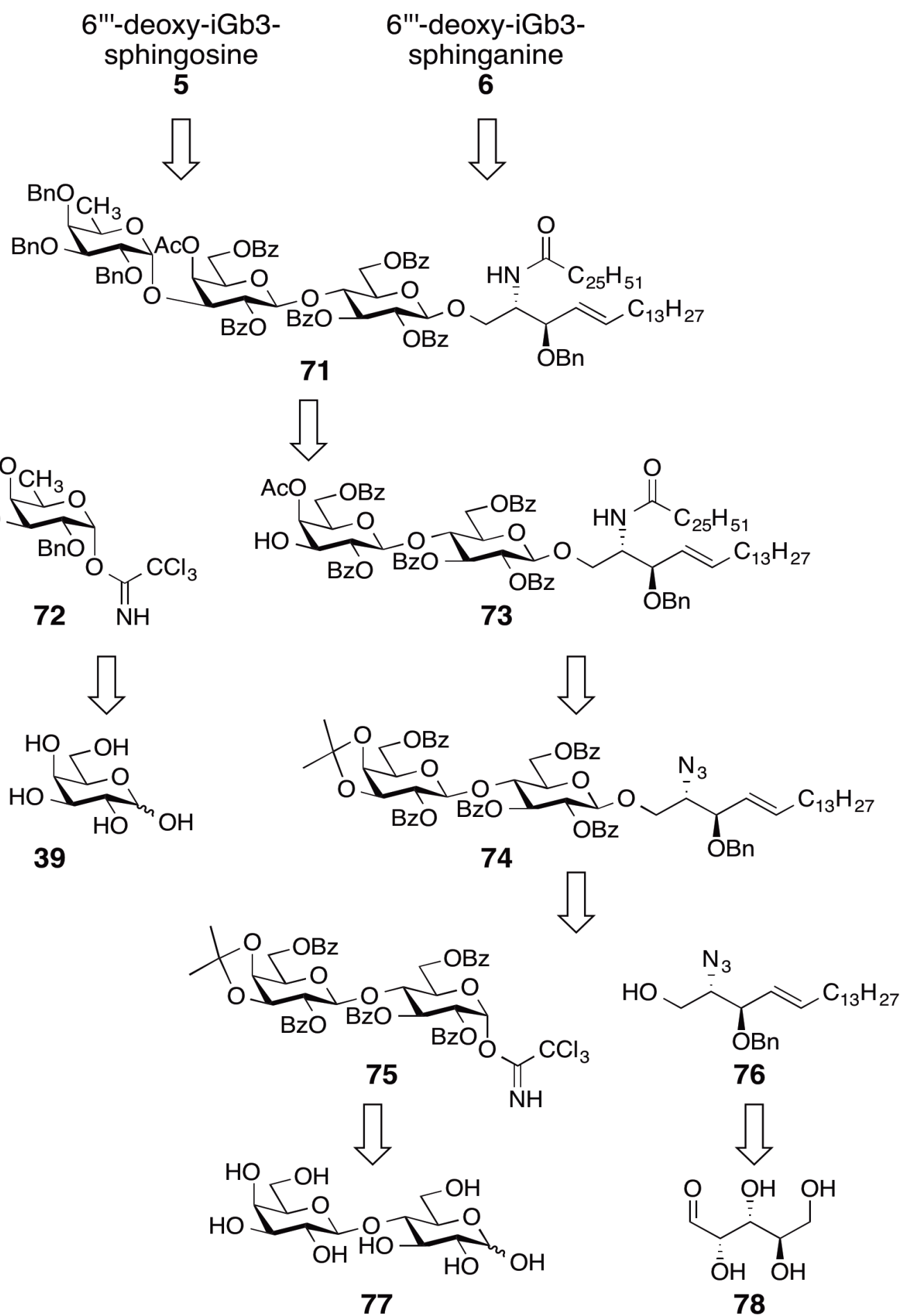

Scheme 4.1. Retrosynthesis of target 6 "'-deoxy-iGb3 sphingosine 5 and 6 "'deoxy-iGb3-sphinganine 6. 


\section{Synthesis of lactosylceramide (LacCer) acceptor}

The synthesis of 6"' -deoxy-iGb3-sphingosine 5 and 6" '-deoxy-iGb3-sphinganine 6 began with the preparation of the appropriately protected lactosyl 2-azido sphingosine intermediate 74 (Scheme 4.2). To this end, D-lactose (77) was peracetylated, followed by the conversion of the anomeric acetate to a thiophenol group and removal of the remaining acetate groups under Zemplén conditions to give thiolactoside $\mathbf{7 9}$ as a crystalline solid according to literature procedure..$^{9,11-14}$ Thiolactoside 79 was then subjected to 2,2-dimethoxypropane and a catalytic amount of $p$-toluenesulfonic acid $(p \mathrm{TsOH})$ to afford the selectively protected $3^{\prime}, 4^{\prime}$-ketal 80. While these conditions generally yield the thermodynamically favoured 5-membered $3^{\prime}, 4^{\prime}$-ketal $\mathbf{8 0}$, some of the kinetic $4^{\prime}, 6^{\prime}$-derivative $\mathbf{8 1}$ was also obtained. ${ }^{14-16}$ Efforts to drive the exclusive formation of the $3^{\prime}, 4^{\prime}-$ ketal $\mathbf{8 0}$ by allowing for a longer reaction time (3 days) was to no avail. Moreover, Xing et al. and Chan et al. also showed that performing the reaction at warmer temperatures did not increase the yield of the desired product significantly. ${ }^{9,17}$ Fortunately, the $3^{\prime}, 4^{\prime}$ - $O$-isopropylidene derivative 80 could be isolated in $56 \%$ yield from the mixture by crystallisation. Subsequent protection of the free hydroxyl groups as benzoate esters afforded the fully protected thioglycoside $\mathbf{8 2}$ in 94\% yield, again as crystalline material. Next, the anomeric thiophenol was hydrolysed with NBS in aqueous acetone ${ }^{9,18}$ followed by the installation of the trichloroacetimidate leaving group ${ }^{9,16,19}$ to give lactosyl imidate donor $\mathbf{7 5}$ in $73 \%$ yield over the two steps. Spectral data of lactosyl imidate donor $\mathbf{7 5}$ matched that previously reported, ${ }^{9,16}$ which was obtained from D-lactose (77) via the use of either an anomeric $p$-toluenethiol group ${ }^{9}$ or an anomeric thexyldimethylsilyl group. ${ }^{16}$ 


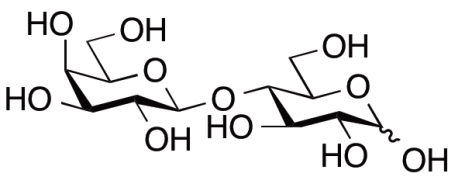

77
1) $\mathrm{Ac}_{2} \mathrm{O}, \mathrm{NaOAC}$

2) $\mathrm{SnCl}_{4}, \mathrm{PhSH}, \mathrm{DCM}$

3) $\mathrm{NaOMe}, \mathrm{MeOH}$

45\% (3 steps)

(2)
79

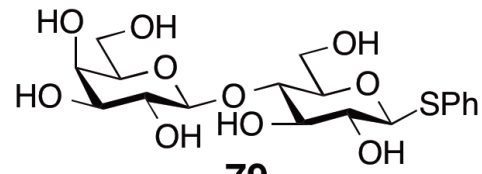

$(\mathrm{MeO})_{2} \mathrm{C}\left(\mathrm{CH}_{3}\right)_{2}, \mathrm{pTsOH}$, acetone, $56 \%$

$\downarrow$

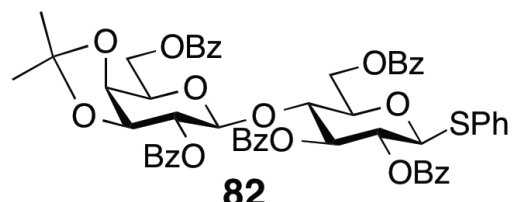

82

BzCl, pyridine, 94\%

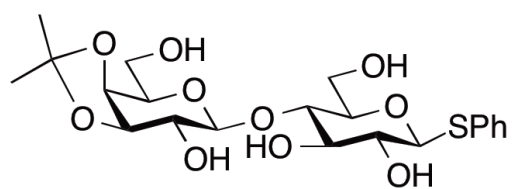

80
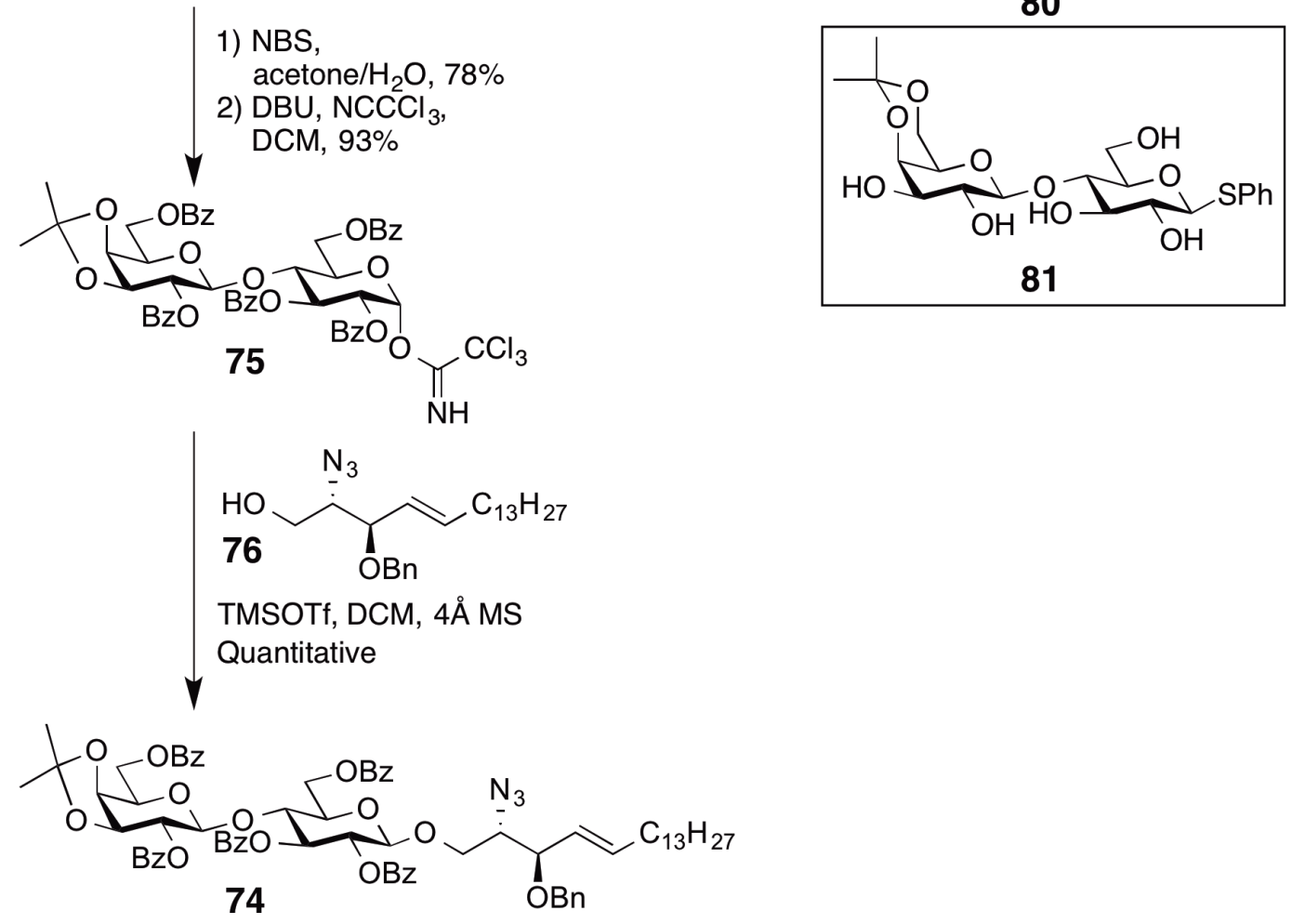

Scheme 4.2. Synthesis of lactosyl 2-azido-sphingosine 74.

With the lactosyl imidate donor $\mathbf{7 5}$ in hand, a coupling reaction to sphingosine acceptor 76 was then performed (Scheme 4.2), whereby sphingosine acceptor 76 was prepared from D-arabinose (78), in nine steps using a strategy previously developed in our group. ${ }^{10}$ The glycosylation reaction utilised TMSOTf as a promoter and proceeded smoothly to give lactosyl 2-azido sphingosine $\mathbf{7 4}$ in quantitative yield. ${ }^{9}{ }^{1} \mathrm{H}$ NMR analysis of the resulting glycolipid revealed a coupling constant of $7.8 \mathrm{~Hz}$ between $\mathrm{H}^{-1}{ }^{\prime}$ and $\mathrm{H}-2^{\prime}$, which confirms the $\beta$ selectivity of the reaction. This stereoselectivity was enabled by the participation of the benzoyl protecting group at C2. Interestingly, when Xing et al. coupled the 
identical lactosyl donor to the full ceramide sphingosine acceptor bearing a C18 acyl lipid under similar activating conditions, the yield was only $60 \%$, presumably due to poorer solubility of the dilipid acceptor. ${ }^{9}$

Following the coupling of the lipid, the azide on the sphingosine backbone was reduced with $\mathrm{PPh}_{3}$ to produce the corresponding amine, which was used without further purification in the condensation with hexacosanoic acid using an EDCI/DMAP-mediated protocol (Scheme 4.3). Under these conditions the fully protected LacCer 83 was produced in 54\% yield over two steps. The acetonide on the 3 "'- and 4" -positions was then removed via hydrolysis under acidic conditions to afford diol 84 in an excellent $96 \%$ yield. The regioselective protection of the 4 "'-OH was then performed by using trimethyl orthoacetate and camphorsulfonic acid to first install a methylorthoacetate protecting group across the 3 "'-OH and 4"'-OH, which subsequently ring opened during an acidic workup to afford the LacCer acceptor 73. 

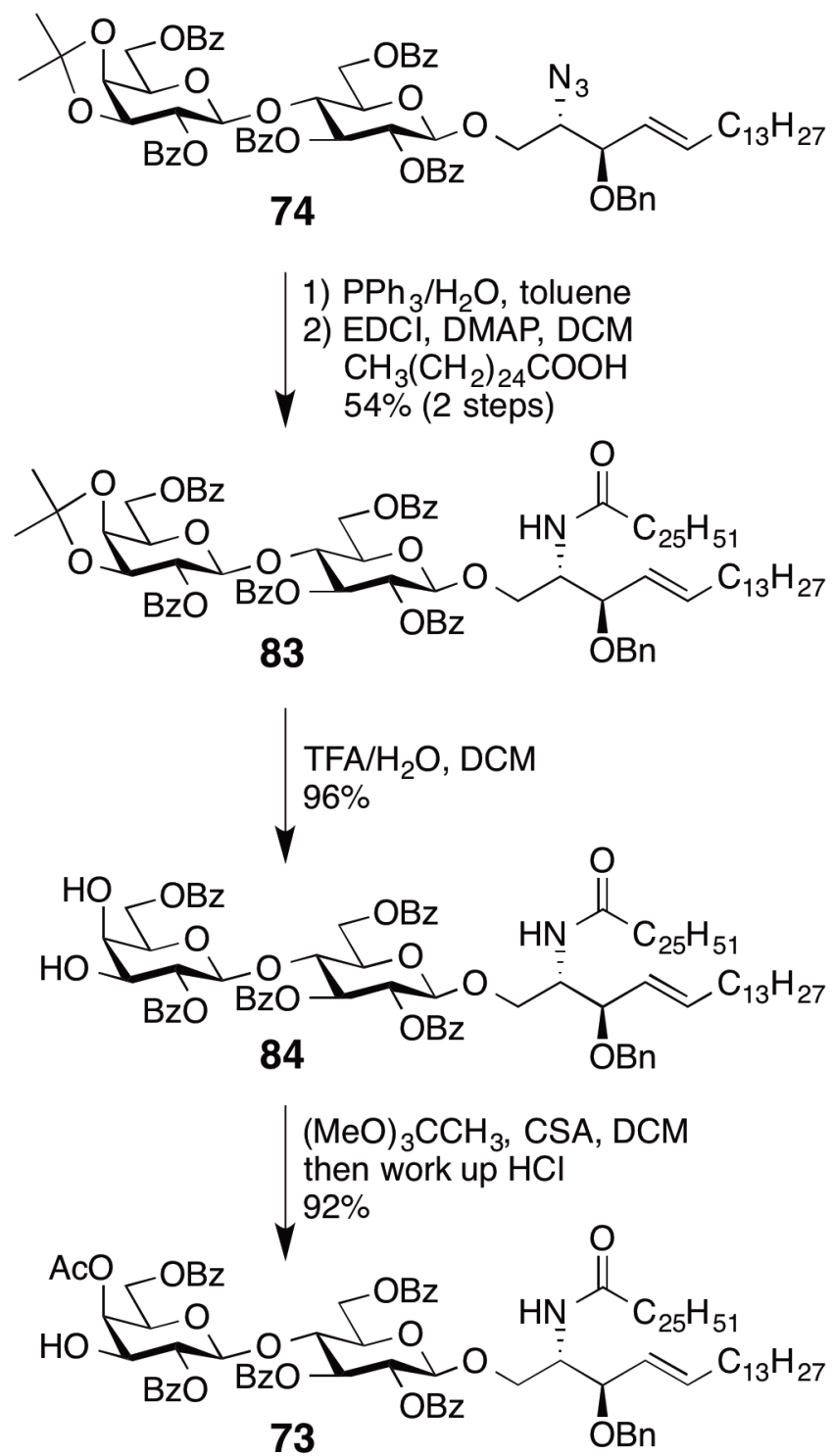

Scheme 4.3. Synthesis of LacCer acceptor 73.

The position of the acetate ester was confirmed, firstly by the chemical shift of the hydrogens at the $3^{\prime \prime}$ and 4"'-positions. As expected, because of the electron withdrawing nature of ester protecting groups, the narrow doublet with a small coupling constant $\left(J_{3^{\prime \prime}, 4^{\prime \prime}}=3.5 \mathrm{~Hz}\right.$, ) characteristic of $\mathrm{H}-4^{\prime \prime}$ is located downfield $(5.22 \mathrm{ppm})$ compared to the doublet of doublets $\left(J_{2^{\prime \prime}, 3^{\prime \prime}}=9.8 \mathrm{~Hz}, J_{3^{\prime \prime}, 4^{\prime \prime}}=3.5 \mathrm{~Hz}\right)$ for H-3"', which is located at $3.82 \mathrm{ppm}$. Additionally, HMBC correlation between the carbonyl carbon of the acetate and the proton at the 4"'-position unambiguously confirms the acetylation of the 4 "'-OH (Figure 4.4). 


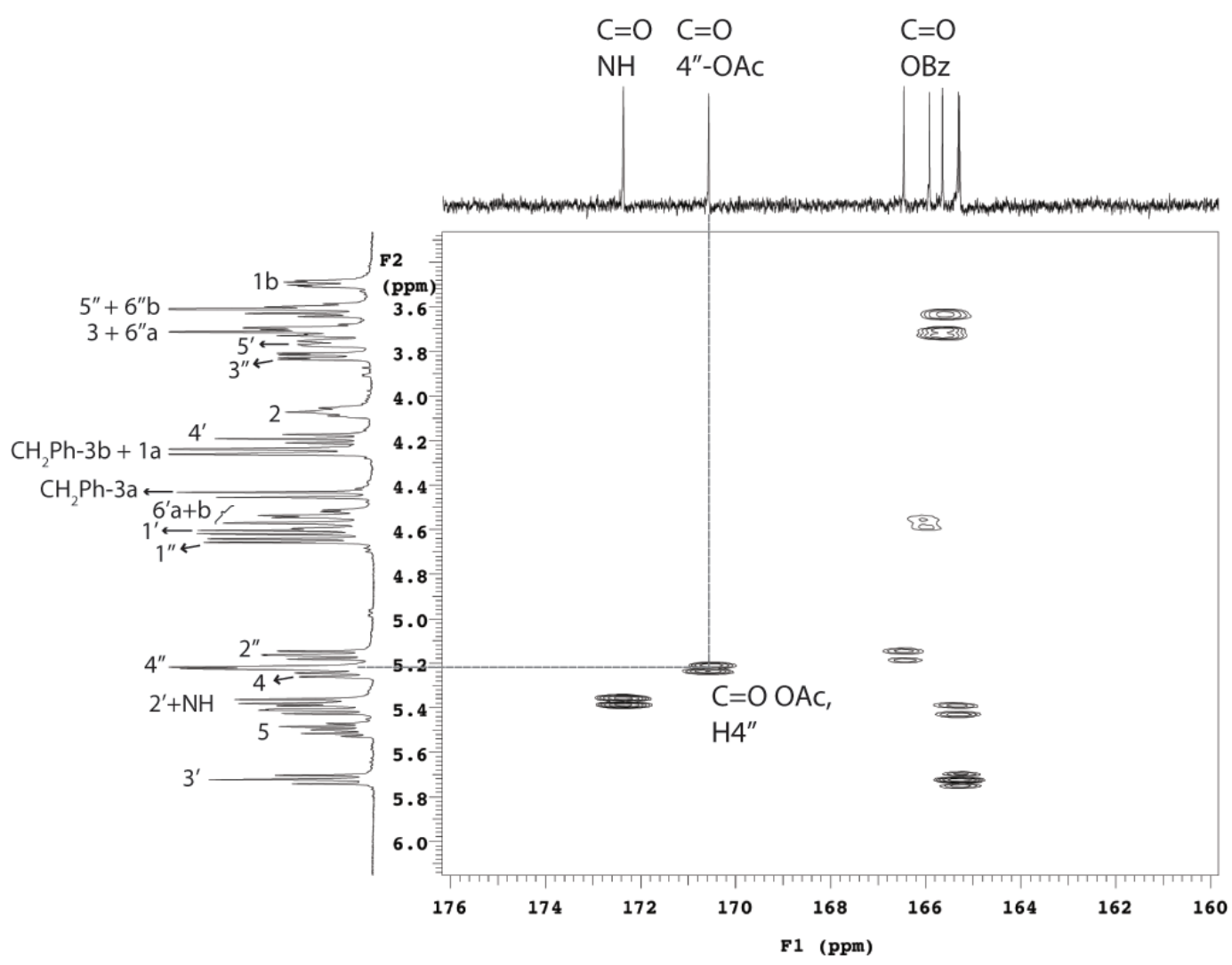

Figure 4.4. HMBC of LacCer acceptor 73 confirming acetylation at position 4".

This versatile approach to acetylate the axial 4-OH over equatorial 3-OH in a cisdiol system was first reported by Lemieux et al. ${ }^{20}$ and the selectivity can be rationalised by stereoelectronic effects. ${ }^{21,22}$ During acidic workup, the methoxy group of intermediate $\mathbf{B}$ is substituted by water (to give tetrahedral intermediate C), and subsequent protonation of either the equatorial oxygen at C-3 (D) or the axial oxygen at C-4 (E) determines the formation of the 4-OAc (F) or 3-OAc (G) product, respectively (Scheme 4.4). The less sterically hindered oxygen at the equatorial position is preferentially protonated over the axial oxygen, ${ }^{23}$ leading to the selective acetylation at the 4-position (F), giving rise to LacCer acceptor 73 . In summary, the LacCer acceptor $\mathbf{7 3}$ was prepared in twelve steps from D-lactose (77) in $8 \%$ overall yield. 


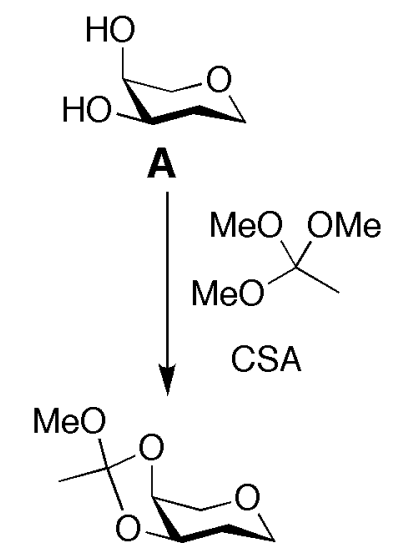

B

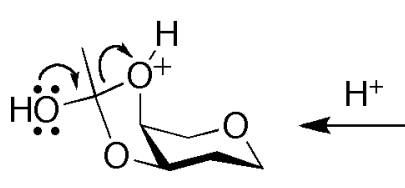

E

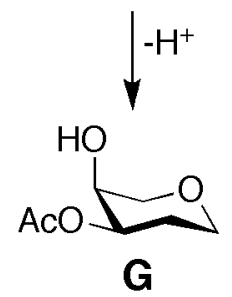$$
\downarrow \mathrm{H}^{+}, \mathrm{H}_{2} \mathrm{O}
$$
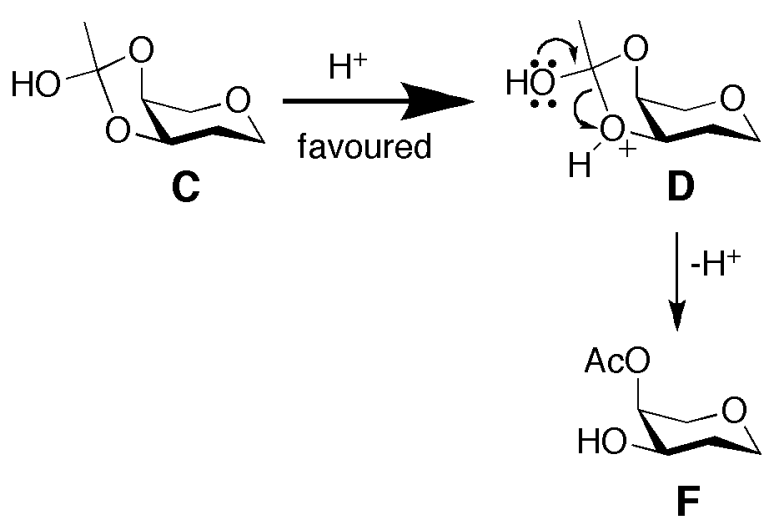

Scheme 4.4. Regioselective acetylation of the 3,4-cis-diol system of galactose. 


\section{Synthesis of thioethyl D-fucoside donor}

Having successfully prepared the LacCer acceptor 73, the next synthetic target was the $\beta$-thioethyl fucose donor 90 (Scheme 4.5). Glycosylation reactions incorporating both D- or L-fucose donors are notorious for poor reaction yields due to the increased acid lability of the $\alpha$-fucosyl linkage. ${ }^{24-26}$ Therefore, mild activating conditions are required for their glycosidation. It was envisioned that $\beta$ thioethyl fucose donor $\mathbf{9 0},{ }^{27,28}$ which can be activated under milder conditions using $\mathrm{CuBr}_{2}$ and $\mathrm{Pr}_{4} \mathrm{NBr}$, will circumvent the problem of low yields. This glycosidation protocol was previously optimised within the group from Ogawa's method. $^{29}$

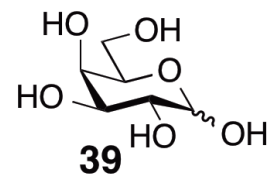

1) Acetone, $\mathrm{ZnCl}_{2}$, $\mathrm{H}_{2} \mathrm{SO}_{4}, 92 \%$

2) $\mathrm{PPh}_{3}, \mathrm{I}_{2}$, Imid. $\checkmark 85 \%$

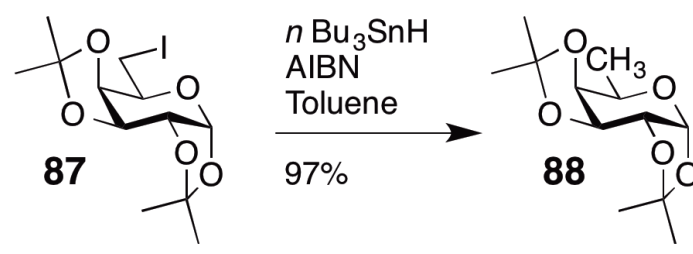

1) $\mathrm{AcOH} / \mathrm{H}_{2} \mathrm{O}$ 2) $\mathrm{Ac}_{2} \mathrm{O}$, pyridine $85 \%$ (2 steps)

1) $\mathrm{NaOMe}, \mathrm{MeOH}$

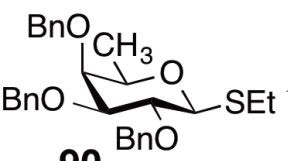

90

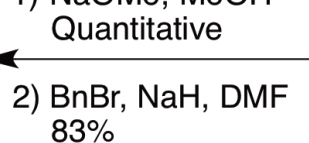

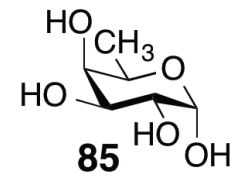

$\mathrm{Ac}_{2} \mathrm{O}$, pyridine Quantitative
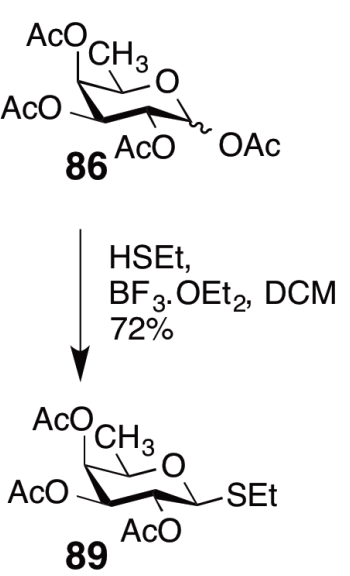

Scheme 4.5. Synthesis of $\beta$-thioethyl fucose donor 90 .

The synthesis of the D-fucose donor building block began with peracetylated Dfucose 86, which can be obtained in quantitative yield by subjecting D-fucose (85) to acetic anhydride and pyridine. However, due to the high cost of the starting 
material D-fucose $(\mathbf{8 5})$, an alternative route to make the peracetylated D-fucose intermediate 86, starting from D-galactose (39) was developed. Iodogalactoside 86 can be made according to methods described earlier (Chapter 2, Scheme 2.3) whereby the 1,2 and 3,4 positions of D-galactose (39) were protected with isopropylidenes followed by the installation of an iodide at the primary position via a modified Appel reaction. ${ }^{30}$ Next, reduction of the iodide under conditions akin to the Barton-McCombie deoxygenation, whereby the radical initiator AIBN and reducing agent tributyltin hydride were used, provided 6-deoxy galactose $\mathbf{8 8}$ in an excellent $97 \%$ yield. ${ }^{31}$ Subsequent hydrolysis of the isopropylidene groups under acidic conditions, followed by peracetylation gave D-fucose tetraacetate $\mathbf{8 6}$ in $85 \%$ yield over two steps. According to this route, D-fucose tetraacetate $\mathbf{8 6}$ was made from D-galactose 39 in 65\% yield over five steps. The robust methodology for the synthesis of D-fucose intermediates, along with the significantly reduced cost of D-galactose (60 times cheaper than D-fucose) provides great incentive to start with the more readily available D-galactose.

Following this, $\beta$-thiofucoside 89 was prepared by converting the anomeric acetate to the thioethyl functionality by using boron trifluoride etherate as the Lewis acid (72\% yield). The remaining acetate protecting groups were then hydrolysed under Zemplén conditions and the resultant free hydroxyls benzylated to give benzylated $\beta$-thiofucoside donor $\mathbf{9 0}$ in $83 \%$ yield over two steps.

\section{Attempted coupling of thioethyl fucoside 90 donor and LacCer acceptor 73}

With the thiofucose donor 90 and LacCer acceptor $\mathbf{7 3}$ in hand, the coupling reaction was attempted by using $\mathrm{CuBr}_{2}$ and $\mathrm{Pr}_{4} \mathrm{NBr}$, but to no avail (Scheme 4.6). No desired product was observed, instead, the self-coupled donor 1,1'-difucose and unreacted acceptor $\mathbf{7 3}$ were obtained. It was speculated that perhaps the lipophilic acceptor was not as nucleophilic while the activated fucose donor was highly reactive, thus leading to the 1,1'-difucose by-product in the presence of trace amount of water. 


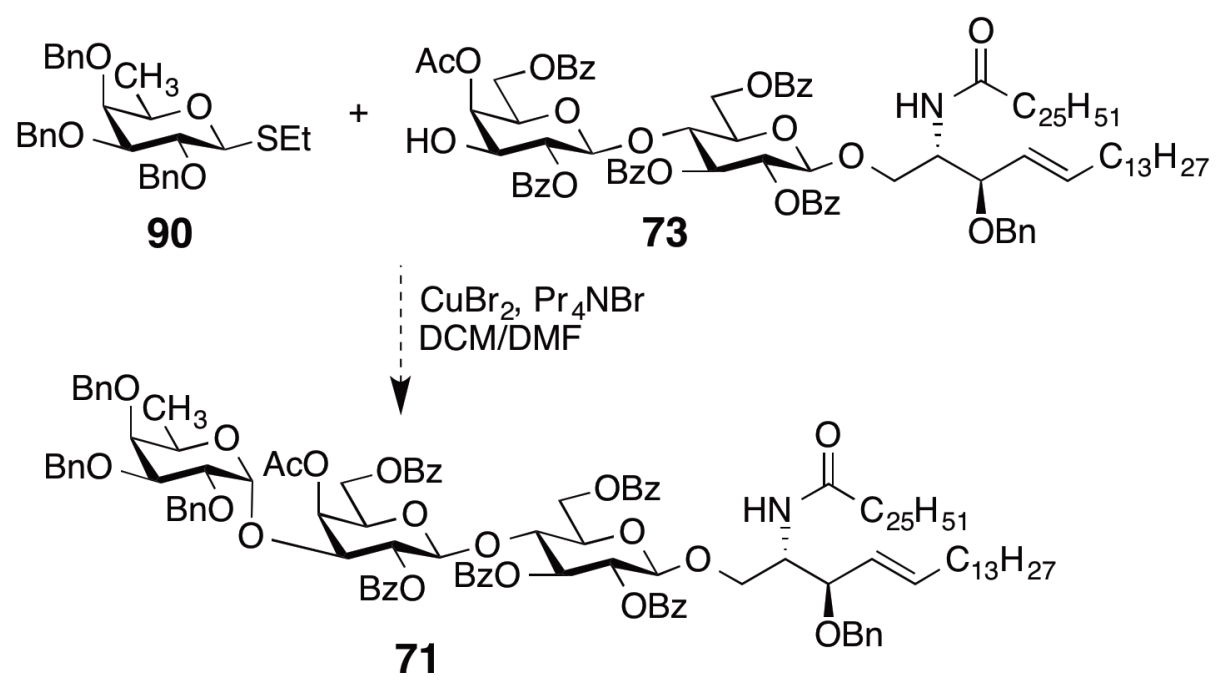

Scheme 4.6. Attempted coupling of thioethyl donor 90 and LacCer acceptor 73.

\section{Modification to the trichloroacetimidate donor 72}

Therefore, an alternative donor, the trichloroacetimidate was synthesised in two steps from the thiofucoside donor 90 (Scheme 4.7). Hydrolysis of the anomeric thioethyl group in the presence of NBS and acetone ${ }^{18}$ afforded the corresponding lactol 91 in 93\% yield. The anomeric hydroxyl was then converted to a trichloroacetimidate group in an excellent 94\% yield to afford the desired fucose donor 72. ${ }^{32}$ Here, both $\alpha$ and $\beta$ anomers were generated in a 2:1 ratio, and they were separable by silica gel column chromatography. Of note, due to the prevalence of L-fucose in biological systems, only L-fucosyl imidate donors have been reported. This work represents the first synthesis of the D-fucosyl imidate. As expected, the ${ }^{1} \mathrm{H}$ and ${ }^{13} \mathrm{C}$ NMR data of $\alpha$-D-fucosyl imidate 72 were identical to that of the enantiomer, $\alpha$-L-fucosyl imidate, ${ }^{33}$ and the optical rotation was equal in value but opposite in magnitude $\left([\alpha]_{\mathrm{D}}^{23} \alpha\right.$-D-fucosyl imidate $\mathbf{7 2}=+58^{\circ} ; \mathrm{c}=1.0$, $\mathrm{CHCl}_{3} ;[\alpha]_{\mathrm{D}}{ }^{20} \alpha$-L-fucosyl imidate $\left.=-60^{\circ} ; \mathrm{c}=1.0, \mathrm{CHCl}_{3}{ }^{33}\right)$

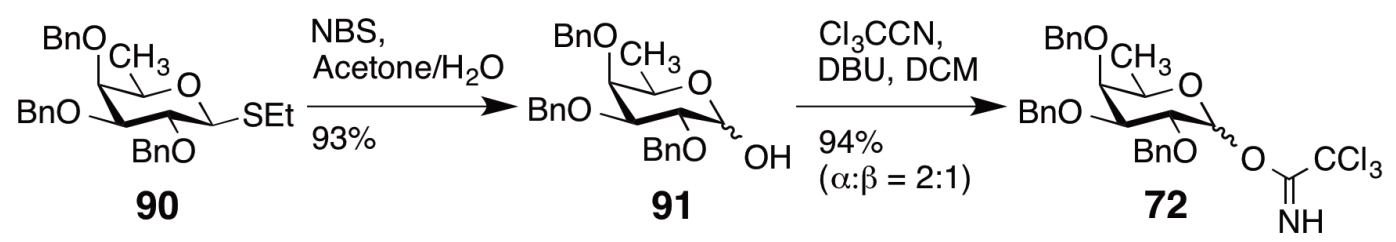

Scheme 4.7. Synthesis of trichloroacetimidate fucose donor $\mathbf{7 2}$. 


\section{Coupling of trichloroacetimidate donor 72 and LacCer acceptor 73 and global deprotection}

Though coupling reactions using either $\beta$-L-fucosyl imidate ${ }^{34,35}$ or $\alpha / \beta$ mixtures of L-fucosyl imidate ${ }^{36}$ have been reported, only the $\alpha$-anomer of $D$-fucosyl imidate 72 was used in the glycosylation reaction with LacCer acceptor 73 so as to follow Schmidt's original coupling conditions, ${ }^{33}$ and to eliminate any ambiguity in the effects of starting with a mixture of anomers on $\alpha / \beta$-selectivity of the coupling reaction.

The coupling of $\alpha$-fucosyl imidate donor $\mathbf{7 2}$ and the LacCer acceptor $\mathbf{7 3}$ was performed with TMSOTf as the catalyst (Scheme 4.8). ${ }^{33}$ Gratifyingly, this reaction proceeded smoothly to afford the fully protected 6"' -deoxy-iGb3 71 in good $69 \%$ yield.

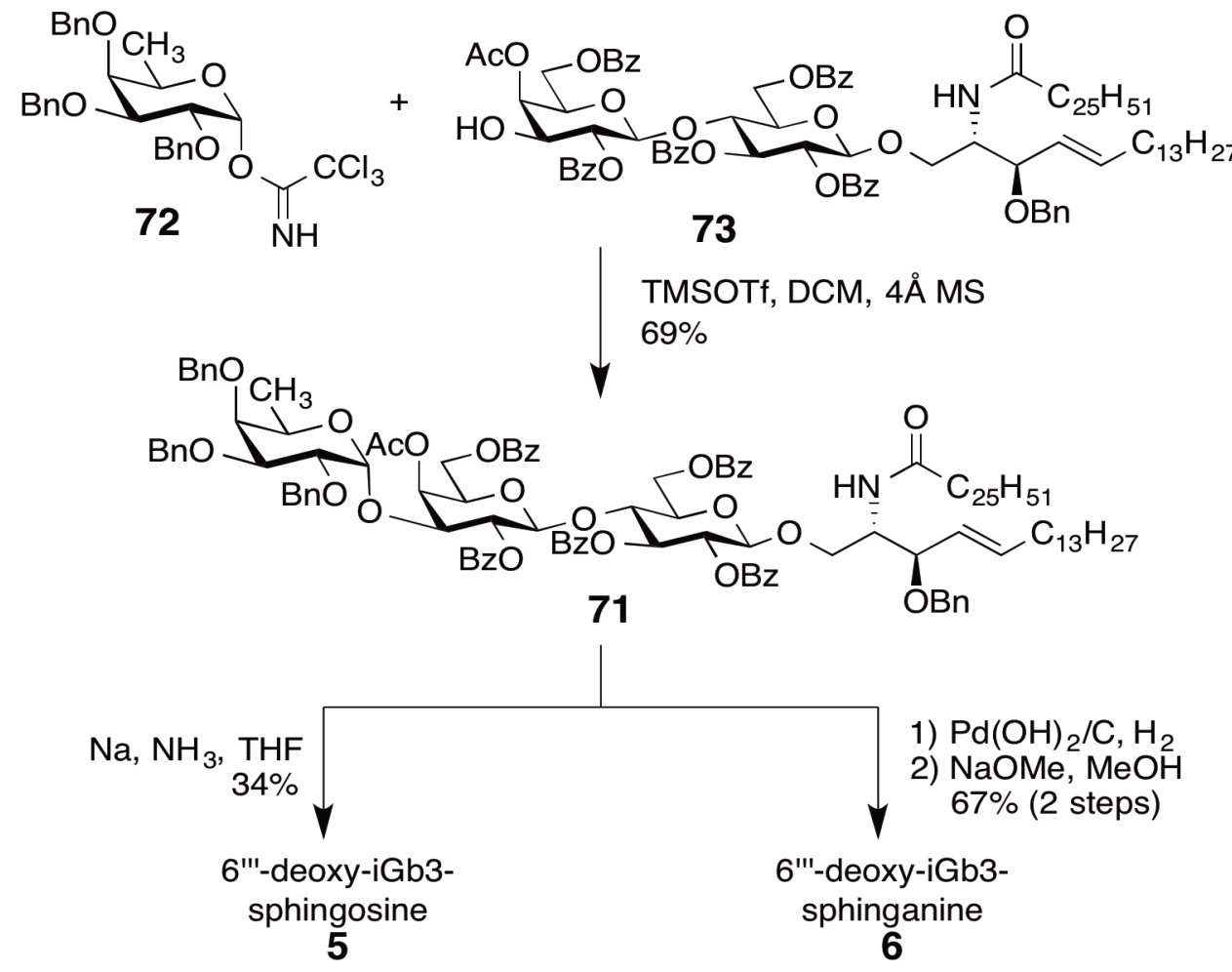

Scheme 4.8. Total syntheses of $6^{\prime \prime \prime}$-deoxy-iGb3-sphingosine 5 and $6{ }^{\prime \prime \prime}$-deoxyiGb3-sphinganine 6.

Analysis of the product by ${ }^{1} \mathrm{H}$ NMR revealed that the coupling constant of $\mathrm{H}-1$ '"' at the newly formed anomeric centre was $3.5 \mathrm{~Hz}$, confirming the formation of the 
$\alpha$-anomer. No $\beta$-coupled product was detected and unreacted acceptor 73 was recovered. Three bond correlations of key glycosidic bonds from HMBC analysis are shown in Figure 4.5. Specifically, the correlation between C-3"' and H-1"' confirms the attachment of the terminal fucose moiety to the 3 " position of LacCer. In addition, an HMBC correlation between H-4" and carbonyl carbon of the acetate further confirms that the LacCer was glycosylated at the 3" (Figure 4.6).

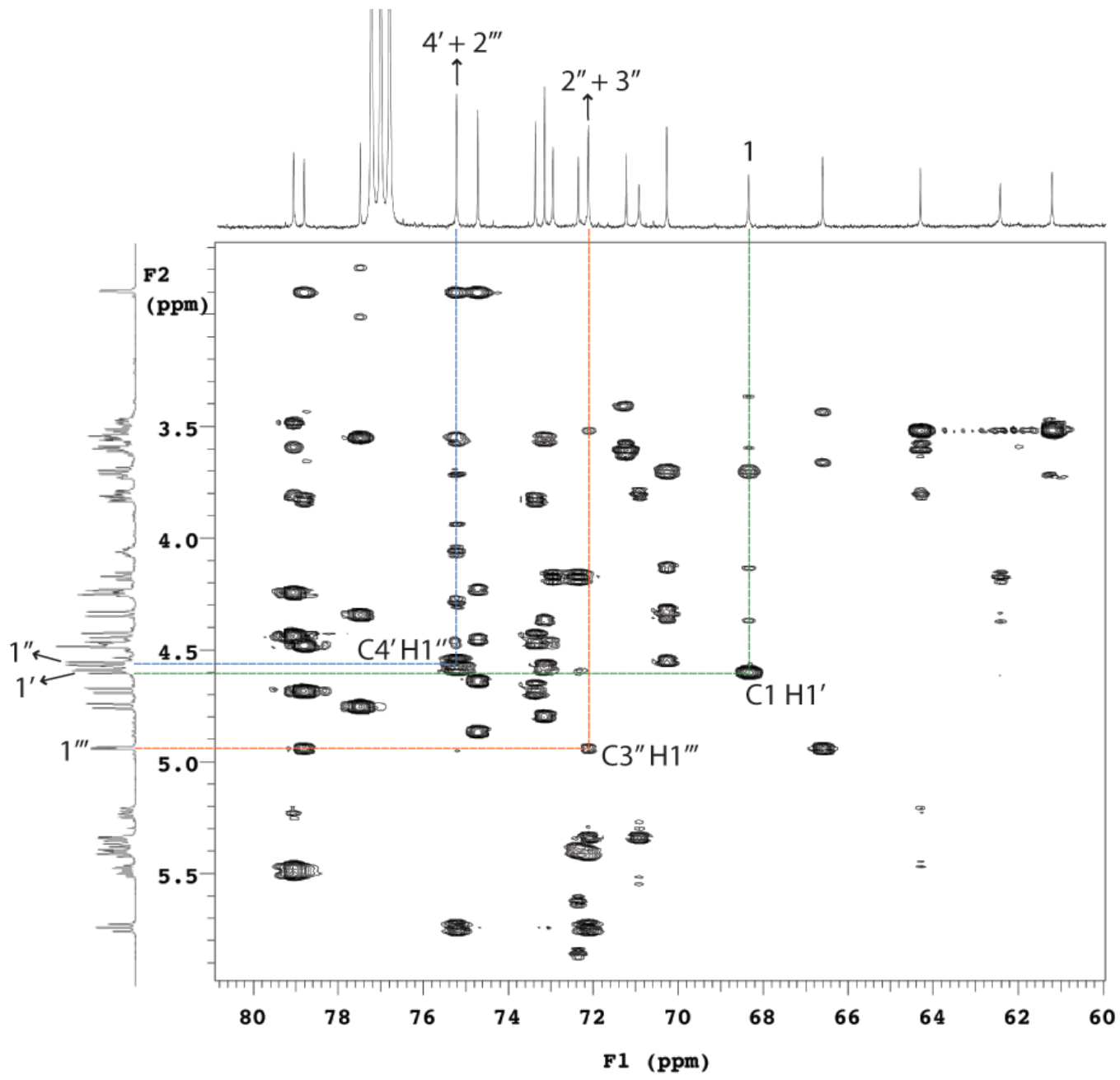

Figure 4.5. HMBC spectrum of 6 "' -deoxy-iGb3 71 confirming the fucosylation of LacCer 73 at the 3"'-position. 


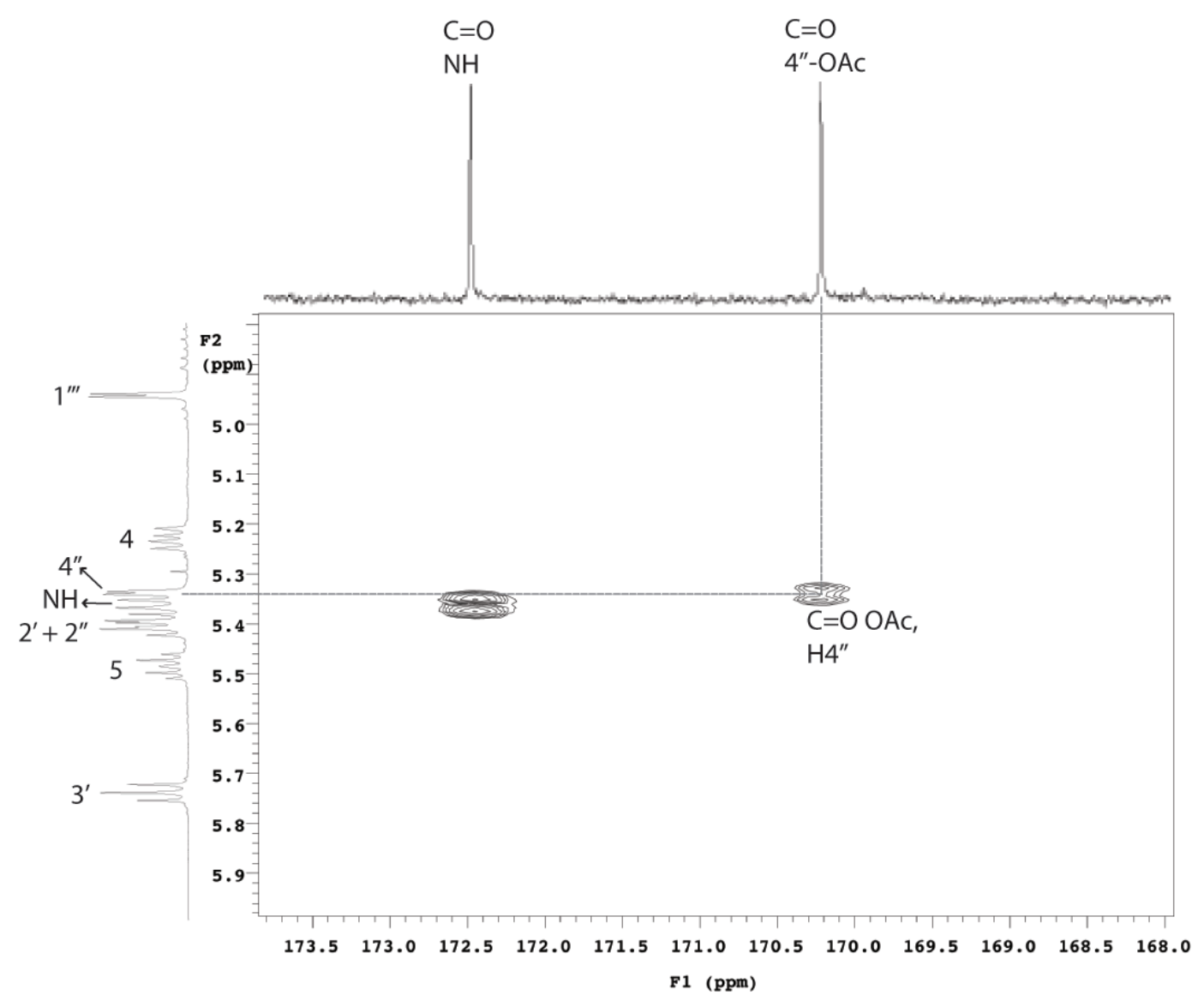

Figure 4.6. HMBC of 6" -deoxy-iGb3 71 confirming acetylation at position 4"'.

With the fully protected 6 "'-deoxy-iGb3 71 at hand, global deprotection under Birch conditions was performed and this allowed for the removal of all ether and ester protecting groups without reducing the alkene. As large amounts of ammonium salts are produced in the Birch reduction, purification by a reverse phase chromatography on a C18 column was attempted, but to no avail as the poor solubility of the amphiphilic glycolipid made the loading of the column rather difficult. Fortunately, upon careful purification by silica gel column chromatography, the target 6 "'-deoxy-iGb3-sphingosine 5 could be isolated in $34 \%$ yield. While the yield of this final step was modest, limited quantities of the fully protected glycolipid $\mathbf{7 1}$ prevented further optimisation of this procedure.

Alternatively, hydrogenation with Pearlman's catalyst followed by the hydrolysis of the esters (acetate and benzoates) under Zemplén condition afforded 6"'deoxy-iGb3-sphinganine 6 in $67 \%$ yield over two steps. In contrast to the sphingosine derivative $\mathbf{5}$, purification of the fully deprotected sphinganine 
analogue 6 was more straightforward as the glycolipid could be obtained by precipitating the glycolipid with methanol, followed by centrifugation of the mixture to form a glycolipid pellet and removal of the sodium methoxidecontaining methanol.

Full characterisation by mass spectrometry, infrared spectroscopy, ${ }^{1} \mathrm{H}$ and ${ }^{13} \mathrm{C}$ NMR confirmed the structure and identity of both target glycolipids. Specifically, analysis by mass spectrometry confirmed the identity of 6 "'-deoxy-iGb3sphingosine 5 (HRMS ESI m/z calcd. for $\left[\mathrm{C}_{62} \mathrm{H}_{117} \mathrm{NO}_{17}+\mathrm{H}\right]^{+}:$1148.8394, obsd.: 1148.8374) and $6^{\prime \prime \prime}$-deoxy-iGb3-sphinganine $6 \mathrm{~m} / \mathrm{z}$ calcd. for $\left[\mathrm{C}_{62} \mathrm{H}_{120} \mathrm{NO}_{17}+\mathrm{H}\right]^{+}:$1150.8551, obsd.: 1150.8553). The key difference between the two target glycolipids is the presence of the $E$-alkene in sphingosine 5, which was confirmed via NMR spectroscopy by the signature doublet of doublets (H-4) and doublet of triplets (H-5) at $6.04 \mathrm{ppm}$ and $5.93 \mathrm{ppm}$, respectively and their corresponding carbon signal at $132.6 \mathrm{ppm}$ and $133.0 \mathrm{ppm} .{ }^{1} \mathrm{H}$ and ${ }^{13} \mathrm{C} \mathrm{NMR}$ data is in agreement with other iGb3 analogues such as the $6^{\prime \prime \prime}$-deoxy-iGb3phytosphingosine $^{4}$ and the iGb3-sphinganine analogue. ${ }^{5}$

\subsection{Conclusion}

In this work, two analogues of iGb3 were synthesised. An iGb3 derivative lacking the 6 "' $-\mathrm{OH}$ on the terminal galactose sugar, 6" 6 -deoxy-iGb3-sphingosine 5 was synthesised in $2 \%$ overall yield over 14 steps (longest linear sequence). A further derivative, 6"'-deoxy-iGb3-sphinganine 6, which lacks the alkene on the ceramide backbone was made in $4 \%$ overall yield over 15 steps (longest linear sequence). The highlight of this synthesis was the high yielding coupling between lactosyl imidate donor and sphingosine acceptor, which proceeded with excellent $\beta$-selectivity. The selective acetylation of the 4"'-position of LacCer also allowed for the regioselective fucosylation of the 3 "' position, which proceeded smoothly when the trichloroacetimidate donor was employed. These 6"'-deoxy-iGb3 derivatives will allow for future assessment of their structure activity relationship in terms of iNKT cell activation, as well as analysis of structural interaction with the TCR of iNKT cells and CD1d by X-ray crystallography. 


\subsection{Experimental}

\section{General procedure}

Unless otherwise stated all reactions were performed under argon. Prior to use, THF (Pancreac) was distilled from sodium and benzophenone, pyridine was distilled and dried over $4 \AA$ molecular sieves ( $4 \AA$ MS), DCM (Pancreac) was distilled from $\mathrm{P}_{2} \mathrm{O}_{5}$, and $\mathrm{H}_{2} \mathrm{O}$ and benzene (Fisher Scientific) were distilled. $\mathrm{SnCl}_{4}$ (Aldrich), PhSH (Koch-Light Laboratories), benzaldehyde dimethyl acetal (Aldrich), $\mathrm{Me}_{2} \mathrm{C}(\mathrm{OMe})_{2}$ (Aldrich), NBS (Aldrich), DBU (Merck), CSA (Acros), $n \mathrm{Bu}_{3} \mathrm{SnCl}$ (Aldrich), AIBN (Aldrich), D-fucose (Aldrich), D-lactose (Aldrich), trityl chloride (Acros), anhydrous $\mathrm{Et}_{2} \mathrm{O}$ (Pancreac), $\mathrm{PPh}_{3}$ (Aldrich), $\mathrm{Pd}(\mathrm{OH})_{2} / \mathrm{C}$ (Aldrich, $20 \mathrm{wt} \%$ ), anhydrous DMF (Acros), TFA (Aldrich), $p \mathrm{TsOH}$ (Aldrich), TMSOTf (Aldrich), $\mathrm{H}_{2} \mathrm{SO}_{4}$ (Lab-Scan), formic acid (Aldrich), AcCl (Aldrich), $\mathrm{BnBr}$ (Fluka), $\mathrm{PMe}_{3}$ (Aldrich, $1 \mathrm{M}$ in THF), $\mathrm{AcOH}$ (Ajax Finechem), $\mathrm{Ac}_{2} \mathrm{O}$ (Peking Reagent), TMSOTf (Aldrich), DiPEA (Aldrich), NaOMe (Janssen Chimica), trichloroacetonitrile (Aldrich), $\mathrm{C}_{25} \mathrm{H}_{51} \mathrm{COOH}$ (Acros), $\mathrm{BzCl}$ (Aldrich, distilled an stored under argon), HBTU (Acros), PyBOP (Aldrich), EDCI (Aldrich), DMAP (Merck), sodium (Aldrich), trimethyl orthoacetate (Aldrich), $\mathrm{LiAlH}_{4}$ (Aldrich), EtOAc (Pancreac), hexanes (Fisher Scientific), petroleum ether (Pure Science), $\mathrm{MeOH}$ (Pure Science), $\mathrm{CHCl}_{3}$ (Pancreac), EtOH (absolute, Pure Science), $\mathrm{NaOH}$ (Pure Science), $\mathrm{NaHCO}_{3}$ (Pure Science), $\mathrm{NaCl}$ (Pancreac), $\mathrm{NH}_{3}$ (BOC gasses) were used as received. All solvents were removed by evaporation under reduced pressure. Reactions were monitored by TLC-analysis on MachereyNagel silica gel coated plastic sheets $\left(0.20 \mathrm{~mm}\right.$, with fluorescent indicator $\left.\mathrm{UV}_{254}\right)$ with detection by UV-absorption (short wave UV - $254 \mathrm{~nm}$; long wave UV - 366 $\mathrm{nm}$ ), by dipping in $10 \% \mathrm{H}_{2} \mathrm{SO}_{4}$ in $\mathrm{EtOH}$ followed by charring at $\sim 150{ }^{\circ} \mathrm{C}$, by dipping in $\mathrm{I}_{2}$ in silica, or by dipping into a solution of ninhydrin in EtOH followed by charring at $\sim 150{ }^{\circ} \mathrm{C}$. Column chromatography was performed on Pure Science silica gel (40-63 micron). AccuBOND II ODS-C18 (Agilent) was used for reverse phase chromatography. Infrared spectra were recorded as thin films using a Bruker Tensor 27 FTIR spectrometer equipped with an Attenuated Total 
Reflectance (ATR) sampling accessory and are reported in wave numbers $\left(\mathrm{cm}^{-1}\right)$. Nuclear magnetic resonance spectra were recorded at $20{ }^{\circ} \mathrm{C}$ in $\mathrm{CD}_{3} \mathrm{OD}, \mathrm{CDCl}_{3}$, or pyridine- $\mathrm{d}_{5}$ using either a Varian INOVA operating at $500 \mathrm{MHz}$ or Varian VNMRS operating at $600 \mathrm{MHz}$. Chemical shifts are given in ppm ( $\delta$ ) relative to TMS. NMR peak assignments were made using COSY, HSQC and HMBC 2D experiments.

\section{Phenyl 4- $O$-( $\beta$-D-galactopyranosyl)-1-thio- $\beta$-D-glucopyranoside (79)}

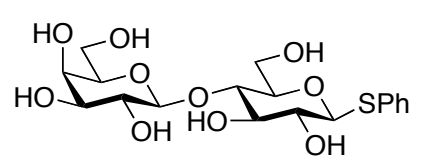

A solution of sodium acetate $(20 \mathrm{~g}, 244 \mathrm{mmol})$ in acetic anhydride $(100 \mathrm{~mL}, 1058 \mathrm{mmol})$ was heated to reflux and D-lactose (77) (40 g, $117 \mathrm{mmol})$ was added in small portions over 30 mins. The resulting mixture was refluxed for $2 \mathrm{~h}$, after which it was cooled to rt and poured over ice and stirred for $1 \mathrm{~h}$. The white solid was filtered and washed with $\mathrm{H}_{2} \mathrm{O}(100 \mathrm{~mL})$. The filtrate was dissolved in Et2O (800 $\mathrm{mL})$ and the organic layer was washed with $\mathrm{H}_{2} \mathrm{O}(2 \times 800 \mathrm{~mL})$, dried $\left(\mathrm{MgSO}_{4}\right)$, filtered and concentrated in vacuo to afford peracetylated D-lactose as a white solid (55 g, $81 \mathrm{mmol}, 70 \%)$. Peracetylated D-lactose (17 g, $25 \mathrm{mmol}$ ) was dissolved in $\mathrm{CH}_{2} \mathrm{Cl}_{2}(125 \mathrm{~mL})$ and thiophenol $(2.09 \mathrm{~mL}, 30 \mathrm{mmol})$ was added. The reaction mixture was then cooled to $0{ }^{\circ} \mathrm{C}, \mathrm{SnCl}_{4}(289 \mu \mathrm{L}, 2.5 \mathrm{mmol})$ added and the resultant mixture stirred at $\mathrm{rt}$ for $4 \mathrm{~d}$. The reaction mixture was diluted with $\mathrm{CH}_{2} \mathrm{Cl}_{2}(200 \mathrm{~mL})$, quenched with sat. aq. $\mathrm{NaHCO}_{3}(150 \mathrm{~mL})$ and sat. aq. $\mathrm{KF}$ $(150 \mathrm{~mL})$ and the organic layer was isolated from the mixture, and further washed with sat. aq. $\mathrm{KF}(150 \mathrm{~mL})$, sat. aq. $\mathrm{NaHCO}_{3}(150 \mathrm{~mL}), \mathrm{H}_{2} \mathrm{O}(150 \mathrm{~mL})$, brine $(150$ $\mathrm{mL})$ and dried $\left(\mathrm{MgSO}_{4}\right)$, filtered and concentrated in vacuo. Crystallisation of the crude mixture from EtOAc/petroleum ether $(9 / 1, \mathrm{v} / \mathrm{v})$ afforded phenyl 4-O$(2,3,4,6$-tetra- $O$-acetyl- $\beta$-D-galactopyranosyl)-2,3,6-tri- $O$-acetyl-1-thio- $\beta$-D-

glucopyranoside as a white solid (11.6 g, $16 \mathrm{mmol}, 64 \%)$. Phenyl 4-O-(2,3,4,6tetra- $O$-acetyl- $\beta$-D-galactopyranosyl)-2,3,6-tri- $O$-acetyl-1-thio- $\beta$-Dglucopyranoside (5 g, $6.86 \mathrm{mmol}$ ) was dissolved in $\mathrm{MeOH}(55 \mathrm{~mL})$, and $\mathrm{NaOMe}$ was added until the $\mathrm{pH}$ of the reaction mixture reached 9.0. The resulting mixture was stirred at $\mathrm{rt}$ for $20 \mathrm{~h}$, after which the reaction mixture was neutralised with Dowex $-\mathrm{H}^{+}$, filtered and concentrated in vacuo. The residue was crystallised from $\mathrm{EtOH} / \mathrm{MeOH}(9.5 / 0.5, \mathrm{v} / \mathrm{v})$ to afford thiolactoside $\mathbf{7 9}^{11,12,14,37}(2.8 \mathrm{~g}, 6.55 \mathrm{mmol}$, 
quantitative) as a white solid. Thiolactoside 79 was obtained in $45 \%$ overall yield in 3 steps from D-lactose (77). Mp 218.2-219.9 ${ }^{\circ} \mathrm{C} ;{ }^{11} \mathrm{R}_{f}: 0.02(\mathrm{DCM} / \mathrm{MeOH}$, $5.7 / 1, \mathrm{v} / \mathrm{v}) ;[\alpha]_{\mathrm{D}}{ }^{25}=-40.0^{\circ}\left(\mathrm{c}=1.0, \mathrm{H}_{2} \mathrm{O}\right) ;^{11} \mathrm{IR}($ film) 3360, 2884, 1644, 1583, 1479, 1439, 1373, 1278, 1117, 1069, 1020,889, 822, 784, 743, $691 \mathrm{~cm}^{-1} ;{ }^{1} \mathrm{H}$ NMR (600 MHz, $\left.\mathrm{D}_{2} \mathrm{O}\right) \delta$ 7.55-7.53 (m, 2H, CH-o), 7.39-7.33 (m, 3H, CH-m, CH-p), $4.78\left(\mathrm{~d}, J_{1,2}=9.1 \mathrm{~Hz}, 1 \mathrm{H}, \mathrm{H}-1\right), 4.40\left(\mathrm{~d}, J_{1^{\prime}, 2^{\prime}}=7.8 \mathrm{~Hz}, 1 \mathrm{H}, \mathrm{H}-1^{\prime}\right), 3.91$ $\left(\mathrm{dd}, J_{6 \mathrm{a}, 6 \mathrm{~b}}=12.6 \mathrm{~Hz}, J_{5,6 \mathrm{a}}=1.7 \mathrm{~Hz}, 1 \mathrm{H}, \mathrm{H}-6 \mathrm{a}\right), 3.87$ (d, $\left.J_{3^{\prime}, 4^{\prime}}=3.4 \mathrm{~Hz}, 1 \mathrm{H}, \mathrm{H}-4^{\prime}\right)$, $3.76\left(\mathrm{dd}, J_{6 \mathrm{a}, 6 \mathrm{~b}}=12.6 \mathrm{~Hz}, J_{5,6 \mathrm{~b}}=5.0 \mathrm{~Hz}, 1 \mathrm{H}, \mathrm{H}-6 \mathrm{~b}\right), 3.76-3.70(\mathrm{~m}, 2 \mathrm{H}, \mathrm{H}-6$ 'a, H6’b), 3.69-3.66 (m, 1H, H-5'), 3.64-3.63 (m, 2H, H-3, H-4), 3.61 (dd, $J_{2^{\prime}, 3^{\prime}}=9.9$ $\left.\mathrm{Hz}, J_{3^{\prime}, 4^{\prime}}=3.4 \mathrm{~Hz}, 1 \mathrm{H}, \mathrm{H}-3^{\prime}\right), 3.59-3.57$ (m, 1H, H-5), 3.49 (dd, $J_{2^{\prime}, 3^{\prime}}=9.9 \mathrm{~Hz}$, $\left.J_{1^{\prime}, 2^{\prime}}=8.0 \mathrm{~Hz}, 1 \mathrm{H}, \mathrm{H}-2^{\prime}\right), 3.37\left(\mathrm{t}, J_{1,2}=9.1 \mathrm{~Hz}, 1 \mathrm{H}, \mathrm{H}-2\right) ;{ }^{13} \mathrm{C}$ NMR $(150 \mathrm{MHz}$, $\left.\mathrm{D}_{2} \mathrm{O}\right) \delta 131.9(\mathrm{C}-i), 131.6,129.3(\mathrm{C}-o, \mathrm{C}-m), 128.1$ (C-p), $102.8\left(\mathrm{C}-1^{\prime}\right), 87.1$ (C1), 78.7 (C-5), 77.8 (C-4), 75.8 (C-3), 75.3 (C-5') 72.5 (C-3'), 71.4 (C-2), 70.9 (C-2'), 68.5 (C-4'), 61.0 (C-6'), 60.0 (C-6); HRMS(ESI) m/z calcd. for $\left[\mathrm{C}_{18} \mathrm{H}_{26} \mathrm{O}_{10} \mathrm{~S}+\mathrm{Na}\right]^{+}: 457.1139$, obsd.: 457.1148 .

\section{Phenyl 4-O-(3,4-O-isopropylidene- $\beta$-D-galactopyranosyl)-1-thio- $\beta$-D-} glucopyranoside (80)

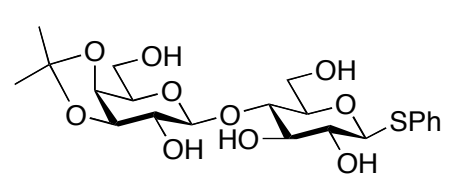

To a solution of thiolactoside 79 (1.98 g, $4.56 \mathrm{mmol})$ in dry DMF $(17 \mathrm{~mL})$ and dry acetone $(34 \mathrm{~mL}), 2,2-$ dimethoxypropane $(923 \mu \mathrm{L})$ and $p \mathrm{TsOH}(81 \mathrm{mg}$, $0.427 \mathrm{mmol}$ ) were added and the resulting solution stirred for $3 \mathrm{~d}$ at $\mathrm{rt}$. The solution was quenched with $\mathrm{NEt}_{3}$ and concentrated under reduced pressure and the residue was then crystalised from hot ethanol to give title compound $\mathbf{8 0}$ as a white crystaline product (1.14 g, $2.40 \mathrm{mmol}, 56 \%)$. Mp 203.2-203.9 ${ }^{\circ} \mathrm{C} \mathrm{R} \mathrm{R}_{f}: 0.30$ $(\mathrm{DCM} / \mathrm{MeOH}, 9 / 1, \mathrm{v} / \mathrm{v}) ;[\alpha]_{\mathrm{D}}{ }^{25}=-27.0^{\circ}(\mathrm{c}=1.0, \mathrm{MeOH}) ; \mathrm{IR}$ (film) 3364, 2946, 2836, 2073, 1653, 1450, 1222, 1119, 1078, 1023, 977, 873, $737 \mathrm{~cm}^{-1} ;{ }^{1} \mathrm{H}$ NMR (500 MHz, $\left.\mathrm{CDCl}_{3} / \mathrm{CD}_{3} \mathrm{OD}, 1 / 1, \mathrm{v} / \mathrm{v}\right) \delta$ 7.52-7.51 (m, 2H, CH-o), 7.30-7.23 (m, $3 \mathrm{H}, \mathrm{CH}-m, \mathrm{CH}-p), 4.58$ (d, $\left.J_{1,2}=9.8 \mathrm{~Hz}, 1 \mathrm{H}, \mathrm{H}-1\right), 4.31$ (d, $J_{1^{\prime}, 2^{\prime}}=8.3 \mathrm{~Hz}, 1 \mathrm{H}, \mathrm{H}-$ $\left.1^{\prime}\right), 4.12\left(\mathrm{dd}, J_{3^{\prime}, 4^{\prime}}=5.6 \mathrm{~Hz}, J_{4^{\prime}, 5^{\prime}}=2.2 \mathrm{~Hz}, 1 \mathrm{H}, \mathrm{H}-4^{\prime}\right), 4.03\left(\mathrm{dd}, J_{2^{\prime}, 3^{\prime}}=7.5 \mathrm{~Hz}\right.$, $\left.J_{3^{\prime}, 4^{\prime}}=5.6 \mathrm{~Hz}, 1 \mathrm{H}, \mathrm{H}-3^{\prime}\right), 3.91-3.88\left(\mathrm{~m}, 1 \mathrm{H}, \mathrm{H}-5^{\prime}\right), 3.86-3.73$ (m, 4H, H-6a, H6b, H-6'a, H-6'b), 3.57 (t, $J_{2,3}=J_{3,4}=8.7 \mathrm{~Hz}, 1 \mathrm{H}, \mathrm{H}-3$ ), 3.53 (t, $J_{3,4}=J_{4,5}=8.7 \mathrm{~Hz}$, 1H, H-4), 3.46 (t, $\left.J_{2^{\prime}, 3^{\prime}}=7.5 \mathrm{~Hz}, 1 \mathrm{H}, \mathrm{H}-2^{\prime}\right), 3.43-3.39$ (m, 1H, H-5), 3.33-3.29 
(m, 1H, H-2), 1.47 (s, 3H, CH $i$ Pr), 1.31 (s, 3H, $\left.\mathrm{CH}_{3} i \mathrm{Pr}\right) ;{ }^{13} \mathrm{C}$ NMR (125 MHz, $\left.\mathrm{CDCl}_{3} / \mathrm{CD}_{3} \mathrm{OD}, 1 / 1, \mathrm{v} / \mathrm{v}\right) \delta 132.6(\mathrm{C}-i), 131.8,128.5$ (C-o, C-m), 127.3 (C-p), 109.9 ( $\left.\mathrm{C}_{\mathrm{q}} i \mathrm{Pr}\right), 102.7\left(\mathrm{C}-1^{\prime}\right), 87.6$ (C-1), 79.7 (C-4), 79.1 (C-3'), 78.5 (C-5), 76.2 (C-3), 73.9 (C-5'), 73.4 (C-4'), 72.7 (C-2'), 71.7 (C-2), 61.1, 60.9 (C-6/C-6'), 27.4, $25.6\left(2 \times \mathrm{CH}_{3} \quad i \mathrm{Pr}\right)$; $\mathrm{HRMS}(\mathrm{ESI}) \mathrm{m} / \mathrm{z}$ calcd. for $\left[\mathrm{C}_{21} \mathrm{H}_{30} \mathrm{O}_{10} \mathrm{~S}+\mathrm{Na}\right]^{+}$: 497.1452, obsd.: 497.1461. Spectral data of 3',4'-isopropylidene protected thiolactoside Error! Reference source not found. matched that previously reported. ${ }^{17}$

\section{Phenyl 4-O-(2,6-di- $O$-benzoyl-3,4- $O$-isopropylidene- $\beta$-D-galactopyranosyl)-} 2,3,6-tri- $O$-benzoyl-1-thio- $\beta$-D-glucopyranoside (82)

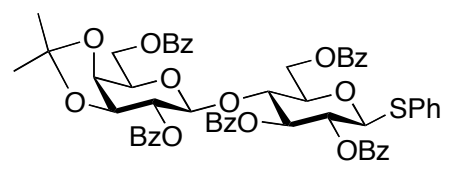

3',4'-isopropylidene protected thiolactoside $\mathbf{8 0}^{14,38}$ (1.10 g, $2.31 \mathrm{mmol})$ was co-evaporated with toluene (x3) and dissolved in pyridine $(23 \mathrm{~mL})$. Benzoyl chloride $(5.9 \mathrm{~mL}, 50.86 \mathrm{mmol})$ and DMAP (0.14 g, $1.16 \mathrm{mmol})$ were added and the reaction was stirred at $\mathrm{rt}$ for $15 \mathrm{~h}$. The reaction mixture was diluted with EtOAc $(100 \mathrm{~mL})$, washed with sat. aq. $\mathrm{NaHCO}_{3}(3 \times 100 \mathrm{~mL}), \mathrm{H}_{2} \mathrm{O}(100 \mathrm{~mL})$ and brine $(100 \mathrm{~mL})$, dried $\left(\mathrm{MgSO}_{4}\right)$, filtered and concentrated under reduced pressure. The product was crystallised from petroleum ether/EtOAc $(2 / 1, \mathrm{v} / \mathrm{v})$ to give the fully protected lactoside $\mathbf{8 2}$ as white fluffy crystals $(1.98 \mathrm{~g}, 1.99 \mathrm{mmol}, 86 \%)$, and the remainder mother liquor was purified by silica flash chromatography (petroleum ether/EtOAc, 3/1, v/v) to afford more product $(0.18 \mathrm{~g}, 0.18 \mathrm{mmol}, 8 \%)$. $\mathrm{R}_{f}: 0.56(\mathrm{PE} / \mathrm{EA}, 1 / 1, \mathrm{v} / \mathrm{v}) ;[\alpha]_{\mathrm{D}}{ }^{25}=$ $+40.0^{\circ}\left(\mathrm{c}=1.0, \mathrm{CHCl}_{3}\right)$; IR (film) 3064, 2988, 2941, 1723, 1602, 1451, 1315, 1265, 1177, 1110, 1083, 1069, 1027, 1000, 753, $708 \mathrm{~cm}^{-1} ;{ }^{1} \mathrm{H}$ NMR (500 MHz, $\left.\mathrm{CDCl}_{3}\right) \delta 8.07\left(\mathrm{~d}, J_{\mathrm{CH}-o, \mathrm{CH}-m}=7.8 \mathrm{~Hz}, 2 \mathrm{H}, \mathrm{CH}-o, \mathrm{OBz}\right), 8.00\left(\mathrm{~d}, J_{\mathrm{CH}-o, \mathrm{CH}-m}=7.8\right.$ $\mathrm{Hz}, 2 \mathrm{H}, \mathrm{CH}-o, \mathrm{OBz}), 7.96\left(\mathrm{~d}, J_{\mathrm{CH}-o, \mathrm{CH}-m}=8.0 \mathrm{~Hz}, 4 \mathrm{H}, 2 \times \mathrm{CH}-o, \mathrm{OBz}\right), 7.92(\mathrm{~d}$, $\left.J_{\mathrm{CH}-o, \mathrm{CH}-m}=7.8 \mathrm{~Hz}, 2 \mathrm{H}, \mathrm{CH}-o, \mathrm{OBz}\right), 7.62-7.09\left(\mathrm{~m}, 20 \mathrm{H}, \mathrm{H}_{\text {arom }}\right.$ ), $5.73\left(\mathrm{t}, J_{3,4}=9.5\right.$ $\mathrm{Hz}, 1 \mathrm{H}, \mathrm{H}-3), 5.40$ (t, $\left.J_{1,2}=9.9 \mathrm{~Hz}, 1 \mathrm{H}, \mathrm{H}-2\right), 5.13$ (t, $J_{1^{\prime}, 2^{\prime}}=7.6 \mathrm{~Hz}, 1 \mathrm{H}, \mathrm{H}-2^{\prime}$ ), $4.88\left(\mathrm{~d}, J_{1,2}=9.9 \mathrm{~Hz}, 1 \mathrm{H}, \mathrm{H}-1\right), 4.65\left(\mathrm{~d}, J_{6 \mathrm{a}, 6 \mathrm{~b}}=12.0 \mathrm{~Hz}, 1 \mathrm{H}, \mathrm{H}-6 \mathrm{a}\right), 4.59$ (d, $J_{1^{\prime}, 2^{\prime}}$ $\left.=7.6 \mathrm{~Hz}, 1 \mathrm{H}, \mathrm{H}-1^{\prime}\right), 4.47\left(\mathrm{dd}, J_{6 \mathrm{a}, 6 \mathrm{~b}}=12.0 \mathrm{~Hz}, J_{5,6 \mathrm{~b}}=5.1 \mathrm{~Hz}, 1 \mathrm{H}, \mathrm{H}-6 \mathrm{~b}\right), 4.26$ 4.21 (m, 2H, H-3', H-6'a), 4.11 (t, $\left.J_{3,4}=9.5 \mathrm{~Hz}, 1 \mathrm{H}, \mathrm{H}-4\right), 4.09$ (d, $J_{4^{\prime}, 5^{\prime}}=5.8 \mathrm{~Hz}$, $\left.1 \mathrm{H}, \mathrm{H}-4^{\prime}\right), 3.89\left(\mathrm{dd}, J_{5,6 \mathrm{a}}=9.6 \mathrm{~Hz}, J_{4,5}=5.0 \mathrm{~Hz}, 1 \mathrm{H}, \mathrm{H}-5\right), 3.83-3.81(\mathrm{~m}, 1 \mathrm{H}, \mathrm{H}-$ 
$\left.5^{\prime}\right), 3.66\left(\mathrm{dd}, J_{6^{\prime} \mathrm{a}, 6^{\prime} \mathrm{b}}=11.2 \mathrm{~Hz}, J_{6^{\prime} \mathrm{a}, 6^{\prime} \mathrm{b}}=7.6 \mathrm{~Hz}, 1 \mathrm{H}, \mathrm{H}-6^{\prime} \mathrm{b}\right), 1.52\left(\mathrm{~s}, 3 \mathrm{H}, \mathrm{CH}_{3} i \mathrm{Pr}\right)$, 1.25 (s, 3H, $\left.\mathrm{CH}_{3} i \mathrm{Pr}\right) ;{ }^{13} \mathrm{C} \mathrm{NMR}\left(125 \mathrm{MHz}, \mathrm{CDCl}_{3}\right) \delta 166.1\left(\mathrm{C}=\mathrm{O}, 66^{\prime}-O-\mathrm{Bn}\right)$, $166.0(\mathrm{C}=\mathrm{O}, 6-O-\mathrm{Bn}), 165.7(\mathrm{C}=\mathrm{O}, 3-O-\mathrm{Bn}), 165.3(\mathrm{C}=\mathrm{O}, 2-O-\mathrm{Bn}), 165.0(\mathrm{C}=\mathrm{O}$, $\left.2^{\prime}-O-\mathrm{Bn}\right), 133.52,133.41,133.30,133.11,133.07$ (C- $\left.p, 5 \mathrm{x} \mathrm{OBz}\right), 132.0$ (C-i, $\mathrm{SPh}), 130.31,130.03,130.00,129.93$ 129.86, 129.72, 129.67, 129.47, 129.40, $128.96,128.83,128.63,128.59,128.54,128.53,128.27,128.24\left(30 \times \mathrm{CH}_{\text {arom }}\right)$, $111.0\left(\mathrm{C}_{\mathrm{q}} i \operatorname{Pr}\right), 100.4\left(\mathrm{C}-1^{\prime}\right), 86.0$ (C-1), 77.4 (C-3'), 77.3 (C-5), 75.5 (C-4), 74.0 (C-3), 73.8 (C-2'), 73.3 (C-4'), 71.5 (C-5'), 70.6 (C-2), 63.0 (C-6), 62.9 (C-6'), 27.6, $26.3\left(2 \times \mathrm{CH}_{3} i \mathrm{Pr}\right) ; \mathrm{HRMS}(\mathrm{ESI}) \mathrm{m} / \mathrm{z}$ calcd. for $\left[\mathrm{C}_{56} \mathrm{H}_{50} \mathrm{O}_{15} \mathrm{~S}+\mathrm{Na}\right]^{+}$: 1017.2763, obsd.: 1017.2774.

\section{4-O-(2,6-Di- $O$-benzoyl-3,4- $O$-isopropylidene- $\beta$-D-galactopyranosyl-2,3,6-tri- $O$-benzoyl- $\alpha$-D-glucopyranose (82a)}

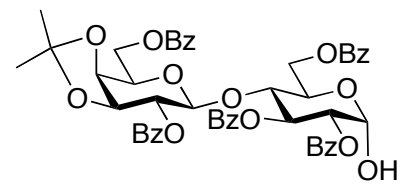

NBS (1.86 g, $10.45 \mathrm{mmol})$ was added to fully protected lactoside $82(2.60 \mathrm{~g}, 2.61 \mathrm{mmol})$ dissolved in acetone $/ \mathrm{H}_{2} \mathrm{O}(9 / 1, \mathrm{v} / \mathrm{v}, 52 \mathrm{~mL})$, and the mixture was stirred at $\mathrm{rt}$ for $50 \mathrm{mins}$. The reaction was quenched with sat. aq. $\mathrm{NaHCO}_{3}(10 \mathrm{~mL})$, diluted with EtOAc (80 $\mathrm{mL})$, washed with sat. aq. $\mathrm{Na}_{2} \mathrm{~S}_{2} \mathrm{O}_{3}(100 \mathrm{~mL})$ and brine $(100 \mathrm{~mL})$, dried $\left(\mathrm{MgSO}_{4}\right)$, filtered and concentrated under reduced pressure. The crude mixture was purified by gradient flash chromatography (petroleum ether/EtOAc, $5 / 1$ to $2 / 1$, v/v) to afford title compound 82a as a clear oil (1.84 g, $2.04 \mathrm{mmol}, 78 \%$ ). For long term storage, the lactol can be crystallised from petroleum ether/EtOAc $(2 / 1, \mathrm{v} / \mathrm{v})$ to give white fluffy crystals. $\mathrm{R}_{f}: 0.50(\mathrm{PE} / \mathrm{EA}, 1 / 1, \mathrm{v} / \mathrm{v}) ;[\alpha]_{\mathrm{D}}{ }^{24}=+71.0^{\circ}(\mathrm{c}=1.0$, $\mathrm{CHCl}_{3}$ ); IR (film) 3064, 1720, 1602, 1452, 1374, 1316, 1265, 1222, 1177, 1109, 1069, , 999, 755, $687 \mathrm{~cm}^{-1} ;{ }^{1} \mathrm{H}$ NMR $\left(500 \mathrm{MHz}, \mathrm{CDCl}_{3}\right) \delta 8.08\left(\mathrm{~d}, J_{\mathrm{CH}-\mathrm{o}, \mathrm{CH}-m}=7.3\right.$ $\mathrm{Hz}, 2 \mathrm{H}, \mathrm{CH}-\mathrm{o}, \mathrm{OBz}), 8.07-7.95$ (m, 8H, 4 x CH-o, OBz), 7.62-7.26 (m, 15H, $\left.\mathrm{H}_{\text {arom }}\right), 6.07\left(\mathrm{t}, J_{2,3}=J_{3,4}=10.0 \mathrm{~Hz}, 1 \mathrm{H}, \mathrm{H}-3\right), 5.58\left(\mathrm{t}, J_{1,2}=3.6 \mathrm{~Hz}, 1 \mathrm{H}, \mathrm{H}-1\right), 5.19$ $\left(\mathrm{dd}, J_{2,3}=10.0 \mathrm{~Hz}, J_{1,2}=3.6 \mathrm{~Hz}, 1 \mathrm{H}, \mathrm{H}-2\right), 5.16\left(\mathrm{t}, J_{1^{\prime}, 2^{\prime}}=J_{2^{\prime}, 3^{\prime}}=7.2 \mathrm{~Hz}, 1 \mathrm{H}, \mathrm{H}-\right.$ $\left.2^{\prime}\right), 4.70\left(\mathrm{~d}, J_{1^{\prime}, 2^{\prime}}=7.4 \mathrm{~Hz}, 1 \mathrm{H}, \mathrm{H}-1^{\prime}\right), 4.60\left(\mathrm{dd}, J_{6 \mathrm{a}, 6 \mathrm{~b}}=12.2 \mathrm{~Hz}, J_{5,6 \mathrm{a}}=1.6 \mathrm{~Hz}\right.$, $1 \mathrm{H}, \mathrm{H}-6 \mathrm{a}), 4.52\left(\mathrm{dd}, J_{6 \mathrm{a}, 6 \mathrm{~b}}=12.2 \mathrm{~Hz}, J_{5,6 \mathrm{~b}}=3.4 \mathrm{~Hz}, 1 \mathrm{H}, \mathrm{H}-6 \mathrm{~b}\right), 4.40-4.38(\mathrm{~m}, 1 \mathrm{H}$, $\mathrm{H}-5), 4.32\left(\mathrm{dd}, J_{6^{\prime} \mathrm{a}, 6^{\prime} \mathrm{b}}=15.3 \mathrm{~Hz}, J_{5,6^{\prime} \mathrm{a}}=8.7 \mathrm{~Hz}, 1 \mathrm{H}, \mathrm{H}-6^{\prime} \mathrm{a}\right), 4.27\left(\mathrm{t}, J_{3^{\prime}, 4^{\prime}}=6.1 \mathrm{~Hz}\right.$, $\left.1 \mathrm{H}, \mathrm{H}-3^{\prime}\right), 4.20\left(\mathrm{t}, J_{3,4}=10.0 \mathrm{~Hz}, 1 \mathrm{H}, \mathrm{H}-4\right), 4.13-4.11$ (m, 1H, H-4'), 3.90-3.85 
(m, 2H, H-5', H-6'b), 2.90 (d, $\left.J_{1, \mathrm{OH}}=3.6 \mathrm{~Hz}, 1 \mathrm{H}, \mathrm{OH}\right), 1.51$ (s, 3H, $\mathrm{CH}_{3} i \mathrm{Pr}$ ), $1.26\left(\mathrm{~s}, 3 \mathrm{H}, \mathrm{CH}_{3} i \mathrm{Pr}\right) ;{ }^{13} \mathrm{C} \mathrm{NMR}\left(125 \mathrm{MHz}, \mathrm{CDCl}_{3}\right) \delta 166.0,166.0(\mathrm{C}=\mathrm{O}, 6-\mathrm{O}-\mathrm{Bn}$, $\left.6^{\prime}-O-\mathrm{Bn}\right), 165.9(\mathrm{C}=\mathrm{O}, 2-O-\mathrm{Bn}), 165.6(\mathrm{C}=\mathrm{O}, 3-O-\mathrm{Bn}), 165.0\left(\mathrm{C}=\mathrm{O}, 2^{\prime}-O-\mathrm{Bn}\right)$, 133.4, 133.4, 133.2, 133.1, 132.9 (C- $p, 5$ x OBz), 130.04, 129.91, 129.77, 129.70, $129.65,129.59,129.52,129.33,128.98,128.60,128.46,128.43,128.36,128.15$ (25 x CHarom), 110.9 ( $\left.\mathrm{C}_{\mathrm{q}} i \operatorname{Pr}\right), 100.0$ (C-1'), 90.3 (C-1), 77.1 (C-3'), 75.4 (C-4), 73.6 (C-2'), 73.6 (C-4'), 72.2 (C-2), 71.3 (C-5'), 69.5 (C-3), 68.6 (C-5), 63.0 (C6'), 62.5 (C-6), 27.4, $26.1\left(2 \times \mathrm{CH}_{3} i \operatorname{Pr}\right) ; \mathrm{HRMS}(\mathrm{ESI}) \mathrm{m} / \mathrm{z}$ calcd. for $\left[\mathrm{C}_{50} \mathrm{H}_{46} \mathrm{O}_{16}+\mathrm{Na}\right]^{+}$: 925.2678, obsd.: 925.2681. Spectral data of lactol 82a matched that previously reported. ${ }^{9}$

\section{$O$-(4-O-(2,6-Di- $O$-benzoyl-3,4- $O$-isopropylidene- $\beta$-D-galactopyranosyl)-2,3,6-} tri-O-benzoyl- $\alpha$-D-glucopyranosyl) trichloroacetimidate (75)

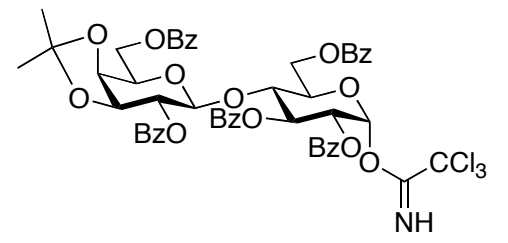

Lactol 82a was co-evaporated with toluene $(\mathrm{x} 3)$ and dissolved in dry $\mathrm{CH}_{2} \mathrm{Cl}_{2} \quad(6 \mathrm{~mL})$. Trichloroacetonitrile $(1.13 \mathrm{~mL}, 11.30 \mathrm{mmol})$ and

DBU $(84 \mu \mathrm{L}, 0.56 \mathrm{mmol})$ were added and the reaction mixture was stirred at $\mathrm{rt}$ for $1 \mathrm{~h}$. Upon completion, the reaction mixture was concentrated and purified immediately by flash column chromatography (petroleum ether/EtOAc/ $\left.\mathrm{CH}_{2} \mathrm{Cl}_{2} / \mathrm{NEt}_{3}, 6 / 1 / 1 / 0.08, \mathrm{v} / \mathrm{v}\right)$ to afford imidate 75 as a colourless foam $(1.08 \mathrm{~g}, 1.03 \mathrm{mmol}, 92 \%)$. $\mathrm{R}_{f}: 0.54(\mathrm{PE} / \mathrm{EA}, 1 / 1, \mathrm{v} / \mathrm{v}) ;[\alpha]_{\mathrm{D}}{ }^{24}=+57.0^{\circ}(\mathrm{c}=$ 1.0, $\mathrm{CHCl}_{3}$ ); IR (film) 3336, 3065, 2986, 2938, 1725, 1677, 1452, 1315, 1265, 1177, 1109, 1070, 1026, 797, 755, $708 \mathrm{~cm}^{-1} ;{ }^{1} \mathrm{H}$ NMR (500 MHz, $\left.\mathrm{CDCl}_{3}\right) \delta 8.55$ $(\mathrm{s}, 1 \mathrm{H}, \mathrm{NH}), 8.07\left(\mathrm{~d}, J_{\mathrm{CH}-o, \mathrm{CH}-m}=7.1 \mathrm{~Hz}, 2 \mathrm{H}, \mathrm{CH}-o, 6^{\prime}-O-\mathrm{Bz}\right), 8.02\left(\mathrm{t}, J_{\mathrm{CH}-o, \mathrm{CH}-m}=\right.$ $8.6 \mathrm{~Hz}, 4 \mathrm{H}, 2 \times \mathrm{CH}-o, \mathrm{OBz}), 7.95$ (t, $\left.J_{\mathrm{CH}-o, \mathrm{CH}-m}=8.7 \mathrm{~Hz}, 4 \mathrm{H}, 2 \times \mathrm{CH}-o, \mathrm{OBz}\right)$, 7.64-7.30 (m, 15H, $\left.\mathrm{H}_{\text {arom }}\right), 6.69\left(\mathrm{~d}, J_{1,2}=3.4 \mathrm{~Hz}, 1 \mathrm{H}, \mathrm{H}-1\right), 6.10\left(\mathrm{t}, J_{3,4}=9.2 \mathrm{~Hz}\right.$, $1 \mathrm{H}, \mathrm{H}-3), 5.50\left(\mathrm{dd}, J_{2,3}=10.2 \mathrm{~Hz}, J_{1,2}=3.4 \mathrm{~Hz}, 1 \mathrm{H}, \mathrm{H}-2\right), 5.16\left(\mathrm{t}, J_{2^{\prime}, 3^{\prime}}=6.9 \mathrm{~Hz}\right.$, $\left.1 \mathrm{H}, \mathrm{H}-2^{\prime}\right), 4.70\left(\mathrm{~d}, J_{1^{\prime}, 2^{\prime}}=7.6 \mathrm{~Hz}, 1 \mathrm{H}, \mathrm{H}-1^{\prime}\right), 4.59$ (d, $\left.J_{6 \mathrm{a}, 6 \mathrm{~b}}=11.8 \mathrm{~Hz}, 1 \mathrm{H}, \mathrm{H}-6 \mathrm{a}\right)$, $4.52\left(\mathrm{~d}, J_{6 \mathrm{a}, 6 \mathrm{~b}}=11.8 \mathrm{~Hz}, 1 \mathrm{H}, \mathrm{H}-6 \mathrm{~b}\right), 4.31-4.24$ (m, 4H, H-4, H-5, H-6'a, H-3'), $4.11\left(\mathrm{~d}, J_{3^{\prime}, 4^{\prime}}=J_{4^{\prime}, 5^{\prime}}=4.4 \mathrm{~Hz}, 1 \mathrm{H}, \mathrm{H}-4^{\prime}\right), 3.81-3.78$ (m, 2H, H-5', H-6'b), 1.50 (s, $\left.3 \mathrm{H}, \mathrm{CH}_{3} i \mathrm{Pr}\right), 1.25\left(\mathrm{~s}, 3 \mathrm{H}, \mathrm{CH}_{3} i \mathrm{Pr}\right) ;{ }^{13} \mathrm{C} \mathrm{NMR}\left(125 \mathrm{MHz}, \mathrm{CDCl}_{3}\right) \delta 165.9(\mathrm{C}=\mathrm{O}$, $\left.6^{\prime}-O-\mathrm{Bn}\right), 165.7(\mathrm{C}=\mathrm{O}, 6-O-\mathrm{Bn}), 165.5(\mathrm{C}=\mathrm{O}, 2-O-\mathrm{Bn}), 165.4(\mathrm{C}=\mathrm{O}, 3-O-\mathrm{Bn})$, 
$165.0\left(\mathrm{C}=\mathrm{O}, 2^{\prime}-O-\mathrm{Bn}\right), 160.7(\underline{\mathrm{C}}=\mathrm{NH}), 133.5,133.4,133.3,133.2,133.1(\mathrm{C}-p, 5 \mathrm{x}$ OBz), 130.1, 129.94, 129.86, 129.78, 129.74, 129.54, 129.50, 129.29, 128.68, $128.63,128.47,128.43,128.41,128.21\left(25 \times \mathrm{CH}_{\text {arom }}\right), 110.8\left(\mathrm{C}_{\mathrm{q}} i \mathrm{Pr}\right), 100.7(\mathrm{C}-$ 1'), 93.1 (C-1), $90.7\left(\mathrm{CCl}_{3}\right), 77.0$ (C-3'), 75.1 (C-4), 73.7 (C-2'), $73.0\left(\mathrm{C}-4^{\prime}\right), 71.4$ (C-5), 71.3 (C-5'), 70.6 (C-2), 70.1 (C-3), 62.8 (C-6'), 62.1 (C-6), 27.3, 26.1 (2 x $\mathrm{CH}_{3} i \mathrm{Pr}$ ); $\mathrm{HRMS}(\mathrm{ESI}) \mathrm{m} / \mathrm{z}$ calcd. for $\left[\mathrm{C}_{52} \mathrm{H}_{46} \mathrm{NO}_{16} \mathrm{Cl}_{3}+\mathrm{H}\right]^{+}: 1046.1955$, obsd.: 1046.1917. Spectral data of lactosyl imidate donor 75 matched that previously reported. $^{9,16}$

(2S,3R,4E)-2-Azido-1-(4-O-(2,6-di- $O$-benzoyl-3,4- $O$-isopropylidene- $\beta$-Dgalactopyranosyl)-2,3,6-tri- $O$-benzoyl- $\beta$-D-glucopyranosyloxy)-3-benzyloxyoctadec-4-ene (74)

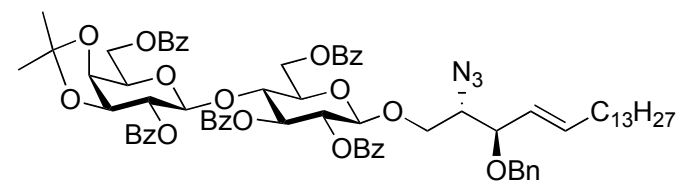

A solution of imidate donor 75 (973 $\mathrm{mg}, 0.93 \mathrm{mmol})$ and lipid acceptor 76 (300 mg, $0.72 \mathrm{mmol})$, co-evaporated with dry toluene (x3), was dissolved in dry $\mathrm{CH}_{2} \mathrm{Cl}_{2}(6 \mathrm{~mL})$ and $4 \AA$ molecular sieves were added. This mixture was cooled to $-20{ }^{\circ} \mathrm{C}$ and a solution of TMSOTf in $\mathrm{CH}_{2} \mathrm{Cl}_{2}(1.38 \mathrm{mmol} / \mathrm{mL}, 157 \mu \mathrm{L}, 0.22 \mathrm{mmol})$ was added slowly dropwise, the resulting solution was stirred $1.5 \mathrm{~h}$ at $-20{ }^{\circ} \mathrm{C}$ until TLC analysis showed complete consumption of lipid acceptor 76. The solution was quenched with $\mathrm{NEt}_{3}(400 \mu \mathrm{L}$, $2.87 \mathrm{mmol}$ ) and concentrated under reduced pressure. The resulting oil was purified by gradient flash chromatography (petroleum ether/EtOAc, 10/1 to $5 / 1$, $\mathrm{v} / \mathrm{v}$ ) to give glycolipid 74 as a colourless oil (936 mg, $0.72 \mathrm{mmol}$, Quantitative). $\mathrm{R}_{f}: 0.49(\mathrm{PE} / \mathrm{EA}, 2 / 1, \mathrm{v} / \mathrm{v}) ;[\alpha]_{\mathrm{D}}^{25}=+10.0^{\circ}\left(\mathrm{c}=1.0, \mathrm{CH}_{2} \mathrm{Cl}_{2}\right)$; IR (film) 3440, 3297, 3067, 2925, 2854, 2361, 2342, 2101, 1720, 1602, 1452, 1373, 1315, 1264, 1177, 1109, 1068, 1027, 825, $707 \mathrm{~cm}^{-1} ;{ }^{1} \mathrm{H}$ NMR (500 MHz, $\left.\mathrm{CDCl}_{3}\right) \delta 8.10$ (d, $\left.J_{\mathrm{CH}-o, \mathrm{CH}-m}=7.3 \mathrm{~Hz}, 2 \mathrm{H}, \mathrm{CH}-o, \mathrm{OBz}\right), 8.03\left(\mathrm{~d}, J_{\mathrm{CH}-o, \mathrm{CH}-m}=7.3 \mathrm{~Hz}, 2 \mathrm{H}, \mathrm{CH}-o\right.$, $\mathrm{OBz}), 8.00\left(\mathrm{~d}, J_{\mathrm{CH}-o, \mathrm{CH}-m}=7.3 \mathrm{~Hz}, 2 \mathrm{H}, \mathrm{CH}-o, \mathrm{OBz}\right), 7.96-7.94(\mathrm{~m}, 4 \mathrm{H}, 2$ x CH-o, OBz), 7.64-7.19 (m, 20H, $\mathrm{H}_{\text {arom }}$ ), 5.73 (t, $\left.J_{2^{\prime}, 3^{\prime}}=9.4 \mathrm{~Hz}, 1 \mathrm{H}, \mathrm{H}-3^{\prime}\right), 5.46-5.39$ (m, $\left.2 \mathrm{H}, \mathrm{H}-2^{\prime}, \mathrm{H}-5\right), 5.40\left(\mathrm{t}, J_{5,6}=6.7 \mathrm{~Hz}, 1 \mathrm{H}, \mathrm{H}-5\right), 5.26\left(\mathrm{dd}, J_{4,5}=15.6 \mathrm{~Hz}, J_{3,4}=8.6\right.$ $\mathrm{Hz}, 1 \mathrm{H}, \mathrm{H}-4), 5.14$ (t, $\left.J_{2^{\prime \prime}, 3^{\prime \prime}}=7.3 \mathrm{~Hz}, 1 \mathrm{H}, \mathrm{H}-2^{\prime \prime}\right), 4.68$ (d, $J_{1^{\prime}, 2^{\prime}}=7.8 \mathrm{~Hz}, 1 \mathrm{H}, \mathrm{H}-$ $\left.1^{\prime}\right), 4.60-4.58$ (m, 2H, H-1' ', H-6'a), 4.47 (dd, $J_{6^{\prime} \mathrm{a}, 6^{\prime} \mathrm{b}}=12.3 \mathrm{~Hz}, J_{5^{\prime}, 6^{\prime} \mathrm{b}}=4.2 \mathrm{~Hz}$, $1 \mathrm{H}, \mathrm{H}-6$ 'b), 4.41 (d, $\left.J_{\mathrm{a}, \mathrm{b}}=12.0 \mathrm{~Hz}, 1 \mathrm{H}, \mathrm{CH}-\mathrm{a}, 3-O-\mathrm{Bn}\right), 4.25-4.20$ (m, 3H, H-4', 
H-3"', H-6" 'a), 4.15 (d, $\left.J_{\mathrm{a}, \mathrm{b}}=12.0 \mathrm{~Hz}, 1 \mathrm{H}, \mathrm{CH}-\mathrm{b}, 3-O-\mathrm{Bn}\right), 4.07$ (dd, $J_{4^{\prime \prime}, 5^{\prime \prime}}=5.6$ $\left.\mathrm{Hz}, J_{3^{\prime \prime}, 4^{\prime \prime}}=1.9 \mathrm{~Hz}, 1 \mathrm{H}, \mathrm{H}-4^{\prime \prime}\right), 3.90\left(\mathrm{dd}, J_{1 \mathrm{a}, 1 \mathrm{~b}}=10.3 \mathrm{~Hz}, J_{1 \mathrm{a}, 2}=5.7 \mathrm{~Hz}, 1 \mathrm{H}, \mathrm{H}-\right.$ 1a), 3.83-3.77 (m, 2H, H-5', H-5' '), 3.73 (dd, $J_{3,4}=8.6 \mathrm{~Hz}, J_{2,3}=5.7 \mathrm{~Hz}, 1 \mathrm{H}, \mathrm{H}-$ 3), $3.65\left(\mathrm{dd}, J_{6^{\prime \prime} \mathrm{a}, 6^{\prime \prime} \mathrm{b}}=11.5 \mathrm{~Hz}, J_{5,6^{\prime \prime} \mathrm{b}}=7.4 \mathrm{~Hz}, 1 \mathrm{H}, \mathrm{H}-6^{\prime \prime} \mathrm{b}\right), 3.60\left(\mathrm{dd}, J_{1,2}=11.2\right.$ $\left.\mathrm{Hz}, J_{2,3}=5.7 \mathrm{~Hz}, 1 \mathrm{H}, \mathrm{H}-2\right), 3.50\left(\mathrm{dd}, J_{1 \mathrm{a}, 1 \mathrm{~b}}=10.3 \mathrm{~Hz}, J_{1 \mathrm{~b}, 2}=5.7 \mathrm{~Hz}, 1 \mathrm{H}, \mathrm{H}-1 \mathrm{~b}\right)$, 1.93-1.91 (m, 2H, H-6), 1.52 (s, 3H, $\left.\mathrm{CH}_{3} i \mathrm{Pr}\right), 1.32-1.26$ (m, 25H, $\mathrm{CH}_{3} i \mathrm{Pr}, \mathrm{H}-7-$ $\mathrm{H}-17), 0.89$ (t, $\left.J_{17,18}=7.0 \mathrm{~Hz}, 3 \mathrm{H}, \mathrm{H}-18\right) ;{ }^{13} \mathrm{C} \mathrm{NMR}\left(125 \mathrm{MHz}, \mathrm{CDCl}_{3}\right) \delta 166.0$ $\left(\mathrm{C}=\mathrm{O}, 6^{\prime \prime}-O-\mathrm{Bn}\right), 165.9\left(\mathrm{C}=\mathrm{O}, 6^{\prime}-O-\mathrm{Bn}\right), 165.7\left(\mathrm{C}=\mathrm{O}, 3^{\prime}-O-\mathrm{Bn}\right), 165.1\left(\mathrm{C}=\mathrm{O}, 2^{\prime}-\right.$ $O-\mathrm{Bn}), 165.0\left(\mathrm{C}=\mathrm{O}, 2^{\prime \prime}-O-\mathrm{Bn}\right), 138.3$ (C-5), 138.1 (C-i, 3-O-Bn), 133.4, 133.3, 133.24, 133.21, 133.0 (C-p, 5 x OBz), 129.88, 129.85, 129.82, 129.8, 129.6, $129.5,129.35,129.28,128.7,128.449,128.44,128.37,128.27,128.17,127.53$, 127.45, 127.44 (30 x $\left.\mathrm{CH}_{\text {arom}}\right), 125.4(\mathrm{C}-4), 110.9$ ( $\left.\mathrm{C}_{\mathrm{q}} i \operatorname{Pr}\right), 101.0\left(\mathrm{C}-1^{\prime}\right), 100.2(\mathrm{C}-$

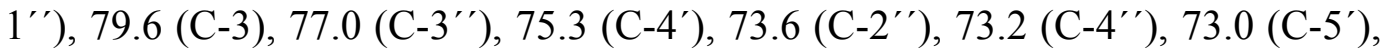
72.7 (C-3'), 71.8 (C-2'), $71.3\left(\mathrm{C}-5^{\prime \prime}\right), 70.0\left(\mathrm{CH}_{2}, 3-\mathrm{O}-\mathrm{Bn}\right), 68.5(\mathrm{C}-1), 63.7$ (C-2), 62.8 (C-6"'), 62.5 (C-6'), 32.3 (C-6), 27.4, 26.2 (2 x CH $\mathrm{CH}_{3}$ Pr), 31.9, 29.70, 29.69, 29.68, 29.66, 29.63, 29.42, 29.37, 29.16, 28.9, 22.7 (C-7-C-17), 14.1 (C-18); HRMS(ESI) $\mathrm{m} / \mathrm{z}$ calcd. for $\left[\mathrm{C}_{75} \mathrm{H}_{85} \mathrm{~N}_{3} \mathrm{O}_{17}+\mathrm{Na}\right]^{+}:$1322.5771, obsd.: 1322.5764 .

\section{$(2 S, 3 R, 4 E)-1-(4-O$-(2,6-Di- $O$-benzoyl-3,4- $O$-isopropylidene- $\beta$-D-}

galactopyranosyl)-2,3,6-tri- $O$-benzoyl- $\beta$-D-glucopyranosyloxy)-3-benzyloxy-

\section{2-hexacosanoylamido-octadecen-4-ene (83)}

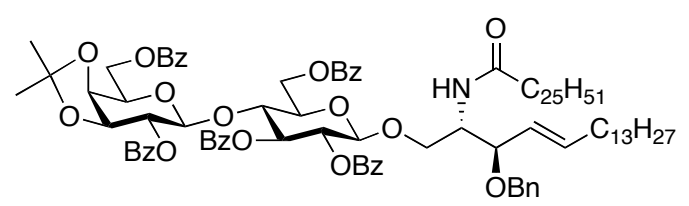

To a solution of glycolipid azide $\mathbf{7 4}$ (100 $\mathrm{mg}, 0.077 \mathrm{mmol})$ in toluene (1

$\mathrm{mL}$ ) triphenylphosphine $(40 \mathrm{mg}, 0.15$

mmol), and distilled water (10 drops) were added, the solution was warmed to 80 ${ }^{\circ} \mathrm{C}$ and stirred overnight. The reaction mixture was then cooled to room temperature, diluted with EtOAc, washed with sat. aq. $\mathrm{NH}_{4} \mathrm{Cl}$, dried $\left(\mathrm{MgSO}_{4}\right)$, filtered and concentrated under reduced pressure to give a colourless oil which was used without further purification. The oil was co-evaporated twice with dry toluene then suspended in DCM (2 mL), EDCI (73 mg, $0.383 \mathrm{mmol})$, DMAP (30 $\mathrm{mg}, 0.246 \mathrm{mmol})$, and hexacosanoic acid $(151 \mathrm{mg}, 0.383 \mathrm{mmol})$ were added and the resulting solution stirred over three nights at room temperature. The reaction mixture was then purified directly by gradient flash chromatography (petroleum 
ether/EtOAc, 10/1 to 2/1, v/v) to give 83 as a colourless oil (92 $\mathrm{mg}, 0.056 \mathrm{mmol}$, $72 \%$ over two steps). $\mathrm{R}_{f}: 0.55(\mathrm{PE} / \mathrm{EA}, 2 / 1, \mathrm{v} / \mathrm{v}) ;[\alpha]_{\mathrm{D}}{ }^{22}=+16.0^{\circ}$ (c = 1.0, $\mathrm{CH}_{2} \mathrm{Cl}_{2}$ ); IR (film) 3323, 3063, 3034, 2921, 2852, 2361, 2341, 1971, 1724, 1646, 1602, 1531, 1452, 1267, 1112, 1068, 1027, $707 \mathrm{~cm}^{-1} ;{ }^{1} \mathrm{H}$ NMR (500 MHz, $\left.\mathrm{CDCl}_{3}\right)$ $\delta 8.09\left(\mathrm{~d}, J_{\mathrm{CH}-o, \mathrm{CH}-m}=7.3 \mathrm{~Hz}, 2 \mathrm{H}, \mathrm{CH}-o, \mathrm{OBz}\right), 8.02\left(\mathrm{~d}, J_{\mathrm{CH}-o, \mathrm{CH}-m}=7.3 \mathrm{~Hz}, 2 \mathrm{H}\right.$, $\mathrm{CH}-o, \mathrm{OBz}), 8.00$ (d, $\left.J_{\mathrm{CH}-o, \mathrm{CH}-m}=7.6 \mathrm{~Hz}, 2 \mathrm{H}, \mathrm{CH}-o, \mathrm{OBz}\right), 7.96\left(\mathrm{~d}, J_{\mathrm{CH}-o, \mathrm{CH}-m}=\right.$ $7.5 \mathrm{~Hz}, 2 \mathrm{H}, \mathrm{CH}-o, \mathrm{OBz}), 7.92$ (d, $\left.J_{\mathrm{CH}-o, \mathrm{CH}-m}=7.3 \mathrm{~Hz}, 2 \mathrm{H}, \mathrm{CH}-o, \mathrm{OBz}\right), 7.62-7.11$ (m, 20H, $\mathrm{H}_{\text {arom }}$ ), 5.73 (t, $\left.J_{2^{\prime}, 3^{\prime}}=9.4 \mathrm{~Hz}, 1 \mathrm{H}, \mathrm{H}-3^{\prime}\right), 5.49$ (dt, $J_{4,5}=15.3 \mathrm{~Hz}, J_{5,6}=$ $6.9 \mathrm{~Hz}, 1 \mathrm{H}, \mathrm{H}-5), 5.393\left(\mathrm{dd}, J_{2^{\prime}, 3^{\prime}}=9.6 \mathrm{~Hz}, J_{1^{\prime}, 2^{\prime}}=8.0 \mathrm{~Hz}, 1 \mathrm{H}, \mathrm{H}-2^{\prime}\right), 5.392$ (d, $\left.J_{\mathrm{NH}, 2}=9.1 \mathrm{~Hz}, 1 \mathrm{H}, \mathrm{NH} \mathrm{C} \mathrm{C}_{26}\right), 5.24\left(\mathrm{dd}, J_{4,5}=15.3 \mathrm{~Hz}, J_{3,4}=8.5 \mathrm{~Hz}, 1 \mathrm{H}, \mathrm{H}-4\right), 5.14$ (t, $\left.J_{1^{\prime \prime}, 2^{\prime \prime}}=7.2 \mathrm{~Hz}, 1 \mathrm{H}, \mathrm{H}-2^{\prime \prime}\right)$, 4.60-4.55 (m, 3H, H-1', H-1" ', H-6'a), 4.48 (dd, $\left.J_{6^{\prime} \mathrm{a}, 6^{\prime} \mathrm{b}}=12.3 \mathrm{~Hz}, J_{5^{\prime}, 6^{\prime}}=4.0 \mathrm{~Hz}, 1 \mathrm{H}, \mathrm{H}-6^{\prime} \mathrm{b}\right), 4.44$ (d, $J_{\mathrm{a}, \mathrm{b}}=11.5 \mathrm{~Hz}, 1 \mathrm{H}, \mathrm{CH}-\mathrm{a}, 3-$ O-Bn), 4.26-4.19 (m, 5H, H-1a, H-4', H-3"', H-6" a, CH-b, 3-O-Bn), 4.09-4.05 (m, 2H, H-2, H-4' ) , 3.81-3.79 (m, 1H, H-5' ), 3.75-3.69 (m, 3H, H-5', H-6' 'b, H-3), $3.47\left(\mathrm{dd}, J_{1 \mathrm{a}, 1 \mathrm{~b}}=9.5 \mathrm{~Hz}, J_{1 \mathrm{~b}, 2}=3.4 \mathrm{~Hz}, 1 \mathrm{H}, \mathrm{H}-1 \mathrm{~b}\right), 1.95-1.92$ (m, 2H, H-6), 1.71-1.61 (m, 2H, $\left.\mathrm{CH}_{2}-\alpha\right), 1.52$ (s, 3H, $\left.\mathrm{CH}_{3} i \mathrm{Pr}\right), 1.55-1.03$ (m, 71H, H-7-H-17, $\left.\mathrm{H}-\beta-\mathrm{H}-(\omega-1), \mathrm{CH}_{3} i \mathrm{Pr}\right), 0.88\left(\mathrm{t}, J_{17,18}=J_{\omega-1, \omega}=6.9 \mathrm{~Hz}, 6 \mathrm{H}, \mathrm{H}-18, \mathrm{H}-\omega\right) ;{ }^{13} \mathrm{C}$ NMR (125 MHz, $\left.\mathrm{CDCl}_{3}\right) \delta 172.4(\mathrm{HNC}=\mathrm{O}), 166.0\left(\mathrm{C}=\mathrm{O}, 6^{\prime \prime}-O-\mathrm{Bn}\right), 165.8$ $\left(\mathrm{C}=\mathrm{O}, 6^{\prime}-O-\mathrm{Bn}\right), 165.6\left(\mathrm{C}=\mathrm{O}, 3^{\prime}-O-\mathrm{Bn}\right), 165.3\left(\mathrm{C}=\mathrm{O}, 2^{\prime}-O-\mathrm{Bn}\right), 165.0\left(\mathrm{C}=\mathrm{O}, 2^{\prime \prime}{ }^{\prime}-\right.$ $O-\mathrm{Bn}$ ), 138.3 (C-i, 3-O-Bn), 137.0 (C-5), 133.5, 133.4, 133.3, 133.2, 133.1 (C-p, 5 x OBz), 129.9, 129.81, 129.78, 129.7, 129.6, 129.5, 129.3, 129.1, 128.7, 128.6, 128.5, 128.4, 128.2, 127.6, 127.4 (30 x $\left.\mathrm{CH}_{\text {arom}}\right), 127.3(\mathrm{C}-4), 110.9\left(\mathrm{C}_{\mathrm{q}} i \operatorname{Pr}\right), 101.3$ (C-1'), $100.1\left(\mathrm{C}-1^{\prime \prime}\right), 79.1$ (C-3), 77.0 (C-3'”), 75.2 (C-4'), 73.6 (C-2'), 73.1 (C$\left.4^{\prime \prime}\right), 73.0$ (C-5'), $72.4\left(\mathrm{C}-3{ }^{\prime}\right), 72.3$ (C-2'), $71.3\left(\mathrm{C}-5^{\prime \prime}\right), 70.3\left(\mathrm{CH}_{2}, 3-O-\mathrm{Bn}\right), 68.3$ (C-1), 62.8 (C-6"'), 62.6 (C-6'), 51.2 (C-2), 36.4 (C- $\alpha$ ), 32.2 (C-6), 31.9, 29.75, 29.73, 29.71, 29.70, 29.66, 29.56, 29.54, 29.40, 29.38, 29.37, 29.27, 29.23, 22.70 (C-7-C-17, C- $\gamma-\mathrm{C}-(\omega-1)), 27.4,26.1\left(2 \mathrm{x} \mathrm{CH}_{3} i \operatorname{Pr}\right), 25.5$ (C- $\beta$ ), 14.1 (C-18, C$\omega) ; \operatorname{HRMS}(E S I) \quad \mathrm{m} / \mathrm{z}$ calcd. for $\left[\mathrm{C}_{101} \mathrm{H}_{137} \mathrm{NO}_{18}+\mathrm{Na}\right]^{+}:$1674.9728, obsd.: 1674.9717. 
(2S,3R,4E)-1-(4-O-(2,6-Di-O-benzoyl- $\beta$-D-galactopyranosyl)-2,3,6-tri- $O$ -

benzoyl- $\beta$-D-glucopyranosyloxy)-3-benzyloxy-2-hexacosanoylamido-octadec4-ene (84)

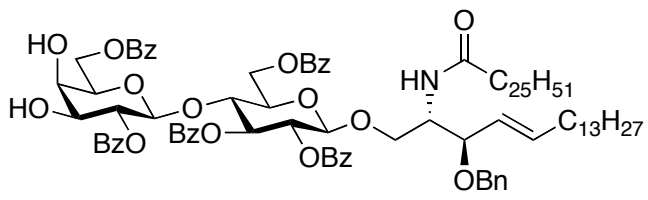

To a solution of fully protected lactosyl ceramide 83 (226 $\mathrm{mg}, 0.14 \mathrm{mmol})$ in $\mathrm{CH}_{2} \mathrm{Cl}_{2}(5 \mathrm{~mL})$ was added $\mathrm{TFA} / \mathrm{H}_{2} \mathrm{O}$ solution $(1 / 1, \mathrm{v} / \mathrm{v}, 0.5 \mathrm{~mL})$ and the resulting solution was stirred at room temperature for $12 \mathrm{~h}$. The solution was diluted with ethyl acetate and the organic layer was then washed with sat. aq. $\mathrm{NaHCO}_{3}$ and brine, dried $\left(\mathrm{MgSO}_{4}\right)$, filtered and concentrated under reduced pressure. The colourless oil was then purified by gradient flash chromatography (petroleum ether/EtOAc, $5 / 1$ to $1 / 1$, v/v) to give diol 84 as a colourless oil (213 mg, 0.13 mmol, 96\%). R $\mathrm{f}_{\mathrm{f}} 0.08$ (PE/EA, 2/1, v/v); $[\alpha]_{\mathrm{D}}^{25}=+15.0^{\circ}\left(\mathrm{c}=1.0, \mathrm{CH}_{2} \mathrm{Cl}_{2}\right)$; IR (film) 3064, 2922, 2852, 2357, 1724, 1512, 1452, 1267, 1176, 1110, 1068, 1028, $708 \mathrm{~cm}^{-1} ;{ }^{1} \mathrm{H}$ NMR $\left(500 \mathrm{MHz}, \mathrm{CDCl}_{3}\right) \delta$ $8.07\left(\mathrm{~d}, J_{\mathrm{CH}-o, \mathrm{CH}-m}=7.3 \mathrm{~Hz}, 2 \mathrm{H}, \mathrm{CH}-o, 2^{\prime \prime}-O-\mathrm{Bz}\right), 8.00\left(\mathrm{t}, J_{\mathrm{CH}-o, \mathrm{CH}-m}=8.7 \mathrm{~Hz}, 4 \mathrm{H}\right.$, $\mathrm{CH}-o, \mathrm{OBz}), 7.94$ (t, $\left.J_{\mathrm{CH}-o, \mathrm{CH}-m}=8.7 \mathrm{~Hz}, 4 \mathrm{H}, 2 \times \mathrm{CH}-o, \mathrm{OBz}\right), 7.59-7.14(\mathrm{~m}, 20 \mathrm{H}$, $\left.\mathrm{H}_{\text {arom }}\right), 5.66\left(\mathrm{t}, J_{2^{\prime}, 3^{\prime}}=J_{3^{\prime}, 4^{\prime}}=9.4 \mathrm{~Hz}, 1 \mathrm{H}, \mathrm{H}-3^{\prime}\right), 5.50\left(\mathrm{dt}, J_{4,5}=15.4 \mathrm{~Hz}, J_{5,6}=6.6\right.$ $\mathrm{Hz}, 1 \mathrm{H}, \mathrm{H}-5), 5.40-5.36$ (m, 2H, H-2', NH), 5.29 (t, $\left.J_{1^{\prime \prime}, 2^{\prime \prime}}=7.8 \mathrm{~Hz}, 1 \mathrm{H}, \mathrm{H}-2^{\prime \prime}\right)$, $5.23\left(\mathrm{dd}, J_{4,5}=15.4 \mathrm{~Hz}, J_{3,4}=8.7 \mathrm{~Hz}, 1 \mathrm{H}, \mathrm{H}-4\right), 4.59\left(\mathrm{~d}, J_{1^{\prime \prime}, 2^{\prime \prime}}=7.8 \mathrm{~Hz}, 1 \mathrm{H}, \mathrm{H}-\right.$ $\left.1^{\prime \prime}\right), 4.56$ (d, $\left.J_{1^{\prime}, 2^{\prime}}=7.8 \mathrm{~Hz}, 1 \mathrm{H}, \mathrm{H}-1^{\prime}\right), 4.53$ (bs, 2H, H-6'a, H-6'b), 4.44 (d, $J_{\mathrm{a}, \mathrm{b}}=$ $11.5 \mathrm{~Hz}, 1 \mathrm{H}, \mathrm{CH}-\mathrm{a}, 3-O-\mathrm{Bn}), 4.24$ (d, $\left.J_{\mathrm{a}, \mathrm{b}}=11.5 \mathrm{~Hz}, 1 \mathrm{H}, \mathrm{CH}-\mathrm{b}, 3-O-\mathrm{Bn}\right), 4.23-$ 4.22 (m, 1H, H-1a), (t, $\left.J_{3^{\prime}, 4^{\prime}}=9.4 \mathrm{~Hz}, 1 \mathrm{H}, \mathrm{H}-4^{\prime}\right), 4.08-4.05$ (m, 1H, H-2), 4.00 $\left(\mathrm{dd}, J_{6 " \mathrm{a}, 6^{\prime \prime} \mathrm{b}}=11.3 \mathrm{~Hz}, J_{5,6^{\prime \prime} \mathrm{a}}=6.8 \mathrm{~Hz}, 1 \mathrm{H}, \mathrm{H}-6{ }^{\prime \prime} \mathrm{a}\right), 3.81$ (bs, $\left.1 \mathrm{H}, \mathrm{H}-4{ }^{\prime \prime}\right), 3.73-$ 3.67 (m, 3H, H-3, H-5', H-3"'), 3.63 (dd, $J_{6^{\prime \prime} \mathrm{a}, 6^{\prime \prime} \mathrm{b}}=11.3 \mathrm{~Hz}, J_{5,6^{\prime \prime} \mathrm{b}}=6.2 \mathrm{~Hz}, 1 \mathrm{H}$, H-6" 'b), 3.48-3.47 (m, 2H, H-1b, H-5' ), 1.95-1.92 (m, 2H, H-6), 1.68-1.60 (m, $\left.2 \mathrm{H}, \mathrm{CH}_{2}-\alpha\right), 1.37-0.90(\mathrm{~m}, 68 \mathrm{H}, \mathrm{H}-7-\mathrm{H}-17, \mathrm{H}-\beta-\mathrm{H}-(\omega-1)), 0.88\left(\mathrm{t}, J_{17,18}=J_{\omega, \omega^{+1}}\right.$ $=6.9 \mathrm{~Hz}, 6 \mathrm{H}, \mathrm{H}-18, \mathrm{H}-\omega) ;{ }^{13} \mathrm{C} \mathrm{NMR}\left(125 \mathrm{MHz}, \mathrm{CDCl}_{3}\right) \delta 172.4(\mathrm{HNC}=\mathrm{O}), 166.4$ $\left(\mathrm{C}=\mathrm{O}, 2^{\prime \prime}-O-\mathrm{Bn}\right), 166.1\left(\mathrm{C}=\mathrm{O}, 6^{\prime \prime}-O-\mathrm{Bn}\right), 165.9\left(\mathrm{C}=\mathrm{O}, 6^{\prime}-O-\mathrm{Bn}\right), 165.8(\mathrm{C}=\mathrm{O}$, $\left.3^{\prime}-O-\mathrm{Bn}\right), 165.3$ (C=O, 2'-O-Bn), 138.3 (C-i, 3-O-Bn), 137.0 (C-5), 133.5, 133.4, 133.3 (C- $p, 5$ x OBz), 129.9, 129.8, 129.7, 129.64, 129.56, 129.54, 129.47, 129.1, 
129.0, 128.59, 128.56, 128.51, 128.4, 128.2, 127.6, 127.4 (30 x $\left.\mathrm{CH}_{\text {arom }}\right), 127.3$ (C4), 101.1 (C-1'), 100.8 (C-1'”), 79.1 (C-3), 75.9 (C-4'), 73.7 (C-2' ), 73.0 (C-5'),

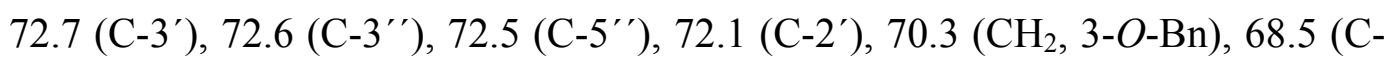
4'), 68.3 (C-1), 62.6 (C-6'), 61.8 (C-6"), 51.2 (C-2), $36.4(\mathrm{C}-\alpha), 32.2$ (C-6), $31.9,29.72,29.71,29.68,29.66,29.56,29.54,29.40,29.37,29.27,29.23,22.7$ (C-7-C-17, C- $\gamma-\mathrm{C}-(\omega-1)), 25.5$ (C- $\beta$ ), 14.1 (C-18, C- $\omega)$; HRMS(ESI) m/z calcd. for $\left[\mathrm{C}_{98} \mathrm{H}_{134} \mathrm{NO}_{18}+\mathrm{H}\right]^{+}: 1612.9595$, obsd.: 1612.9612 .

(2S,3R,4E)-1-(4-O-(4-O-Acetyl-2,6-di- $O$-benzoyl- $\beta$-D-galactopyranosyl)-2,3,6tri-O-benzoyl- $\beta$-D-glucopyranosyloxy)-3-benzyloxy-2-hexacosanoylamidooctadec-4-ene (73)

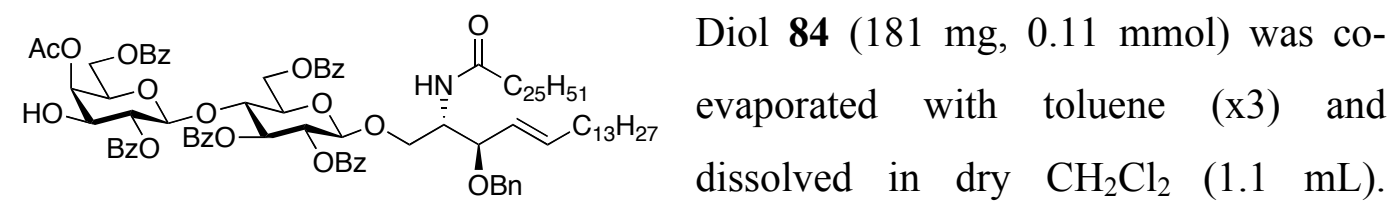

Trimethyl orthoacetate $(84 \mu \mathrm{L}, 0.67 \mathrm{mmol})$ and CSA (52 $\mathrm{mg}, 0.22 \mathrm{mmol})$ were added and the reaction mixture was stirred at $\mathrm{rt}$ for $16 \mathrm{~h}$. The reaction mixture was diluted with EtOAc $(30 \mathrm{~mL})$, washed with $1 \mathrm{M} \mathrm{HCl}$ solution $(3 \times 30 \mathrm{~mL})$, sat. aq. $\mathrm{NaHCO}_{3}(30 \mathrm{~mL})$ and brine $(30 \mathrm{~mL})$, dried $\left(\mathrm{MgSO}_{4}\right)$, filtered and concentrated in vacuo. The residue was purified by gradient flash chromatography (petroleum ether/EtOAc, $10 / 1$ to $1 / 1, \mathrm{v} / \mathrm{v})$ to afford acetate 73 as a clear oil $(170 \mathrm{mg}, 0.26$ mmol, 92\%). R $\mathrm{R}_{f} 0.24(\mathrm{PE} / \mathrm{EA}, 2 / 1, \mathrm{v} / \mathrm{v}) ;[\alpha]_{\mathrm{D}}{ }^{24}=+4.0^{\circ}\left(\mathrm{c}=1.0, \mathrm{CH}_{2} \mathrm{Cl}_{2}\right)$; IR (film) 3325, 3064, 2923, 2853, 2359, 1966, 1726, 1452, 1372, 1265, 1177, 1108, 1094, 1069, 1027, 975, 736, $708 \mathrm{~cm}^{-1}$; ${ }^{1} \mathrm{H} \mathrm{NMR}\left(500 \mathrm{MHz}, \mathrm{CDCl}_{3}\right) \delta 8.06\left(\mathrm{~d}, J_{\mathrm{CH}-}\right.$ $o, \mathrm{CH}-m=7.8 \mathrm{~Hz}, 2 \mathrm{H}, \mathrm{CH}-o, \mathrm{OBz}), 8.03\left(\mathrm{~d}, J_{\mathrm{CH}-o, \mathrm{CH}-m}=7.6 \mathrm{~Hz}, 4 \mathrm{H}, 2 \times \mathrm{CH}-o\right.$, $\mathrm{OBz}), 7.96\left(\mathrm{~d}, J_{\mathrm{CH}-o, \mathrm{CH}-m}=7.3 \mathrm{~Hz}, 2 \mathrm{H}, \mathrm{CH}-o, \mathrm{OBz}\right), 7.96\left(\mathrm{~d}, J_{\mathrm{CH}-o, \mathrm{CH}-m}=7.8 \mathrm{~Hz}\right.$, $2 \mathrm{H}, \mathrm{CH}-o, \mathrm{OBz}), 7.60-7.13\left(\mathrm{~m}, 20 \mathrm{H}, \mathrm{H}_{\text {arom }}\right), 5.72\left(\mathrm{t}, J_{2^{\prime}, 3^{\prime}}=J_{3^{\prime}, 4^{\prime}}=9.7 \mathrm{~Hz}, 1 \mathrm{H}, \mathrm{H}-\right.$ $\left.3^{\prime}\right), 5.50\left(\mathrm{dt}, J_{4,5}=15.6 \mathrm{~Hz}, J_{5,6}=6.6 \mathrm{~Hz}, 1 \mathrm{H}, \mathrm{H}-5\right), 5.41\left(\mathrm{dd}, J_{1^{\prime}, 2^{\prime}}=8.0 \mathrm{~Hz}, J_{2^{\prime}, 3^{\prime}}\right.$ $\left.=9.7 \mathrm{~Hz}, 1 \mathrm{H}, \mathrm{H}-2^{\prime}\right), 5.37\left(\mathrm{~d}, J_{\mathrm{NH}, 2^{\prime}}=9.3 \mathrm{~Hz}, 1 \mathrm{H}, \mathrm{NH}\right), 5.26-5.22(\mathrm{~m}, 1 \mathrm{H}, \mathrm{H}-4)$, $5.22\left(\mathrm{~d}, J_{3^{\prime \prime}, 4^{\prime \prime}}=3.5 \mathrm{~Hz}, 1 \mathrm{H}, \mathrm{H}-4^{\prime \prime}\right), 5.16\left(\mathrm{dd}, J_{2^{\prime \prime}, 3^{\prime \prime}}=9.8 \mathrm{~Hz}, J_{1^{\prime \prime}, 2^{\prime \prime}}=8.0 \mathrm{~Hz}, 1 \mathrm{H}\right.$, $\left.\mathrm{H}-2^{\prime \prime}\right), 4.65$ (d, $\left.J_{1^{\prime \prime}, 2^{\prime \prime}}=8.0 \mathrm{~Hz}, 1 \mathrm{H}, \mathrm{H}-1^{\prime \prime}\right), 4.61$ (d, $\left.J_{1^{\prime}, 2^{\prime}}=8.0 \mathrm{~Hz}, 1 \mathrm{H}, \mathrm{H}-1^{\prime}\right)$, 4.60-4.51 (m, 2H, H-6'a, H-6'b), 4.44 (d, $\left.J_{\mathrm{a}, \mathrm{b}}=11.5 \mathrm{~Hz}, 1 \mathrm{H}, \mathrm{CH}-\mathrm{a}, 3-O-\mathrm{Bn}\right)$, $4.25\left(\mathrm{~d}, J_{\mathrm{a}, \mathrm{b}}=11.5 \mathrm{~Hz}, 1 \mathrm{H}, \mathrm{CH}-\mathrm{b}, 3-O-\mathrm{Bn}\right), 4.19\left(\mathrm{t}, J_{3^{\prime}, 4^{\prime}}=9.7 \mathrm{~Hz}, 1 \mathrm{H}, \mathrm{H}-4^{\prime}\right), 4.07$ $(\mathrm{m}, 1 \mathrm{H}, \mathrm{H}-2), 3.82\left(\mathrm{dd}, J_{2^{\prime \prime}, 3^{\prime \prime}}=9.8 \mathrm{~Hz}, J_{3^{\prime \prime}, 4^{\prime \prime}}=3.5 \mathrm{~Hz}, 1 \mathrm{H}, \mathrm{H}-3^{\prime \prime}\right), 3.76\left(\mathrm{~d}, J_{4^{\prime}, 5^{\prime}}=\right.$ 
$\left.7.8 \mathrm{~Hz}, 1 \mathrm{H}, \mathrm{H}-5^{\prime}\right), 3.73-3.68$ (m, 2H, H-3, H-6"'a), 3.64-3.59 (m, 2H, H-5'", H6" 'b), 3.50 (dd, $\left.J_{1 \mathrm{a}, 1 \mathrm{~b}}=9.8 \mathrm{~Hz}, J_{1 \mathrm{~b}, 2}=3.5 \mathrm{~Hz}, 1 \mathrm{H}, \mathrm{H}-1 \mathrm{~b}\right), 1.99$ (s, 3H, OAc), 1.981.92 (m, 2H, H-6), 1.69-1.60 (m, 2H, $\mathrm{CH}_{2}-\alpha$ ), 1.37-1.03 (m, 68H, H-7-H-17, H$\beta-\mathrm{H}-(\omega-1)), 0.88\left(\mathrm{t}, J_{17,18}=J_{\omega-1, \omega}=6.9 \mathrm{~Hz}, 6 \mathrm{H}, \mathrm{H}-18, \mathrm{H}-\omega\right) ;{ }^{13} \mathrm{C}$ NMR $(125$ $\left.\mathrm{MHz}, \mathrm{CDCl}_{3}\right) \delta 172.4(\mathrm{HNC}=\mathrm{O}), 170.6(\mathrm{C}=\mathrm{O}, \mathrm{OAc}), 166.5\left(\mathrm{C}=\mathrm{O}, 2^{\prime \prime}-\mathrm{O}-\mathrm{Bn}\right)$, $165.9\left(\mathrm{C}=\mathrm{O}, 6^{\prime}-O-\mathrm{Bn}\right), 165.6\left(\mathrm{C}=\mathrm{O}, 6^{\prime \prime}-O-\mathrm{Bn}\right), 165.3\left(\mathrm{C}=\mathrm{O}, 2^{\prime}-O-\mathrm{Bn}\right), 165.3$ $\left(\mathrm{C}=\mathrm{O}, 3^{\prime}-O-\mathrm{Bn}\right), 138.3(\mathrm{C}-i, 3-O-\mathrm{Bn}), 137.0(\mathrm{C}-5), 133.6,133.5,133.4,133.2(\mathrm{C}-$ p, $5 \mathrm{x} \mathrm{OBz}), 129.89,120.82,129.76,129.62,129.51,129.41,129.06,128.9$, 128.6, 128.58, 128.26, 128.19, 127.63, 127.40, 127.30 (30 x $\mathrm{CH}_{\text {arom }}$ ), $101.3(\mathrm{C}-$ 1'), 100.3 (C-1'”), 79.1 (C-3), 75.3 (C-4'), 73.5 (C-2'”), 73.0 (C-5'), 72.4 (C-3'), 72.1 (C-2'), $71.5\left(\mathrm{C}-3^{\prime \prime}\right), 71.1$ (C-5'), $70.3\left(\mathrm{CH}_{2}, 3-O-\mathrm{Bn}\right), 69.3$ (C-4'), 68.3 (C1), 62.5 (C-6'), 61.2 (C-6"'), 21.1 (C-2), 36.4 (C- $\alpha$ ), 32.2 (C-6), 31.92, 29.72, $29.70,29.68,29.66,29.55,29.54,29.39,29.37,29.36,29.26,29.22,25.45,22.69$ (C-7-C-17, C- $\beta-\mathrm{C}-(\omega-1)), 20.6$ (OAc), 14.1 (C-18, C- $\omega)$; HRMS(ESI) m/z calcd. for $\left[\mathrm{C}_{100} \mathrm{H}_{135} \mathrm{NO}_{19}+\mathrm{Na}\right]^{+}: 1676.9521$, obsd.: 1676.9526 .

\section{6-Deoxy-1,2:3,4-di- $O$-isopropylidene- $\alpha$-D-galactopyranose $(88)^{31}$}

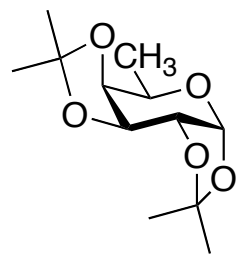

$\mathrm{LiAlH}_{4}(350 \mathrm{mg}, 9.22 \mathrm{mmol})$ was carefully added to a solution of $\mathrm{Bu}_{3} \mathrm{SnCl}(3 \mathrm{~g}, 9.22 \mathrm{mmol})$ in dry $\mathrm{Et}_{2} \mathrm{O}(20 \mathrm{~mL})$ at $0{ }^{\circ} \mathrm{C}$. The reaction mixture was stirred at $\mathrm{rt}$ for $3 \mathrm{~h}$, and ice water $(10 \mathrm{~mL})$ was added and stirred for a further 5 mins. The mixture was filtered through a celite pad and the organic layer washed with $\mathrm{H}_{2} \mathrm{O}(2 \times 20 \mathrm{~mL})$, dried $\left(\mathrm{MgSO}_{4}\right)$ and concentrated in vacuo to give $\mathrm{Bu}_{3} \mathrm{SnH}$ as an oil, which was set aside for the next step.

6-Deoxy-6-iodo-1,2:3,4-di- $O$-isopropylidene- $\alpha$-D-galactopyranose, $\mathbf{8 7}^{30,39,40}(1.0$ $\mathrm{g}, 2.84 \mathrm{mmol})$ was co-evaporated with toluene (x3) and dissolved in toluene (1.8 $\mathrm{mL}) . \mathrm{Bu}_{3} \mathrm{SnH}(1.53 \mathrm{~mL}, 5.67 \mathrm{mmol})$ and AIBN (70 mg, $\left.0.43 \mathrm{mmol}\right)$ were added and stirred at $100{ }^{\circ} \mathrm{C}$ for $1.5 \mathrm{~h}$, after which the reaction mixture was diluted with EtOAc $(80 \mathrm{~mL})$, the organic layer washed with water $(80 \mathrm{~mL})$ and brine $(80 \mathrm{~mL})$, dried $\left(\mathrm{MgSO}_{4}\right)$, filtered and concentrated in vacuo. The resultant oil was purified by gradient flash chromatography (petroleum ether to petroleum ether/EtOAc, $30 / 1$ to 3/1, v/v) to afford 6-deoxy-galactose 88 as a clear oil (672 $\mathrm{mg}, 2.75 \mathrm{mmol}$, 97\%). $\mathrm{R}_{f}: 0.27(\mathrm{PE} / \mathrm{EA}, 10 / 1, \mathrm{v} / \mathrm{v}) ;[\alpha]_{\mathrm{D}}^{25}=-37.0^{\circ}\left(\mathrm{c}=1.0, \mathrm{CHCl}_{3}\right) ; \mathrm{IR}($ film$)$ 
3027, 2930, 1369, 1321, 1230, 1170, 1045, 1020, 904, $669 \mathrm{~cm}^{-1} ;{ }^{1} \mathrm{H}$ NMR (500 $\left.\mathrm{MHz}, \mathrm{CDCl}_{3}\right) \delta 5.51\left(\mathrm{~d}, J_{1,2}=5.1 \mathrm{~Hz}, 1 \mathrm{H}, \mathrm{H}-1\right), 4.58\left(\mathrm{dd}, J_{3,4}=8.0 \mathrm{~Hz}, J_{2,3}=2.2\right.$ $\mathrm{Hz}, 1 \mathrm{H}, \mathrm{H}-3), 4.27\left(\mathrm{dd}, J_{1,2}=5.1 \mathrm{~Hz}, J_{2,3}=2.2 \mathrm{~Hz}, 1 \mathrm{H}, \mathrm{H}-2\right), 4.07$ (dd, $J_{3,4}=8.0$ $\left.\mathrm{Hz}, J_{4,5}=1.8 \mathrm{~Hz}, 1 \mathrm{H}, \mathrm{H}-4\right), 3.90\left(\mathrm{dq}, J_{5,6}=6.6 \mathrm{~Hz}, J_{4,5}=1.8 \mathrm{~Hz}, 1 \mathrm{H}, \mathrm{H}-5\right), 1.51$ (s, 3H, $\mathrm{CH}_{3} i \mathrm{Pr}$ ), 1.45 (s, 3H, $\mathrm{CH}_{3} i \mathrm{Pr}$ ), 1.34 (s, 3H, $\mathrm{CH}_{3} i \mathrm{Pr}$ ), 1.32 (s, 3H, $\mathrm{CH}_{3}$ $i \operatorname{Pr}), 1.24\left(\mathrm{~d}, J_{5,6}=6.5 \mathrm{~Hz}, 1 \mathrm{H}, \mathrm{H}-6\right) ;{ }^{13} \mathrm{C} \mathrm{NMR}\left(125 \mathrm{MHz}, \mathrm{CDCl}_{3}\right) \delta 108.9\left(\mathrm{C}_{\mathrm{q}}\right.$ $i \operatorname{Pr}-3,4), 108.2$ (C $\mathrm{C}_{\mathrm{q}}$ Pr-1,2), 96.5 (C-1), 73.5 (C-4), 70.9 (C-3), 70.3 (C-2), 63.4 (C-5), $26.0\left(2 \mathrm{x} \mathrm{CH}_{3} i \mathrm{Pr}\right), 26.0\left(\mathrm{CH}_{3} i \mathrm{Pr}\right), 24.9\left(\mathrm{CH}_{3} i \mathrm{Pr}\right), 17.5$ (C-6); HRMS(ESI) $\mathrm{m} / \mathrm{z}$ calcd. for $\left[\mathrm{C}_{12} \mathrm{H}_{20} \mathrm{O}_{5}+\mathrm{NH}_{4}\right]^{+}: 262.1649$, obsd.: 262.1655 .

\section{1,2,3,4-Tetra- $O$-acetyl-6-deoxy- $\alpha / \beta$-D-galactopyranose (86)}

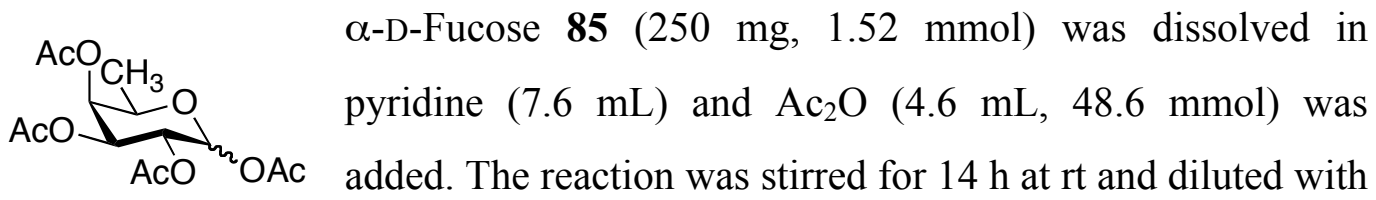
$\mathrm{CH}_{2} \mathrm{Cl}_{2}$, washed with $\mathrm{H}_{2} \mathrm{O}(2 \times 50 \mathrm{~mL})$, sat. aq. $\mathrm{NaHCO}_{3}(50 \mathrm{~mL})$, brine $(50 \mathrm{~mL})$ and dried over $\mathrm{MgSO}_{4}$. The $\mathrm{MgSO}_{4}$ was filtered off, the filtrate concentrated in vacuo and purified by gradient flash chromatography (petroleum ether/EtOAc, $10: 1$ to $3: 1, \mathrm{v} / \mathrm{v})$ to afford an $\alpha / \beta$ mixture (1:1.4) of peracetylated D-fucose 86 as a clear oil (506 mg, $1.52 \mathrm{mmol}$, Quantitative).

Alternative method starting from 6-deoxy-1,2:3,4-di-O-isopropylidene- $\alpha-D$ galactopyranose, $\boldsymbol{8 8}^{31}$ :

A solution of $\mathrm{AcOH} / \mathrm{H}_{2} \mathrm{O}(4 / 1, \mathrm{v} / \mathrm{v}, 5 \mathrm{~mL})$ was added to 6-deoxy galactoside $\mathbf{8 8}$ and stirred for $19 \mathrm{~h}$, after which the reaction mixture was warmed to $105^{\circ} \mathrm{C}$ and stirred for further $2 \mathrm{~h}$. The reaction mixture was concentrated, and the residue coevaporated with pyridine $(3 \times 8 \mathrm{~mL})$, dissolved in pyridine $(5 \mathrm{~mL})$ and $\mathrm{Ac}_{2} \mathrm{O}(0.46$ $\mathrm{ml}, 4.86 \mathrm{mmol}$ ). After stirring at $\mathrm{rt}$ for $16 \mathrm{~h}$, the reaction mixture was taken up in EtOAc $(50 \mathrm{~mL})$, washed with $0.5 \mathrm{M} \mathrm{HCl}$ solution $(50 \mathrm{~mL})$, sat. aq. $\mathrm{NaHCO}_{3}(50$ $\mathrm{mL})$, water $(50 \mathrm{~mL})$ and brine $(50 \mathrm{~mL})$, dried $\left(\mathrm{MgSO}_{4}\right)$, filtered and concentrated in vасио. Purification by gradient flash chromatography (petroleum ether/EtOAc, 10:1 to $3: 1, \mathrm{v} / \mathrm{v}$ ) afforded an $\alpha / \beta$ mixture (1:5) of peracetylated D-fucose 86 as a clear oil (273 mg, $0.82 \mathrm{mmol}, 85 \%)$. R $\mathrm{R}_{f} 0.51(\mathrm{PE} / \mathrm{EA}, 1 / 2, \mathrm{v} / \mathrm{v}) ;[\alpha]_{\mathrm{D}}{ }^{25}=59.0^{\circ}(\mathrm{c}$ $=1.0, \mathrm{CHCl}_{3}$ ); IR (film) 3027, 2942, 2880, 1746, 1433, 1369, 1321, 1212, 1167, 
1052, 1023, 904, $668 \mathrm{~cm}^{-1} ;{ }^{1} \mathrm{H}$ NMR $\left(500 \mathrm{MHz}, \mathrm{CDCl}_{3}\right) \delta 5.66\left(\mathrm{~d}, J_{1,2}=5.7 \mathrm{~Hz}\right.$, $1 \mathrm{H}, \mathrm{H}-1), 5.29$ (t, $\left.J_{2,3}=10.3 \mathrm{~Hz}, 1 \mathrm{H}, \mathrm{H}-2\right), 5.25$ (d, $\left.J_{3,4}=3.2 \mathrm{~Hz}, 1 \mathrm{H}, \mathrm{H}-4\right), 5.05$ $\left(\mathrm{dd}, J_{2,3}=10.3 \mathrm{~Hz}, J_{3,4}=3.2 \mathrm{~Hz}, 1 \mathrm{H}, \mathrm{H}-3\right), 3.94$ (q, $\left.J_{5,6}=6.4 \mathrm{~Hz}, 1 \mathrm{H}, \mathrm{H}-5\right), 2.17$ (s, 3H, 4-OAc), 2.09 (s, 3H, 1-OAc), 2.02 (s, 3H, 2-OAc), 1.97 (s, 3H, 3-OAc), $1.20\left(\mathrm{~d}, J_{5,6}=6.4 \mathrm{~Hz}, 1 \mathrm{H}, \mathrm{H}-6\right) ;{ }^{13} \mathrm{C}$ NMR $\left(125 \mathrm{MHz}, \mathrm{CDCl}_{3}\right) \delta 170.5(\mathrm{C}=\mathrm{O}, 4-$ OAc), $170.0(\mathrm{C}=\mathrm{O}, 3-\mathrm{OAc}), 169.5(\mathrm{C}=\mathrm{O}, 2-\mathrm{OAc}), 169.2(\mathrm{C}=\mathrm{O}, 1-\mathrm{OAc}), 92.1(\mathrm{C}-$ 1), 71.2 (C-3), 70.2 (C-5), 69.9 (C-4), 67.9 (C-2), 20.8 (1-OAc), 20.7 (2-OAc), 20.63 (4-OAc), 20.56 (3-OAc), 15.9 (C-6); HRMS(ESI) $\mathrm{m} / \mathrm{z}$ calcd. for $\left[\mathrm{C}_{14} \mathrm{H}_{20} \mathrm{O}_{9}+\mathrm{NH}_{4}\right]^{+}: 350.1446$, obsd.: 350.1450 .

\section{Ethyl 2,3,4-tri-O-acetyl-6-deoxy-1-thio- $\beta$-D-galactopyranoside (89)}

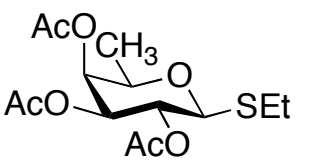

Peracetylated D-fucose 86 (620 mg, $1.87 \mathrm{mmol})$ was coevaporated with toluene (x3) and dissolved in dry $\mathrm{CH}_{2} \mathrm{Cl}_{2}(10$ $\mathrm{mL})$. Ethanethiol $(0.41 \mathrm{~mL}, 5.60 \mathrm{mmol})$ was added at $\mathrm{rt}$, followed by freshly distilled $48 \% \mathrm{BF}_{3} . \mathrm{OEt}_{2}(0.71 \mathrm{~mL}, 2.80 \mathrm{mmol})$ and stirred for $2 \mathrm{~h}$. The reaction mixture was diluted with $\mathrm{CH}_{2} \mathrm{Cl}_{2}(80 \mathrm{~mL})$ and washed with $\mathrm{H}_{2} \mathrm{O}$ $(2 \times 50 \mathrm{~mL})$, sat. aq. $\mathrm{NaHCO}_{3}(50 \mathrm{~mL})$, brine $(50 \mathrm{~mL})$ and dried over $\mathrm{MgSO}_{4}$ and filtered. The concentrated filtrate was purified by flash column chromatography (petroleum ether/EtOAc, 5:1, v/v) to give $\beta$-thiofucoside 89 as a clear oil (452 mg, $1.35 \mathrm{mmol}, 72 \%) . \mathrm{R}_{f}: 0.55(\mathrm{PE} / \mathrm{EA}, 1 / 1, \mathrm{v} / \mathrm{v}) ;[\alpha]_{\mathrm{D}}{ }^{25}=+49.0^{\circ}\left(\mathrm{c}=1.0, \mathrm{CHCl}_{3}\right) ; \mathrm{IR}$ (film) 3023, 2904, 1746, 1331, 1245, 1218, 1084, 1055, 1020, 863, 749, $667 \mathrm{~cm}^{-1}$; ${ }^{1} \mathrm{H} \mathrm{NMR}\left(500 \mathrm{MHz}, \mathrm{CDCl}_{3}\right) \delta 5.23\left(\mathrm{~d}, J_{3,4}=3.5 \mathrm{~Hz}, 1 \mathrm{H}, \mathrm{H}-4\right), 5.17\left(\mathrm{t}, J_{1,2}=J_{2,3}=\right.$ $10.0 \mathrm{~Hz}, 1 \mathrm{H}, \mathrm{H}-2), 5.00\left(\mathrm{dd}, J_{2,3}=10.0 \mathrm{~Hz}, J_{3,4}=3.5 \mathrm{~Hz}, 1 \mathrm{H}, \mathrm{H}-3\right), 4.42\left(\mathrm{~d}, J_{1,2}=\right.$ $10.0 \mathrm{~Hz}, 1 \mathrm{H}, \mathrm{H}-1), 3.79$ (q, $\left.J_{5,6}=6.4 \mathrm{~Hz}, 1 \mathrm{H}, \mathrm{H}-5\right), 2.75-2.62\left(\mathrm{~m}, 2 \mathrm{H}, \mathrm{CH}_{2} \mathrm{CH}_{3}\right)$, 2.13 (s, 3H, 4-OAc), 2.02 (s, 3H, 2-OAc), 1.94 (s, 3H, 3-OAc), $1.23\left(\mathrm{t}, J_{\mathrm{CH} 2, \mathrm{CH} 3}=\right.$ $7.5 \mathrm{~Hz}, 3 \mathrm{H}, \mathrm{CH}_{2} \mathrm{CH}_{3}$ ) 1.17 (d, $\left.J_{5,6}=6.6 \mathrm{~Hz}, 3 \mathrm{H}, \mathrm{H}-6\right) ;{ }^{13} \mathrm{C} \mathrm{NMR}(125 \mathrm{MHz}$, $\left.\mathrm{CDCl}_{3}\right) \delta 170.6(\mathrm{C}=\mathrm{O}, 4-\mathrm{OAc}), 170.1(\mathrm{C}=\mathrm{O}, 3-\mathrm{OAc}), 169.6(\mathrm{C}=\mathrm{O}, 2-\mathrm{OAc}), 83.5$ (C-1), 73.1 (C-5), 72.3 (C-3), 70.4 (C-4), 67.3 (C-2), $24.1\left(\mathrm{CH}_{2} \mathrm{CH}_{3}\right), 20.8$ (2OAc), 20.7 (4-OAc), 20.6 (3-OAc), 16.4 (C-6), $14.7\left(\mathrm{CH}_{2} \mathrm{CH}_{3}\right)$; HRMS(ESI) m/z calcd. for $\left[\mathrm{C}_{14} \mathrm{H}_{22} \mathrm{O}_{7} \mathrm{~S}+\mathrm{NH}_{4}\right]^{+}: 352.1424$, obsd.: 352.1430 . 
Ethyl 2,3,4-tri- $O$-benzyl-6-deoxy-1-thio- $\beta$-D-galactopyranoside $(90)^{28}$

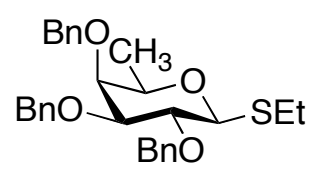

$\beta$-thiofucoside 89 (389 $\mathrm{mg}, 1.16 \mathrm{mmol}$ ) was dissolved in methanol and $\mathrm{NaOMe}$ was added until the solution reached $\mathrm{pH}$ 9.0. The reaction mixture was stirred at $\mathrm{rt}$ for $1 \mathrm{~h}$, neutralized with Dowex $\mathrm{H}^{+}$, filtered and concentrated to give the pure triol as a colourless oil (242 mg, $1.16 \mathrm{mmol})$, which was used without further purification. The triol (243 mg, $1.16 \mathrm{mmol}$ ) was co-evaporated with toluene (x3) and dissolved in dry DMF $(12 \mathrm{~mL})$ and benzyl bromide $(0.55 \mathrm{~mL}, 4.66 \mathrm{mmol})$ was added. The mixture was cooled to $0{ }^{\circ} \mathrm{C}$ and $\mathrm{NaH}$ (60\% in oil suspension, $233 \mathrm{mg}, 5.82 \mathrm{mmol}$ ) was added. The reaction was stirred for $17 \mathrm{~h}$ at $\mathrm{rt}$, quenched with $\mathrm{MeOH}(5 \mathrm{~mL})$ and concentrated to remove the DMF. The crude oil was redissolved in EtOAc (50 $\mathrm{mL})$, and washed with sat. aq. $\mathrm{NaHCO}_{3}(50 \mathrm{~mL})$ and brine $(50 \mathrm{~mL})$, dried $\left(\mathrm{MgSO}_{4}\right)$, filtered and concentrated in vacuo. The residue was purified by gradient flash chromatography (petroleum ether/EtOAc, 20:1 to 10:1, v/v) to afford benzylated thiofucoside 90 as a clear oil (464 mg, $0.97 \mathrm{mmol}, 83 \%$ ). $\mathrm{R}_{f}: 0.73$ $(\mathrm{PE} / \mathrm{EA}, 2 / 1, \mathrm{v} / \mathrm{v}) ;[\alpha]_{\mathrm{D}}{ }^{27}=-6.0^{\circ}\left(\mathrm{c}=1.0, \mathrm{CHCl}_{3}\right)$; IR (film) 3064, 2978, 2868, 1606, 1497, 1454, 1357, 1208, 1124, 1087, 1066, 1047, 875, 745, 732, $697 \mathrm{~cm}^{-1}$; ${ }^{1} \mathrm{H}$ NMR (500 MHz, $\left.\mathrm{CDCl}_{3}\right) \delta 7.44-7.29\left(\mathrm{~m}, 15 \mathrm{H}, \mathrm{H}_{\text {arom }}\right), 5.03\left(\mathrm{~d}, J_{\mathrm{a}, \mathrm{b}}=11.8 \mathrm{~Hz}\right.$, $1 \mathrm{H}, \mathrm{CH}-\mathrm{a}, 4-O-\mathrm{Bn}), 4.93\left(\mathrm{~d}, J_{\mathrm{a}, \mathrm{b}}=10.3 \mathrm{~Hz}, 1 \mathrm{H}, \mathrm{CH}-\mathrm{a}, 2-O-\mathrm{Bn}\right), 4.84$ (d, $J_{\mathrm{a}, \mathrm{b}}=$ $10.3 \mathrm{~Hz}, 1 \mathrm{H}, \mathrm{CH}-\mathrm{b}, 2-O-\mathrm{Bn}), 4.80$ (d, $\left.J_{\mathrm{a}, \mathrm{b}}=12.0 \mathrm{~Hz}, 1 \mathrm{H}, \mathrm{CH}-\mathrm{a}, 3-O-\mathrm{Bn}\right), 4.77$ (d, $\left.J_{\mathrm{a}, \mathrm{b}}=12.0 \mathrm{~Hz}, 1 \mathrm{H}, \mathrm{CH}-\mathrm{b}, 3-O-\mathrm{Bn}\right), 4.73$ (d, $\left.J_{\mathrm{a}, \mathrm{b}}=11.8 \mathrm{~Hz}, 1 \mathrm{H}, \mathrm{CH}-\mathrm{b}, 4-O-\mathrm{Bn}\right)$, $4.43\left(\mathrm{~d}, J_{1,2}=9.5 \mathrm{~Hz}, 1 \mathrm{H}, \mathrm{H}-1\right), 3.86\left(\mathrm{t}, J_{1,2}=J_{2,3}=9.5 \mathrm{~Hz}, 1 \mathrm{H}, \mathrm{H}-2\right), 3.64\left(\mathrm{~d}, J_{3,4}\right.$ $=2.6 \mathrm{~Hz}, 1 \mathrm{H}, \mathrm{H}-4), 3.60\left(\mathrm{dd}, J_{2,3}=9.5 \mathrm{~Hz}, J_{3,4}=2.6 \mathrm{~Hz}, 1 \mathrm{H}, \mathrm{H}-3\right), 3.51\left(\mathrm{q}, J_{5,6}=\right.$ $6.4 \mathrm{~Hz}, 1 \mathrm{H}, \mathrm{H}-5), 2.84-2.71\left(\mathrm{~m}, 2 \mathrm{H}, \mathrm{C}_{2} \mathrm{CH}_{3}\right), 1.33\left(\mathrm{t}, J_{\mathrm{CH} 2, \mathrm{CH} 3}=7.5 \mathrm{~Hz}, 2 \mathrm{H}\right.$, $\left.\mathrm{C}_{2} \mathrm{CH}_{3}\right), 1.24$ (d, $\left.J_{5,6}=6.4 \mathrm{~Hz}, 1 \mathrm{H}, \mathrm{H}-6\right) ;{ }^{13} \mathrm{C} \mathrm{NMR}\left(125 \mathrm{MHz}, \mathrm{CDCl}_{3}\right) \delta 138.7$ (C-i, 4-O-Bn), 138.5 (C-i, 3-O-Bn), 138.4 (C-i, 2-O-Bn), 128.6, 128.5, 128.34, $128.29,128.22,128.19,128.0,127.73,127.69,127.59,127.54$ (15 x $\left.\mathrm{CH}_{\text {arom }}\right), 85.0$ (C-1), 84.5 (C-3), 78.4 (C-2), 76.5 (C-4), 75.7 ( $\left.\mathrm{CH}_{2}, 2-\mathrm{O}-\mathrm{Bn}\right), 75.6\left(\mathrm{C}-5, \mathrm{CH}_{2}, 4-\right.$ $O-\mathrm{Bn}), 72.9\left(\mathrm{CH}_{2}, 3-\mathrm{O}-\mathrm{Bn}\right), 24.7\left(\underline{\mathrm{CH}}_{2} \mathrm{CH}_{3}\right), 17.3(\mathrm{C}-6), 15.0\left(\mathrm{CH}_{2} \underline{\mathrm{CH}}_{3}\right)$; HRMS(ESI) m/z calcd. for $\left[\mathrm{C}_{29} \mathrm{H}_{34} \mathrm{NO}_{4} \mathrm{~S}+\mathrm{NH}_{4}\right]^{+}:$496.2516, obsd.: 496.2517. 


\section{2,3,4-Tri- $O$-benzyl-6-deoxy- $\alpha / \beta$-D-galactopyranose $(91)^{41}$}

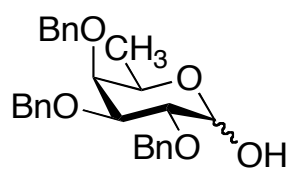

Thiofucoside 90 (183 $\mathrm{mg}, 0.38 \mathrm{mmol}$ ) was dissolved in acetone $/ \mathrm{H}_{2} \mathrm{O}(7.6 \mathrm{~mL}, 9: 1, \mathrm{v} / \mathrm{v})$ and NBS $(238 \mathrm{mg}, 1.34 \mathrm{mmol})$ was added. The reaction mixture was stirred at rt for $10 \mathrm{mins}$ and diluted with ethyl acetate $(30 \mathrm{~mL})$ and the organic layer was then washed with sat. aq. $\mathrm{NaHCO}_{3}(30 \mathrm{~mL})$, and brine $(30 \mathrm{~mL})$, dried $\left(\mathrm{MgSO}_{4}\right)$, filtered and concentrated under reduced pressure. The residue was purified by gradient flash chromatography (petroleum ether/EtOAc, $2: 1, \mathrm{v} / \mathrm{v}$ ) to afford an $\alpha / \beta$ mixture (2.5:1) of lactol 91 as a colourless oil (154 mg, $0.35 \mathrm{mmol}, 93 \%)$. $\mathrm{R}_{f}: 0.16$ $(\mathrm{PE} / \mathrm{EA}, 2 / 1, \mathrm{v} / \mathrm{v}) ;[\alpha]_{\mathrm{D}}{ }^{27}=+18.0^{\circ}\left(\mathrm{c}=1.0, \mathrm{CHCl}_{3}\right.$, value obtained for $2.5: 1 \alpha / \beta$ mixture); IR (film) 3398, 3063, 3030, 2877, 1497, 1454, 1359, 1309, 1211, 1170, 1091, 1061, 1027, 950, 912, 815, 734, 696, $666 \mathrm{~cm}^{-1} ; \alpha:{ }^{1} \mathrm{H}$ NMR $(500 \mathrm{MHz}$, $\left.\mathrm{CDCl}_{3}\right) \delta 7.41-7.28$ (m, 15H, $\left.\mathrm{H}_{\text {arom }}\right), 5.27\left(\mathrm{~d}, J_{1,2}=3.7 \mathrm{~Hz}, 1 \mathrm{H}, \mathrm{H}-1\right), 4.98\left(\mathrm{~d}, J_{\mathrm{a}, \mathrm{b}}\right.$ $=11.6 \mathrm{~Hz}, 1 \mathrm{H}, \mathrm{CH}-\mathrm{a}, 4-O-\mathrm{Bn}), 4.84\left(\mathrm{~d}, J_{\mathrm{a}, \mathrm{b}}=11.5 \mathrm{~Hz}, 1 \mathrm{H}, \mathrm{CH}-\mathrm{a}, 3-O-\mathrm{Bn}\right), 4.82$ (d, $\left.J_{\mathrm{a}, \mathrm{b}}=10.5 \mathrm{~Hz}, 1 \mathrm{H}, \mathrm{CH}-\mathrm{a}, 2-O-\mathrm{Bn}\right), 4.76\left(\mathrm{~d}, J_{\mathrm{a}, \mathrm{b}}=11.5 \mathrm{~Hz}, 1 \mathrm{H}, \mathrm{CH}-\mathrm{b}, 3-O-\mathrm{Bn}\right)$, $4.72\left(\mathrm{~d}, J_{\mathrm{a}, \mathrm{b}}=10.5 \mathrm{~Hz}, 1 \mathrm{H}, \mathrm{CH}-\mathrm{b}, 2-O-\mathrm{Bn}\right), 4.67$ (d, $J_{\mathrm{a}, \mathrm{b}}=11.7 \mathrm{~Hz}, 1 \mathrm{H}, \mathrm{CH}-\mathrm{b}, 4-$ $O$-Bn), 4.10 (q, $\left.J_{5,6}=6.5 \mathrm{~Hz}, 1 \mathrm{H}, \mathrm{H}-5\right), 4.04$ (dd, $J_{2,3}=9.9 \mathrm{~Hz}, J_{1,2}=3.7 \mathrm{~Hz}, 1 \mathrm{H}$, $\mathrm{H}-2), 3.90$ (dd, $\left.J_{2,3}=9.9 \mathrm{~Hz}, J_{3,4}=2.6 \mathrm{~Hz}, 1 \mathrm{H}, \mathrm{H}-3\right), 3.67$ (bs, $\left.1 \mathrm{H}, \mathrm{H}-4\right), 1.14$ (d, $\left.J_{5,6}=6.5 \mathrm{~Hz}, 1 \mathrm{H}, \mathrm{H}-6\right) ;{ }^{13} \mathrm{C}$ NMR $\left(125 \mathrm{MHz}, \mathrm{CDCl}_{3}\right) \delta 138.7$ (C-i, 3-O-Bn), 138.5 (C-i, 4-O-Bn), 138.2 (C-i, 2-O-Bn), 128.47, 128.45, 128.44, 128.35, 128.26, 128.21, 128.1, 127.8, 127.69, 127.68, 127.62, 127.60, 127.5 (15 x CH $\left.\mathrm{CH}_{\text {arom }}\right), 91.9$ (C-1), 79.1 (C-3), 77.4 (C-4), 76.5 (C-2), $74.8\left(\mathrm{CH}_{2}, 4-\mathrm{O}-\mathrm{Bn}\right), 73.5\left(\mathrm{CH}_{2}, 2-\mathrm{O}-\right.$ $\mathrm{Bn}), 73.0\left(\mathrm{CH}_{2}, 3-O-\mathrm{Bn}\right), 66.7$ (C-5), 16.8 (C-6); HRMS(ESI) m/z calcd. for $\left[\mathrm{C}_{27} \mathrm{H}_{30} \mathrm{O}_{5}+\mathrm{NH}_{4}\right]^{+}: 452.2431$, obsd.: 452.2436 . 
$O$-(2,3,4-Tri- $O$-benzyl-6-deoxy- $\alpha / \beta$-D-galactopyranosyl) trichloroacetimidate (72) $)^{32,33}$

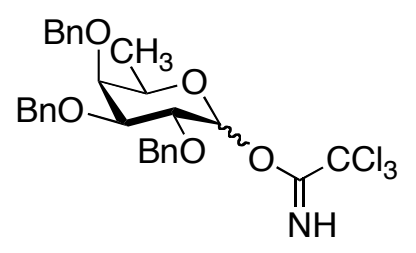

Lactol 91 (153 mg, $0.35 \mathrm{mmol}$ ) was co-evaporated with toluene (x3) and dissolved in dry $\mathrm{CH}_{2} \mathrm{Cl}_{2}(3.5 \mathrm{~mL})$. DBU $(79 \mu \mathrm{L}, 0.53 \mathrm{mmol})$ and trichloroacetonitrile $(353$ $\mu \mathrm{L}, 3.52 \mathrm{mmol}$ ) were added at $\mathrm{rt}$ and the mixture was stirred for $1 \mathrm{~h}$. The reaction mixture was concentrated in vacuo and the residue purified by gradient flash chromatography $\left(1 \% \mathrm{NEt}_{3}+\right.$ petroleum ether to petroleum ether/EtOAc, 20:1, v/v). Both the $\alpha$-isomer 72 (126 mg, $0.22 \mathrm{mmol}$, $62 \%$ ) and the $\beta$-isomer ( $66 \mathrm{mg}, 0.11 \mathrm{mmol}, 32 \%$ ) were obtained as clear oils. $\mathrm{R}_{f}$ : $0.61(\mathrm{PE} / \mathrm{EA}, 2 / 1, \mathrm{v} / \mathrm{v}) ; \alpha:[\alpha]_{\mathrm{D}}^{23}=+58.0^{\circ}\left(\mathrm{c}=1.0, \mathrm{CHCl}_{3}\right)$; IR (film) 3343, 30064, 3030, 2935, 2904, 2874,1731, 1669, 1603, 1497, 1454, 1356, 1293, 1216, 1103, 1066, 1027, 968, 943, 838, 794, 735, 697, $644 \mathrm{~cm}^{-1} ;{ }^{1} \mathrm{H}$ NMR (500 MHz, $\left.\mathrm{CDCl}_{3}\right) \delta 8.53(\mathrm{~s}, 1 \mathrm{H}, \mathrm{NH}), 7.41-7.29\left(\mathrm{~m}, 15 \mathrm{H}, \mathrm{H}_{\text {arom }}\right), 6.55\left(\mathrm{~d}, J_{1,2}=3.4 \mathrm{~Hz}, 1 \mathrm{H}\right.$, $\mathrm{H}-1), 5.04\left(\mathrm{~d}, J_{\mathrm{a}, \mathrm{b}}=11.5 \mathrm{~Hz}, 1 \mathrm{H}, \mathrm{CH}-\mathrm{a}, 4-O-\mathrm{Bn}\right), 4.89$ (d, $J_{\mathrm{a}, \mathrm{b}}=11.7 \mathrm{~Hz}, 1 \mathrm{H}, \mathrm{CH}-$ a, 3-O-Bn), $4.80-4.78$ (m, 3H, CH-b, 3-O-Bn, CH-a, CH-b, 2-O-Bn), 4.71 (d, $J_{\mathrm{a}, \mathrm{b}}$ $=11.5 \mathrm{~Hz}, 1 \mathrm{H}, \mathrm{CH}-\mathrm{b}, 4-O-\mathrm{Bn}), 4.27$ (dd, $\left.J_{2,3}=10.0 \mathrm{~Hz}, J_{1,2}=3.4 \mathrm{~Hz}, 1 \mathrm{H}, \mathrm{H}-2\right)$, $4.12\left(\mathrm{q}, J_{5,6}=6.4 \mathrm{~Hz}, 1 \mathrm{H}, \mathrm{H}-5\right), 4.06\left(\mathrm{dd}, J_{2,3}=10.0 \mathrm{~Hz}, J_{3,4}=2.6 \mathrm{~Hz}, 1 \mathrm{H}, \mathrm{H}-3\right)$, 3.74 (bs, $1 \mathrm{H}, \mathrm{H}-4), 1.19$ (d, $\left.J_{5,6}=6.4 \mathrm{~Hz}, 1 \mathrm{H}, \mathrm{H}-6\right) ;{ }^{13} \mathrm{C} \mathrm{NMR}\left(125 \mathrm{MHz}, \mathrm{CDCl}_{3}\right)$ $\delta 161.3(\underline{\mathrm{C}}=\mathrm{NH}), 138.6(\mathrm{C}-i, 3-O-\mathrm{Bn}), 138.5(\mathrm{C}-i, 2-O-\mathrm{Bn}), 138.4(\mathrm{C}-i, 4-O-\mathrm{Bn})$, $128.5,128.42,128.37,128.29,128.26,127.74,127.70,127.6,127.5(15 \mathrm{x}$ $\left.\mathrm{CH}_{\text {arom}}\right), 95.3(\mathrm{C}-1), 91.6\left(\mathrm{CCl}_{3}\right), 78.3(\mathrm{C}-3), 77.3(\mathrm{C}-4), 75.8(\mathrm{C}-2), 75.0\left(\mathrm{CH}_{2}, 4-\right.$ $O-\mathrm{Bn}), 73.2\left(\mathrm{CH}_{2}, 3-O-\mathrm{Bn}\right), 72.9\left(\mathrm{CH}_{2}, 2-O-\mathrm{Bn}\right), 69.6(\mathrm{C}-5), 16.7(\mathrm{C}-6)$; HRMS(ESI) $\mathrm{m} / \mathrm{z}$ calcd. for $\left[\mathrm{C}_{29} \mathrm{H}_{30} \mathrm{NO}_{5} \mathrm{Cl}_{3}+\mathrm{Na}\right]^{+}:$600.1082, obsd.: 600.1092 . 
(2S,3R,4E)-1-(4-O-(4-O-Acetyl-2,6-di-O-benzoyl-3-O-(2,3,4-tri- $O$-benzyl-6deoxy- $\alpha$-D-galactopyranosyl)- $\beta$-D-galactopyranosyl)-2,3,6-tri- $O$-benzoyl- $\beta$-Dglucopyranosyloxy)-3-benzyloxy-2-hexacosanoylamido-octadec-4-ene (71)

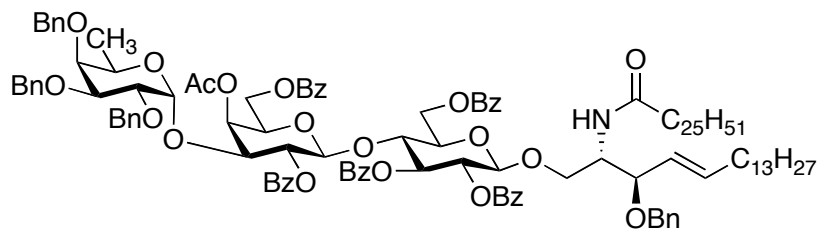

Fucosyl imidate donor 72 (32 $\mathrm{mg}, 0.055 \mathrm{mmol})$ and lactosyl ceramide acceptor 73 (44.7 mg, $0.027 \mathrm{mmol})$ were coevaporated with toluene $(\mathrm{x} 3)$, dissolved in dry $\mathrm{CH}_{2} \mathrm{Cl}_{2}(1 \mathrm{~mL})$ and stirred with activated $4 \AA$ molecular sieves for $3 \mathrm{~h}$. The reaction mixture was cooled to $-40{ }^{\circ} \mathrm{C}$, and a solution of TMSOTf in $\mathrm{CH}_{2} \mathrm{Cl}_{2}(0.28 \mathrm{mmol} / \mathrm{mL}, 9.8 \mu \mathrm{L}, 0.0027 \mathrm{mmol})$ was added and stirred for $1 \mathrm{~h}$. The reaction was warmed to $-20{ }^{\circ} \mathrm{C}$ and further TMSOTf solution $(0.28 \mathrm{mmol} / \mathrm{mL}, 5.9 \mu \mathrm{L}, 0.0016 \mathrm{mmol})$ was added. The reaction mixture was allowed to warm slowly to $\mathrm{rt}$ while stirring overnight ( $20 \mathrm{~h}$ ), upon which it was quenched with $\mathrm{NEt}_{3}(40 \mu \mathrm{L}, 0.29 \mathrm{mmol})$, filtered and concentrated in vacuo. The resultant oil was purified by gradient flash chromatography (3\% to $8 \%$ EtOAc in toluene) to afford the fully protected triglycosyl ceramide 71 as a clear oil $(38.6 \mathrm{mg}, 0.019 \mathrm{mmol}, 69 \%) . \mathrm{R}_{f}: 0.49$ $(\mathrm{PE} / \mathrm{EA}, 2 / 1, \mathrm{v} / \mathrm{v}) ;[\alpha]_{\mathrm{D}}{ }^{24}=+12.0^{\circ}\left(\mathrm{c}=1.0, \mathrm{CHCl}_{3}\right)$; IR (film) 3091, 2923, 2853, 1730, 1672, 1602, 1497, 1453, 1364, 1315, 1267, 1176, 1097, 1069, 1028, 978, $755,710,641 \mathrm{~cm}^{-1} ;{ }^{1} \mathrm{H}$ NMR $\left(600 \mathrm{MHz}, \mathrm{CDCl}_{3}\right) \delta 8.02\left(\mathrm{~d}, J_{\mathrm{CH}-o, \mathrm{CH}-m}=7.3 \mathrm{~Hz}\right.$, $\left.2 \mathrm{H}, \mathrm{CH}-o, 6^{\prime \prime}-O-\mathrm{Bz}\right), 8.01\left(\mathrm{~d}, J_{\mathrm{CH}-o, \mathrm{CH}-m}=7.0 \mathrm{~Hz}, 2 \mathrm{H}, \mathrm{CH}-o, 3^{\prime}-O-\mathrm{Bz}\right), 7.98(\mathrm{~d}$, $\left.J_{\mathrm{CH}-o, \mathrm{CH}-m}=7.1 \mathrm{~Hz}, 2 \mathrm{H}, \mathrm{CH}-o, 2^{\prime}-O-\mathrm{Bz}\right), 7.95\left(\mathrm{~d}, J_{\mathrm{CH}-o, \mathrm{CH}-m}=7.1 \mathrm{~Hz}, 2 \mathrm{H}, \mathrm{CH}-o\right.$, $\left.2^{\prime \prime}-O-\mathrm{Bz}\right), 7.93\left(\mathrm{~d}, J_{\mathrm{CH}-o, \mathrm{CH}-m}=7.1 \mathrm{~Hz}, 2 \mathrm{H}, \mathrm{CH}-o, 6{ }^{\prime}-O-\mathrm{Bz}\right), 7.61-7.13(\mathrm{~m}, 35 \mathrm{H}$, $\left.\mathrm{H}_{\text {arom }}\right), 5.74\left(\mathrm{t}, J_{3^{\prime}, 4^{\prime}}=9.6 \mathrm{~Hz}, 1 \mathrm{H}, \mathrm{H}-3^{\prime}\right), 5.49\left(\mathrm{dt}, J_{4,5}=15.3 \mathrm{~Hz}, J_{5,6}=6.7 \mathrm{~Hz}, 1 \mathrm{H}\right.$, $\mathrm{H}-5), 5.41\left(\mathrm{t}, J_{1^{\prime}, 2^{\prime}}=J_{2^{\prime}, 3^{\prime}}=7.5 \mathrm{~Hz}, 1 \mathrm{H}, \mathrm{H}-2^{\prime}\right), 5.40\left(\mathrm{t}, J_{1^{\prime \prime}, 2^{\prime \prime}}=J_{2^{\prime \prime}, 3^{\prime \prime}}=7.5 \mathrm{~Hz}, 1 \mathrm{H}\right.$, $\left.\mathrm{H}-2^{\prime \prime}\right), 5.36\left(\mathrm{~d}, J_{\mathrm{NH}, 2^{\prime}}=9.1 \mathrm{~Hz}, 1 \mathrm{H}, \mathrm{NH}\right), 5.34\left(\mathrm{~d}, J_{3^{\prime \prime}, 4^{\prime \prime}}=3.4 \mathrm{~Hz}, 1 \mathrm{H}, \mathrm{H}-4^{\prime \prime}\right), 5.23$ (dt, $\left.J_{4,5}=15.3 \mathrm{~Hz}, J_{3,4}=8.8 \mathrm{~Hz}, 1 \mathrm{H}, \mathrm{H}-4\right), 4.94\left(\mathrm{~d}, J_{1^{\prime \prime \prime}, 2^{\prime \prime}}=3.5 \mathrm{~Hz}, 1 \mathrm{H}, \mathrm{H}-1^{\prime \prime \prime}\right)$, $4.75\left(\mathrm{~d}, J_{\mathrm{a}, \mathrm{b}}=11.3 \mathrm{~Hz}, 1 \mathrm{H}, \mathrm{CH}-\mathrm{a}, 4^{\prime \prime \prime}-O-\mathrm{Bn}\right), 4.72\left(\mathrm{~d}, J_{\mathrm{a}, \mathrm{b}}=11.9 \mathrm{~Hz}, 1 \mathrm{H}, \mathrm{CH}-\mathrm{a}\right.$, $\left.3^{\prime \prime \prime}-O-\mathrm{Bn}\right), 4.60$ (d, $\left.J_{1^{\prime}, 2^{\prime}}=7.9 \mathrm{~Hz}, 1 \mathrm{H}, \mathrm{H}-1^{\prime}\right), 4.56$ (d, $\left.J_{1^{\prime \prime}, 2^{\prime \prime}}=8.2 \mathrm{~Hz}, 1 \mathrm{H}, \mathrm{H}-1^{\prime \prime}\right)$, 4.58-4.54 (m, 2H, CH-a, CH-b, 2"' -O-Bn), 4.48 (d, $J_{\mathrm{a}, \mathrm{b}}=11.9 \mathrm{~Hz}, 1 \mathrm{H}, \mathrm{CH}-\mathrm{b}$, 3'"'-O-Bn), 4.48-4.47 (m, 2H, H-6'a, H-6'b), 4.44 (d, $J_{\mathrm{a}, \mathrm{b}}=11.5 \mathrm{~Hz}, 1 \mathrm{H}, \mathrm{CH}-\mathrm{a}, 3-$ $O$-Bn), $4.34\left(\mathrm{~d}, J_{\mathrm{a}, \mathrm{b}}=11.3 \mathrm{~Hz}, 1 \mathrm{H}, \mathrm{CH}-\mathrm{b}, 4^{\prime \prime \prime}-O-\mathrm{Bn}\right), 4.25\left(\mathrm{dd}, J_{1 \mathrm{a}, 1 \mathrm{~b}}=9.8 \mathrm{~Hz}\right.$, 
$\left.J_{1 \mathrm{a}, 2}=2.7 \mathrm{~Hz}, 1 \mathrm{H}, \mathrm{H}-1 \mathrm{a}\right), 4.24\left(\mathrm{~d}, J_{\mathrm{a}, \mathrm{b}}=11.5 \mathrm{~Hz}, 1 \mathrm{H}, \mathrm{CH}-\mathrm{b}, 3-O-\mathrm{Bn}\right), 4.17$ (t, $J_{3^{\prime}, 4^{\prime}}$

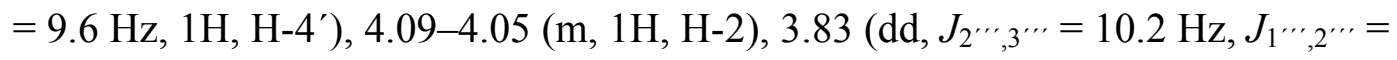
$\left.3.5 \mathrm{~Hz}, 1 \mathrm{H}, \mathrm{H}-2^{\prime \prime \prime}\right), 3.80$ (dd, $\left.J_{2^{\prime \prime}, 3^{\prime \prime}}=10.3 \mathrm{~Hz}, J_{3^{\prime \prime}, 4^{\prime \prime}}=3.4 \mathrm{~Hz}, 1 \mathrm{H}, \mathrm{H}-3^{\prime \prime}\right), 3.74$ 3.69 (m, 2H, H-5', H-3), 3.62-3.59 (m, 2H, H-6" 'a, H-6" 'b), 3.57-3.55 (m, 2H, H$\left.5^{\prime \prime \prime}, \mathrm{H}-3^{\prime \prime \prime}\right), 3.52\left(\mathrm{t}, J_{5^{\prime \prime}, 6^{\prime \prime}}=6.9 \mathrm{~Hz}, 1 \mathrm{H}, \mathrm{H}-5^{\prime \prime}\right), 3.48\left(\mathrm{dd}, J_{1 \mathrm{a}, 1 \mathrm{~b}}=9.8 \mathrm{~Hz}, J_{1 \mathrm{a}, 2}=\right.$

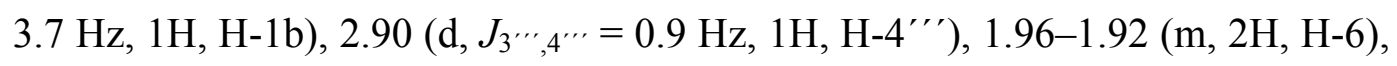
1.70-1.62 (m, 2H, $\left.\mathrm{CH}_{2}-\alpha\right), 1.63$ (s, 3H, OAc), 1.31-1.04 (m, 68H, H-7-H-17, H$\beta-\mathrm{H}-(\omega-1)), 0.88\left(\mathrm{t}, J_{17,18}=J_{\omega-1, \omega}=7.0 \mathrm{~Hz}, 6 \mathrm{H}, \mathrm{H}-18, \mathrm{H}-\omega\right), 0.79\left(\mathrm{~d}, J_{5^{\prime \prime \prime}, 6^{\prime \prime \prime}}=\right.$ $\left.6.5 \mathrm{~Hz}, 3 \mathrm{H}, \mathrm{H}-6{ }^{\prime \prime \prime}\right) ;{ }^{13} \mathrm{C} \mathrm{NMR}\left(150 \mathrm{MHz}, \mathrm{CDCl}_{3}\right) \delta 172.5(\mathrm{HNC}=\mathrm{O}), 170.2$ $(\mathrm{C}=\mathrm{O}, \mathrm{OAc}), 166.0\left(\mathrm{C}=\mathrm{O}, 6^{\prime}-O-\mathrm{Bn}\right), 165.8\left(\mathrm{C}=\mathrm{O}, 6^{\prime \prime}-O-\mathrm{Bn}\right), 165.5\left(\mathrm{C}=\mathrm{O}, 2^{\prime \prime}-O\right.$ $\mathrm{Bn}), 165.3\left(\mathrm{C}=\mathrm{O}, 3^{\prime}-O-\mathrm{Bn}\right), 164.5\left(\mathrm{C}=\mathrm{O}, 2^{\prime}-O-\mathrm{Bn}\right), 139.0\left(\mathrm{C}-i, 3^{\prime \prime \prime}-O-\mathrm{Bn}\right), 138.7$ (C-i, 2"' $-O-\mathrm{Bn}), 138.5$ (C-i, 4"' -O-Bn), 138.4 (C-i, 3-O-Bn), 137.2 (C-5), 133.7, 133.6, 133.55, 133.52, 133.3 (C-p, 5 x OBz), 129.97, 129.89, 129.87, 129.78, $129.67,129.60,129.48,129.18,128.75,128.71,128.57,128.44,128.34,128.32$, $128.22,128.17,128.09,128.05,127.85,127.76,127.61,127.59,127.57,127.52$, 127.41 (45 x $\left.\mathrm{CH}_{\text {arom}}\right), 127.44$ (C-4), 101.5 (C-1'), 100.9 (C-1"'), 94.2 (C-1"'), 79.2 (C-3), 79.0 (C-3"'), 77.6 (C-4"'), 75.4 (C-4', C-2"'), $74.9\left(\mathrm{CH}_{2}, 4^{\prime \prime \prime}-\mathrm{O}-\right.$ $\mathrm{Bn}), 73.5\left(\mathrm{CH}_{2}, 2^{\prime \prime \prime}-\mathrm{O}-\mathrm{Bn}\right), 73.3\left(\mathrm{CH}_{2}, 3^{\prime \prime \prime}-\mathrm{O}-\mathrm{Bn}\right), 73.1\left(\mathrm{C}-5{ }^{\prime}\right), 72.5\left(\mathrm{C}-3{ }^{\prime}\right), 72.3$ $\left(\mathrm{C}-2^{\prime \prime}, \mathrm{C}-3^{\prime \prime}\right), 71.4\left(\mathrm{C}-5^{\prime \prime}\right), 71.1\left(\mathrm{C}-2^{\prime}\right), 70.4\left(\mathrm{CH}_{2}, 3-O-\mathrm{Bn}\right), 68.5(\mathrm{C}-1), 66.7(\mathrm{C}-$ 5'"'), 64.4 (C-4"'), 62.5 (C-6'), 61.3 (C-6"'), 51.3 (C-2), 36.6 (C- $\alpha), 32.4(\mathrm{C}-6)$, $32.07,30.17,29.89,29.87,29.85,29.82,29.80,29.70,29.68,29.53,29.52,29.51$, 29.41, 29.39, 29.36, 27.22, 25.58, 22.84 (C-7-C-17, C- $\beta-\mathrm{C}-(\omega-1)), 20.4$ (OAc), 16.2 (C-6"'), 14.3 (C-18, C- $\omega$ ); HRMS(ESI) m/z calcd. for $\left[\mathrm{C}_{127} \mathrm{H}_{163} \mathrm{NO}_{23}+\mathrm{Na}\right]^{+}$: 2093.1508, obsd.: 2093.1463. 
$(2 S, 3 R, 4 E)-1-(4-O-(3-O-(6-D e o x y-\alpha-D-g a l a c t o p y r a n o s y l)-\beta-D-$

galactopyranosyl)- $\beta$-D-glucopyranosyloxy)-2-hexacosanoylamido-3-hydroxyoctadec-4-ene (5)

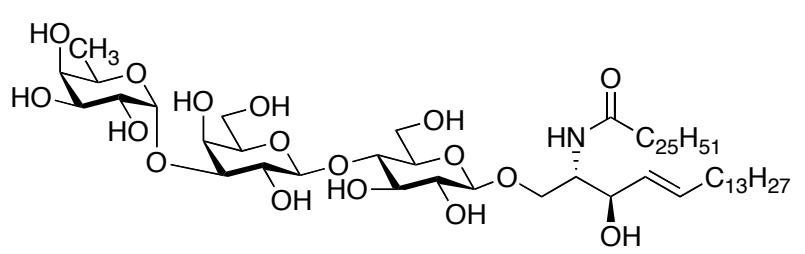

Fully protected triglycosyl ceramide $71 \quad(33 \quad \mathrm{mg}, \quad 0.016$ mmol) was dissolved in dry THF $(2 \mathrm{~mL})$ and $\mathrm{NH}_{3}(10 \mathrm{~mL})$ was condensed into the reaction vessel at $-78{ }^{\circ} \mathrm{C}$. Small pieces of $\mathrm{Na}(\mathrm{s})$ were added carefully until the solution remained deep blue and the reaction mixture was stirred for 30 mins. The reaction was then quenched with a few drops of $\mathrm{MeOH}$ and $\mathrm{Na}$ (s) was added again until the deep blue colour persisted, and the reaction mixture stirred for a further 30 mins. The reaction was quenched with $10 \mathrm{~mL}$ $\mathrm{MeOH}$, and the reaction warmed slowly to rt to allow the ammonia to evaporate. Trace ammonia was removed with an $\mathrm{Ar}$ stream. The reaction mixture was quenched to $\mathrm{pH} 7$ with Dowex- $\mathrm{H}^{+}$, filtered and washed with pyridine and concentrated in vacuo. The resultant oil was purified by gradient flash chromatography (DCM/MeOH, 20/1 to $10 / 1$, v/v) to afford fully deprotected 6"' deoxy-iGb3-sphingosine 5 as an amorphous white solid $(6.2 \mathrm{mg}, 0.0054 \mathrm{mmol}$, 34\%). $\mathrm{R}_{f}: 0.29(\mathrm{DCM} / \mathrm{MeOH}, 5.7 / 1, \mathrm{v} / \mathrm{v}) ;[\alpha]_{\mathrm{D}}^{27}=+37.0^{\circ}(\mathrm{c}=0.1$, pyridine); IR (film) 3402, 2922, 1653, 1558, 1541, 1458, 1134, 1099, 1072, 1038, 890, 814, 791, 694, 648, $633 \mathrm{~cm}^{-1} ;{ }^{1} \mathrm{H}$ NMR (500 MHz, pyridine-d $\left.\mathrm{d}_{5}\right) \delta 8.45\left(\mathrm{~d}, J_{2, \mathrm{NH}}=8.2\right.$ $\mathrm{Hz}, 1 \mathrm{H}, \mathrm{NH}), 6.04\left(\mathrm{dd}, J_{4,5}=15.2 \mathrm{~Hz}, J_{3,4}=16.4 \mathrm{~Hz}, 1 \mathrm{H}, \mathrm{H}-4\right), 5.93\left(\mathrm{dt}, J_{4,5}=\right.$ $\left.15.2 \mathrm{~Hz}, J_{5,6}=6.9 \mathrm{~Hz}, 1 \mathrm{H}, \mathrm{H}-5\right), 5.59\left(\mathrm{~d}, J_{1^{\prime \prime}, 2^{\prime \prime \prime}}=3.7 \mathrm{~Hz}, 1 \mathrm{H}, \mathrm{H}-1^{\prime \prime \prime}\right), 5.11(\mathrm{~d}$, $\left.J_{1^{\prime \prime}, 2^{\prime \prime}}=7.9 \mathrm{~Hz}, 1 \mathrm{H}, \mathrm{H}-1^{\prime \prime}\right), 4.95$ (q, $\left.J_{5^{\prime \prime \prime}, 6^{\prime \prime \prime}}=6.7 \mathrm{~Hz}, 1 \mathrm{H}, \mathrm{H}-5^{\prime \prime \prime}\right), 4.89$ (d, $J_{1^{\prime}, 2^{\prime}}=$ $7.9 \mathrm{~Hz}, 1 \mathrm{H}, \mathrm{H}-1^{\prime}$ ), 4.81-4.79 (m, 3H, H-1a, H-2, H-3), 4.66 (dd, $J_{2^{\prime \prime}, 3^{\prime \prime \prime}}=9.9 \mathrm{~Hz}$, $\left.J_{1^{\prime \prime \prime}, 2^{\prime \prime \prime}}=3.7 \mathrm{~Hz}, 1 \mathrm{H}, \mathrm{H}-2^{\prime \prime \prime}\right), 4.59$ (d, $\left.J_{3^{\prime \prime}, 4^{\prime \prime}}=2.1 \mathrm{~Hz}, 1 \mathrm{H}, \mathrm{H}-4^{\prime \prime}\right), 4.56-4.53$ (m,

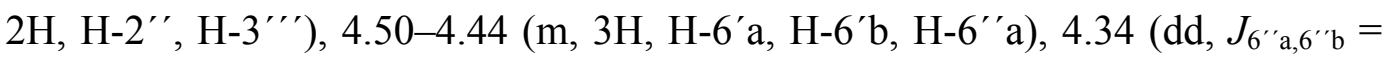
$\left.10.9 \mathrm{~Hz}, J_{5^{\prime \prime}, 6^{\prime \prime} \mathrm{b}}=3.4 \mathrm{~Hz}, 1 \mathrm{H}, \mathrm{H}-6^{\prime \prime} \mathrm{b}\right), 4.30-4.23$ (m, 3H, H-4', H-3"', H-3'), 4.18-4.17 (m, 1H, H-1b), 4.14 (bs, 1H, H-4"' ), 4.10-4.05 (m, 2H, H-5'", H-2'), 3.87-3.85 (m, 1H, H-5'), $2.46\left(\mathrm{t}, J_{\alpha, \beta}=7.4 \mathrm{~Hz}, 2 \mathrm{H}, \mathrm{CH}_{2}-\alpha\right), 2.08\left(\mathrm{dd}, J_{6,7}=13.9\right.$ $\left.\mathrm{Hz}, J_{5,6}=6.9 \mathrm{~Hz}, 2 \mathrm{H}, \mathrm{H}-6\right), 1.87-1.81\left(\mathrm{~m}, 2 \mathrm{H}, \mathrm{CH}_{2}-\beta\right), 1.59\left(\mathrm{~d}, J_{5^{\prime \prime} \prime} 6^{\prime \prime \prime}=6.6 \mathrm{~Hz}\right.$,

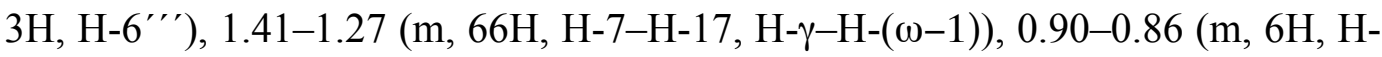


18, H- $\omega) ;{ }^{13} \mathrm{C}$ NMR (125 MHz, pyridine-d $)_{5} \delta 173.7$ (HNC $\left.=\mathrm{O}\right), 133.0$ (C-5), 132.6 (C-4), 105.84, 105.79 (C-1', C-1' '), 97.9 (C-1'"'), 82.4 (C-4'), 80.4 (C-3' '), 77.00 (C-3'), 76.95 (C-5'), 76.8 (C-5'), 75.1 (C-2'), 73.8 (C-4'”'), 73.0 (C-3), 72.0 (C-3"'), 70.8 (C-1, C-2"), 70.4 (C-2"'), 68.1 (C-5"'), 66.3 (C-4"), 62.3 (C6'), 62.2 (C-6"'), 55.2 (C-2), 37.3 (C- $\alpha), 33.1$ (C-6), 26.8 (C- $\beta$ ), 32.48, 32.46, 30.38, 30.36, 30.33, 30.25, 30.14, 30.07, 29.98, 29.94, 23.29, 23.27 (C-7-C-17, C- $\gamma-\mathrm{C}-(\omega-1)), 17.6$ (C-6"'), 14.6 (C-18, C- $\omega)$; HRMS(ESI) m/z calcd. for $\left[\mathrm{C}_{62} \mathrm{H}_{117} \mathrm{NO}_{17}+\mathrm{H}\right]^{+}:$1148.8394, obsd.: 1148.8374 .

\section{(2S,3R,4E)-1-(4-O-(3-O-(6-Deoxy- $\alpha$-D-galactopyranosyl)- $\beta$-D-}

galactopyranosyl)- $\beta$-D-glucopyranosyloxy)-2-hexacosanoylamido-3-hydroxyoctadecane (6)

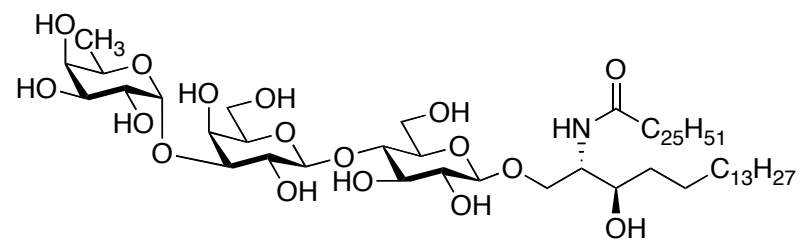

$\mathrm{Pd}(\mathrm{OH})_{2} / \mathrm{C}(2 \mathrm{~mol} \%)$ was added to a solution of fully protected triglycosyl ceramide $71(22 \mathrm{mg}$, $0.010 \mathrm{mmol})$ dissolved in $\mathrm{CHCl}_{3} / \mathrm{EtOH}(3 / 2, \mathrm{v} / \mathrm{v})$. The reaction mixture was stirred under $\mathrm{H}_{2}$ atmosphere for $4 \mathrm{~h}$, filtered through a celite pad and concentrated under reduced pressure. The resultant oil was purified by gradient flash chromatography $(2.5 \%$ to $5 \% \mathrm{MeOH}$ in DCM) to afford debenzylated 6"'-deoxy-iGb3-sphinganine (13.4 mg, 0.0078 mmol, 75\%), which was subsequently dissolved in MeOH/DCM (2/1, v/v, 4.5 $\mathrm{mL}$ ). $\mathrm{NaOMe}$ was added until the reaction mixture reached $\mathrm{pH} 9$ and stirred at $\mathrm{rt}$ for $17 \mathrm{~h}$. The reaction mixture was warmed to $40{ }^{\circ} \mathrm{C}$ and stirred for further $3 \mathrm{~h}$, then neutralised to $\mathrm{pH} 7$ with Dowex $-\mathrm{H}^{+}$. The resin was removed by filtration and washed successively with pyridine and concentrated under reduced pressure. $\mathrm{MeOH}(5 \mathrm{~mL})$ was added to the crude mixture and cooled to $-4{ }^{\circ} \mathrm{C}$ to give a suspension of glycolipid in solution. The precipitate was collected by centrifuge and washed with $\mathrm{MeOH}(2 \times 5 \mathrm{~mL})$, to afford the fully deprotected 6"' -deoxyiGb3-sphinganine 6 as an amorphous white solid (6.1 mg, $0.0053 \mathrm{mmol}, 70 \%)$. More desired product was precipitated from the combined MeOH supernatant (1.7 $\mathrm{mg}, 0.0015 \mathrm{mmol}, 19 \%$ ). The total yield of $6^{\prime \prime \prime}$-deoxy-iGb3-sphinganine 6 was $89 \%$ (7.8 mg, $0.0068 \mathrm{mmol}) . \mathrm{R}_{f}: 0.23(\mathrm{DCM} / \mathrm{MeOH}, 5 / 1, \mathrm{v} / \mathrm{v}) ;[\alpha]_{\mathrm{D}}^{27}=+42.0^{\circ}(\mathrm{c}$ $=0.1$, pyridine); IR (film) 3342, 2919, 2850, 1626, 1551, 1468, 1379, 1165, 1081, 
1038, 899, 813, 778, 722, 677, 651, $632 \mathrm{~cm}^{-1} ;{ }^{1} \mathrm{H}$ NMR (500 MHz, pyridine-d $\left.\mathrm{d}_{5}\right) \delta$ $8.45\left(\mathrm{~d}, J_{2, \mathrm{NH}}=9.0 \mathrm{~Hz}, 1 \mathrm{H}, \mathrm{NH}\right), 7.64\left(\mathrm{~d}, J_{2^{\prime}, \mathrm{OH}}=3.0 \mathrm{~Hz}, 1 \mathrm{H}, \mathrm{H}-2^{\prime}-\mathrm{OH}\right), 7.36(\mathrm{~d}$, $\left.J_{2 " \prime 3} 3^{\prime \prime \prime}, \mathrm{OH}=5.5 \mathrm{~Hz}, 1 \mathrm{H}, \mathrm{H}-2^{\prime \prime} / 3^{\prime \prime \prime}-\mathrm{OH}\right), 7.08\left(\mathrm{~d}, J_{2}{ }^{\prime \prime}, \mathrm{OH}=5.5 \mathrm{~Hz}, 1 \mathrm{H}, \mathrm{H}-2^{\prime \prime \prime}-\mathrm{OH}\right)$, $6.68\left(\mathrm{~d}, J_{6^{\prime \prime}, \mathrm{OH}}=5.0 \mathrm{~Hz}, 1 \mathrm{H}, \mathrm{H}-6^{\prime \prime}-\mathrm{OH}\right), 6.56\left(\mathrm{~d}, J_{2^{\prime \prime} / 3^{\prime \prime \prime}, \mathrm{OH}}=5.4 \mathrm{~Hz}, 1 \mathrm{H}, \mathrm{H}-\right.$ $\left.2^{\prime \prime} / 3^{\prime \prime \prime}-\mathrm{OH}\right), 6.48\left(\mathrm{~d}, J_{6^{\prime}, \mathrm{OH}}=6.2 \mathrm{~Hz}, 1 \mathrm{H}, \mathrm{H}-6^{\prime}-\mathrm{OH}\right), 6.30\left(\mathrm{~d}, J_{3, \mathrm{OH}}=6.3 \mathrm{~Hz}, 1 \mathrm{H}\right.$, H-3-OH), 6.26 (d, $\left.J_{4}{ }^{\prime \prime \prime}, \mathrm{OH}=3.8 \mathrm{~Hz}, 1 \mathrm{H}, \mathrm{H}-4^{\prime \prime \prime}-\mathrm{OH}\right), 6.18$ (s,1H, H-3'-OH), 5.83 $\left(\mathrm{s}, 1 \mathrm{H}, \mathrm{H}-4^{\prime \prime}-\mathrm{OH}\right), 5.60$ (d, $\left.J_{1^{\prime \prime}, 2^{\prime \prime \prime}}=3.3 \mathrm{~Hz}, 1 \mathrm{H}, \mathrm{H}-1^{\prime \prime \prime}\right), 5.10\left(\mathrm{~d}, J_{1^{\prime \prime}, 2^{\prime \prime}}=8.0 \mathrm{~Hz}\right.$, $\left.1 \mathrm{H}, \mathrm{H}-1^{\prime \prime}\right), 4.95$ (q, $\left.J_{5^{\prime \prime \prime}, 6^{\prime \prime}}=6.5 \mathrm{~Hz}, 1 \mathrm{H}, \mathrm{H}-5^{\prime \prime \prime}\right), 4.89$ (d, $J_{1^{\prime}, 2^{\prime}}=7.6 \mathrm{~Hz}, 1 \mathrm{H}, \mathrm{H}-$ $\left.1^{\prime}\right), 4.80\left(\mathrm{dd}, J_{1 \mathrm{a}, 1 \mathrm{~b}}=10.4 \mathrm{~Hz}, J_{1 \mathrm{a}, 2}=4.4 \mathrm{~Hz}, 1 \mathrm{H}, \mathrm{H}-1 \mathrm{a}\right), 4.73-4.69(\mathrm{~m}, 1 \mathrm{H}, \mathrm{H}-2)$, 4.68-4.64 (m, 1H, H-2'"'), 4.59 (bs, 1H. H-4"'), 4.57-4.53 (m, 2H, H-2"', H-3"')), 4.51-4.49 (m, 2H, H-6'a, H-6'b), 4.46 (dd, $J_{6^{\prime \prime} \mathrm{a}, 6^{\prime \prime} \mathrm{b}}=12.2 \mathrm{~Hz}, J_{5,6^{\prime \prime} \mathrm{a}}=5.6 \mathrm{~Hz}, 1 \mathrm{H}$, H-6"'a), 4.37-4.33 (m, 1H, H-6' 'b), 4.29-4.21 (m, 4H, H-3', H-4', H-3'”, H-3), $4.18\left(\mathrm{dd}, J_{1 \mathrm{a}, 1 \mathrm{~b}}=10.4 \mathrm{~Hz}, J_{1 \mathrm{~b}, 2}=3.2 \mathrm{~Hz}, 1 \mathrm{H}, \mathrm{H}-1 \mathrm{~b}\right), 4.14$ (bs, 1H, H-4"' ), 4.104.06 (m, 2H, H-5' $\left.{ }^{\prime \prime}, \mathrm{H}-2^{\prime}\right), 4.89-4.87\left(\mathrm{~m}, 1 \mathrm{H}, \mathrm{H}-5^{\prime}\right), 2.48$ (t, $J_{\alpha, \beta}=7.4 \mathrm{~Hz}, 2 \mathrm{H}$, $\left.\mathrm{CH}_{2}-\alpha\right)$, 1.96-1.80 (m, 4H, H-4, $\left.\mathrm{CH}_{2}-\beta\right), 1.60$ (d, $\left.J_{5^{\prime \prime}, 6^{\prime \prime}}=6.5 \mathrm{~Hz}, 3 \mathrm{H}, \mathrm{H}-6^{\prime \prime \prime}\right)$, 1.41-1.27 (m, 70H, H-5-H-17, H- $\gamma-\mathrm{H}-(\omega-1)), 0.89-0.87$ (m, 6H, H-18, H- $\omega$ ); ${ }^{13} \mathrm{C}$ NMR (125 MHz, pyridine-d $\left.{ }_{5}\right) \delta 173.6(\mathrm{HNC}=\mathrm{O}), 105.9$ (C-1', C-1' '), 98.0 (C$\left.1^{\prime \prime \prime}\right), 82.5$ (C-4'), 80.5 (C-3' ), 77.1 (C-3'), 77.0 (C-5'), 76.9 (C-5'), 75.1 (C-2'), 73.8 (C-4"'), 72.0 (C-3"'), 71.7 (C-3), 71.1 (C-1), 70.9 (C-2'), 70.4 (C-2"'), 68.1 (C-5"'), 66.3 (C-4'), 62.4 (C-6'), 62.2 (C-6"'), 55.3 (C-2), 37.3 (C- $\alpha$ ), 35.3 (C-4), 26.9 (C- $\beta$ ), 32.49, 32.48, 30.59, 30.48, 30.40, 30.38, 30.30, 30.27, 30.22, 30.12, 29.99, 29.96, 26.79, 23.30, 23.29 (C-7-C-17, C- $\gamma-\mathrm{C}-(\omega-1)), 17.6$ (C-6" ' ), $14.6(\mathrm{C}-18, \mathrm{C}-\omega)$; HRMS(ESI) $\mathrm{m} / \mathrm{z}$ calcd. for $\left[\mathrm{C}_{62} \mathrm{H}_{119} \mathrm{NO}_{17}+\mathrm{H}\right]^{+}:$1150.8551, obsd.: 1150.8553 . 


\subsection{References}

1. Zajonc, D. M.; Savage, P. B.; Bendelac, A.; Wilson, I. A.; Teyton, L., J. Mol. Biol. 2008, 377, 1104-1116.

2. Pellicci, D. G.; Clarke, A. J.; Patel, O.; Mallevaey, T.; Beddoe, T.; Le Nours, J.; Uldrich, A. P.; McCluskey, J.; Besra, G. S.; Porcelli, S. A.; Gapin, L.; Godfrey, D. I.; Rossjohn, J., Nat. Immunol. 2011, 12, 827-833.

3. Yu, E. D.; Girardi, E.; Wang, J.; Zajonc, D. M., J. Immunol. 2011, 187, 2079-2083.

4. Chen, W.; Xia, C.; Wang, J.; Thapa, P.; Li, Y.; Talukdar, A.; Nadas, J.; Zhang, W.; Zhou, D.; Wang, P. G., J. Org. Chem. 2007, 72, 9914-9923.

5. Xia, C.; Schümann, J.; Emmanuel, R.; Zhang, Y.; Chen, W.; Zhang, W.; De Libero, G.; Wang, P. G., J. Med. Chem. 2007, 50, 3489-3496.

6. Banchet-Cadeddu, A.; Hénon, E.; Dauchez, M.; Renault, J.-H.; Monneaux, F.; Haudrechy, A., Org. Biomol. Chem. 2011, 9, 3080-3104.

7. Li, Y.; Thapa, P.; Hawke, D.; Kondo, Y.; Furukawa, K.; Furukawa, K.; Hsu, F.-F.; Adlercreutz, D.; Weadge, J.; Palcic, M. M.; Wang, P. G.; Levery, S. B.; Zhou, D., J. Proteome Res. 2009, 8, 2740-2751.

8. Leung, L.; Tomassi, C.; Van Beneden, K.; Decruy, T.; Trappeniers, M.; Elewaut, D.; Gao, Y.; Elliott, T.; Al-Shamkhani, A.; Ottensmeier, C.; Werner, J. M.; Williams, A.; Van Calenbergh, S.; Linclau, B., ChemMedChem 2009, 4, 329-334.

9. Xing, G.-W.; Chen, L.; Liang, F.-F., Eur. J. Org. Chem. 2009, 2009, 59635970.

10. Dangerfield, E. M.; Cheng, J. M. H.; Knight, D. A.; Weinkove, R.; Dunbar, P. R.; Hermans, I. F.; Timmer, M. S. M.; Stocker, B. L., ChemBioChem 2012, 13, 1349-1356.

11. Purves, C. B., J. Am. Chem. Soc. 1929, 51, 3619-3627.

12. Nicolaou, K. C.; Caulfield, T.; Kataoka, H.; Kumazawa, T., J. Am. Chem. Soc. 1988, 110, 7910-7912.

13. Chen, L.; Liang, F.; Xu, M.; Xing, G.; Deng, Z., Acta Chimica Sinica 2009, 67, 1355-1362.

14. Müller, D.; Vic, G.; Critchley, P.; Crout, D. H. G.; Lea, N.; Roberts, L.; Lord, J. M., J. Chem. Soc., Perkin Trans. 1 1998, 2287-2294.

15. Catelani, G.; Colonna, F.; Marra, A., Carbohydr. Res. 1988, 182, 297-300.

16. Lay, L.; Windmüller, R.; Reinhardt, S.; Schmidt, R. R., Carbohydr. Res. 1997, 303, 39-49.

17. Chan, J.; Lewis, A. R.; Indurugalla, D.; Schur, M.; Wakarchuk, W.; Bennet, A. J., J. Am. Chem. Soc. 2012, 134, 3748-3757.

18. Motawia, M. S.; Marcussen, J.; Moller, B. L., J. Carbohydr. Chem. 1995, 14, 1279-1294.

19. Windmuller, R.; Schmidt, R. R., Tetrahedron Lett. 1994, 35, 7927-7930.

20. Lemieux, R. U.; Driguez, H., J. Am. Chem. Soc. 1975, 97, 4069-4075.

21. Dong, H.; Zhou, Y.; Pan, X.; Cui, F.; Liu, W.; Liu, J.; Ramström, O., J. Org. Chem. 2012, 77, 1457-1467.

22. Binkley, R. W.; Goewey, G. S.; Johnston, J. C., J. Org. Chem. 1984, 49, 992-996. 
23. King, J. F.; Allbutt, A. D., Can. J. Chem. 1970, 48, 1754-1769.

24. Kunz, H.; Unverzagt, C., Angew. Chem. Int. Ed. 1988, 27, 1697-1699.

25. Unverzagt, C.; Kunz, H., Bioorg. Med. Chem. 1994, 2, 1189-1201.

26. Peters, S.; Lowary, T. L.; Hindsgaul, O.; Meldal, M.; Bock, K., J. Chem. Soc. Perkin Trans. 1 1995, 3017-3022.

27. Zhiyuan, Z.; Magnusson, G., Carbohydr. Res. 1994, 262, 79-101.

28. Boulineau, F. P.; Wei, A., J. Org. Chem. 2004, 69, 3391-3399.

29. Sato, S.; Mori, M.; Ito, Y.; Ogawa, T., Carbohydr. Res. 1986, 155, C6-C10.

30. Cheng, J. M. H.; Chee, S. H.; Knight, D. A.; Acha-Orbea, H.; Hermans, I. F.; Timmer, M. S. M.; Stocker, B. L., Carbohydr. Res. 2011, 346, 914-926.

31. Yu, Z.; Ya-Peng, L.; Li, Z., J. Carbohydr. Chem. 2008, 27, 113-119.

32. Schmidt, R. R., Angew. Chem. Int. Ed. 1986, 25, 212-235.

33. Wegmann, B.; Schmidt, R. R., Carbohydr. Res. 1988, 184, 254-261.

34. Wang, C.-C.; Lee, J.-C.; Luo, S.-Y.; Fan, H.-F.; Pai, C.-L.; Yang, W.-C.; Lu, L.-D.; Hung, S.-C., Angew. Chem. Int. Ed. 2002, 41, 2360-2362.

35. Meloncelli, P. J.; Lowary, T. L., Carbohydr. Res. 2010, 345, 2305-2322.

36. Zhang, P.; Ng, K.; Ling, C.-C., Org. Biomol. Chem. 2010, 8, 128-136.

37. Nicolaou, K. C.; Caulfield, T. J.; Katoaka, H., Carbohydr. Res. 1990, 202, 177-191.

38. Yan, F.; Wakarchuk, W. W.; Gilbert, M.; Richards, J. C.; Whitfield, D. M., Carbohydr. Res. 2000, 328, 3-16.

39. Banks, M. R.; Blake, A. J.; Cadogan, J. I. G.; Dawson, I. M.; Gaur, S.; Gosney, I.; Gould, R. O.; Grant, K. J.; Hodgson, P. K. G., J. Chem. Soc., Chem. Commun. 1993, 1146-1148.

40. Ballou, C. E.; Fischer, H. O. L., J. Am. Chem. Soc. 1954, 76, 3188-3193.

41. Lunau, N.; Meier, C., Eur. J. Org. Chem. 2012, 2012, 6260-6270. 


\section{Chapter 5. Synthesis of acyl chain homologues of iGb3}

\subsection{Introduction}

The $N$-acyl lipids of CD1d-binding glycolipids have been reported to impact glycolipid activity by altering the ability of the glycolipids to activate iNKT cells and also by affecting the cytokine profile induced. ${ }^{1-6}$ The acyl lipid lodges itself into the hydrophobic $\mathrm{A}^{\prime}$-pocket of $\mathrm{CD} 1 \mathrm{~d}^{7,8}$ and along with the ceramide backbone, is responsible for the positioning of the sugar headgroup for recognition by the iNKT cell TCR. ${ }^{5}$ In addition, changes to the lipid portion of $\alpha$ GalCer have been suggested to affect its solubility, leading to variations in in vivo distribution and pharmacology of the glycolipids. ${ }^{3}$ This could alter glycolipid processing and presentation by various subsets of dendritic cells, ultimately resulting in the specific iNKT cell activation.

While the influence of $\alpha$-GalCer analogues with acyl chains of varying length, saturation and substitution patterns have been extensively studied, ${ }^{1,2,4-6,9}$ no systematic analysis has been carried out for $N$-acyl chain homologues of isoglobotrihexosylceramide, iGb3, 4 (Figure 5.1). To date, the short acyl chain derivatives of iGb3 4 (i.e. $\mathrm{C} 8$ and $\mathrm{C} 18$ derivatives), have been used for crystallographic analysis, but they have not been compared side-by-side for their structure activity relationships. The C26 acyl lipid is notorious for being poorly soluble, therefore, in the synthesis of glycosphingolipids, the original C26 acyl chain, as found in $\alpha$-GalCer and iGb3, is often substituted by shorter variants (C16, C18, C24). This presumably has the advantage of increasing the yield of the amide coupling with the more readily soluble fatty acids, and consequently, of improving the solubility of the final glycosphingolipids, which assists with purification and characterisation. 


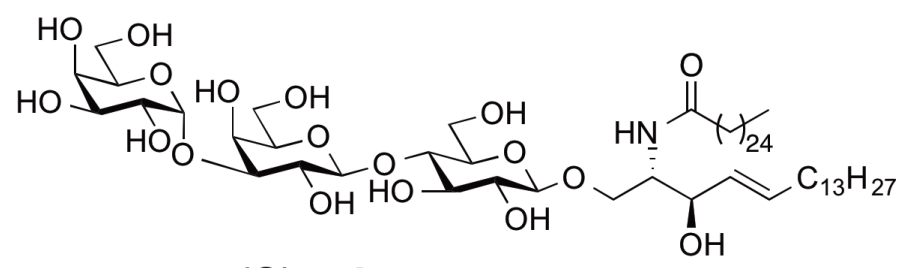

iGb3, 4

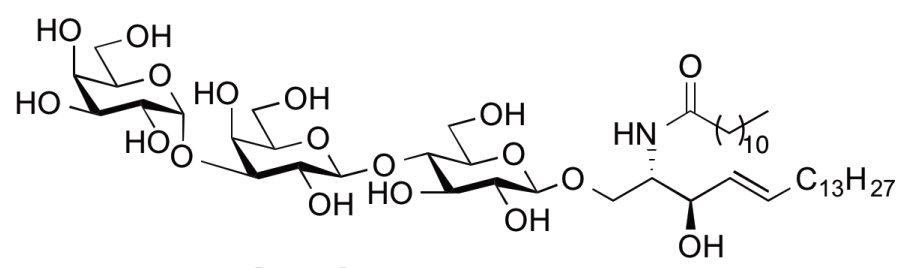

iGb3-C12, 7

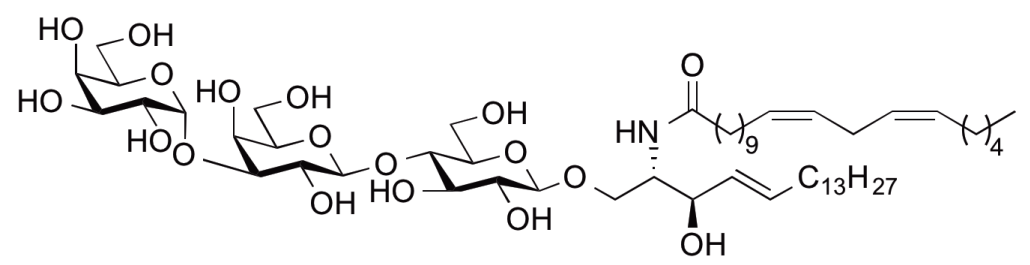

iGb3-C20:2, 8

Figure 5.1. iGb3 (4) and acyl chain homologues, iGb3-C12 (7) and iGb3-C20:2 (8).

Access to a panel of acyl chain homologues of iGb3 (4) will provide a useful means by which to probe the structure activity relationship between iNKT cell activation and acyl lipid length and saturation. To this end, the novel iGb3-C12 analogue 7 will provide a platform to further test how the truncated lipid can affect the orientation of the sugar headgroup and hence activity (Figure 5.1). While shorter acyl chain homologues of iGb3 such as the $\mathrm{C} 8,{ }^{10,11} \mathrm{C} 16,{ }^{12} \mathrm{C} 18,{ }^{13}$ and $\mathrm{C} 24^{14}$ homologues have previously been made, no systematic structure activity relationship studies were performed on this series. In addition, $\alpha$-GalCer with a C20:2 acyl lipid has been found to have superior activity compared to the saturated analogue C20, and can skew the immune system towards an antiinflammatory (Th2) cytokine response., ${ }^{2,15,16}$ Accordingly, the unprecedented C20:2 derivative of iGb3 8, will be prepared. Through synthesis, subsequent structure activity relationship studies and crystal structure analysis, it will thus be possible to understand the influence that the unsaturated acyl chain has on the presentation of triglycosylceramides. 
One other point to keep in mind is that while $\alpha$-GalCer remains the glycolipid adjuvant of choice in DC based cancer immunotherapy, the activity of this glycolipid is so potent that anergy can be induced. ${ }^{17-20}$ Thus, while it is a long way off, there is also the potential to use iGb3 analogues in cancer immunotherapy or, through structural modification and the judicious induction of a different cytokine profile, as a therapy for diseases other than cancer, such as infectious diseases and autoimmune diseases. ${ }^{21-23}$ Furthermore, there has been increasing appreciation for the role that $\beta$-linked glycolipids play in activating iNKT cells, beyond just serving as the endogenous ligands responsible for positive selection of iNKT cells. $^{24}$ Brigl et al. convincingly illustrated that in a microbial infection setting, iNKT cells were able to respond to inflammatory stimulus in the absence of known microbial-derived ligands for iNKT TCR. ${ }^{25}$ Innate stimulus such as the TLR signal stimulates the production of IL-12, causing cytokine-mediated activation of iNKT cells, presumably through the activity of endogenous glycolipids. While the exact identity of the self-glycolipids remains to be determined, $\beta$-glucosylceramide ( $\beta$-GlcCer) was isolated in abundance and was shown to accumulate in the lymphoid tissues of mice and humans during infection. ${ }^{26}$ Much remains to be answered about the exact role of $\beta$-linked self glycolipids, but these findings point towards the possibility of using $\beta$-linked glycolipids, such as iGb3 analogues, to activate iNKT cells not just for cancer, but also for other disease settings. 


\subsection{Synthesis of acyl chain homologues of iGb3}

\section{Retrosynthesis}

To synthesise the acyl chain homologues of iGb3, iGb3-C12 7 and iGb3-C20:2 8, a highly convergent strategy was chosen, whereby both glycolipids $\mathbf{7}$ and $\mathbf{8}$ could be obtained from the triglycosyl 2-azido-sphingosine intermediate 92 via azide reduction, amide coupling with the desired fatty acid, then global deprotection via Birch reduction (Scheme 5.1). In turn, azide 92 could be made by the glycosylation reaction between galactosyl imidate donor 93 and lactosylsphingosine (LacSphing) acceptor 94, while the galactosyl imidate donor could be obtained in several steps from D-galactose (39). Finally, the LacSphing acceptor 94 could be obtained from lactosyl 2-azido-sphingosine 74, where the synthesis of lactosyl 2-azido-sphingosine $\mathbf{7 4}$ is described in Chapter 4 (Scheme 4.2). This strategy differs from the approach used to synthesise 6"'-deoxy-iGb3, in that the complete trisaccharide core is prepared prior to the addition of $\mathrm{N}$-acyl chain. The advantage of this approach is that the late stage azide intermediate $\mathbf{9 2}$ could be functionalised with desired fatty acids, followed immediately by global deprotection to afford the target iGb3 analogues. 


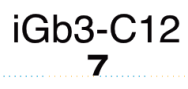

$\underset{\mathbf{R}}{\text { iGb3-C20:2 }}$<smiles>[CH]1C=CC1</smiles><smiles>C=CCCC</smiles>

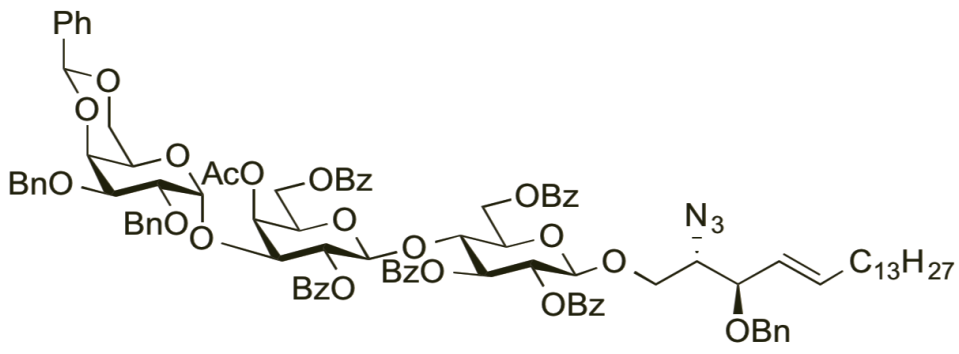

92<smiles>C/C=C/Cc1ccccc1</smiles>

93<smiles>C1CCC1</smiles><smiles>CC=CCCCC</smiles>
$3 n$<smiles>[CH]1C=CC1</smiles>

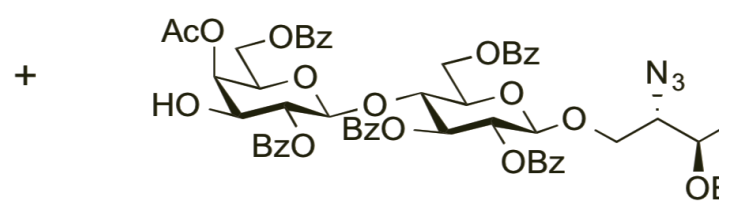

94<smiles>C1=CCCC1</smiles>

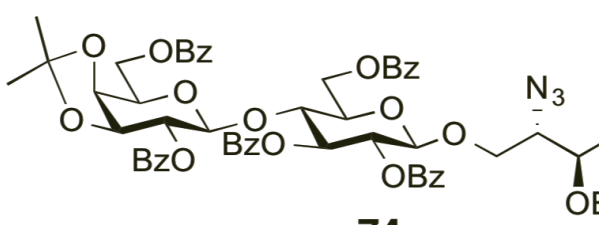

74

Scheme 5.1. Retrosynthesis of target iGb3 homologues, iGb3-C12 (7) and iGb3C20:2 (8).

\section{Synthesis of galactosyl imidate donor 93}

The synthesis of target iGb3 homologues $\mathbf{7}$ and $\mathbf{8}$ began with the synthesis of galactosyl imidate donor 93 (Scheme 5.1). ${ }^{27-30}$ Here, D-galactose (39) was peracetylated, then subjected to the Helferich glycosidation using tin(IV) chloride as the Lewis acid, ${ }^{31}$ and the acetate groups were then removed using sodium 
methoxide in methanol to afford thiogalactoside 95. Next, the 4- and 6-hydroxyls were selectively protected with a benzylidene group, which was previously anticipated to favour $\alpha$-glycosylation, ${ }^{28,32,33}$ and the remaining hydroxyls were then benzylated to afford the fully protected thiogalactoside 96 in 53\% yield over two steps. Initial attempts at synthesising iGb3 by other members of our group using a thiol donor to attach the terminal sugar led to poor yields and a mixture of products, ${ }^{34}$ so accordingly, an imidate donor was chosen. To this end, the anomeric thiophenol group in $\mathbf{9 6}$ was hydrolysed using NBS in the presence of acetone and water to provide lactol 97 in quantitative yield. ${ }^{35}$ The anomeric hydroxyl was then converted into a trichloroacetimidate group to afford galactosyl imidate donor 93 as crystalline material in $86 \%$ yield. In summary, the galactose donor building block 93 was made from D-galactose (39) in seven steps and in an overall yield of $45 \%$, which is comparable to Wong and co-workers' synthesis via the $1-S$ - $p$-methylphenyl intermediate. ${ }^{28}$
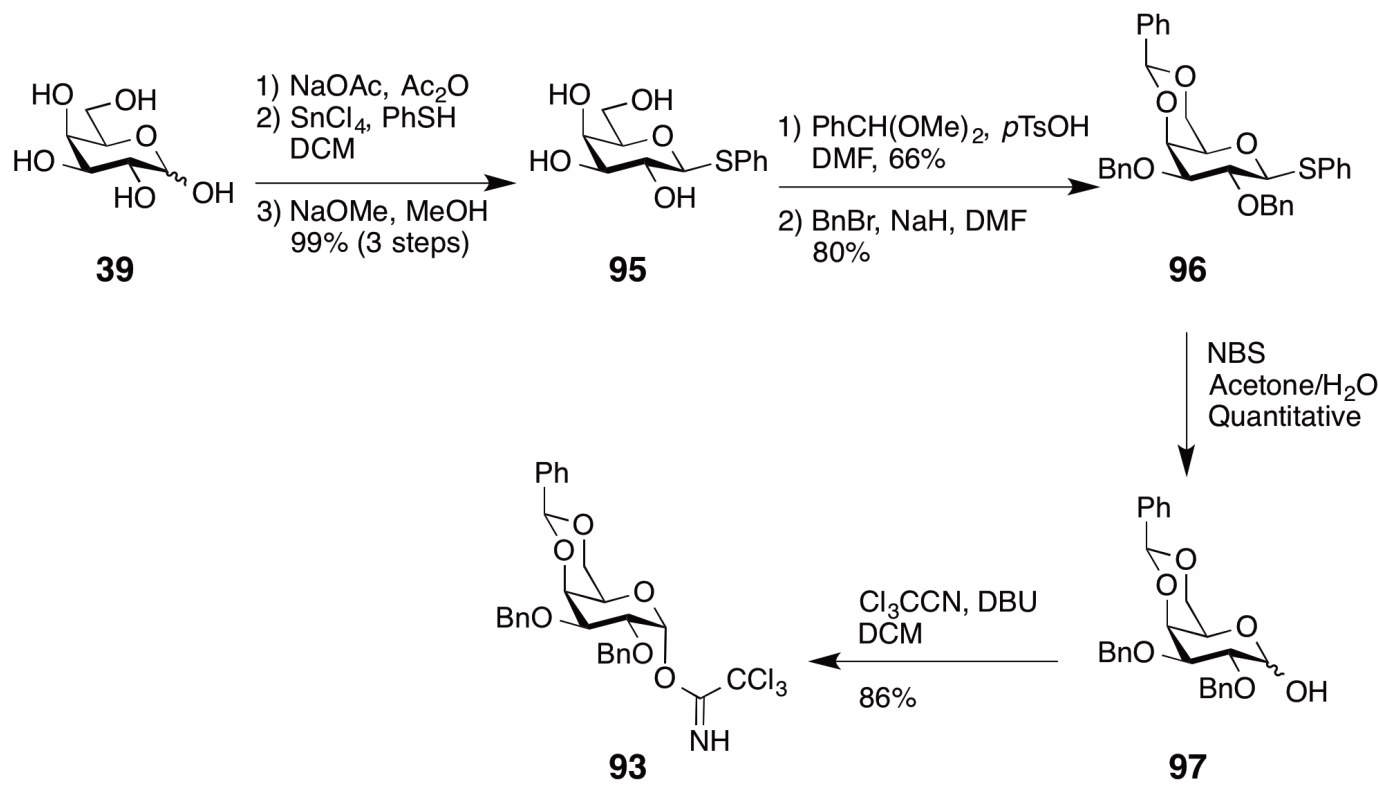

Scheme 5.2. Synthesis of galactosyl imidate donor 93 . 


\section{Triglycosyl 2-azido-sphingosine 92}

With the galactose building block in hand, attention was then turned towards the synthesis of triglycosyl 2-azido-sphingosine 92, which commenced with lactosyl 2-azido-sphingosine 74, an intermediate from the synthesis of 6"' -deoxy-iGb3 analogues (cf. Chapter 4, Scheme 4.2). Accordingly, the isopropylidene group in 74 was first hydrolysed in the presence of acid to afford diol 98 in an excellent $99 \%$ yield (Scheme 5.3). Next, the 4 "'-OH was regioselectively protected as an acetate by employing the same strategy used in Chapter 4, whereby trimethyl orthoacetate and camphorsulfonic acid were used to first install a methyl orthoacetate protecting group at 3 "'-OH and 4" $-\mathrm{OH}$, which subsequently ringopened upon acidic workup. In this way, LacSphing acceptor 94 was prepared in an excellent $94 \%$ yield. The successful acetylation at the $44^{\prime \prime}$-position was confirmed by a HMBC between the carbonyl carbon of the acetate and H-4" . 

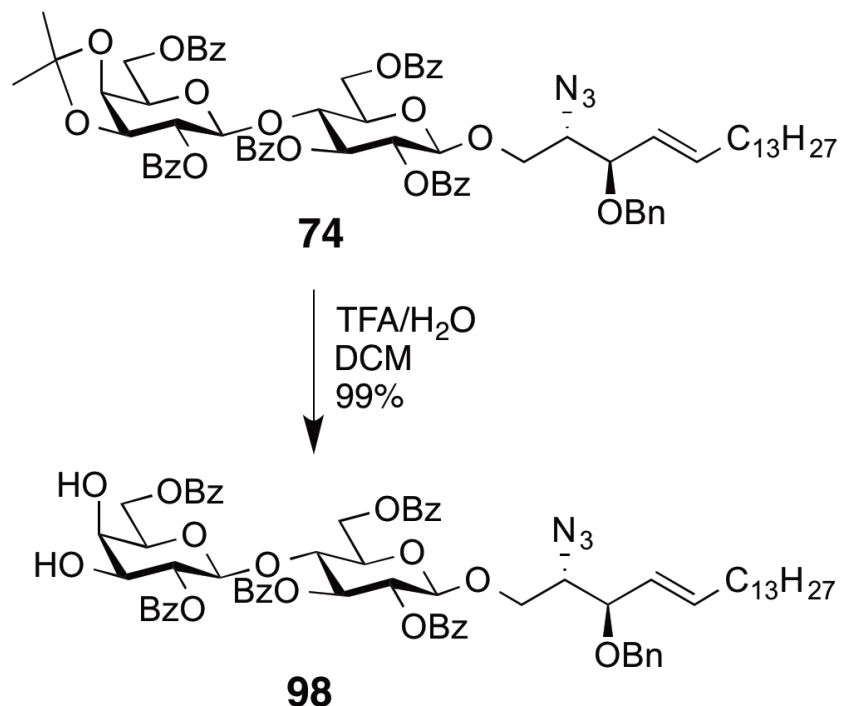

98
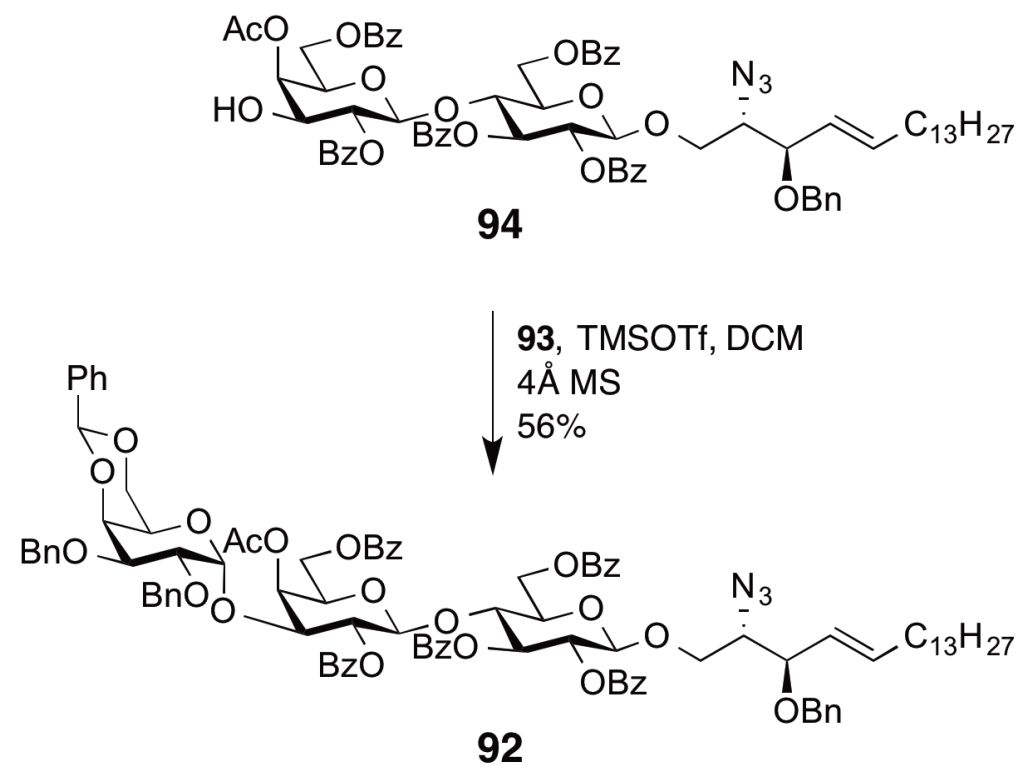

Scheme 5.3. Synthesis of triglycosyl 2-azido-sphingosine 92 .

Using galactose donor 93 and the LacSphing acceptor 94 a glycosylation reaction was then performed with TMSOTf as the promoter. Initially, the reaction was carried out at at $-40{ }^{\circ} \mathrm{C}$, however this proved unsuccessful and no desired product was formed. When the reaction was repeated at warmer temperatures (activation at $-20{ }^{\circ} \mathrm{C}$ followed by stirring from $0{ }^{\circ} \mathrm{C}$ to rt over $2.5 \mathrm{~h}$ ) the desired $\alpha$-isomer of 92 was formed (56\% yield), however, a significant amount (ca. 40\%) of unreacted acceptor 94 was also isolated. Despite efforts to push the reaction to completion 
by sequentially adding more equivalents of the donor to the reaction mixture, the glycosylation yield could not be improved. Indeed, the best yields were obtained when the donor was added in one portion. The glycosylation at the 3 " position was confirmed by HMBC between C-3"' and H-1"' (Figure 5.2).

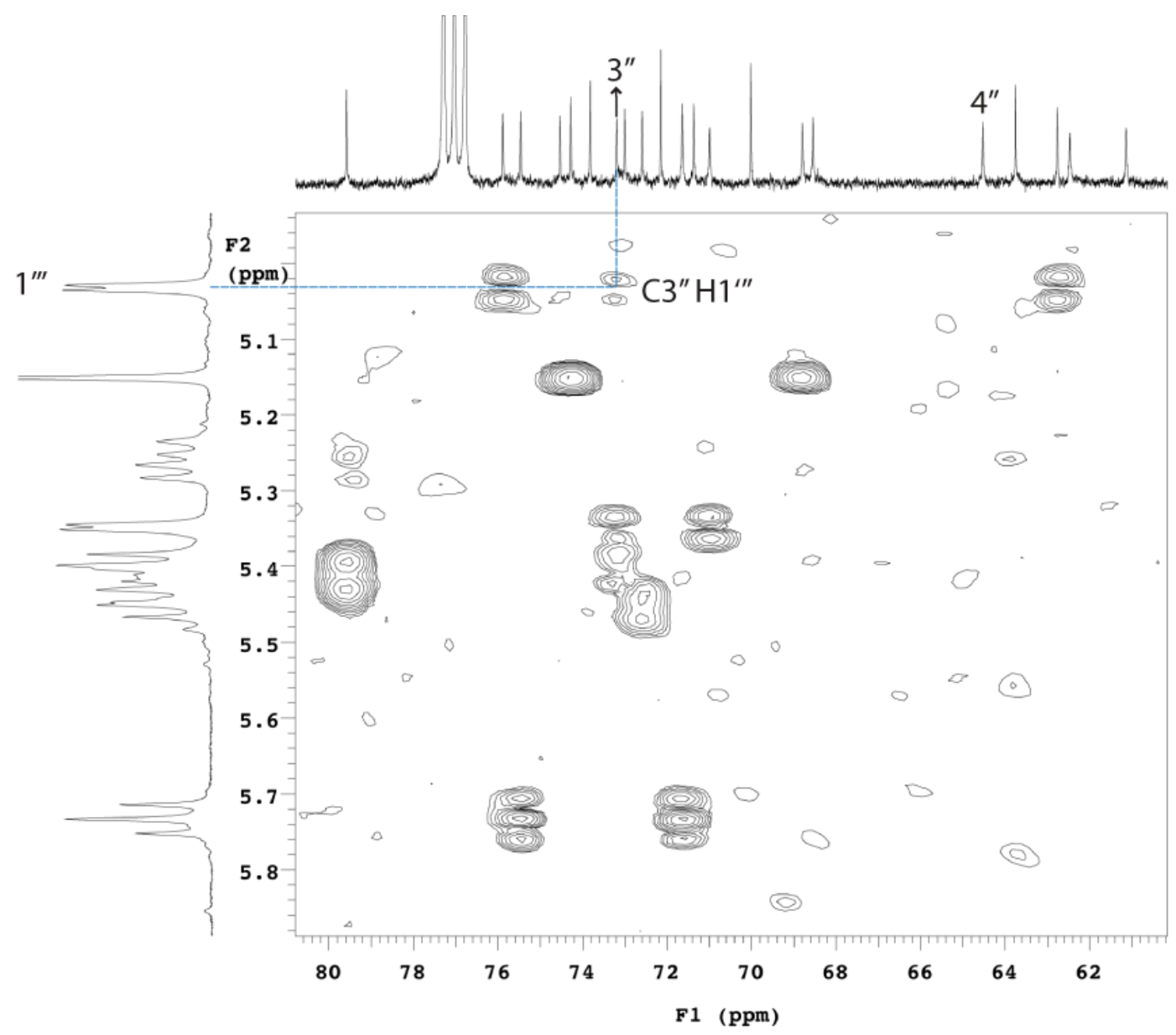

Figure 5.2. HMBC spectrum of triglycosyl 2-azido-sphingosine 92. 


\section{Coupling of acyl lipids and global deprotection}

Having prepared triglycosyl 2-azido-sphingosine 92, the next step was to functionalise the sphingosine backbone with fatty acids bearing the $\mathrm{C} 12$ or the C20:2 lipid. The reduction of the azide to the amine using triphenylphosphine and water was then undertaken according to previously published procedures, ${ }^{36,37}$ and this provided amine 99, which was used without further purification (Scheme 5.4). Dodecanoic $(\mathrm{C} 12)$ acid was coupled to the amine by using an EDCI/DMAP coupling protocol to provide the fully protected iGb3-C12 100 in an excellent $75 \%$ yield over two steps. An identical coupling strategy was carried out for the C20:2 analogue, whereby azide reduction to the amine 99 , followed by immediate coupling with 11Z,14Z-eicosadienoic (C20:2) acid afforded the fully protected iGb3-C20:2 101 in 28\% yield over two steps. The lower yield of this second acylation is in part attributed to the poorer solubility of the C20:2 lipid compared to the shorter chain $\mathrm{C} 12$ variant, which causes it to be less reactive. In addition, it has also been reported that skipped dienes are particularly prone to autoxidation to form conjugated diene hydroperoxides. ${ }^{38,39}$ Extensive studies on linoleic (C18:2) acid, which has the same unsaturation pattern as the C20:2 acid, have shown that 1,3-conjugated diene hydroperoxides are formed from the incorporation of atmospheric oxygen. ${ }^{39,40}$ Exposure to oxygen from the atmosphere for too long leads to autoxidation products as observed for the starting material C20:2 acid whereby $\mathrm{m} / \mathrm{z}$ corresponding to the 1,3-conjugated diene alcohol $(\mathrm{m} / \mathrm{z}$ calcd. for $\mathrm{C}_{20} \mathrm{H}_{35} \mathrm{O}_{3}{ }^{-}: 323.2592$, obsd.: 323.2587$)$ and the 1,3-conjugated diene ketone $(\mathrm{m} / \mathrm{z}$ calcd. for $\mathrm{C}_{20} \mathrm{H}_{33} \mathrm{O}_{3}{ }^{-}: 321.2435$, obsd.: 321.2435) were detected by mass spectrometry. Effort to minimise the degradation of the C20:2 glycolipid by autoxidation was taken by ensuring that the compounds are always kept under an inert, oxygen-free atmosphere. It should also be noted that none of the byproducts were in the purified fractions of the fully protected iGb3-C20:2 101. 

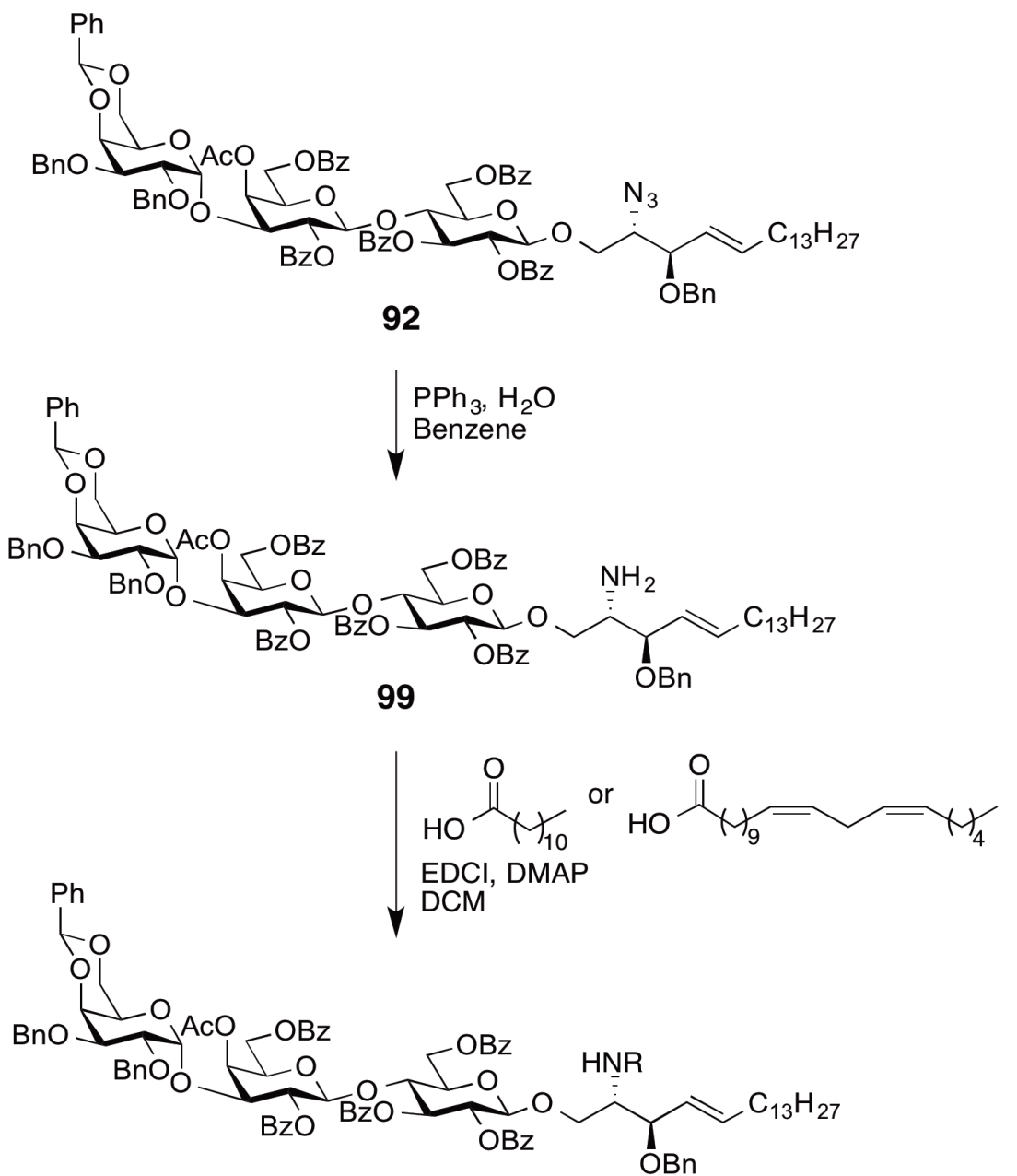

$$
100, \mathrm{R}=101, \mathrm{R}=
$$

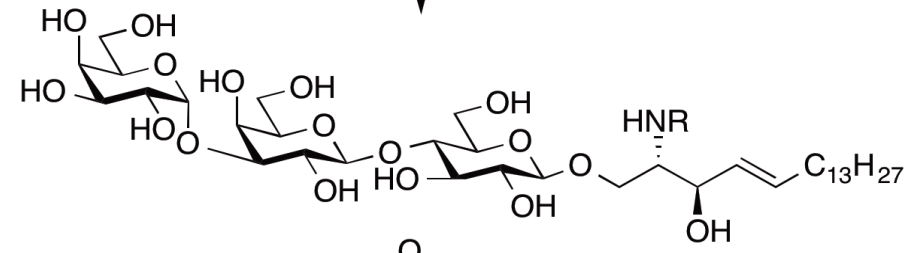

\footnotetext{
iGb3-C12, $7 \mathrm{R}=$ \&

iGb3-C20:2, $8 \mathrm{R}=$
}

Scheme 5.4. Coupling of acyl lipid and global deprotection to form iGb3-C12 (7) and iGb3-C20:2 (8). 
Next, the global deprotection of the fully protected iGb3-C12 100 was carried out with a Birch reaction to remove all ether and ester protecting groups, but leaving the alkene intact. The reaction was performed under standard conditions for glycolipids, ${ }^{41,42}$ yet surprisingly, mass spectrometry analysis of the crude reaction mixture before and after quenching with Dowex $-\mathrm{H}^{+}$revealed the presence the desired product iGb3-C12 (7) (m/z calcd. for $\left[\mathrm{C}_{48} \mathrm{H}_{89} \mathrm{NO}_{18}+\mathrm{H}\right]^{+}$: 968.6152, obsd.: 968.6159) along with the hydrolysed by-products LacCer-C12 (m/z calcd. for $\left[\mathrm{C}_{42} \mathrm{H}_{78} \mathrm{NO}_{13}+\mathrm{H}\right]^{+}:$806.5624, obsd.: 806.5632), GlcCer-C12 (m/z calcd. for $\left[\mathrm{C}_{36} \mathrm{H}_{68} \mathrm{NO}_{8}+\mathrm{H}\right]^{+}:$644.5096, obsd.: 644.5100) and Cer-C12 (m/z calcd. for $\left[\mathrm{C}_{30} \mathrm{H}_{58} \mathrm{NO}_{3}+\mathrm{H}\right]^{+}:$482.4568, obsd.: 482.4573). As glycosidic linkages are generally considered to be stable under basic conditions, this result was surprising. The yields of Birch reactions performed on glycolipids varies, ${ }^{41,42}$ and indeed, others have reported modest yields, ${ }^{41-43}$ fragmentation under Birch conditions has not been widely reported, though the cleavage of $\beta$-mannosyl glycosidic bonds have been observed by several others. ${ }^{44,45}$ Further systematic studies need to be undertaken to understand why this hydrolysis occurred and it would be valuable if others were to comment on observed by-products to assist with understanding the mechanisms that are taking place.

Despite the formation of the by-products, purification of the crude residue by silica gel column chromatography, followed by reverse phase column chromatography afforded the desired target iGb3-C12 7 in 24\% yield. GlcCerC12 was also isolated (34\% yield) along with a mixture of semi-deprotected glycolipid (with intact benzylidene and the benzyl protecting groups) as identified by mass spectrometry $\left(\mathrm{m} / \mathrm{z}\right.$ calcd. for $\left[\mathrm{C}_{76} \mathrm{H}_{111} \mathrm{NO}_{3}+\mathrm{H}\right]^{+}: 1326.7874$, obsd.: 1326.7851). The incomplete deprotection of the ether protecting groups was puzzling as the Birch protocol employed involved two rounds of addition of sodium, with quenching by methanol in between each addition of sodium, however due to the limited availability of the intermediate triglycosyl 2-azidosphingosine 92, this reaction was not further optimised. Nonetheless, the desired target iGb3-C12 7 was obtained in sufficient quantities for characterisation and biological evaluation. 
The same global deprotection procedure was applied to the fully protected iGb3C20:2 101. Again, like the C12 analogue 100, careful mass spectrometry analysis of the crude reaction mixture before and after neutralisation with Dowex $-\mathrm{H}^{+}$ revealed the presence of the desired product iGb3-C20:2 (8) $(\mathrm{m} / \mathrm{z}$ calcd. for $\left[\mathrm{C}_{56} \mathrm{H}_{101} \mathrm{NO}_{18}+\mathrm{H}\right]^{+}:$1076.7091, obsd.: 1076.7090) along with by-products from hydrolysis of the glycosidic linkages, namely LacCer-C20:2 (m/z calcd. for $\left[\mathrm{C}_{50} \mathrm{H}_{91} \mathrm{NO}_{13}+\mathrm{H}\right]^{+}:$914.6563, obsd.: 914.6575), GlcCer-C20:2 (m/z calcd. for $\left[\mathrm{C}_{44} \mathrm{H}_{81} \mathrm{NO}_{8}+\mathrm{H}\right]^{+}:$752.6035, obsd.: 752.6042) and Cer-C20:2 (m/z calcd. for $\left[\mathrm{C}_{38} \mathrm{H}_{71} \mathrm{NO}_{3}+\mathrm{H}\right]^{+}:$590.5507, obsd.:m/z 590.5524). The incompletely deprotected intermediate with ether groups intact were not detected. Purification of the crude reaction mixture by silica gel column chromatography afforded the desired target iGb3-C20:2 (8) in 48\% yield.

Having successfully prepared the $\mathrm{C} 12$ and $\mathrm{C} 20: 2$ iGb3 homologues, and in Chapter 4 the $6^{\prime \prime \prime}$-deoxy derivatives, it would be interesting to compare the syntheses. Three key transformations for both routes are the acyl chain coupling, the terminal sugar coupling and the formation of the 3 "'-OH glycolipid acceptor, and as the yields for these key transformations in both strategies are very comparable, it is difficult to say whether one of these approaches is better. The advantage to the synthetic strategy employed across these two different series of iGb3 homologues, however, is the fact that the late stage key intermediate, lactosyl 2-azido-sphingosine glycolipid 74, is easily accessible from D-lactose and can be functionalised accordingly to obtain both terminal sugar homologues and acyl chain homologues from a common intermediate. Taken as a whole, the strategy here differs from all other iGb3 syntheses previously reported, whereby a trisaccharide donor is coupled directly to either the complete ceramide ${ }^{41,46}$ or in two steps, to the sphingosine backbone followed by $N$-acylation of the lipid backbone. ${ }^{37,47,48}$ While these reported strategies may be more convergent for the synthesis of one target, the route designed in this work is robust and versatile for the synthesis of multiple targets of either the terminal sugar or the acyl chain series. 


\subsection{Conclusion}

In summary, two novel acyl chain homologues of iGb3, iGb3-C12 (7) and iGb3C20:2 (8) were synthesised in $2 \%$ and 1\% overall yield, respectively, over 12 steps from D-lactose (longest linear sequence). Key to the synthesis is the coupling of the terminal galactose sugar donor 93 to the lactosylsphingosine (LacSphing) acceptor 94, which proceeded in good selectivity giving only the $\alpha$ isomer in 56\% yield. Acceptor 94 itself was synthesised from the common intermediate lactosyl 2-azido sphingosine 74, which was used for the synthesis of 6"' -deoxy-iGb3 analogues described in Chapter 4. In addition, the triglycosyl sphingosine intermediate 92 represents a useful intermediate for functionalisation with desired acyl chains, as was illustrated in this work, whereby the C12 and C20:2 lipids were coupled. It was observed that amide coupling with shorter chain homologues (i.e. C12), proceeds more smoothly compared to their longer chain counterparts, such as the C20:2, and also the C26 lipid used the Chapter 4. In addition, the sensitivity of C20:2 lipid to autoxidation means that extra care had to be taken when working with this lipid. From this work, the successful synthesis of these two acyl chain homologues will allow for further structure activity relationship studies to be performed by our collaborators so that more insight can be gained on the influence of lipid length and saturation patterns for iNKT cell activity. Moreover, crystal structure studies of these glycolipids in complex with CD1d and TCR of iNKT cells will shed light on the favourable or unfavourable interactions giving rise to the activity observed. 


\subsection{Experimental}

\section{General procedure}

Unless otherwise stated all reactions were performed under argon. Prior to use, THF (Pancreac) was distilled from sodium and benzophenone, pyridine was

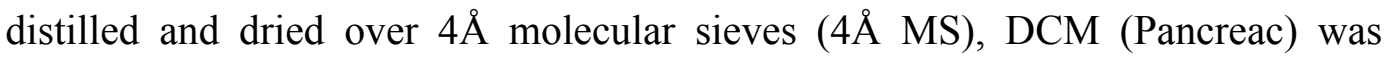
distilled from $\mathrm{P}_{2} \mathrm{O}_{5}$, and $\mathrm{H}_{2} \mathrm{O}$ and benzene (Fisher Scientific) were distilled. $\mathrm{SnCl}_{4}$ (Aldrich), PhSH (Koch-Light Laboratories), benzaldehyde dimethyl acetal (Aldrich), $\mathrm{Me}_{2} \mathrm{C}(\mathrm{OMe})_{2}$ (Aldrich), NBS (Aldrich), DBU (Merck), CSA (Acros), $n \mathrm{Bu}_{3} \mathrm{SnCl}$ (Aldrich), AIBN (Aldrich), D-fucose (Aldrich), D-lactose (Aldrich), trityl chloride (Acros), anhydrous $\mathrm{Et}_{2} \mathrm{O}$ (Pancreac), $\mathrm{PPh}_{3}$ (Aldrich), $\mathrm{Pd}(\mathrm{OH})_{2} / \mathrm{C}$ (Aldrich, $20 \mathrm{wt} \%$ ), anhydrous DMF (Acros), TFA (Aldrich), $p \mathrm{TsOH}$ (Aldrich), TMSOTf (Aldrich), $\mathrm{H}_{2} \mathrm{SO}_{4}$ (Lab-Scan), formic acid (Aldrich), AcCl (Aldrich), $\mathrm{BnBr}$ (Fluka), $\mathrm{PMe}_{3}$ (Aldrich, $1 \mathrm{M}$ in THF), $\mathrm{AcOH}$ (Ajax Finechem), $\mathrm{Ac}_{2} \mathrm{O}$ (Peking Reagent), TMSOTf (Aldrich), DiPEA (Aldrich), NaOMe (Janssen Chimica), trichloroacetonitrile (Aldrich), lauric acid (Hopkin \& Williams), 11Z,14Z-eicosadienoic acid (Allichem LLC), BzCl (Aldrich, distilled an stored under argon), HBTU (Acros), PyBOP (Aldrich), EDCI (Aldrich), DMAP (Merck), sodium (Aldrich), trimethyl orthoacetate (Aldrich), $\mathrm{LiAlH}_{4}$ (Aldrich), EtOAc (Pancreac), hexanes (Fisher Scientific), petroleum ether (Pure Science), $\mathrm{MeOH}$ (Pure Science), $\mathrm{CHCl}_{3}$ (Pancreac), EtOH (absolute, Pure Science), $\mathrm{NaOH}$ (Pure Science), $\mathrm{NaHCO}_{3}$ (Pure Science), $\mathrm{NaCl}$ (Pancreac), $\mathrm{NH}_{3}$ (BOC gasses) were used as received. All solvents were removed by evaporation under reduced pressure. Reactions were monitored by TLC-analysis on Macherey-Nagel silica gel coated plastic sheets $\left(0.20 \mathrm{~mm}\right.$, with fluorescent indicator $\left.\mathrm{UV}_{254}\right)$ with detection by UV-absorption (short wave UV - $254 \mathrm{~nm}$; long wave UV - $366 \mathrm{~nm}$ ), by dipping in $10 \% \mathrm{H}_{2} \mathrm{SO}_{4}$ in EtOH followed by charring at $\sim 150{ }^{\circ} \mathrm{C}$, by dipping in $\mathrm{I}_{2}$ in silica, or by dipping into a solution of ninhydrin in $\mathrm{EtOH}$ followed by charring at $\sim 150{ }^{\circ} \mathrm{C}$. Column chromatography was performed on Pure Science silica gel (40-63 micron). AccuBOND II ODS-C18 (Agilent) was used for reverse phase chromatography. Infrared spectra were recorded as thin films using a Bruker Tensor 27 FTIR spectrometer equipped with an Attenuated Total 
Reflectance (ATR) sampling accessory and are reported in wave numbers $\left(\mathrm{cm}^{-1}\right)$. Nuclear magnetic resonance spectra were recorded at $20{ }^{\circ} \mathrm{C}$ in $\mathrm{CD}_{3} \mathrm{OD}, \mathrm{CDCl}_{3}$, or pyridine- $\mathrm{d}_{5}$ using either a Varian INOVA operating at $500 \mathrm{MHz}$ or Varian VNMRS operating at $600 \mathrm{MHz}$. Chemical shifts are given in ppm ( $\delta$ ) relative to TMS. NMR peak assignments were made using COSY, HSQC and HMBC 2D experiments.

\section{Phenyl 2,3,4,6-tetra- $O$-acetyl-1-thio- $\beta$-D-galactopyranoside}

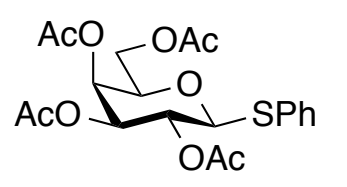

1,2,3,4,6-penta- $O$-acetyl- $\beta$-D-galactopyranose $(20 \mathrm{~g}, 51.24$ mmol) was co-evaporated with toluene (x2), dissolved in $\mathrm{CH}_{2} \mathrm{Cl}_{2}(150 \mathrm{~mL})$ and cooled to $0{ }^{\circ} \mathrm{C}$. Thiophenol $(6.3 \mathrm{~mL}$, $61.42 \mathrm{mmol})$ and tin(IV) chloride $(0.6 \mathrm{~mL}, 5.12 \mathrm{mmol})$ was added to the reaction mixture under argon and stirred for $3 \mathrm{~h}$. The reaction was quenched with a $3 \mathrm{M}$ solution of potassium fluoride (100 mL, 6 equiv.) and the organic layer was washed with water, saturated sodium bicarbonate solution, brine and dried over $\mathrm{MgSO}_{4}$, filtered and concentrated in vacuo. The residue was taken up in ethyl acetate and passed through a plug of silica gel to remove residual tin by-products. This afforded title compound as a yellow oil (23.9 g). $\mathrm{R}_{f}$ : 0.73 (PE/EA, 1/1, v/v); $[\alpha]^{23}{ }_{\mathrm{D}}=+4.0^{\circ}\left(\mathrm{c}=1.0, \mathrm{CHCl}_{3}\right)$; IR (thin film) 3060, 2917, 2849, 2400, 2000, 1747, 1583, 1480, 1439, 1369, 1200, 1054, 917, 736, $702 \mathrm{~cm}^{-1} ;{ }^{1} \mathrm{H}$ NMR (500 $\left.\mathrm{MHz}, \mathrm{CDCl}_{3}\right) \delta 7.51(\mathrm{~m}, 2 \mathrm{H}, \mathrm{CH}-o \mathrm{Ph}), 7.31(\mathrm{~m}, 3 \mathrm{H}, \mathrm{CH}-m \mathrm{Ph}), 5.41\left(\mathrm{~d}, 1 \mathrm{H}, J_{3,4}\right.$ $=3.4 \mathrm{~Hz}, \mathrm{H}-4), 5.24\left(\mathrm{t}, 1 \mathrm{H}, J_{1,2}=J_{2,3}=10.0 \mathrm{~Hz}, \mathrm{H}-2\right), 5.05\left(\mathrm{dd}, 1 \mathrm{H}, J_{2,3}=10.0\right.$ $\left.\mathrm{Hz}, J_{3,4}=3.4 \mathrm{~Hz}, \mathrm{H}-3\right), 4.72\left(\mathrm{~d}, 1 \mathrm{H}, J_{1,2}=10.0 \mathrm{~Hz}, \mathrm{H}-1\right), 4.18\left(\mathrm{dd}, 1 \mathrm{H}, J_{6 \mathrm{a}, 6 \mathrm{~b}}=\right.$ $\left.11.4 \mathrm{~Hz}, J_{5,6 \mathrm{a}}=7.1 \mathrm{~Hz}, \mathrm{H}-6 \mathrm{a}\right), 4.11\left(\mathrm{dd}, 1 \mathrm{H}, J_{6 \mathrm{a}, 6 \mathrm{~b}}=11.4 \mathrm{~Hz}, J_{5,6 \mathrm{~b}}=6.2 \mathrm{~Hz}, \mathrm{H}-6 \mathrm{~b}\right)$, $3.94\left(\mathrm{t}, 1 \mathrm{H}, J_{5,6 a}=7.1 \mathrm{~Hz}, J_{5,6 \mathrm{~b}}=6.2 \mathrm{~Hz}, \mathrm{H}-5\right), 2.11\left(\mathrm{~s}, 3 \mathrm{H}, \mathrm{COCH}_{3}\right), 2.09$ (s, 3H, $\left.\mathrm{COCH}_{3}\right), 2.04\left(\mathrm{~s}, 3 \mathrm{H}, \mathrm{COCH}_{3}\right), 1.97\left(\mathrm{~s}, 3 \mathrm{H}, \mathrm{COCH}_{3}\right) ;{ }^{13} \mathrm{C} \mathrm{NMR}(500 \mathrm{MHz}$, $\left.\mathrm{CDCl}_{3}\right) \delta 170.4,170.2,170.1,169.4\left(\mathrm{COCH}_{3}\right), 132.5(\mathrm{CH}-o \mathrm{Ph}) 132.5(\mathrm{C}-i \mathrm{Ph})$, 128.9 (CH- $m$ Ph), 128.2 (CH-p Ph), 86.6 (C-1), 74.4 (C-5), 72.0 (C-3), 67.22, $67.20(\mathrm{C}-2$ and $\mathrm{C}-4), 61.6(\mathrm{C}-6), 20.8\left(\mathrm{COCH}_{3}\right), 20.7\left(\mathrm{COCH}_{3}\right), 20.64\left(\mathrm{COCH}_{3}\right)$, $20.59\left(\mathrm{COCH}_{3}\right)$; HRMS(ESI) $\mathrm{m} / \mathrm{z}$ calcd. for $\left[\mathrm{C}_{20} \mathrm{H}_{24} \mathrm{O}_{9} \mathrm{~S}+\mathrm{Na}\right]^{+}:$463.1034, obsd.: 463.1034 . 


\section{Phenyl 1-thio- $\beta$-D-galactopyranoside (95)}

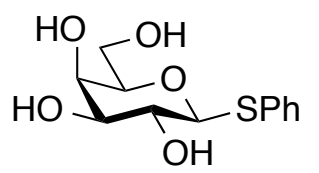

Sodium methoxide was added slowly to a suspension of phenyl 2,3,4,6-tetra- $O$-acetyl-1-thio- $\beta$-D-galactopyranoside $(23.9 \mathrm{~g})$ in methanol $(200 \mathrm{~mL})$ until the solution reached $\mathrm{pH}$ 13. The reaction mixture was stirred for $4 \mathrm{~h}$ and neutralised with Dowex- $\mathrm{H}^{+}$. The resin was removed by filtration and the methanolic solution was concentrated to afford 95 as a white solid (13.9 g, 99\% over two steps), which was used without further purification. $\mathrm{R}_{f}: 0.37(\mathrm{EA} / \mathrm{MeOH}, 9 / 1, \mathrm{v} / \mathrm{v}) ;[\alpha]^{21}{ }_{\mathrm{D}}=-37.0^{\circ}(\mathrm{c}=0.1$, $\mathrm{MeOH}$ ); IR (thin film) 3383, 2895, 1583, 1480, 1439, 1057, 865, 743, $692 \mathrm{~cm}^{-1}$; ${ }^{1} \mathrm{H}$ NMR (500 MHz, CD $\left.3 \mathrm{OD}\right) \delta$ 7.56-7.53 (m, 2H, CH-o Ph), 7.31-7.27 (m, 2H, CH- $m$ Ph), 7.24-7.22 (m, 1H, CH-p Ph), 4.60 (d, 1H, $J_{1,2}=9.7$ Hz, H-1), 3.91 (bs, $1 \mathrm{H}, \mathrm{H}-4), 3.77\left(\mathrm{dd}, 1 \mathrm{H}, J_{6 \mathrm{a}, 6 \mathrm{~b}}=11.5 \mathrm{~Hz}, J_{5,6 \mathrm{a}}=7.0 \mathrm{~Hz}, \mathrm{H}-6 \mathrm{a}\right), 3.72\left(\mathrm{dd}, 1 \mathrm{H}, J_{6 \mathrm{a}, 6 \mathrm{~b}}\right.$ $\left.=11.5 \mathrm{~Hz}, J_{5,6 \mathrm{~b}}=5.5 \mathrm{~Hz}, \mathrm{H}-6 \mathrm{~b}\right), 3.60\left(\mathrm{t}, 1 \mathrm{H}, J_{1,2}=J_{2,3}=9.7 \mathrm{~Hz}, \mathrm{H}-2\right), 3.56(\mathrm{t}, 1 \mathrm{H}$, $\left.J_{4,5}=J_{5,6 \mathrm{~b}}=5.5 \mathrm{~Hz}, \mathrm{H}-5\right), 3.49\left(\mathrm{dd}, 1 \mathrm{H}, J_{2,3}=9.5 \mathrm{~Hz}, J_{3,4}=3.0 \mathrm{~Hz}, \mathrm{H}-3\right) ;{ }^{13} \mathrm{C}$ NMR (500 MHz, CD 3 OD) $\delta 135.9$ (Ci SPh) 131.9 (CH-o Ph), 129.8 (CH-m Ph), 127.9 (CH-p Ph), 90.2 (C-1), 80.5 (C-5), 76.2 (C-3), 70.9 (C-2), 70.3 (C-4), 62.6 (C-6); HRMS(ESI) m/z calcd. for $\left[\mathrm{C}_{12} \mathrm{H}_{16} \mathrm{O}_{5} \mathrm{~S}+\mathrm{Na}\right]^{+}: 295.0611$, obsd.: 295.0613.

\section{Phenyl 4,6-O-benzylidene-1-thio- $\beta$-D-galactopyranoside}

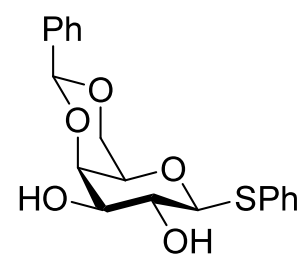

Thioglycoside 95 (13.9 g, $51.0 \mathrm{mmol})$ was co-evaporated with dry DMF (x2) and dissolved in dry DMF (180 mL). Under an argon atmosphere, benzaldehyde dimethyl acetal $(11.5 \mathrm{~mL}$, $76.6 \mathrm{mmol})$ was added followed by $p \mathrm{TsOH}(1.0 \mathrm{~g}, 5.1 \mathrm{mmol})$ and the reaction mixture was stirred at $\mathrm{rt}$ overnight. The reaction mixture was neutralised with triethylamine, concentrated in vacuo and redissolved in ethyl acetate. The organic layer was washed with water, sat. aq. $\mathrm{NaHCO}_{3}$, brine, dried over $\mathrm{MgSO}_{4}$, fillered and concentrated in vacuo. Crystallisation from ethyl acetate/petroleum ether $(1: 2, \mathrm{v} / \mathrm{v})$ afforded title compound as fluffy white crystals $(12.2 \mathrm{~g}, 33.8 \mathrm{mmol}, 66 \%)$. Mp $154-156{ }^{\circ} \mathrm{C}$; $\mathrm{R}_{f}$. $0.69(\mathrm{EA} / \mathrm{MeOH}, 9 / 1, \mathrm{v} / \mathrm{v}) ;[\alpha]^{22}{ }_{\mathrm{D}}=-34.5^{\circ}\left(\mathrm{c}=1.0, \mathrm{CHCl}_{3}\right)$; IR (thin film) 3401, 3061, 2976, 2869, 1583, 1480, 1451, 1439, 1362, 1098, 1070, 1041, 1027, 925, 898, 866, 734, $696 \mathrm{~cm}^{-1} ;{ }^{1} \mathrm{H}$ NMR (500 MHz, $\left.\mathrm{CDCl}_{3}\right) \delta 7.70(\mathrm{~m}, 2 \mathrm{H}$, aromatic H), 7.40-7.29 (m, $8 \mathrm{H}$, aromatic H), $5.52(\mathrm{~s}, 1 \mathrm{H}, \mathrm{CH}-\mathrm{Ph}), 4.52\left(\mathrm{~d}, 1 \mathrm{H}, J_{1,2}=9.0 \mathrm{~Hz}\right.$, 
H-1), $4.40\left(\mathrm{~d}, 1 \mathrm{H}, J_{6 \mathrm{a}, 6 \mathrm{~b}}=12.5 \mathrm{~Hz}, \mathrm{H}-6 \mathrm{a}\right) 4.22\left(\mathrm{~d}, 1 \mathrm{H}, J_{3,4}=1.5 \mathrm{~Hz}, \mathrm{H}-4\right), 4.05$ $\left(\mathrm{dd}, 1 \mathrm{H}, J_{6 \mathrm{a}, 6 \mathrm{~b}}=12.5 \mathrm{~Hz}, J_{5,6 \mathrm{~b}}=1.0 \mathrm{~Hz}, \mathrm{H}-6 \mathrm{~b}\right), 3.71-3.70(\mathrm{~m}, 2 \mathrm{H}, \mathrm{H}-2$, and H-3), 3.57 (bs, 1H, H-5), 2.60 (s, 1H, HO-3), 2.58 (d, 1H, $J=8.5 \mathrm{~Hz}, \mathrm{HO}-2) ;{ }^{13} \mathrm{C}$ NMR $\left(500 \mathrm{MHz}, \mathrm{CDCl}_{3}\right) \delta 137.5,130.6(\mathrm{C}-i \mathrm{Ph}), 133.8,129.4,129.0,128.3,126.5(8 \mathrm{x}$ $\mathrm{CH}_{\text {arom}}$ ), $101.4\left(\mathrm{PhCHO}_{2}\right), 87.0$ (C-1), 75.3 (C-4), 73.8 (C-2), 70.1 (C-5), 69.3 (C6), 68.8 (C-3); HRMS(ESI) m/z calcd. for $\left[\mathrm{C}_{19} \mathrm{H}_{20} \mathrm{O}_{5} \mathrm{~S}+\mathrm{Na}\right]^{+}: 383.0924$, obsd.: 383.0927 .

\section{Phenyl 2,3-O-benzyl-4,6-benzylidene-1-thio- $\beta$-D-galactopyranoside (96)}

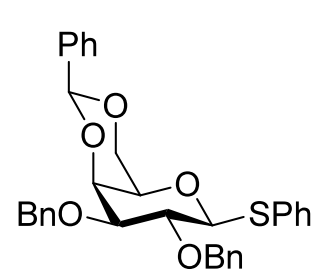

Phenyl 4,6-O-benzylidene-1-thio- $\beta$-D-galactopyranoside ( $2 \mathrm{~g}$, $5.55 \mathrm{mmol})$ was dissolved in dry DMF (25 $\mathrm{mL})$ and benzyl bromide (1.6 mL, $13.3 \mathrm{mmol})$ was added under nitrogen. The reaction mixture was cooled to $0{ }^{\circ} \mathrm{C}$ and sodium hydride ( $60 \%$ in oil suspension) $(0.72 \mathrm{~g}, 18.0 \mathrm{mmol})$ was added slowly. The reaction mixture was stirred overnight at $\mathrm{rt}$ after which time methanol $(15 \mathrm{~mL})$ was added slowly to quench the reaction. The reaction mixture was diluted with diethyl ether and ethyl acetate and washed with water, sat. aq. $\mathrm{NaHCO}_{3}$, brine and dried over $\mathrm{MgSO}_{4}$. The organic layer was concentrate in vacuo and the product was crystallised from ethyl acetate/petroleum ether to afford 96 as white fluffy crystals (2.39 g, 4.42 mmol, 80\%). Mp 171.2-171.9 ${ }^{\circ} \mathrm{C} ; \mathrm{R}_{f}: 0.49$ (PE/EA, 3/1, v/v); $[\alpha]^{22}{ }_{\mathrm{D}}$ $=-18.9^{\circ}\left(\mathrm{c}=1.0, \mathrm{CHCl}_{3}\right)$; IR (thin film) 2817, 2849, 1977, 1584, 1453, 1399, 1092, 1057, 1026, 815, 759, 732, $696 \mathrm{~cm}^{-1} ;{ }^{1} \mathrm{H}$ NMR (500 MHz, $\left.\mathrm{CDCl}_{3}\right) \delta 7.73-$ 7.71 (m, 2H, CH-o $\mathrm{PhCHO}_{2}$ ), 7.55-7.53 (m, 2H, CH-o SPh), 7.43-7.19 (m, 16H, aromatic $\mathrm{H}), 5.50\left(\mathrm{~s}, 1 \mathrm{H}, \mathrm{PhCHO}_{2}\right), 4.72\left(\mathrm{~m}, 4 \mathrm{H}, 2 \times \mathrm{CH}_{2} \mathrm{Ph}\right), 4.63\left(\mathrm{~d}, 1 \mathrm{H}, J_{1,2}=\right.$ $9.3 \mathrm{~Hz}, \mathrm{H}-1), 4.39$ (d, 1H, $\left.J_{6 \mathrm{a}, 6 \mathrm{~b}}=12.5 \mathrm{~Hz}, \mathrm{H}-6 \mathrm{a}\right), 4.17$ (d, 1H, $\left.J_{3,4}=3.4 \mathrm{~Hz}, \mathrm{H}-4\right)$, $4.01(\mathrm{dd}, 1 \mathrm{H}, \mathrm{J}=1.3,12.0 \mathrm{~Hz}, \mathrm{H}-6 \mathrm{a}), 3.91\left(\mathrm{t}, 1 \mathrm{H}, J_{1,2}=J_{2,3}=9.3 \mathrm{~Hz}, \mathrm{H}-2\right), 3.64$ $\left(\mathrm{dd}, J_{2,3}=9.3 \mathrm{~Hz}, 1 \mathrm{H}, J_{3,4}=3.4 \mathrm{~Hz}, \mathrm{H}-3\right), 3.44(\mathrm{bs}, 1 \mathrm{H}, \mathrm{H}-5) ;{ }^{13} \mathrm{C}$ NMR $(500$ $\left.\mathrm{MHz}, \mathrm{CDCl}_{3}\right) \delta 138.5,138.1,137.9$ (C-i Ph), 132.8 (CH-o SPh), 132.7, 129.1, $128.9,128.4,128.4,128.24,128.19,127.8,127.7,127.5,126.6$ (19 x $\left.\mathrm{CH}_{\text {arom }}\right)$, $101.4\left(\mathrm{PhCHO}_{2}\right), 86.5$ (C-1), 81.4 (C-3), 75.5 (2- $\left.\mathrm{OCH}_{2} \mathrm{Ph}\right), 75.4$ (C-2), 73.7 (C4), $71.9\left(3-\mathrm{OCH}_{2} \mathrm{Ph}\right), 69.9$ (C-5), 69.4 (C-6); HRMS(ESI) m/z calcd. for $\left[\mathrm{C}_{19} \mathrm{H}_{20} \mathrm{O}_{5} \mathrm{~S}+\mathrm{Na}\right]^{+}:$563.1868, obsd.: 563.1871 . 


\section{2,3-Di-O-benzyl-4,6-O-benzylidene-D-galactose (97)}

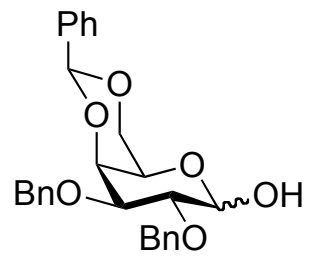

NBS (1.73 g, $9.71 \mathrm{mmol})$ was added to a solution of thioglycoside $96(1.5 \mathrm{~g}, 2.77 \mathrm{mmol})$ in acetone $(111 \mathrm{~mL})$ and water $(5.5 \mathrm{~mL})$, and the reaction was stirred at room temperature for $10 \mathrm{~min}$. The reaction mixture was diluted with EtOAc $(80 \mathrm{ml})$, and washed sat. aq. $\mathrm{Na}_{2} \mathrm{~S}_{2} \mathrm{O}_{3}(80 \mathrm{~mL})$, water $(80 \mathrm{~mL})$ and brine $(80 \mathrm{~mL})$, dried over $\mathrm{MgSO}_{4}$, filtered and concentrated under reduced pressure. The resulting oil was purified by gradient flash chromatography (Petroleum ether/EtOAc, $5 / 1$ to $0 / 100, \mathrm{v} / \mathrm{v}$ ) then crystallised (Petroleum ether/EtOAc, 2/1, v/v) to give 97 as fluffy white crystals (1.24 g, $2.76 \mathrm{mmol}$, Quantitative). $\mathrm{R}_{f}=0.08(\mathrm{PE} / \mathrm{EA}, 5 / 1, \mathrm{v} / \mathrm{v}) . \mathrm{Mp}=165.5-166.4{ }^{\circ} \mathrm{C} ;[\alpha]_{\mathrm{D}}{ }^{24}=+41.0$ $\left(c=1.0, \mathrm{CHCl}_{3}\right)$. IR (film) 3408, 3064, 3032, 2865, 1454, 1400, 1364, 1249, 1097, 1052, 1027, 996, 909, 732, $697 \mathrm{~cm}^{-1} ;{ }^{1} \mathrm{H}$ NMR (500 MHz, $\left.\mathrm{CDCl}_{3}\right) \delta 7.57-$ $7.53(\mathrm{~m}, 3 \mathrm{H}$, aromatics), 7.43-7.29 (m, 12H, aromatics), 5.50 (s, 1H, CHbenzylidene), 5.38 (s, 1H, H-1), 4.93-4.86 (m, 1H, CH-a, 2-O-Bn), 4.82-4.76 (m, 2H, CH-a, CH-b, 3-O-Bn), 4.73-4.69 (m, 1H, CH-a, 2-O-Bn), $4.24\left(\mathrm{~d}, J_{6 \mathrm{a}, 6 \mathrm{~b}}=\right.$ $12.5 \mathrm{~Hz}, 1 \mathrm{H}, \mathrm{H}-6 \mathrm{a}), 4.23$ (s, 1H, H-4), 4.07 (dd, $\left.J_{1,2}=3.4, J_{2,3}=9.7 \mathrm{~Hz}, 1 \mathrm{H}, \mathrm{H}-2\right)$, $4.03\left(\mathrm{~d}, J_{6 \mathrm{a}, 6 \mathrm{~b}}=12.5 \mathrm{~Hz}, 1 \mathrm{H}, \mathrm{H}-6 \mathrm{~b}\right), 3.98\left(\mathrm{dd}, J_{3,4}=3.4, J_{2,3}=9.7 \mathrm{~Hz}, 1 \mathrm{H}, \mathrm{H}-3\right)$, 3.88 (s, 1H, H-5), 2.90 (s, 1H, 1-OH); ${ }^{13} \mathrm{C}$ NMR (125 MHz, $\left.\mathrm{CDCl}_{3}\right) \delta 138.5$, $138.2,137.8,128.9,128.7,128.6,128.5,128.45,128.39,128.21,128.18,128.14$, 128.1, 128.9, 128.8, 128.7, 126.3 (18 x CHarom), $101.1\left(\mathrm{PhCHO}_{2}\right), 92.2(\mathrm{C} 1)$, $75.73(\mathrm{C} 2), 75.71(\mathrm{C} 3), 74.3(\mathrm{C} 4), 73.9\left(2-\mathrm{OCH}_{2} \mathrm{Ph}\right), 71.8\left(3-\mathrm{OCH}_{2} \mathrm{Ph}\right), 69.5$ (C6), 62.8 (C5); HRMS(ESI) $\mathrm{m} / \mathrm{z}$ calcd. for $\left[\mathrm{C}_{27} \mathrm{H}_{28} \mathrm{O}_{6}+\mathrm{Na}\right]^{+}:$471.1778, obsd.: 471.1774 .

\section{2,3-di- $O$-benzyl-4,6-O-benzylidene- $\alpha$-D-galactosyl trichloroacetimidate (93)}

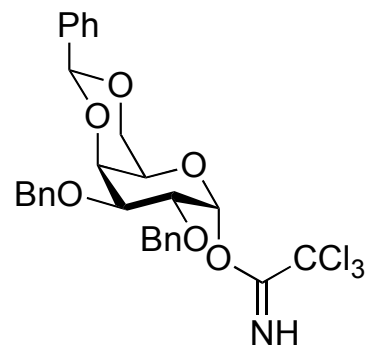

To a solution of lactol $97(240 \mathrm{mg}, 0.535 \mathrm{mmol}$, coevaporated 3 times dry toluene) in dry $\mathrm{CH}_{2} \mathrm{Cl}_{2}(5.4 \mathrm{~mL})$ was added trichloroacetonitrile $(537 \mu \mathrm{L}, 0.77 \mathrm{~g}, 5.35$ mmol) followed by 1,8-diazabicycloundec-7-ene $(120 \mu \mathrm{L}$, $0.12 \mathrm{~g}, 0.80 \mathrm{mmol}$ ) and the resulting brown solution

stirred $45 \mathrm{~min}$ at $\mathrm{rt}$. The solution was concentrated under reduced pressure and 
purified by gradient flash chromatography (Petroleum ether/EtOAc/NEt $3,95 / 5 / 1$ to $1 / 1 / 0, \mathrm{v} / \mathrm{v} / \mathrm{v})$ to give trichloroacetimidate 93 as a colourless oil $(273 \mathrm{mg}, 0.46$ mmol, 86\%). $\mathrm{R}_{f}=0.69(\mathrm{PE} / \mathrm{EA}, 1 / 2, \mathrm{v} / \mathrm{v}) ; \mathrm{Mp}=140.8-142.1{ }^{\circ} \mathrm{C} ;[\alpha]_{\mathrm{D}}{ }^{24}=+93.0$ $\left(c=1.0, \mathrm{CHCl}_{3}\right)$. IR (film) 3339, 3065, 2907, 2864, 1671, 1497, 1456, 1368, 1290, 1249, 1175, 1120, 1098, 1027, 968, 846, 794, 738, $697 \mathrm{~cm}^{-1} .{ }^{1} \mathrm{H}$ NMR (500

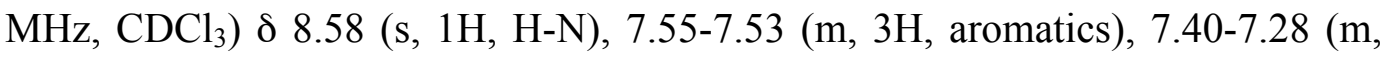
$15 \mathrm{H}$, aromatics), 6.65 (d, $\left.J_{1,2}=3.4 \mathrm{~Hz}, 1 \mathrm{H}, \mathrm{H}-1\right), 5.53$ (s, 1H, CH-benzylidene), $4.84\left(\mathrm{~d}, J_{\mathrm{a}, \mathrm{b}}=11.9 \mathrm{~Hz}, 1 \mathrm{H}, \mathrm{CH}-\mathrm{a}, 3-O-\mathrm{Bn}\right), 4.81\left(\mathrm{~d}, J_{\mathrm{a}, \mathrm{b}}=8.8 \mathrm{~Hz}, 1 \mathrm{H}, \mathrm{CH}-\mathrm{a}, 2-O-\right.$ $\mathrm{Bn}), 4.79\left(\mathrm{~d}, J_{\mathrm{a}, \mathrm{b}}=8.8 \mathrm{~Hz}, 1 \mathrm{H}, \mathrm{CH}-\mathrm{b}, 2-O-\mathrm{Bn}\right), 4.77$ (d, $J_{\mathrm{a}, \mathrm{b}}=11.9 \mathrm{~Hz}, 1 \mathrm{H}, \mathrm{CH}-\mathrm{b}$, 3-O-Bn), 4.31-4.27 (m, 3H, H-2, H-4 and H-6a), 4.09 (dd, $J_{3,4}=3.5, J_{2,3}=10.0$ $\mathrm{Hz}, 1 \mathrm{H}, \mathrm{H}-3), 4.02$ (dd, $\left.J_{5,6 \mathrm{~b}}=1.5, J_{6 \mathrm{a}, 6 \mathrm{~b}}=12.7 \mathrm{~Hz}, 1 \mathrm{H}, \mathrm{H}-6 \mathrm{~b}\right), 3.85$ (s, 1H, H-5); ${ }^{13} \mathrm{C}$ NMR $\left(125 \mathrm{MHz}, \mathrm{CDCl}_{3}\right) \delta 161.0(\underline{\mathrm{C}}=\mathrm{NH}), 138.4,138.3,137.6,129.0,128.3$, $128.26,128.2,128.0,127.7,127.5,127.4,126.4$ (18 x $\left.\mathrm{CH}_{\text {arom }}\right), 101.1(\mathrm{CH}-$ benzylidene), $95.6(\mathrm{C} 1), 91.4\left(\mathrm{CCl}_{3}\right), 75.1$ (C2), 74.7 (C3), $74.5(\mathrm{C} 4), 73.1$ (2$\left.\mathrm{OCH}_{2} \mathrm{Ph}\right), 72.2\left(3-\mathrm{OCH}_{2} \mathrm{Ph}\right), 69.1$ (C6), 65.3 (C5); HRMS(ESI) m/z calcd. for $\left[\mathrm{C}_{29} \mathrm{H}_{28} \mathrm{NO}_{6} \mathrm{Cl}_{3}+\mathrm{Na}\right]^{+}:$614.0874, obsd.: 614.0872 .

(2S,3R,4E)-2-Azido-1-(4- $O$-(2,6-di- $O$-benzoyl- $\beta$-D-galactopyranosyl)-2,3,6-tri$O$-benzoyl- $\beta$-D-glucopyranosyloxy)-3-benzyloxy-octadec-4-ene (98)

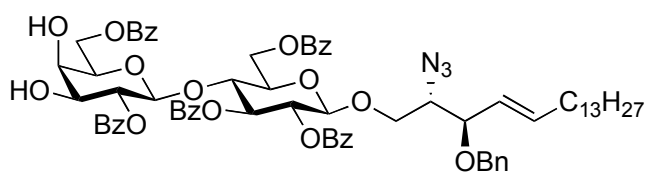

To a solution of lactosyl 2-azidsphingosine 74 (144 $\mathrm{mg}, 0.11 \mathrm{mmol})$ in $\mathrm{CH}_{2} \mathrm{Cl}_{2}(4 \mathrm{~mL})$ was added TFA $/ \mathrm{H}_{2} \mathrm{O}(1 / 1$, $\mathrm{v} / \mathrm{v}, 0.4 \mathrm{~mL}$ ) and the resulting solution was stirred at room temperature for $20 \mathrm{~h}$. The solution was diluted with EtOAc and the organic layer was then washed with sat. aq. $\mathrm{NaHCO}_{3}(\mathrm{x} 3)$ and brine, dried $\left(\mathrm{MgSO}_{4}\right)$, filtered and concentrated under reduced pressure. The colourless oil was then purified by gradient flash chromatography (petroleum ether/EtOAc, 5/1 to 1/1, v/v) to give diol $\mathbf{9 8}$ as a colourless oil (139 mg, $0.11 \mathrm{mmol}, 99 \%)$. $\mathrm{R}_{f}: 0.46(\mathrm{PE} / \mathrm{EA}, 1 / 1, \mathrm{v} / \mathrm{v}) ;[\alpha]_{\mathrm{D}}{ }^{21}=$ $+17.0^{\circ}$ (c = 1.0, $\mathrm{CHCl}_{3}$ ); IR (film) 3448, 3066, 2925, 2854, 2102, 1720, 1602, $1585,1452,1315,1270,1177,1113,1095,1069,1028,976,756,708 \mathrm{~cm}^{-1} ;{ }^{1} \mathrm{H}$ NMR (500 MHz, $\left.\mathrm{CDCl}_{3}\right) \delta 8.07\left(\mathrm{~d}, J_{\mathrm{CH}-o, \mathrm{CH}-m}=7.8 \mathrm{~Hz}, 2 \mathrm{H}, \mathrm{CH}-o, 2^{\prime \prime}-O-\mathrm{Bz}\right)$, 8.02-8.00 (m, 4H, 2 x CH-o, OBz), 7.97 (d, $\left.J_{\mathrm{CH}-o, \mathrm{CH}-m}=7.8 \mathrm{~Hz}, 2 \mathrm{H}, \mathrm{CH}-o, \mathrm{OBz}\right)$, $7.93\left(\mathrm{~d}, J_{\mathrm{CH}-o, \mathrm{CH}-m}=7.8 \mathrm{~Hz}, 2 \mathrm{H}, \mathrm{CH}-o, 2^{\prime}-O-\mathrm{Bz}\right), 7.61-7.19\left(\mathrm{~m}, 20 \mathrm{H}, \mathrm{H}_{\text {arom }}\right), 5.67$ 
(t, $\left.J_{2^{\prime}, 3^{\prime}}=J_{3^{\prime}, 4^{\prime}}=9.4 \mathrm{~Hz}, 1 \mathrm{H}, \mathrm{H}-3^{\prime}\right), 5.46-5.39\left(\mathrm{~m}, 2 \mathrm{H}, \mathrm{H}-2^{\prime}, \mathrm{H}-5\right), 5.30-5.23(\mathrm{~m}$, $\left.2 \mathrm{H}, \mathrm{H}-2^{\prime \prime}, \mathrm{H}-4\right), 4.66$ (d, $\left.J_{1^{\prime}, 2^{\prime}}=7.8 \mathrm{~Hz}, 1 \mathrm{H}, \mathrm{H}-1^{\prime}\right), 4.60$ (d, $J_{1^{\prime \prime}, 2^{\prime \prime}}=7.8 \mathrm{~Hz}, 1 \mathrm{H}$, $\left.\mathrm{H}-1^{\prime \prime}\right), 4.59-4.58\left(\mathrm{~m}, 1 \mathrm{H}, \mathrm{H}-6^{\prime} \mathrm{a}\right), 4.52\left(\mathrm{dd}, J_{6^{\prime} \mathrm{a}, 6^{\prime} \mathrm{b}}=12.0 \mathrm{~Hz}, J_{5^{\prime}, 6^{\prime} \mathrm{b}}=4.4 \mathrm{~Hz}, 1 \mathrm{H}\right.$, H-6’b), 4.40 (d, $\left.J_{\mathrm{a}, \mathrm{b}}=11.8 \mathrm{~Hz}, \mathrm{CH}-\mathrm{a}, 3-\mathrm{O}-\mathrm{Bn}\right), 4.19-4.14$ (m, 2H, H-4', CH-b, 3$O-\mathrm{Bn}), 4.04\left(\mathrm{dd}, J_{6^{\prime \prime}, 6^{\prime \prime} \mathrm{b}}=11.4 \mathrm{~Hz}, J_{5^{\prime \prime}, 6^{\prime \prime}}=6.7 \mathrm{~Hz}, 1 \mathrm{H}, \mathrm{H}-6^{\prime \prime}{ }^{\prime} \mathrm{a}\right), 3.90$ (dd, $J_{1 \mathrm{a}, 1 \mathrm{~b}}=$ $\left.10.2 \mathrm{~Hz}, J_{1 \mathrm{a}, 2}=5.8 \mathrm{~Hz}, 1 \mathrm{H}, \mathrm{H}-1 \mathrm{a}\right), 3.82-3.79\left(\mathrm{~m}, 1 \mathrm{H}, \mathrm{H}-5^{\prime}\right), 3.77$ (d, $J_{3^{\prime \prime}, 4^{\prime \prime}}=3.3$ $\mathrm{Hz}, 1 \mathrm{H}, \mathrm{H}-4^{\prime \prime}$ ), 3.72 (dd, $\left.J_{3,4}=8.5 \mathrm{~Hz}, J_{2,3}=5.6 \mathrm{~Hz}, 1 \mathrm{H}, \mathrm{H}-3\right), 3.66$ (dd, $J_{2^{\prime \prime}, 3^{\prime \prime}}=$ $\left.9.8 \mathrm{~Hz}, J_{3^{\prime \prime}, 4^{\prime \prime}}=3.3 \mathrm{~Hz}, 1 \mathrm{H}, \mathrm{H}-3^{\prime \prime}\right), 3.60-3.56$ (m, 2H, H-2, H-6" 'b), 3.51 (dd, $\left.J_{1 \mathrm{a}, 1 \mathrm{~b}}=10.2 \mathrm{~Hz}, J_{1 \mathrm{~b}, 2}=5.4 \mathrm{~Hz}, 1 \mathrm{H}, \mathrm{H}-1 \mathrm{~b}\right), 3.46$ (t, $\left.J_{5,6}=6.7 \mathrm{~Hz}, 1 \mathrm{H}, \mathrm{H}-5^{\prime \prime}\right), 1.93-$ 1.90 (m, 2H, H-6), 1.31-1.25 (m, 22H, H-7-H-17), 0.88 (t, $J_{17,18}=7.0$ Hz, 3H, H18); ${ }^{13} \mathrm{C}$ NMR $\left(125 \mathrm{MHz}, \mathrm{CDCl}_{3}\right) \delta 166.5\left(\mathrm{C}=\mathrm{O}, 2{ }^{\prime \prime}-O-\mathrm{Bn}\right), 166.2\left(\mathrm{C}=\mathrm{O}, 6{ }^{\prime \prime}-O-\right.$ $\mathrm{Bn}), 166.1\left(\mathrm{C}=\mathrm{O}, 6^{\prime}-O-\mathrm{Bn}\right), 166.0\left(\mathrm{C}=\mathrm{O}, 3^{\prime}-O-\mathrm{Bn}\right), 165.2\left(\mathrm{C}=\mathrm{O}, 2^{\prime}-O-\mathrm{Bn}\right), 138.4$ (C-5), 138.2 (C-i, 3-O-Bn), 133.6, 133.5, 133.4, 133.3 (C-p, 5 x OBz), 130.06, $129.95,129.85,129.79,129.76,129.70,129.68,129.66,129.42,129.22,128.73$, 128.62, 128.49, 128.39, 127.58 (30 x $\mathrm{CH}_{\text {arom }}$ ), 125.6 (C-4), 101.0 (C-1', C-1' '), 79.7 (C-3), 76.2 (C-4'), 73.8 (C-2' '), 73.1 (C-3', C-5'), 72.7 (C-3'”, C-5' ), 71.7 $\left(\mathrm{C}-2^{\prime}\right), 70.1\left(\mathrm{CH}_{2}, 3-O-\mathrm{Bn}\right), 68.6\left(\mathrm{C}-1, \mathrm{C}-4^{\prime \prime}\right), 63.9$ (C-2), 62.7 (C-6'), $61.9(\mathrm{C}-$ $6^{\prime \prime}$ ), 32.4 (C-6), 32.0, 29.83, 29.81, 29.80, 29.79, 29.76, 29.55, 29.49, 29.29, 29.03, 22.8 (C-7-C-17), $14.3 \quad$ (C-18); HRMS(ESI) $\mathrm{m} / \mathrm{z}$ calcd. for $\left[\mathrm{C}_{72} \mathrm{H}_{81} \mathrm{~N}_{3} \mathrm{O}_{17}+\mathrm{Na}\right]^{+}:$1282.5458, obsd.: 1282.5453 .

(2S,3R,4E)-1-(4-O-(4-O-Acetyl-2,6-di- $O$-benzoyl- $\beta$-D-galactopyranosyl)-2,3,6tri- $O$-benzoyl- $\beta$-D-glucopyranosyloxy)-2-azido-3-benzyloxy-octadec-4-ene (94)

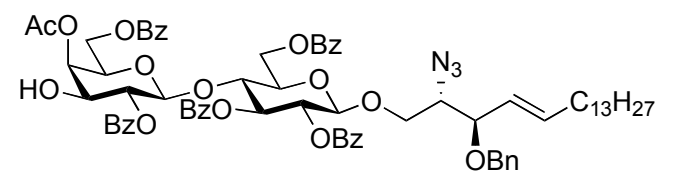

Diol 98 (62 mg, $0.049 \mathrm{mmol})$ was coevaporated with toluene (x3) and dissolved in dry $\mathrm{CH}_{2} \mathrm{Cl}_{2}(0.8 \mathrm{~mL})$.

Trimethyl orthoacetate $(19 \mu \mathrm{L}, 0.15 \mathrm{mmol})$ and CSA $(5.7 \mathrm{mg}, 0.025 \mathrm{mmol})$ were added and the reaction mixture was stirred at $\mathrm{rt}$ for $5 \mathrm{~h}$. The reaction mixture was diluted with EtOAc (30 mL), washed with $1 \mathrm{M} \mathrm{HCl}\left(30 \mathrm{~mL}\right.$ x 3), sat. aq. $\mathrm{NaHCO}_{3}$ $(30 \mathrm{~mL})$ and brine $(30 \mathrm{~mL})$, dried $\left(\mathrm{MgSO}_{4}\right)$, filtered and concentrated in vacuo. The residue was purified by gradient flash chromatography (petroleum ether/EtOAc, 10:1 to 3:1, v/v) to afford acetate 94 as a clear oil (59 mg, 0.046 
mmol, 94\%). Rf: $0.63(\mathrm{PE} / \mathrm{EA}, 1 / 1, \mathrm{v} / \mathrm{v}) ;[\alpha]_{\mathrm{D}}^{25}=+2.0^{\circ}\left(\mathrm{c}=1.0, \mathrm{CHCl}_{3}\right)$; IR (film) 3475,2102 , 1727, 1602, 1452, 1371, 1315, 1269, 1177, 1110, 1095, 1070, 1028, 976, $709 \mathrm{~cm}^{-1} ;{ }^{1} \mathrm{H}$ NMR $\left(500 \mathrm{MHz}, \mathrm{CDCl}_{3}\right) \delta 8.06\left(\mathrm{~d}, J_{\mathrm{CH}-o, \mathrm{CH}-m}=8.3 \mathrm{~Hz}, 2 \mathrm{H}\right.$, $\left.\mathrm{CH}-o, 2^{\prime \prime}-O-\mathrm{Bz}\right), 8.04-8.02$ (m, 4H, 2 x CH-o, 3'-O-Bz, 6"' $\left.O-\mathrm{Bz}\right), 7.98$ (d, $J_{\mathrm{CH}-}$ $\left.o, \mathrm{CH}-m=7.8 \mathrm{~Hz}, 2 \mathrm{H}, \mathrm{CH}-o, 6^{\prime}-O-\mathrm{Bz}\right), 7.95\left(\mathrm{~d}, J_{\mathrm{CH}-o, \mathrm{CH}-m}=7.8 \mathrm{~Hz}, 2 \mathrm{H}, \mathrm{CH}-o, 2^{\prime}-O-\right.$ Bz), 7.62-7.19 (m, 20H, Harom), $5.72\left(\mathrm{t}, J_{2^{\prime}, 3^{\prime}}=J_{3^{\prime}, 4^{\prime}}=9.6 \mathrm{~Hz}, 1 \mathrm{H}, \mathrm{H}-3^{\prime}\right), 5.46(\mathrm{dd}$, $\left.J_{2^{\prime}, 3^{\prime}}=9.6 \mathrm{~Hz}, J_{1^{\prime}, 2^{\prime}}=7.9 \mathrm{~Hz}, 1 \mathrm{H}, \mathrm{H}-2^{\prime}\right), 5.41\left(\mathrm{dt}, J_{4,5}=15.7 \mathrm{~Hz}, J_{5,6}=6.6 \mathrm{~Hz}, 1 \mathrm{H}\right.$, $\mathrm{H}-5), 5.26\left(\mathrm{dd}, J_{4,5}=15.7 \mathrm{~Hz}, J_{3,4}=8.6 \mathrm{~Hz}, 1 \mathrm{H}, \mathrm{H}-4\right), 5.21\left(\mathrm{~d}, J_{3^{\prime \prime}, 4^{\prime \prime}}=3.4 \mathrm{~Hz}, 1 \mathrm{H}\right.$, $\left.\mathrm{H}-4^{\prime \prime}\right), 5.17\left(\mathrm{dd}, J_{2^{\prime \prime}, 3^{\prime \prime}}=9.8 \mathrm{~Hz}, J_{1^{\prime \prime}, 2^{\prime \prime}}=8.0 \mathrm{~Hz}, 1 \mathrm{H}, \mathrm{H}-2^{\prime \prime}\right), 4.69$ (d, $J_{1^{\prime}, 2^{\prime}}=7.9$ $\left.\mathrm{Hz}, 1 \mathrm{H}, \mathrm{H}-1^{\prime}\right), 4.65$ (d, $\left.J_{1^{\prime \prime}, 2^{\prime \prime}}=8.0 \mathrm{~Hz}, 1 \mathrm{H}, \mathrm{H}-1^{\prime \prime}\right), 4.62$ (d, $J_{6^{\prime} \mathrm{a}, 6^{\prime} \mathrm{b}}=12.0 \mathrm{~Hz}, 1 \mathrm{H}$, H-6'a), $4.53\left(\mathrm{dd}, J_{6^{\prime} \mathrm{a}, 6^{\prime} \mathrm{b}}=12.0 \mathrm{~Hz}, J_{5,6^{\prime} \mathrm{b}}=4.3 \mathrm{~Hz}, 1 \mathrm{H}, \mathrm{H}-6^{\prime} \mathrm{b}\right), 4.41\left(\mathrm{~d}, J_{\mathrm{a}, \mathrm{b}}=11.7\right.$ $\mathrm{Hz}, 1 \mathrm{H}, \mathrm{CH}-\mathrm{a}, 3-O-\mathrm{Bn}), 4.21\left(\mathrm{t}, J_{3^{\prime}, 4^{\prime}}=9.6 \mathrm{~Hz}, 1 \mathrm{H}, \mathrm{H}^{-} 4^{\prime}\right), 4.15\left(\mathrm{~d}, J_{\mathrm{a}, \mathrm{b}}=11.7 \mathrm{~Hz}\right.$, $1 \mathrm{H}, \mathrm{CH}-\mathrm{b}, 3-O-\mathrm{Bn}), 3.92\left(\mathrm{dd}, J_{1 \mathrm{a}, 1 \mathrm{~b}}=10.0 \mathrm{~Hz}, J_{1 \mathrm{a}, 2}=5.9 \mathrm{~Hz}, 1 \mathrm{H}, \mathrm{H}-1 \mathrm{a}\right), 3.83-$ 3.81 (m, 2H, H-5', H-3' ) , 3.76-3.72 (m, 2H, H-3, H-6"'a), 3.61-3.56 (m, 2H, H2, H-5' ), 3.55-3.50 (m, 2H, H-6" b, H-1b), 2.01 (s, 3H, OAc), 1.98-1.91 (m, 2H, $\mathrm{H}-6), 1.32-1.21$ (m, 22H, H-7-H-17), 0.88 (t, $\left.J_{17,18}=6.9 \mathrm{~Hz}, 3 \mathrm{H}, \mathrm{H}-18\right) ;{ }^{13} \mathrm{C}$ NMR (125 MHz, $\left.\mathrm{CDCl}_{3}\right) \delta 170.7(\mathrm{C}=\mathrm{O}, \mathrm{OAc}), 166.6\left(\mathrm{C}=\mathrm{O}, 2^{\prime \prime}-O-\mathrm{Bn}\right), 166.1$ $\left(\mathrm{C}=\mathrm{O}, 6^{\prime}-O-\mathrm{Bn}\right), 165.8\left(\mathrm{C}=\mathrm{O}, 6^{\prime \prime}-O-\mathrm{Bn}\right), 165.5\left(\mathrm{C}=\mathrm{O}, 3^{\prime}-O-\mathrm{Bn}\right), 165.1\left(\mathrm{C}=\mathrm{O}, 2^{\prime}-\right.$ $O$-Bn), 138.4 (C-5), 138.2 (C-i, 3-O-Bn), 133.7, 133.6, 133.5, 133.4, 133.3 (C-p, 5 x OBz), 130.02, 130.30, 129.88, 129.74, 129.67, 129.65, 129.60, 129.43, $129.02,128.80,128.71,128.70,128.50,128.40,128.37,127.58\left(30 \times \mathrm{CH}_{\text {arom }}\right)$, 125.6 (C-4), $101.2\left(\mathrm{C}-1^{\prime}\right), 100.5$ (C-1' ), 79.7 (C-3), 75.6 (C-4'), 73.6 (C-2')), 73.1 (C-5'), 72.8 (C-3'), 71.8 (C-2'), $71.7\left(\mathrm{C}-3^{\prime \prime}\right), 71.3\left(\mathrm{C}-5^{\prime \prime}\right), 70.1\left(\mathrm{CH}_{2}, 3-O-\right.$ Bn), 69.4 (C-4" ), 68.7 (C-1), 63.9 (C-2), 62.7 (C-6'), 61.3 (C-6"'), 32.4 (C-6), 32.0, 29.83, 29.81, 29.80, 29.79, 29.76, 29.55, 29.49, 29.29, 29.03, 22.8 (C-7-C17), 20.7 (OAc), 14.3 (C-18); HRMS(ESI) m/z calcd. for $\left[\mathrm{C}_{72} \mathrm{H}_{83} \mathrm{~N}_{3} \mathrm{O}_{18}+\mathrm{NH}_{4}\right]^{+}$: 1319.6010, obsd.: 1319.5966 . 
(2S,3R,4E)-1-(4-O-(4-O-Acetyl-2,6-di- $O$-benzoyl-3-O-(2,3-di- $O$-benzyl-4,6-Obenzylidene- $\alpha$-D-galactopyranosyl)- $\beta$-D-galactopyranosyl)-2,3,6-tri- $O$ benzoyl- $\beta$-D-glucopyranosyloxy)-2-azido-3-benzyloxy-octadec-4-ene (92)

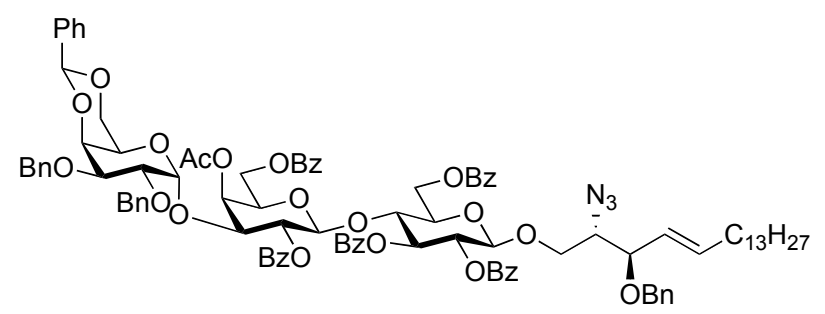

Galactosyl imidate donor 93 (165 $\mathrm{mg}, 0.278 \mathrm{mmol}$ ) and lactosyl 2-azidosphingosine acceptor 94 (145 mg, $0.111 \mathrm{mmol})$ were co-evaporated with toluene (x3), dissolved in dry $\mathrm{CH}_{2} \mathrm{Cl}_{2}(1 \mathrm{~mL})$ and stirred with activated $4 \AA$ molecular sieves for 30 mins. The reaction mixture was cooled to $-20{ }^{\circ} \mathrm{C}$, and a solution of TMSOTf in $\mathrm{CH}_{2} \mathrm{Cl}_{2}(0.55 \mathrm{mmol} / \mathrm{mL}, 50 \mu \mathrm{L}, 0.027 \mathrm{mmol})$ was added and the mixture stirred at $0{ }^{\circ} \mathrm{C}$ for $2 \mathrm{~h}$. The reaction was warmed to $\mathrm{rt}$ and stirred for another 30 mins, upon which it was diluted with EtOAc $(30 \mathrm{~mL})$, washed with sat. aq. $\mathrm{NaHCO}_{3}(30 \mathrm{~mL})$ and brine $(30 \mathrm{~mL})$, dried $\left(\mathrm{MgSO}_{4}\right)$, filtered and concentrated in vacuo. The resultant oil was purified by gradient flash chromatography (petroleum ether/EtOAc, 10:1 to $4: 1, \mathrm{v} / \mathrm{v}$ ) to afford the fully protected triglycosyl ceramide 92 as a clear oil (109 mg, $0.0629 \mathrm{mmol}, 56 \%)$. $\mathrm{R}_{f}$ : $0.82(\mathrm{PE} / \mathrm{EA}, 1 / 1, \mathrm{v} / \mathrm{v}) ;[\alpha]_{\mathrm{D}}{ }^{26}=+58.0^{\circ}\left(\mathrm{c}=1.0, \mathrm{CHCl}_{3}\right)$; IR (film) 3089, 3065, 2925, 2854, 2102, 1731, 1602, 1585, 1452, 1364, 1315, 1268, 1177, 1097, 1069, 1028, 979, 755, $710 \mathrm{~cm}^{-1}$; ${ }^{1} \mathrm{H}$ NMR $\left(500 \mathrm{MHz}, \mathrm{CDCl}_{3}\right) \delta 8.05-7.94(\mathrm{~m}, 10 \mathrm{H}, 5 \mathrm{x}$ $\left.\mathrm{CH}-o, 2^{\prime}-O-\mathrm{Bz}, 3^{\prime}-O-\mathrm{Bz}, 6^{\prime}-O-\mathrm{Bz}, 2^{\prime \prime}-O-\mathrm{Bz}, 6^{\prime \prime}-O-\mathrm{Bz}\right), 7.63-7.19$ (m, 35H, $\left.\mathrm{H}_{\text {arom }}\right), 5.73\left(\mathrm{t}, J_{2^{\prime}, 3^{\prime}}=J_{3^{\prime}, 4^{\prime}}=9.4 \mathrm{~Hz}, 1 \mathrm{H}, \mathrm{H}-3^{\prime}\right), 5.45\left(\mathrm{dd}, J_{2^{\prime}, 3^{\prime}}=9.4 \mathrm{~Hz}, J_{1^{\prime}, 2^{\prime}}=8.0\right.$ $\mathrm{Hz}, 1 \mathrm{H}, \mathrm{H}-2^{\prime}$ ), 5.43-5.38 (m, 2H, H-5, H-2' $), 5.35$ (d, $J_{3^{\prime \prime}, 4^{\prime \prime}}=3.1 \mathrm{~Hz}, 1 \mathrm{H}, \mathrm{H}-$ $\left.4^{\prime \prime}\right), 5.26\left(\mathrm{dd}, J_{4,5}=15.4 \mathrm{~Hz}, J_{3,4}=8.6 \mathrm{~Hz}, 1 \mathrm{H}, \mathrm{H}-4\right), 5.15\left(\mathrm{~s}, 1 \mathrm{H}, \mathrm{PhCHO}_{2}\right), 5.03$ $\left(\mathrm{d}, J_{4,5}=3.2 \mathrm{~Hz}, 1 \mathrm{H}, \mathrm{H}-1^{\prime \prime \prime}\right), 4.68\left(\mathrm{~d}, J_{1^{\prime}, 2^{\prime}}=8.0 \mathrm{~Hz}, 1 \mathrm{H}, \mathrm{H}-1^{\prime}\right), 4.65\left(\mathrm{~d}, J_{\mathrm{a}, \mathrm{b}}=\right.$ $\left.11.5 \mathrm{~Hz}, 1 \mathrm{H}, \mathrm{CH}-\mathrm{a}, 2^{\prime \prime \prime}-\mathrm{O}-\mathrm{Bn}\right), 4.64$ (d, $\left.J_{\mathrm{a}, \mathrm{b}}=12.2 \mathrm{~Hz}, 1 \mathrm{H}, \mathrm{CH}-\mathrm{a}, 3^{\prime \prime \prime}-O-\mathrm{Bn}\right)$, $4.59\left(\mathrm{~d}, J_{1^{\prime \prime}, 2^{\prime \prime}}=8.0 \mathrm{~Hz}, 1 \mathrm{H}, \mathrm{H}-1^{\prime \prime}\right), 4.55$ (d, $\left.J_{\mathrm{a}, \mathrm{b}}=11.5 \mathrm{~Hz}, 1 \mathrm{H}, \mathrm{CH}-\mathrm{b}, 2^{\prime \prime \prime}-O-\mathrm{Bn}\right)$, $4.52-4.50$ (m, 3H, H-6'a, H-6'b, CH-b, 3'"'-O-Bn), 4.40 (d, $J_{\mathrm{a}, \mathrm{b}}=11.7 \mathrm{~Hz}, 1 \mathrm{H}$, CH-a, 3-O-Bn), 4.20-4.14 (m, 2H, H-4', CH-b, 3-O-Bn), 3.92 (dd, $J_{1 \mathrm{a}, 1 \mathrm{~b}}=10.2$ 
$\left.\mathrm{Hz}, J_{1 \mathrm{a}, 2}=5.8 \mathrm{~Hz}, 1 \mathrm{H}, \mathrm{H}-1 \mathrm{a}\right), 3.90-3.88$ (m, 2H, H-2"', $\left.\mathrm{H}-6{ }^{\prime \prime \prime} \mathrm{a}\right), 3.81-3.78$ (m, 2H, H-5', H-3' ), 3.73 (dd, $\left.J_{3,4}=8.6 \mathrm{~Hz}, J_{2,3}=5.6 \mathrm{~Hz}, 1 \mathrm{H}, \mathrm{H}-3\right), 3.67-3.64$ (m, $2 \mathrm{H}, \mathrm{H}-6$ "'a, H-3"') 3.59 (q, $\left.J_{1,2}=J_{2,3}=5.5 \mathrm{~Hz}, 1 \mathrm{H}, \mathrm{H}-2\right), 3.55-3.49$ (m, 3H, H-

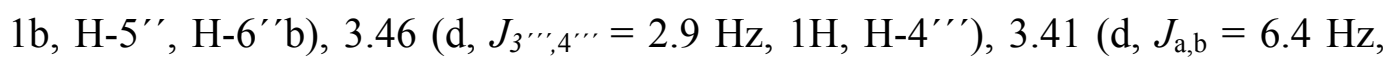
$1 \mathrm{H}, \mathrm{H}-6$ "'b), 3.29 (s, 1H, H-5"'), 1.93-1.90 (2H, H-6"), 1.63 (s, 3H, OAc), $1.26-0.92(\mathrm{~m}, 22 \mathrm{H}, \mathrm{H}-7-\mathrm{H}-17), 0.89$ (t, $\left.J_{17,18}=6.9 \mathrm{~Hz}, 3 \mathrm{H}, \mathrm{H}-18\right) ;{ }^{13} \mathrm{C}$ NMR $(125$ $\mathrm{MHz}^{\left.\mathrm{CDCl}_{3}\right)} \delta 170.1(\mathrm{C}=\mathrm{O}, \mathrm{OAc}), 166.1\left(\mathrm{C}=\mathrm{O}, 6^{\prime}-O-\mathrm{Bn}\right), 165.8\left(\mathrm{C}=\mathrm{O}, 6^{\prime \prime}-O-\right.$ $\mathrm{Bn}), 165.5\left(\mathrm{C}=\mathrm{O}, 3^{\prime}-O-\mathrm{Bn}\right), 165.2\left(\mathrm{C}=\mathrm{O}, 2^{\prime}-O-\mathrm{Bn}\right), 164.4\left(\mathrm{C}=\mathrm{O}, 2^{\prime \prime}-O-\mathrm{Bn}\right)$, 138.9, 138.7 (C-i, 2"' -O-Bn, 3"' -O-Bn), 138.4 (C-5), 138.2 (C-i, 3-O-Bn), 137.8 (C-i, benzylidene), 133.7, 133.58, 133.55, 133.4, 133.3 (C-p, 5 x OBz), 129.97, 129.86, 129.77, 129.70, 129.68, 129.64, 129.55, 129.44, 129.18, 129.00, 128.90, $128.80,218.74,128.51,128.39,128.33,128.26,128.21,128.19,127.72,127.59$, 127.57, 127.57, 127.50 (43 x $\mathrm{CH}_{\text {arom}}$ ), 126.4 (C-o, benzylidene), 125.5 (C-4), 101.2 (C-1'), 101.0 (C-1" , CH-benzylidene), 95.4 (C-1"'), 79.7 (C-3), 76.0 (C3'”), 75.6 (C-4'), 74.7 (C-2'"'), $74.4\left(\mathrm{C}-4^{\prime \prime \prime}\right), 73.9\left(\mathrm{CH}_{2}, 2^{\prime \prime \prime}-\mathrm{O}-\mathrm{Bn}\right), 73.3$ (C3') 73.1 (C-5'), 72.7 (C-3'), $72.3\left(\mathrm{CH}_{2}, 3^{\prime \prime \prime}-\mathrm{O}-\mathrm{Bn}\right), 71.8$ (C-2'), $71.5\left(\mathrm{C}-5^{\prime \prime}\right)$, 71.1 (C-2'), $70.1\left(\mathrm{CH}_{2}, 3-\mathrm{O}-\mathrm{Bn}\right), 68.9\left(\mathrm{C}-6^{\prime \prime \prime}\right), 68.7(\mathrm{C}-1), 64.6\left(\mathrm{C}-4{ }^{\prime \prime}\right), 63.9(\mathrm{C}-$ 2), 62.9 (C-5"'”), 62.6 (C-6'), 61.3 (C-6"'), 32.4 (C-6), 32.06, 29.83, 29.82, 29.80, 29.79, 29.76, 29.55, 29.49, 29.29, 29.04, 22.83 (C-7-C-17), 20.3 (OAc), 14.3 (C18); HRMS(ESI) $\mathrm{m} / \mathrm{z}$ calcd. for $\left[\mathrm{C}_{101} \mathrm{H}_{109} \mathrm{~N}_{3} \mathrm{O}_{23}+\mathrm{NH}_{4}\right]^{+}:$1749.7790, obsd. 1749.7802. 
(2S,3R,4E)-1-(4-O-(4-O-Acetyl-2,6-di- $O$-benzoyl-3-O-(2,3-di- $O$-benzyl-4,6-Obenzylidene- $\alpha$-D-galactopyranosyl)- $\beta$-D-galactopyranosyl)-2,3,6-tri- $O$ benzoyl- $\beta$-D-glucopyranosyloxy)-3-benzyloxy-2-dodecanoylamido-octadec-4ene (100)

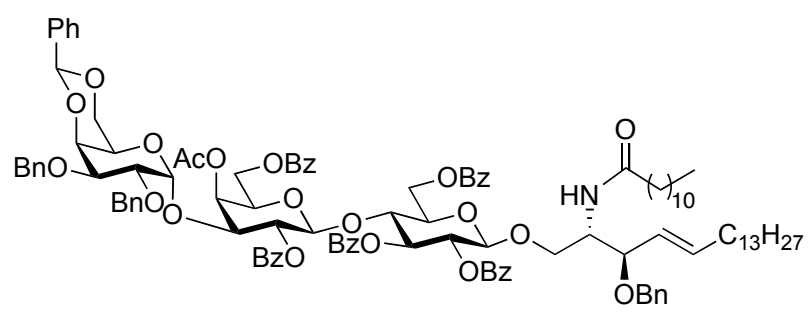

To a solution of glycolipid azide $92(68 \mathrm{mg}, 0.039 \mathrm{mmol})$ in dry benzene $(0.8 \mathrm{~mL})$ triphenylphosphine $(21 \mathrm{mg}, 0.078 \mathrm{mmol})$, and distilled water $(30 \mu \mathrm{L})$ were added, the solution was warmed to $45^{\circ} \mathrm{C}$ and stirred overnight. The reaction mixture was then cooled to room temperature, diluted with EtOAc $(20 \mathrm{~mL})$, washed with sat. aq. $\mathrm{NH}_{4} \mathrm{Cl},(5 \mathrm{~mL})$, dried $\left(\mathrm{MgSO}_{4}\right)$, filtered and concentrated under reduced pressure to give a colourless oil which was used without further purification. Half of the amine intermediate obtained was coupled to the $\mathrm{C} 12$ fatty acid as described below.

The oil was co-evaporated twice with dry toluene then suspended in DCM (1 mL), EDCI (11.3 mg, $0.0589 \mathrm{mmol})$, DMAP (9.6mg, $0.0785 \mathrm{mmol})$, and lauric acid (11.8 $\mathrm{mg}, 0.0589 \mathrm{mmol})$ were added and the resulting solution stirred over 4 days at room temperatur, after which it was diluted with EtOAc $(30 \mathrm{~mL})$, washed with sat. aq. $\mathrm{NaHCO}_{3}(30 \mathrm{~mL})$ and brine $(30 \mathrm{~mL})$, dried $\left(\mathrm{MgSO}_{4}\right)$, filtered and concentrated in vacuo. The reaction mixture was then purified directly by gradient flash chromatography (petroleum ether/EtOAc, 10/1 to 2/1, v/v) to give $\mathbf{1 0 0}$ as a colourless oil (28 mg, $0.015 \mathrm{mmol}, 75 \%$ over two steps). $\mathrm{R}_{f}: 0.68$ (PE/EA, 1/1, $\mathrm{v} / \mathrm{v}) ;[\alpha]_{\mathrm{D}}{ }^{24}=+62.0^{\circ}\left(\mathrm{c}=0.1, \mathrm{CHCl}_{3}\right) ;$ IR (film) 2924, 2854, 1733, 1718, 1576, 1558, 1541, 1473, 1177, 1098, 1070, 1028, 756, 633, $620 \mathrm{~cm}^{-1} ;{ }^{1} \mathrm{H}$ NMR $(500$ $\left.\mathrm{MHz} \mathrm{CDCl}_{3}\right) \delta 8.04-7.93\left(\mathrm{~m}, 10 \mathrm{H}, 5\right.$ x CH-o, 2' $-O-\mathrm{Bz}, 3^{\prime}-O-\mathrm{Bz}, 6^{\prime}-O-\mathrm{Bz}, 2^{\prime \prime}-O-$ $\left.\mathrm{Bz}, 6^{\prime \prime}-O-\mathrm{Bz}\right), 7.56-7.18\left(\mathrm{~m}, 35 \mathrm{H}, \mathrm{H}_{\mathrm{arom}}\right), 5.74\left(\mathrm{t}, J_{2^{\prime}, 3^{\prime}}=J_{3^{\prime}, 4^{\prime}}=9.6 \mathrm{~Hz}, 1 \mathrm{H}, \mathrm{H}-\right.$ $\left.3^{\prime}\right), 5.49\left(\mathrm{dt}, J_{4,5}=15.2 \mathrm{~Hz}, J_{5,6}=7.0 \mathrm{~Hz}, 1 \mathrm{H}, \mathrm{H}-5\right), 5.42-5.38$ (m, 3H, H-2', H- 
$\left.2^{\prime \prime}, \mathrm{NH}\right), 5.36\left(\mathrm{~m}, 1 \mathrm{H}, \mathrm{H}-4^{\prime \prime}\right), 5.24\left(\mathrm{dd}, J_{4,5}=15.2 \mathrm{~Hz}, J_{3,4}=8.8 \mathrm{~Hz}, 1 \mathrm{H}, \mathrm{H}-4\right)$,

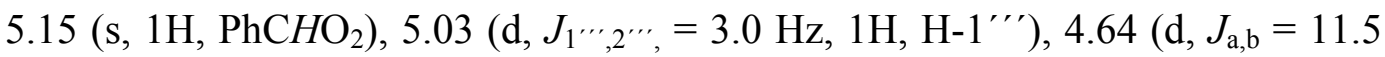
$\left.\mathrm{Hz}, 1 \mathrm{H}, \mathrm{CH}-\mathrm{a}, 3^{\prime \prime \prime}-\mathrm{O}-\mathrm{Bn}\right), 4.63$ (d, $\left.J_{\mathrm{a}, \mathrm{b}}=12.5 \mathrm{~Hz}, 1 \mathrm{H}, \mathrm{CH}-\mathrm{a}, 2^{\prime \prime \prime}-O-\mathrm{Bn}\right), 4.61(\mathrm{~d}$, $\left.J_{1^{\prime}, 2^{\prime}}=8.0 \mathrm{~Hz}, 1 \mathrm{H}, \mathrm{H}-1^{\prime}\right), 4.59\left(\mathrm{~d}, J_{1^{\prime \prime}, 2^{\prime \prime}}=8.1 \mathrm{~Hz}, 1 \mathrm{H}, \mathrm{H}-1^{\prime \prime}\right), 4.51\left(\mathrm{~d}, J_{\mathrm{a}, \mathrm{b}}=11.6\right.$ $\mathrm{Hz}, 1 \mathrm{H}, \mathrm{CH}-\mathrm{b}, 3^{\prime \prime \prime}-\mathrm{O}-\mathrm{Bn}$ ), 4.49-4.43 (m, 3H, H-6'a, H-6'b, CH-b, 2'"'-O-Bn), $4.44\left(\mathrm{~d}, J_{\mathrm{a}, \mathrm{b}}=11.6 \mathrm{~Hz}, 1 \mathrm{H}, \mathrm{CH}-\mathrm{a}, 3-O-\mathrm{Bn}\right), 4.26-4.24$ (m, 2H, CH-b, 3-O-Bn, H1a), 4.17 (t, $\left.J_{2^{\prime}, 3^{\prime}}=J_{3^{\prime}, 4^{\prime}}=9.6 \mathrm{~Hz}, 1 \mathrm{H}, \mathrm{H}-4^{\prime}\right), 4.09-4.06$ (m, 1H, H-2), 3.90-3.88 (m, 2H, H-2"' , H-6"' a), 3.80 (dd, $\left.J_{2^{\prime \prime}, 3^{\prime \prime}}=10.0 \mathrm{~Hz}, J_{3^{\prime \prime}, 4^{\prime \prime}}=3.3 \mathrm{~Hz}, 1 \mathrm{H}, \mathrm{H}-3^{\prime \prime}\right)$, 3.75-3.70 (m, 2H, H-3, H-5 ), 3.65 (dd, $J_{2}{ }^{\prime \prime \prime}, 3^{\prime \prime \prime}=10.0 \mathrm{~Hz}, J_{3^{\prime \prime \prime}, 4^{\prime \prime \prime}} 3.1 \mathrm{~Hz}, 1 \mathrm{H}, \mathrm{H}-$ $\left.3^{\prime \prime \prime}\right), 3.62$ (d, $\left.J_{5^{\prime \prime}, 6^{\prime \prime}}=6.6 \mathrm{~Hz}, 2 \mathrm{H}, \mathrm{H}-6^{\prime \prime} \mathrm{a}, \mathrm{H}-6^{\prime \prime} \mathrm{b}\right), 3.53-3.48$ (m, 2H, H-1b, H-

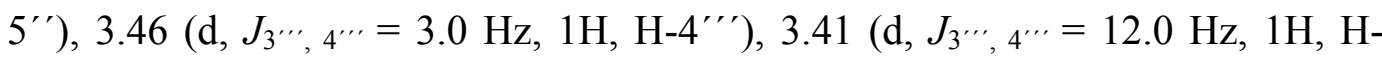
$\left.6^{\prime \prime \prime} \mathrm{b}\right), 3.29$ (s, 1H, H-5'"'), 1.96-1.92 (m, 2H, H-6), 1.68-1.63 (m, 2H, $\mathrm{CH}_{2}-\alpha$ ), 1.61 (s, 3H, OAc), 1.29-1.03 (m, 40H, H-7-H-17, H- $\beta-\mathrm{H}-(\omega-1)), 0.89-0.87$ (m, $6 \mathrm{H}, \mathrm{H}-18, \mathrm{H}-\omega) ;{ }^{13} \mathrm{C}$ NMR $\left(125 \mathrm{MHz}, \mathrm{CDCl}_{3}\right) \delta 172.5(\mathrm{HNC}=\mathrm{O}), 170.1(\mathrm{C}=\mathrm{O}$, OAc), $166.1\left(\mathrm{C}=\mathrm{O}, 6^{\prime}-O-\mathrm{Bn}\right), 165.8\left(\mathrm{C}=\mathrm{O}, 6^{\prime \prime}-O-\mathrm{Bn}\right), 165.5\left(\mathrm{C}=\mathrm{O}, 3^{\prime}-O-\mathrm{Bn}\right)$, $165.4\left(\mathrm{C}=\mathrm{O}, 2^{\prime}-O-\mathrm{Bn}\right), 164.4\left(\mathrm{C}=\mathrm{O}, 2^{\prime \prime}-O-\mathrm{Bn}\right), 138.9,138.7\left(\mathrm{C}-i, 2^{\prime \prime \prime}-O-\mathrm{Bn}\right.$, 3"' -O-Bn), 138.5 (C-i, 3-O-Bn), 137.8 (C-i, benzylidene), 137.2 (C-5), 133.8, 133.6, 133.5, 133.3 (C- $p, 5$ x OBz), 132.99, 129.97, 129.89, 129.86, 129.78, $129.69,129.59$, 129.49, 129.20, 129.17, 128.99, 128.91, 128.76, 128.74, 128.34, $128.31,128.24,128.20,128.18,127.73,127.71,127.58,127.51,127.49,127.44$, 126.37 (45 x CHarom, C-4), 101.5 (C-1'), 101.0 (C-1' '), 100.9 (CH-benzylidene), 95.4 (C-1"'), 79.3 (C-3), 76.0 (C-3'”'), 75.5 (C-4'), 74.6 (C-2'”), 74.4 (C-4"'), $73.9\left(\mathrm{CH}_{2}, 3^{\prime \prime \prime}-\mathrm{O}-\mathrm{Bn}\right), 73.3\left(\mathrm{C}-3^{\prime \prime}\right), 73.1\left(\mathrm{C}-5^{\prime}\right), 72.5\left(\mathrm{C}-3{ }^{\prime}\right), 72.3\left(\mathrm{CH}_{2}, 2^{\prime \prime \prime}-O-\right.$ Bn, C-2'/C-2'), $71.5\left(\mathrm{C}-2^{\prime} / \mathrm{C}-2^{\prime \prime}\right), 71.1$ (C-5'), $70.4\left(\mathrm{CH}_{2}, 3-O-\mathrm{Bn}\right), 68.9$ (C$\left.6^{\prime \prime \prime}\right), 68.5$ (C-1), 64.6 (C-4"'), 62.9 (C-5"'), 62.6 (C-6'), 61.2 (C-6"), 51.4 (C-2), 36.6 (C- $\alpha$ ), 32.3 (C-6), 32.05, 29.84, 29.83, 29.83, 29.80, 29.78, 29.66, 29.49, $29.39,29.34,25.69,25.57,23.99,23.96,22.82$ (C-7-C-17, C- $\beta-\mathrm{C}-(\omega-1)), 20.3$ (OAc), 14.3 (C-18, C- $\omega)$; HRMS(ESI) m/z calcd. for $\left[\mathrm{C}_{113} \mathrm{H}_{133} \mathrm{NO}_{24}+\mathrm{H}\right]^{+}$: 1888.9290, obsd. 1888.9282 
$(2 S, 3 R, 4 E)-2-D o d e c a n o y l a m i d o-1-(4-O-(3-O-\alpha-D-g a l a c t o p y r a n o s y l)-\beta-D-$ galactopyranosyl)- $\beta$-D-glucopyranosyloxy)-3-hydroxy-octadec-4-ene (7)

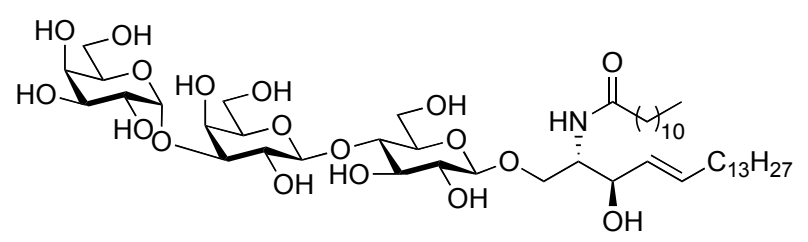

Fully protected triglycosyl ceramide $100(25 \mathrm{mg}, 0.0132 \mathrm{mmol})$ was dissolved in dry THF $(2 \mathrm{~mL})$ and $\mathrm{NH}_{3}(10 \mathrm{~mL})$ was condensed into the reaction vessel at -78 ${ }^{\circ} \mathrm{C}$. Small pieces of $\mathrm{Na}(\mathrm{s})$ were added carefully until the solution remained deep blue and the reaction mixture was stirred for 30 mins. The reaction was then quenched with a few drops of $\mathrm{MeOH}$ and $\mathrm{Na}$ (s) was added again until the deep blue colour persisted, and the reaction mixture stirred for a further 30 mins. The reaction was quenched with $10 \mathrm{~mL} \mathrm{MeOH}$, and the reaction warmed slowly to $\mathrm{rt}$ to allow the ammonia to evaporate. Trace ammonia was removed with an Ar stream. The reaction mixture was quenched to $\mathrm{pH} 7$ with Dowex- $\mathrm{H}^{+}$, filtered and washed with pyridine and concentrated in vacuo. The resultant oil was purified by gradient flash chromatography (DCM/MeOH, 20/1 to 5/1, v/v) to afford fully deprotected iGb3-C12 7 as an amorphous white solid (3.1 mg, $0.0032 \mathrm{mmol}$, 24\%). $\mathrm{R}_{f}: 0.07(\mathrm{DCM} / \mathrm{MeOH}, 5 / 1, \mathrm{v} / \mathrm{v}) ;[\alpha]_{\mathrm{D}}{ }^{24}=+29.0^{\circ}(\mathrm{c}=0.1$, pyridine $)$; IR (film) 3347, 2956, 2922, 2852, 1641, 1552, 1466, 1377, 1266, 1150, 1076, 1029, 973, 803, 774, 720, 693, $662 \mathrm{~cm}^{-1} ;{ }^{1} \mathrm{H}$ NMR (600 MHz, pyridine-d $\left.\mathrm{d}_{5}\right) \delta 8.45(\mathrm{~d}$, $\left.J_{2, \mathrm{NH}}=8.4 \mathrm{~Hz}, 1 \mathrm{H}, \mathrm{NH}\right), 7.46(\mathrm{~d}, J=5.3 \mathrm{~Hz}, 1 \mathrm{H}, \mathrm{OH}), 6.78\left(\mathrm{~d}, J_{4,5}=4.7 \mathrm{~Hz}, 1 \mathrm{H}\right.$, $\mathrm{OH}), 6.71,6.64,6.56(3 \mathrm{x}$ bs, 3H, 3 x OH), 6.51-6.49 (m, 1H, OH), 6.19 (bs, 1H, $\mathrm{OH}), 6.03\left(\mathrm{dd}, J_{4,5}=15.4 \mathrm{~Hz}, J_{3,4}=6.6 \mathrm{~Hz}, 1 \mathrm{H}, \mathrm{H}-4\right), 5.95-5.90(\mathrm{~m}, 1 \mathrm{H}, \mathrm{H}-5)$, $5.70(\mathrm{~s}, 1 \mathrm{H}, \mathrm{OH}), 5.68\left(\mathrm{~d}, J_{1^{\prime \prime}, 2^{\prime \prime \prime}}=3.8 \mathrm{~Hz}, 1 \mathrm{H}, \mathrm{H}-1^{\prime \prime \prime}\right), 5.11\left(\mathrm{~d}, J_{1^{\prime \prime}, 2^{\prime \prime}}=7.7 \mathrm{~Hz}\right.$, $\left.1 \mathrm{H}, \mathrm{H}-1^{\prime \prime}\right), 5.08$ (t, $\left.J_{5^{\prime \prime \prime}, 6^{\prime \prime \prime}}=6.2 \mathrm{~Hz}, 1 \mathrm{H}, \mathrm{H}-5^{\prime \prime \prime}\right), 4.90$ (d, $J_{1^{\prime}, 2^{\prime}}=7.9 \mathrm{~Hz}, 1 \mathrm{H}, \mathrm{H}-$ $1^{\prime}$ ), 4.83-4.79 (m, 3H, H-1a, H-2, H-3), 4.75 (dd, $J_{2}{ }^{\prime \prime \prime}, 3^{\prime \prime \prime}=9.6 \mathrm{~Hz}, J_{1^{\prime \prime}, 2^{\prime \prime}=3.8}$ Hz, 1H, H-2"'), 4.69 (bs, 1H, H-4"' ), 4.59 (bs, 1H, H-4"'), 4.57-4.43 (m, 2H, H2"', H-3"') , 4.47-4.45 (m, 3H, H-6"'a, H-6"' b, H-6"'a), 4.34-4.31 (m, 2H, H6'a, H-6' 'b), 4.28-4.23 (m, 4H, H-6'b, H-3', H-4', H-3' '), 4.17 (dd, $J_{1 \mathrm{a}, 1 \mathrm{~b}}=9.7$ $\left.\mathrm{Hz}, J_{1 \mathrm{~b}, 2}=2.9 \mathrm{~Hz}, 1 \mathrm{H}, \mathrm{H}-1 \mathrm{~b}\right), 4.08-4.03$ (m, 2H, H-2', H-5' $)$, 3.88-3.85 (m, 1H, 
H-5'), $2.45\left(\mathrm{t}, J_{\alpha, \beta}=7.1 \mathrm{~Hz}, 2 \mathrm{H}, \mathrm{CH}_{2}-\alpha\right), 2.07\left(\mathrm{dd}, J_{6,7}=14.3 \mathrm{~Hz}, J_{5,6}=6.6 \mathrm{~Hz}\right.$, 1H, H-6), 1.88-1.88 (m, 2H, $\left.\mathrm{CH}_{2}-\beta\right), 1.37-1.22$ (m, 38H, H-7-H-11, H- $\gamma-\mathrm{H}-$ $(\omega-1)), 0.88\left(\mathrm{t}, J_{11,12}=J_{\omega-1, \omega}=6.6 \mathrm{~Hz}, 6 \mathrm{H}, \mathrm{H}-12, \mathrm{H}-\omega\right) ;{ }^{13} \mathrm{C} \mathrm{NMR}(125 \mathrm{MHz}$, pyridine-d $\left.\mathrm{d}_{5}\right) \delta 173.7(\mathrm{HNC}=\mathrm{O}), 133.0(\mathrm{C}-4), 132.6(\mathrm{C}-5), 105.8\left(\mathrm{C}-1^{\prime}, \mathrm{C}-1^{\prime \prime}\right)$, 98.0 (C-1'"'), 82.4 (C-4'), 80.4 (C-3'”), 77.0 (C-5'), 76.92 (C-3'), 76.85 (C-5'), 75.1 (C-2'), 73.2 (C-5"'), 73.0 (C-3), 72.0 (C-3"'), 71.2 (C-4"'), 70.8 (C-1, C$\left.2^{\prime \prime}\right), 70.7$ (C-2'"'), 66.3 (C-4'), 62.6 (C-6"' ), 62.29 (C-6"'), 62.17 (C-6'), 55.2 (C-2), 37.3 (C- $\alpha$ ), 33.1 (C-6), 32.5, 30.37, 30.34, 30.31, 30.28, 30.25, 30.23, 30.21, 30.12, 30.07, 29.96, 23.28 (C7-C-11, C- $\gamma-\mathrm{C}-(\omega-1)), 26.8$ (C- $\beta$ ), 14.6 (C12, C- $\omega$ ); HRMS(ESI) $\mathrm{m} / \mathrm{z}$ calcd. for $\left[\mathrm{C}_{48} \mathrm{H}_{89} \mathrm{NO}_{18}+\mathrm{H}\right]^{+}$: 968.6152, obsd.: 968.6159.

(2S,3R,4E)-1-(4-O-(4-O-Acetyl-2,6-di- $O$-benzoyl-3- $O$-(2,3-di- $O$-benzyl-4,6-Obenzylidene- $\alpha$-D-galactopyranosyl)- $\beta$-D-galactopyranosyl)-2,3,6-tri- $O$ benzoyl- $\beta$-D-glucopyranosyloxy)-3-benzyloxy-2-(11Z,14Zeicosadienoylamido)-octadec-4-ene (101)

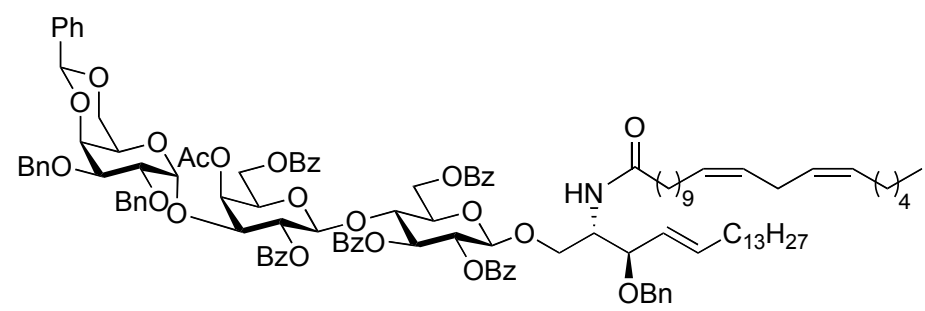

To a solution of glycolipid azide $92(23 \mathrm{mg}, 0.013 \mathrm{mmol})$ in dry benzene $(0.8 \mathrm{~mL})$ triphenylphosphine (7 $\mathrm{mg}, 0.026 \mathrm{mmol})$, and distilled water $(5 \mu \mathrm{L})$ were added, the solution was warmed to $45^{\circ} \mathrm{C}$ and stirred overnight. The reaction mixture was then cooled to room temperature, diluted with EtOAc $(15 \mathrm{~mL})$, washed with sat. aq. $\mathrm{NH}_{4} \mathrm{Cl}(5 \mathrm{~mL})$, dried $\left(\mathrm{MgSO}_{4}\right)$, filtered and concentrated under reduced pressure to give a colourless oil which was used without further purification. The oil was co-evaporated twice with dry toluene then suspended in DCM (1 mL), EDCI (11.3 mg, $0.0589 \mathrm{mmol})$, DMAP (9.6mg, $0.0785 \mathrm{mmol})$, and 11Z,14Zeicosadienoic acid $(11.8 \mathrm{mg}, 0.0589 \mathrm{mmol})$ were added and the resulting solution stirred over 2 days at room temperature, after which it was diluted with EtOAc $(20 \mathrm{~mL})$, washed with sat. aq. $\mathrm{NaHCO}_{3}(20 \mathrm{~mL})$ and brine $(20 \mathrm{~mL})$, dried 
$\left(\mathrm{MgSO}_{4}\right)$, filtered and concentrated in vacuo. The reaction mixture was then purified directly by gradient flash chromatography (petroleum ether/EtOAc, 10/1 to $2 / 1, \mathrm{v} / \mathrm{v})$ to give $\mathbf{1 0 1}$ as a colourless oil $(7.5 \mathrm{mg}, 0.0038 \mathrm{mmol}, 28 \%$ over two steps). The product was kept under inert argon atmosphere at all time to prevent degradation by autoxidation. $\mathrm{R}_{f}: 0.39(\mathrm{PE} / \mathrm{EA}, 2 / 1, \mathrm{v} / \mathrm{v}) ;[\alpha]_{\mathrm{D}}{ }^{20}=+40.0^{\circ}(\mathrm{c}=1.0$, $\mathrm{CHCl}_{3}$ ); IR(film) 3062, 30111, 2956, 2926, 1730, 1453, 1438, 1270, 1177, 1119 , 1070, 1028, 998, 754, 711, $634 \mathrm{~cm}^{-1} ;{ }^{1} \mathrm{H} \mathrm{NMR}\left(500 \mathrm{MHz}, \mathrm{CDCl}_{3}\right) \delta 8.04-7.93$ $\left(\mathrm{m}, 10 \mathrm{H}, 5\right.$ x CH-o, 2'-O-Bz, 3'-O-Bz, 6'-O-Bz, 2' $\left.-O-\mathrm{Bz}, 6^{\prime \prime}-O-\mathrm{Bz}\right), 7.61-7.12$ $\left(\mathrm{m}, 35 \mathrm{H}, \mathrm{H}_{\text {arom }}\right), 5.73\left(\mathrm{t}, J_{2^{\prime}, 3^{\prime}}=J_{3^{\prime}, 4^{\prime}}=9.6 \mathrm{~Hz}, 1 \mathrm{H}, \mathrm{H}-3^{\prime}\right), 5.49\left(\mathrm{dt}, J_{4,5}=15.6 \mathrm{~Hz}\right.$, $\left.J_{5,6}=6.7 \mathrm{~Hz}, 1 \mathrm{H}, \mathrm{H}-5\right), 5.41-5.30$ (m, 4H, H-2', H-2' $, \mathrm{NH}, \mathrm{H}-4^{\prime \prime}$ ), 5.23 (dd, $J_{4,5}$ $\left.=15.3 \mathrm{~Hz}, J_{3,4}=8.6 \mathrm{~Hz}, 1 \mathrm{H}, \mathrm{H}-4\right), 5.15\left(\mathrm{~s}, 1 \mathrm{H}, \mathrm{PhCHO}_{2}\right), 5.03\left(\mathrm{~d}, J_{1^{\prime \prime \prime}, 2^{\prime \prime \prime},}=3.0\right.$ $\left.\mathrm{Hz}, 1 \mathrm{H}, \mathrm{H}-1^{\prime \prime \prime}\right), 4.62$ (d, $\left.J_{\mathrm{a}, \mathrm{b}}=11.3 \mathrm{~Hz}, 1 \mathrm{H}, \mathrm{CH}-\mathrm{a}, 3^{\prime \prime \prime}-O-\mathrm{Bn}\right), 4.63\left(\mathrm{~d}, J_{\mathrm{a}, \mathrm{b}}=12.4\right.$ $\left.\mathrm{Hz}, 1 \mathrm{H}, \mathrm{CH}-\mathrm{a}, 2^{\prime \prime \prime}-O-\mathrm{Bn}\right), 4.61$ (d, $\left.J_{1^{\prime}, 2^{\prime}}=8.0 \mathrm{~Hz}, 1 \mathrm{H}, \mathrm{H}-1^{\prime}\right), 4.58\left(\mathrm{~d}, J_{1^{\prime \prime}, 2^{\prime \prime}}=8.0\right.$ $\left.\mathrm{Hz}, 1 \mathrm{H}, \mathrm{H}-1^{\prime \prime}\right), 4.53$ (d, $\left.J_{\mathrm{a}, \mathrm{b}}=11.8 \mathrm{~Hz}, 1 \mathrm{H}, \mathrm{CH}-\mathrm{b}, 3^{\prime \prime \prime}-\mathrm{O}-\mathrm{Bn}\right), 4.49-4.47$ (m, 3H, H-6'a, H-6'b, CH-b, 2'"'-O-Bn), 4.43 (d, $\left.J_{\mathrm{a}, \mathrm{b}}=11.7 \mathrm{~Hz}, 1 \mathrm{H}, \mathrm{CH}-\mathrm{a}, 3-O-\mathrm{Bn}\right)$, 4.25-4.24 (m, 2H, CH-b, 3-O-Bn, H-1a), 4.16 (t, $\left.J_{2^{\prime}, 3^{\prime}}=J_{3^{\prime}, 4^{\prime}}=9.5 \mathrm{~Hz}, 1 \mathrm{H}, \mathrm{H}-4^{\prime}\right)$,

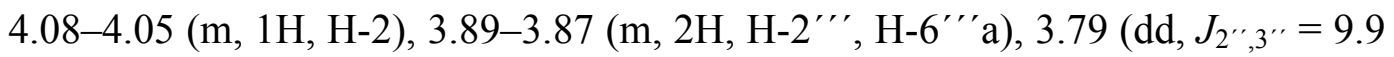
$\left.\mathrm{Hz}, J_{3^{\prime \prime}, 4^{\prime \prime}}=3.1 \mathrm{~Hz}, 1 \mathrm{H}, \mathrm{H}-3^{\prime \prime}\right), 3.74-3.69$ (m, 2H, H-3, H-5'), 3.65 (dd, $J_{2^{\prime \prime \prime}, 3^{\prime \prime \prime}=}=$ $10.0 \mathrm{~Hz}, J_{3^{\prime \prime}, 4^{\prime \prime \prime}} 3.1 \mathrm{~Hz}, 1 \mathrm{H}, \mathrm{H}-3^{\prime \prime \prime}$ ), 3.61 (d, $J_{5^{\prime \prime}, 6^{\prime \prime}}=6.6 \mathrm{~Hz}, 2 \mathrm{H}, \mathrm{H}-6$ "a, H-6" 'b),

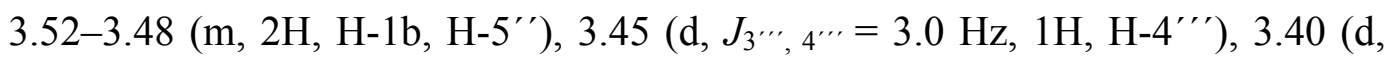

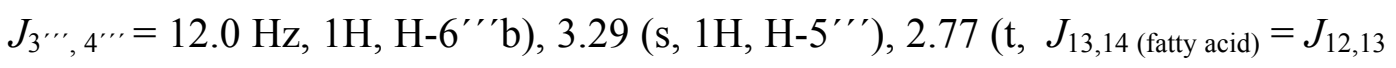
(fatty acid) $=6.9 \mathrm{~Hz}, 2 \mathrm{H}, \mathrm{H}-13$ fatty acid), 2.05 (m, 4H, H-10, H-16 fatty acid), 1.951.92 (m, 2H, H-6), 1.68-1.63 (m, 2H, $\mathrm{CH}_{2}-\alpha$ ), 1.61 (s, 3H, OAc), 1.35-1.23 (m, $42 \mathrm{H}, \mathrm{H}-7-\mathrm{H}-17, \mathrm{H}-\beta-\mathrm{H}-(\omega-1)), 0.89-0.86$ (m, 6H, H-18, H- $\omega) ;{ }^{13} \mathrm{C}$ NMR $(125$ $\left.\mathrm{MHz}, \mathrm{CDCl}_{3}\right) \delta 172.5(\mathrm{HN} \underline{\mathrm{C}}=\mathrm{O}), 170.1(\mathrm{C}=\mathrm{O}, \mathrm{OAc}), 166.1\left(\mathrm{C}=\mathrm{O}, 6^{\prime}-O-\mathrm{Bn}\right)$, $165.8\left(\mathrm{C}=\mathrm{O}, 6^{\prime \prime}-O-\mathrm{Bn}\right), 165.5\left(\mathrm{C}=\mathrm{O}, 3^{\prime}-O-\mathrm{Bn}\right), 165.4\left(\mathrm{C}=\mathrm{O}, 2^{\prime}-O-\mathrm{Bn}\right), 164.4$ $\left(\mathrm{C}=\mathrm{O}, 22^{\prime \prime}-O-\mathrm{Bn}\right), 138.9,138.7$ (C-i, 2"' $\left.-O-\mathrm{Bn}, 3^{\prime \prime \prime}-O-\mathrm{Bn}\right), 138.5$ (C-i, 3-O-Bn), 137.8 (C-i, benzylidene), 137.2 (C-5), 133.8, 133.7, 133.6, 133.5, 133.3 (C- $p, 5$ x OBz), 130.35, 13.27, 129.97, 129.91, 129.88, 129.79, 129.70, 129.67, 129.61, 129.49 , 129.20 ,129.16, 129.02, 128.92, 128.78, 128.76, 128.70, 128.62, 128.40, $128.36,128.32$, 128.26, 128.22, 128.19, 128.12, 128.06, 127.74, 127.73, 127.61, 127.53, 127.50, 126.39 ( $\mathrm{CH}_{\text {arom }}, \mathrm{C}-4, \mathrm{C}-11, \mathrm{C}-12, \mathrm{C}-14, \mathrm{C}-15$ fatty acid $), 101.5$ (C-1', C-1'”), 100.9 (CH-benzylidene), 95.4 (C-1'"'), 79.3 (C-3), 76.0 (C-3'"'), 
75.5 (C-4'), 74.6 (C-2"'), 74.4 (C-4"'), $73.9\left(\mathrm{CH}_{2}, 3^{\prime \prime \prime}-O-\mathrm{Bn}\right), 73.3$ (C-3"'), 73.1 (C-5'), 72.5 (C-3'), 72.3 ( $\left.\mathrm{CH}_{2}, 2^{\prime \prime \prime}-O-\mathrm{Bn}, \mathrm{C}-2^{\prime} / \mathrm{C}-2^{\prime \prime}\right), 71.5$ (C-2'/C-2'), 71.1 (C5'), $70.4\left(\mathrm{CH}_{2}, 3-\mathrm{O}-\mathrm{Bn}\right), 69.0\left(\mathrm{C}-6^{\prime \prime \prime}\right), 68.5(\mathrm{C}-1), 64.6\left(\mathrm{C}-4^{\prime \prime}\right), 62.9\left(\mathrm{C}-5^{\prime \prime \prime}\right)$, 62.6 (C-6'), 61.2 (C-6'), 51.4 (C-2), 36.6 (C-2 fatty acid), 32.4 (C-6), 32.07, $31.66,29.86,29.85,29.82$, 29.74, 29.68, 29.66, 29.51, 29.49, 29.39, 29.37, 27.40, 27.34, 27.23, 25.77, 25.64, 25.58, 25.19, 23.92, 22.84, 22.72, 20.21 (C-7-C-17, C-3-C-10 fatty acid, C16-C19 fatty acid), 20.3 (OAc), 14.3 (C-18, C20 fatty acid); HRMS(ESI) $\mathrm{m} / \mathrm{z}$ calcd. for $\left[\mathrm{C}_{121} \mathrm{H}_{145} \mathrm{NO}_{24}+\mathrm{H}+\mathrm{NH}_{4}\right]^{2+}: 1007.5284$, obsd. 1007.5262 .

\section{(2S,3R,4E)-2-(11Z,14Z-Eicosadienoylamido)-1-(4-O-(3-O- $\alpha-\mathrm{D}-$}

galactopyranosyl)- $\beta$-D-galactopyranosyl)- $\beta$-D-glucopyranosyloxy)-3-hydroxyoctadec-4-ene (8)

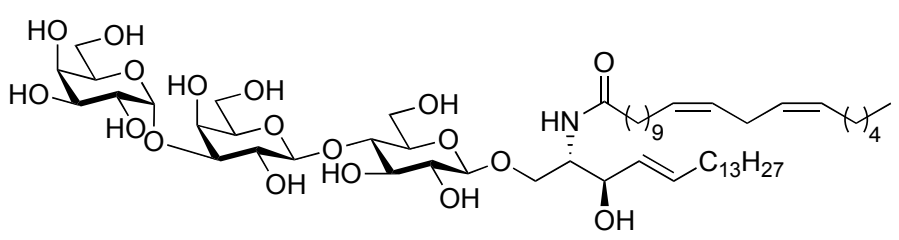

Fully protected triglycosyl ceramide $101(20 \mathrm{mg}, 0.010 \mathrm{mmol})$ was dissolved in dry THF $(1 \mathrm{~mL})$ and $\mathrm{NH}_{3}(10 \mathrm{~mL})$ was condensed into the reaction vessel at -78 ${ }^{\circ} \mathrm{C}$. Small pieces of $\mathrm{Na}(\mathrm{s})$ were added carefully until the solution remained deep blue and the reaction mixture was stirred for 30 mins. The reaction was then quenched with a few drops of $\mathrm{MeOH}$ and $\mathrm{Na}$ (s) was added again until the deep blue colour persisted, and the reaction mixture stirred for a further $30 \mathrm{mins}$. The reaction was quenched with $10 \mathrm{~mL} \mathrm{MeOH}$, and the reaction warmed slowly to $\mathrm{rt}$ to allow the ammonia to evaporate. Trace ammonia was removed with an Ar stream. The reaction mixture was quenched to $\mathrm{pH} 7$ with Dowex $-\mathrm{H}^{+}$, filtered and washed with pyridine and concentrated in vacuo. The resultant oil was purified by gradient flash chromatography (DCM/MeOH, 20/1 to $5 / 1, \mathrm{v} / \mathrm{v}$ ) to afford fully deprotected iGb3-C12 8 as an amorphous white solid (5.2 $\mathrm{mg}, 0.0048 \mathrm{mmol}$, 48\%). $\mathrm{R}_{f}: 0.13(\mathrm{DCM} / \mathrm{MeOH}, 4 / 1, \mathrm{v} / \mathrm{v}) ;[\alpha]_{\mathrm{D}}^{24}=+26.0^{\circ}(\mathrm{c}=0.1$, pyridine); IR (film) 3360, 3010, 2923, 2853, 1640, 1548, 1464, 1377, 1255, 1150, 1075, 1030, $971,895,804,632 \mathrm{~cm}^{-1} ;{ }^{1} \mathrm{H}$ NMR $\left.(600 \mathrm{MHz} \text {, pyridine-d })_{5}\right) 8.49\left(\mathrm{~d}, J_{2, \mathrm{NH}}=8.3\right.$ $\mathrm{Hz}, 1 \mathrm{H}, \mathrm{NH}), 6.04$ (dd, $\left.J_{4,5}=15.2 \mathrm{~Hz}, J_{3,4}=6.3 \mathrm{~Hz}, 1 \mathrm{H}, \mathrm{H}-4\right), 5.93\left(\mathrm{dd}, J_{4,5}=15.2\right.$ 
$\left.\mathrm{Hz}, J_{5,6}=6.7 \mathrm{~Hz}, \mathrm{H}-5\right), 5.68$ (d, $\left.J_{1^{\prime \prime}, 2^{\prime \prime}}=3.6 \mathrm{~Hz}, 1 \mathrm{H}, \mathrm{H}-1^{\prime \prime \prime}\right), 5.54-5.48$ (m, 4H, H-11, H-12, H-14, H-15 fatty acid), 5.09 (d, $\left.J_{1^{\prime \prime}, 2^{\prime \prime}}=8.0 \mathrm{~Hz}, 1 \mathrm{H}, \mathrm{H}-1^{\prime \prime}\right), 5.06$ (t, $\left.J_{5^{\prime \prime \prime}, 6^{\prime \prime \prime}}=5.9 \mathrm{~Hz}, 1 \mathrm{H}, \mathrm{H}-5^{\prime \prime \prime}\right), 4.90\left(\mathrm{~d}, J_{1^{\prime}, 2^{\prime}}=7.9 \mathrm{~Hz}, 1 \mathrm{H}, \mathrm{H}-1^{\prime}\right), 4.84-4.79$ (m,

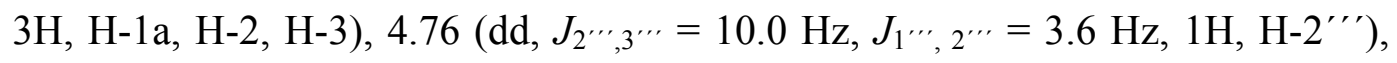
4.68 (bs, 1H, H-4"'), 4.59 (bs, 1H, H-4"'), 4.58-4.52 (m, 2H, H-2", H-3"'), 4.51-4.43 (m, 3H, H-6"' a, H-6" 'b, H-6"'a), 4.33 (dd, $J_{a, b}=10.9$ Hz, $J_{a, b}=4.9$ Hz, 2H, H-6'a, H-6' 'b), 4.29-4.24 (m, 4H, H-6'b, H-3', H-4', H-3' ), 4.18-4.16 (m, 1H, H-1b), 4.08-4.03 (m, 2H, H-2', H-5' ), 3.87-3.85 (m, 1H, H-5'), 2.93 (t, $J_{13,14 \text { (fatty acid) }}=J_{12,13 \text { (fatty acid) }}=5.3 \mathrm{~Hz}, 2 \mathrm{H}, \mathrm{H}-13$ fatty acid), $2.46\left(\mathrm{t}, J_{\alpha, \beta}=7.6 \mathrm{~Hz}\right.$, 2H, H-2 fatty acid), 2.15-2.06 (m, 6H, H-10, H-16 fatty acid, H-6), 1.86-1.80 (m, $\left.2 \mathrm{H}, \mathrm{CH}_{2}-\beta\right), 1.36-1.23$ (m, 42H, H-3-H-9 fatty acid, H-17-H-19 fatty acid, H717), 0.89-0.86 (m, 6H, H-18, H-20 fatty acid); ${ }^{13} \mathrm{C}$ NMR (125 MHz, pyridine- $\mathrm{d}_{5}$ ) ठ 173.7 (HNC=O), 133.0 (C-4), 132.6 (C-5), 130.8, 128.7 (C-11, C-12, C-14, C15 fatty acid), 105.8 (C-1', C-1' ), 98.0 (C-1'"'), 82.3 (C-4'), 80.4 (C-3' '), 76.97 (C-5'), 76.92 (C-3'), 76.82 (C-5'”), 75.1 (C-2'), 73.2 (C-5'”), 73.0 (C-3), 72.0 (C3"') 71.2 (C-4"'), 70.8 (C-1, C-2'), 70.7 (C-2'"'), 66.2 (C-4"'), 62.6, 62.2 (C6"', C-6"', C-6'), 55.2 (C-2), 37.3 (C- $\alpha$ ), 33.1 (C-6), 30.30, 30.27, 30.24, 30.21, 30.12 , 30.05, 29.96, 29.94, 293.92, 29.83, 27.90, 27.79, 27.38, 26.74, 26.37, 23.27, 23.14 (C7-C-17, C-2-C-10 fatty acid, C16-C19 fatty acid), 14.6, 14.56 (C18, C-20 fatty acid); HRMS(ESI) m/z calcd. for $\left[\mathrm{C}_{56} \mathrm{H}_{101} \mathrm{NO}_{18}+\mathrm{H}\right]^{+}:$1076.7091, obsd.: 1076.7090). 


\subsection{References}

1. Goff, R. D.; Gao, Y.; Mattner, J.; Zhou, D.; Yin, N.; Cantu, C.; Teyton, L.; Bendelac, A.; Savage, P. B., J. Am. Chem. Soc. 2004, 126, 13602-13603.

2. Yu, K. O. A.; Im, J. S.; Molano, A.; Dutronc, Y.; Illarionov, P. A.; Forestier, C.; Fujiwara, N.; Arias, I.; Miyake, S.; Yamamura, T.; Chang, Y.t.; Besra, G. S.; Porcelli, S. A., Proc. Natl. Acad. Sci. U. S. A. 2005, 102, 3383-3388.

3. Zajonc, D. M.; Cantu, C.; Mattner, J.; Zhou, D.; Savage, P. B.; Bendelac, A.; Wilson, I. A.; Teyton, L., Nat. Immunol. 2005, 6, 810-818.

4. Chang, Y.-J.; Huang, J.-R.; Tsai, Y.-C.; Hung, J.-T.; Wu, D.; Fujio, M.; Wong, C.-H.; Yu, A. L., Proc. Natl. Acad. Sci. U. S. A. 2007, 104, 1029910304.

5. McCarthy, C.; Shepherd, D.; Fleire, S.; Stronge, V. S.; Koch, M.; Illarionov, P. A.; Bossi, G.; Salio, M.; Denkberg, G.; Reddington, F.; Tarlton, A.; Reddy, B. G.; Schmidt, R. R.; Reiter, Y.; Griffiths, G. M.; van der Merwe, P. A.; Besra, G. S.; Jones, E. Y.; Batista, F. D.; Cerundolo, V., J. Exp. Med. 2007, 204, 1131-1144.

6. Tashiro, T.; Hongo, N.; Nakagawa, R.; Seino, K.-i.; Watarai, H.; Ishii, Y.; Taniguchi, M.; Mori, K., Bioorg. Med. Chem. 2008, 16, 8896-8906.

7. Zeng, Z.; Castaño, A. R.; Segelke, B. W.; Stura, E. A.; Peterson, P. A.; Wilson, I. A., Science 1997, 277, 339-345.

8. Koch, M.; Stronge, V.; Shepherd, D.; Gadola, S.; Mathew, B.; Ritter, G.; Fersht, A.; Besra, G.; Schmidt, R.; Jones, E.; Cerundolo, V., Nat. Immunol. 2005, 6, 819-826.

9. Liu, Y.; Goff, R.; Zhou, D.; Mattner, J.; Sullivan, B.; Khurana, A.; Cantu, C.; Ravkov, E.; Lbegbu, C.; Altman, J.; Teyton, L.; BendelaC, A.; Savage, P., J. Immunol. Methods 2006, 312, 34-39.

10. Zajonc, D. M.; Savage, P. B.; Bendelac, A.; Wilson, I. A.; Teyton, L., J. Mol. Biol. 2008, 377, 1104-1116.

11. Adlercreutz, D.; Weadge, J. T.; Petersen, B. O.; Duus, J. Ø.; Dovichi, N. J.; Palcic, M. M., Carbohydr. Res. 2010, 345, 1384-1388.

12. Qiu, D.; Schmidt, R. R., Liebigs Ann. Chem. 1992, 1992, 217-224.

13. Kimura, A.; Imamura, A.; Ando, H.; Ishida, H.; Kiso, M., Synlett 2006, 2379-2382.

14. Koike, K.; Sugimoto, M.; Sato, S.; Ito, Y.; Nakahara, Y.; Ogawa, T., Carbohydr. Res. 1987, 163, 189-208.

15. Im, J. S.; Arora, P.; Bricard, G.; Molano, A.; Venkataswamy, M. M.; Baine, I.; Jerud, E. S.; Goldberg, M. F.; Baena, A.; Yu, K. O. A.; Ndonye, R. M.; Howell, A. R.; Yuan, W.; Cresswell, P.; Chang, Y.-t.; Illarionov, P. A.; Besra, G. S.; Porcelli, S. A., Immunity 2009, 30, 888-898.

16. Yu, E. D.; Girardi, E.; Wang, J.; Mac, T.-T.; Yu, K. O. A.; Van Calenbergh, S.; Porcelli, S. A.; Zajonc, D. M., J. Biol. Chem. 2012, 287, 1269-1278.

17. Zhou, Z.; Zhang, C.; Xia, C.; Chen, W.; Zhu, H.; Shang, P.; Ma, F.; Wang, P. G.; Zhang, J.; Xu, W.; Tian, Z., Mol. Cancer Ther. 2011, 10, 1375-1384.

18. Wherry, E. J., Nat. Immunol. 2011, 12, 492-499. 
19. Zhang, W.; Zheng, X.; Xia, C.; Perali, R. S.; Yao, Q.; Liu, Y.; Zheng, P.; Wang, P. G., ChemBioChem 2008, 9, 1423-1430.

20. Singh, A. K.; Wilson, M. T.; Hong, S.; Olivares-Villagómez, D.; Du, C.; Stanic, A. K.; Joyce, S.; Sriram, S.; Koezuka, Y.; Van Kaer, L., J. Exp. Med. 2001, 194, 1801-1811.

21. Yu, K. O. A.; Porcelli, S. A., Immunol. Lett. 2005, 100, 42-55.

22. Bendelac, A.; Savage, P. B.; Teyton, L., Annu. Rev. Immunol. 2007, 25, 297-336.

23. van den Heuvel, M. J.; Garg, N.; Van Kaer, L.; Haeryfar, S. M. M., Trends Mol Med 2011, 17, 65-77.

24. Godfrey, D. I.; Pellicci, D. G.; Rossjohn, J., Nat. Immunol. 2011, 12, 1135 1137.

25. Brigl, M.; Tatituri, R. V. V.; Watts, G. F. M.; Bhowruth, V.; Leadbetter, E. A.; Barton, N.; Cohen, N. R.; Hsu, F.-F.; Besra, G. S.; Brenner, M. B., J. Exp. Med. 2011, 208, 1163-1177.

26. Brennan, P. J.; Tatituri, R. V. V.; Brigl, M.; Kim, E. Y.; Tuli, A.; Sanderson, J. P.; Gadola, S. D.; Hsu, F.-F.; Besra, G. S.; Brenner, M. B., Nat. Immunol. 2011, 12, 1202-1211.

27. Figueroa-Perez, S.; Schmidt, R., Carbohydr. Res. 2000, 328, 95-102.

28. Plettenburg, O.; Bodmer-Narkevitch, V.; Wong, C. H., J. Org. Chem. 2002, 67, 4559-4564.

29. Banchet-Cadeddu, A.; Hénon, E.; Dauchez, M.; Renault, J.-H.; Monneaux, F.; Haudrechy, A., Org. Biomol. Chem. 2011, 9, 3080-3104.

30. Dangerfield, E. M.; Cheng, J. M. H.; Knight, D. A.; Weinkove, R.; Dunbar, P. R.; Hermans, I. F.; Timmer, M. S. M.; Stocker, B. L., ChemBioChem 2012, 13, 1349-1356.

31. Helferich, B.; Schmitz-Hillebrecht, E., Ber. 1933, 66, 378-383.

32. Lee, A.; Farrand, K. J.; Dickgreber, N.; Hayman, C. M.; Jurs, S.; Hermans, I. F.; Painter, G. F., Carbohydr. Res. 2006, 341, 2785-2798.

33. Morales-Serna, J. A.; Boutureira, O.; Díaz, Y.; Matheu, M. I.; Castillón, S., Carbohydr. Res. 2007, 342, 1595-612.

34. Dangerfield, E.; Timmer, M.; Stocker, B., Victoria University of Wellington, New Zealand. 2011.

35. Motawia, M. S.; Marcussen, J.; Moller, B. L., J. Carbohydr. Chem. 1995, 14, 1279-1294.

36. Nicolaou, K. C.; Caulfield, T. J.; Katoaka, H., Carbohydr. Res. 1990, 202, 177-191.

37. Yao, Q.; Song, J.; Xia, C.; Zhang, W.; Wang, P. G., Org. Lett. 2006, 8, 911914.

38. Porter, N. A.; Caldwell, S. E.; Mills, K. A., Lipids 1995, 30, 277-290.

39. Pratt, D. A.; Tallman, K. A.; Porter, N. A., Acc Chem Res 2011, 44, 458467.

40. Chan, H. W. S.; Levett, G.; Matthew, J. A., Chem. Phys. Lipids 1979, 24 , 245-256.

41. Yin, N.; Long, X.; Goff, R. D.; Zhou, D.; Cantu, C.; Mattner, J.; Mezard, P. S.; Teyton, L.; Bendelac, A.; Savage, P. B., ACS Chem. Biol. 2009, 4, 191197.

42. Horlacher, T.; Oberli, M. A.; Werz, D. B.; Kröck, L.; Bufali, S.; Mishra, R.; Sobek, J.; Simons, K.; Hirashima, M.; Niki, T.; Seeberger, P. H., ChemBioChem 2010, 11, 1563-1573. 
43. Zhou, X.; Forestier, C.; Goff, R.; Li, C.; Teyton, L.; Bendelac, A.; Savage, P. B., Org. Lett. 2002, 4, 1267-1270.

44. Oberli, M. A.; Bindschadler, P.; Werz, D. B.; Seeberger, P. H., Org. Lett. 2008, 10, 905-908.

45. Walvoort, M. T. C.; Lodder, G.; Overkleeft, H. S.; Codée, J. D. C.; Van Der Marel, G. A., J. Org. Chem. 2010, 75, 7990-8002.

46. Morales-Serna, J. A.; Díaz, Y.; Matheu, M. I.; Castillón, S., Eur. J. Org. Chem. 2009, 2009, 3849-3852.

47. Chen, W.; Xia, C.; Wang, J.; Thapa, P.; Li, Y.; Talukdar, A.; Nadas, J.; Zhang, W.; Zhou, D.; Wang, P. G., J. Org. Chem. 2007, 72, 9914-9923.

48. Xia, C.; Schümann, J.; Emmanuel, R.; Zhang, Y.; Chen, W.; Zhang, W.; De Libero, G.; Wang, P. G., J. Med. Chem. 2007, 50, 3489-3496. 


\section{Chapter 6. Summary and future prospects}

Glycosphingolipids play a crucial role in modulating the immune response, and in particular, the glycolipid adjuvant $\alpha$-galactosyl ceramide ( $\alpha$-GalCer) is capable of enhancing the body's ability to detect and destroy cancerous cells during dendritic cell (DC)-based cancer immunotherapy. In stimulating an anti-cancer immune response, $\alpha$-GalCer is presented by the CD1d molecule found on DCs to the TCR of iNKT cells, which was discussed in the introduction (Chapter 1) to this thesis. In addition to foreign $\alpha$-linked glycolipids, $\beta$-linked glycosphingolipids, such as isoglobotrihexosylceramide (iGb3), can also be recognised by the TCR of NKT cells when it is bound to CD1d. Accordingly, iGb3 analogues have much potential as immunomodulatory compounds, as was described in Chapter 1.

Various analogues of $\alpha$-GalCer have been synthesised with the aim of fine-tuning the immune response generated during DC-based cancer treatment. Hence, a comprehensive understanding of how $\alpha$-GalCer interacts with the immune system is crucial in order to guide efforts towards improved vaccination strategies. One of the ways to study the uptake and trafficking of $\alpha$-GalCer in vitro and in vivo is by using fluorescent $\alpha$-GalCer probes. In Chapter 2, the synthesis of the fluorescent probe, dansyl- $\alpha$-GalCer was described. Biological evaluation of dansyl- $\alpha$-GalCer revealed that it was able to activate DCs and iNKT cells in a similar fashion to the parent glycolipid $\alpha$-GalCer and its activity was CD1d-dependent. It was also shown that DCs that have taken up dansyl- $\alpha-G a l C e r$ in vitro were detectable by flow cytometry. Unfortunately, the fluorescence of dansyl- $\alpha$-GalCer was too weak to be visualised by fluorescent microscopy due to photobleaching of the dye. Accordingly, another $\alpha$-GalCer probe bearing a brighter fluorescent group, BODIPY, was synthesised, as was discussed in Chapter 3. The dansyl- $\alpha-G a l C e r$ and BODIPY- $\alpha$-GalCer probes were made via two different synthetic strategies, which reflects the different chemistries that the respective dyes are compatible with. It is envisioned that the brighter BODIPY fluorophore will allow for its use in in vivo studies, however, care must be taken to avoid the photo-oxidation of the 
dye upon prolonged exposure to light and oxygen. Indeed, this photo-oxidation under relatively mild conditions has not been previously reported for the BODIPY fluorophore and future work will include exploring this phenomenon further. Both dansyl- $\alpha$-GalCer and BODIPY- $\alpha$-GalCer were sent to our collaborator, Hans van der Vliet (VU University Medical Centre, Amsterdam) for further studies to understand glycolipid uptake by human DCs. It will be exciting to see the results from this work and how the probes have been used to address biological questions.

In Chapter 4 the synthesis of two iGb3 analogues, 6"'-deoxy-iGb3-sphingosine and 6 "'-deoxy-iGb3-sphinganine was described. The highlight of this route was the highly efficient synthesis of a lactosyl 2-azido-sphingosine acceptor which, in this chapter, was $N$-acylated then functionalised with a D-fucose moiety. The use of two different deprotection strategies performed on the late stage intermediate (i.e. the fully protected 6 "'-deoxy-iGb3), allowed for the generation of 6 "' deoxy-iGb3 analogues with the sphingosine or the sphinganine lipid backbone. 6 "'-deoxy-iGb3-sphingosine will be valuable to unambiguously ascertain the importance of the 6-hydroxyl group of the terminal sugar in the interaction with CD1d. The other analogue, 6"'-deoxy-iGb3-sphinganine, which has a fully saturated lipid backbone, is a valuable glycolipid to determine if the removal of the rigid $E$-alkene on the lipid backbone alters the glycolipid activity. Both glycolipids have been sent to our collaborators, Jamie Rossjohn (Monash University) and Dale Godfrey (University of Melbourne) for testing in iNKT cell assays, as well as crystal structure studies in the context of CD1d and iNKT cells.

In Chapter 5 the synthesis of two novel iGb3 analogues bearing either the dodecanoic acid (iGb3-C12) or the di-unsaturated 11Z,14Z-eicosadienoic acid (iGb3-C20:2) was presented. The synthetic route made use of the common intermediate lactosyl 2-azido-sphingosine, previously described in Chapter 4. The key synthetic steps included the coupling of the terminal galactose sugar, which occurred in high yield, followed by functionalisation of the lipid backbone with the two desired fatty acids. The iGb3 analogues will allow for the study of structure activity relationship and crystal structure analyses, which will provide 
insight into how lipid chain length and unsaturation can influence glycolipid activity.

Taken together, Chapter 4 and Chapter 5 describe an elegant, robust and convergent synthetic route that allows for the generation of two series of iGb3 analogues; namely the terminal sugar series and the $N$-acyl chain series. In the future, it would be valuable to extend the repertoire of these two series to further probe the structure-activity relationships. For the terminal sugar series, it would be interesting to attach D-galactose in a $\beta 1-3$ linkage, instead of the original $\alpha 1-4$ linkage found in $\mathrm{iGb3}$, to assess if the orientation of the terminal sugar affects how it interacts with the CD1d molecule and the TCR of iNKT cells, and subsequently the effect that this has on the quality of iNKT cell activation. In addition, it would also be valuable to substitute the terminal galactose with an $\mathrm{N}$ acetyl galactosamine moiety to generate $\mathrm{iGb3}$ analogues that are akin to gangliosides, which is another class of biologically important glycosphingolipids that contain $N$-acetylgalactosamine attached to a sialylated lactosylceramide. The effect of an $\mathrm{N}$-acetyl group on CD1d binding and subsequent iNKT cell activation could potentially lead to an additional series of biologically active glycosphingolipids.

In the future, it would also be interesting to extend the series of acyl chain homologues by attaching fatty acids of different lengths. Of particular interest would be the saturated C20 acid which, together with the iGb3-C20:2 synthesised in Chapter 5, will provide insight into the influence that rigid double bonds have on the iNKT cell activity. These are just some of the many glycosphingolipids that can be prepared and studied in order to find an optimum glycolipid adjuvant for use in the treatment of diseases, such as cancer. 

APPENDIX 



\section{List of Publications}

A divergent approach to the synthesis of iGb3 sugar and lipid analogues via a lactosyl 2-azido-sphingosine intermediate

Cheng, J. M. H.; Dangerfield, E. M.; Timmer, M. S. M.; Stocker, B. L., Org. Biomol. Chem., 2014, 12, 2729-2736.

Species-specific activity of glycolipid ligands for invariant NKT cells.

Dangerfield, E. M.; Cheng, J. M. H.; Knight, D. A.; Weinkove, R.; Dunbar, P. R.; Hermans, I. F.; Timmer, M. S. M.; Stocker, B. L., ChemBioChem, 2012, 13, 1349-1356.

An improved synthesis of dansylated $\alpha$-galactosylceramide and its use as a fluorescent probe for the monitoring of glycolipid uptake by cells

Cheng, J. M. H.; Chee, S. H.; Knight, D. A.; Acha-Orbea, H.; Hermans, I. F.; Timmer, M. S. M.; Stocker, B. L., Carbohydrate Research, 2011, 346, 914-926.

Endogenous and exogenous CD1-binding glycolipids

Cheng, J. M. H.; Khan, A. K.; Stocker, B. L.; Timmer, M. S. M., International Journal of Carbohydrate Chemistry, 2011, vol. 2011, Article ID 749591, 13 pages.

Rapid synthesis of 1-deoxygalactonojirimycin using a carbamate annulation Timmer, M. S. M.; Dangerfield, E. M.; Cheng, J. M. H.; Gulab, S. A.; Stocker, B. L., Tetrahedron Letters, 2011, 52, 4803-4805.

Methyl 6-azido-6-deoxy- $\alpha$-D-galactoside

Cheng, J. M. H.; Gulab, S. A.; Timmer, M. S. M.; Stocker, B. L.; Gainsford, G. J., Acta Crystallographica Section E, 2011, E67, o1941-1942.

Methyl 6-deoxy-6-iodo- $\alpha$-D-galactoside

Gulab, S. A.; Cheng, J. M. H.; Timmer, M. S. M.; Stocker, B. L.; Gainsford, G. J., Acta Crystallographica Section E, 2010, E66, o1724.

Glycolipids and CD1: The crossroad between chemistry and immunology Khan, A. K.; Cheng, J. M. H.; Timmer, M. S. M.; Stocker, B. L., NZIC Chemistry in New Zealand, 2010, 74, 57-62. 



\section{${ }^{1} \mathrm{H} \&{ }^{13} \mathrm{C}$ NMR Spectra \\ Chapter 2}


1-O-(2,3,4-tri-O-benzyl-6-deoxy-6-[ $N$-(5-[dimethylamino]napth-1-ylsulfonyl)amido]- $\alpha$-D-galactopyranosyl)-2-hexacosanoylamido-Dribo-octadecane-1,3,4-triol (50) ${ }^{1} \mathrm{H} \mathrm{NMR}, \mathrm{CDCl}_{3}, 500 \mathrm{MHz}$

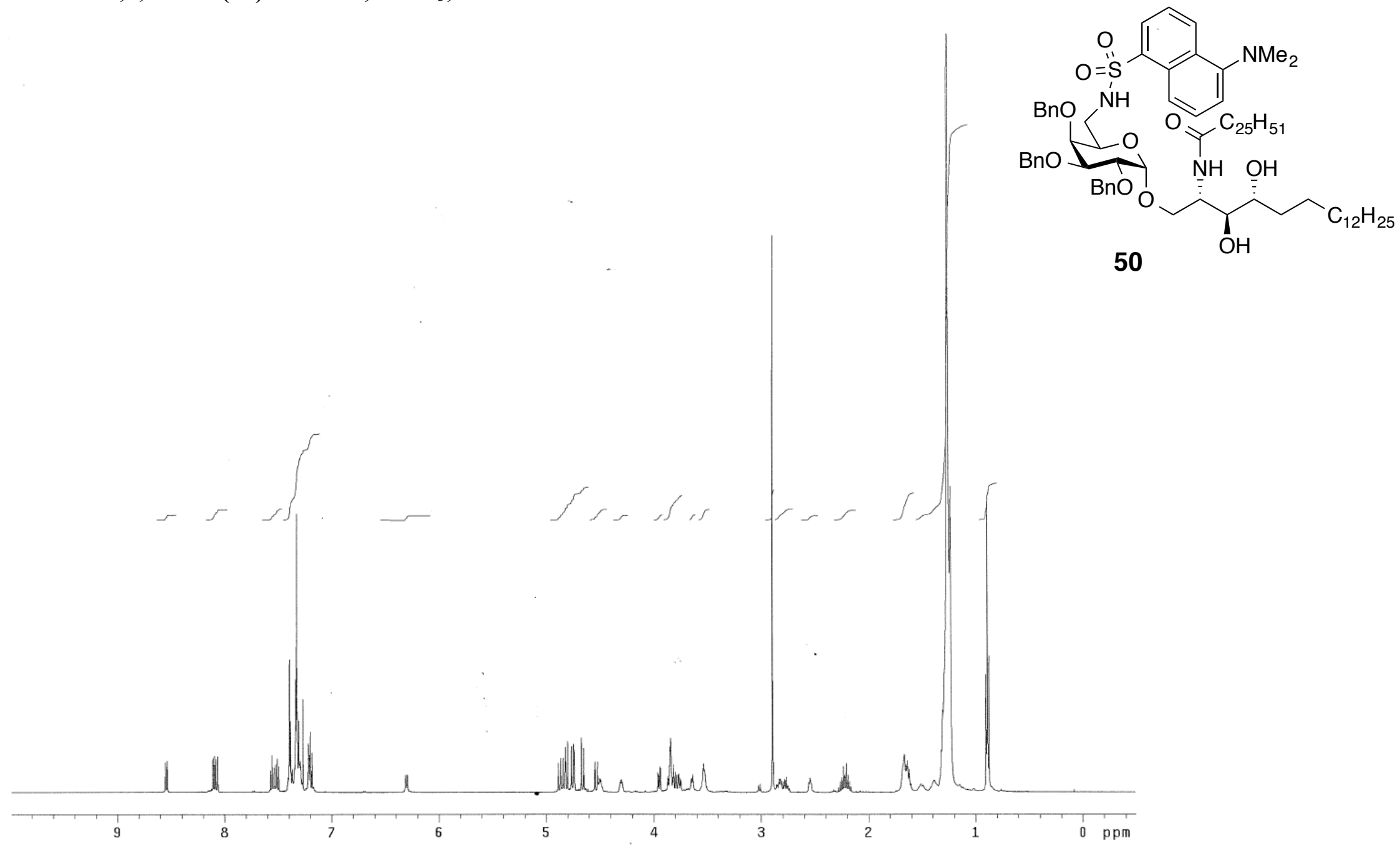


1-O-(2,3,4-tri- $O$-benzyl-6-deoxy-6-[ $N$-(5-[dimethylamino]napth-1-ylsulfonyl)amido]- $\alpha$-D-galactopyranosyl)-2-hexacosanoylamido-Dribo-octadecane-1,3,4-triol (50) ${ }^{13} \mathrm{C} \mathrm{NMR,} \mathrm{CDCl}_{3}, 125 \mathrm{MHz}$

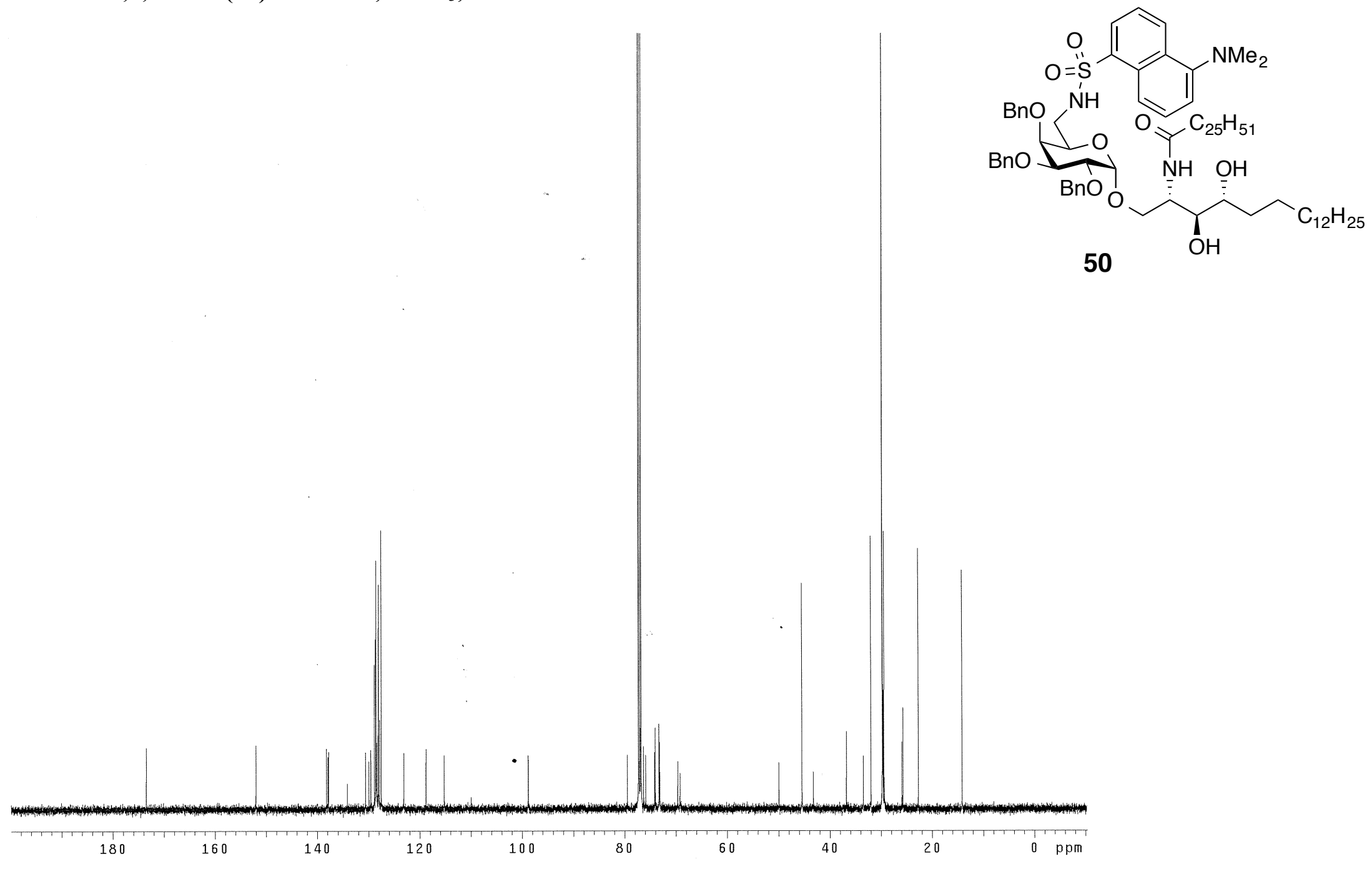


1-O-[6-deoxy-6-[N-(5-[dimethylamino]napth-1-ylsulfonyl)amido]- $\alpha$-D-galactopyranosyl]-2-hexacosanoylamido-D-ribo-octadecane1,3,4-triol (2) ${ }^{1} \mathrm{H}$ NMR, pyridine- $\mathrm{d}_{5}, 600 \mathrm{MHz}$

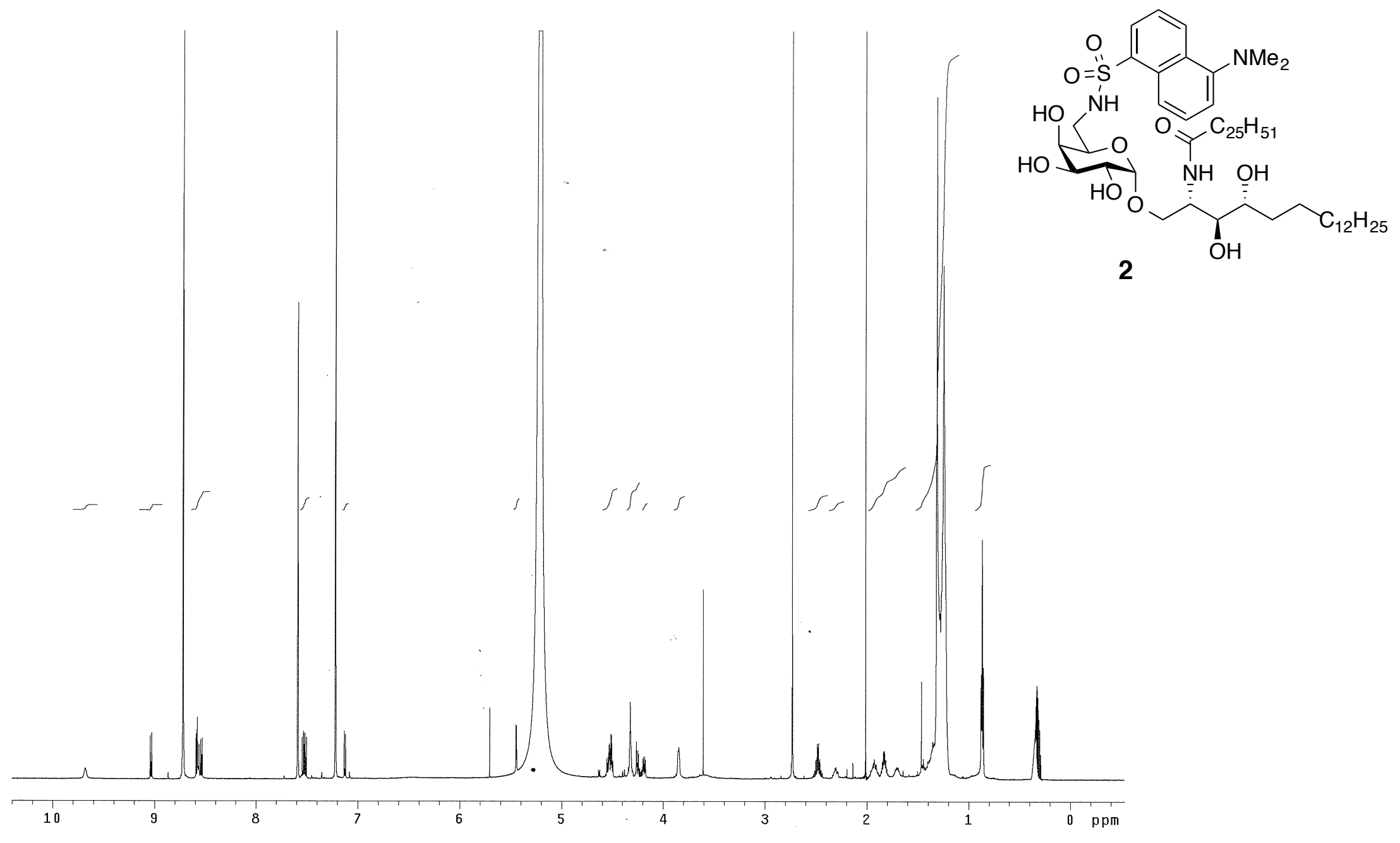


1-O-[6-deoxy-6-[N-(5-[dimethylamino]napth-1-ylsulfonyl)amido]- $\alpha$-D-galactopyranosyl]-2-hexacosanoylamido-D-ribo-octadecane1,3,4-triol (2) ${ }^{13} \mathrm{C}$ NMR, pyridine- $\mathrm{d}_{5}, 150 \mathrm{MHz}$

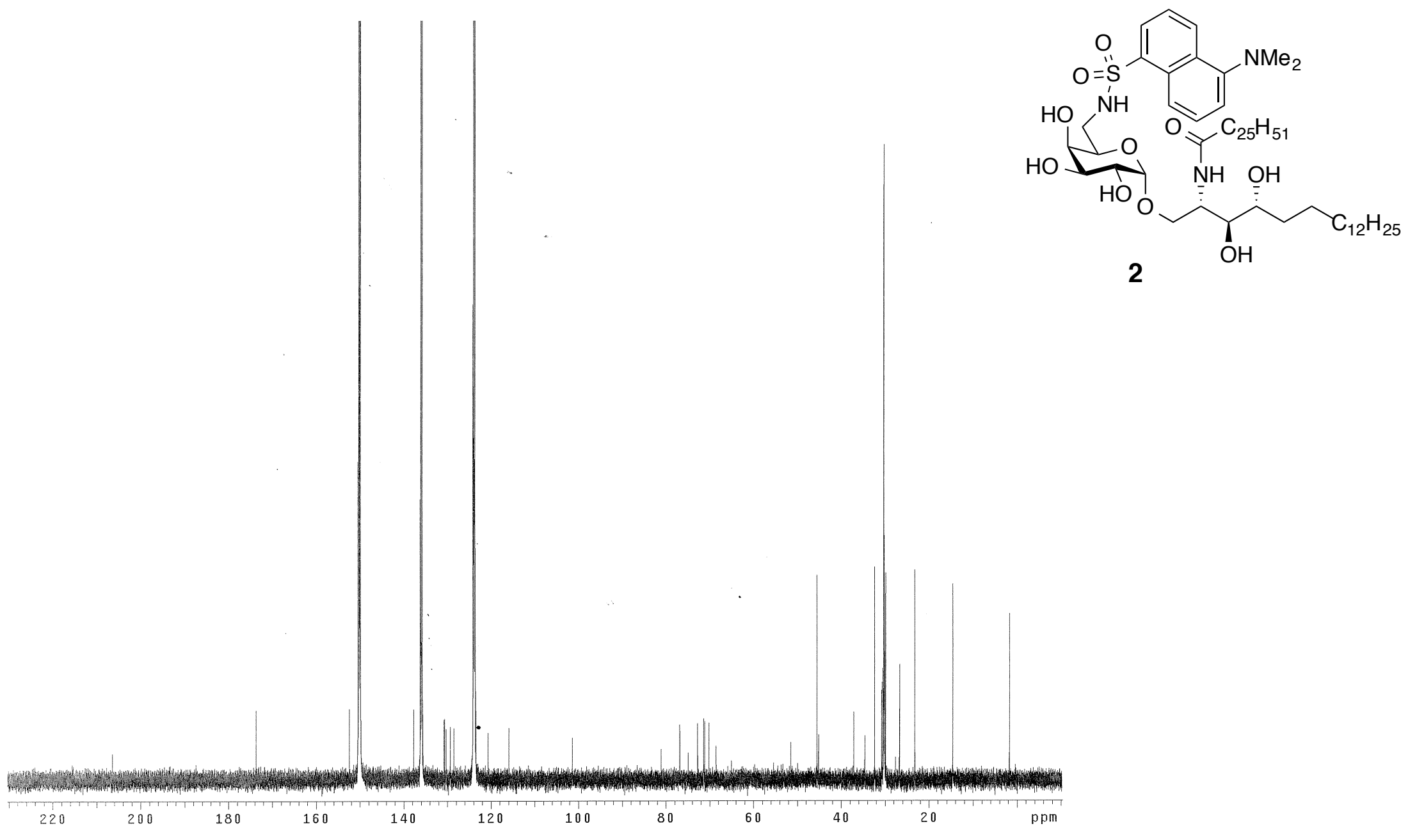




\section{${ }^{1} \mathrm{H} \&{ }^{13} \mathrm{C}$ NMR Spectra \\ Chapter 3}


6-Azido-2,3,4-tri- $\boldsymbol{O}$-benzyl-6-deoxy- $\boldsymbol{\alpha}$-D-galactose (59) ${ }^{1} \mathrm{H} \mathrm{NMR}, \mathrm{CDCl}_{3}, 500 \mathrm{MHz}$

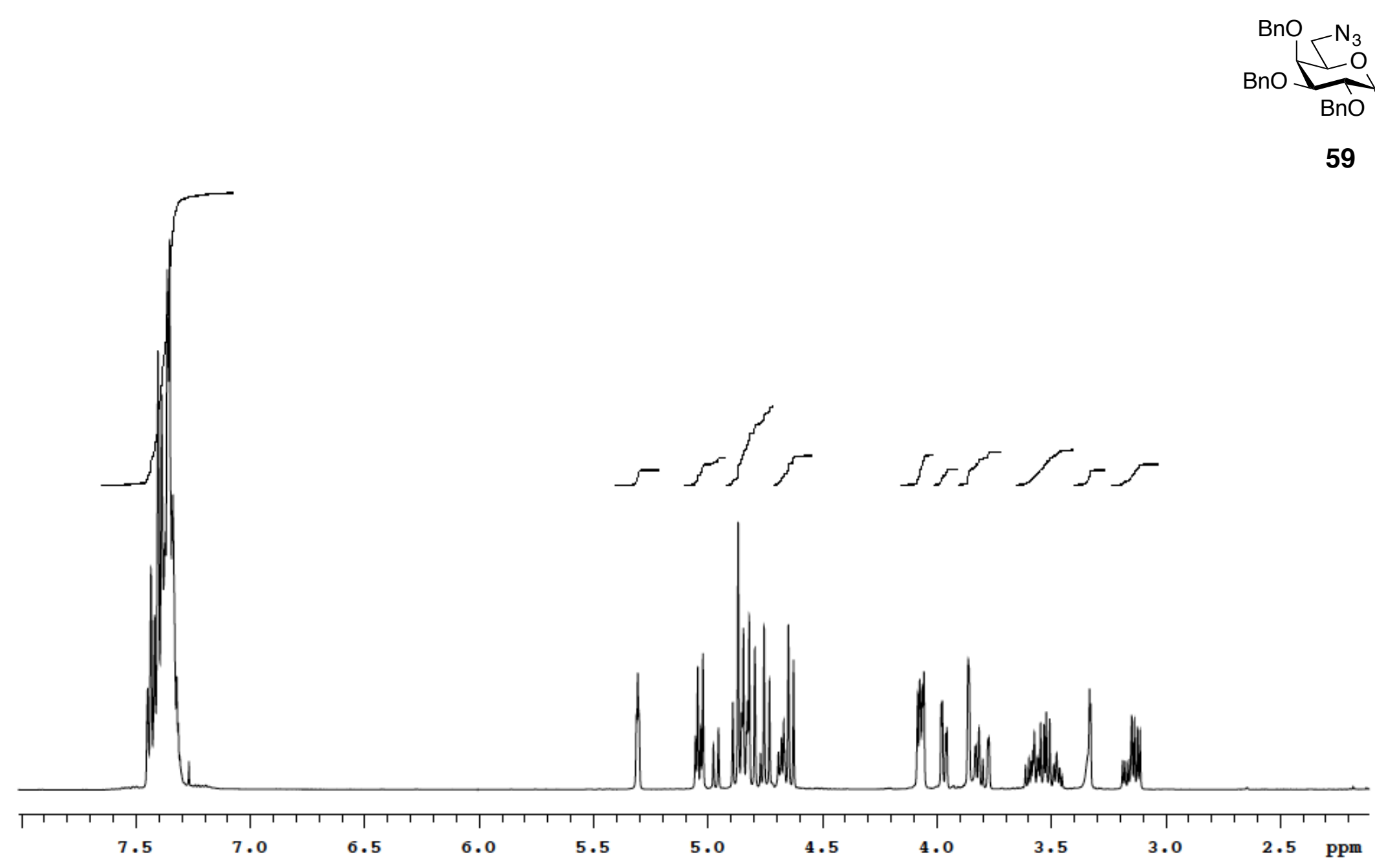


6-Azido-2,3,4-tri- $\boldsymbol{O}$-benzyl-6-deoxy- $\boldsymbol{\alpha}$-D-galactose (59) ${ }^{13} \mathrm{C} \mathrm{NMR,} \mathrm{CDCl}_{3}, 125 \mathrm{MHz}$

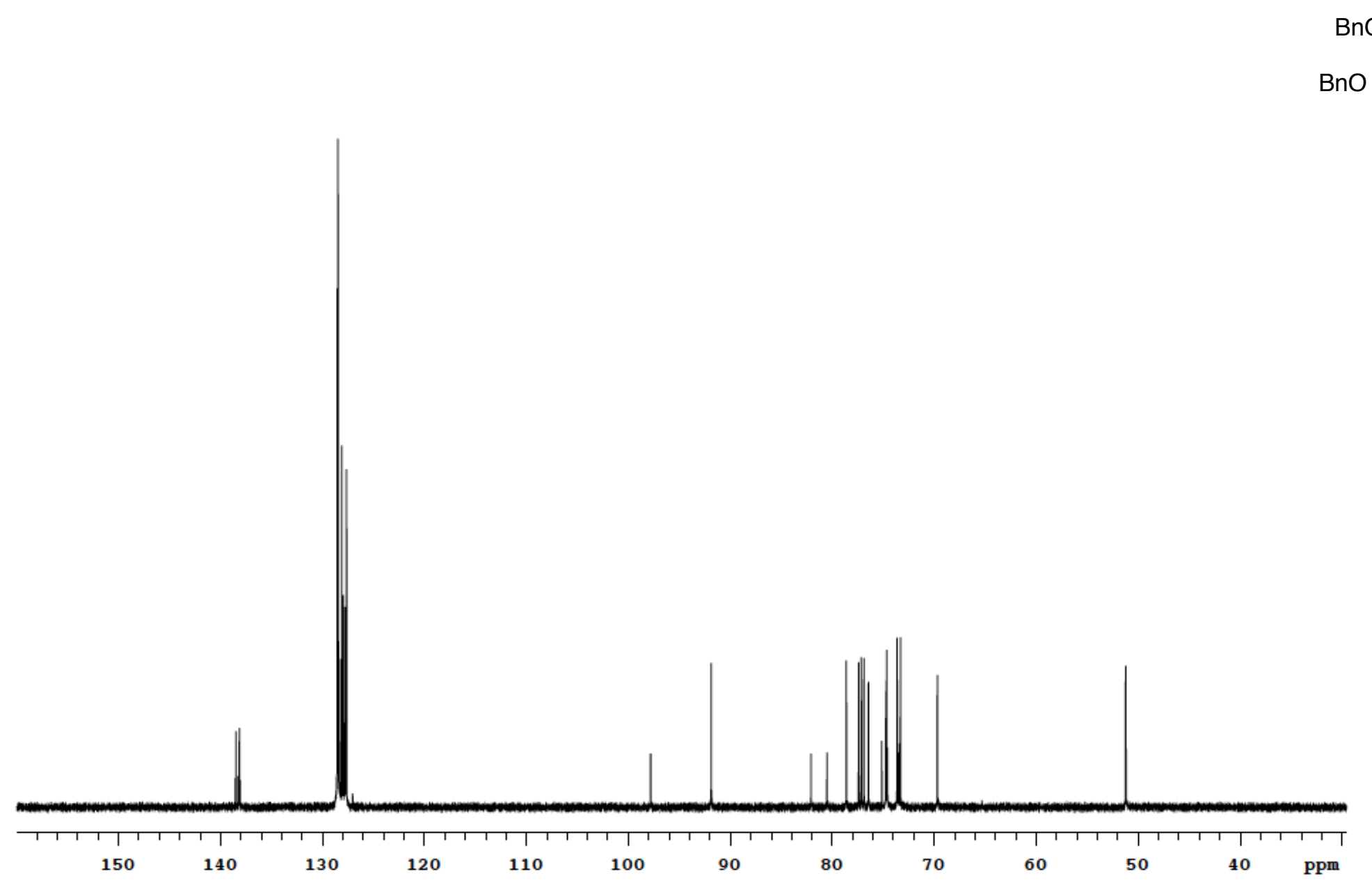


$\boldsymbol{O}$-(6-Azido-2,3,4-tri- $\boldsymbol{O}$-benzyl-6-deoxy- $\boldsymbol{\alpha}$-D-galactosyl) trichloroacetimidate (56) ${ }^{1} \mathrm{H} \mathrm{NMR,} \mathrm{CDCl}_{3}, 500 \mathrm{MHz}$

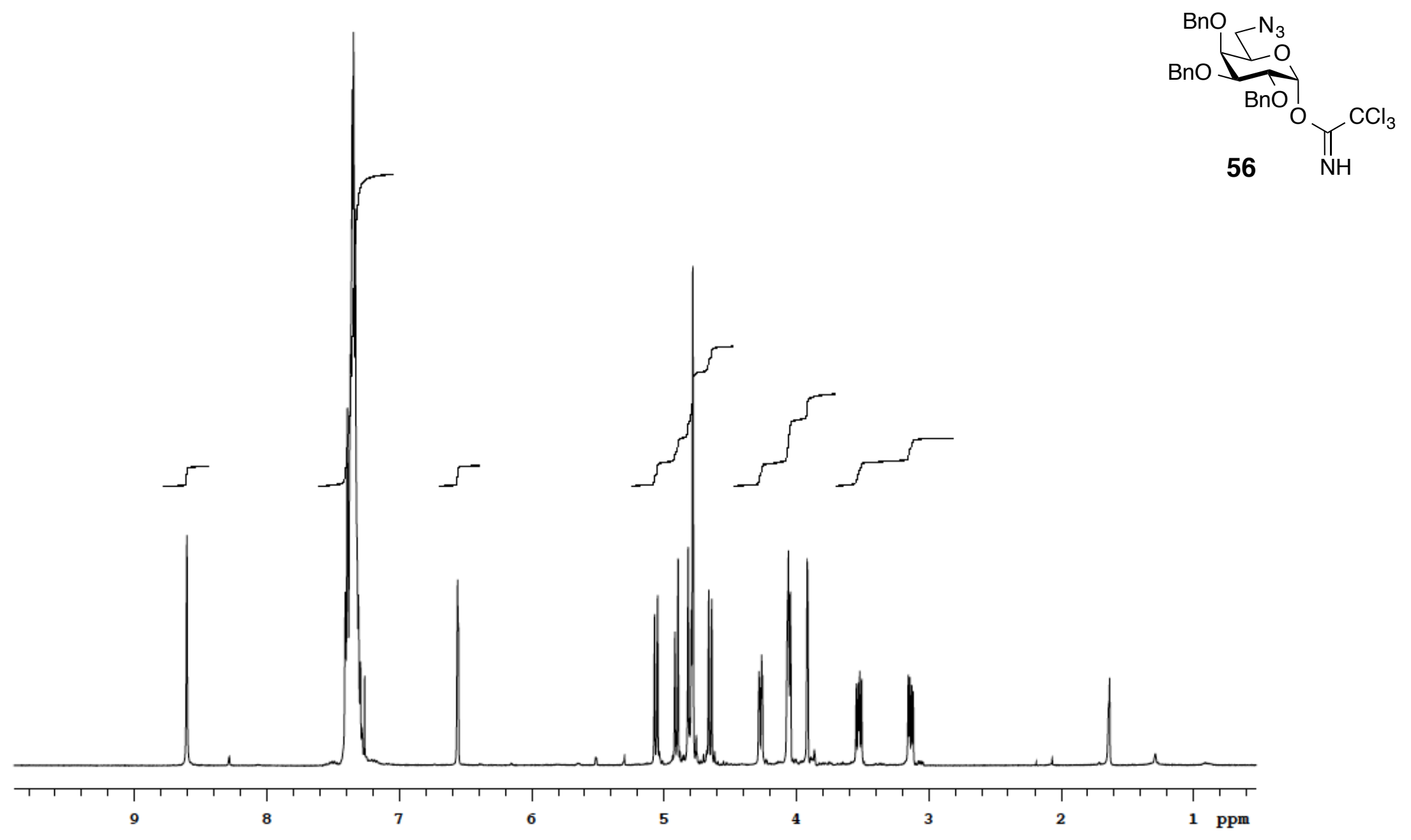


$\boldsymbol{O}$-(6-Azido-2,3,4-tri- $\boldsymbol{O}$-benzyl-6-deoxy- $\boldsymbol{\alpha}$-D-galactosyl) trichloroacetimidate (56) ${ }^{13} \mathrm{C} \mathrm{NMR,} \mathrm{CDCl}_{3}, 125 \mathrm{MHz}$

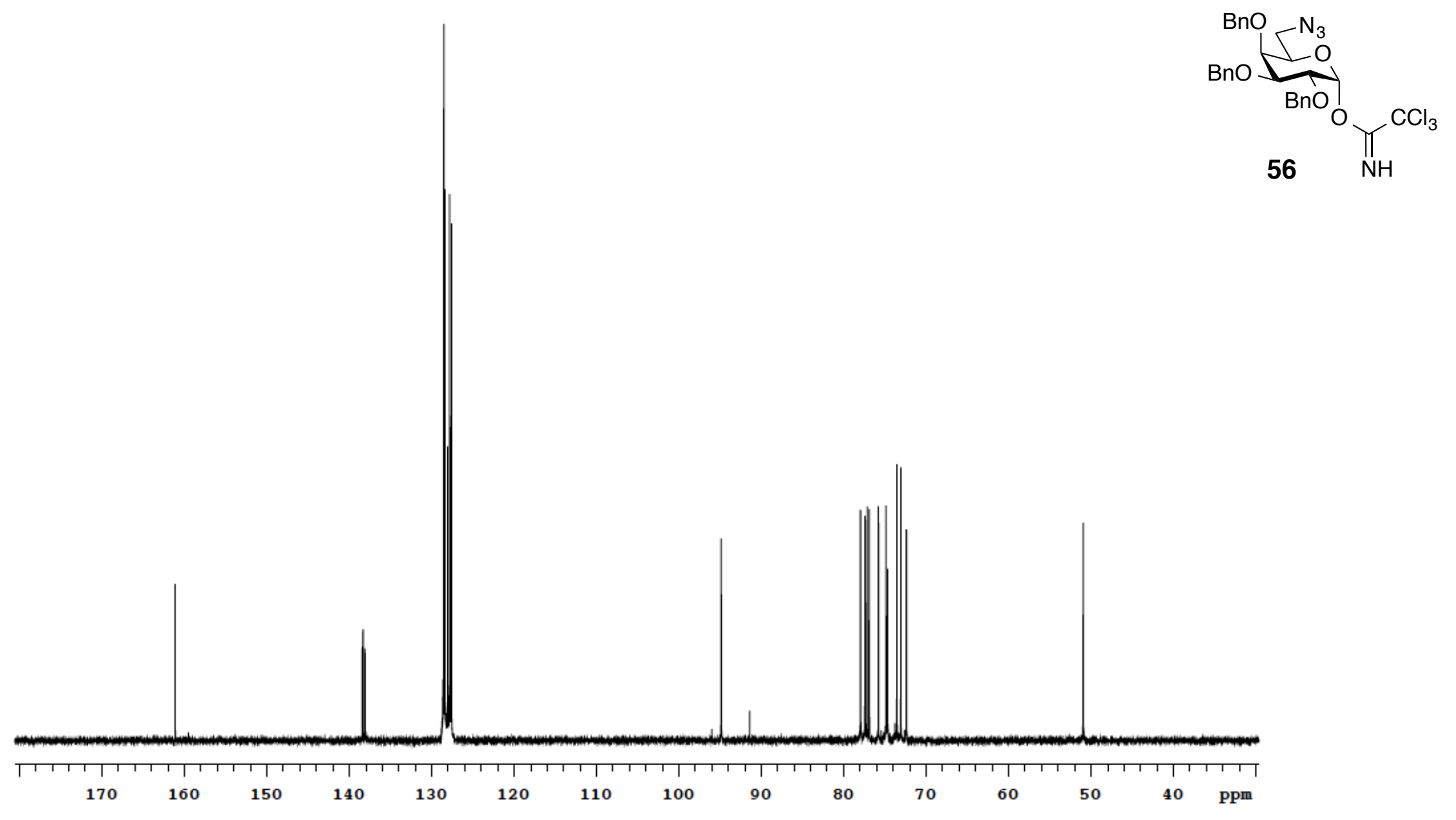


4-(4,4-Difluoro-1,3,5,7-tetramethyl-3a,4a-diaza-4-bora-s-indacen-8-yl)benzoic acid (52) ${ }^{1} \mathrm{H}$ NMR, $\mathrm{CD}_{3} \mathrm{OD}, 500 \mathrm{MHz}$

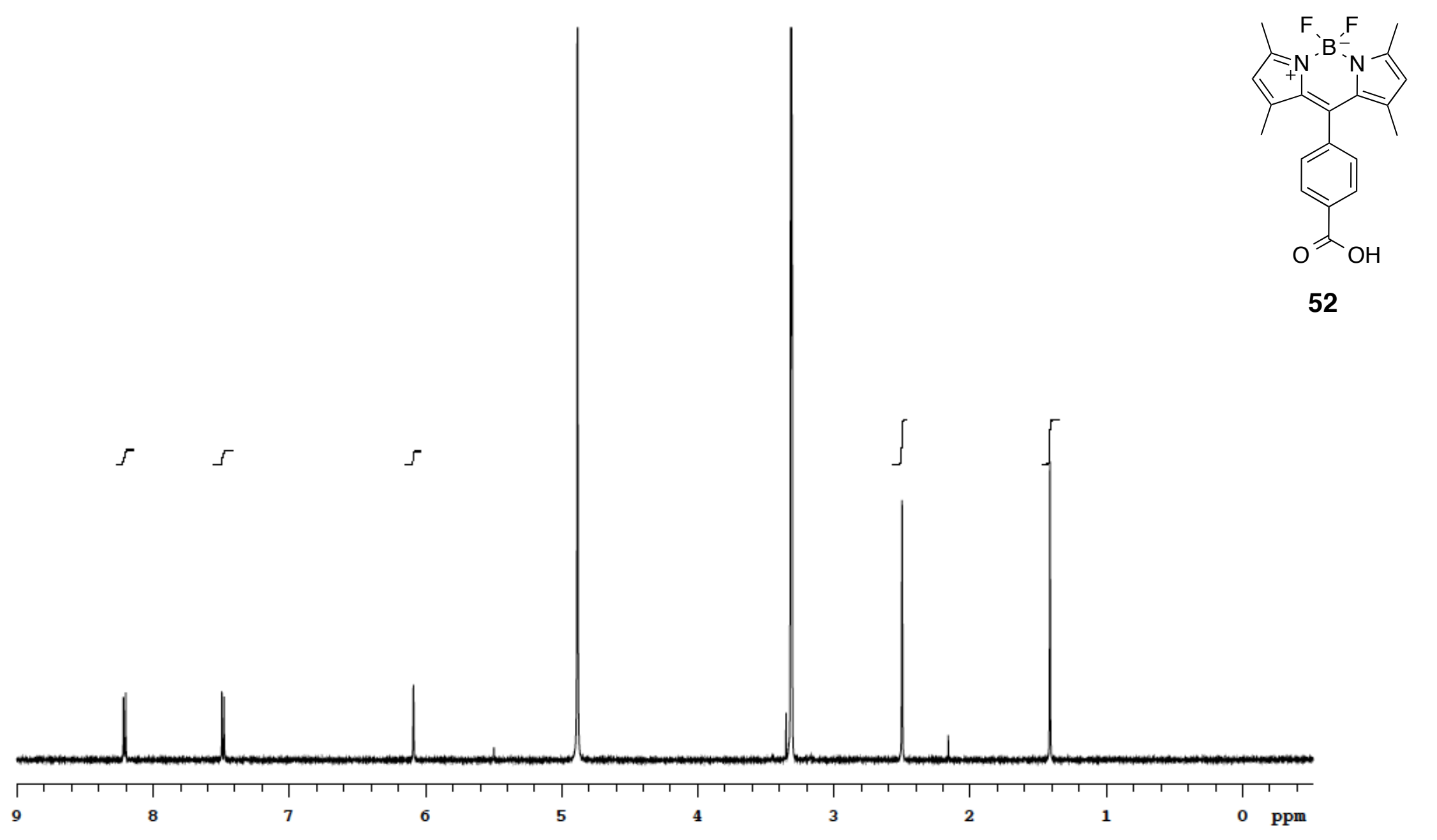


4-(4,4-Difluoro-1,3,5,7-tetramethyl-3a,4a-diaza-4-bora-s-indacen-8-yl)benzoic acid (52) ${ }^{13} \mathrm{C} \mathrm{NMR}, \mathrm{CD}_{3} \mathrm{OD}, 125 \mathrm{MHz}$
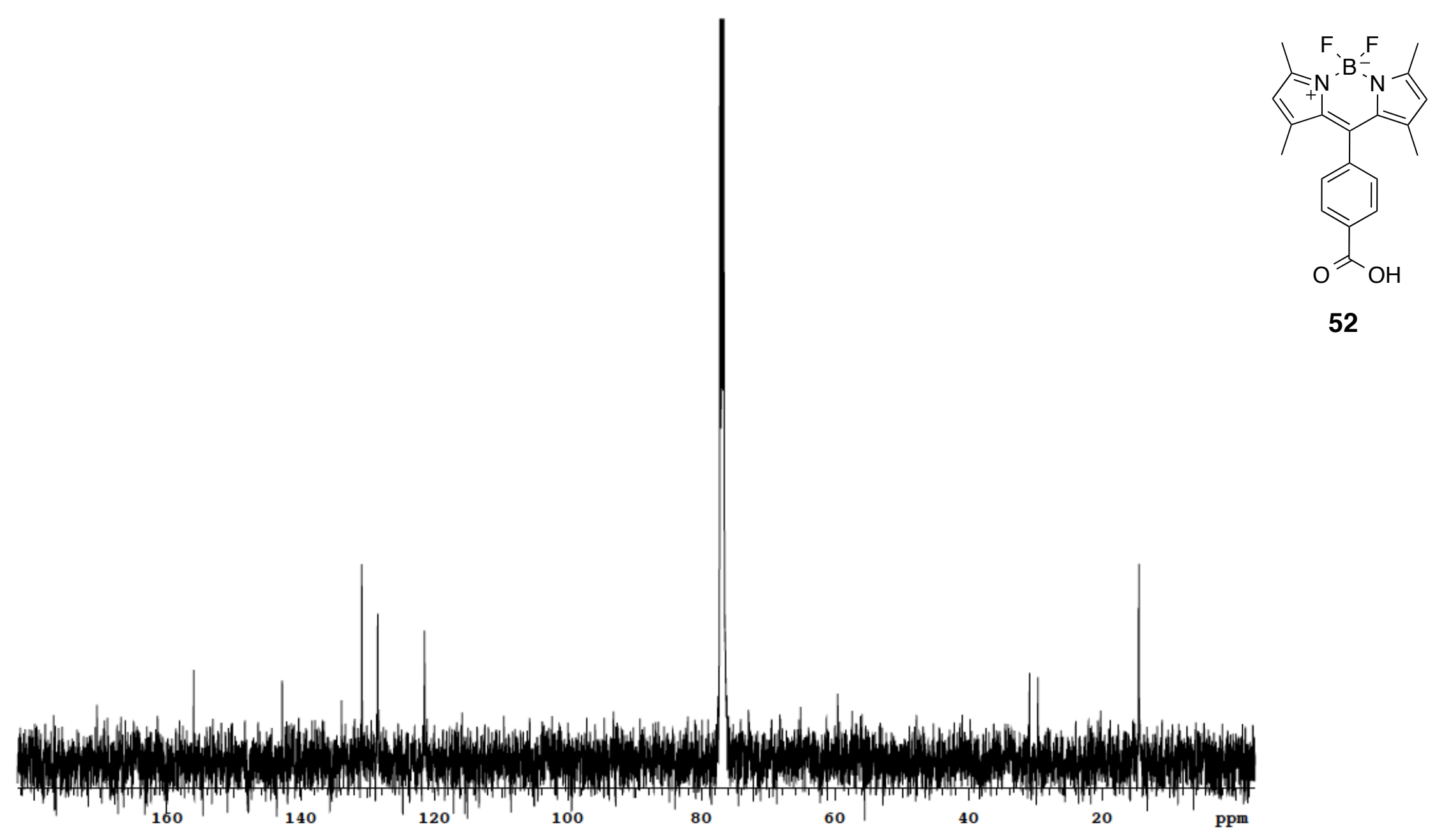
2,5-Dioxopyrrolidin-1-yl-4-(4,4-difluoro-1,3,5,7-tetramethyl-3a,4a-diaza-4-bora-s-indacen-8-yl)benzoate (70) ${ }^{1} \mathrm{H} \mathrm{NMR}, \mathrm{CDCl}_{3}, 500 \mathrm{MHz}$

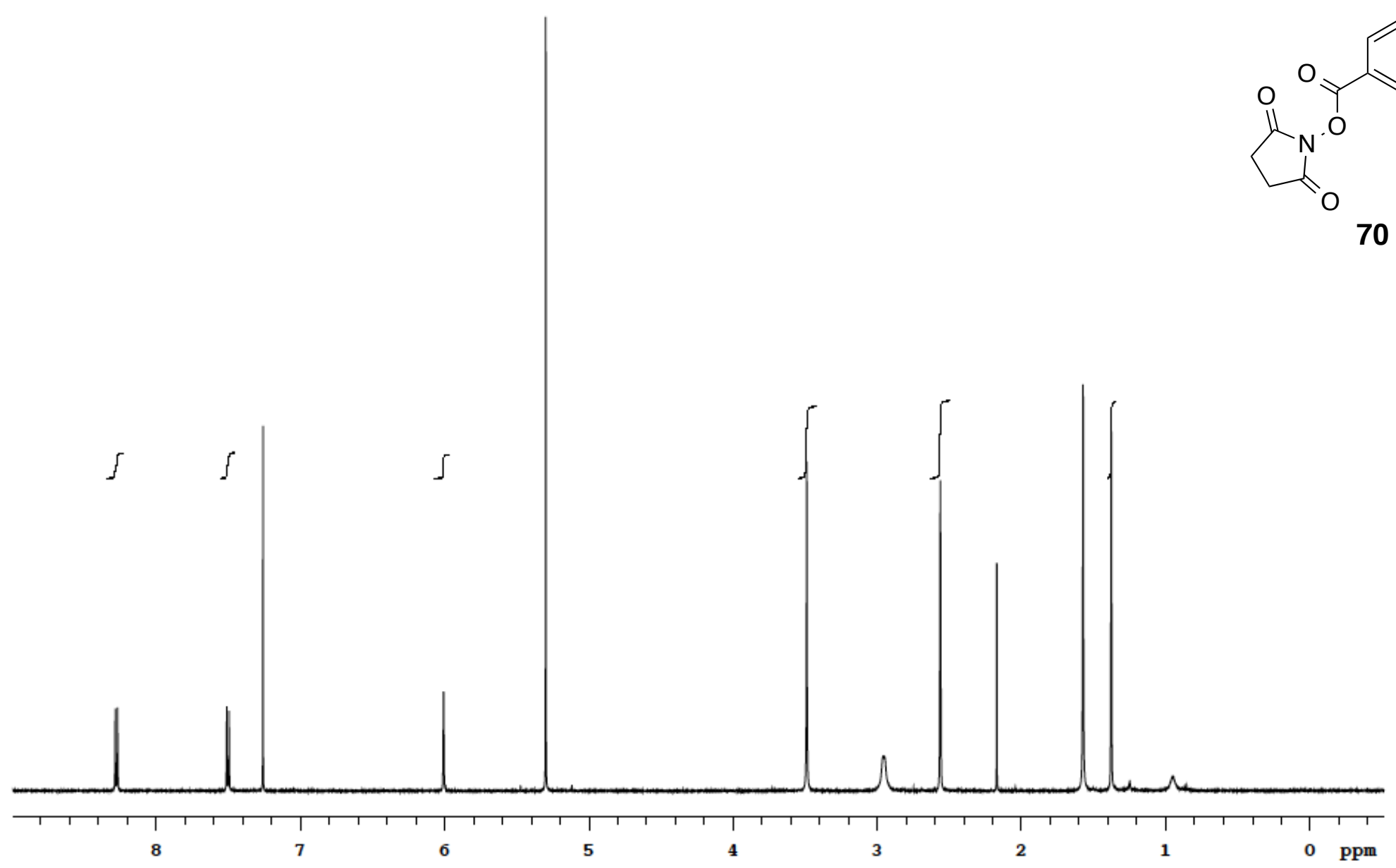


2,5-Dioxopyrrolidin-1-yl-4-(4,4-difluoro-1,3,5,7-tetramethyl-3a,4a-diaza-4-bora-s-indacen-8-yl)benzoate (70)

${ }^{13} \mathrm{C} \mathrm{NMR}, \mathrm{CDCl}_{3}, 125 \mathrm{MHz}$
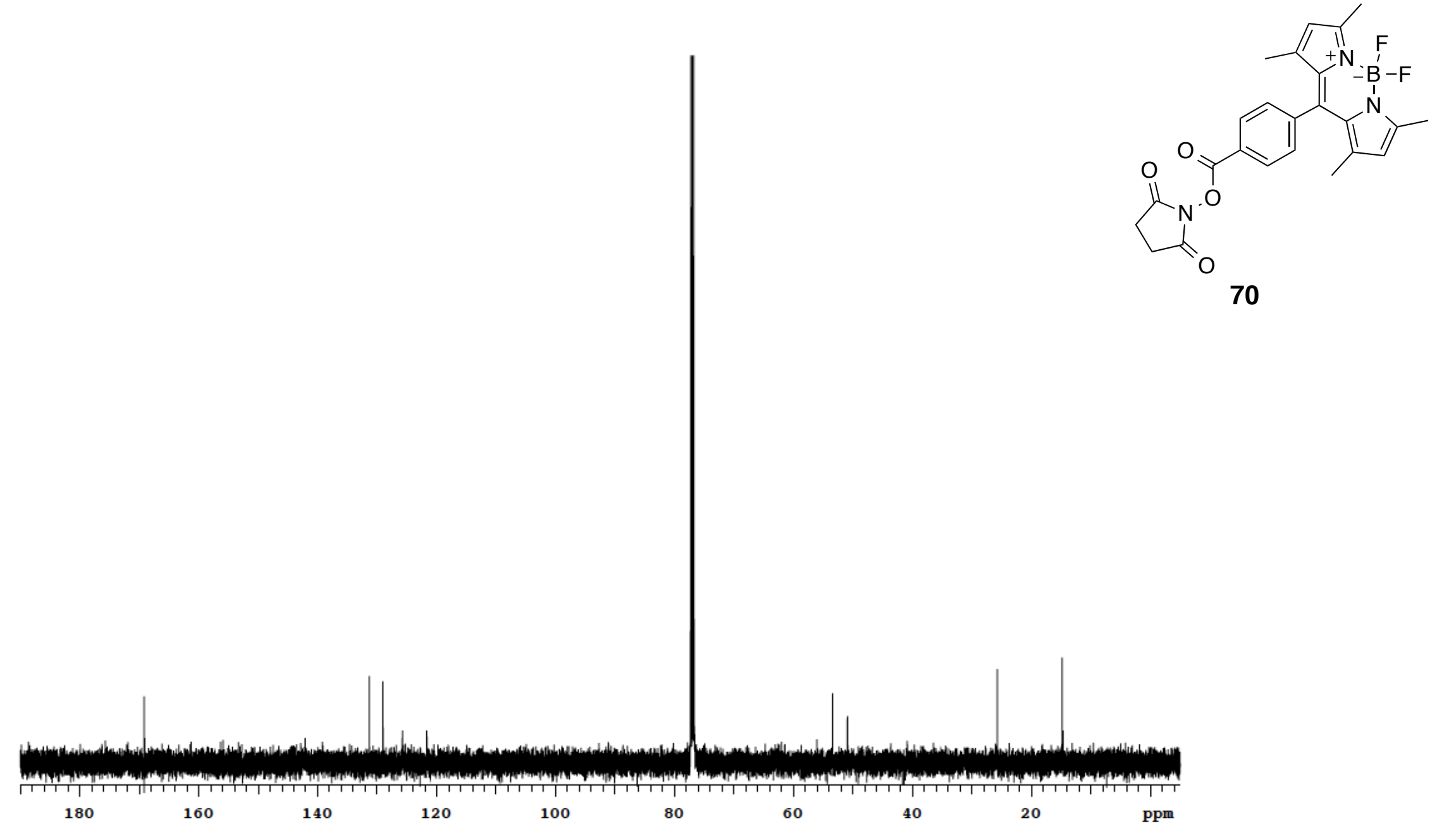
(2S,3S,4R)-2-Hexacosanoylamido-D-ribo-octadecane-1,3,4-triol (67) ${ }^{1} \mathrm{H} \mathrm{NMR}, 10 \% \mathrm{CD}_{3} \mathrm{OD}$ in $\mathrm{CDCl}_{3}, 300 \mathrm{MHz}$

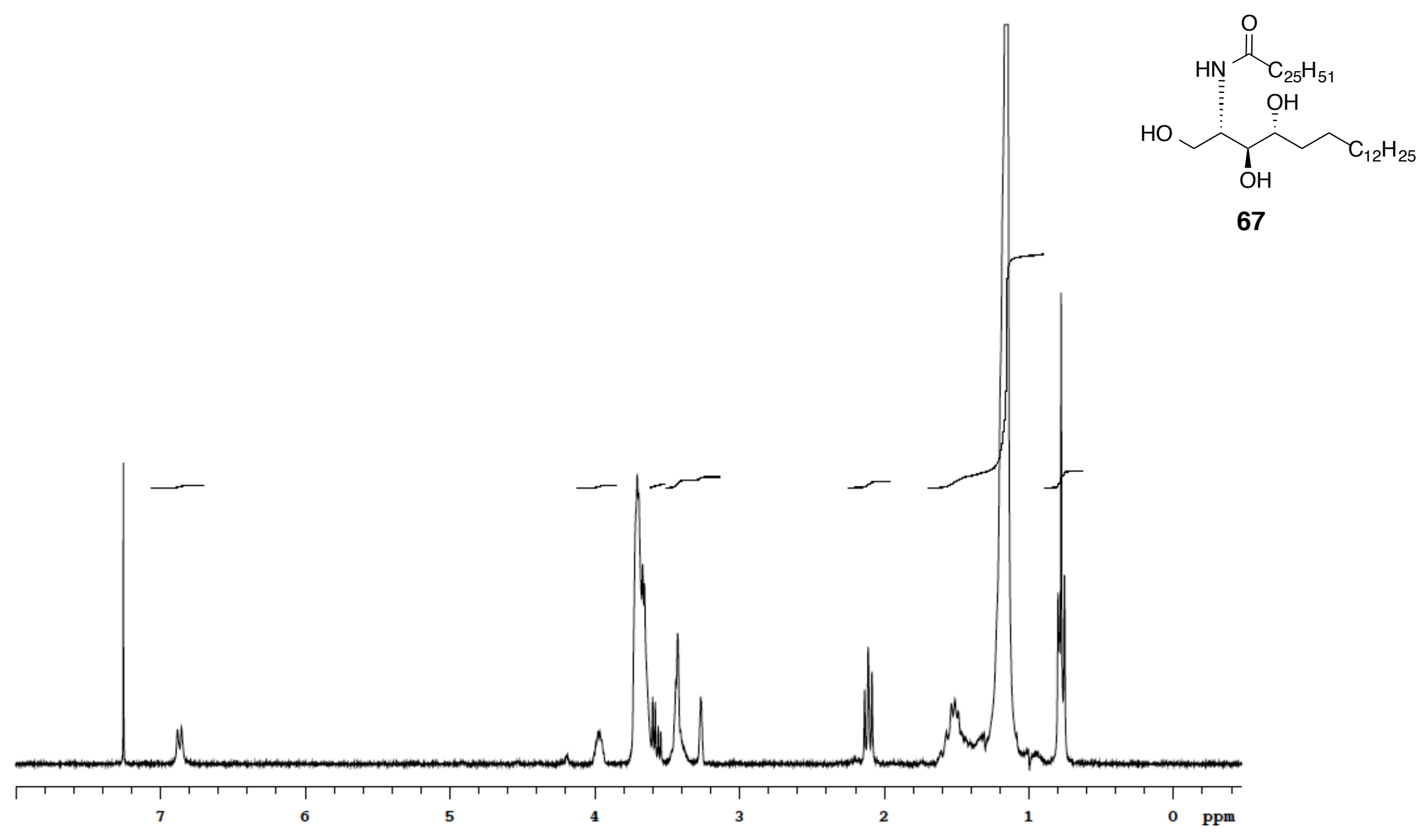


(2S,3S,4R)-2-Hexacosanoylamido-D-ribo-octadecane-1,3,4-triol (67) ${ }^{13} \mathrm{C} \mathrm{NMR,} \mathrm{10 \%} \mathrm{CD}_{3} \mathrm{OD}$ in $\mathrm{CDCl}_{3}, 125 \mathrm{MHz}$

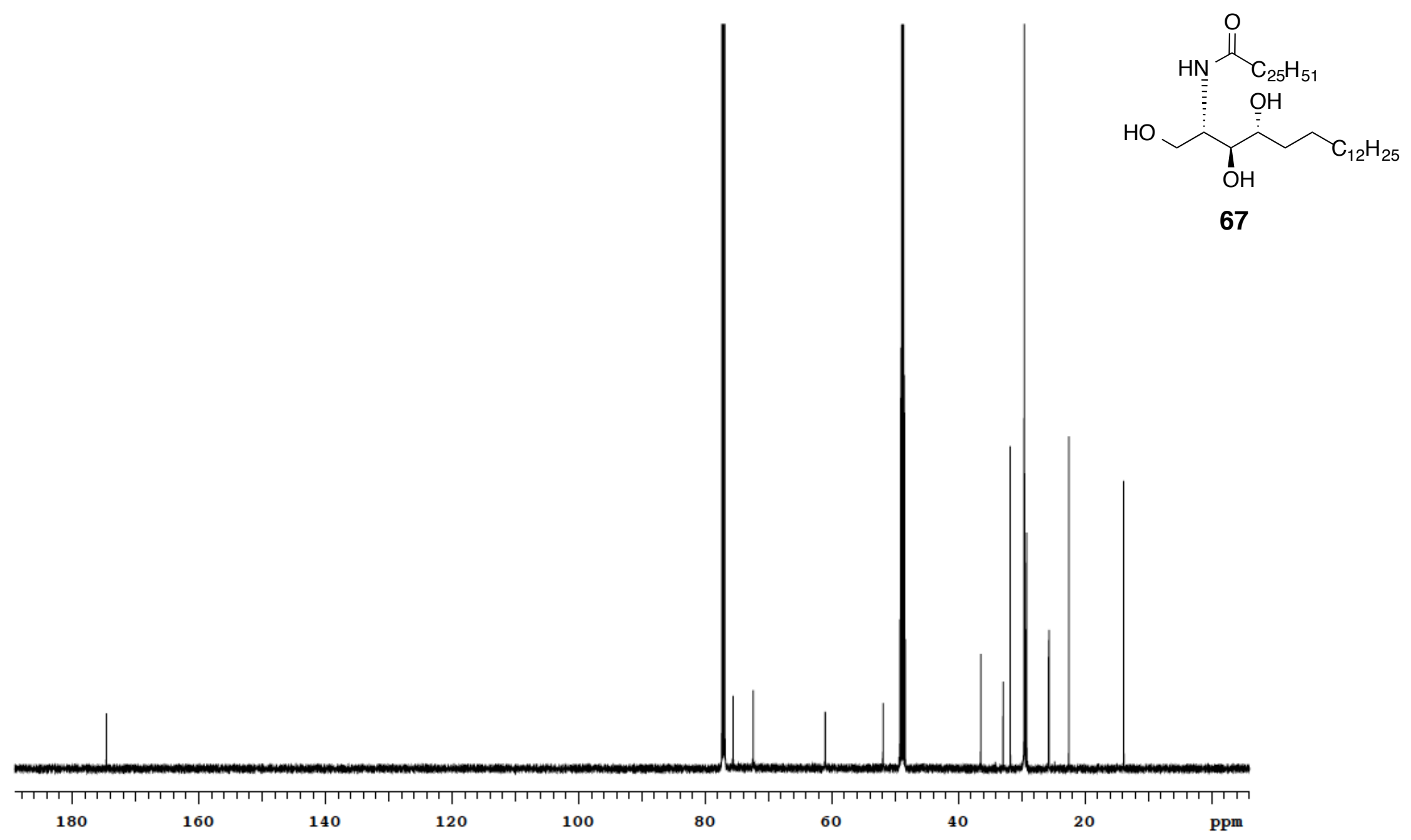


(2S,3S,4R)-2-Hexacosanoylamido-1-triphenylmethoxy-octadecane-3,4-diol (68) ${ }^{1} \mathrm{H} \mathrm{NMR}^{\mathrm{CDCl}} \mathrm{CD}_{3}, 500 \mathrm{MHz}$

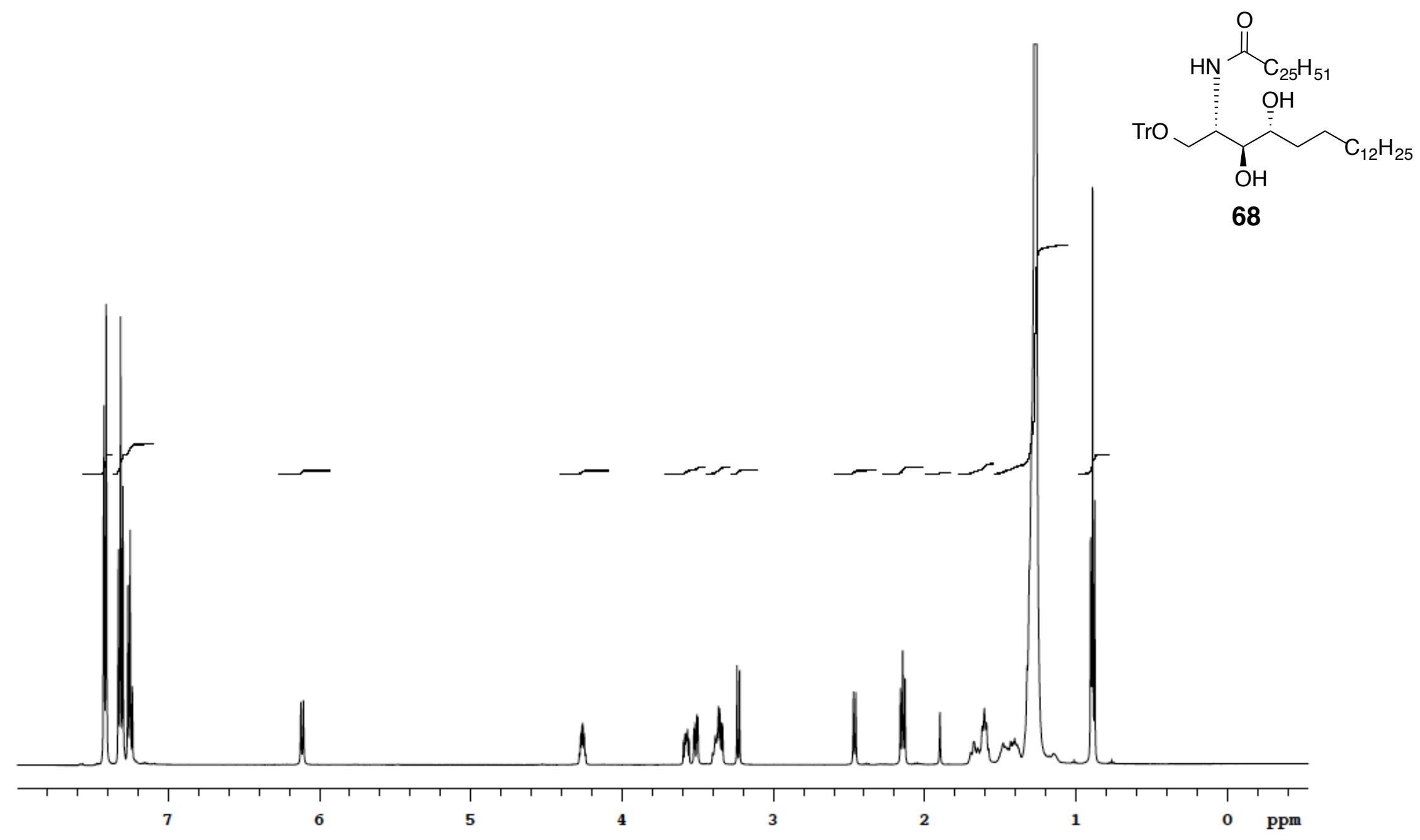


(2S,3S,4R)-2-Hexacosanoylamido-1-triphenylmethoxy-octadecane-3,4-diol (68) ${ }^{13} \mathrm{C} \mathrm{NMR}^{\mathrm{CDCl}} \mathrm{CD}_{3}, 125 \mathrm{MHz}$

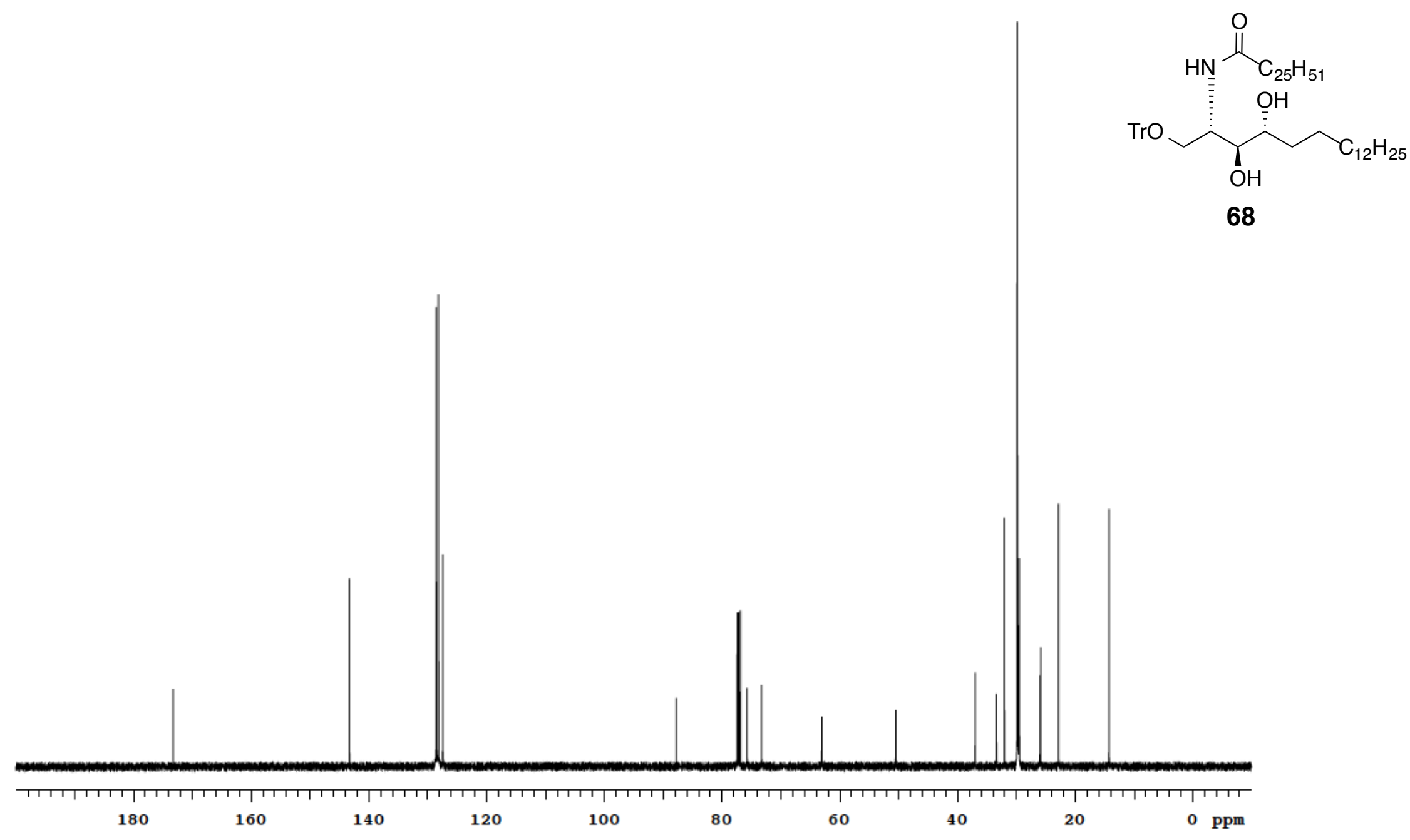


(2S,3S,4R)-3,4-Dibenzoyloxy-2-hexacosanoylamido-1-triphenylmethyoxy-octadecane ${ }^{1} \mathrm{H} \mathrm{NMR,} \mathrm{CDCl}_{3}, 500 \mathrm{MHz}$

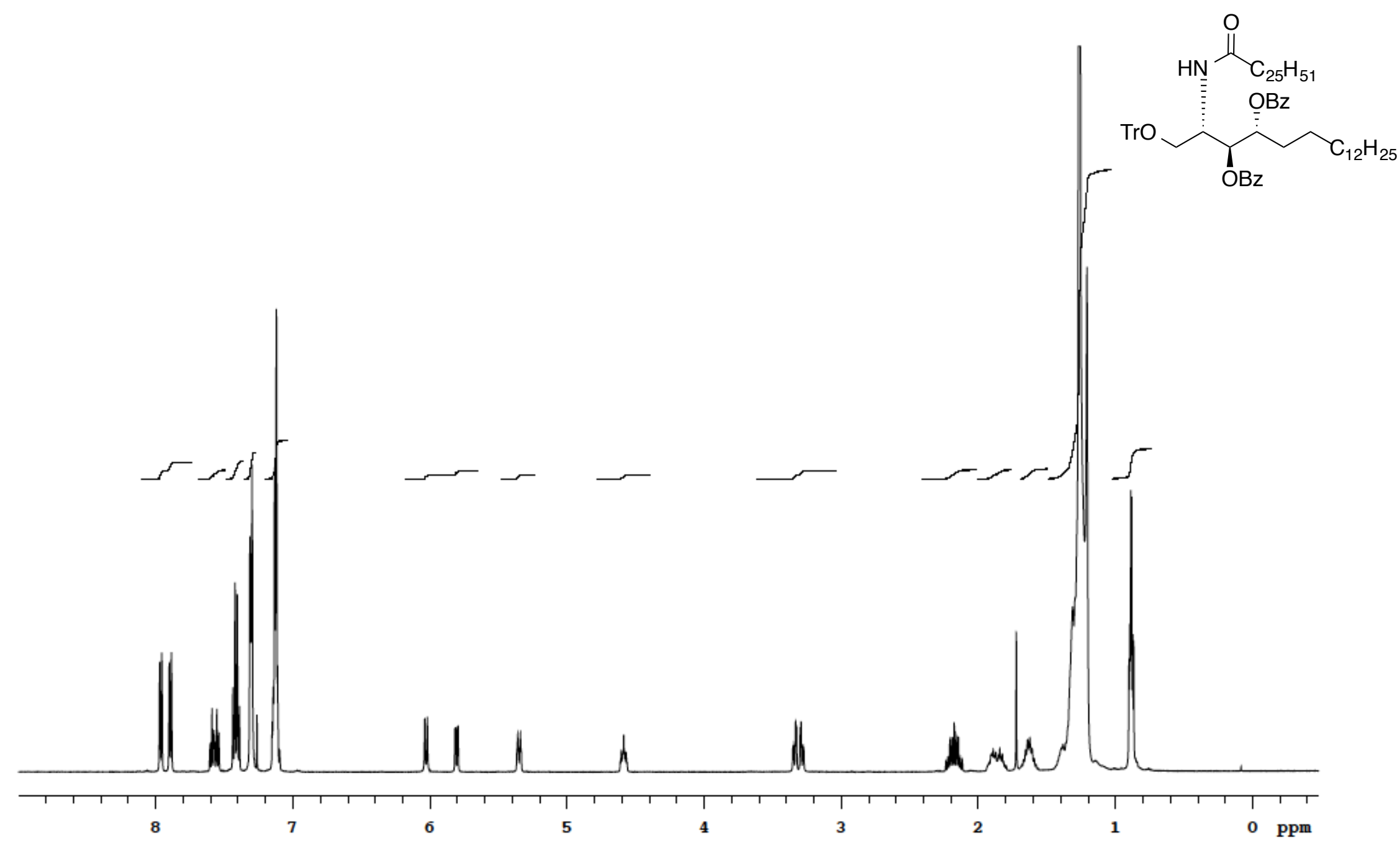


(2S,3S,4R)-3,4-Dibenzoyloxy-2-hexacosanoylamido-1-triphenylmethyoxy-octadecane ${ }^{13} \mathrm{C} \mathrm{NMR,} \mathrm{CDCl}_{3}, 125 \mathrm{MHz}$

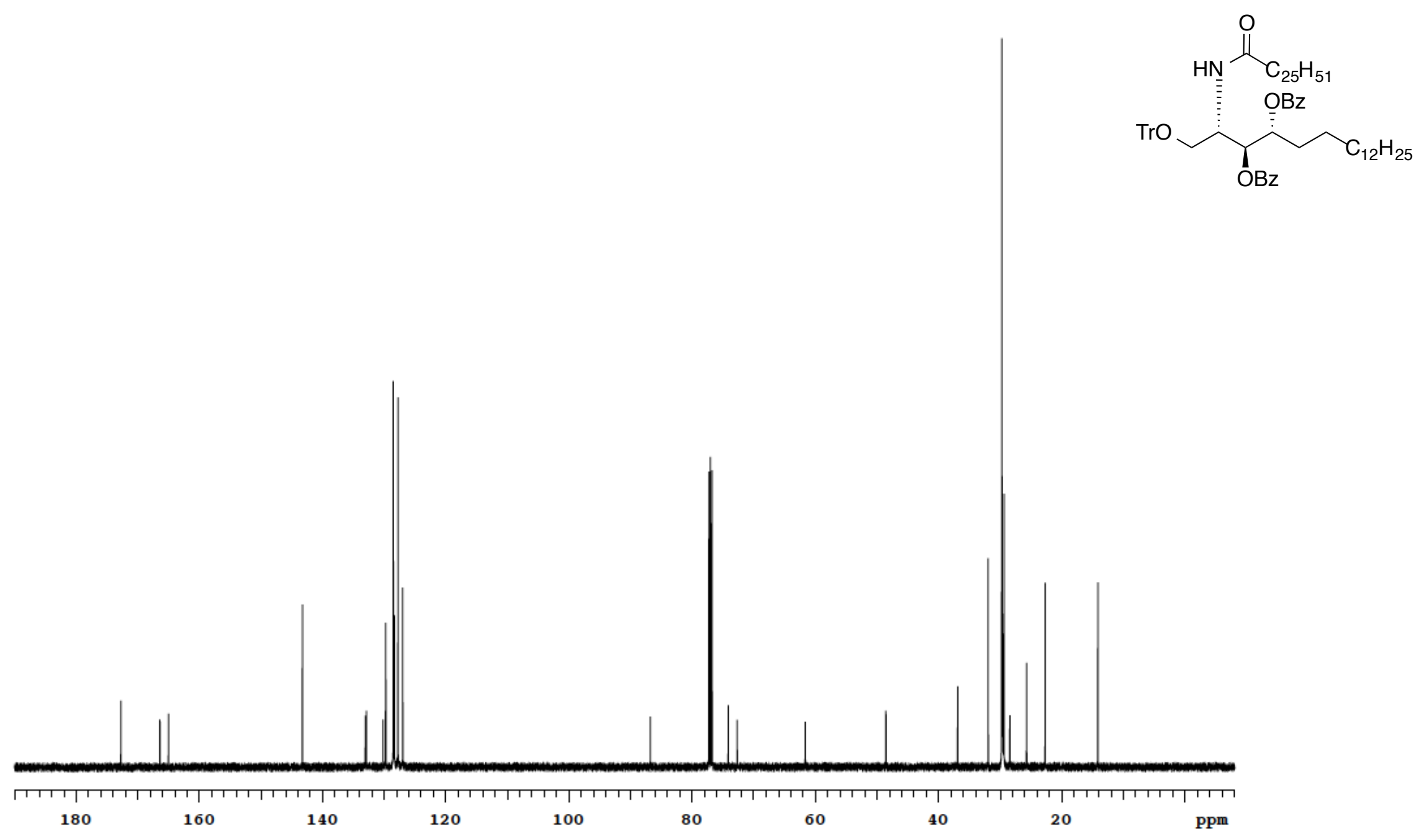


(2S,3S,4R)-3,4-Dibenzoyloxy-2-hexacosanoylamido-octadecan-1-ol (57) ${ }^{1} \mathrm{H} \mathrm{NMR}, \mathrm{CDCl}_{3}, 500 \mathrm{MHz}$

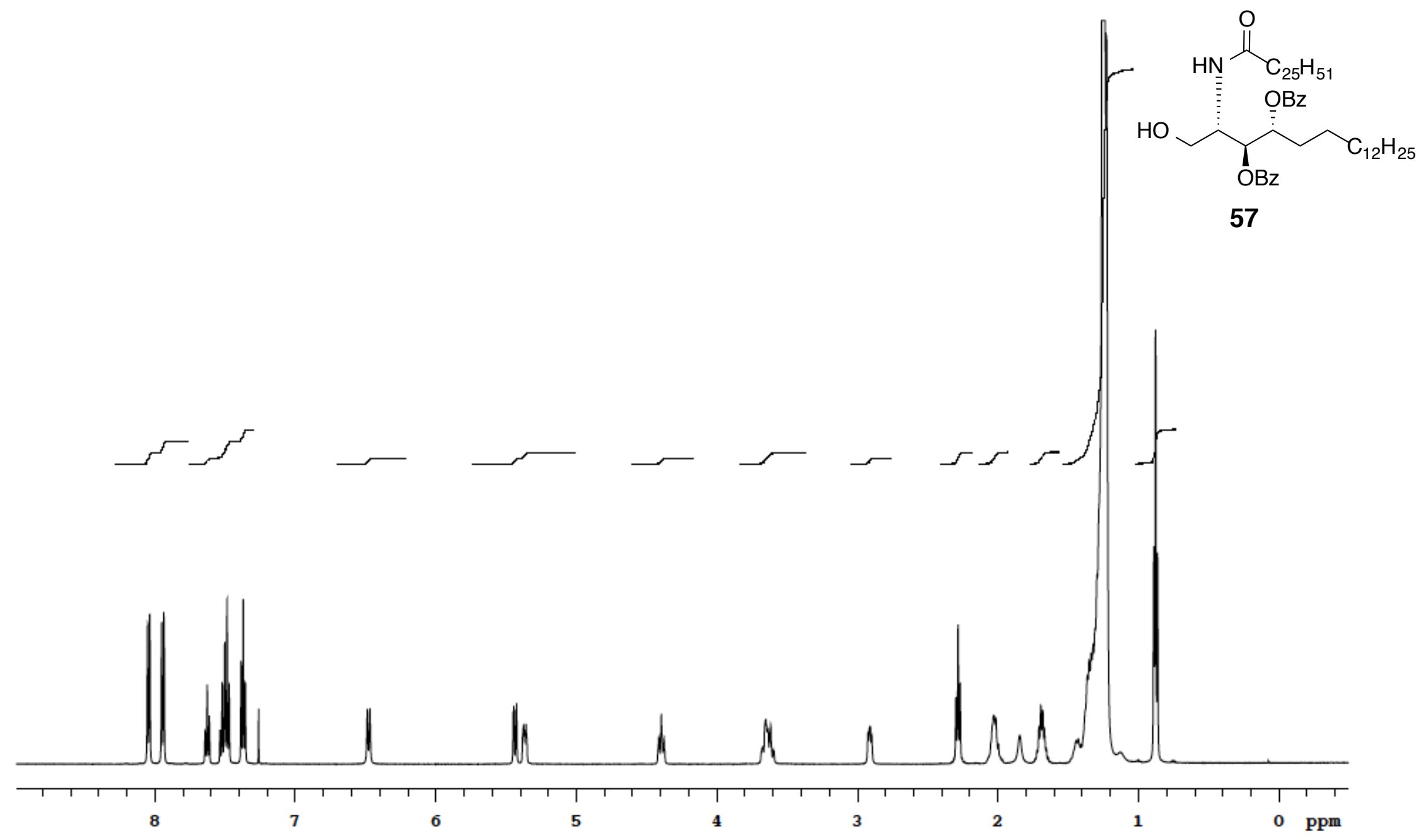


(2S,3S,4R)-3,4-Dibenzoyloxy-2-hexacosanoylamido-octadecan-1-ol (57) ${ }^{13} \mathrm{C} \mathrm{NMR,} \mathrm{CDCl}_{3}, 125 \mathrm{MHz}$

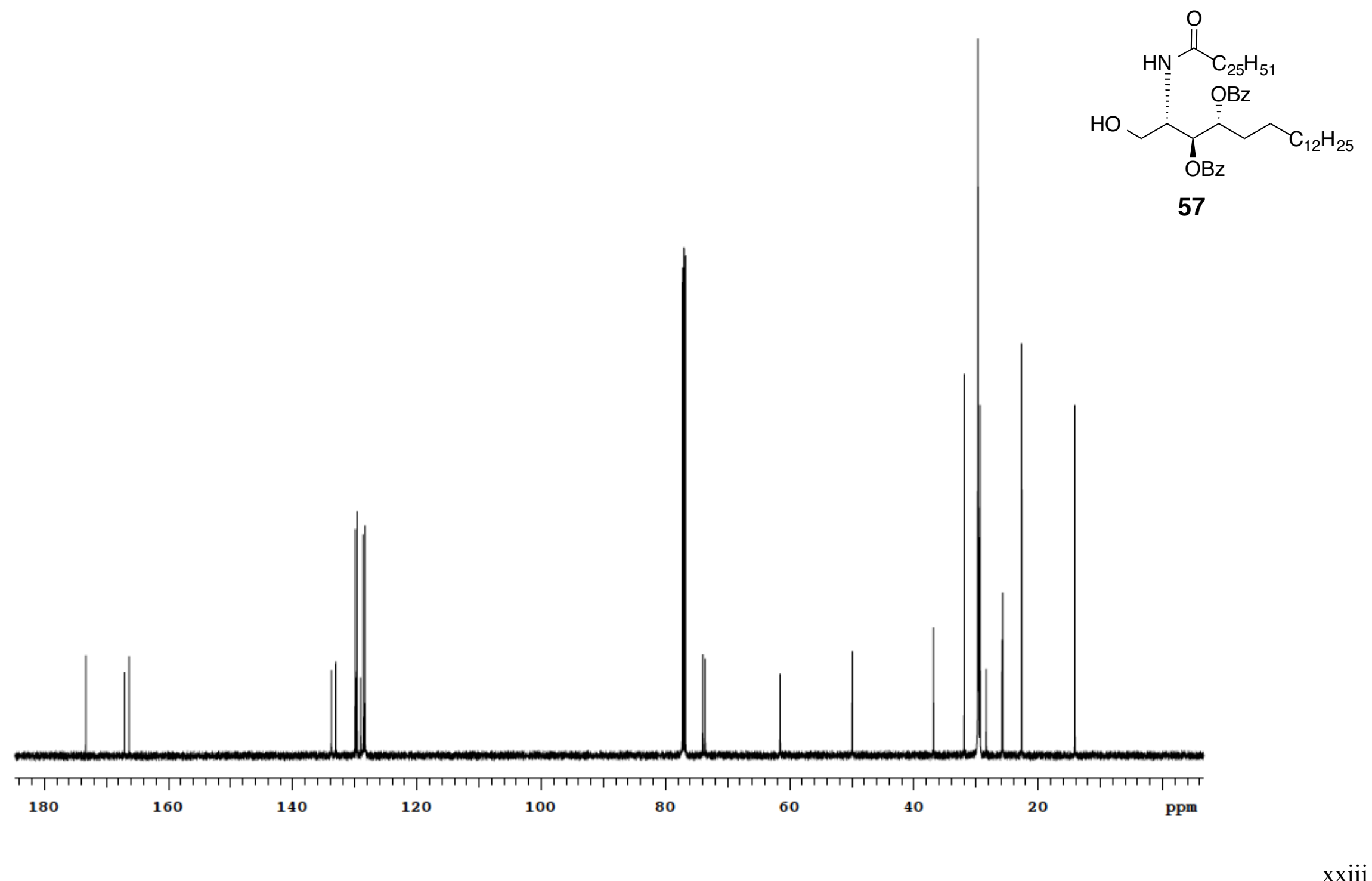


(2S,3S,4R)-3,4-Dibenzoyloxy-1-(2,3,4-tri- $O$-benzyl-6-deoxy-6-azido- $\alpha$-D-galactopyranosyloxy)-2-hexacosanoylamido-octadecane (55) ${ }^{1} \mathrm{H} \mathrm{NMR}, \mathrm{CDCl}_{3}, 500 \mathrm{MHz}$

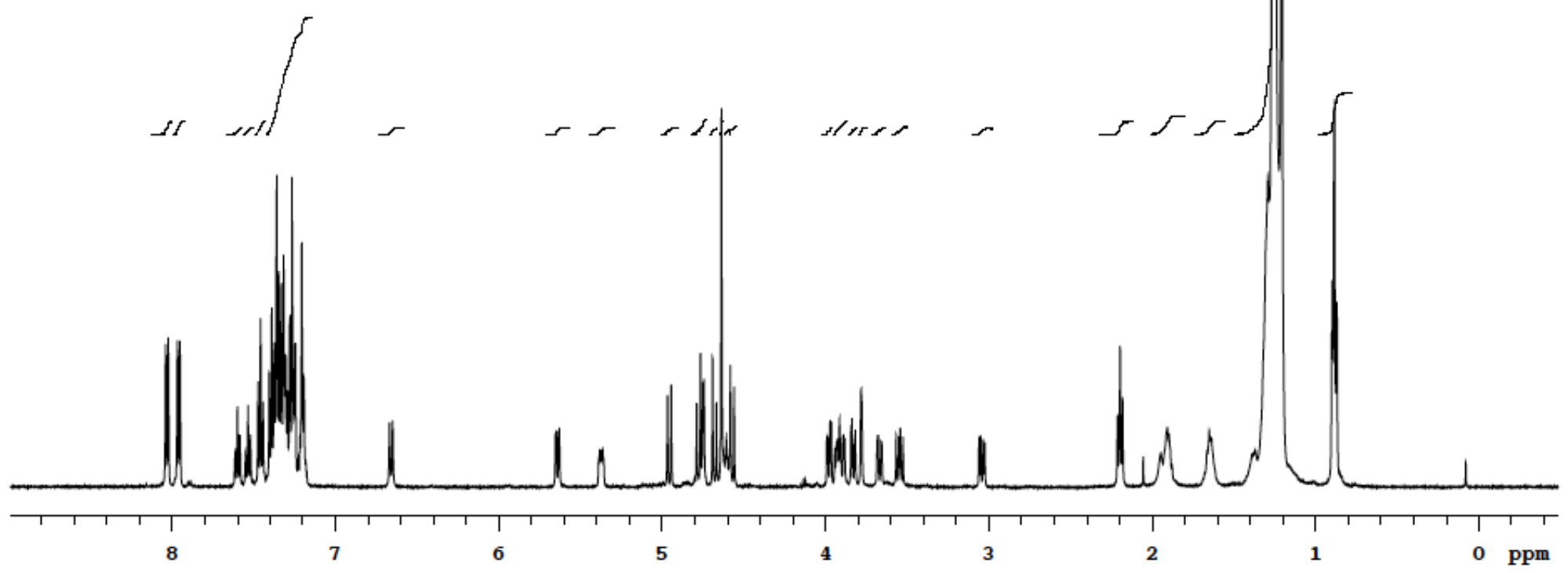

xxiv 
(2S,3S,4R)-3,4-Dibenzoyloxy-1-(2,3,4-tri- $O$-benzyl-6-deoxy-6-azido- $\alpha$-D-galactopyranosyloxy)-2-hexacosanoylamido-octadecane (55)

${ }^{13} \mathrm{C} \mathrm{NMR}, \mathrm{CDCl}_{3}, 125 \mathrm{MHz}$

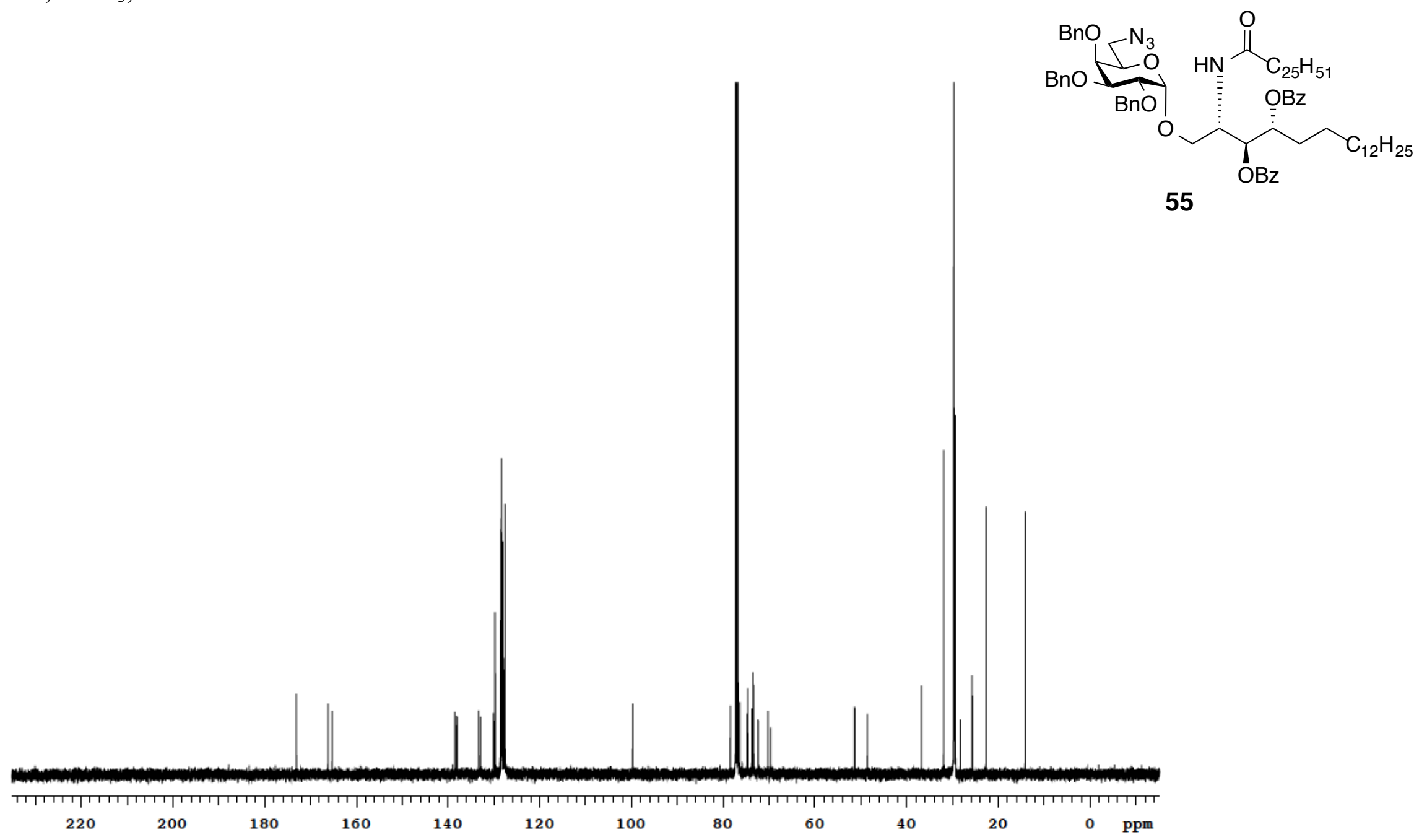


(2S,3S,4R)-2-Hexacosanoylamido-(2,3,4-tri-O-benzyl-6-deoxy-6-azido- $\alpha$-D-galactopyranosyloxy)-octadecane-3,4-diol (69)

${ }^{1} \mathrm{H} \mathrm{NMR}, \mathrm{CDCl}_{3}, 500 \mathrm{MHz}$

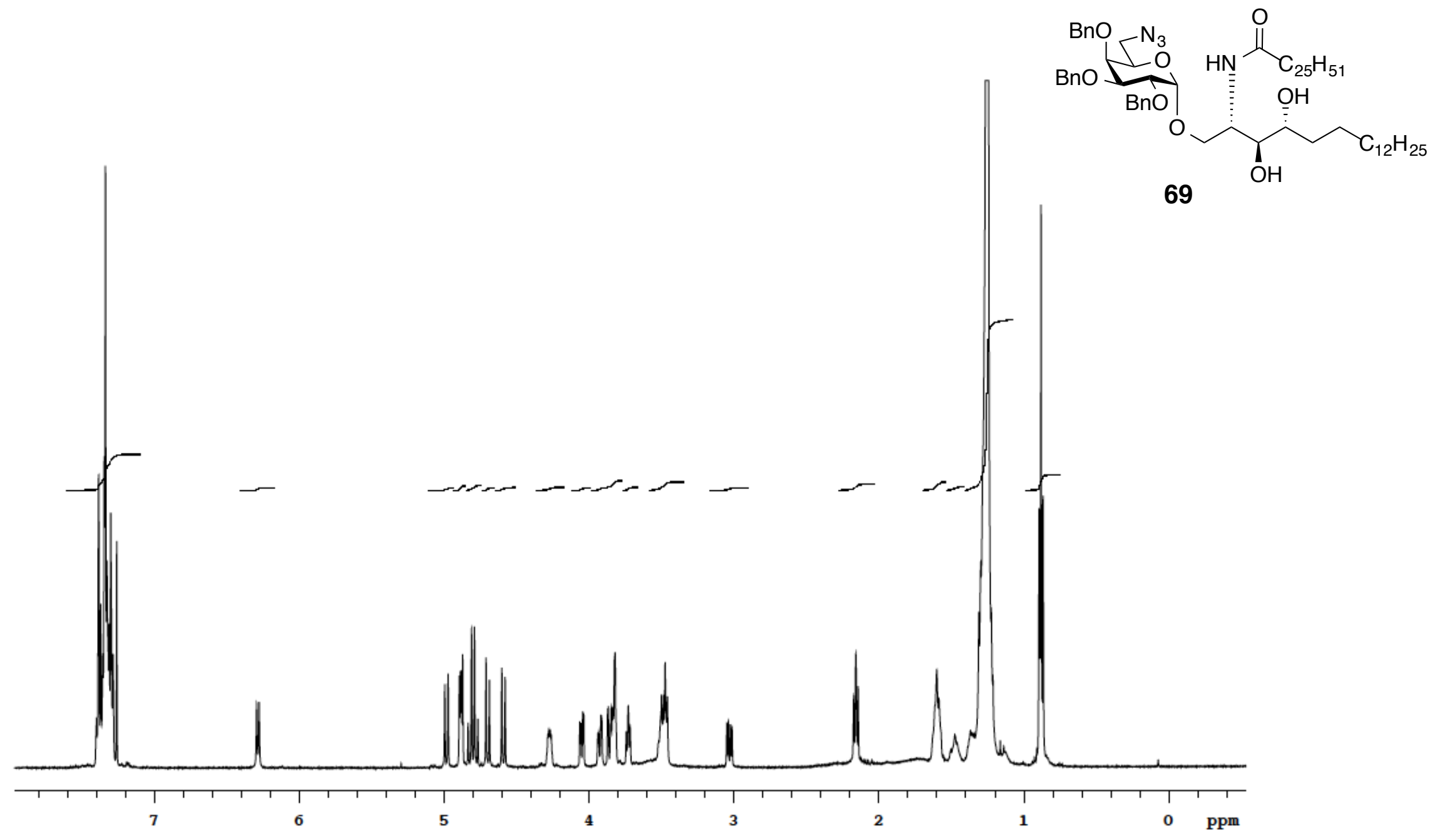


(2S,3S,4R)-2-Hexacosanoylamido-(2,3,4-tri- $O$-benzyl-6-deoxy-6-azido- $\alpha$-D-galactopyranosyloxy)-octadecane-3,4-diol (69)

${ }^{13} \mathrm{C} \mathrm{NMR}, \mathrm{CDCl}_{3}, 125 \mathrm{MHz}$

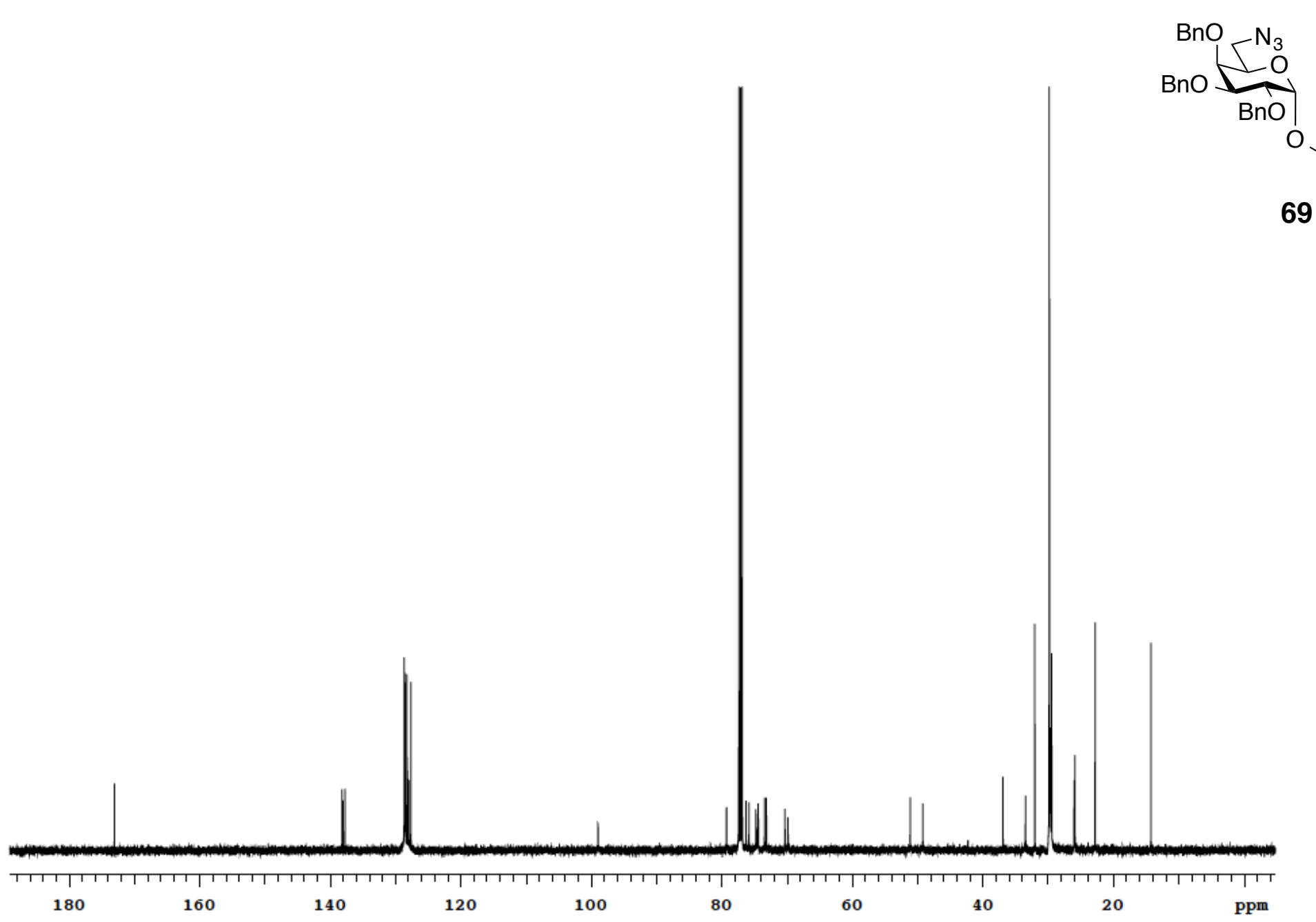

xxvii 
(2S,3S,4R)-1-(6-Amino-6-deoxy- $\alpha$-D-galactopyranosyloxy)-2-hexacosanoylamido-octadecane-3,4-diol (51)

${ }^{1} \mathrm{H} \mathrm{NMR}, \mathrm{CDCl}_{3}, 500 \mathrm{MHz}$

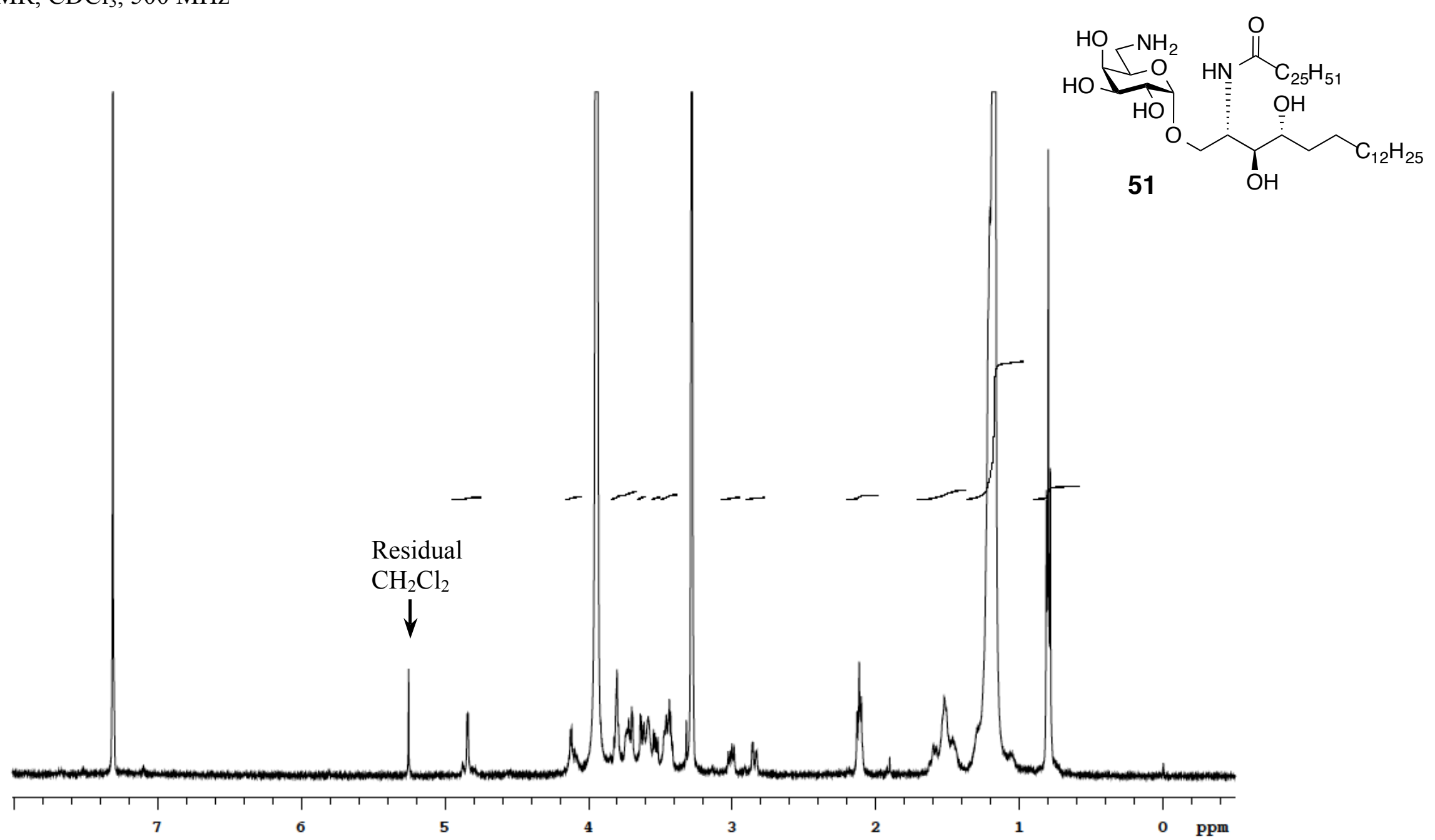


(2S,3S,4R)-1-(6-Deoxy-6-[4-(4,4-difluoro-1,3,5,7-tetramethyl-3a,4a-diaza-4-bora-s-indacen-8-yl)benzylamido])- $\alpha$-Dgalactopyranosyloxy)-2-hexacosanoylamido-octadecane-3,4-diol (3) ${ }^{1} \mathrm{H} \mathrm{NMR}$, pyridine- $\mathrm{d}_{5}, 600 \mathrm{MHz}$

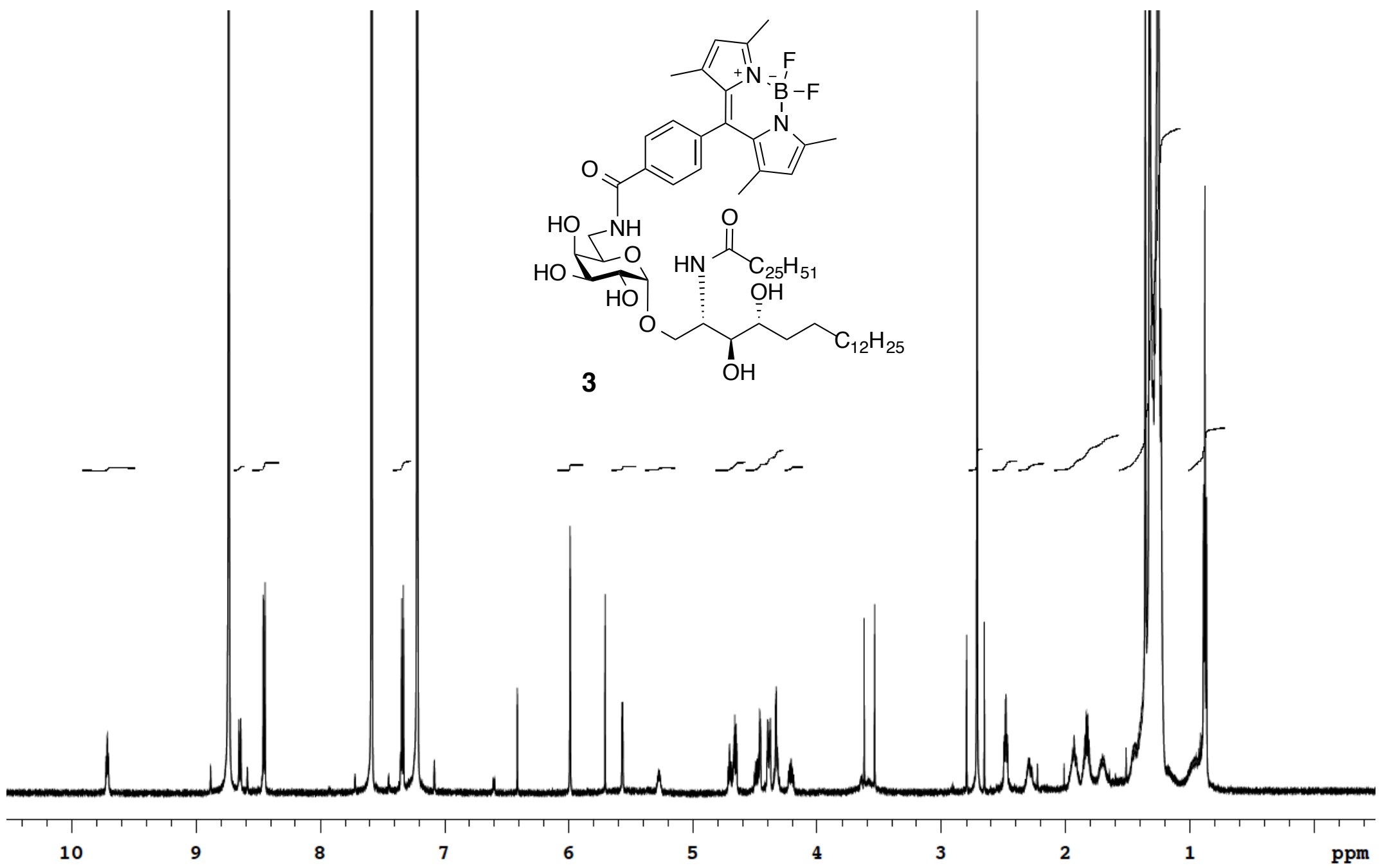


(2S,3S,4R)-1-(6-Deoxy-6-[4-(4,4-difluoro-1,3,5,7-tetramethyl-3a,4a-diaza-4-bora-s-indacen-8-yl)benzylamido])- $\alpha$-Dgalactopyranosyloxy)-2-hexacosanoylamido-octadecane-3,4-diol (3) ${ }^{13} \mathrm{C} \mathrm{NMR}$, pyridine- $\mathrm{d}_{5}, 150 \mathrm{MHz}$

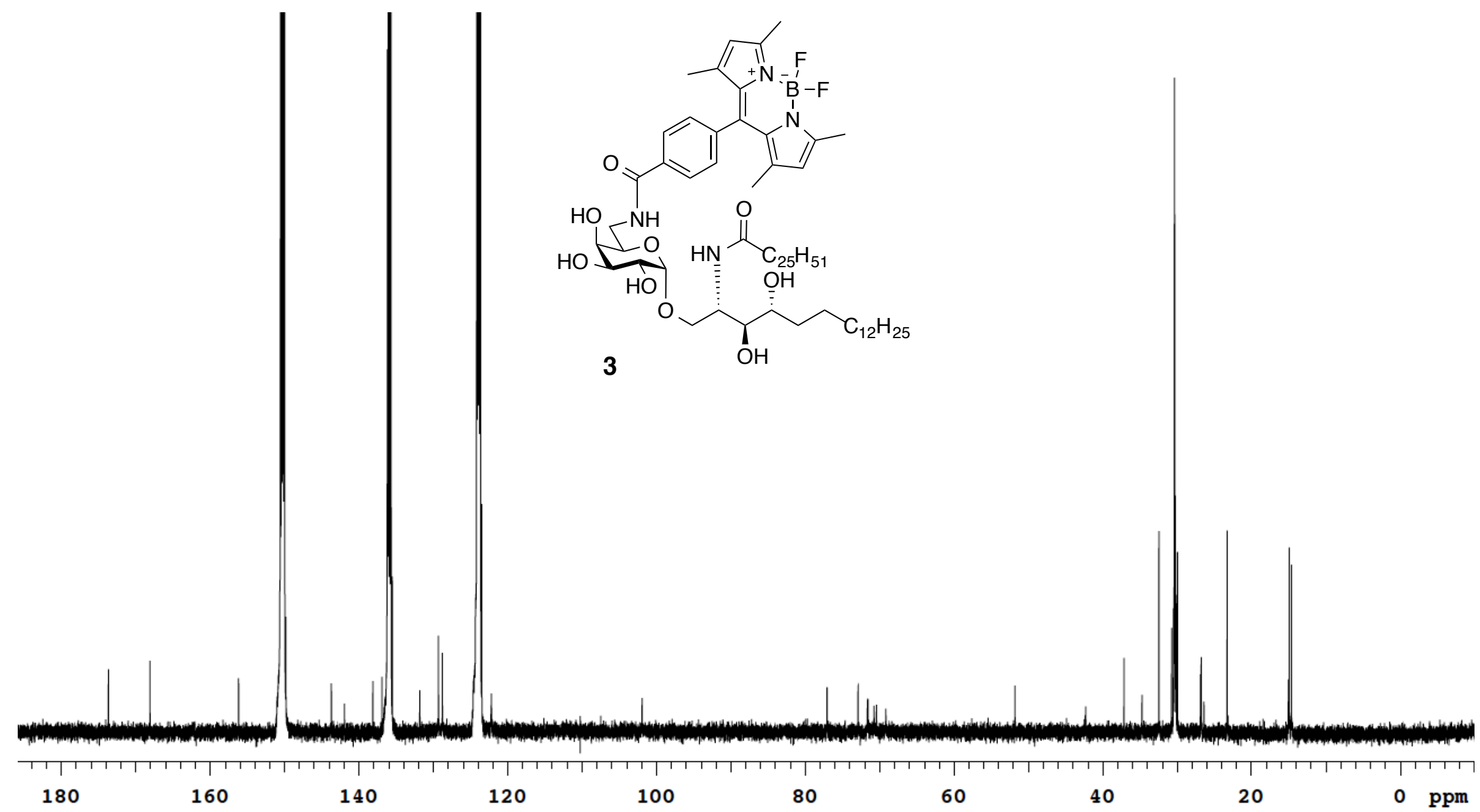


${ }^{1} \mathrm{H} \&{ }^{13} \mathrm{C}$ NMR Spectra

Chapter 4 
Phenyl 4- $\boldsymbol{O}$-( $\boldsymbol{\beta}$-D-galactopyranosyl)-1-thio- $\boldsymbol{\beta}$-D-glucopyranoside (79) ${ }^{1} \mathrm{H} \mathrm{NMR}, \mathrm{D}_{2} \mathrm{O}, 600 \mathrm{MHz}$

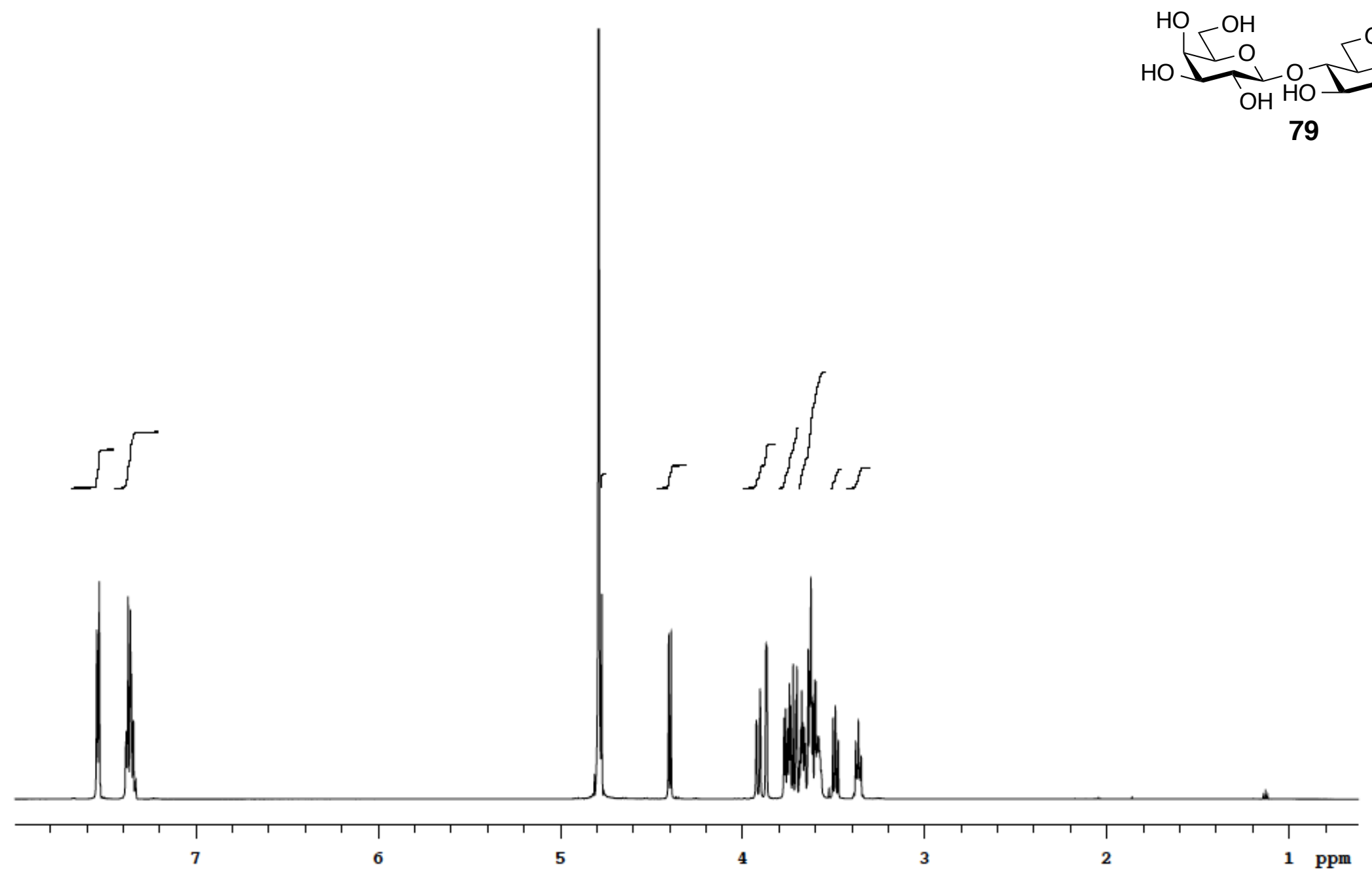


Phenyl 4- $\boldsymbol{O}$-( $\boldsymbol{\beta}$-D-galactopyranosyl)-1-thio- $\boldsymbol{\beta}$-D-glucopyranoside (79) ${ }^{13} \mathrm{C}$ NMR, $\mathrm{D}_{2} \mathrm{O}, 150 \mathrm{MHz}$
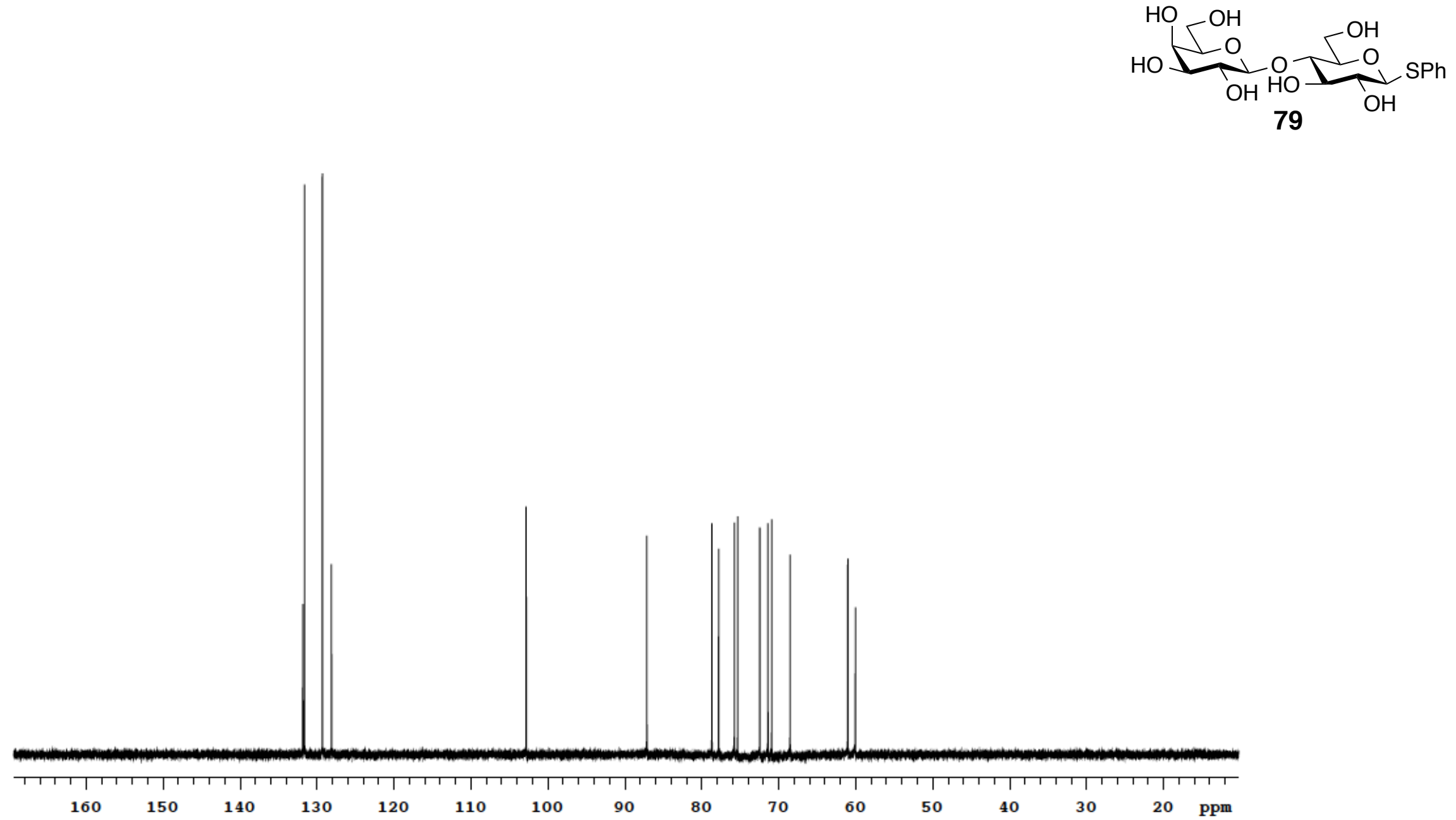
Phenyl 4-O-(3,4-O-isopropylidene- $\beta$-D-galactopyranosyl)-1-thio- $\beta$-D-glucopyranoside (80)

${ }^{1} \mathrm{H} \mathrm{NMR}, \mathrm{CDCl}_{3} / \mathrm{CD}_{3} \mathrm{OD}, 1 / 1$, v/v, $500 \mathrm{MHz}$

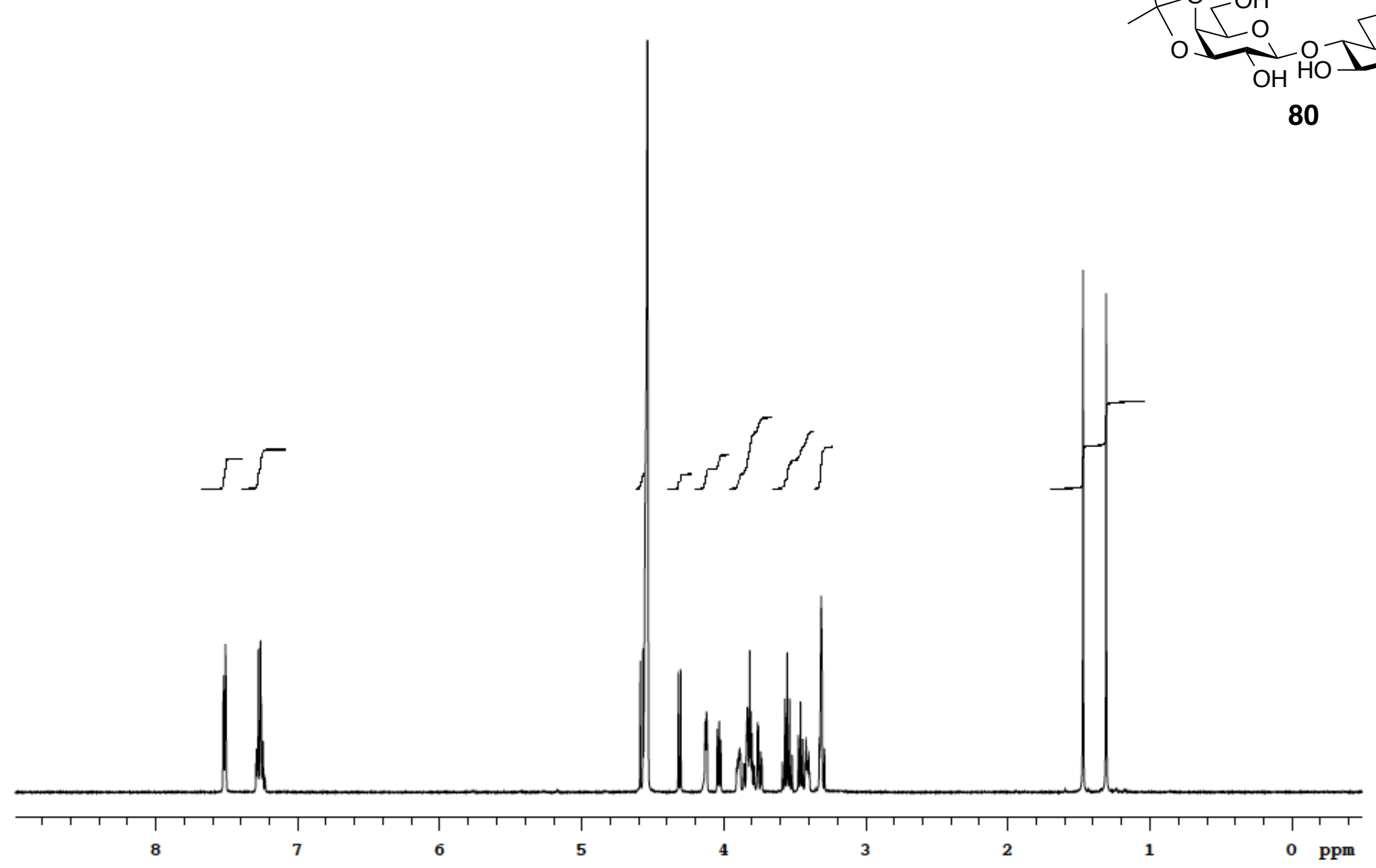


Phenyl 4-O-(3,4-O-isopropylidene- $\beta$-D-galactopyranosyl)-1-thio- $\beta$-D-glucopyranoside (80)

${ }^{13} \mathrm{C} \mathrm{NMR}, \mathrm{CDCl}_{3} / \mathrm{CD}_{3} \mathrm{OD}, 1 / 1, \mathrm{v} / \mathrm{v}, 125 \mathrm{MHz}$

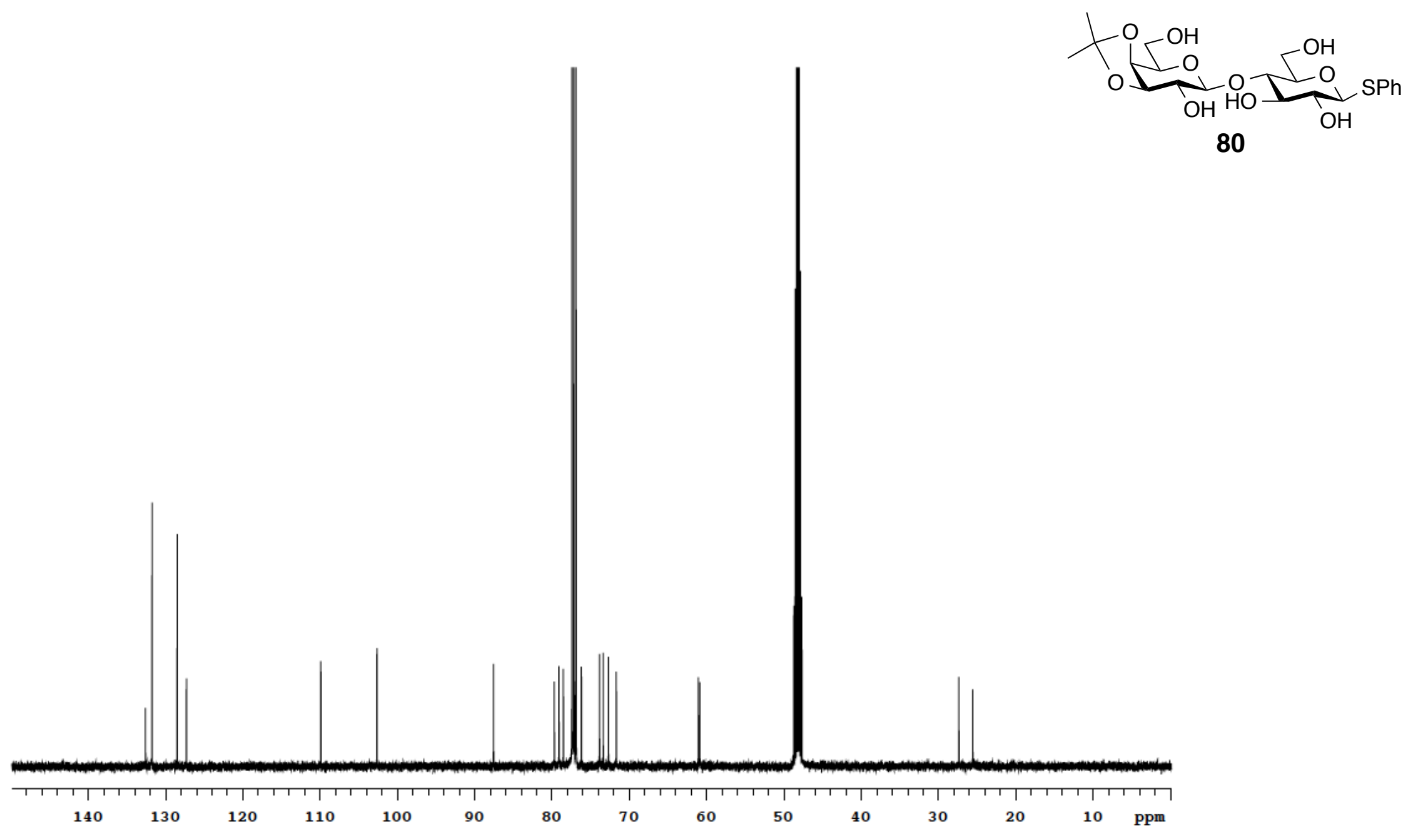


Phenyl 4- $O$-(2,6-di- $O$-benzoyl-3,4- $O$-isopropylidene- $\beta$-D-galactopyranosyl)-2,3,6-tri- $O$-benzoyl-1-thio- $\beta$-D-glucopyranoside (82) ${ }^{1} \mathrm{H} \mathrm{NMR}, \mathrm{CDCl}_{3}, 500 \mathrm{MHz}$
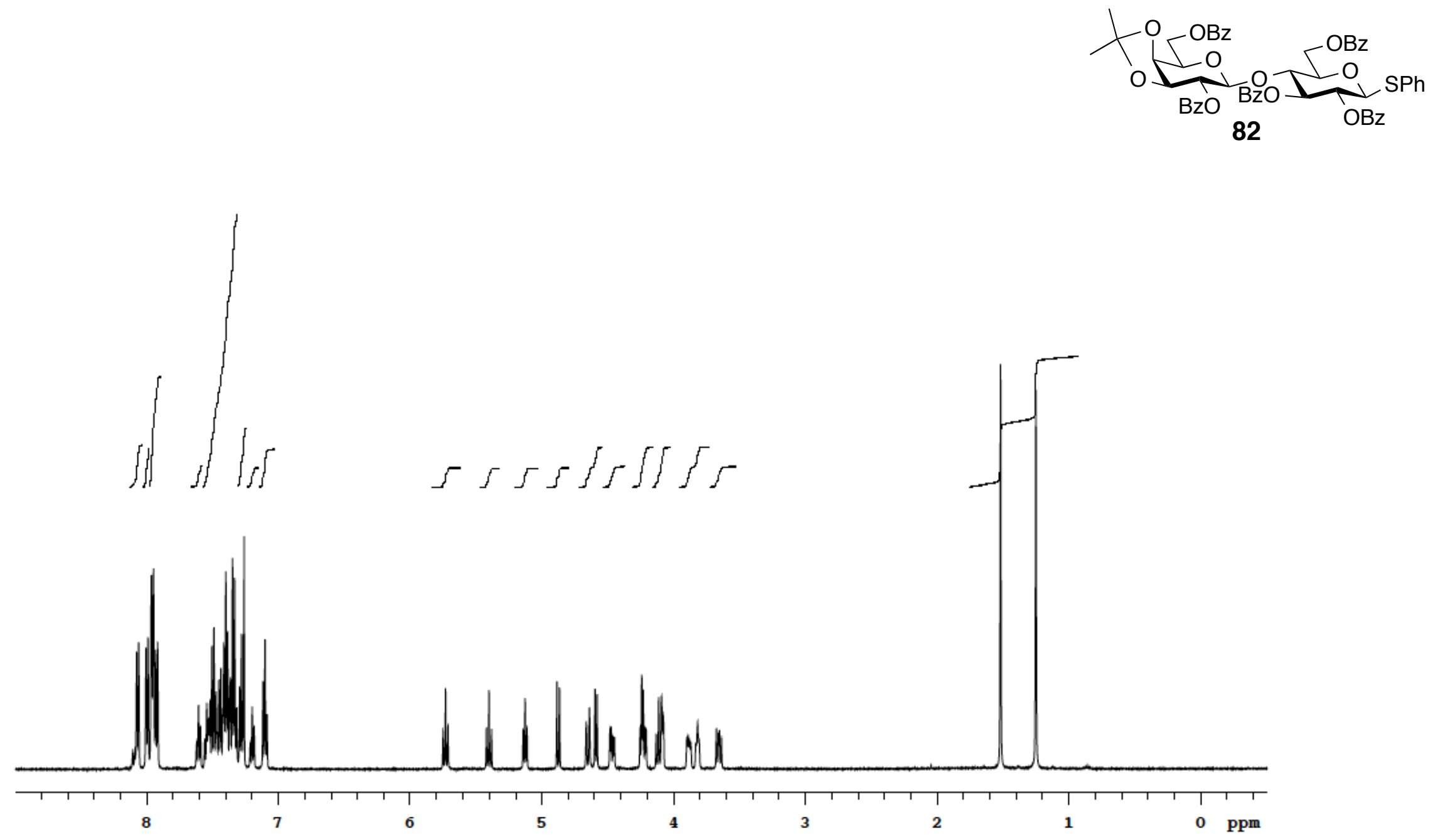
Phenyl 4- $O$-(2,6-di- $O$-benzoyl-3,4- $O$-isopropylidene- $\beta$-D-galactopyranosyl)-2,3,6-tri- $O$-benzoyl-1-thio- $\beta$-D-glucopyranoside (82)

${ }^{13} \mathrm{C} \mathrm{NMR}, \mathrm{CDCl}_{3}, 125 \mathrm{MHz}$

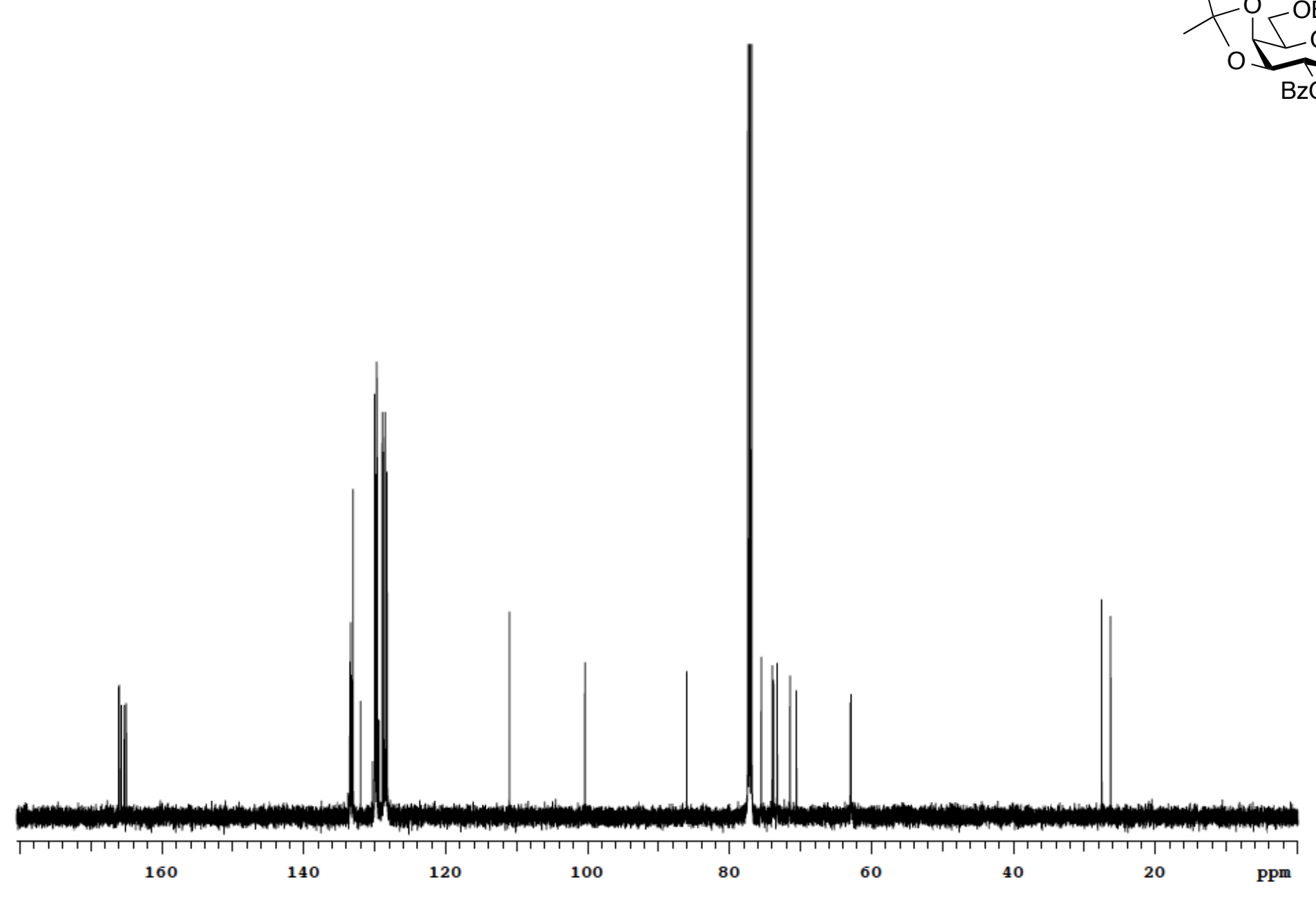


4-O-(2,6-Di- $O$-benzoyl-3,4- $O$-isopropylidene- $\beta$-D-galactopyranosyl-2,3,6-tri- $O$-benzoyl- $\alpha$-D-glucopyranose (82a)

${ }^{1} \mathrm{H} \mathrm{NMR}, \mathrm{CDCl}_{3}, 500 \mathrm{MHz}$

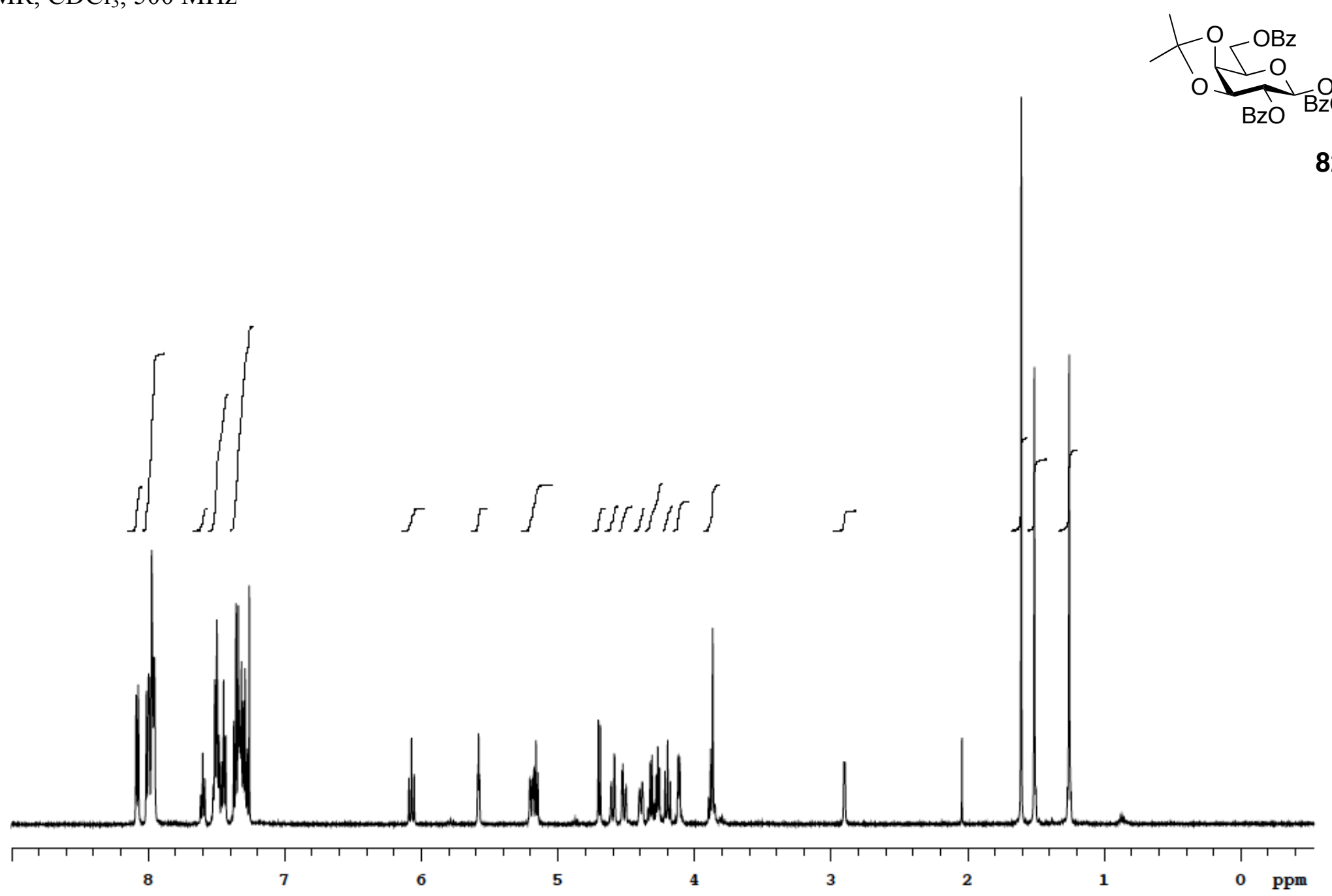


4-O-(2,6-Di- $O$-benzoyl-3,4- $O$-isopropylidene- $\beta$-D-galactopyranosyl-2,3,6-tri- $O$-benzoyl- $\alpha$-D-glucopyranose (82a)

${ }^{13} \mathrm{C} \mathrm{NMR}, \mathrm{CDCl}_{3}, 125 \mathrm{MHz}$

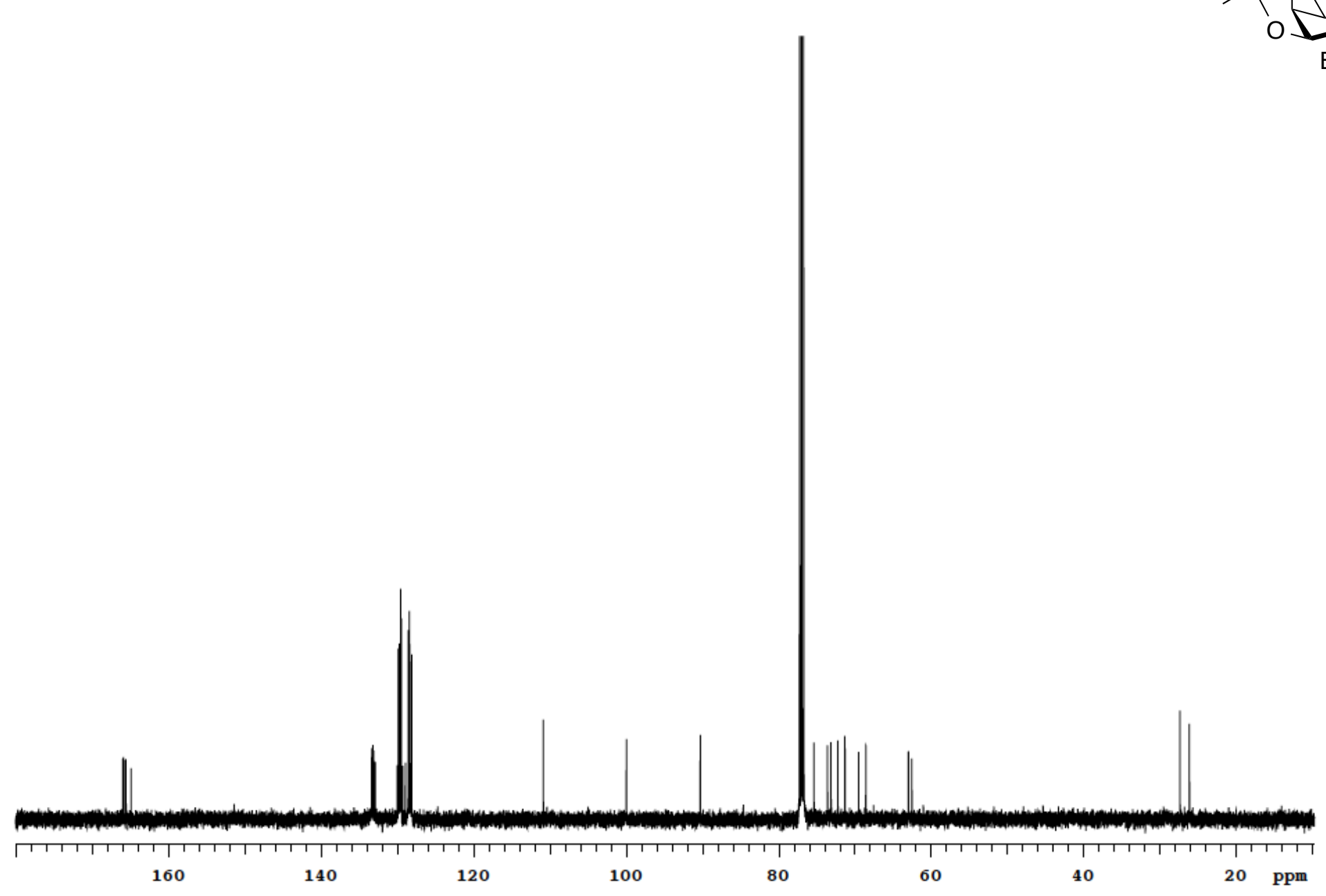


$O$-(4-O-(2,6-Di- $O$-benzoyl-3,4- $O$-isopropylidene- $\beta$-D-galactopyranosyl)-2,3,6-tri- $O$-benzoyl- $\alpha$-D-glucopyranosyl) trichloroacetimidate (75) ${ }^{1} \mathrm{H} \mathrm{NMR}, \mathrm{CDCl}_{3}, 500 \mathrm{MHz}$
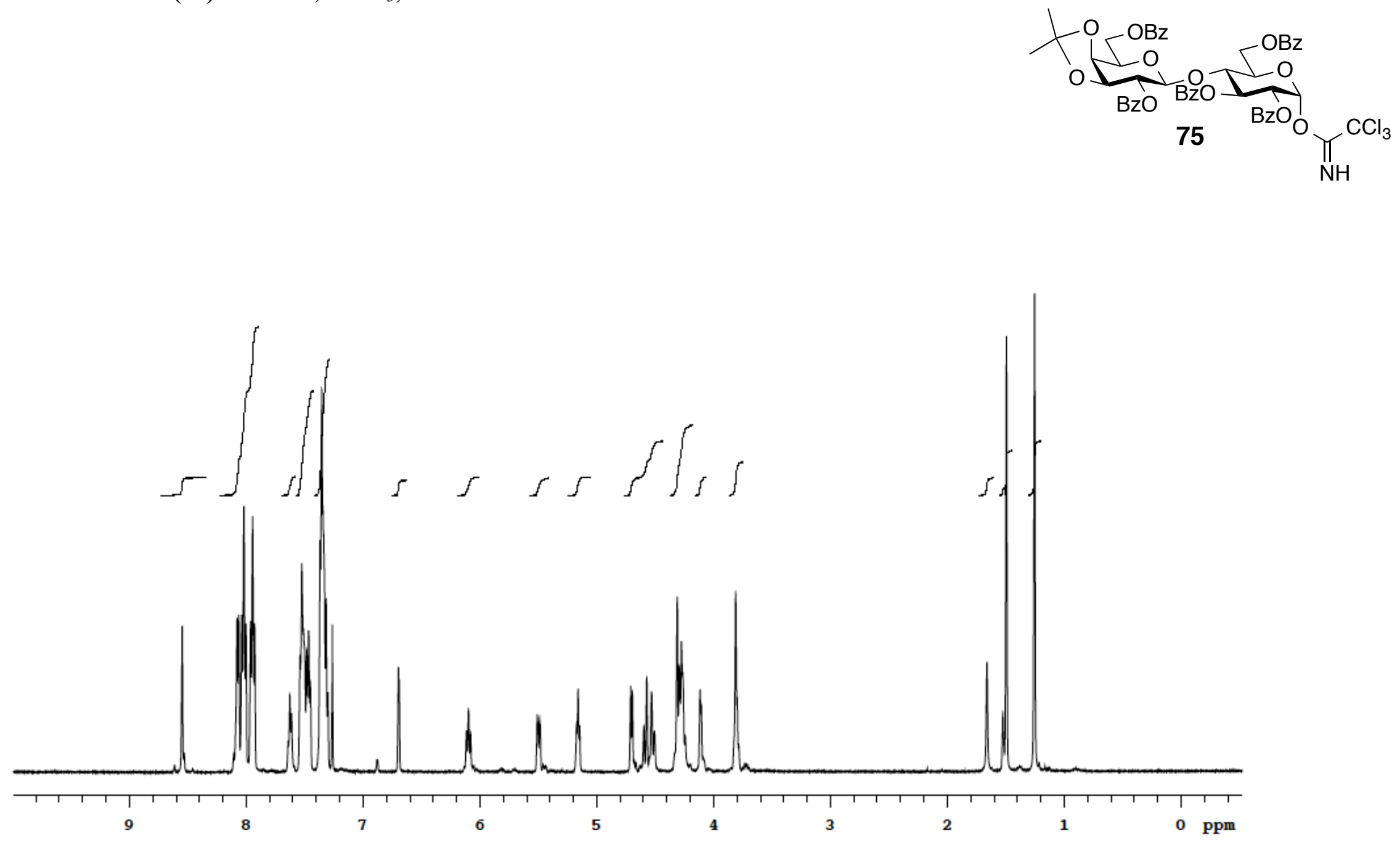
$O$-(4-O-(2,6-Di- $O$-benzoyl-3,4- $O$-isopropylidene- $\beta$-D-galactopyranosyl)-2,3,6-tri- $O$-benzoyl- $\alpha$-D-glucopyranosyl) trichloroacetimidate (75) ${ }^{13} \mathrm{C} \mathrm{NMR}, \mathrm{CDCl}_{3}, 125 \mathrm{MHz}$

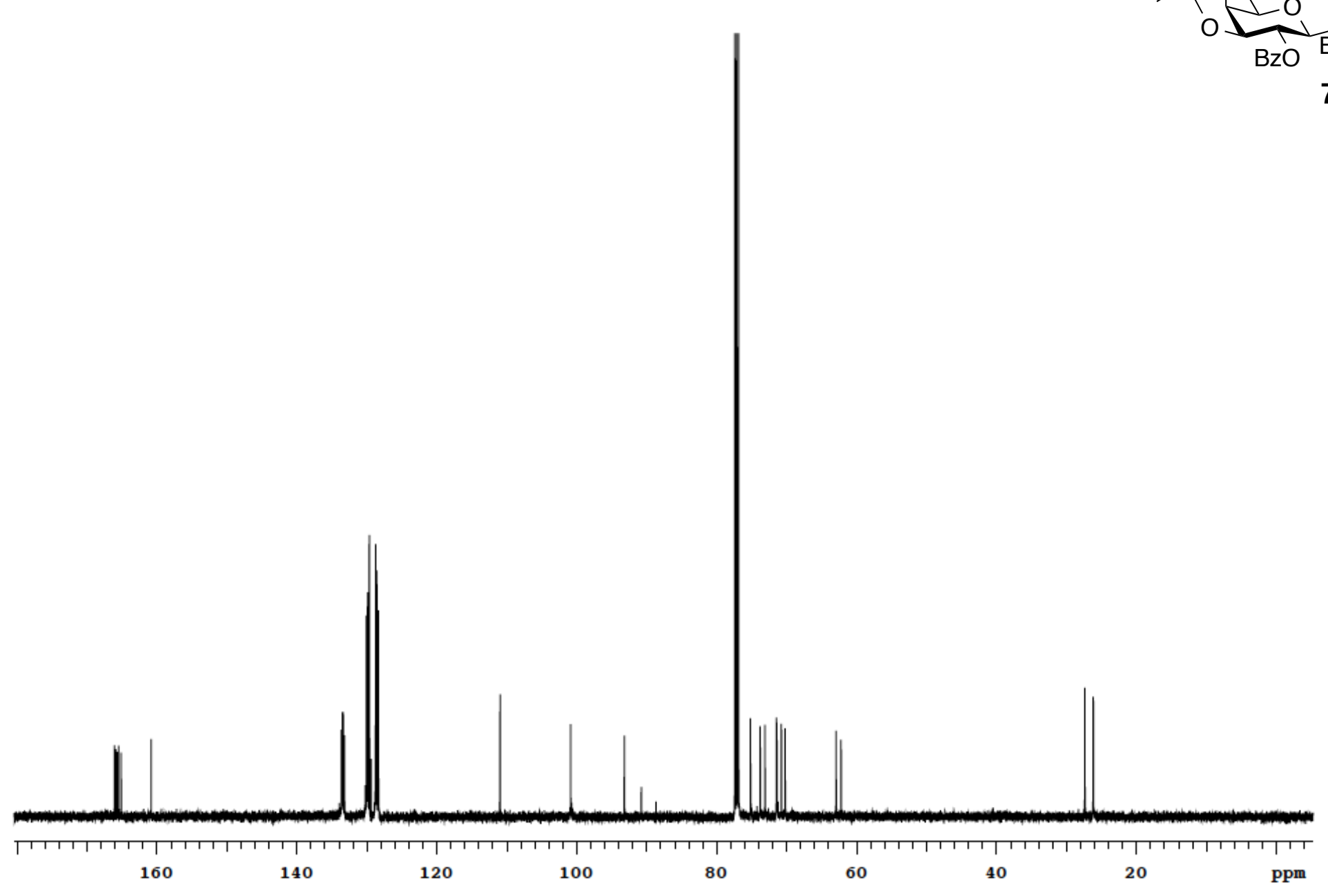


(2S,3R,4E)-2-Azido-1-(4- $O$-(2,6-di- $O$-benzoyl-3,4- $O$-isopropylidene- $\beta$-D-galactopyranosyl)-2,3,6-tri- $O$-benzoyl- $\beta$-Dglucopyranosyloxy)-3-benzyloxy-octadec-4-ene (74) ${ }^{1} \mathrm{H} \mathrm{NMR}, \mathrm{CDCl}_{3}, 500 \mathrm{MHz}$
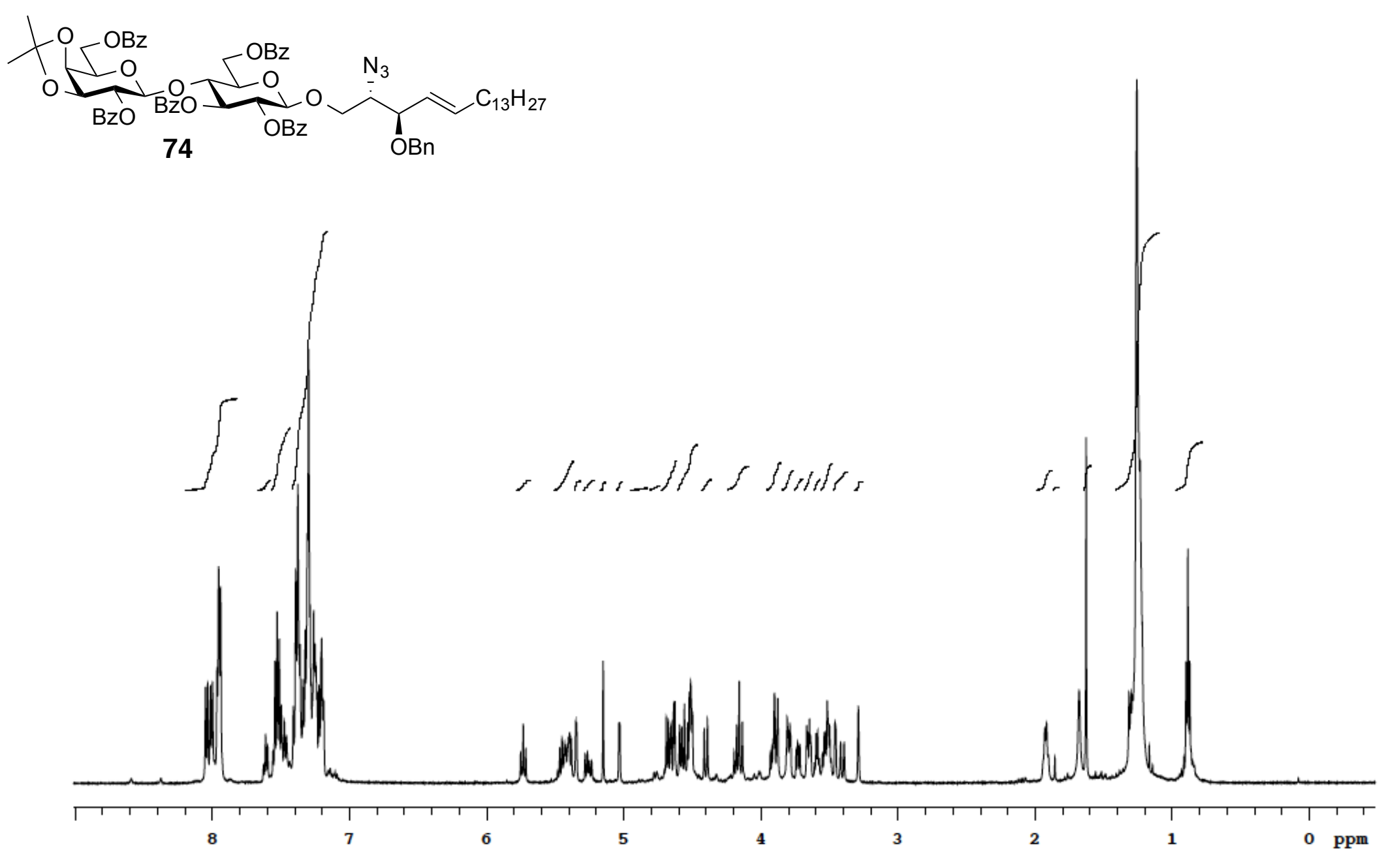
(2S,3R,4E)-2-Azido-1-(4- $O$-(2,6-di- $O$-benzoyl-3,4- $O$-isopropylidene- $\beta$-D-galactopyranosyl)-2,3,6-tri- $O$-benzoyl- $\beta$-Dglucopyranosyloxy)-3-benzyloxy-octadec-4-ene (74) ${ }^{13} \mathrm{C} \mathrm{NMR}, \mathrm{CDCl}_{3}, 125 \mathrm{MHz}$
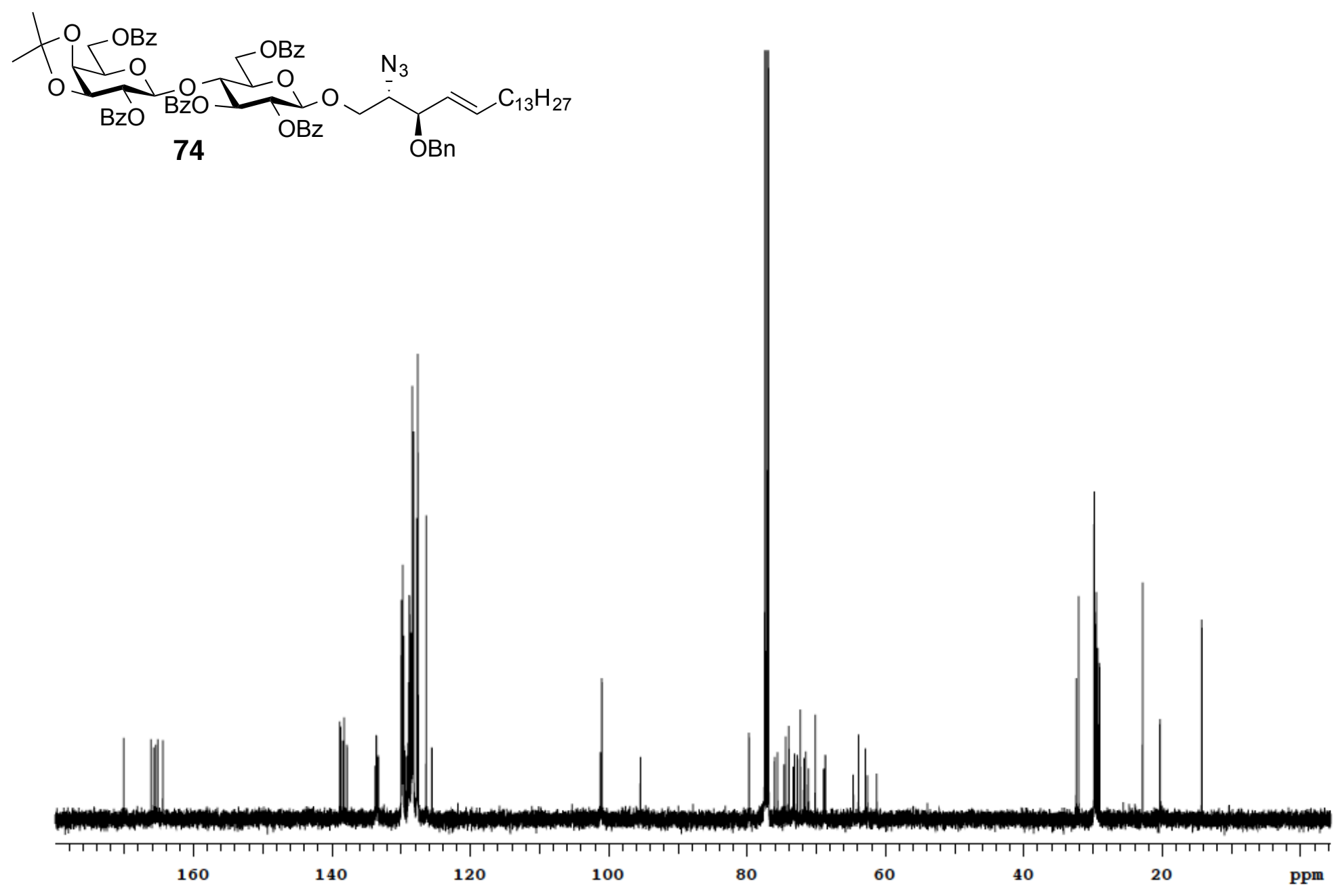
$(2 S, 3 R, 4 E)-1-(4-O$-(2,6-Di- $O$-benzoyl-3,4- $O$-isopropylidene- $\beta$-D-galactopyranosyl)-2,3,6-tri- $O$-benzoyl- $\beta$-D-glucopyranosyloxy)-3benzyloxy-2-hexacosanoylamido-octadecen-4-ene (83) ${ }^{1} \mathrm{H} \mathrm{NMR}, \mathrm{CDCl}_{3}, 500 \mathrm{MHz}$
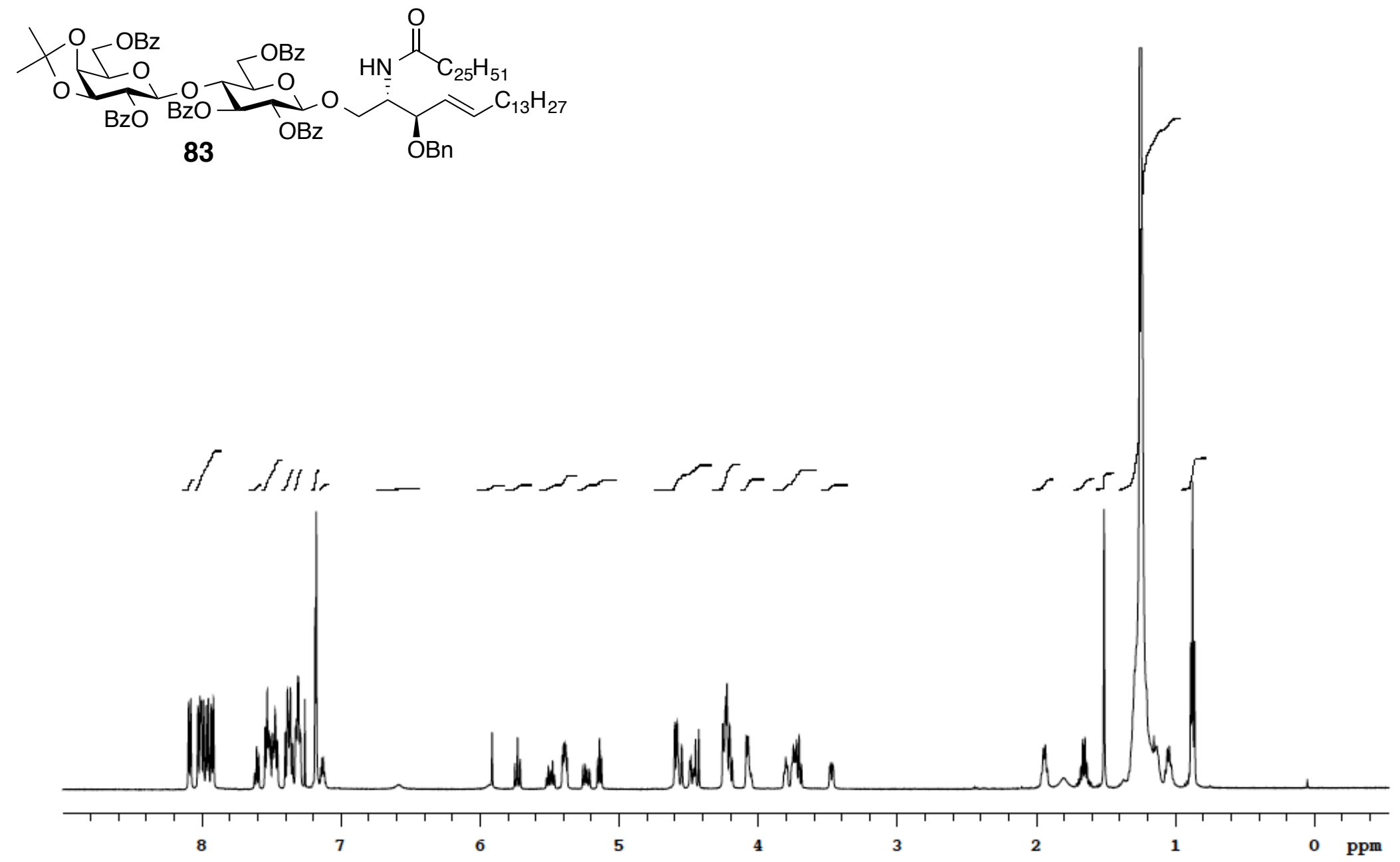

xliv 
(2S,3R,4E)-1-(4-O-(2,6-Di-O-benzoyl-3,4- $O$-isopropylidene- $\beta$-D-galactopyranosyl)-2,3,6-tri- $O$-benzoyl- $\beta$-D-glucopyranosyloxy)-3benzyloxy-2-hexacosanoylamido-octadecen-4-ene (83) ${ }^{13} \mathrm{C} \mathrm{NMR}, \mathrm{CDCl}_{3}, 125 \mathrm{MHz}$

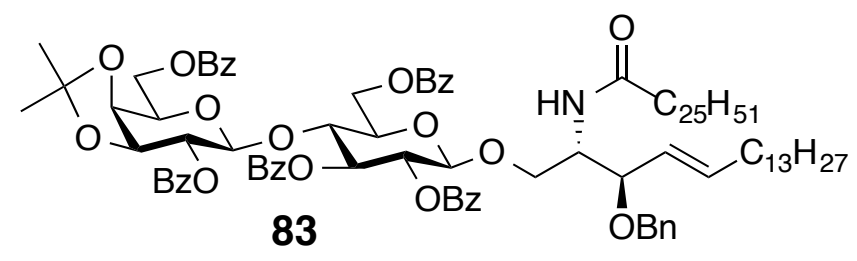

83

OBn

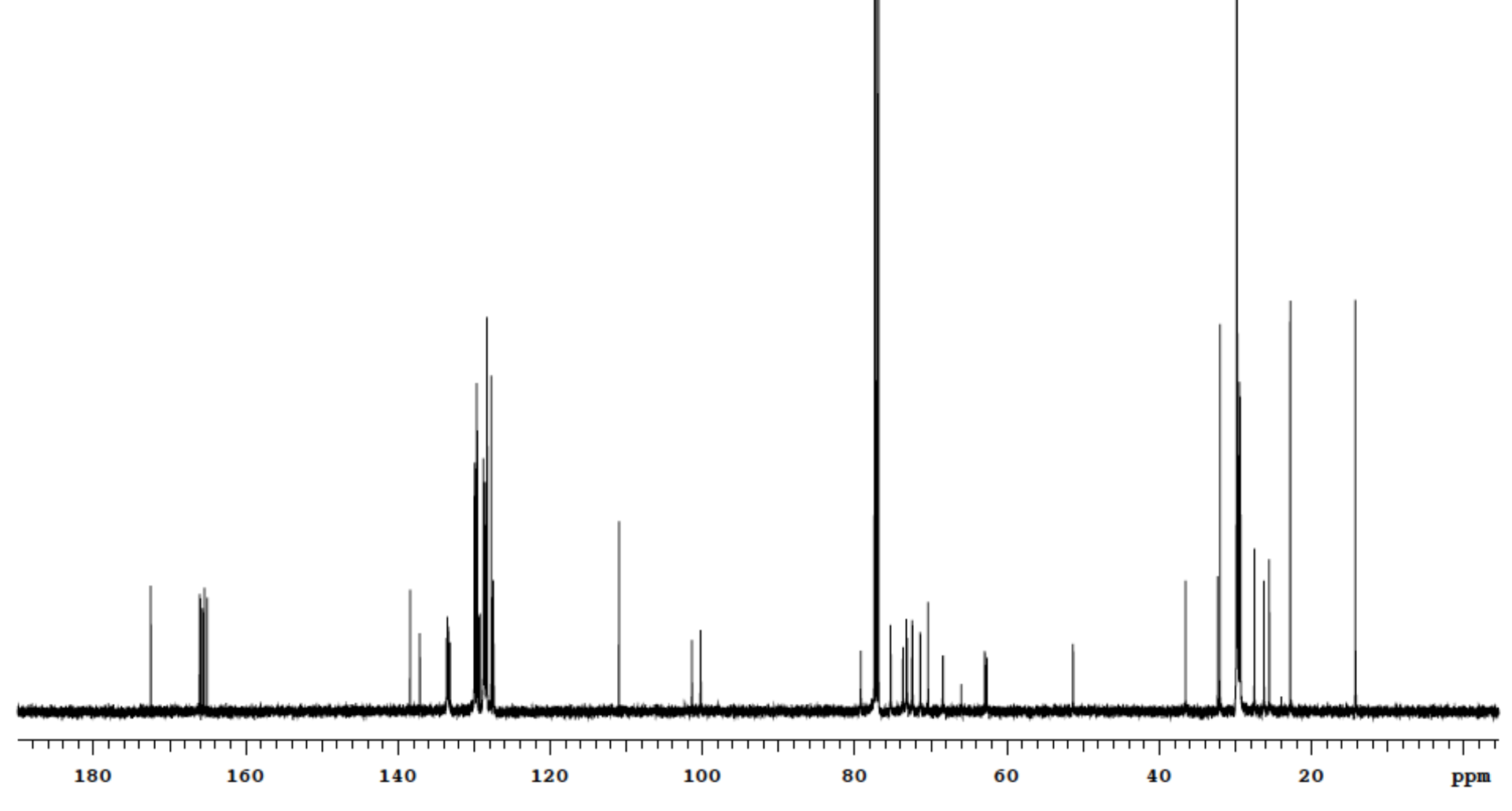


$(2 S, 3 R, 4 E)-1-(4-O$-(2,6-Di- $O$-benzoyl- $\beta$-D-galactopyranosyl)-2,3,6-tri- $O$-benzoyl- $\beta$-D-glucopyranosyloxy)-3-benzyloxy-2hexacosanoylamido-octadec-4-ene (84) ${ }^{1} \mathrm{H} \mathrm{NMR}, \mathrm{CDCl}_{3}, 500 \mathrm{MHz}$
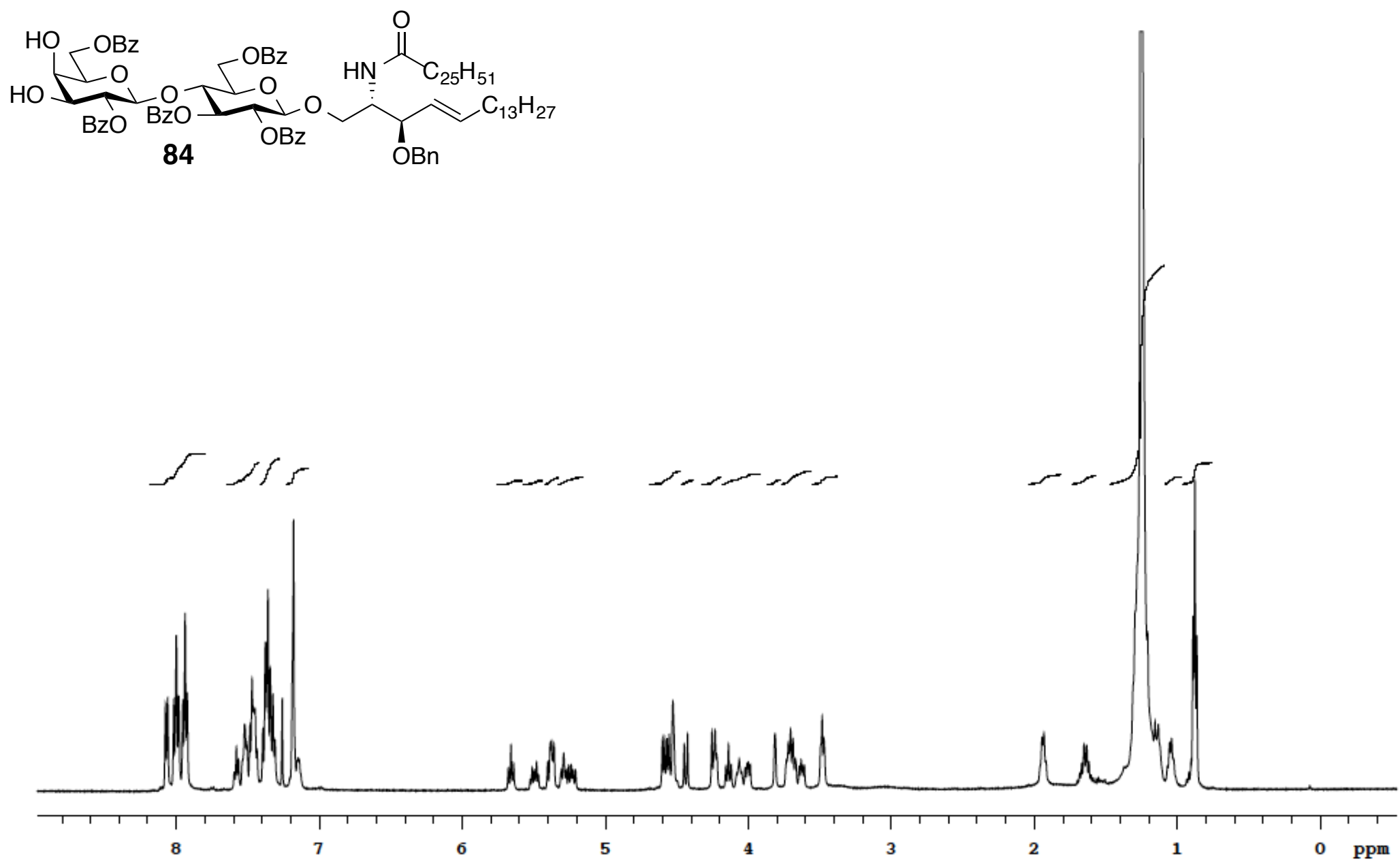

xlvi 
(2S,3R,4E)-1-(4-O-(2,6-Di-O-benzoyl- $\beta$-D-galactopyranosyl)-2,3,6-tri- $O$-benzoyl- $\beta$-D-glucopyranosyloxy)-3-benzyloxy-2hexacosanoylamido-octadec-4-ene (84) ${ }^{13} \mathrm{C} \mathrm{NMR}, \mathrm{CDCl}_{3}, 125 \mathrm{MHz}$
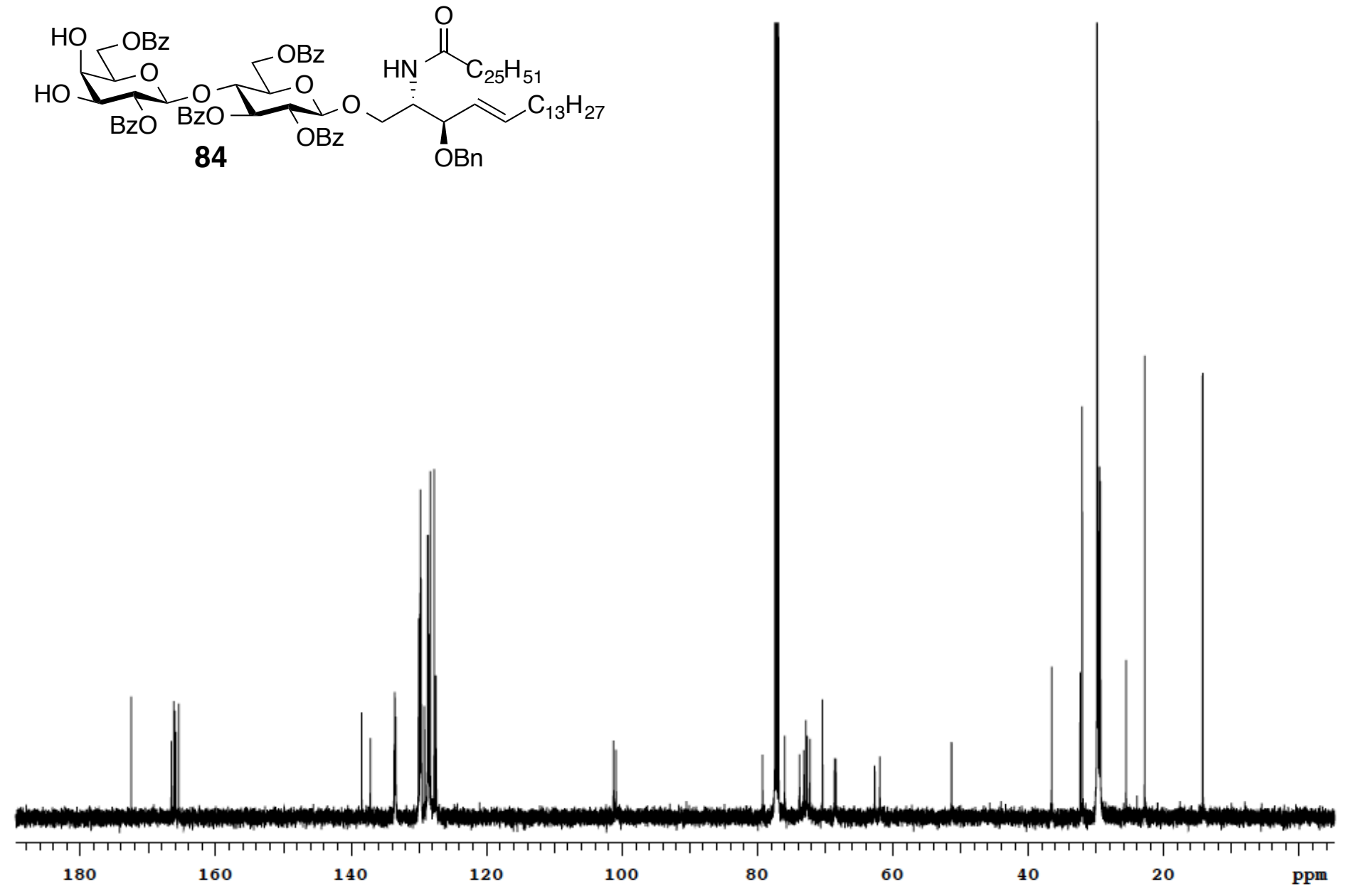
(2S,3R,4E)-1-(4-O-(4-O-Acetyl-2,6-di- $O$-benzoyl- $\beta$-D-galactopyranosyl)-2,3,6-tri- $O$-benzoyl- $\beta$-D-glucopyranosyloxy)-3-benzyloxy-2hexacosanoylamido-octadec-4-ene (73) ${ }^{1} \mathrm{H} \mathrm{NMR}, \mathrm{CDCl}_{3}, 500 \mathrm{MHz}$
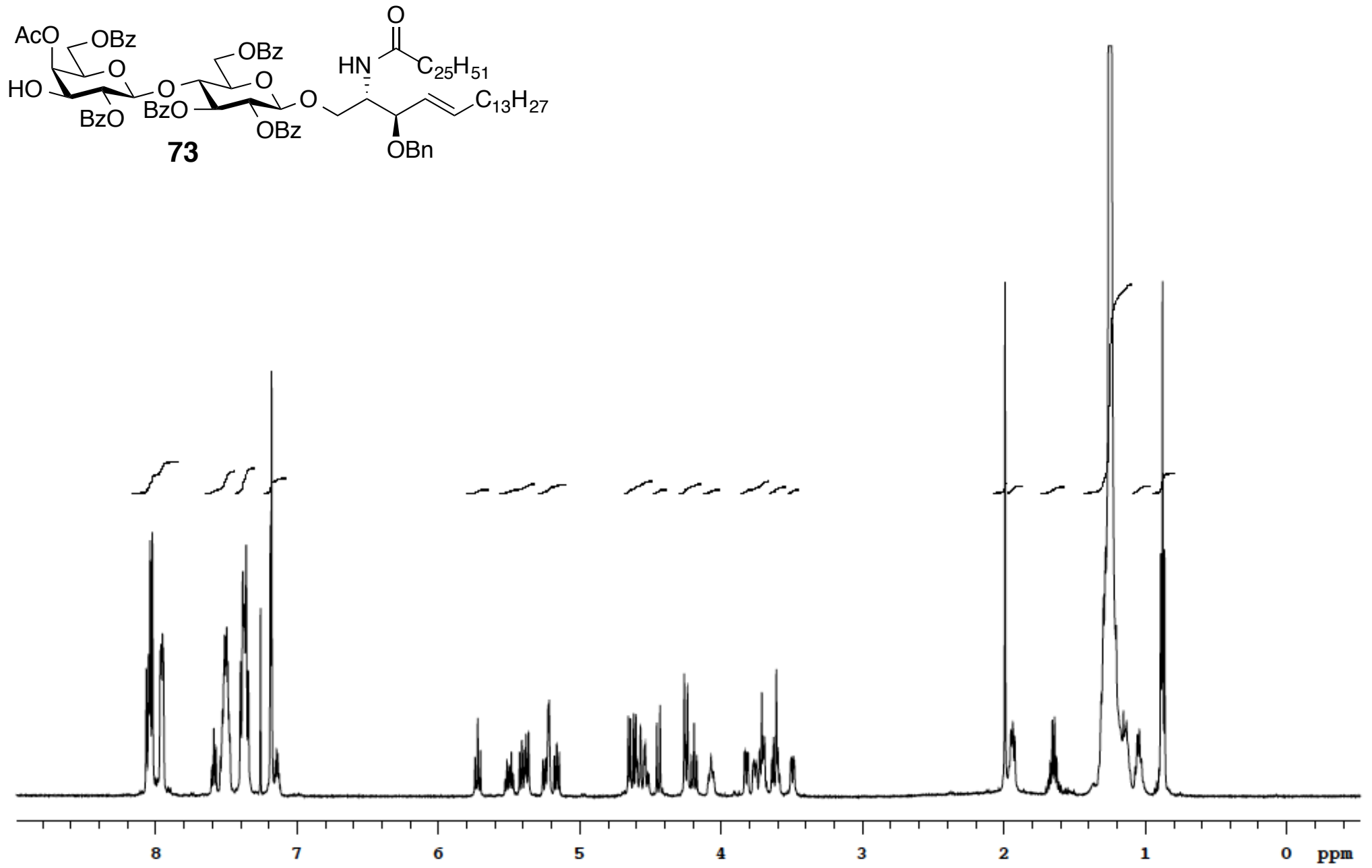
(2S,3R,4E)-1-(4-O-(4-O-Acetyl-2,6-di- $O$-benzoyl- $\beta$-D-galactopyranosyl)-2,3,6-tri- $O$-benzoyl- $\beta$-D-glucopyranosyloxy)-3-benzyloxy-2hexacosanoylamido-octadec-4-ene (73) ${ }^{13} \mathrm{C} \mathrm{NMR}, \mathrm{CDCl}_{3}, 125 \mathrm{MHz}$
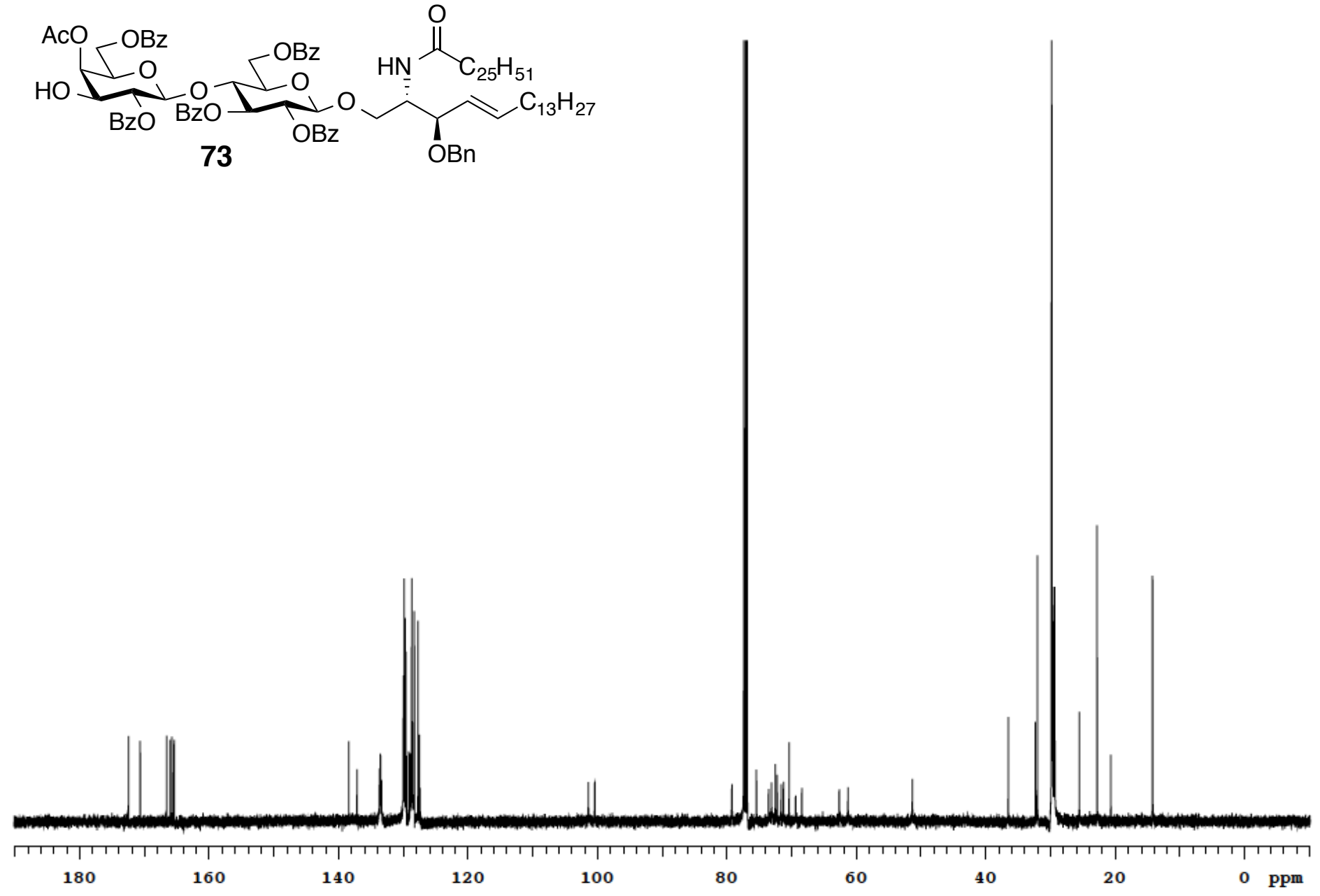
6-Deoxy-1,2:3,4-di- $\boldsymbol{O}$-isopropylidene- $\boldsymbol{\alpha}$-D-galactopyranose (88) ${ }^{1} \mathrm{H} \mathrm{NMR}, \mathrm{CDCl}_{3}, 500 \mathrm{MHz}$
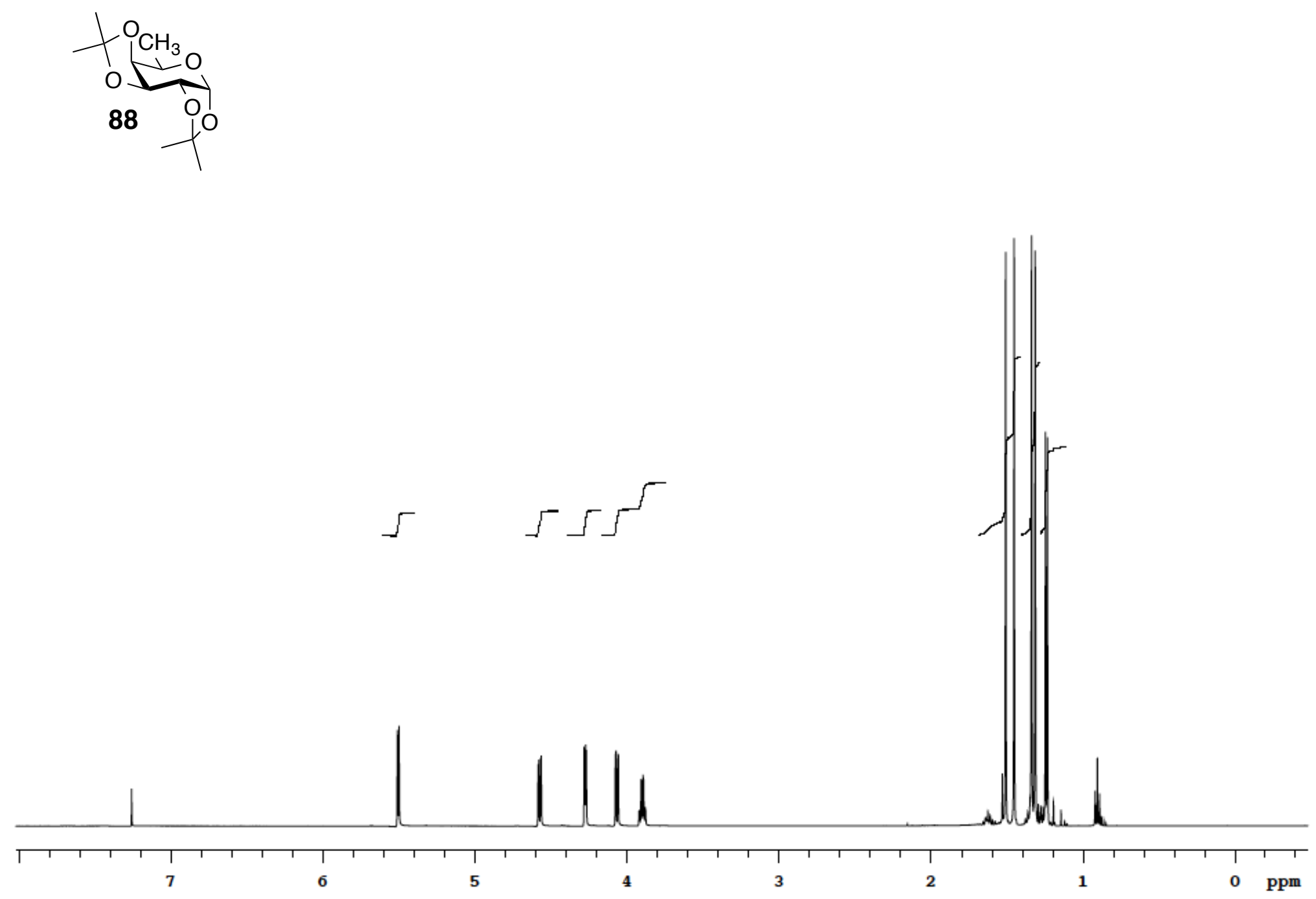
6-Deoxy-1,2:3,4-di- $\boldsymbol{O}$-isopropylidene- $\boldsymbol{\alpha}$-D-galactopyranose (88) ${ }^{13} \mathrm{C} \mathrm{NMR}^{\mathrm{N}} \mathrm{CDCl}_{3}, 125 \mathrm{MHz}$
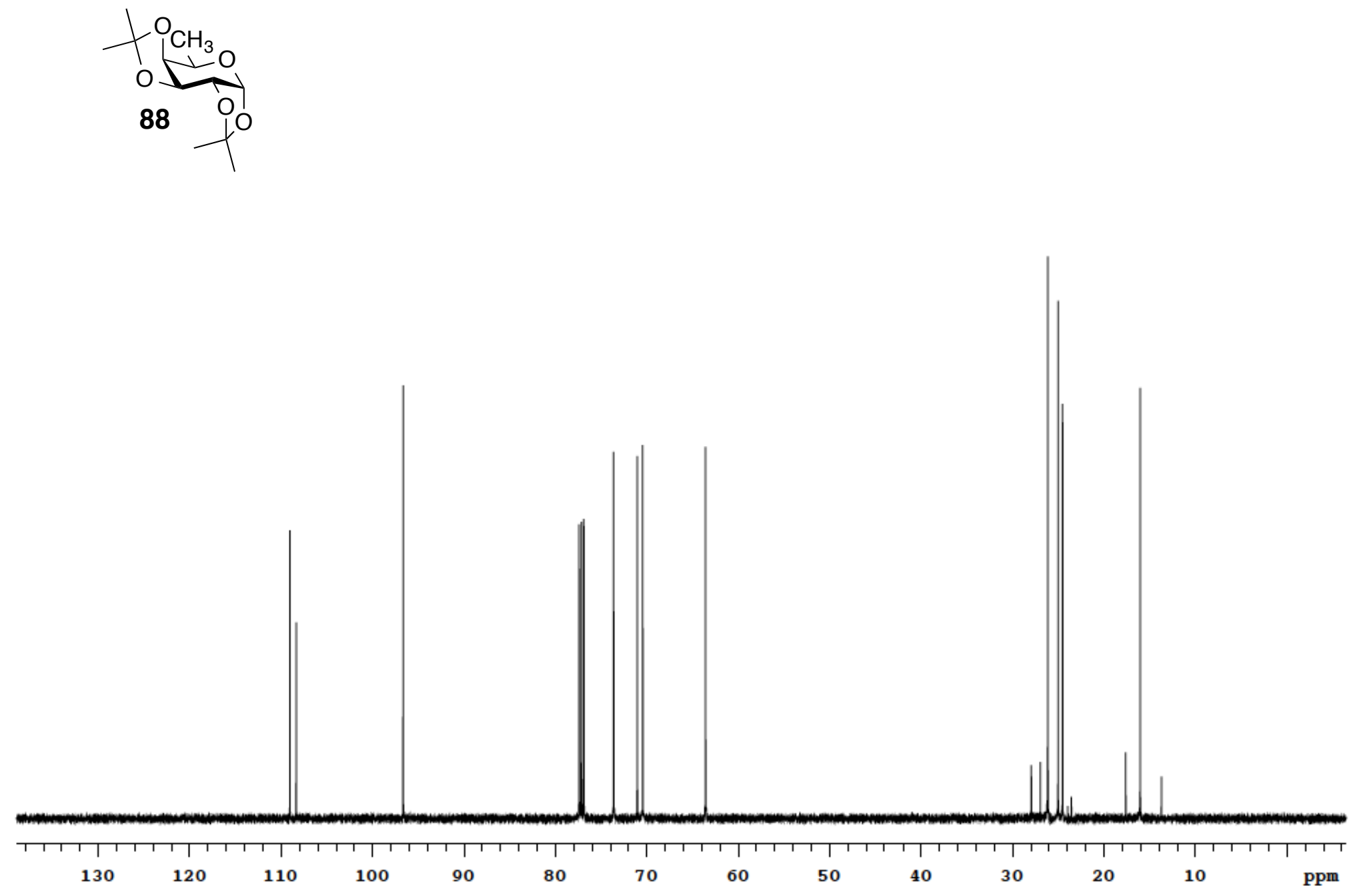
1,2,3,4-Tetra- $\boldsymbol{O}$-acetyl-6-deoxy- $\boldsymbol{\alpha} / \boldsymbol{\beta}$-D-galactopyranose (86) ${ }^{1} \mathrm{H} \mathrm{NMR}, \mathrm{CDCl}_{3}, 500 \mathrm{MHz}$

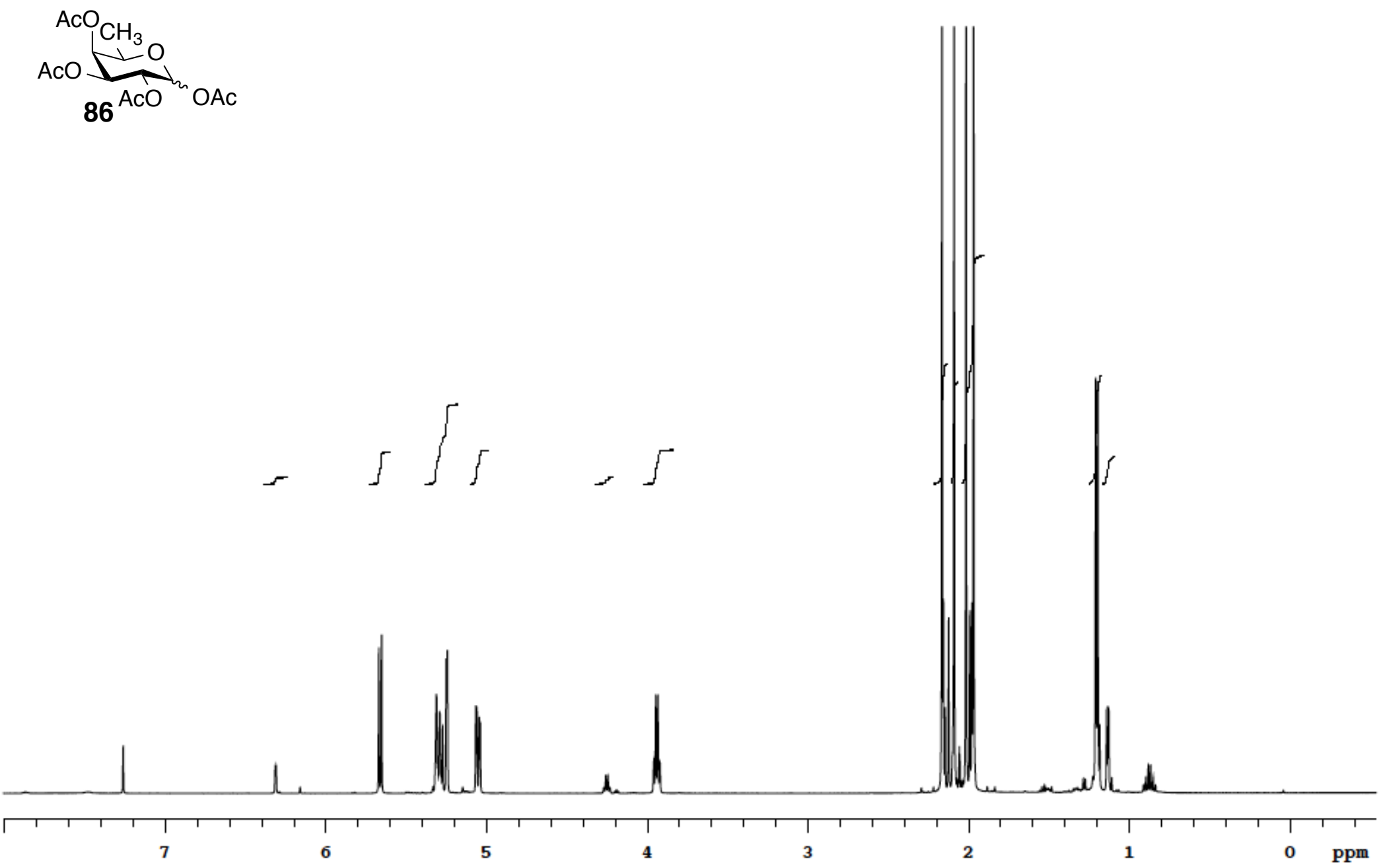


1,2,3,4-Tetra- $\boldsymbol{O}$-acetyl-6-deoxy- $\boldsymbol{\alpha} / \boldsymbol{\beta}$-D-galactopyranose (86) ${ }^{13} \mathrm{C} \mathrm{NMR,} \mathrm{CDCl}_{3}, 125 \mathrm{MHz}$
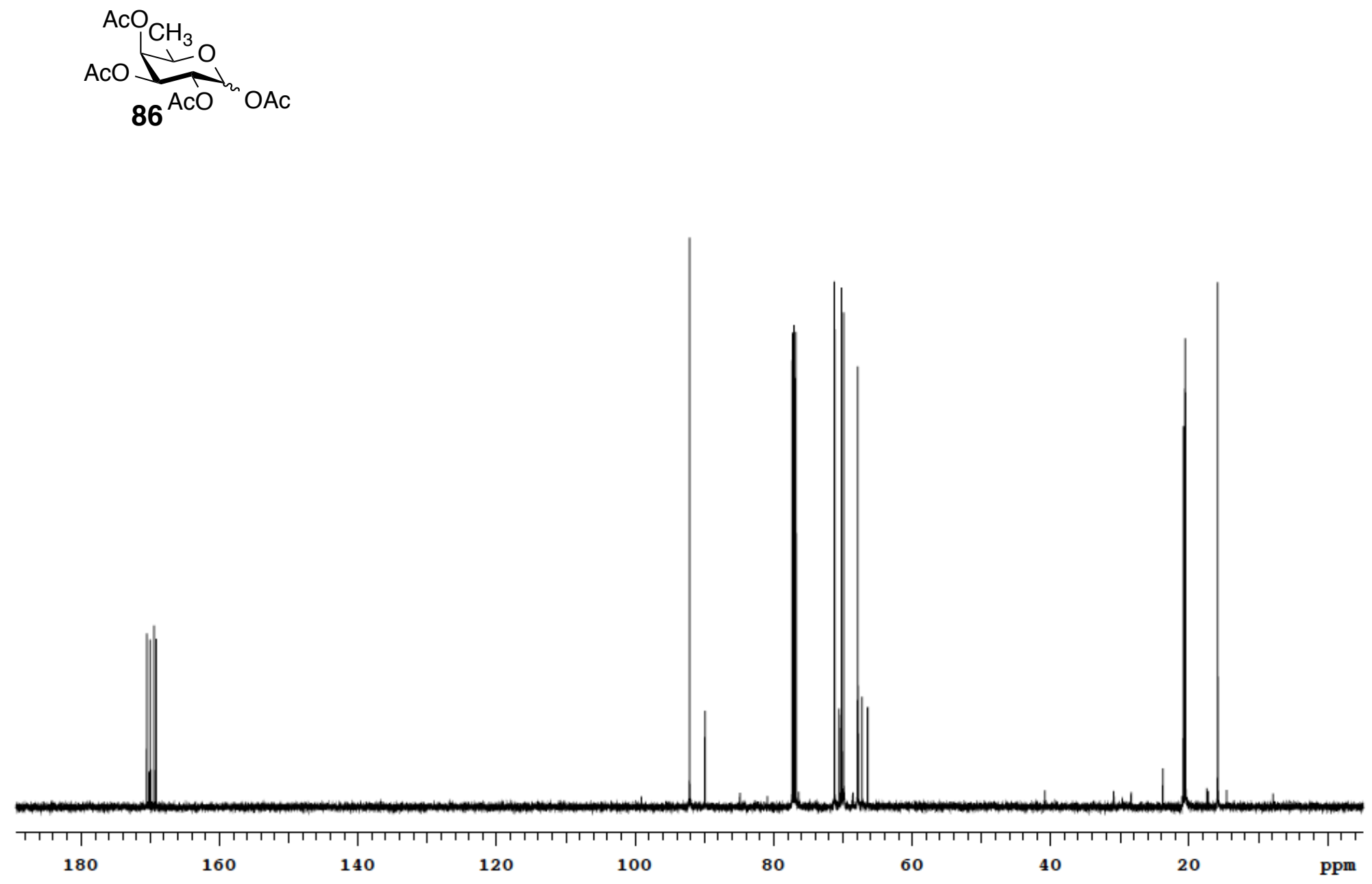
Ethyl 2,3,4-tri- $\boldsymbol{O}$-acetyl-6-deoxy-1-thio- $\boldsymbol{\beta}$-D-galactopyranoside (89) ${ }^{1} \mathrm{H} \mathrm{NMR}, \mathrm{CDCl}_{3}, 500 \mathrm{MHz}$
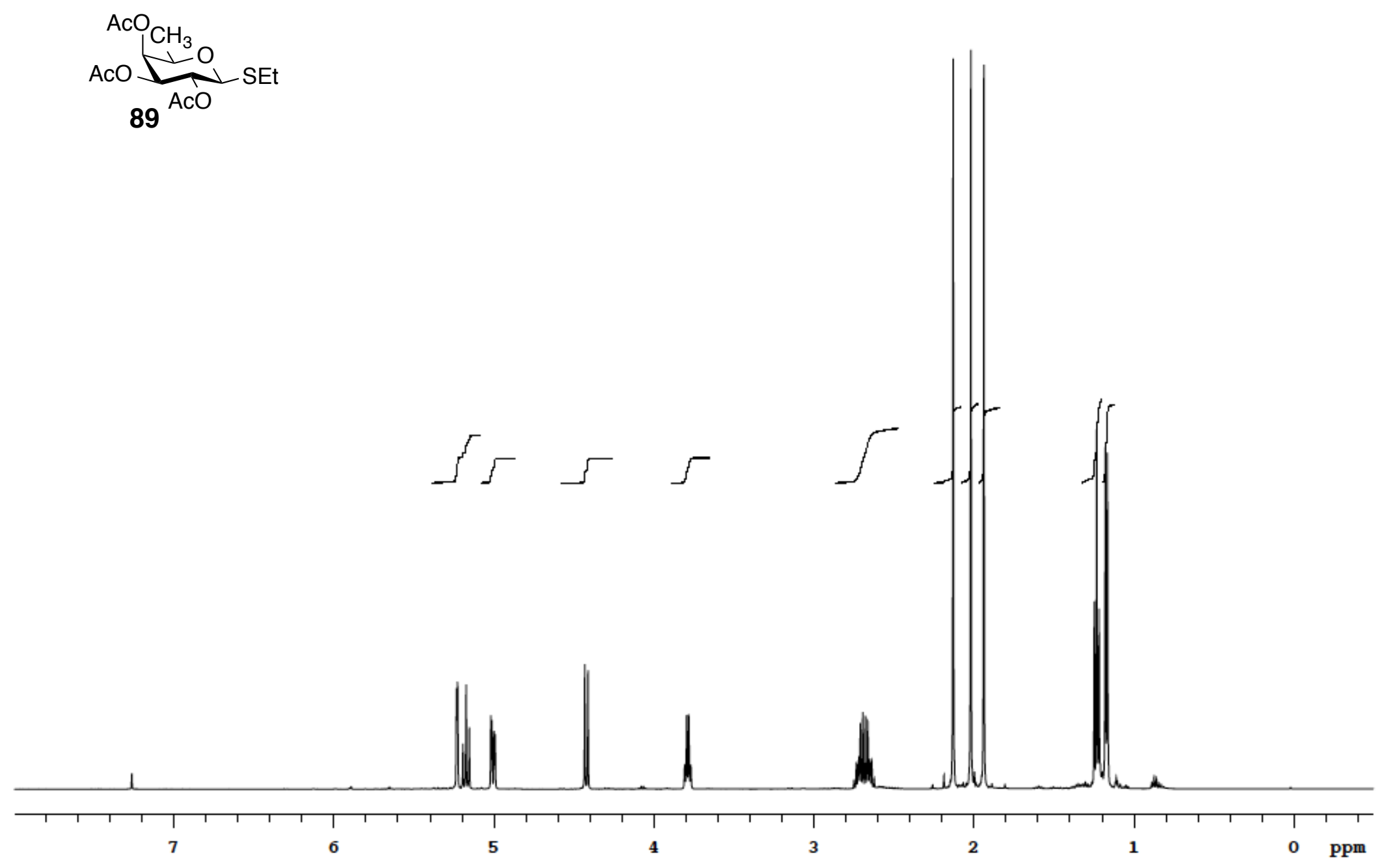
Ethyl 2,3,4-tri-O-acetyl-6-deoxy-1-thio- $\boldsymbol{\beta}$-D-galactopyranoside (89) ${ }^{13} \mathrm{C} \mathrm{NMR} \mathrm{CDCl}_{3}, 125 \mathrm{MHz}$
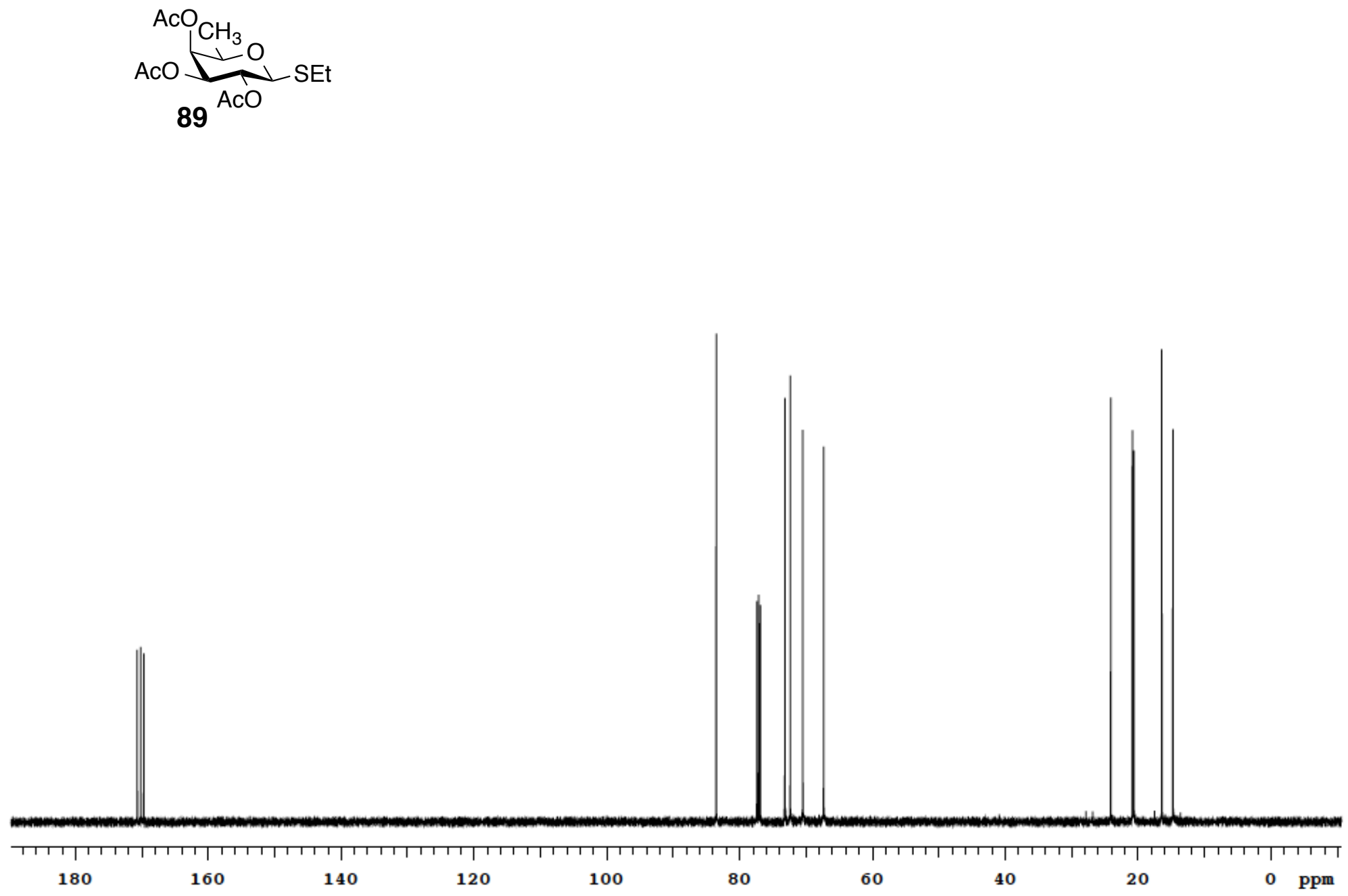
Ethyl 2,3,4-tri-O-benzyl-6-deoxy-1-thio- $\boldsymbol{\beta}$-D-galactopyranoside (90) ${ }^{1} \mathrm{H} \mathrm{NMR}, \mathrm{CDCl}_{3}, 500 \mathrm{MHz}$
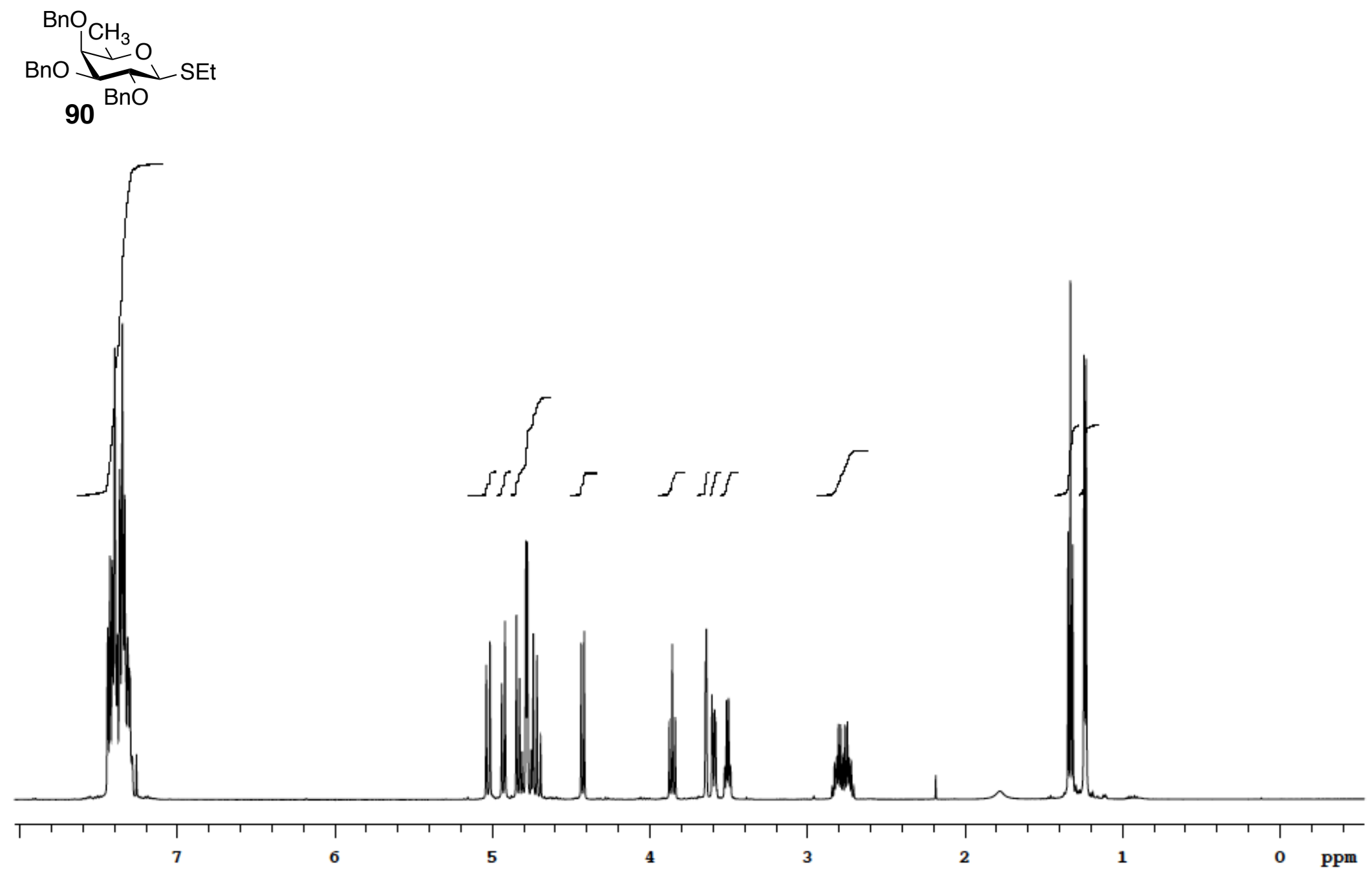
Ethyl 2,3,4-tri-O-benzyl-6-deoxy-1-thio- $\boldsymbol{\beta}$-D-galactopyranoside (90) ${ }^{13} \mathrm{C} \mathrm{NMR,} \mathrm{CDCl}_{3}, 125 \mathrm{MHz}$

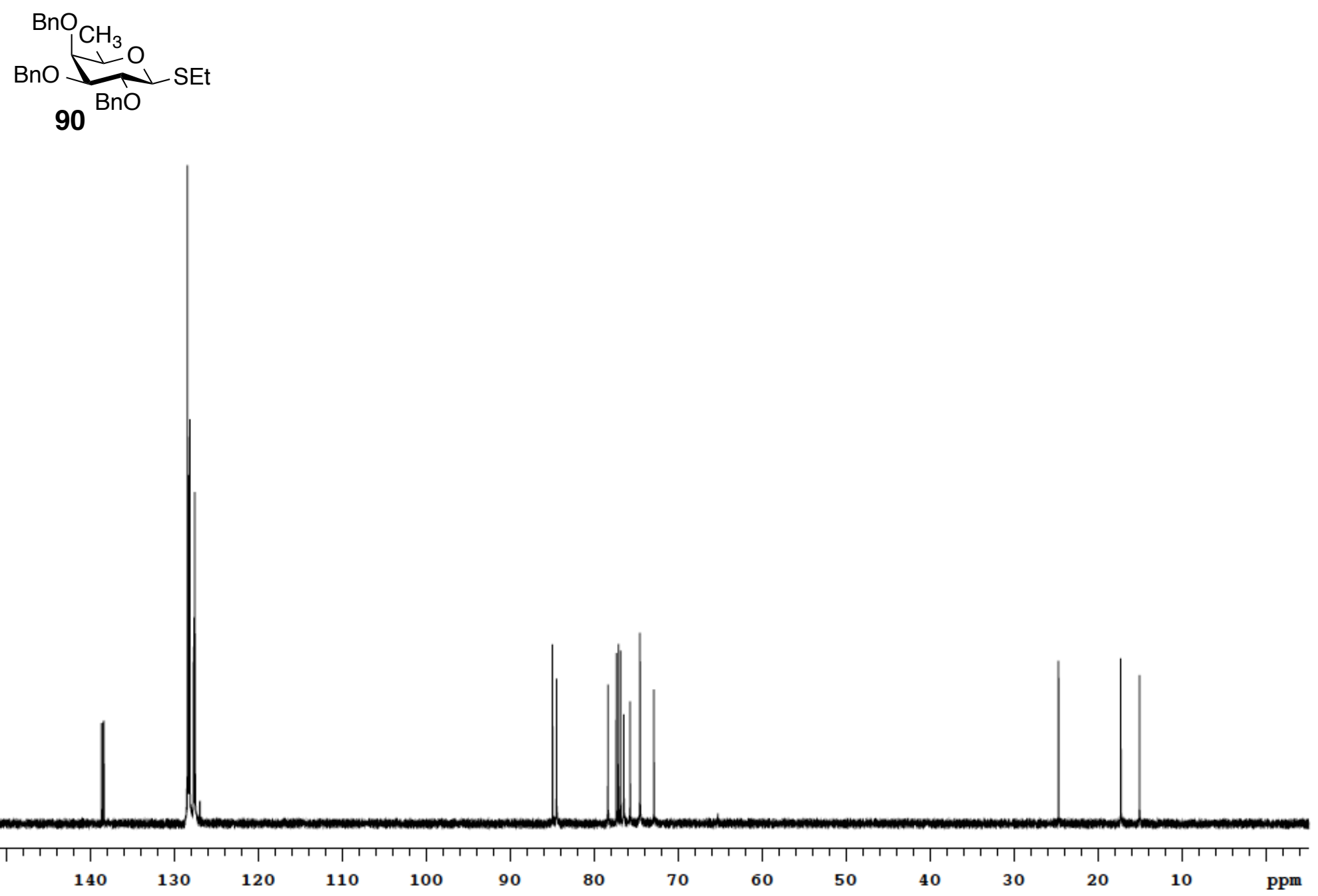


2,3,4-Tri-O-benzyl-6-deoxy- $\boldsymbol{\alpha} / \boldsymbol{\beta}$-D-galactopyranose (91) ${ }^{1} \mathrm{H} \mathrm{NMR}, \mathrm{CDCl}_{3}, 500 \mathrm{MHz}$
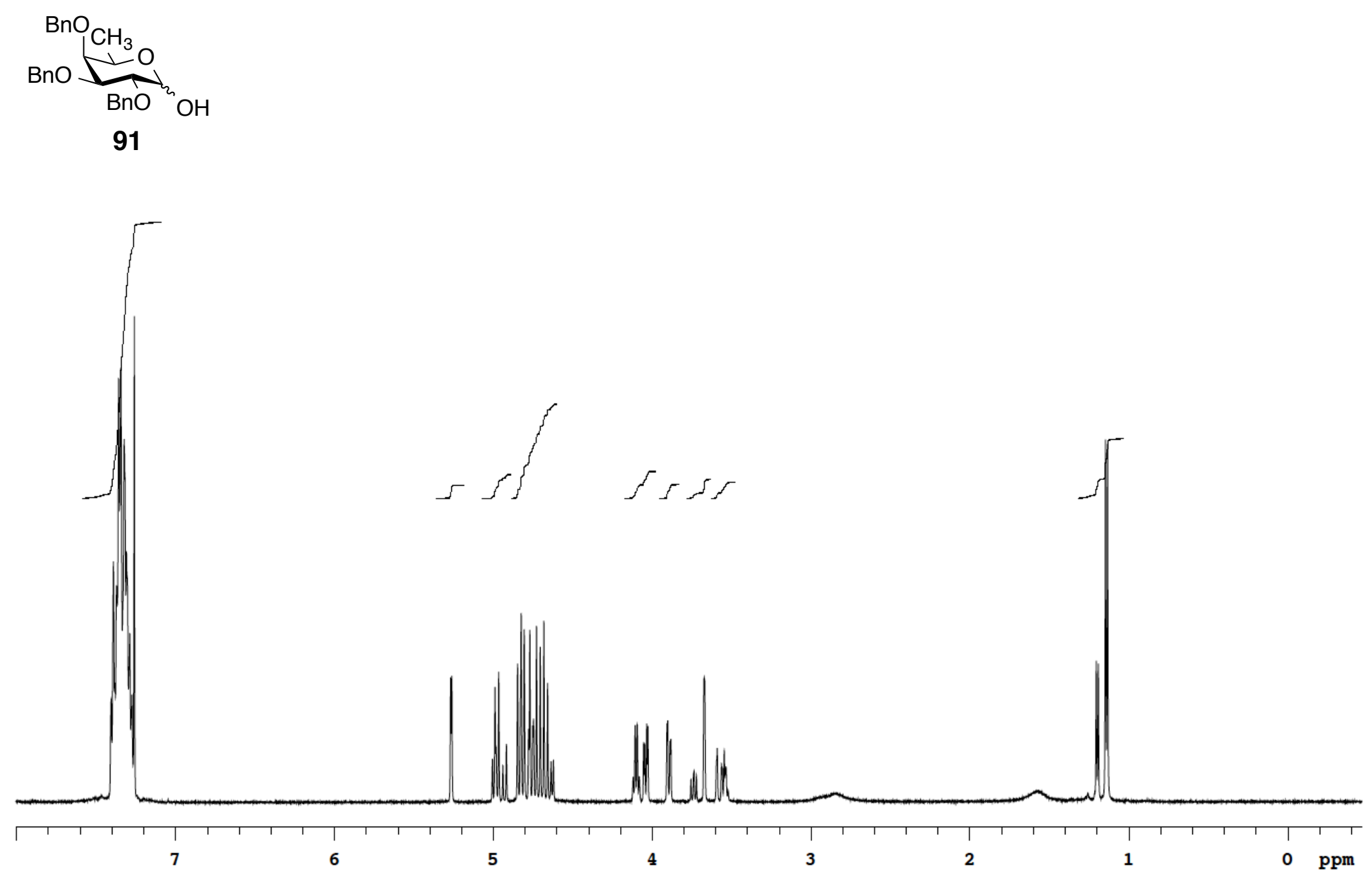
2,3,4-Tri- $\boldsymbol{O}$-benzyl-6-deoxy- $\boldsymbol{\alpha} / \boldsymbol{\beta}$-D-galactopyranose (91) ${ }^{13} \mathrm{C} \mathrm{NMR}^{\mathrm{CDCl}} \mathrm{CD}_{3}, 125 \mathrm{MHz}$

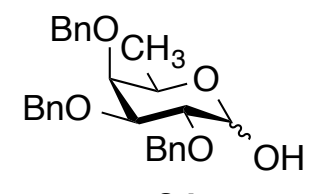

91

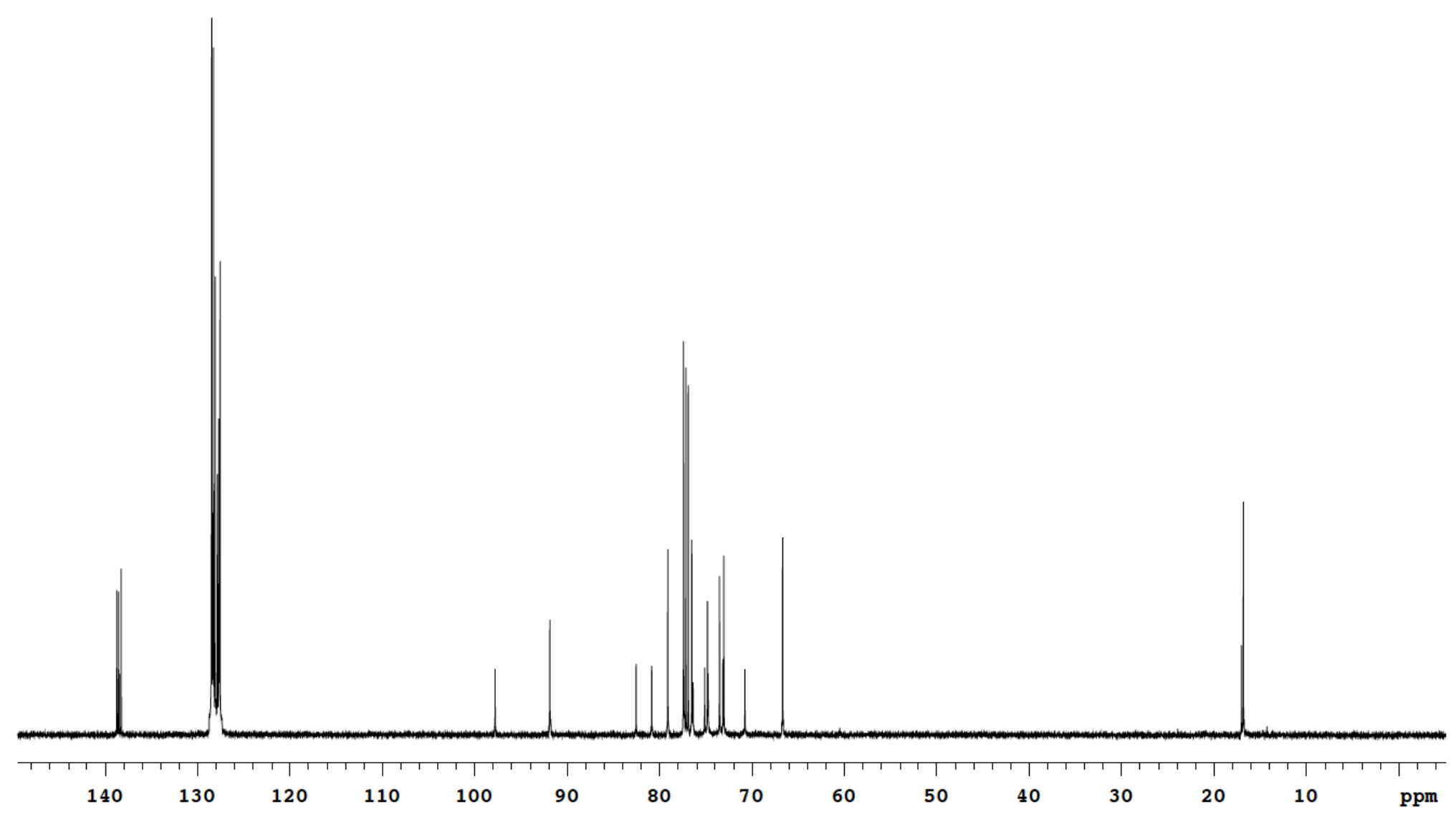


$\boldsymbol{O}$-(2,3,4-Tri- $\boldsymbol{O}$-benzyl-6-deoxy- $\boldsymbol{\alpha} / \boldsymbol{\beta}$-D-galactopyranosyl) trichloroacetimidate (72) ${ }^{1} \mathrm{H} \mathrm{NMR}, \mathrm{CDCl}_{3}, 500 \mathrm{MHz}$
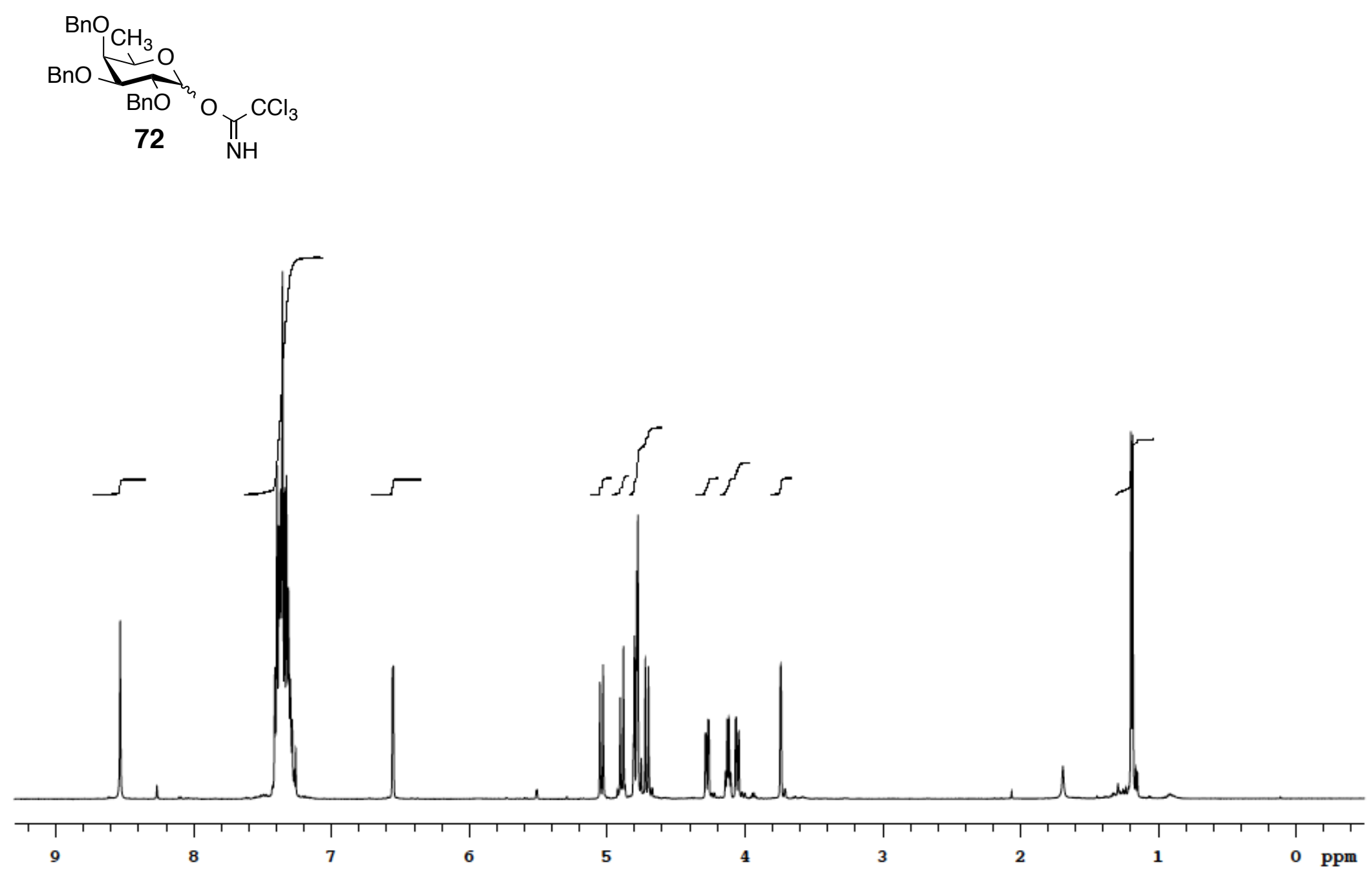
$\boldsymbol{O}$-(2,3,4-Tri-O-benzyl-6-deoxy- $\boldsymbol{\alpha} / \boldsymbol{\beta}$-D-galactopyranosyl) trichloroacetimidate (72) ${ }^{13} \mathrm{C} \mathrm{NMR,} \mathrm{CDCl}_{3}, 125 \mathrm{MHz}$
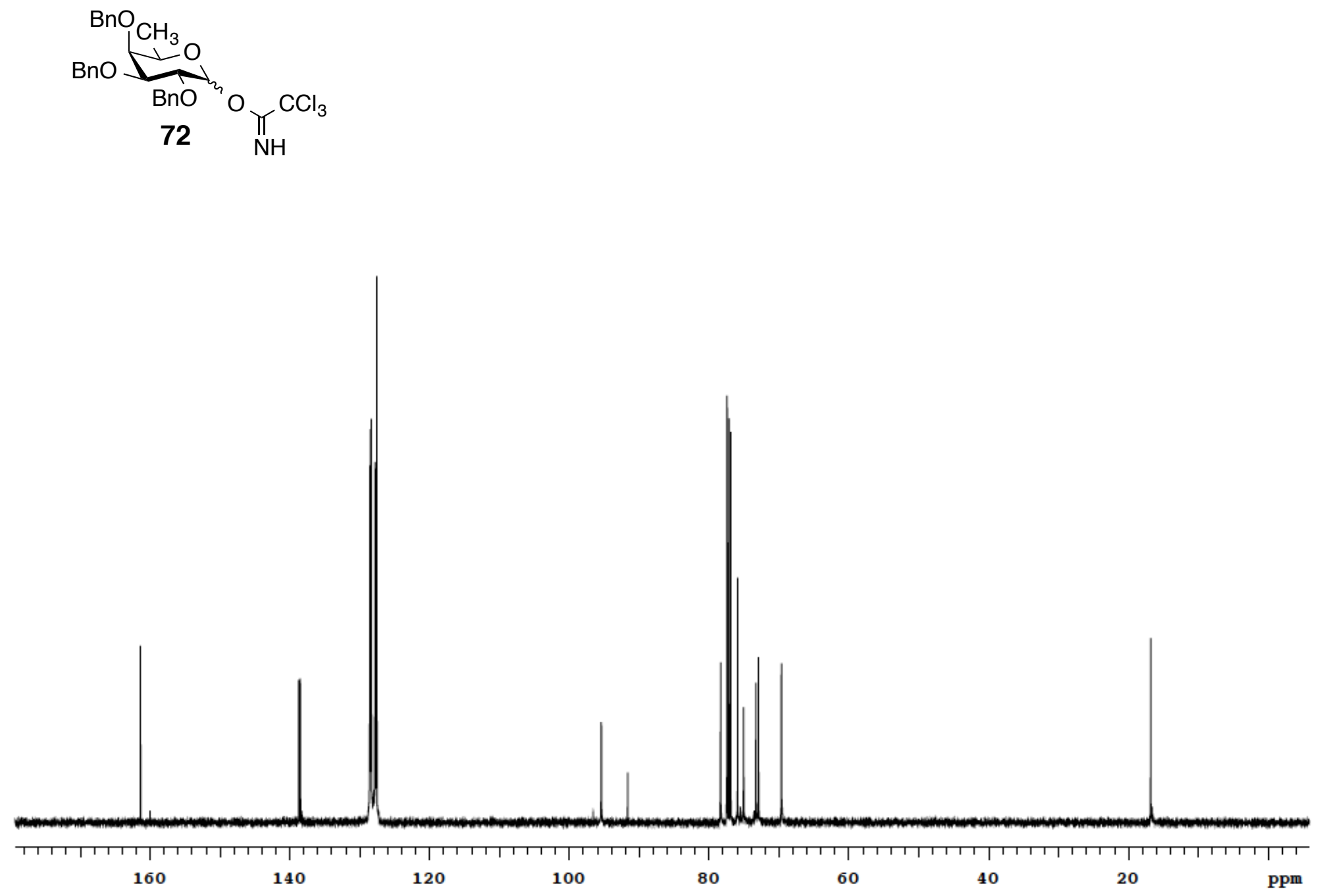
(2S,3R,4E)-1-(4-O-(4-O-Acetyl-2,6-di- $O$-benzoyl-3-O-(2,3,4-tri- $O$-benzyl-6-deoxy- $\alpha$-D-galactopyranosyl)- $\beta$-D-galactopyranosyl)-2,3,6tri- $\boldsymbol{O}$-benzoyl- $\boldsymbol{\beta}$-D-glucopyranosyloxy)-3-benzyloxy-2-hexacosanoylamido-octadec-4-ene (71) ${ }^{1} \mathrm{H} \mathrm{NMR}, \mathrm{CDCl}_{3}, 500 \mathrm{MHz}$

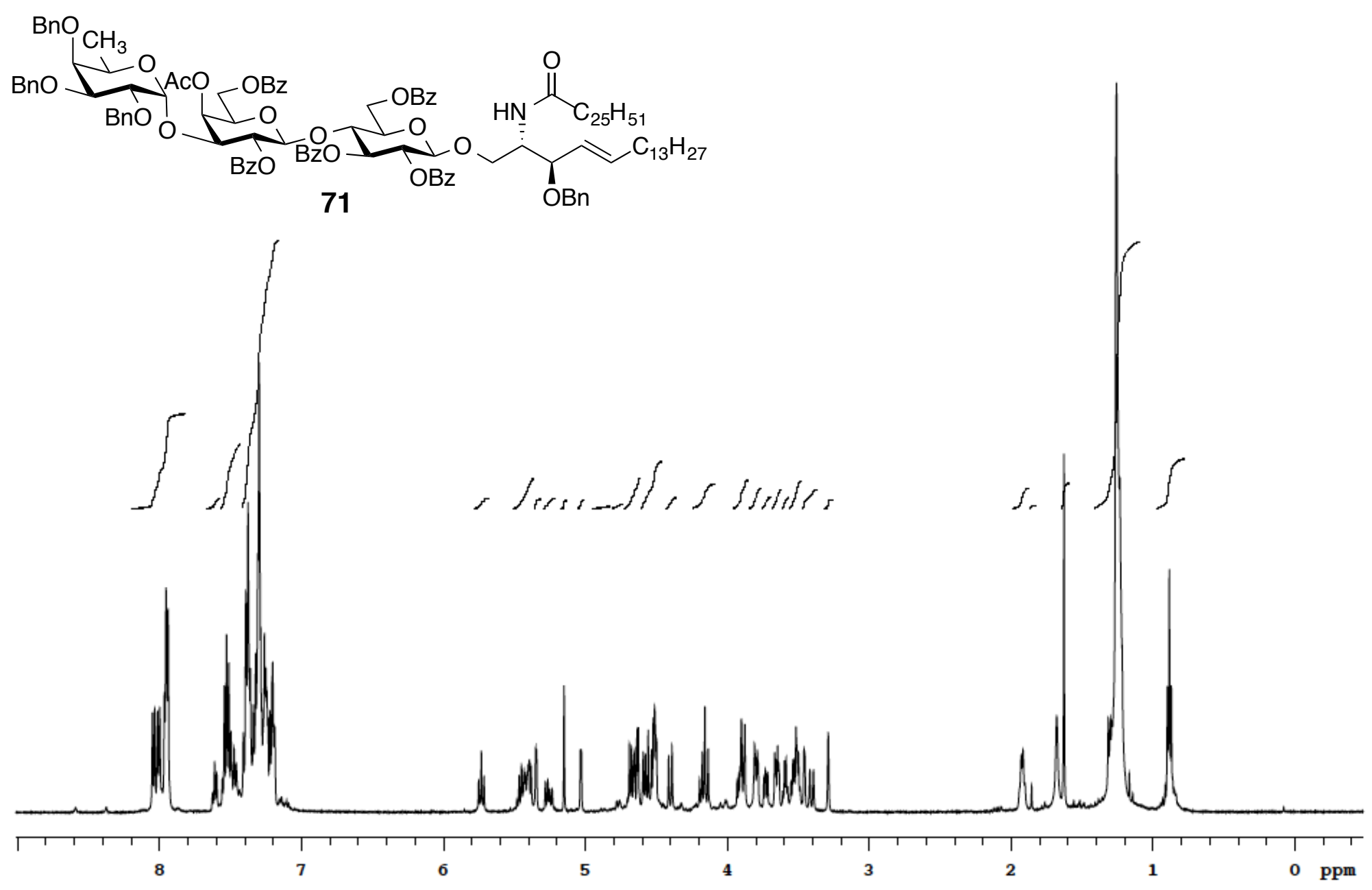


$(2 S, 3 R, 4 E)-1-(4-O$-(4-O-Acetyl-2,6-di- $O$-benzoyl-3-O-(2,3,4-tri- $O$-benzyl-6-deoxy- $\alpha$-D-galactopyranosyl)- $\beta$-D-galactopyranosyl)2,3,6-tri- $\boldsymbol{O}$-benzoyl- $\boldsymbol{\beta}$-D-glucopyranosyloxy)-3-benzyloxy-2-hexacosanoylamido-octadec-4-ene (71) ${ }^{13} \mathrm{C} \mathrm{NMR}, \mathrm{CDCl}_{3}, 125 \mathrm{MHz}$

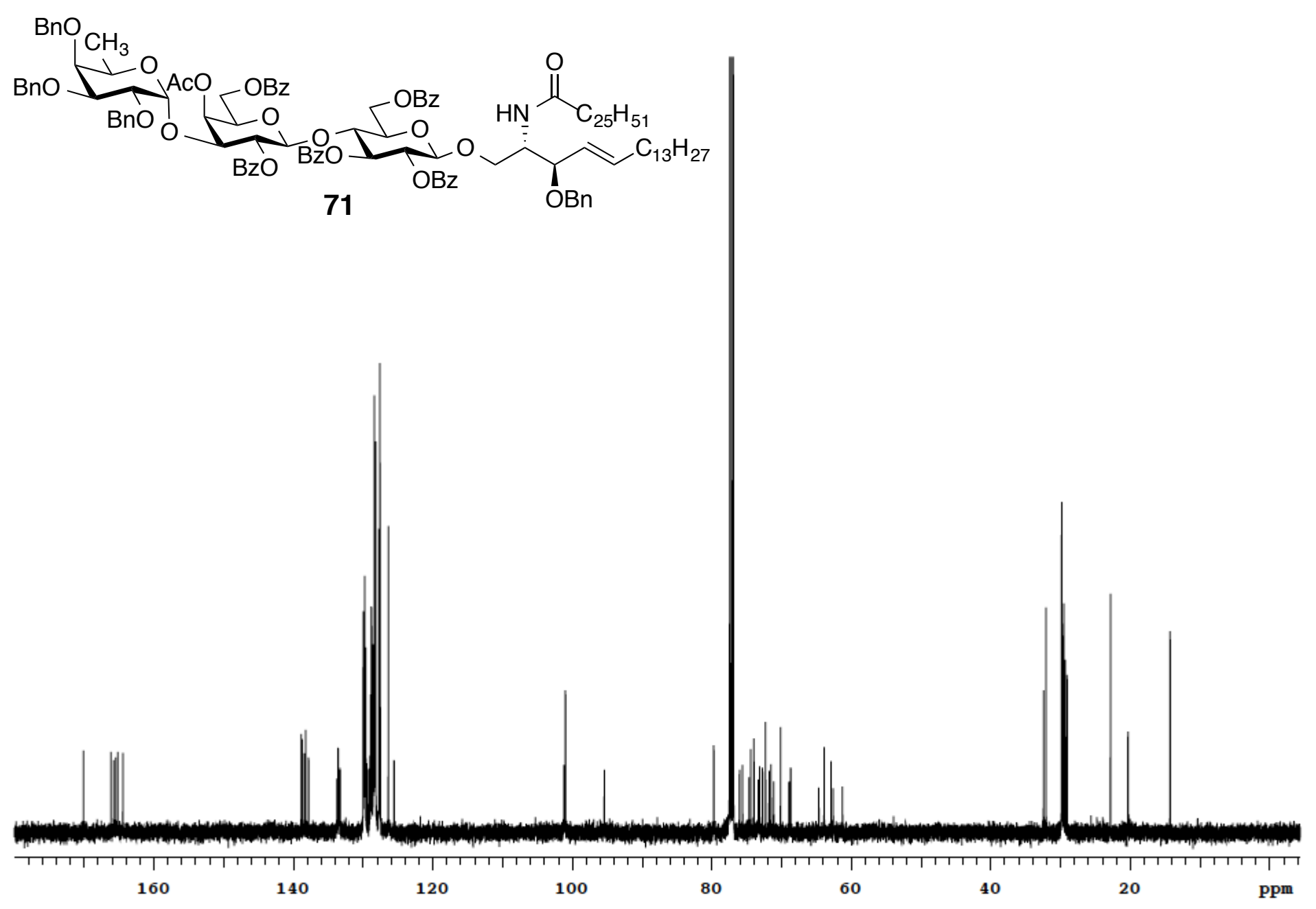


(2S,3R,4E)-1-(4-O-(3-O-(6-Deoxy- $\alpha$-D-galactopyranosyl)- $\beta$-D-galactopyranosyl)- $\beta$-D-glucopyranosyloxy)-2-hexacosanoylamido-3hydroxy-octadec-4-ene (5) ${ }^{1} \mathrm{H}$ NMR, pyridine- $\mathrm{d}_{5}, 500 \mathrm{MHz}$

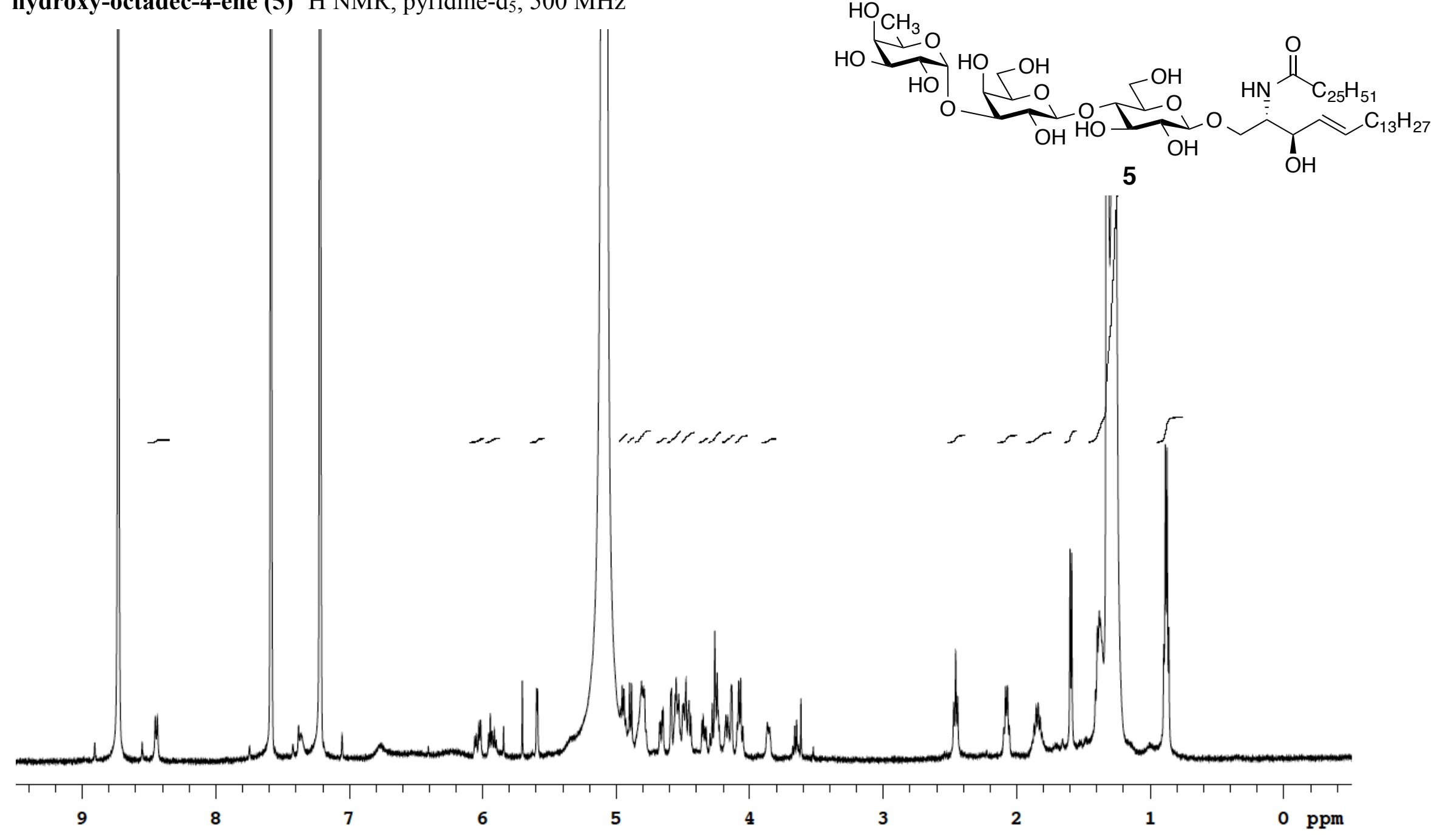


(2S,3R,4E)-1-(4-O-(3-O-(6-Deoxy- $\alpha$-D-galactopyranosyl)- $\beta$-D-galactopyranosyl)- $\beta$-D-glucopyranosyloxy)-2-hexacosanoylamido-3hydroxy-octadec-4-ene (5) ${ }^{13} \mathrm{C}$ NMR, pyridine- $\mathrm{d}_{5}, 125 \mathrm{MHz}$

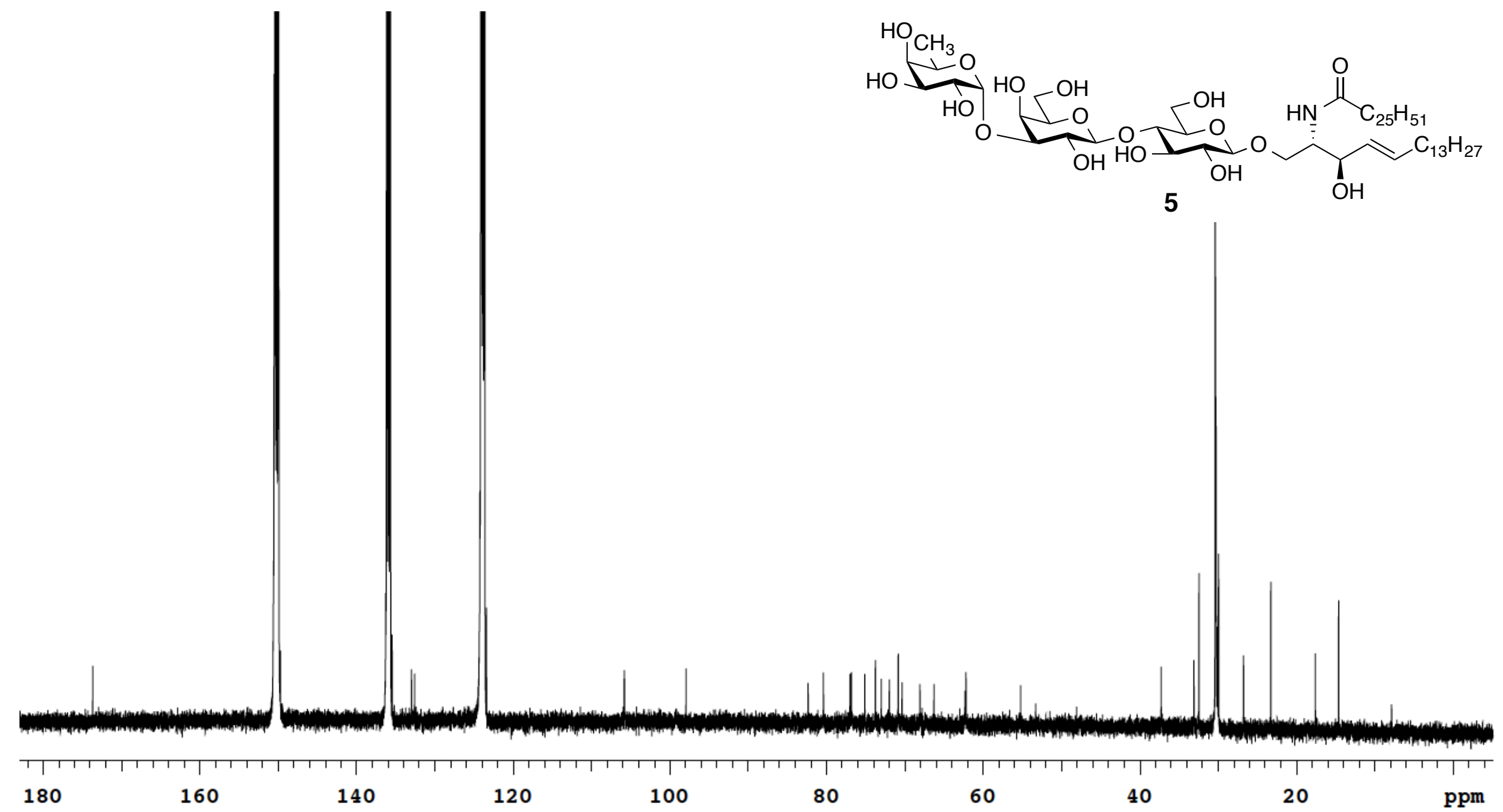


(2S,3R,4E)-1-(4-O-(3-O-(6-Deoxy- $\alpha$-D-galactopyranosyl)- $\beta$-D-galactopyranosyl)- $\beta$-D-glucopyranosyloxy)-2-hexacosanoylamido-3-

hydroxy-octadecane (6) ${ }^{1} \mathrm{H}$ NMR, pyridine- $\mathrm{d}_{5}, 500 \mathrm{MHz}$

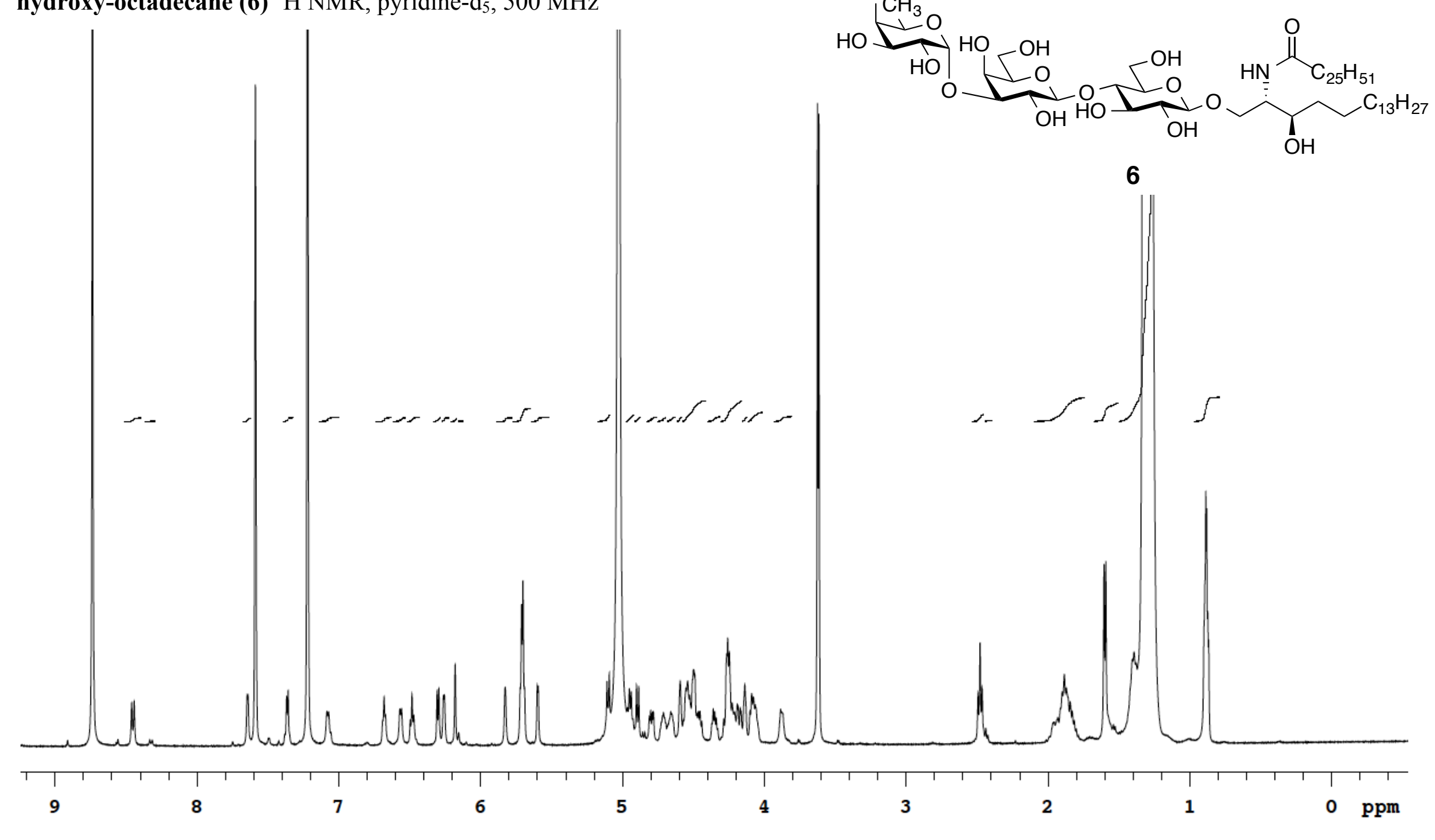


(2S,3R,4E)-1-(4-O-(3-O-(6-Deoxy- $\alpha$-D-galactopyranosyl)- $\beta$-D-galactopyranosyl)- $\beta$-D-glucopyranosyloxy)-2-hexacosanoylamido-3hydroxy-octadecane (6) ${ }^{13} \mathrm{C}$ NMR, pyridine- $\mathrm{d}_{5}, 125 \mathrm{MHz}$

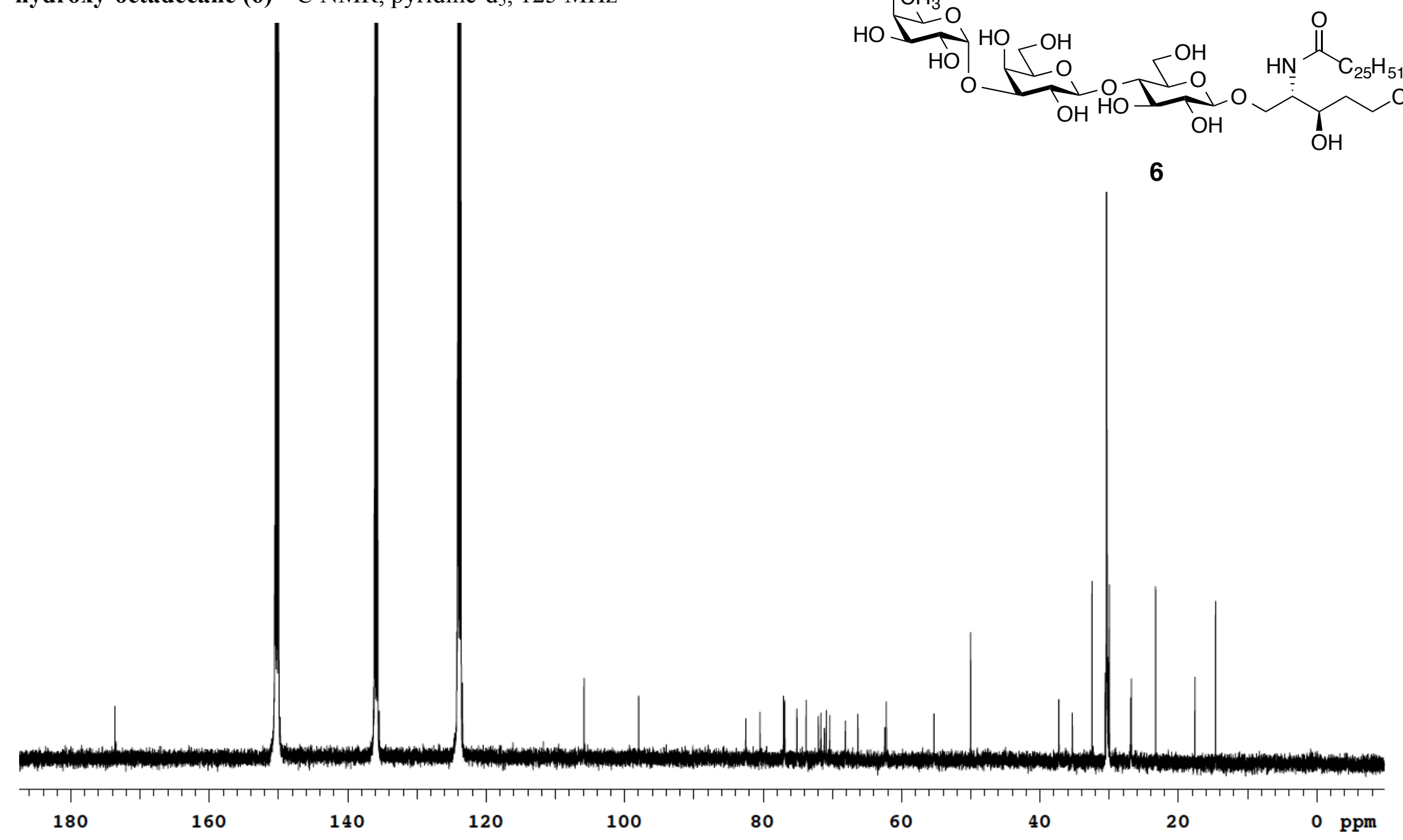




\section{${ }^{1} \mathrm{H} \&{ }^{13} \mathrm{C}$ NMR Spectra}

Chapter 5 
(2S,3R,4E)-2-Azido-1-(4- $O$-(2,6-di- $O$-benzoyl- $\beta$-D-galactopyranosyl)-2,3,6-tri- $O$-benzoyl- $\beta$-D-glucopyranosyloxy)-3-benzyloxyoctadec-4-ene (98) ${ }^{1} \mathrm{H} \mathrm{NMR}, \mathrm{CDCl}_{3}, 500 \mathrm{MHz}$

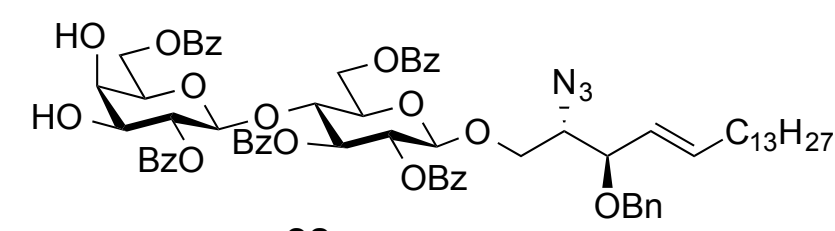

98

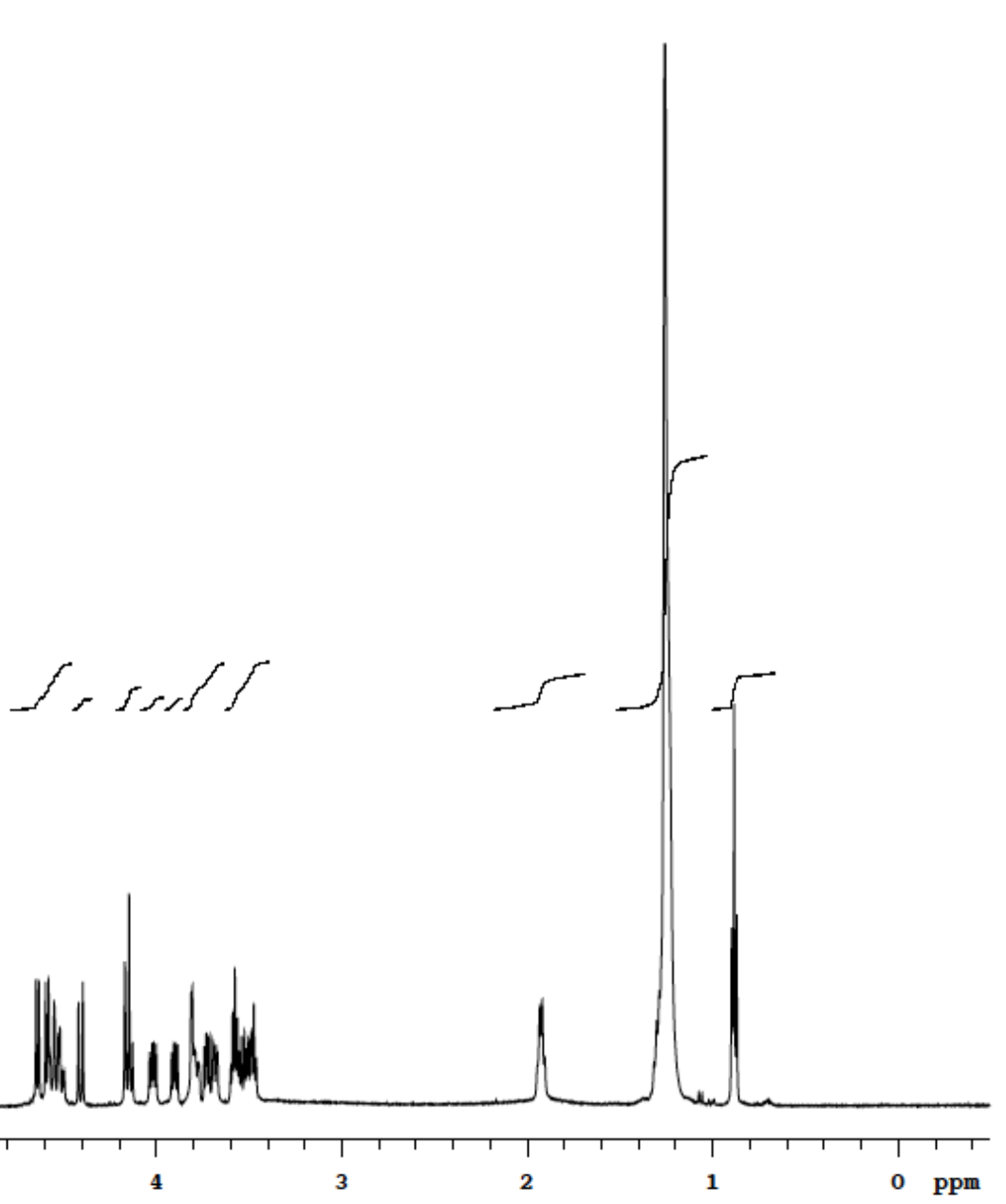


(2S,3R,4E)-2-Azido-1-(4-O-(2,6-di- $O$-benzoyl- $\beta$-D-galactopyranosyl)-2,3,6-tri- $O$-benzoyl- $\beta$-D-glucopyranosyloxy)-3-benzyloxyoctadec-4-ene (98) ${ }^{13} \mathrm{C} \mathrm{NMR,} \mathrm{CDCl}_{3}, 125 \mathrm{MHz}$

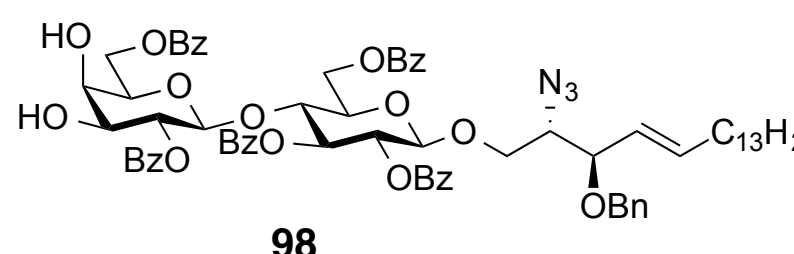

98

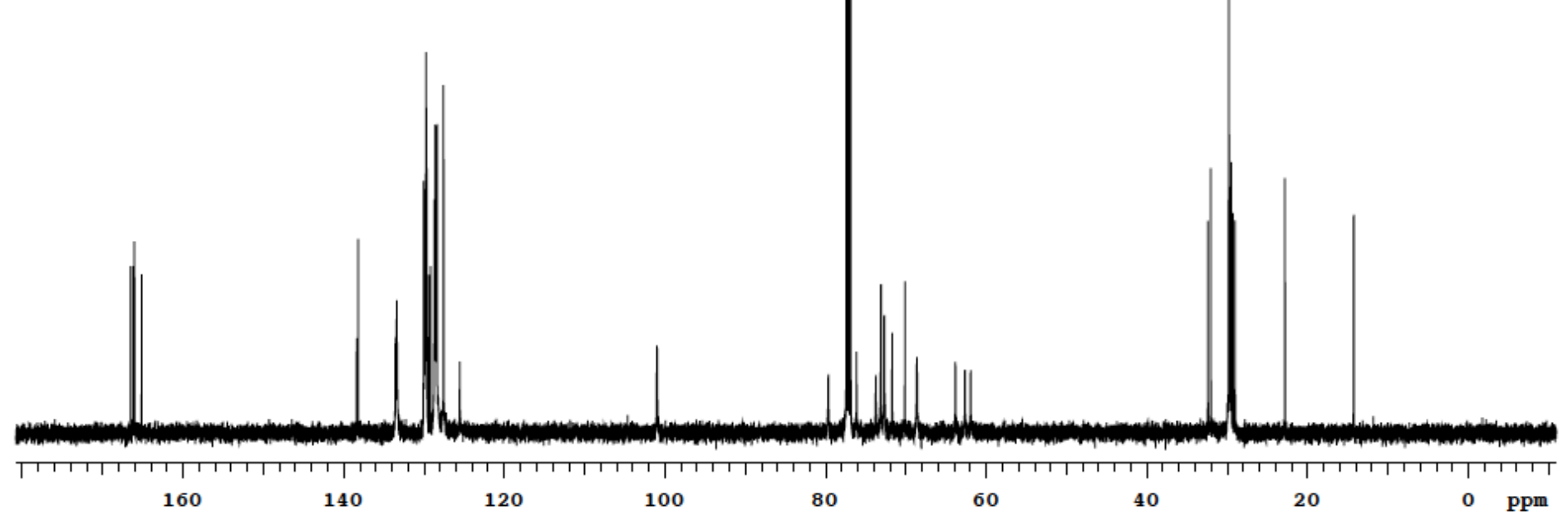


$(2 S, 3 R, 4 E)-1-(4-O$-(4- $O$-Acetyl-2,6-di- $O$-benzoyl- $\beta$-D-galactopyranosyl)-2,3,6-tri- $O$-benzoyl- $\beta$-D-glucopyranosyloxy)-2-azido-3benzyloxy-octadec-4-ene (94) ${ }^{1} \mathrm{H} \mathrm{NMR}, \mathrm{CDCl}_{3}, 500 \mathrm{MHz}$

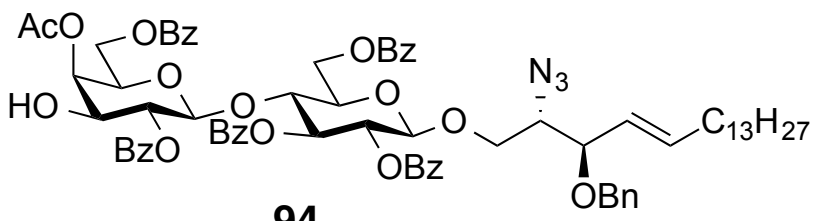

94

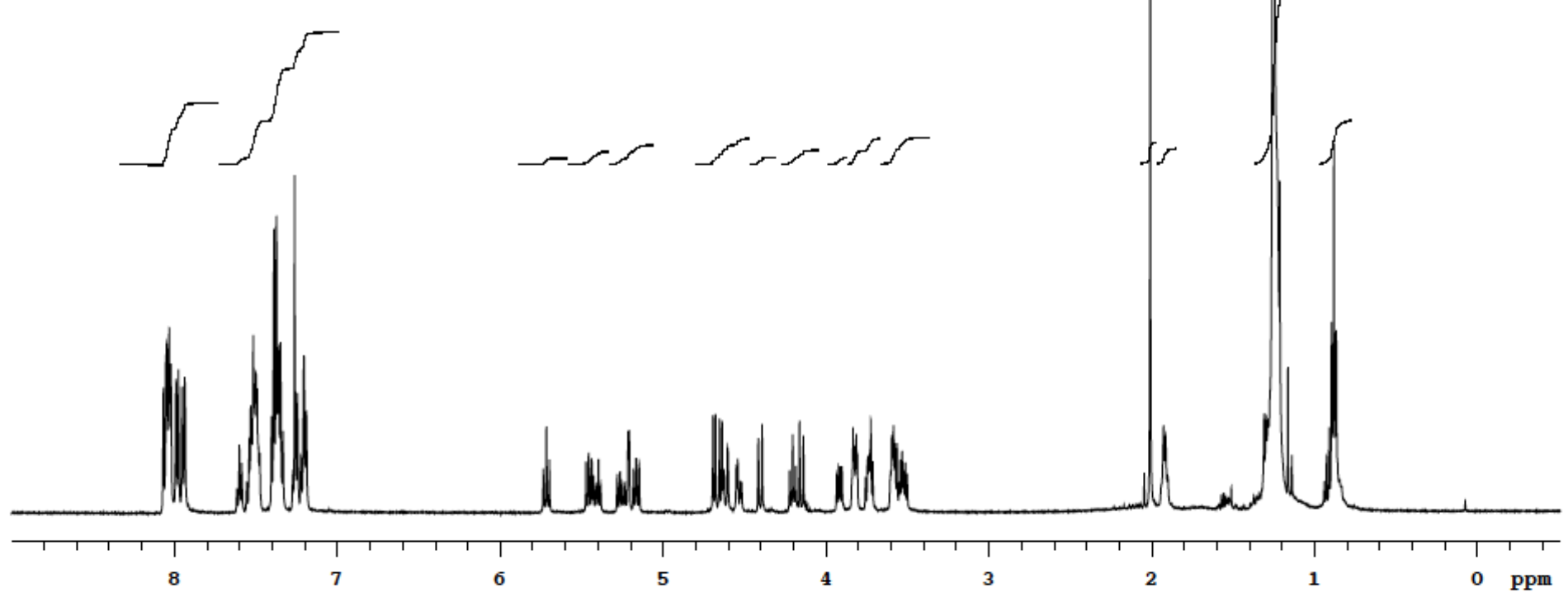

1xxii 
(2S,3R,4E)-1-(4-O-(4-O-Acetyl-2,6-di- $O$-benzoyl- $\beta$-D-galactopyranosyl)-2,3,6-tri- $O$-benzoyl- $\beta$-D-glucopyranosyloxy)-2-azido-3benzyloxy-octadec-4-ene (94) ${ }^{13} \mathrm{C} \mathrm{NMR}, \mathrm{CDCl}_{3}, 125 \mathrm{MHz}$

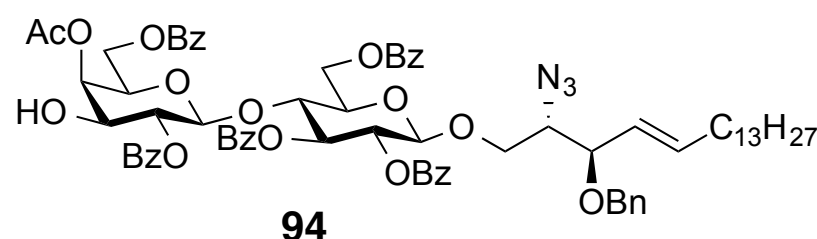

94

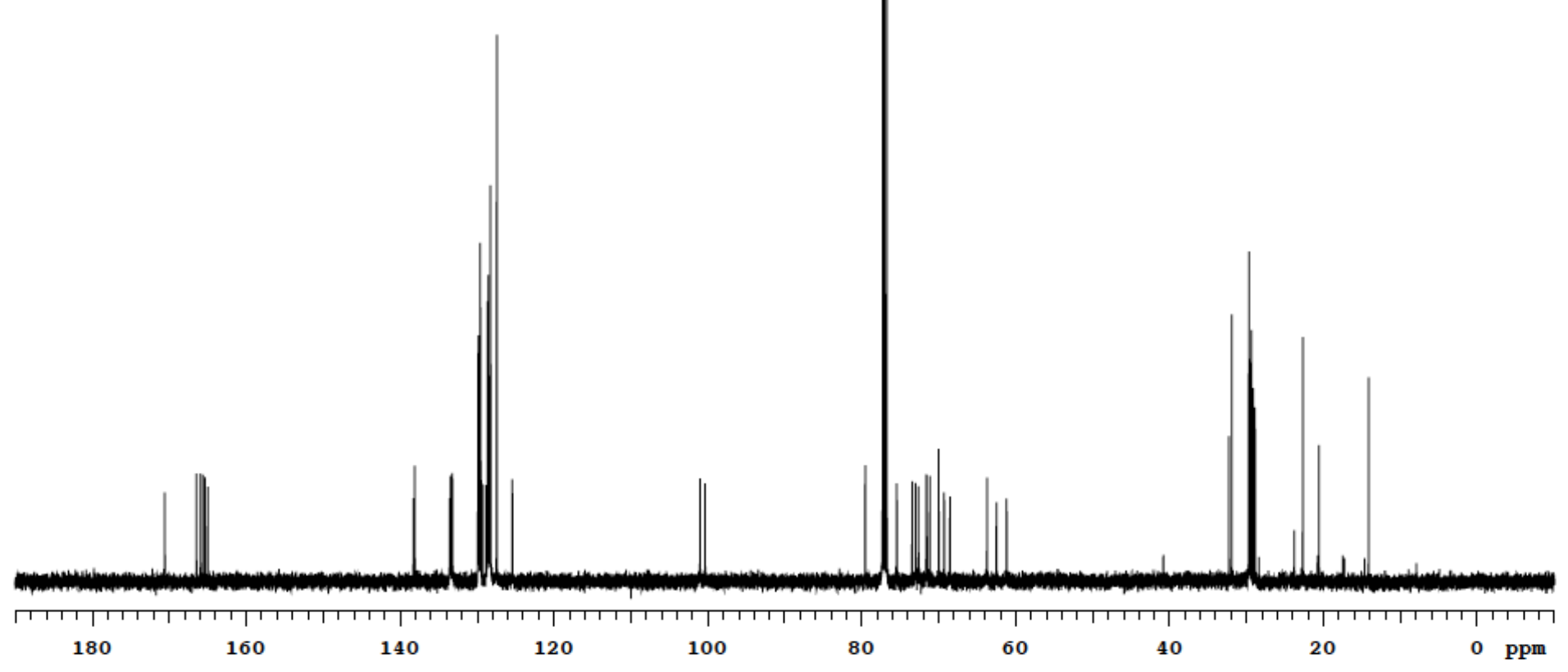


(2S,3R,4E)-1-(4-O-(4-O-Acetyl-2,6-di- $O$-benzoyl-3-O-(2,3-di- $O$-benzyl-4,6- $O$-benzylidene- $\alpha$-D-galactopyranosyl)- $\beta$-Dgalactopyranosyl)-2,3,6-tri- $\boldsymbol{O}$-benzoyl- $\beta$-D-glucopyranosyloxy)-2-azido-3-benzyloxy-octadec-4-ene (92) ${ }^{1} \mathrm{H} \mathrm{NMR,} \mathrm{CDCl}_{3}, 500 \mathrm{MHz}$

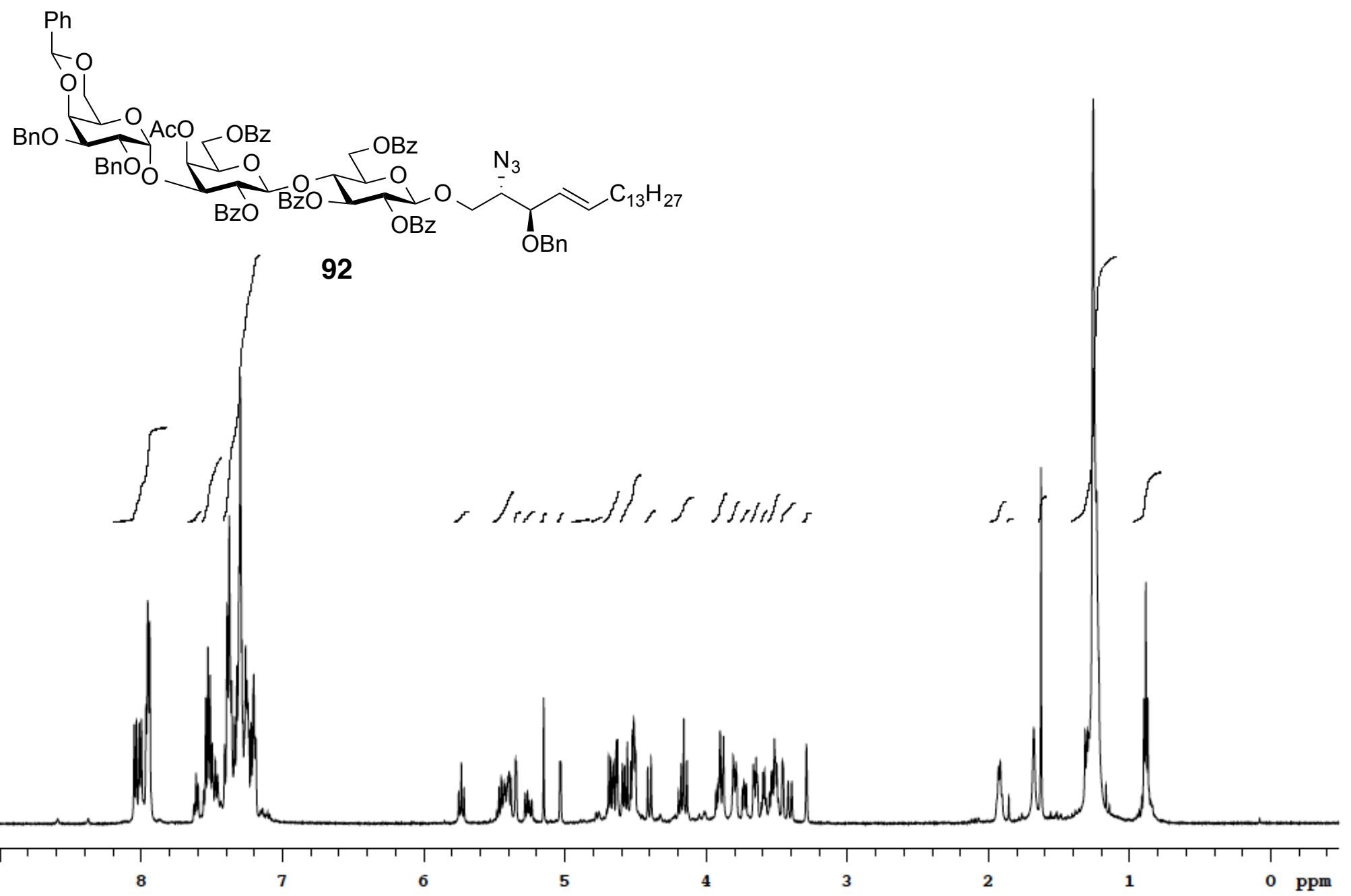


(2S,3R,4E)-1-(4-O-(4-O-Acetyl-2,6-di- $O$-benzoyl-3-O-(2,3-di- $O$-benzyl-4,6- $O$-benzylidene- $\alpha$-D-galactopyranosyl)- $\beta$-Dgalactopyranosyl)-2,3,6-tri- $\boldsymbol{O}$-benzoyl- $\boldsymbol{\beta}$-D-glucopyranosyloxy)-2-azido-3-benzyloxy-octadec-4-ene (92) ${ }^{13} \mathrm{C} \mathrm{NMR}, \mathrm{CDCl}_{3}, 125 \mathrm{MHz}$

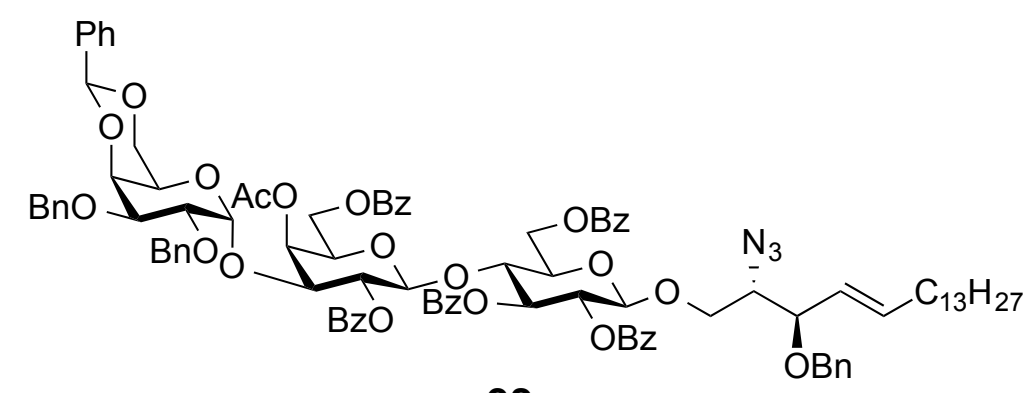

92

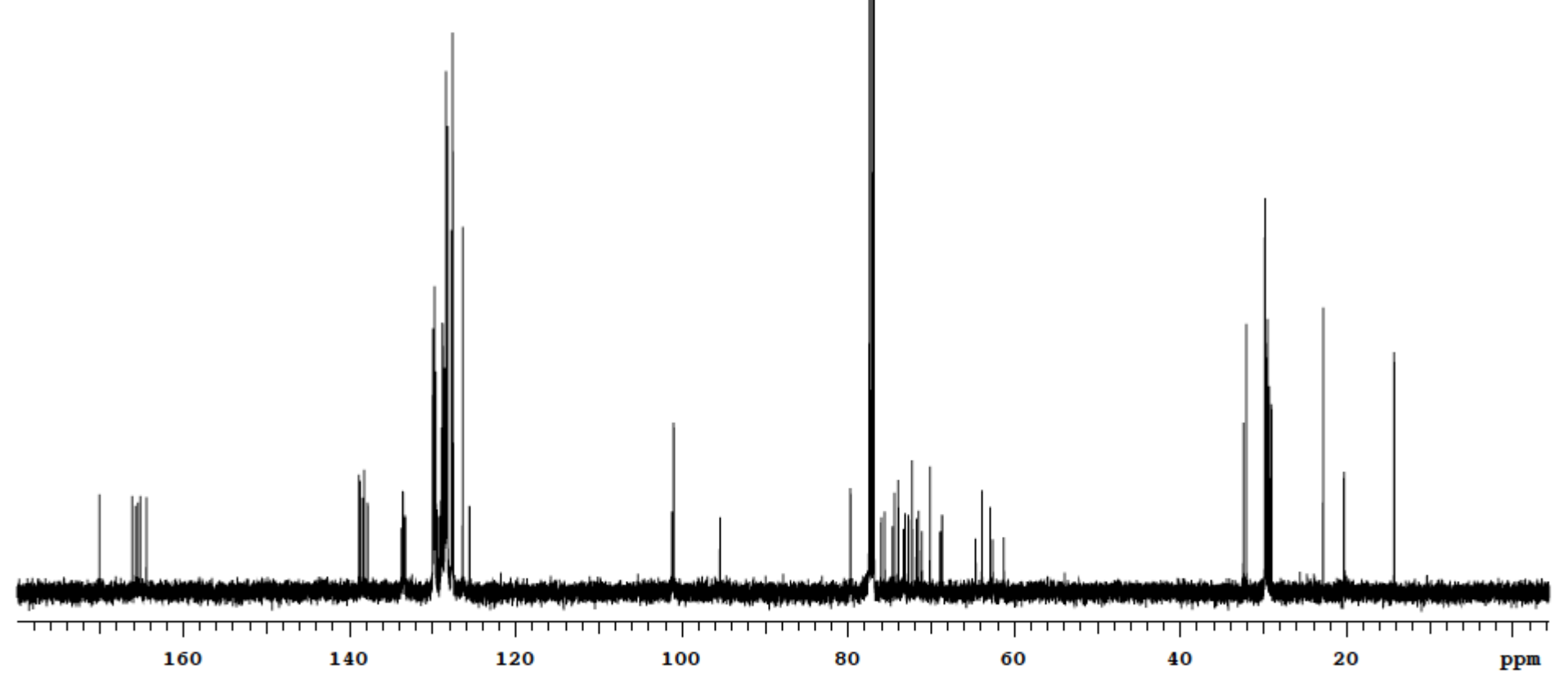


(2S,3R,4E)-1-(4-O-(4-O-Acetyl-2,6-di- $O$-benzoyl-3- $O$-(2,3-di- $O$-benzyl-4,6- $O$-benzylidene- $\alpha$-D-galactopyranosyl)- $\beta$-Dgalactopyranosyl)-2,3,6-tri- $O$-benzoyl- $\beta$-D-glucopyranosyloxy)-3-benzyloxy-2-dodecanoylamido-octadec-4-ene (100)

${ }^{1} \mathrm{H} \mathrm{NMR}, \mathrm{CDCl}_{3}, 500 \mathrm{MHz}$
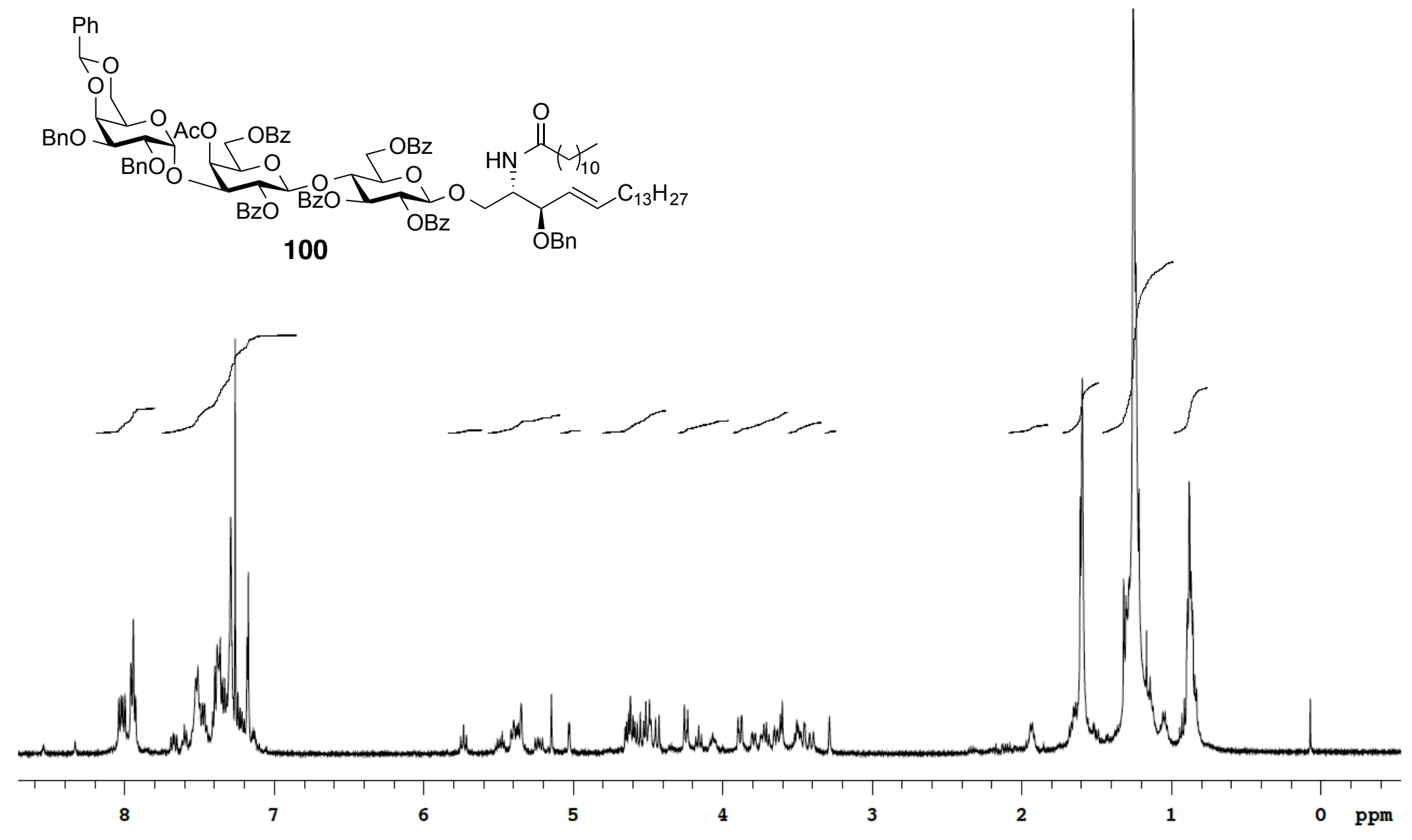
$(2 S, 3 R, 4 E)-1-(4-O$-(4- $O$-Acetyl-2,6-di- $O$-benzoyl-3- $O$-(2,3-di- $O$-benzyl-4,6- $O$-benzylidene- $\alpha$-D-galactopyranosyl)- $\beta$-Dgalactopyranosyl)-2,3,6-tri- $O$-benzoyl- $\beta$-D-glucopyranosyloxy)-3-benzyloxy-2-dodecanoylamido-octadec-4-ene (100)

${ }^{13} \mathrm{C} \mathrm{NMR}, \mathrm{CDCl}_{3}, 125 \mathrm{MHz}$

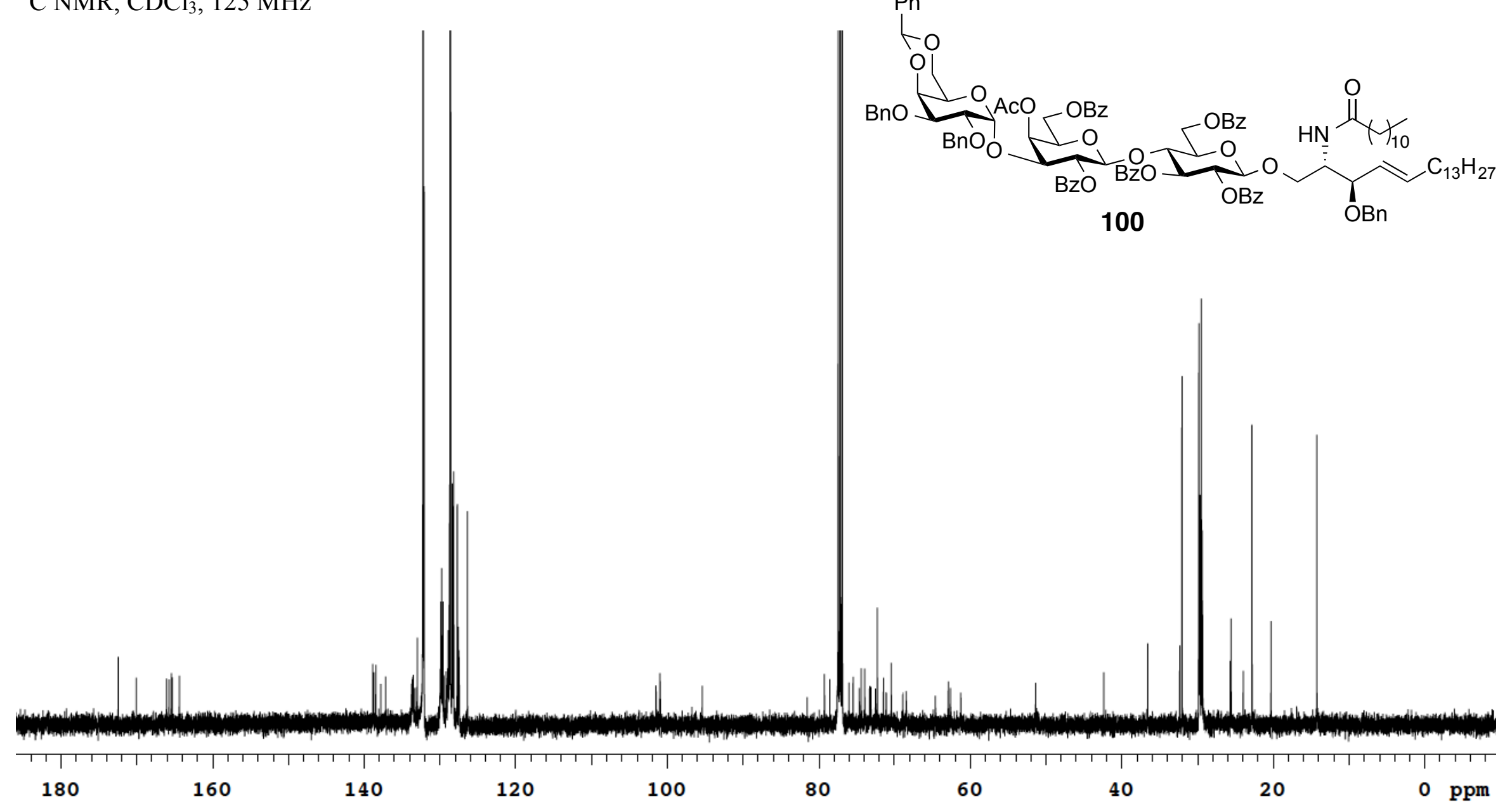


(2S,3R,4E)-2-Dodecanoylamido-1-(4- $O$-(3- $O$ - $\alpha$-D-galactopyranosyl)- $\beta$-D-galactopyranosyl)- $\beta$-D-glucopyranosyloxy)-3-hydroxyoctadec-4-ene (7) ${ }^{1} \mathrm{H}$ NMR, pyridine- $\mathrm{d}_{5}, 600 \mathrm{MHz}$

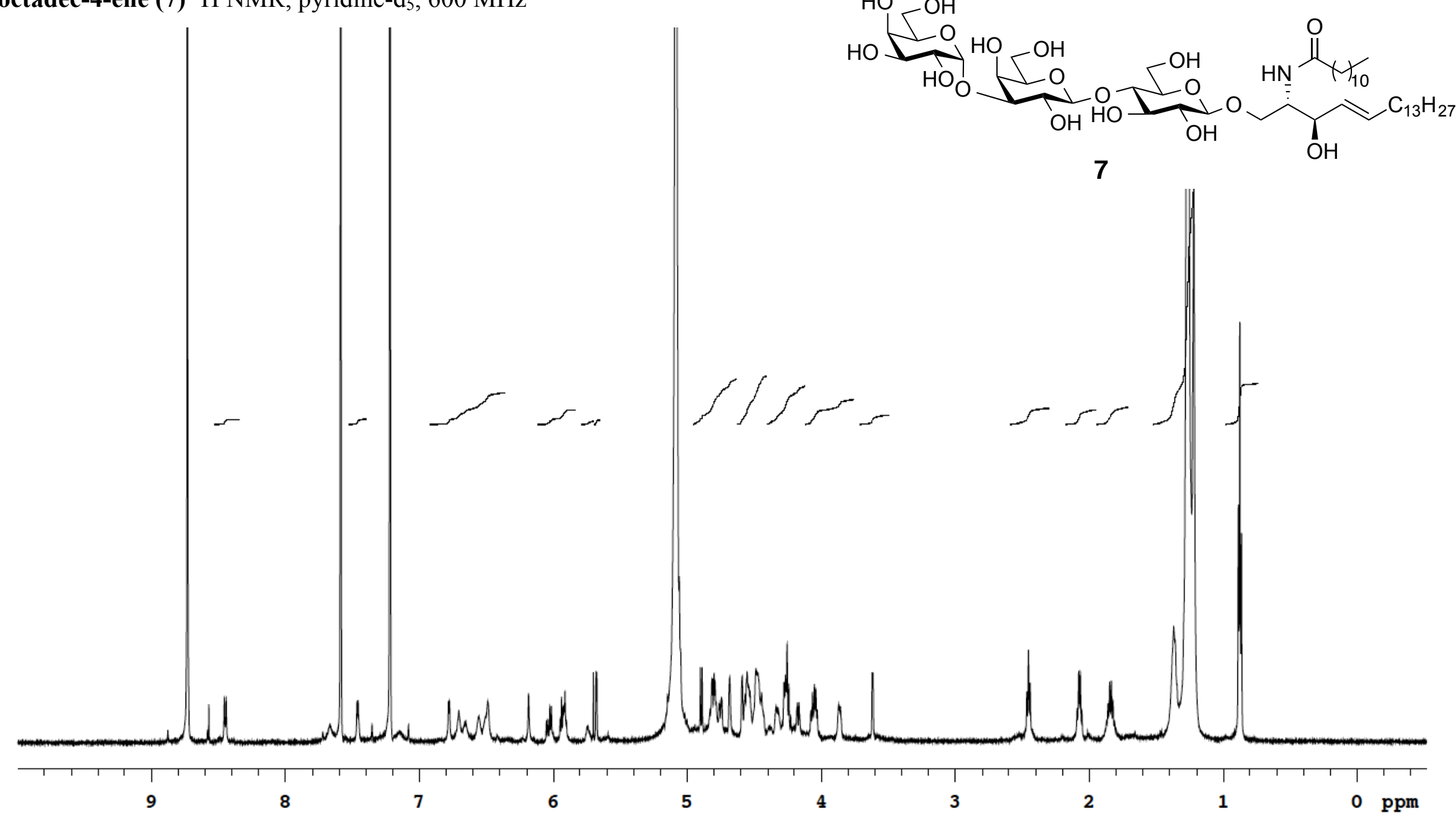


(2S,3R,4E)-2-Dodecanoylamido-1-(4-O-(3-O- $\alpha$-D-galactopyranosyl)- $\beta$-D-galactopyranosyl)- $\beta$-D-glucopyranosyloxy)-3-hydroxyoctadec-4-ene (7) ${ }^{13} \mathrm{C}$ NMR, pyridine- $\mathrm{d}_{5}, 125 \mathrm{MHz}$

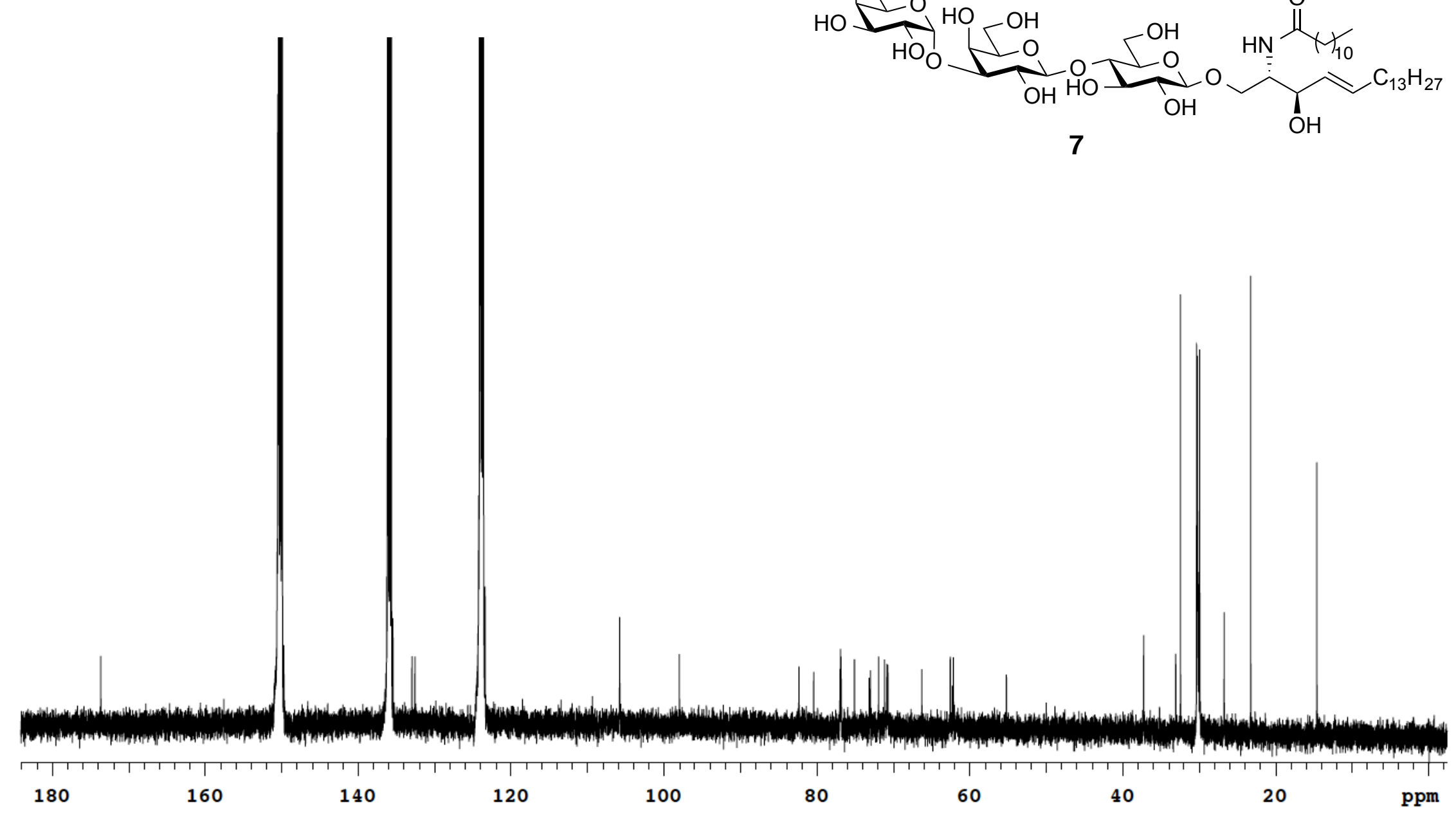


$(2 S, 3 R, 4 E)-1-(4-O-(4-O$-Acetyl-2,6-di- $O$-benzoyl-3-O-(2,3-di-O-benzyl-4,6- $O$-benzylidene- $\alpha$-D-galactopyranosyl)- $\beta$-D-

galactopyranosyl)-2,3,6-tri- $O$-benzoyl- $\beta$-D-glucopyranosyloxy)-3-benzyloxy-2-(11Z,14Z-eicosadienoylamido)-octadec-4-ene (101)

${ }^{1} \mathrm{H} \mathrm{NMR}, \mathrm{CDCl}_{3}, 500 \mathrm{MHz}$

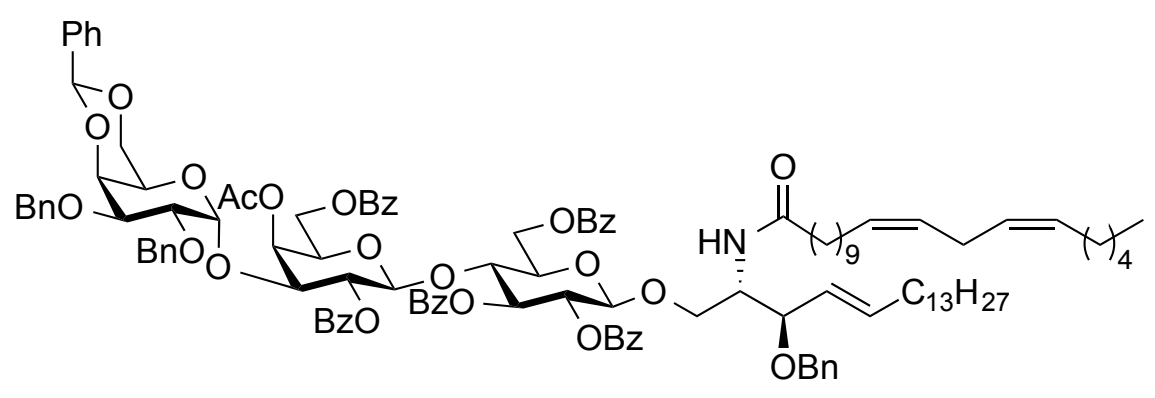

101

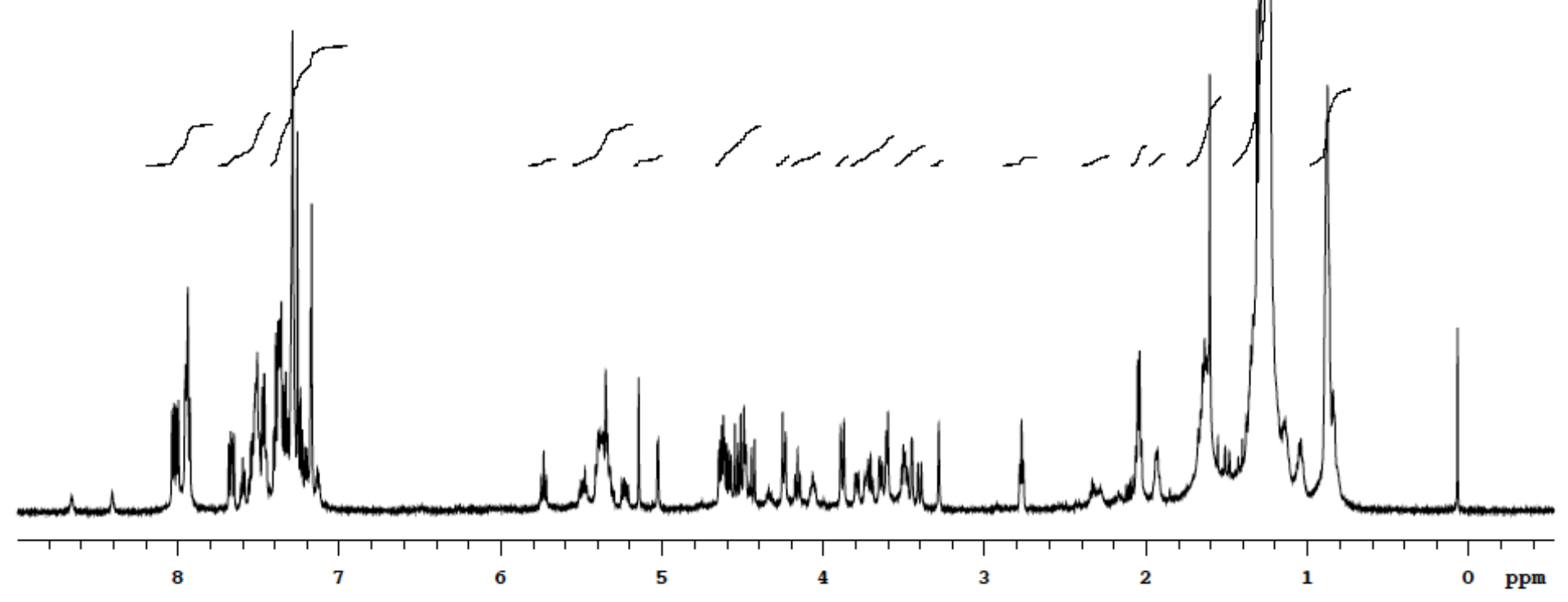

1xxx 
(2S,3R,4E)-1-(4-O-(4-O-Acetyl-2,6-di- $O$-benzoyl-3-O-(2,3-di- $O$-benzyl-4,6-O-benzylidene- $\alpha$-D-galactopyranosyl)- $\beta$-D-galactopyranosyl)2,3,6-tri- $O$-benzoyl- $\beta$-D-glucopyranosyloxy)-3-benzyloxy-2-(11Z,14Z-eicosadienoylamido)-octadec-4-ene (101)

${ }^{13} \mathrm{C} \mathrm{NMR}, \mathrm{CDCl}_{3}, 125 \mathrm{MHz}$
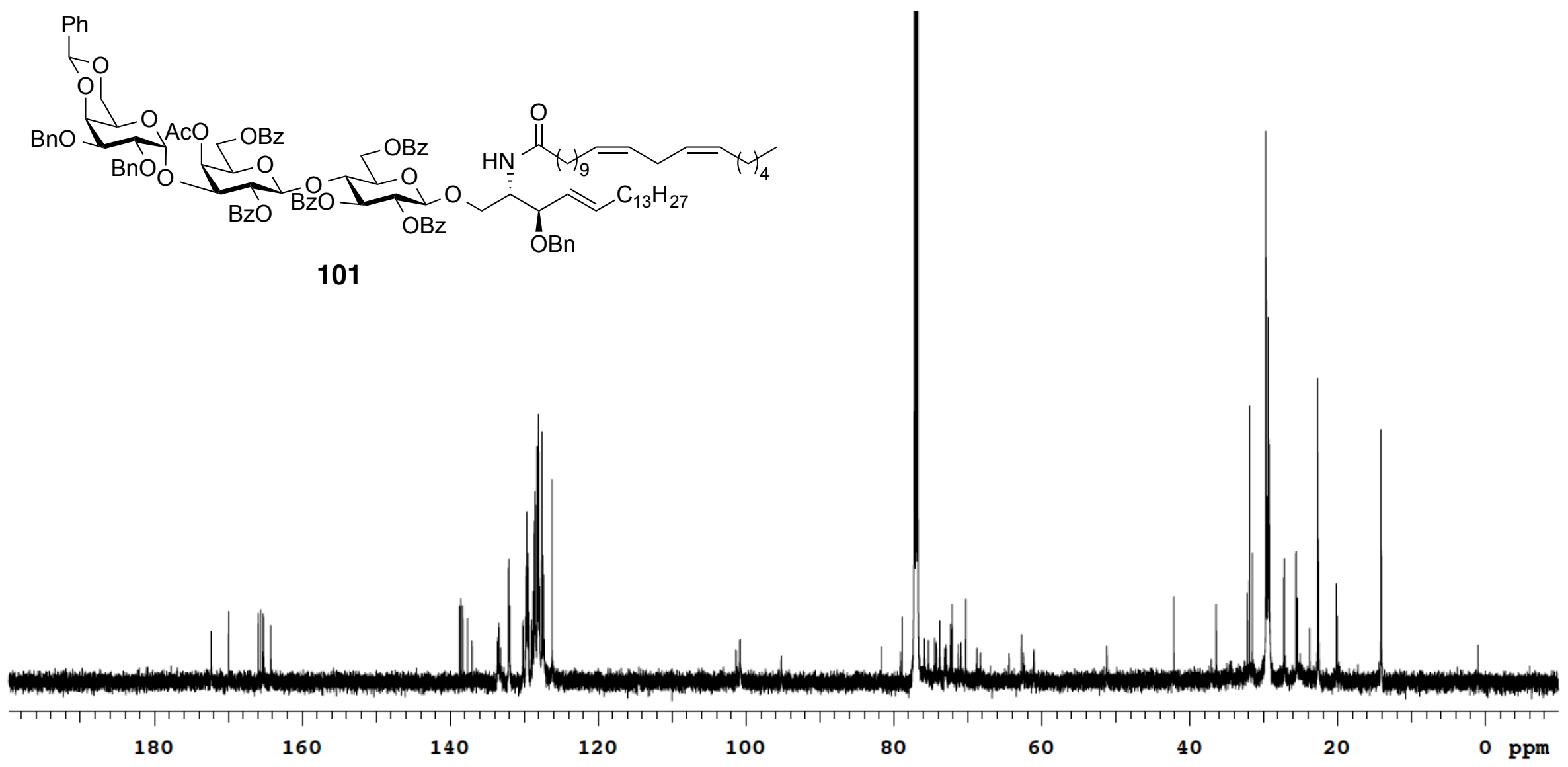
(2S,3R,4E)-2-(11Z,14Z-Eicosadienoylamido)-1-(4-O-(3-O- $\alpha$-D-galactopyranosyl)- $\beta$-D-galactopyranosyl)- $\beta$-D-glucopyranosyloxy)-3hydroxy-octadec-4-ene (8) ${ }^{1} \mathrm{H}$ NMR, pyridine- $\mathrm{d}_{5}, 600 \mathrm{MHz}$

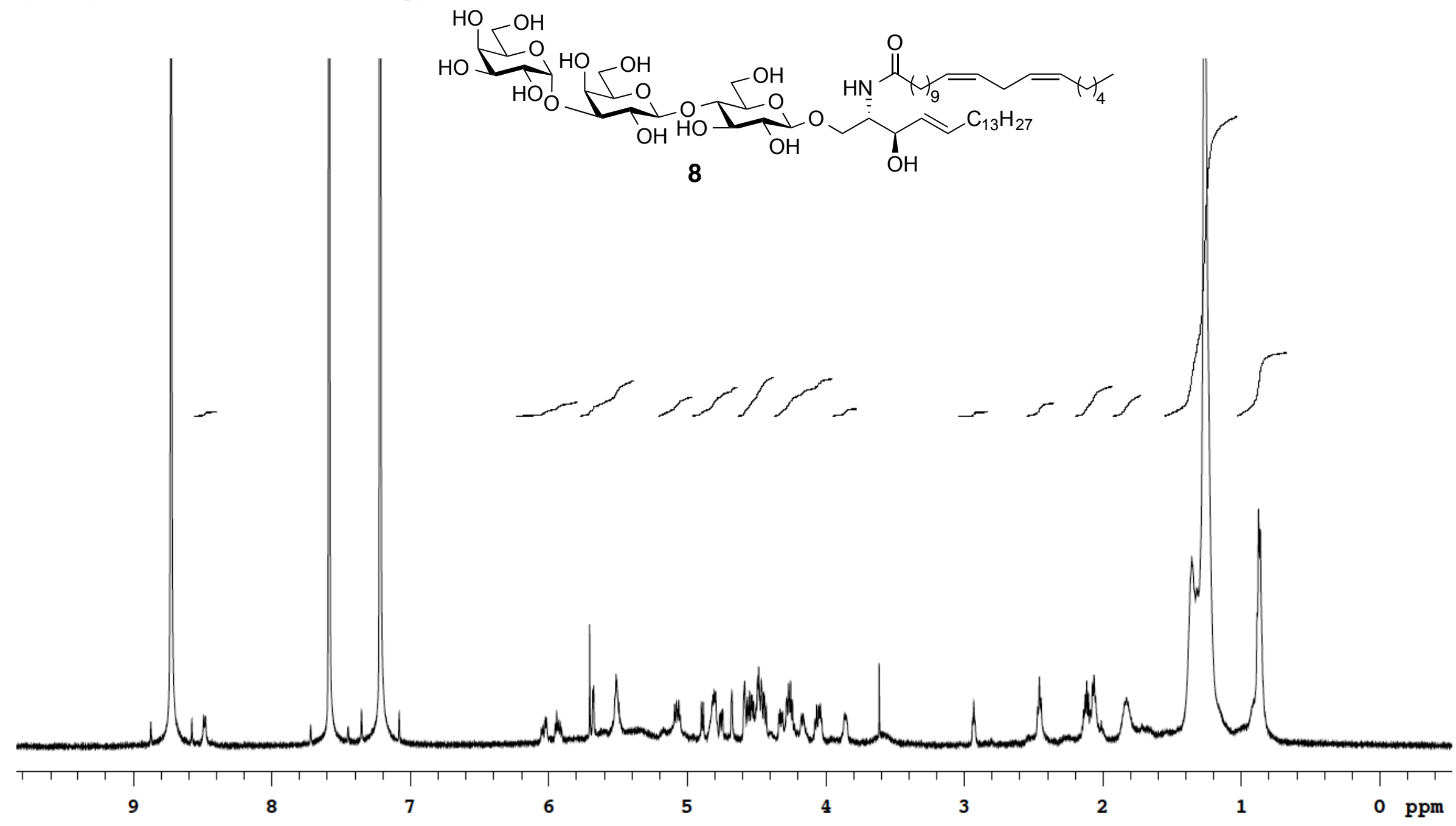


(2S,3R,4E)-2-(11Z,14Z-Eicosadienoylamido)-1-(4-O-(3-O- $\alpha$-D-galactopyranosyl)- $\beta$-D-galactopyranosyl)- $\beta$-D-glucopyranosyloxy)-3hydroxy-octadec-4-ene (8) ${ }^{13} \mathrm{C}$ NMR, pyridine- $\mathrm{d}_{5}, 150 \mathrm{MHz}$

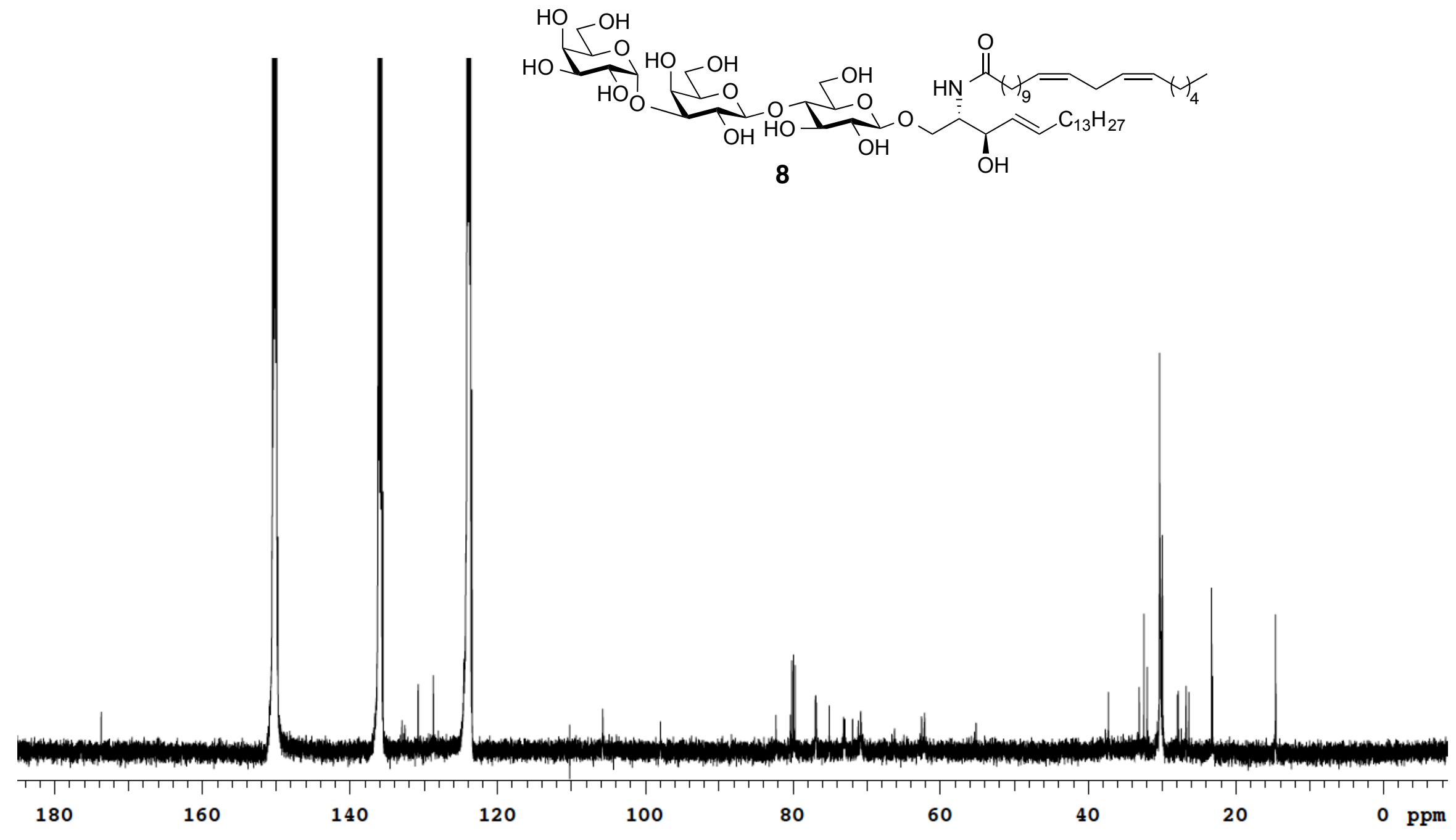

